\title{
Cost and Throughput Modeling of Manual and Automated Order Fulfillment Systems
}

\author{
Mardi L. Russell \\ Thesis submitted to the Faculty of the \\ Virginia Polytechnic Institute and State University \\ in partial fulfillment of the requirements for the degree of \\ Master of Science \\ in \\ Engineering
}

Russell D. Meller, Chair
C. Patrick Koelling
William G. Sullivan

May 11, 2001

Blacksburg, Virginia

Keywords: Order Fulfillment, Sortation Systems, Warehousing, Distribution Centers Copyright 2001, Mardi L. Russell 


\title{
Cost and Throughput Modeling of Manual and Automated Order Fulfillment Systems
}

\author{
Mardi L. Russell
}

\section{(ABSTRACT)}

The e-commerce revolution has brought about the need for more efficient order fulfillment. Guidelines are needed to determine the order fulfillment system design, and whether a manual or automated system should be implemented. A spreadsheet-based descriptive model has been developed based on demand levels, labor rates, and order sizes. We propose to combine the descriptive model with simulation output to demonstrate reactions of the system to fluctuations in demand, labor rates, and order sizes. The ultimate research goal is to provide a design aid to companies engaged in order fulfillment systems, emphasizing the decision of whether or not to automate the sorting process.

This work was supported by the National Science Foundation under Dr. Russell D. Meller's NSF CAREER Grant DMII 9623605/9996444. 


\section{Acknowledgments}

There are many people who helped me throughout my graduate studies at Virginia Tech. First and foremost, I would like to thank my advisor, Dr. Russell D. Meller. Everyone should get to have an advisor like him. Without his support and guidance, I never would have made it through this year. Many thanks to my committee members as well, Dr. Pat Koelling and Dr. Bill Sullivan.

I would also like to thank John Krakora at J. Crew in Lynchburg, Virginia and Marcy Kegley at Hanover Direct in Roanoke, Virginia for all the valuable information and tours of their distribution centers. In addition, I would like to thank Larry Smith for allowing me to perform multiple time studies at Cross Creek Apparel in Mount Airy, North Carolina.

My parents are the two most important people in my life, and without their support, longdistance calls, and several 8-hour trips, I never would have survived such a long way from home.

My friends at Virginia Tech have been extremely supportive and helpful throughout graduate school. I would like to especially thank my roommate, Katie Flahive, for listening to me complain, and Latanya Walker, for introducing me to most of my friends here and feeding me home-cooked meals each week. Most importantly, I want to thank my boyfriend, Morgan Stewart, for his support and patience with me during this time.

I would like to express my gratitude to Dr. Rich Cassady, who nudged me this way from Mississippi State and always assured me that I was smart enough to be here.

Throughout the past year or two, my fellow students in the ISE department have become 
my good friends. I want to thank Barb Fraticelli for making me feel welcome when I first started school here, helping me with many assignments, and letting me borrow her zip drive to back up my work. I also want to thank Greg Beskow for working on every project with me and for all his work with INFORMs. Finally, I would like to thank John Klote for making me look organized and being a good friend.

Finally, I would like to thank my best friends, Robin White, Janna Smith, Hillary Haynes, and Tara Cochrane. 


\section{Contents}

1 Introduction $\quad 1$

1.1 Distribution Center Description . . . . . . . . . . . . . . . 2

1.1.1 Sortation System Description . . . . . . . . . . . . . 3

1.2 Types of Sortation Systems _ . . . . . . . . . . . . . . . 5

2 Literature Review $\quad 8$

2.1 General Warehousing . . . . . . . . . . . . . . . 8

2.2 Storage . . . . . . . . . . . . . . . . . . . 11

2.2.1 Automated Storage and Retrieval Systems . . . . . . . . . . . 12

2.3 Order-picking . . . . . . . . . . . . . . . . . . . . 13

2.3.1 Order-picking Methods . . . . . . . . . . . . . . 13

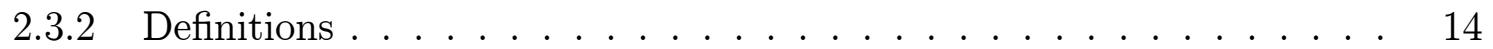

2.3.3 Routing Policies . . . . . . . . . . . . . . . . . 15

2.3.4 Storage Policies . . . . . . . . . . . . . . . 17

2.3.5 Order-Batching Policies ................. 17

2.3.6 Combined Methods of Travel Distance/Time Reduction . . . . . . . . 18

2.3.7 Comments . . . . . . . . . . . . . . . . . . . . . . 19 
2.4 Sortation Systems . . . . . . . . . . . . . . . . . . . . . . . . . . . . 19

2.4.1 Recirculating vs. Nonrecirculating Shipping Systems _ . . . . . . . 20

2.4.2 Recirculating Systems in Shipping Systems . . . . . . . . . . . . . . 21

2.4.3 Non-Recirculating Systems in Order Fulfillment . . . . . . . . . . 22

2.4 .4 Conveyor Systems . . . . . . . . . . . . . . . . . . . . . . . . 24

3 Problem Statement $\quad 26$

3.1 Tradeoffs Between Automated and Manual Sortation . . . . . . . . . . 26

3.2 Research Goals . . . . . . . . . . . . . . . . . . . . . . . . 27

3.3 Theoretical Efficiency Curve . . . . . . . . . . . . . . . . . . . . . . . . . . 29

3.4 Actual Efficiency Curve $\ldots \ldots \ldots . \ldots \ldots$

3.4 .1 Picking Efficiency . . . . . . . . . . . . . . . . . . . . . 31

$3.4 .2 \quad$ Packing Efficiency . . . . . . . . . . . . . . . . . . . 35

3.5 Forming the Efficiency Curve from Simulation Data . . . . . . . . . . . 38

3.6 Picking vs. Packing Efficiencies $\ldots \ldots \ldots \ldots$

3.7 Company Data and Modeling . . . . . . . . . . . . . . . . 43

4 Deterministic Model $\quad 45$

4.1 Fundamental Relationships . . . . . . . . . . . . . . . . . . . 46

4.1 .1 Notation and Illustrated Relationships _. . . . . . . . . . . . 46

4.1 .2 Assumptions . . . . . . . . . . . . . . . . . . . . 51

4.2 Manual Sortation System . . . . . . . . . . . . . . . . . . . 53

4.3 Automated Sortation System _ . . . . . . . . . . . . . . . 54

4.4 Example . . . . . . . . . . . . . . . . . . . 55 


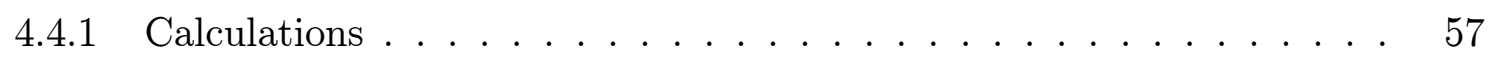

4.5 Model Experiments . . . . . . . . . . . . . . . . . . 59

5 Sensitivity Analysis $\quad 61$

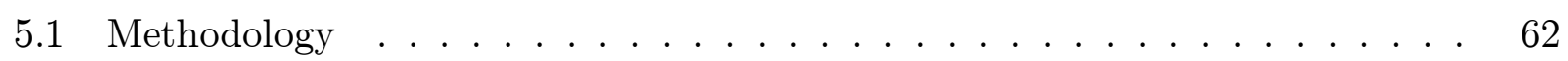

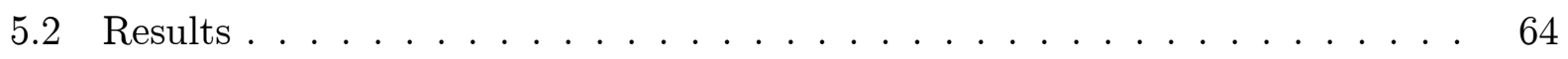

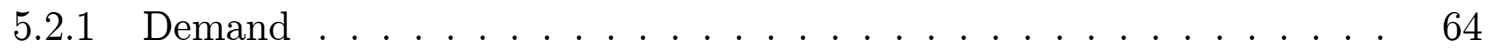

5.2 .2 Labor Costs .............................. 66

5.2.3 Pack Station Costs . . . . . . . . . . . . . . . 66

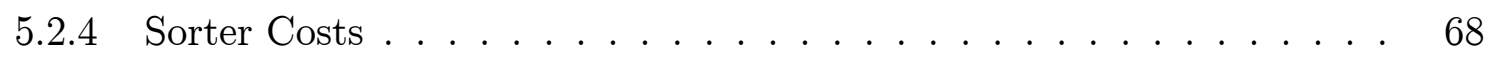

5.2 .5 Induction Rates . . . . . . . . . . . . . . . . . 69

5.2.6 Combination of Parameters ................. 69

5.3 Results for Order-Batching in a Manual System . . . . . . . . . . . . . 70

5.3.1 Points Along a Curve ................... 70

5.3.2 Approximate Model to Determine Optimal Order-Batching . . . . . . 73

5.4 Results for Order-Batching in an Automated System . . . . . . . . . . . 81

6 Throughput Simulation Results $\quad 83$

6.1 Methodology .......................... 86

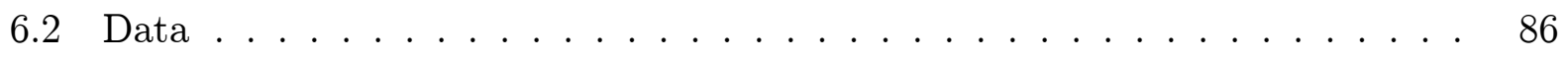

7 Conclusions and Future Research 101

7.1 Conclusions . . . . . . . . . . . . . . . . . . 101

7.2 Future Research . . . . . . . . . . . . . . . . . 102 


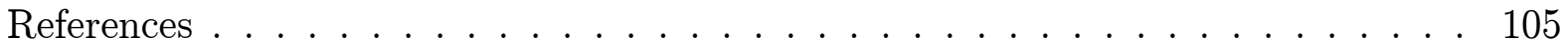

$\begin{array}{ll}\text { A Time Study Data and Results } & 108\end{array}$

B Verification Simulation Results 113

$\begin{array}{ll}\text { C Deterministic Spreadsheet Model Results } & 183\end{array}$

D Sensitivity Analysis Data and Results 185

E Proof of Result 1. 190

F Throughput Simulation Results $\quad 194$

G Adjusted Induction Model $\quad 224$ 


\section{List of Figures}

1.1 Distribution Center Flow $[40] \ldots \ldots \ldots$

1.2 Packing Sorter Installation $[1] \ldots \ldots \ldots$

1.3 Tilt-tray Sorter $[3] \ldots \ldots \ldots \ldots \ldots \ldots \ldots$

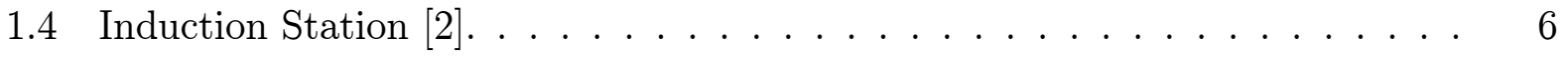

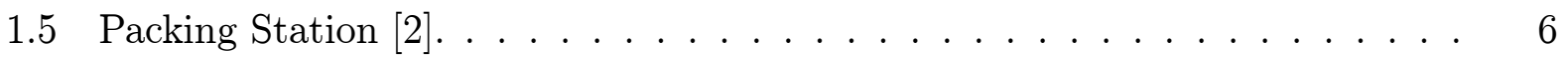

3.1 Manual Sortation System. . . . . . . . . . . . . . . . . . . . . . 28

3.2 Automated Sortation System $[27] \ldots \ldots \ldots$

3.3 Manual and Automated Efficiency Curves. . . . . . . . . . . . . . . . 30

3.4 Decreasing Automated Efficiency Curves. . . . . . . . . . . . . . . . . 31

3.5 Effects of Batching Orders on Travel Time. . . . . . . . . . . . . . 33

3.6 Picking Rates vs Batch Size. . . . . . . . . . . . . . . . . . . . . . . . 34

3.7 Number of Orders vs Total Pack Time (No Overlap). . . . . . . . . . . . 37

3.8 Number of Bins Packed. . . . . . . . . . . . . . . . . . . . . . . . 38

3.9 Packing Time Study Results. . . . . . . . . . . . . . . . . . . . . . 39

3.10 Actual Efficiency Curve with Complete Wave Overlap. . . . . . . . . . . . 41

3.11 Actual Efficiency Curve with No Wave Overlap. . . . . . . . . . . . . . . 42 
3.12 Actual Efficiency Curve with No Wave Overlap (Decrease due to Walking Times Only). . . . . . . . . . . . . . . . . . 42

4.1 Decreasing Automated Picking Efficiency Curves. . . . . . . . . . . . . 53

4.2 Example Picking vs. Packing Efficiency Curve. . . . . . . . . . . . . . . 57

5.1 Sensitivity Data at Demand $=550$ Orders/Hour. . . . . . . . . . . . 67

5.2 Sensitivity Data at Demand $=3300$ Orders/Hour. . . . . . . . . . . . 68

5.3 Estimated Picking vs. Packing Relationship. . . . . . . . . . . . . . 73

5.4 Picking Rate vs. Number of Employees. . . . . . . . . . . . . . . . 75

5.5 Case 1 - Minimum is Included in Picking Boundaries. . . . . . . . . . . . . 78

5.6 Case 2 - Minimum is Lower than Picking Boundaries. . . . . . . . . . . 78

5.7 Case 3 - Minimum is Higher than Picking Boundaries. . . . . . . . . . . 79

5.8 Example Objective Functions. . . . . . . . . . . . . . . . 80 


\section{List of Tables}

3.1 Cross Creek Picking Efficiency Data. . . . . . . . . . . . . . . . 32

3.2 Wave Length Effects on Picking Efficiency. . . . . . . . . . . . . . . 35

4.1 Sortation System Costs [4] . . . . . . . . . . . . . . . 52

4.2 Example Parameters. . . . . . . . . . . . . . . . . 56

4.3 Example Calculations for the Manual System. . . . . . . . . . . . . . 58

4.4 Example Calculations for the Automated System. . . . . . . . . . . . . . . 59

5.1 Sensitivity Analysis. . . . . . . . . . . . . . . . . 62

5.2 Sensitivity Analysis Experiments for Sorter Cost of $\$ 250,000 \ldots$. . . . . . . . 63

5.3 Example Picking and Packing Boundaries and Resulting Optimal Rates ( $m$ does not change). . . . . . . . . . . . . . . . . . 80

5.4 Example Picking and Packing Boundaries and Resulting Optimal Rates ( $m$

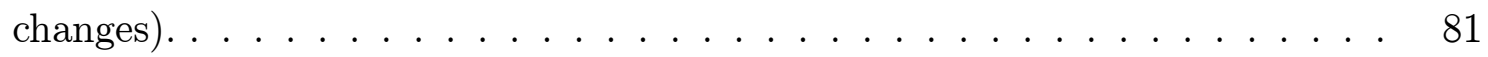

6.1 Input Data for Deterministic Spreadsheet Model. . . . . . . . . . . . . . . 87

6.2 Output Data for Deterministic Spreadsheet Model with Overlapping Waves. 88

6.3 Output Data for Deterministic Spreadsheet Model with Non-Overlapping Waves. 88

6.4 Effects of Order-Batching on Subsystem Throughput. . . . . . . . . . . . . 90 
6.5 Example Effects of the Order of Setting Subsystem Rates for Example Trial 2. 92

6.6 Output Data from Throughput Simulation Model (Overlapping Waves). . . . 94

6.7 Output Data from Throughput Simulation Model (Non-overlapping Waves). 94

6.8 Results from Throughput Simulation Model (Overlapping Waves). . . . . . . 95

6.9 Results from Throughput Simulation Model (Non-overlapping Waves). . . . 95

6.10 Comparison of Induction Results with High and Low Variation (Overlapping

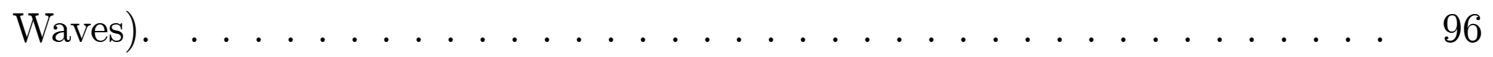

6.11 Comparison of Induction Results with High and Low Variation (Non-overlapping

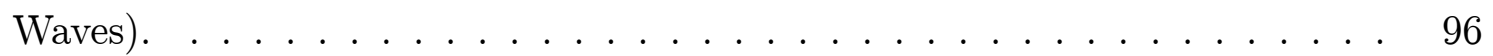

6.12 Output Data — Best Case Scenario (Overlapping Waves). . . . . . . . . . 97

6.13 Output Data — Best Case Scenario (Non-overlapping Waves). . . . . . . . 98

6.14 Results — Best Case Scenario (Overlapping Waves) . . . . . . . . . . . . . 98

6.15 Results - Best Case Scenario (Non-overlapping Waves). . . . . . . . . . . . 99

A.1 Zone Picking Time Study Data $($ Batch Size = 20) . . . . . . . . . . . . 109

A.2 Zone Picking Time Study Data $($ Batch Size = 10) . . . . . . . . . . . . . 109

A.3 Zone Picking Time Study Data $($ Batch Size = 5) . . . . . . . . . . . . 109

A.4 Zone Picking Time Study Data $($ Batch Size =1). . . . . . . . . . . . . . 110

A.5 Picking Time Study Summary. . . . . . . . . . . . . . . . . . . . . 110

A.6 Packing Time Study Data (Batch Size $=20$ Orders) . . . . . . . . . . . 111

A.7 Packing Time Study Data (Batch Size $=10$ Orders) . . . . . . . . . . . 111

A.8 Packing Time Study Data (Batch Size $=5$ Orders) . . . . . . . . . . . 111

A.9 Packing Time Study Data (Batch Size $=4$ Orders) . . . . . . . . . . . 111

A.10 Packing Time Study Data (Batch Size $=2$ Orders) . . . . . . . . . . . . 112 
A.11 Packing Time Study Summary. . . . . . . . . . . . . . . . . . . . . . 112

B.1 Simulation Data for Picking Times (Medium Rate). . . . . . . . . . . . . 114

B.2 Simulation Data for Picking Times (High Rate). . . . . . . . . . . . 132

B.3 Simulation Data for Picking Times (Low Rate). . . . . . . . . . . . . 150

B.4 Manual Packing Times and Rates. . . . . . . . . . . . . . . . . 168

B.5 Simulation Data for Automated Packing with Overlapping Waves. . . . . . . 171

B.6 Simulation Data for Automated Packing with Non-Overlapping Waves. . . . 178

B.7 Manual Picking and Packing Rate Combinations. . . . . . . . . . . . . . 182

B.8 Automated Picking and Packing Rate Combinations. . . . . . . . . . . . . 182

C.1 Automated System Deterministic Model Calculations (Demand $=1100$ orders $/$ hr, Sorter Cost $=\$ 250,000) \ldots \ldots \ldots \ldots$

C.2 Manual System Deterministic Model Calculations. . . . . . . . . . . . . . . 184

D.1 Sensitivity Analysis for Demand $=550$ orders/hour . . . . . . . . 186

D.2 Sensitivity Analysis for Demand $=1100$ orders/hour. $\ldots \ldots \ldots \ldots$

D.3 Sensitivity Analysis for Demand $=2200$ orders/hour. . . . . . . . . . 188

D.4 Sensitivity Analysis for Demand $=3300$ orders $/$ hour $\ldots \ldots \ldots \ldots$

F.1 Automated System Prescriptive Model Calculations (High Picking and High Packing Levels, Demand $=550$ orders $/$ hr, Sorter Cost $=\$ 250,000) \ldots \ldots$

F.2 Manual System Prescriptive Model Calculations (High Picking and High Packing Levels). . . . . . . . . . . . . . . . . . . 196

F.3 Automated System Prescriptive Model Calculations (High Picking and Low Packing Levels, Demand $=550$ orders $/$ hr, Sorter Cost $=\$ 1,000,000) \ldots \ldots$ 
F.4 Manual System Prescriptive Model Calculations (High Picking and Low Packing Levels). . . . . . . . . . . . . . . . . . . . . . . . 197

F.5 Automated System Prescriptive Model Calculations (Medium Picking and Medium Packing Levels, Demand $=2200$ orders $/$ hr, Sorter Cost $=\$ 250,000) .198$

F.6 Manual System Prescriptive Model Calculations (Medium Picking and Medium Packing Levels). . . . . . . . . . . . . . . . . . . . . . . . . 198

F.7 Automated System Prescriptive Model Calculations (Low Picking and High Packing Levels, Demand $=3300$ orders $/$ hr, Sorter Cost $=\$ 250,000) . \quad$. . . 199

F.8 Manual System Prescriptive Model Calculations (Low Picking and High Packing Levels). . . . . . . . . . . . . . . . . . . . . . . . . 199

F.9 Automated System Prescriptive Model Calculations (Low Picking and Low Packing Levels, Demand $=2200$ orders $/$ hr, Sorter Cost $=\$ 500,000) . \quad \ldots$. . 200

F.10 Manual System Prescriptive Model Calculations (Low Picking and Low Packing Levels). . . . . . . . . . . . . . . . . . . . . 200

F.11 Throughput Simulation Picking Times (Overlapping Waves). . . . . . . . . 201

F.12 Throughput Simulation Packing Times (Overlapping Waves). . . . . . . . . 202

F.13 Throughput Simulation Picking Times (Non-Overlapping Waves). . . . . . . 204

F.14 Throughput Simulation Packing Times (Non-Overlapping Waves). . . . . . 206

F.15 Throughput Simulation Scaled Picking Times (Overlapping Waves). . . . . . 208

F.16 Throughput Simulation Scaled Packing Times (Overlapping Waves). . . . . . 209

F.17 Throughput Simulation Scaled Picking Times (Non-Overlapping Waves). . . 211

F.18 Throughput Simulation Scaled Packing Times (Non-Overlapping Waves). . . 213

F.19 Throughput Simulation Induction Times (Overlapping Waves - c.v. =0.2). 215

F.20 Throughput Simulation Induction Times (Non-Overlapping Waves - c.v. =

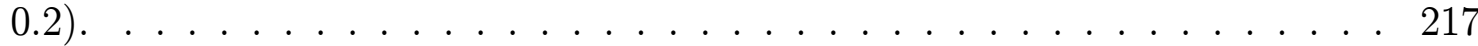


F.21 Throughput Simulation Induction Times (Overlapping Waves - c.v. = 0.4). 219

F.22 Throughput Simulation Induction Times (Non-Overlapping Waves - c.v. =

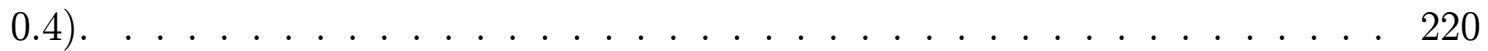

F.23 Number of Items Inducted (Overlapping Waves) . . . . . . . . . . . . . . . 223

F.24 Number of Items Inducted (Non-overlapping Waves) . . . . . . . . . . . . . . 223

G.1 Effective Induction Rates for Conveyor Speed of 100 Trays per Minute. . . . 226

G.2 Effective Induction Rates for Conveyor Speed of 150 Trays per Minute. . . . 227

G.3 Effective Induction Rates for Conveyor Speed of 200 Trays per Minute. . . . 228

G.4 Input Data for Example Experiments. . . . . . . . . . . . . . . . . 229

G.5 Induction Results from Example Experiments. . . . . . . . . . . . . . . . . 229

G.6 Inductor Throughput for Example Experiments. . . . . . . . . . . . . . . . 229

G.7 Induction Times for Example Experiments. . . . . . . . . . . . . . . . . 230 


\section{Chapter 1}

\section{Introduction}

Sortation systems are becoming more and more common in distribution centers. With the explosion of the e-commerce revolution, more emphasis is placed on customer delivery. Customers have high expectations for internet delivery. The orders are becoming smaller. Where a bricks-and-mortar company might send a case of one item to the retailer, an e-business will have many orders with one or two different items each. Customers use the World Wide Web for convenience, but are often disappointed by poor delivery performance. For instance, the Christmas season of 1999 was disastrous for many e-businesses. These companies were not prepared for the mad rush of customers to the internet. A research study of 50 e-commerce sites showed that $20 \%$ of packages ordered arrived late or never made it [38].

E-businesses soon recognized that on-time delivery could be achieved if distribution centers (DCs) became more efficient. Sortation systems are proving to be a way to achieve the desired levels of efficiency. Catalog distributors also use sortation systems as a means to sort through thousands of orders efficiently. In fact, sortation systems are becoming common in every type of fulfillment centers. 


\subsection{Distribution Center Description}

In order to fully understand the role of sortation systems in distribution centers, an overview of the entire distribution system should be understood as well. Distribution centers generally consist of five main areas: receiving, the reserve area, the forward area, packing, and shipping. Areas such as inspection, returns, and administration are part of the system as well.

In the receiving area, trucks bring shipments in to be unloaded and placed in the reserve area. Paperwork is completed, and samples are removed for inspection. Cases of items are then placed in storage locations in the reserve area. In this area, different types of storage assignment policies are used, including dedicated storage, random storage, and class-based storage. Items remain in the reserve area until they are needed in the picking or forward area.

The forward area is the storage area where cases are opened and items are removed as needed. Pickers receive a list of orders to be picked in a certain time period (wave). They travel up and down aisles, picking items from cases as needed. As in the reserve area, several types of picking configurations can be seen. In addition, pickers use many differing methods to collect items for orders. There are three main types of order-picking: zone picking, batch picking, and picking single orders to totes. Items travel from the picking area to the packing area via conveyors or totes.

Sortation occurs at this stage in the distribution process. The amount of sortation required depends heavily on the type of picking used. For instance, batch picking requires much more sortation than single-order picking. Once sorted, the orders are packaged and sent to the shipping area. In this area, packages are separated into trucks for final distribution (e.g., UPS, RPS, USPS, common carrier, etc.). Figure 1.1 illustrates the flow of a distribution center. 


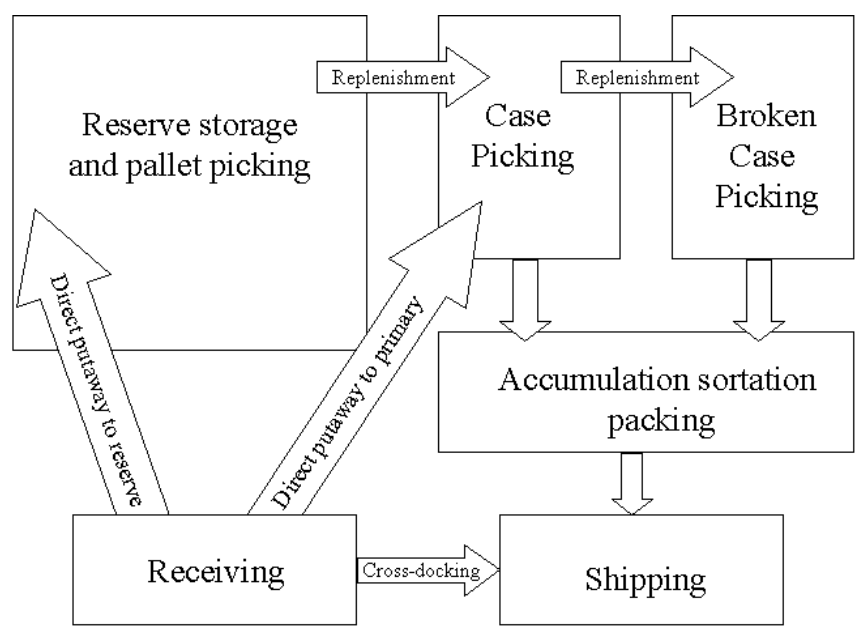

Figure 1.1: Distribution Center Flow [40].

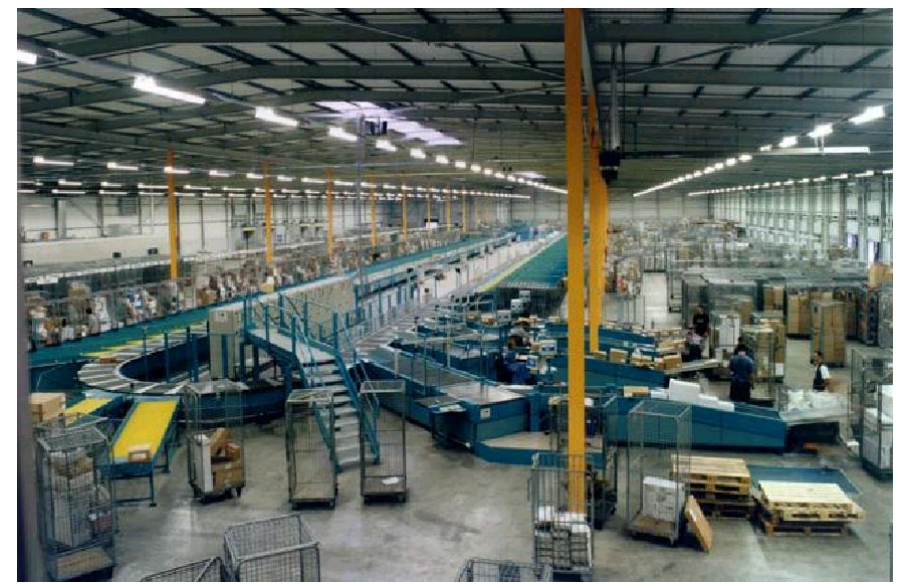

Figure 1.2: Packing Sorter Installation [1].

\subsubsection{Sortation System Description}

Sortation systems are conveyor systems that circulate around packing areas. Items arriving from the picking area of the DC are inducted onto the conveyor and then sorted to the correct packing station based on their respective order number. The drop mechanism is instructed to discharge the item to a particular packing station by a computer system. Sorters of this type can sort between 100 and 400 items per minute, depending on their size and conveyor speed. Figure 1.2 shows the installation of a packing sorter.

Now that companies realize that sortation systems can be the answer to their efficiency problems, several questions should be answered. Sortation systems are large and expensive, 
and may not be feasible for the company considering it. The first consideration the company must make is the feasibility of the system. The size of the required system, as well as the number of employees needed to operate the system must be considered. This requirement can help determine if automated sortation will be more cost effective than manual sortation. After determining if the system should be implemented, smaller decisions such as the number of orders filled per wave must be answered.

\section{Wave Size}

The common method of dividing orders within a distribution system is to utilize waves. A wave is basically a period of time in which a group of orders is either picked, sorted, or packed. A wave can range in length from 20 minutes to 2 hours, depending on the capacity of the sorter, the number of employees, and the volume of products. Waves are used to balance the workload in the picking and packing areas as well as to synchronize these activities [7]. Workers only work on the orders assigned to that particular wave.

Wave length is an important factor in distribution system design. Studies have been conducted to determine when the next wave should be released into the system and how this affects the throughput of the system. Varying wave lengths can have an effect on picker's and packer's efficiency, as well as the size of the sorter that would be required. In this research, we focus on the effects of wave length on system cost.

Setting the wave length can have a significant effect on a system configuration. For instance, a short wave length results in less efficient pickers, since the number of orders that can be batched is smaller. However, a packer may be more efficient in the manual system since he is receiving fewer orders. A long wave length allows for more intelligent batching, therefore increasing the pickers' efficiency. Longer wave lengths also result in larger sorters, which effects the capital costs of the system. 


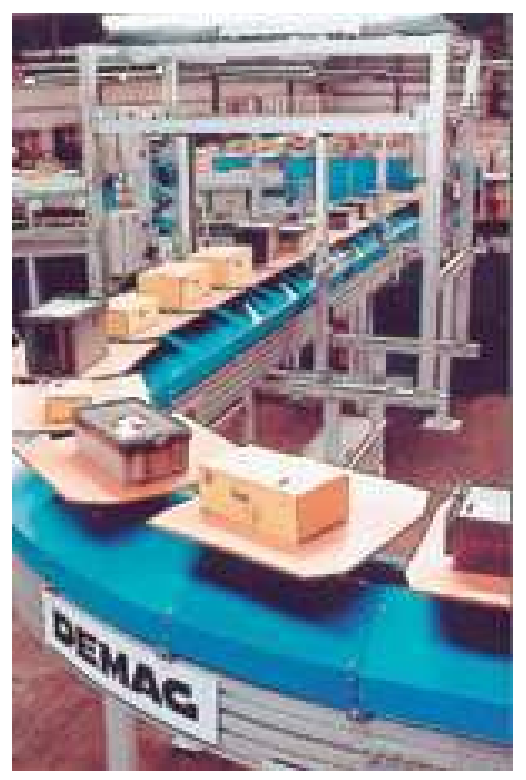

Figure 1.3: Tilt-tray Sorter [3].

\section{$1.2 \quad$ Types of Sortation Systems}

There are several types of sortation systems, each based on their configuration and uses. The most common are tilt-tray sorters, bomb-bay sorters, and cross-belt sorters. A company will select one of these types of sorters based mainly on the nature of the product needing sortation. For instance, a product such as a comforter can handle a drop from a tilt-tray or bomb-bay sorter, while a cross-belt sorter would be best suited to handle items that are more fragile, such as a video tape or compact disc. A tilt-tray sorter is shown in Figure 1.3. Each automated sortation system consists of three parts: the conveyor (including trays or belts), the induction area, and the packing area. The induction station includes a chute, conveyor, or staging area for parts arriving from picking and a platform for the workers. Sortation systems can have one induction station or split stations to increase productivity. At the induction station, items arrive from the picking area, and inductors place items onto the trays so that the scanner can read the item information from its label. The scanner then sends this information to the computer. Induction stations and packing stations are shown in Figures 1.4 and 1.5, respectively.

The part then travels on the conveyor to the pack station designated by the central computer 


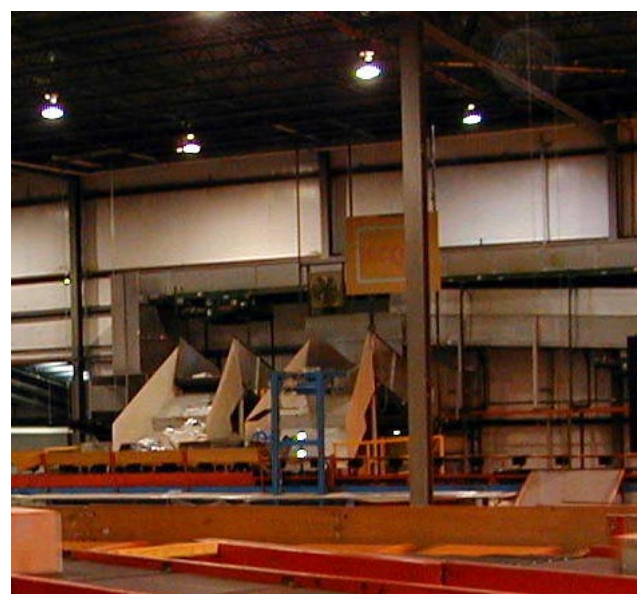

Figure 1.4: Induction Station [2].

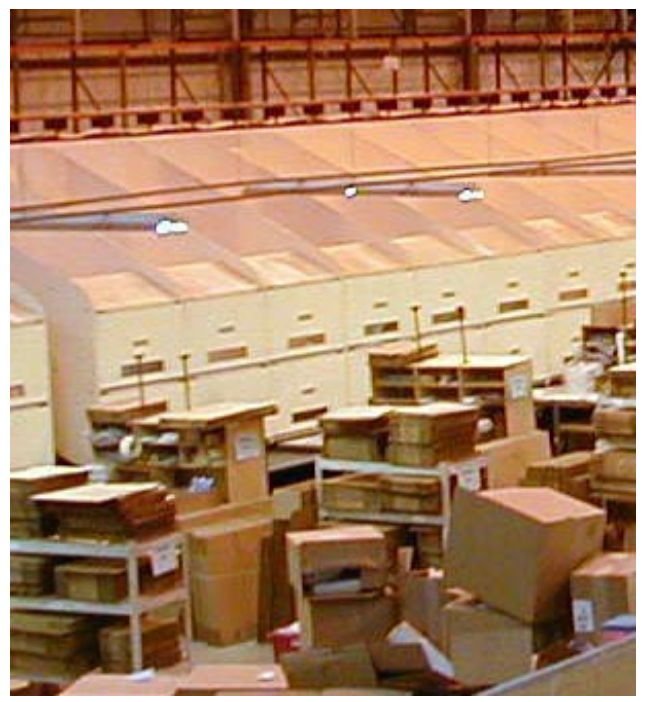

Figure 1.5: Packing Station [2].

system. As the item arrives to the pack station, it is discharged to a chute leading to the packing work area. There are generally several chutes coming into one pack station. Each chute can hold several orders, or a single order depending on how the system is configured. From this area, the parts are packed by order and the packaged orders are sent to the next processing area (typically shipping).

Automated sortation systems can be used for several purposes. The most obvious use is sorting items to be packaged. However, many companies use the system to sort returned items back into picking zones or rows. Another common use is sorting packages to be shipped. 
Automated sortation systems can either be designed to accommodate recirculation or not. Recirculating systems allow items to travel around the conveyor several times to allow sortation lanes to accommodate more than one order per sorting wave. This type of system is commonly used in shipping areas. In a recirculating system, the number of lanes is typically less than the number of orders. Recirculation is therefore necessary since each incoming item will not find an available lane. Another reason for using a recirculating system is to use the loop to accumulate all items in an order before the order is assigned to a lane.

The focus of this research is on non-recirculating systems (NRS) used in packing areas. In this type of system, the sortation conveyor is empty before reaching the induction station again since each sortation lane accommodates only one order per sorting wave.

In Chapter 2, literature related to distribution systems and sortation issues is discussed. This chapter provides a general overview of distribution research as well as specific research related to automated sortation. 


\section{Chapter 2}

\section{Literature Review}

Because literature concerning sortation systems is somewhat limited, an overview of warehousing and distribution systems research will be reviewed. This list includes general warehousing issues, storage policies, automated storage/retrieval systems (AS/RS), order-picking, sortation systems, and conveyor systems.

This overview will provide background and insights into research that has taken place up to this point, and will lead to the need for research into sortation system design concerns.

\subsection{General Warehousing}

According to Cormier and Gunn [11] there are three major warehousing problems: throughput capacity, storage capacity, and warehouse design. Much effort has been placed on maximizing throughput. Batching and picking policies, as well as storage assignment policies, have been aimed at this objective. Storage capacity models, on the other hand, have been developed with the objective of minimizing total discounted costs. Other objectives in these models include minimizing material handling costs, inventory costs, and holding costs.

Cormier and Gunn [11] review research relevant to each of these warehousing problems. They conclude that the model used in the decision-making process should be determined 
based on the type of decision that is being made. For instance, a strategic decision that will have a long-term impact, such as storage capacity design, should use an optimal model if possible. Although models that yield optimal results are more complex and difficult to solve, the effort is justifiable based on the importance of the decision. In contrast, an operational decision such as assignment of products to storage areas may be more suitable for a good, fast decision from a heuristic.

Gray et al. [24] developed a method of warehouse design using a hierarchical approach. Their research studies the entire design and operation of the system. Decisions such as layout, equipment, storage, order-picking, and technology are considered. Since the entire design is too complex to be determined by a single model, the method used is a hierarchical decision structure. The main decision levels include facility design and technology selection, item allocation, and operating policies. Within these levels are smaller decisions such as assigning items to zones, order batch sizes, and other similar decisions. The solution to this problem is found by iterating between the three main levels until a solution is found that minimized the total cost. This decision structure considers the interaction and fundamental relationships between each decision as well as the individual decisions. The authors propose using the analytical models within each level of detail to eliminate poor solutions. Once this is completed, simulation can be used to examine the subset of solutions reasonably.

Cormier and Gunn [10] developed a discounted cost model that establishes a warehouse size and inventory policy. Under the assumption of constant demand, this model integrates the two factors to determine a design that is optimal for both. The model resulting from the coordination of warehouse sizing issues with inventory policy is best applied at the conceptual stage of design, mainly due to the simplifying assumptions made.

Distribution design can also involve determining the optimal number of distribution centers for a particular company. Erlebacher and Meller [19] developed a model that solves a location-inventory problem with a large number of customers. This model will determine the optimal number and locations of DCs, and which customers should be served by each DC based on inventory costs as well as transportation costs. Because of the difficulty of this problem, a heuristic procedure was developed to solve the problem. 
Recently, van den Berg completed a literature review survey of warehousing systems [41]. This review summarizes new methods, technologies, and models used to deal with customers that are requesting smaller orders and shorter response times. In order to improve accuracy and quality, warehouse operations must be improved to respond to these changes. Literature related to the planning and control of warehousing systems was reviewed.

Planning of warehouse operations consists mainly of storage location policies. Decisions related to storage location include how products are distributed throughout the warehouse, what products are clustered, how products should be stored in order to balance workload, and the assignment of products to locations. Distribution of products into order-picking areas and reserve areas, as well as order-picking methods in these areas has been studied. Correlated products that are often ordered together can be clustered in order to reduce travel time. Research regarding the proper amount of clustering and its effects has been conducted. Storage assignment policies that have been developed will be discussed in a later section of this review.

Control of warehouse operations include batching of orders as well as order-picking routing and sequencing. Batching orders reduces the average travel time per order. Routing and sequencing orders in an intelligent manner can also reduce the travel time required to pick orders. Methods of batching, routing, and sequencing orders will be discussed in the review of order-picking techniques.

Many methods used to reduce travel time and increase throughput have been discussed in this literature review. However, little research has been completed concerning the use of automated sortation systems to increase throughput. Automated sortation systems allow the use of more sophisticated order-picking techniques that have been proven to reduce travel time required to pick an order. Although research regarding automated sortation systems exists, to our knowledge no research has shown when this type of system should be implemented based on throughput and associated costs. 


\subsection{Storage}

Warehouse objectives include maximizing space and minimizing travel time required for order-picking operations. Storage policies can have a significant effect on these objectives. There are three main storage policies commonly used in practice: randomized storage, dedicated storage, and class-based storage. With randomized storage, parts are placed in a location based on available storage space at the time of storage. Over time, even if parts are assigned to the closest open location, the distribution of parts over the warehouse appears random [6]. With dedicated storage, parts are assigned a particular location in the warehouse, where each location is dedicated to one part. Class-based storage assigns locations to parts based on a class of items with similar properties (e.g., demand, product type, and size).

Although dedicated and class-based storage policies can reduce material handling distances by locating fast movers at locations close to the input/output point, a tradeoff must be made between this reduction and the amount of storage space required for these policies. With randomized storage, items can be placed in any available location. In dedicated and class-based storage, a certain amount of excess storage space has to account for variations in demand. Randomized storage requires a much smaller amount of excess space than either dedicated or class-based storage. Malmborg [29] developed a model that determines the space requirements for a randomized storage system based on a probability distribution based on item activity levels. This model allows for a detailed analysis of the tradeoffs between using randomized or dedicated storage policies. An application of this model for a 25-aisle storage system resulted in lower average retrieval costs for randomized storage in certain scenarios. With randomized storage, this analysis also resulted in space requirement savings of an average of $30 \%$ of that required using dedicated storage [29].

Optimal arrangement of stock in the order-picking area depends on several factors: size requirements, correlated products, demand frequency, and type of order-picking used. Elsayed [17] examined four heuristic algorithms for order-picking using an automated storage and retrieval system. Each algorithm seeks to minimize material handling distance in a picking 
tour. This problem is harder since the order-picker must perform a tour instead of an outand-back trip as in the reserve area. Storage in order-picking areas will be discussed further in the review of order-picking.

Another method of storage is COI-based storage assignment. Cube-per-order index (COI) is the ratio of the amount of storage space required for a particular SKU to the order frequency [9]. In this storage assignment policy, items with low COI ratios are stored closer to the input/output point than items with high COIs. The effects of this storage assignment policy with different routing methods will be discussed in the order-picking review.

Larson et al. [28] developed a heuristic approach to warehouse layout in a facility with class-based storage. This heuristic consists of three phases: Phase 1 (determination of aisle layout and dimensions), Phase 2 (assignment of material to a storage medium such as a flow rack in the forward area or the reserve area), and Phase 3 (allocation of floor space). Floor space is allocated based on the storage medium. The use of a class-based storage policy provides flexibility to deal with demand fluctuations, and allows for decreased travel time and material handling costs without leaving as much excess storage space as a dedicated storage policy requires. Significant reductions in material handling distances are a result of using this heuristic warehouse layout approach.

\subsubsection{Automated Storage and Retrieval Systems}

An automated storage and retrieval system (AS/RS) is a storage system that is guided by a set of rails on which machines move to and from storage racks on a fixed path [40]. AS/RS can perform single or dual command operations. In a single command operation, a location is visited and either a pickup or a delivery is executed. In a dual command operation, both a pickup and a delivery are executed in the same cycle. AS/RS cranes are unique because they can travel horizontally and vertically simultaneously. This can help to reduce material handling times, especially in very high racks.

Hausman, Schwarz, and Graves [25] studied optimal storage assignment policies with automated warehousing systems such as AS/RSs. They considered random assignment, turnover- 
based assignment, and class-based turnover assignment. Analytical expressions were developed to determine the travel time for each system. It was found that turnover-based assignment results in significant reductions in travel times in an AS/RS. Elsayed [17] developed four algorithms (discussed further in order-picking review) that seeked to minimize total costs of picking orders within a warehouse that utilizes an AS/RS.

Bozer and White [6] developed design algorithms to determine the near-minimum number of orderpickers for several system configurations. Each configuration is some version of an end-of-aisle AS/RS. Configurations include one aisle per picker or multiple pickers per aisle. The algorithm attempts to minimize the number of pickers subject to system throughput, storage capacity, and constraints on resources that must take on integer values. This design algorithm can be used to evaluate system configurations when used with a cost model.

Meller and Mungwattana [33] developed analytical models to determine the throughput of multi-shuttle AS/RSs. Multi-shuttle systems increase throughput, resulting in cost savings up to a point where the capital investment exceeds this savings. In addition, several operating policies were modeled to evaluate the performance of the policy. For instance, the nearest neighbor policy and reverse nearest neighbor policies were examined. Each reduced the travel time between storage and retrieval locations. By increasing the number of commands per cycle and using intelligent storage and retrieval strategies, significant increases in throughput were seen over single-shuttle AS/RSs.

\subsection{Order-picking}

\subsubsection{Order-picking Methods}

Order-picking is the most time consuming activity that takes place in the distribution process. According to Drury [16], it may account for up to $60 \%$ of all labor activities. This activity is also quite costly, making up approximately $65 \%$ of the warehouse's total operating cost [12]. Because of these alarming statistics, order-picking has become a popular research field. The majority of this research focuses on minimizing the total travel distance 
in a picking tour, or a set of orders picked at a particular time. Many methods of reducing this distance have been examined. Some methods involve sequencing the pick tour in order to provide a tour with the shortest possible distance. Others have attempted to solve this problem by optimally storing items in the picking area. Finally, methods of order-batching are used to develop efficient pick lists.

\subsubsection{Definitions}

In order to fully understand the research encompassing order-picking strategies, several terms should be defined.

Traversal: Aisle travel policy that requires the picker to enter the aisle at one end, pick all items from that aisle, and exit the other end without reversing.

Return: Aisle travel policy that allows the picker to enter and leave the aisle at the same end.

Largest Gap: Aisle travel policy where pickers use a return policy, but in the first and last aisles entered, the picker travels to the position of the largest gap between the two adjacent locations in the aisle [15].

I/O Point: Area in the warehouse where pickers receive picking lists and drop off completed orders (see Central Depot).

Central Depot: Specified location in a picking area where pickers pick up order lists and drop off completed orders.

Picking Tour: The sequence of locations at which items are picked to fill a specified number of orders.

Order-picking By Order: Method where pickers in the warehouse pick one order at a time.

Batch Picking: Method where pickers in the warehouse pick items needed for several orders, accumulating the orders with other pickers after all items have been picked. 
Sort-While Pick: Method where pickers retrieve items needed several orders and sort items into orders as they are picking.

\subsubsection{Routing Policies}

Routing policies determine the sequence used to pick parts to complete orders on a pick list. Several routing policies have been developed over the years. However, many of these policies are not commonly used in practice because they are not well known. Policies have been examined and evaluated for between-aisle travel and within-aisle travel.

There are two basic routing policies: the traversal policy and the return policy. Goetschalckx and Ratliff [22] developed an optimum traversal aisle model for picking within the aisle. This model was compared to the routing policy used commonly in practice, the ' $\mathrm{Z}$ ' pick policy. This policy requires pickers to visit slots within aisles in a Z-pattern, stopping at only the locations of items on the pick list. The ' $\mathrm{Z}$ ' policy is commonly used because only one picking pattern is required for every pick tour. In order to compare the two policies, the authors determined the length of the optimal Z-pattern tour, which is normally determined arbitrarily (i.e., non-optimally). The Z-pattern tour was outperformed by the optimum traversal aisle model by an average of $12 \%$ [22]. The optimum traversal aisle model was also studied with the all return model. While both models using optimum traversal instead of ' $\mathrm{Z}$ ' pick were superior, the traversal policy has a shorter pick tour in all practical situations.

Caron et al. [9] combined a COI-based storage policy with different routing policies. This storage policy assigns items with a low ratio of required storage space to order frequency close to the I/O point. COI-based storage and random storage are combined with traversal and return routing policies. With COI-based storage, traversal routing policies generally outperform the return policy. Return policies outperform traversal policies in situations when the number of picks per aisle is low or there are many picks in few aisles (skewed inventory curves). With random storage, traversal is at least as good as return. The authors concluded that the average number of picks in an aisle is a key factor in determining the best routing policy with COI-based storage. 
Ratliff and Rosenthal [39] developed an algorithm to determine the optimal picking route that minimizes the picking time. Their method uses graph theory to find a minimum length tour subgraph from a graph with vertices representing each pick location and aisle end, and edges representing distances between each vertex. For a rectangular warehouse, the runtime of this method increases linearly in the number of aisles.

Routing orderpickers was again studied by DeKoster and Van Der Poort [14] in order to compare an extended version of Ratliff and Rosenthal's optimal order-picking route with a commonly used S-shape heuristic. The algorithm developed by Ratliff and Rosenthal was extended to the case with a central depot. The authors determined that the major factors affecting the performance of the routing policy are the savings in time traveling in and out of aisles, and the warehouse layout. The layout can affect the time needed to enter an aisle. For instance, a wide aisle usually means an order-picking truck will travel in and out, and saving trips in and out of the aisle can considerably reduce the travel time. The optimal order-picking model developed by DeKoster and Van Der Poort outperforms the S-shape heuristic. When the number of stops per aisle is larger, the heuristic is only slightly worse than the optimal policy.

Another strategy being used to minimize travel distances or times for picking tours is the addition of cross aisles [14]. Cross aisles may be added in order to provide intermediate entry and exits points in an aisle. Cross aisles provide flexibility in the picking route, as well as shorter travel distance opportunities. The authors warn against adding cross aisles excessively, as this may actually increase travel time due to walking across extra aisles. Additional costs such as the cost of increasing the facility size or lost storage space may make this option unattractive. Their experimentation showed that the addition of cross aisles reduced the tour distance by up to $30 \%$ [14]. In fact, the only case shown not to reduce this distance is one in which only one pick per aisle is required. 


\subsubsection{Storage Policies}

Efficient product layout is key in the reduction of travel time in pick tours. Jarvis and McDowell [26] take a different approach to the order-picking efficiency problem by attempting to optimally locate the items as opposed to developing an optimal picking tour. Results showed that the shape of the inventory curve has the greatest impact on the time and distance required to complete the pick tour. For instance, if the curve were skewed so that one product makes up a large proportion of the orders to be picked, fewer aisles must be traversed.

\subsubsection{Order-Batching Policies}

Order-batching is another method of reducing travel time in picking operations. According to DeKoster et al. [15], many companies batch orders according to a first-come, first-serve (FCFS) policy. This policy clusters orders as they arrive to the picking area. They argue that a more intelligent method of order-batching will result in significant reductions in travel distances. Several heuristic algorithms, including seed algorithms and savings algorithms were tested and compared with the FCFS policy of order-batching. The authors developed robust algorithms that did not depend on the type of order, size of order, material handling systems, or capacity of the warehouse.

According to De Koster et al. [15], seed algorithms begin by selecting a seed order, and adding consecutive orders until the picker's capacity is full. Orders are added based on either the minimum distance from the first order, the number of aisles to be visited, or the order that will save the most time when compared to picking the same orders separately. Savings algorithms base the orders added to the batch by the amount of time savings resulting from batching those orders. Using both S-shape and largest gap routing rules, the different algorithms were compared and evaluated based on total average travel time [15].

The results [15] from extensive simulation experimentation indicate savings algorithms proved to be superior when the number of orders per batch is small or the largest gap routing rule is used. Seed algorithms are superior when the S-shape strategy is used. Seed algorithms 
should also be used if there are many orders in the batch, simply due to the amount of calculations that must be made.

Gibson and Sharp [21] also present order-batching procedures based on heuristic algorithms. They used spacefilling curves (SFC) to batch orders. This method compares the closeness of each item in an order to all other items in the order. Each item is assigned a rank, and using the minimum and maximum item rank in an order, all orders are compared in the same way. The results of the order comparison determine the rank of the entire order. Once the orders are ranked, a FCFS method is used to batch the orders on the sorted list. This method completes a batch by taking orders off the sorted list until the desired batch size has been reached.

Another method Gibson and Sharp developed was the Sequential Minimum Distance (SMD) batching heuristic [21]. This method uses a greedy algorithm to place orders in batches. Orders are placed by the distance from the seed order to each of the other orders. Many factors were manipulated to determine their effects on the algorithms (FCFS, SFC, and SMD). The FCFS method remained fairly consistent regardless of most factors. However, skewed item location did significantly reduce the tour lengths. This effect was even more noticeable with SFC and SMD (up to a $44 \%$ reduction). Results in [21] showed that the SFC method is preferable with Euclidean, rectilinear, and Chebyshev metrics, but SMD is superior when using the aisle metric to measure the distance between orders.

\subsubsection{Combined Methods of Travel Distance/Time Reduction}

It is not uncommon to combine a variety of methods commonly used to reduce travel distance in picking operations. As mentioned earlier, COI-based storage combined with intelligent routing policies was proven to reduce travel distance even more so than with using either method individually [9]. Also, within aisle and overall routing algorithms, such as optimum aisle traversal using an all traversal or return routing policy, have been combined to reduce distances in routing sequences.

Finally, Daniels et al. [13] used a method of inventory assignment in conjunction with picking 
sequences to determine ways to reduce travel distances. The inventory assignment method the authors developed takes advantage of new technology as a way to locate inventory. Computer tracking of inventory allows for storage of items in multiple locations, therefore giving the sequencing algorithm more choices of locations from which to select items. The Traveling Salesman Problem formulation is modified to include the decision of which inventory location or locations should be visited in the tour. Several order-picking methods were modified, including the Modified Nearest Neighbor method, the Shortest Arc method, Randomized Construction, and Tabu Search. In each method, the sequence is determined by the lowest cost based on travel time or distance to the inventory location. At each move, the computer updates the inventory to reflect the quantity retrieved.

\subsubsection{Comments}

Order-picking methods can have a significant impact on the need for sortation. Although the improved routing of orderpickers can provide substantial gains in efficiency, routing policies may not be the area with the largest potential gains. The area with the largest potential gains may be using order-batching methods. If no batching of orders is performed, a worker will have to make a stop at a location to pick an item each time that item occurs in the separate orders. However, if batch picking is used with large numbers of orders, substantial gains in picking will result since multiple visits to the same location over multiple orders will be batched into one visit. With batching, sortation systems will be required in order to achieve the desired throughput. While sortation systems are large and expensive, the increased throughput often justifies the purchase of such a system. We now review research on sortation systems.

\subsection{Sortation Systems}

As mentioned earlier, only a small amount of research has been published concerning sortation systems. This section reviews the work that has been completed to date. 


\subsubsection{Recirculating vs. Nonrecirculating Shipping Systems}

Bozer and Sharp [8] provided insights into design alternatives of shipping sortation systems. Several different design factors and control strategies were evaluated based on throughput. Both recirculating and nonrecirculating systems were examined. Many systems were compared on the basis of the performance measure, throughput ratio, which is the ratio of observed throughput to maximum throughput.

In a shipping sortation system, if the destination shipping lanes do not have enough capacity, the throughput ratio can be increased by recirculating items. However, if the lanes will hold many items, recirculation does not increase the throughput ratio very much. For a given lane capacity, increasing the lane capacity further causes the relative improvement in throughput to decrease in recirculating systems. If lane capacity is increased to very high levels, little or no improvement in throughput is shown.

Through simulation experiments, results in [8] showed that increasing the number of lanes while holding the lane capacity constant does not greatly affect throughput ratio for recirculating systems, but decreases throughput ratio for nonrecirculating systems.

Bozer and Sharp [8] also examined the effects of clustering items into lanes. A group of clustered items have a better chance of being sent to a particular set of lanes rather than having equal probability of being sent to any of the lanes. In other words, the assumption of uniformity in lane assignment no longer holds. Items arrive in clusters for many reasons. Clustering generally occurs because of the methods of order-picking, labeling, or unloading of items. Usually when items are clustered, the subset of lanes to which the clustered items are sent varies over time in order to balance the lanes. However, in rare cases where one subset of lanes tends to receive more clusters, the system will have to be modified in order to achieve balance. The clustering phenomena has a negative impact on the system. With a nonrecirculating system, clustering drastically reduced the throughput capacity. Clustering also reduces the throughput in recirculating systems, but the effect is not as extreme unless the lane capacity is small.

A final sortation system design aspect that was explored in [8] was the control strategy where 
the decision to divert was made at the actual divert point as opposed to the induction point. With small lane capacities, the throughput was improved using this method in a recirculating system. However, with large lane capacities, little or no improvement was shown.

\subsubsection{Recirculating Systems in Shipping Systems}

In [7], Bozer, Quiroz, and Sharp focused their study to recirculating sortation systems to determine the effects of the lane assignment and wave release strategies. Simulation was used to obtain the throughput ratio based on different combinations of several factors, including the distribution of orders in a wave, the number of sortation lanes, the lane assignment strategy, and the wave release strategy.

The alternative lane assignment strategies tested were the "order completion enforced" strategy, the "order completion relaxed" strategy, the "advance priority ranking" strategy, and the "incidental assignment" strategy. "Order completion enforced" means that all of the totes in an order must be on the conveyor loop before the order is "eligible" for lane assignment. "Order completion relaxed" means that lanes can be assigned as soon as the first tote of the order is on the conveyor loop. "Advance priority ranking" assigns orders as soon as the location of all the totes in the order is known. "Incidental assignment" means that each tote is scanned at the scanner before the lanes, and if it belongs to an eligible order, and if a lane is unassigned, a lane is assigned to that order.

The wave release strategies studied by Bozer et al. [7] included overlapping and non-overlapping waves. The non-overlapping waves strategy was used as a base comparison since it is the most conservative strategy. Waves are overlapped by allowing the release of the next wave when a predetermined percentage of the previous wave of orders has been diverted into the assigned lane. This method reduces idle time, but may cause congestion.

The conclusions of the study in [7] showed that the best lane assignment strategy based on throughput was "incidental assignment." Overlapping waves appeared to be a good strategy, using $90 \%$ or more as the predetermined percentage diverted as defined above.

An analytical model of sorting time in a recirculating sortation system was developed by 
Johnson [27]. He compared two common sorting strategies: fixed priority schemes (FPS) and the next available rule (NAR). FPS sorts the orders from largest to smallest or vice versa on some measure. For instance, the "advance priority ranking" is a fixed priority scheme, using a priority index to sort orders. NAR does not use a predetermined sorted list, but rather uses information based on the system as the assignment must be made. An example of NAR is the "incidental assignment" strategy. In Johnson's model, the spread and gap were used to determine the total expected sorting time. Spread is the time required to sort an individual order, and gap is the time between the completion of one order and the beginning of the next order. The analytical model, combined with simulation, showed that NAR outperforms FPS (which formalizes the simulation results presented in [7]. This outcome was significant since most companies use a fixed priority scheme. However, the effect of using a particular lane assignment strategy is less obvious when lane blocking occurs.

Meller [30] studied the lane assignment problem with recirculating systems. An algorithm was developed to optimally assign orders to lanes. This algorithm minimizes the maximum time that any item is recirculating on the conveyor subject to constraints such as lane capacity, one order per lane, and proper order sequencing within a lane for LIFO (last infirst out) truck loading. The algorithm developed shows that by optimally assigning orders to lanes on the basis of the random item arrival sequence, the throughput of the system can be increased.

\subsubsection{Non-Recirculating Systems in Order Fulfillment}

Few papers exist that study solely non-recirculating sortation systems. This system is different than nonrecirculating systems. In this type of system, the inducted items are not allowed to recirculate, therefore each item must be diverted to a lane on the first trip around the conveyor and thus, no conveyor blocking can ever occur. However, in the nonrecirculating systems studied by Bozer et al. [7], items must recirculate in a loop if the accumulation lane is full. This loop is separate from the accumulation lane and acts simply as a queue that allows the following items to attempt to be inducted. 
One of the first papers that looks at non-recirculating systems provides an analytical model of system throughput based on different induction configurations. Meller and Johnson [31] developed this model based on a queueing approximation. Non-recirculation is ensured by making the maximum number of items in a wave equal to the number of bays or chutes. A variety of system configurations were modeled, including fast induction (faster than the speed of the conveyor) and slow induction. Side-by-side (SBS) as well as split induction stations (SPL) were also examined. Finally, the system was analyzed when a tote setup time was included. In all cases, inductor blocking was modeled.

Inductor blocking occurs when the inductor has to wait for a full tray to go by before an item can be inducted onto an available tray. For instance, if there are several inductors at one station, the first inductor will see only empty trays, and will be able to induct at his maximum rate. However, the next inductor down the line will see some full trays that have been loaded onto by the first inductor, and will have to wait for the next empty tray. This continues down the line, with the last inductor at the station seeing the fewest empty trays. Side-by-side inductors work on a single platform at one end of the conveyor, while split inductors have multiple induction stations, usually equally spread around the loop. At each station, multiple inductors may be working. The advantage of splitting inductors is a reduction in conveyor blocking, and increased throughput when the conveyor is the bottleneck. Many induction layouts were modeled and simulated to determine the throughput capabilities. In addition, multiple inductors were simulated at the same rate and at different rates. In all cases, the analytical model provided very accurate approximations [31].

Results from their model [31] showed that splitting induction stations only increases throughput appreciably with fast induction (i.e., the conveyor is the bottleneck), and the maximum possible throughput is two times the speed of the conveyor (this is only achievable with many SPL stations). The model also shows that sortation systems work best when item commonality between orders is present.

Meller and Johnson [32] also developed a preliminary design model for non-recirculating sortation systems. Their model utilizes a mixed-integer program to minimize non-recirculating system costs subject to a throughput constraint and a wave time constraint. They examined 
key parameters such as the number of bins and induction stations to determine the system design that would minimize the total system cost. The number of bins limits the number of orders that can be simultaneously sorted as well as the time to sort a wave. The wave composition also affects the total sortation time. Wave composition algorithms should take advantage of the item commonality within a wave.

\subsubsection{Conveyor Systems}

Conveyor theory is an important basis for understanding sortation systems. Work completed in the late seventies and early eighties analyzed closed-loop conveyor systems. The conveyor system consists of a single loading station, a single unloading station, belts, and a conveyor to transport and hold parts.

\section{Modeling Material Flow with Difference Equations}

Muth [34] first analyzed closed-loop conveyor systems using difference equations. He expressed continuous material flow in terms of space and time in order to determine the compatibility of sets of conveyor parameters with operating requirements. Once these conditions were established, a set of compatible conveyor parameters and operating requirements can be selected in order to minimize costs subject to certain constraints. An extension to this analysis included the discrete material flow case [35]. Another extension modeled random material flow [36]. In this model, the amount of material unloaded from the conveyor was defined by a difference equation. Results from this model proved that adequate conveyor capacity can minimize the variance in the output flow.

\section{Modeling Conveyors using Queueing Theory}

El Sayed et al. [18] used multiple channel queueing theory to develop performance measures for closed-loop conveyor systems with Poisson inputs and outputs. They investigated conveyors with two types of arrivals, singlets and doublets, and two-channel unloading stations. 
Singlets are one arrival, while doublets are two simultaneous arrivals. Expressions for performance measures such as the probability that all channels are idle, the probability of a lost item due to busy channels, and the expected number of units in the system were established. The conveyor design can then be optimized by minimizing the probability of lost arrivals. This can be done by maintaining a low value of doublet arrivals and decreasing the number of singlet arrivals. Obviously, if the probability of both types of arrivals are decreased or at low values, there will rarely be a lost arrival. Conversely, the expected number of units in the system can be increased by maintaining a high value of doublet arrivals while increasing the number of singlet arrivals.

Proctor et al. [37] again used queueing theory to study systems with three service channels and dual input. Homogeneous servers (equal rates) and heterogeneous servers (unequal rates) were both studied. Cases where unserviced units were either lost or allowed to recirculate were examined. Conclusions showed that as more doublet arrivals occurred, system performance improved. System performance measures include the probability of the system being idle, the expected number of units in the system, the probability of a lost item, and the probability that the first server is busy. System performance improves as the probability of lost items, an idle system, and a busy first server decrease.

Regardless of the type of arrival, conclusions showed that utilization of the service channels decreased according to their order in the loop. In other words, the first server was utilized more than the second. Results showed that recirculation improved system performance but does not affect server utilization [37]. 


\section{Chapter 3}

\section{Problem Statement}

\subsection{Tradeoffs Between Automated and Manual Sorta- tion}

Although sortation systems increase the throughput of a distribution center, it is not always feasible for a company to implement this type of system. Many factors must be carefully weighed when making the decision to purchase a sortation system. Tradeoffs exist between the capital cost of the automated system and the labor savings usually realized with respect to a manual system. The capital costs include the hardware, software, and square footage needed to operate the system. The labor includes the pickers, packers, and inductors.

While picking efficiency can increase with the sortation system, the labor savings may not be enough to justify the cost of the sorter. An average tilt-tray sorter (including variable costs) can cost $\$ 250,000$ to $\$ 1,000,000$ depending on the number of chutes, pack stations, and induction stations. Pack station costs for manual and automated systems are quite different as well. A pack station for a manual system includes a basic table and any accessories needed to hold boxes, tape, and other items used in the packing operation. However, a pack station for an automated system includes all this plus the chutes leading from the sorter trays to the packing tables. In addition, labor is required for induction in an automated system and 
no induction is needed in a manual system.

Some companies are physically required to use a sorter. For instance, at the distribution center for Nine West, there simply was not enough room to add more pickers to increase throughput. The addition of a tilt-tray sortation system allowed the company to add a new line of accessories to its distribution center while remaining in its current location [5]. For companies like Nine West, keeping the sorter within their budget is still important. The focus of their decision should be placed on the configuration and size of the sorter.

Many companies base the decision to buy a sorter on return-on-investment (ROI) [20]. After determining the throughput that could be achieved with such a system, the ROI can be calculated. If the ROI is not feasible to the company, the sortation system should be abandoned for a manual sortation system. This decision is especially important for some start-up internet companies who are struggling to make a profit.

Figure 3.1 shows a diagram of a manual sortation system. Parts arrive on a conveyor from the picking area (usually in totes), where they travel to a packing station. The worker then removes a tote and places it out of the way until he or she can complete the previous set of orders. Once the orders are boxed and ready to ship, the worker (usually) places the box onto another conveyor leading to the shipping area (sometimes the conveyor is directly beneath the incoming conveyor to save space, as in Figure 3.1).

Figure 3.2 shows a diagram of an automated sortation system. Section 1.2 provided a detailed description of the flow in this system.

\subsection{Research Goals}

Automated sortation is becoming a popular way to deal with efficiency problems in growing DCs. E-commerce businesses, such as toysrus.com, use sortation systems to provide direct delivery of high volumes of small orders. Many e-businesses model their DCs after catalog fulfillment centers. These companies have been using practices that provide good examples of quick delivery and efficiency for years. Catalog companies such as J. Crew and L.L. Bean 


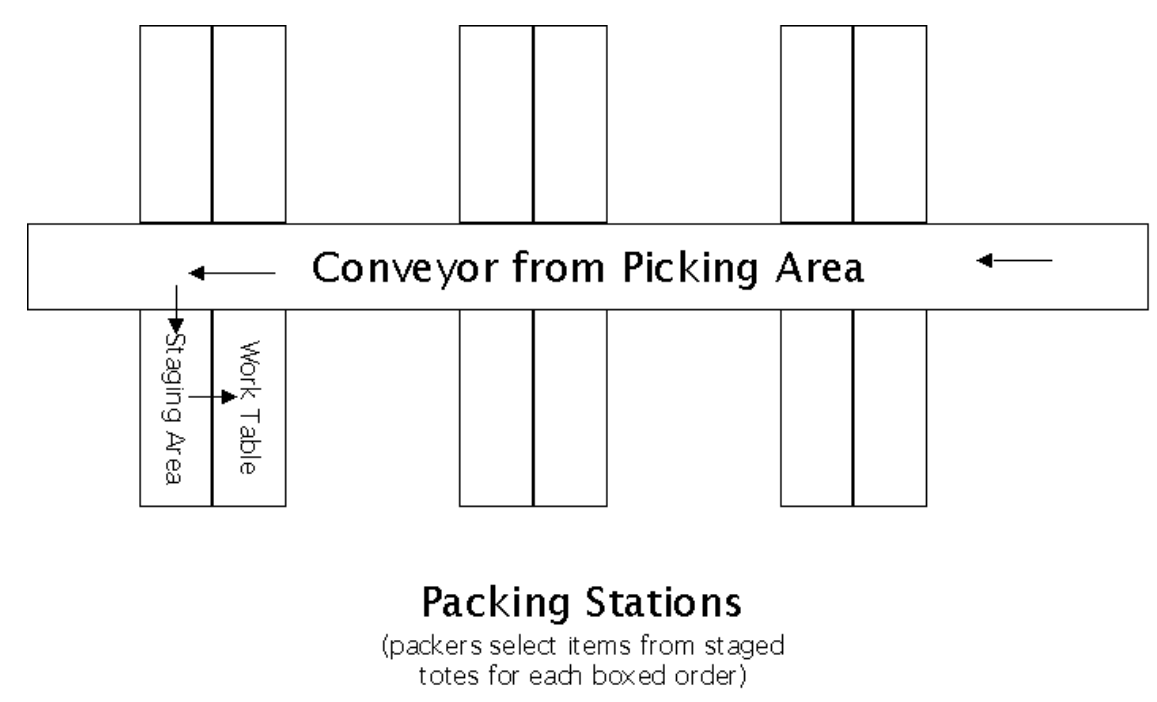

Figure 3.1: Manual Sortation System.

use sortation systems to increase their throughput. Of course, these companies are now also using the internet to collect orders. Sortation systems are also being found in third-party logistics providers. Companies who need to accommodate heavy volumes of products, such as Nike, use sortation systems to increase throughput as well. It is obvious that a wide variety of companies are considering the conversion from manual to automated sortation systems. This shift in technology presents a need for a tool to provide guidelines for implementing such a system.

The purpose of this research is to provide a method to answer three main questions regarding the selection of a sortation system. First, the company should determine if the sortation process should be manual or automated. This question is answered by looking at all tradeoffs between manual and automated systems, and then determining which system is more cost effective. Second, if an automated system is preferred, the company should determine if this system is feasible based on their current economic situation. Third, the system should be designed in order to meet the specific needs of the company, such as room for growth, peaks in demand, product volume, the types of products that will use the sorter, and square footage available in the facility. All these factors must be considered when designing the sortation system. In this analysis, economic factors as well as the throughput of the system are considered. 


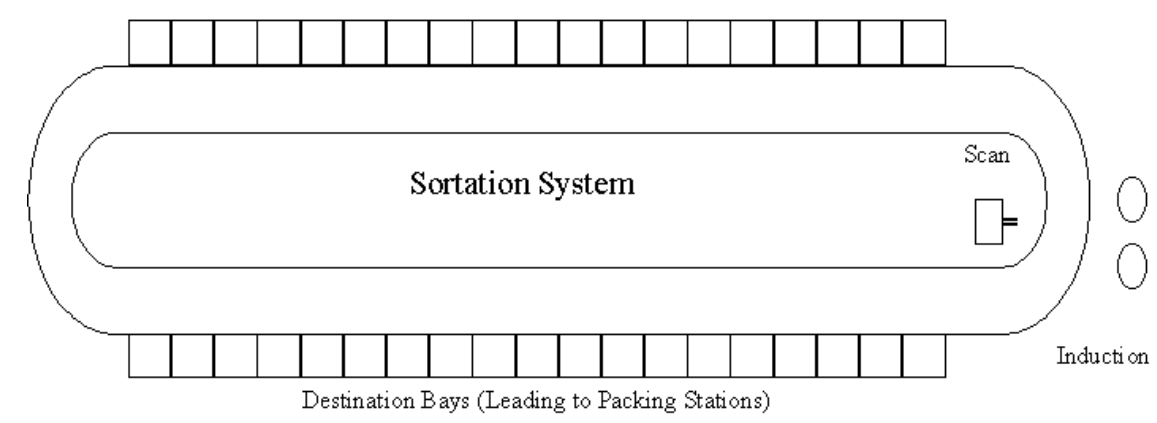

Figure 3.2: Automated Sortation System [27].

\subsection{Theoretical Efficiency Curve}

In order to understand the relationship between the two main costs of a system, picking and packing labor, an efficiency curve was developed. An efficiency curve depicts the important relationship between the picking standard and the packing standard. Namely, for a manual sortation system, as the batch size being picked increases, the packing standard will decrease. For instance, if the pickers pick individual orders, instead of batches, the packers have nothing to sort through, and therefore their packing standard is very high. In contrast, if a large batch is picked from zones, the packers may have many orders' worth of items to sort through before they can begin packing the orders. On the other hand, for the automated sorting system, the packing standard will remain approximately constant for all levels of picking standards due to the sortation performed to the orders as they arrive to the pack stations. That is, regardless of how the orders are picked, they will arrive to the pack stations in terms of orders already sorted.

These relationships for manual and automated systems are carefully reflected in the model so as to make an accurate comparison between the two systems. For each efficiency curve, several common wave lengths are examined. Figure 3.3 illustrates efficiency curves for automated and manual sortation systems over a range of wave lengths. 
Points on the curve for a wave length represent a change in order-batching for the pickers. As more orders are batched together, the pickers' efficiency increases, but the packers' efficiency decreases. This is represented in Figure 3.3 by having the picking standard increase while the packing standard decreases.

It is important to note the relationships between the various curves as well. As the wave length increases, we can still assign the same number of orders to each picker. Since the number of orders the packer must sort through remains the same, the packing standard will remain the same. However, due to the longer wave, the picker assignments can now be improved. That is, since there are more orders to choose from in a longer wave, the assignment of orders to pickers can be made to improve the picker's efficiency. Thus, the curves shift simultaneously upward and to the right.

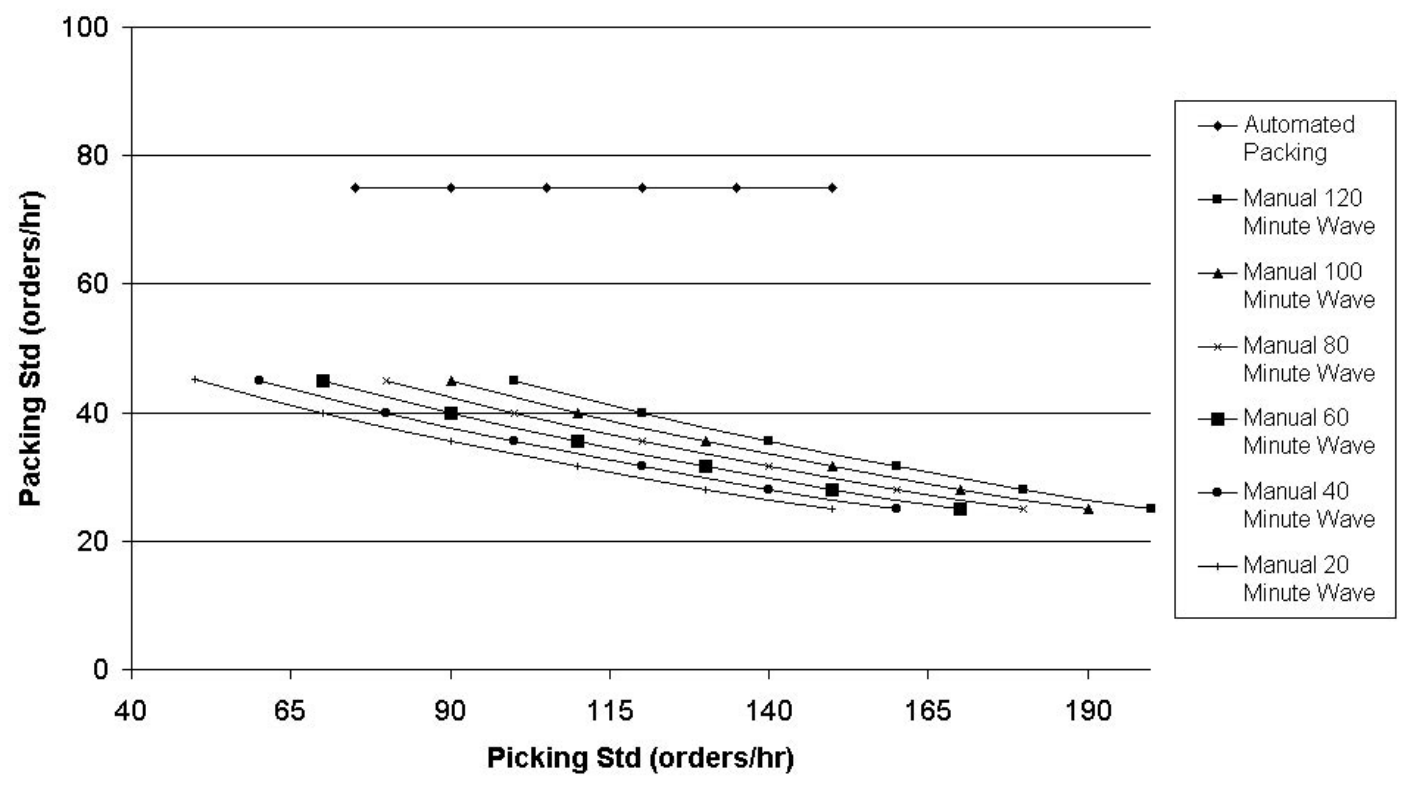

Figure 3.3: Manual and Automated Efficiency Curves.

In Figure 3.4 the same relationship is presented, as well as an additional relationship in the automated system. Realistically, if the wave length increases, the packing efficiency will decrease slightly for the automated system as the picking standard increases. Although the sorter works at a constant rate, the packers will be required to move between more packing 


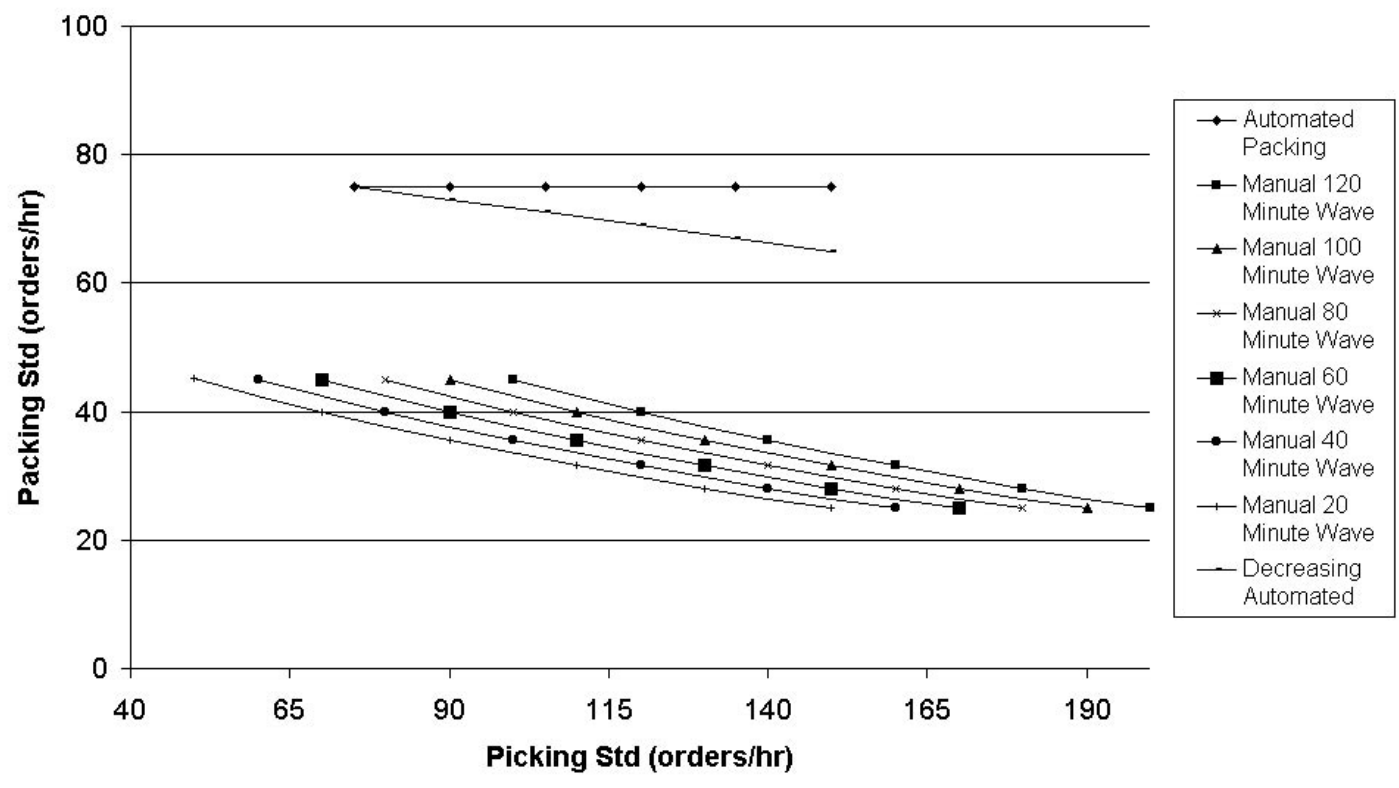

Figure 3.4: Decreasing Automated Efficiency Curves.

stations. Due to the randomness of order-completion times, this will cause the slight decrease in the packing efficiency.

\subsection{Actual Efficiency Curve}

Although we believe the relationships represented in the theoretical efficiency curves presented in the previous section exist, it is important to examine them in an actual or simulated context. Time study data are presented in Appendix A and simulation results are presented in Appendix B. The purpose of this section is to present our results and compare the theoretical efficiency curve with an actual one.

\subsubsection{Picking Efficiency}

With an effective sortation system, picker efficiency in the DC can increase. Since the sorter can efficiently sort many orders at once, the pickers can use more of a batch picking technique. 
It has been reported that batch picking can save approximately $40 \%$ of required labor [23]. This technique will save walking time by allowing the pickers to retrieve identical items for several orders at once, instead of traveling to its location each time an order requires that item.

In order to verify the relationship between order-batching and picking efficiency, several experiments were completed. First, a time study was conducted in order to obtain realistic data demonstrating the benefit of batching orders. This time study was conducted at Cross Creek Apparel in Mount Airy, North Carolina. Twenty orders were picked in several different batch sizes, and travel times were recorded for each set of orders. The data collected is tabulated in Table 3.1 and is presented graphically in Figure 3.5. In Figure 3.5, the scaled number of stops is simply the number of stops from Table 3.1 scaled for presentation purposes. As can be seen, when no batching is performed (batch size equal to one), the picker must make multiple stops at a SKU location each time that SKU appears in an order. However, as the amount of batching increases, the number of stops and the travel time for the entire picking tour decrease since the picker can pick for several orders at that particular SKU's location. Notice when all orders are batched together (batch size equals all 20 orders), the number of stops exactly equals the number of SKUs, therefore minimizing the total travel time. The picking time study results are presented in full in Tables A.1 through A.4 in Appendix A.

Although this time study verified that batching orders increases picking efficiency, it does not account for different inventory arrangements. For instance, one distribution center might put the items that account for $80 \%$ of the items picked in the $20 \%$ of the picking area closest to the

Table 3.1: Cross Creek Picking Efficiency Data.

\begin{tabular}{cccc} 
Batch Size & Total Time $(\mathrm{min})$ & Number of Stops & Number of SKUs \\
\hline 20 & 38.35 & 300 & 300 \\
10 & 54.82 & 354 & 300 \\
5 & 67.35 & 393 & 300 \\
1 & 99.44 & 437 & 300 \\
\hline
\end{tabular}




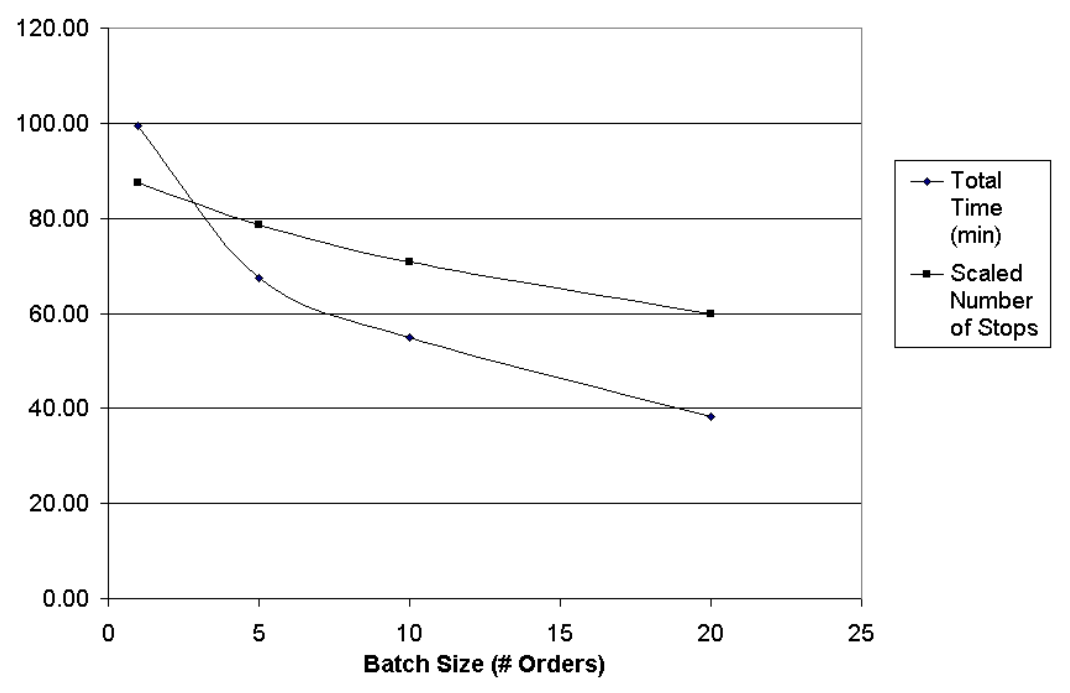

Figure 3.5: Effects of Batching Orders on Travel Time.

I/O point. However, other distribution centers might arrange the items evenly throughout the picking area. In order to capture the effects of several configurations, a simulation study was conducted.

The picking simulation was designed to capture the effects of batching on picking efficiency for three different inventory curves: $80 / 20,60 / 40$, and 50/50. In the simulation, a single picker is given batches ranging from 1 order to 150 orders. An average of 3 items per order was used to represent an average-sized order placed on the internet. A pick list of all items to be picked in the batch is generated, and then the row each item is in is determined. The picker then uses a traversal strategy to retrieve items in the pick list. The picking time per order is calculated and compared for each batch size. Simulation results are presented in full in Tables B.1 through B.6 in Appendix B.

Figure 3.6 shows this relationship between batch size and pick time per order for each of the three inventory curves. From this graph we can see that the picking time decreases rapidly over small batches and then tapers as the batch size increases. As expected, the 80/20 curve has a smaller picking time decrease than the 50/50 curve. An 80/20 curve has most of its items concentrated in a small portion of the picking area, so batching orders will not be as effective with this setup than with a 50/50 curve. In a 50/50 curve, the picker will save more 


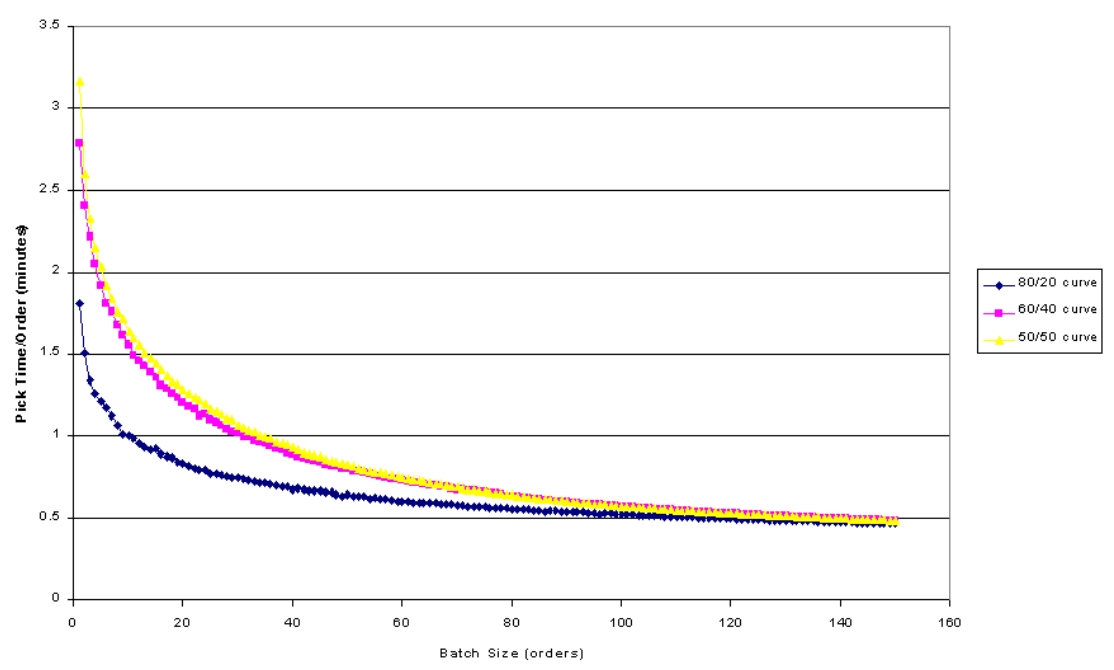

Figure 3.6: Picking Rates vs Batch Size.

aisle travel since the items are spread evenly throughout the picking area. For instance, with a single order in a batch, the average pick time per order over 100 replications was 1.81 minutes for an $80 / 20$ curve, 2.78 minutes for a $60 / 40$ curve, and 3.16 minutes for a 50/50 curve. After increasing the batch size to five orders, the average pick time decreases to 1.21 for the $80 / 20$ curve, 1.92 for the $60 / 40$ curve, and 2.03 for the 50/50 curve. The improvement is then $0.59,0.86$, and 1.13 , respectively. The $80 / 20$ curve will be the curve considered in all calculations from this point on. This curve is indicative of most companies and storing items based on turnover is used in most picking areas.

Not only can order-batching be effective with automated sortation systems, but also with manual systems. As the wave length increases, the picker can be more selective in the orders that are batched. Orders can be batched within a wave to decrease picking time per order, but also across wave length increases. A longer wave length provides more orders for the picker to select from when batching. The batches can be formed in a way that reduces the picking time even further. For example, selecting orders with items in a few rows that are close together, or a zone, can further reduce picking time. This type of batching was not included in the simulation, but results were estimated using the picking time study results combined with the simulation results as described next. 
Table 3.2: Wave Length Effects on Picking Efficiency.

\begin{tabular}{rr}
$\begin{array}{r}\text { Wave Length } \\
\text { (minutes) }\end{array}$ & Rate Increase from \\
Previous Wave (\%) \\
\hline 20 & $\mathrm{~N} / \mathrm{A}$ \\
40 & 14.04 \\
60 & 8.21 \\
80 & 5.83 \\
100 & 4.52 \\
120 & 3.69 \\
\hline
\end{tabular}

The picking time study results were gathered for pick lists separated by zones. Each zone represents a portion of the picking area with rows that are close together, therefore reducing the distance that must be traveled in each picking wave. From the results gathered in this time study, a reduction in picking time when using zone picking was estimated for wave length increases from 20 minutes to 120 minutes. The results from this calculation provided the shift in efficiency curves for each wave length. This shift shows the efficiency gains from intelligent batching while keeping the number of orders a packer is responsible for the same. Table 3.2 shows the percentage increase in picking rate based on the wave length increase. Notice that as the wave length increases, this percentage decreases due to the fact that more aisles will be entered for longer wave lengths.

\subsubsection{Packing Efficiency}

Packing efficiencies vary based on wave length, batch sizes, and whether a system is automated or manual. In general, as the batch size increases, the packing efficiency decreases. However, the system configuration has a large effect on the amount of efficiency lost by batching orders. For instance, an automated system does not lose much efficiency when orders are batched, especially if waves are overlapped. A manual system loses a great deal of packer efficiency with each increase of batch size. 


\section{Automated Systems}

Packers in an automated system are usually assigned to several pack stations. Each pack station can have one or more bins where the items for an order fall from the sorter trays. When a bin is ready to be packed, a light or a similar indicator alerts the packer that that bin is ready. Packers may have to walk between several pack stations to reach a bin that is ready to be packed. Because of this, as the packer is assigned more and more pack stations, this results in more and more walking. The amount of walking that is required results in a slight packing rate decrease.

As mentioned before, the decrease in packing rates due to walking between pack stations varies greatly based on the wave setup. If waves are not overlapped, the packer must wait for bins to become ready to be packed. Items arrive to the bins at some time period between the beginning of the wave and the end of the wave. However, with waves overlapped, the packer would not have to wait for bins to become ready to be packed. Some of the bins should be ready at the beginning of the wave, and by the time the packer has completed those bins, the packer should not have to wait for other bins to receive all the items in the order.

Both overlap scenarios discussed above were modeled using simulation. The wave overlap situation was modeled as if there were complete overlap. In other words, all bins are ready at the beginning of the wave, so no time is spent waiting for bins to become ready to pack. The packer simply packs the first bin and then walks to the next bin. With no wave overlap, the packer must wait for the orders to become ready to pack, then decide which order should be packed next. In the simulation model developed, the packer packs an order, then checks the indicators to see if any other orders are ready to be packed. If no orders are ready at that time, the packer waits until the next order is ready and walks to that bin. However, if one or more orders are ready, the packer walks to the closest bin that is ready and packs the order. In this situation, the time spent waiting for orders to get ready and walking between bins decreases the packing rate.

Figures 3.7 and 3.8 present the amount of orders that can be packed in a wave for both 


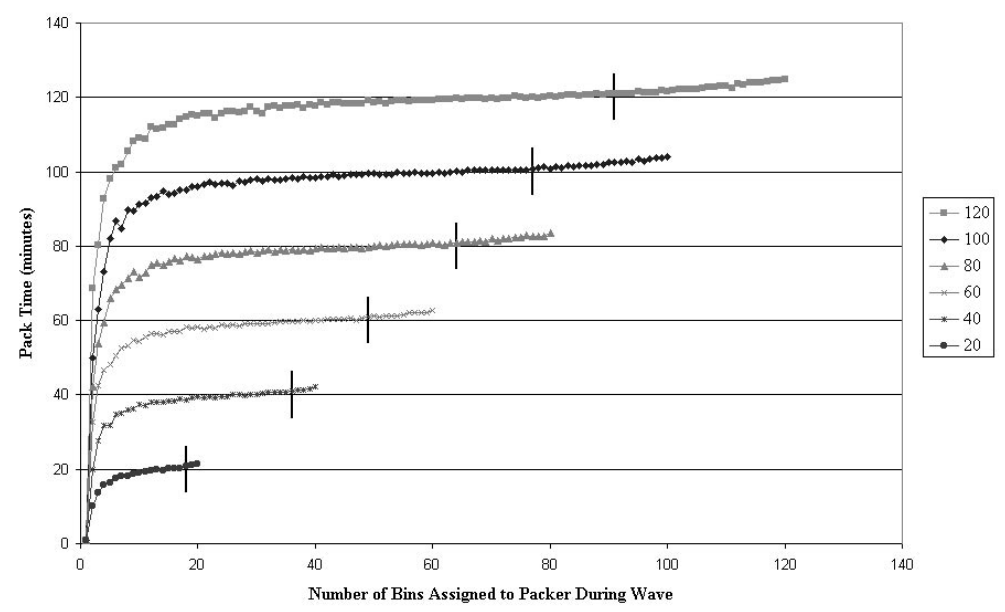

Figure 3.7: Number of Orders vs Total Pack Time (No Overlap).

overlap scenarios. The vertical lines on each curve in Figure 3.7 represent the maximum bin assignment that can be completed within the wave length for no overlap. Figure 3.8 also illustrates this result for the no overlap scenario, but also illustrates the results with overlap. Note that due to the minimum amount of walking, the expected packing rates with complete overlap are higher than the expected packing rates with no overlap. Also, the packer can sometimes pack more than the expected amount due to randomness present in the simulation model. Overall, since the walking time between one bin is so small, the decrease in packing efficiency as the wave length or batch size increases is not significant under complete overlap.

\section{Manual System}

With a manual sortation system, as the batch size delivered to the packer increases, the packer's efficiency will decrease. The packer is responsible for sorting through the orders before packing them. As the batch size increases, sorting the items down to individual orders becomes more and more difficult. A time study was conducted at Cross Creek Apparel to verify the relationship between batch size and pack time per order. In this study, twenty orders were picked and brought to the packing station. These orders were divided into random batches of 20,10, 5, 4, and 2 orders and the sort and pack time was recorded for each batch. Using this data, the packing time was estimated for batches of different sizes. 


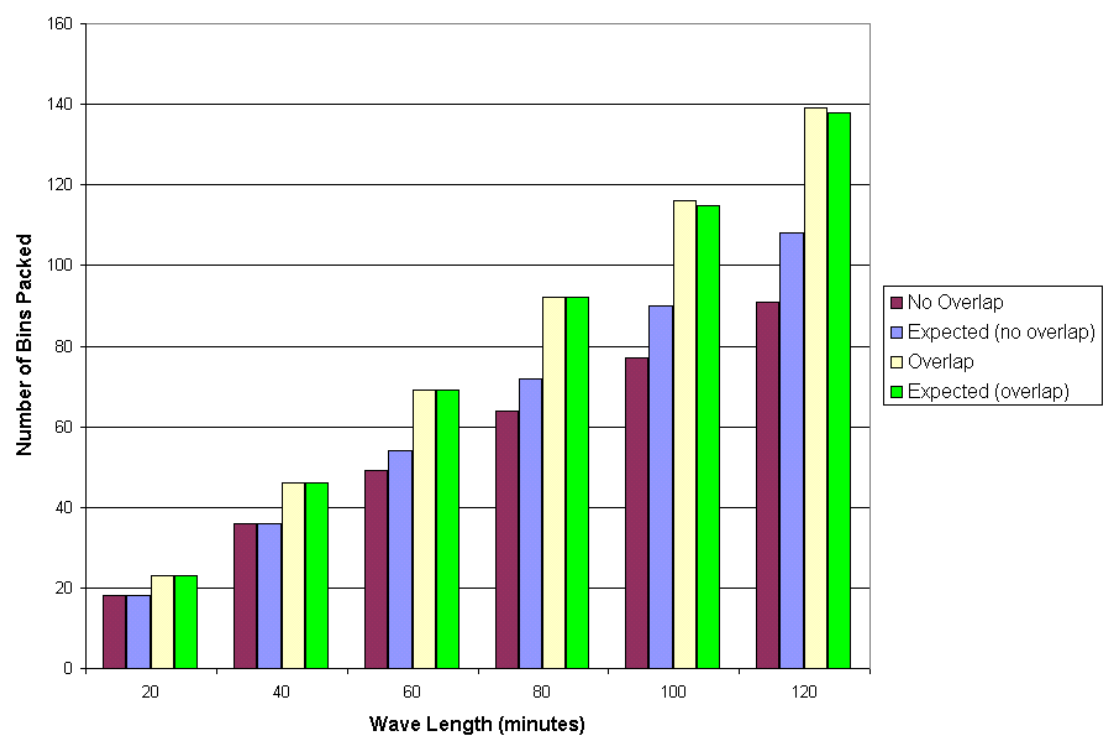

Figure 3.8: Number of Bins Packed.

The packing time study data is shown in Figure 3.9.

\subsection{Forming the Efficiency Curve from Simulation Data}

Once all simulation and time study data were collected, the information was used to develop the actual efficiency curve. First, the picking rates were determined for each wave length using the zone picking time study data. That is, the estimated gain in efficiency due to batching orders over a longer period of time was determined from the time study data, where the zone picking method provides information about the gain of intelligent batching. In addition to the picking rates, the time to pick the orders assigned to the picker that is associated with that particular rate was calculated. Using this time, the highest possible rate that can be achieved in a wave was determined. For instance, a picker working within a 20minute wave can pick a certain number of orders in slightly less than 20 minutes. Therefore, this picking rate was used as an upper bound for a 20-minute wave. The corresponding manual packing rate was then determined by taking the rate that corresponds with the same batch size. We assume that the picker and packer work with an identical batch size, however, the pickers will be assigned more batches in order to even out the work within a wave (since, 


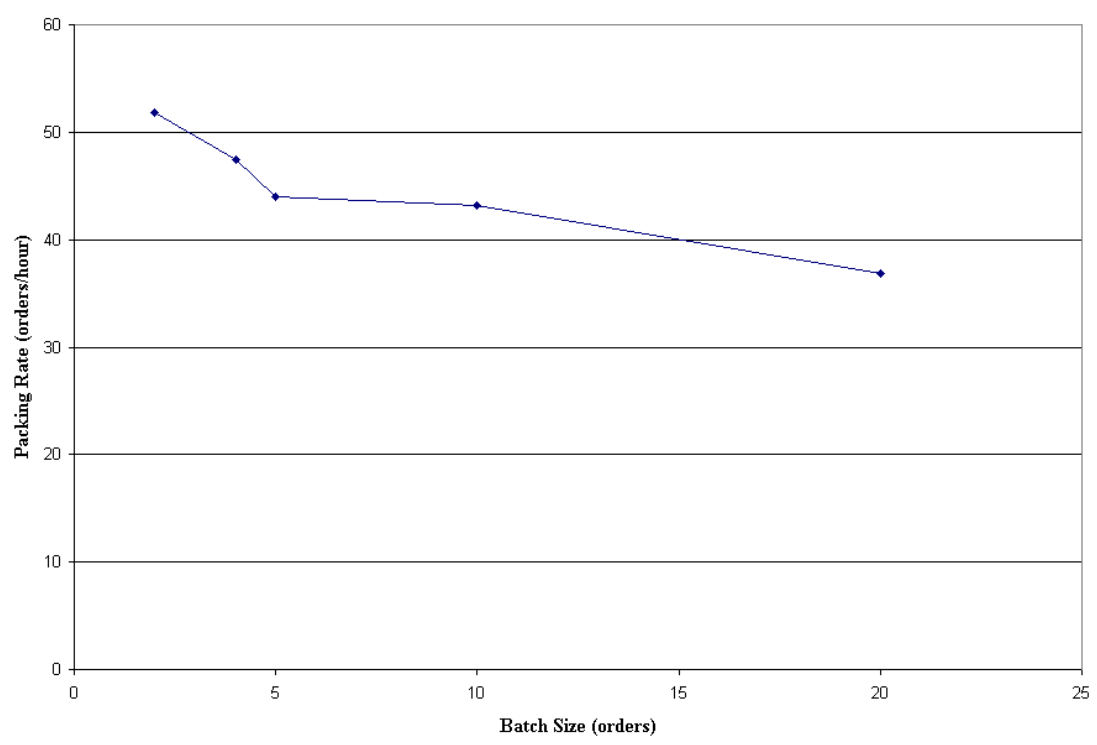

Figure 3.9: Packing Time Study Results.

in general, pickers work at a higher rate than packers). For example, consider a wave with a batch size equal to 34 orders. Pickers can work at a rate of 99 orders per hour and packers can work at a rate of 41 orders per hour given this batch size. This system would require more than twice as many packers than pickers working at this rate.

To obtain other picking and packing rate combinations for the manual system, we selected six rate combinations for each wave length. In other words, for each wave length, the maximum number of orders that can be picked within a specified wave length was determined, and the corresponding picking and packing rates were obtained for this batch size. This was chosen as the sixth combination on the curve (i.e., the point furthest to the right on the efficiency curve). The other five combinations were developed by dividing the total time so that the first combination completes six batches of a size that can be picked in one-sixth of the wave length, the second combination completes five batches of a size that can be picked in one-fifth of the wave length, and so forth. The packing rates are then determined by the rate at which that batch size can be completed. These combinations were used for both the spreadsheet model and the efficiency curve. However, every combination of picking and packing rates were used to develop the curve more accurately. In other words, batch sizes ranging from one to the maximum number of orders that can be completed within a wave 
were simulated to fill in points between the six points evaluated in the spreadsheet model. This process was completed for every wave length considered.

The automated system curve was developed in much the same way. However, since picking and packing are not dependent on one another, they were selected separately. For each wave length, a corresponding picking and packing rate were again calculated. The batch size corresponding to the highest time under the specified wave length (or highest efficiency) was used as input in the spreadsheet model for the picking rate. The packing rate was determined for each wave length independently. The picking and packing rates for each wave length were then plotted.

Data used to develop the efficiency curves is presented in Appendix B for different levels of picking and packing rates. In particular, Tables B.1 through B.3 present the picking rates and times generated for each batch size. Table B.4 presents the packing rates and times for manual packing system simulations, and Tables B.5 and B.6 present the packing results for simulated automated systems. This will be discussed more thoroughly in Chapter 6 . Tables B.7 and B.8 summarize the efficiency curve data for manual and automated systems, respectively.

\subsection{Picking vs. Packing Efficiencies}

Now that the relationships between batch size and picking efficiency and packing efficiency have been verified, the relationships between picking efficiency and packing efficiency can be verified as well. For an automated system, as the wave length increases, more orders are batched, therefore increasing the picking efficiency. Since the sorting is automated and at a constant rate, the packers can pack at approximately the same rate regardless of the number of orders batched together. Figure 3.10 shows the system with waves that overlap completely. This system results in an efficiency curve similar to the theoretical curve in Figure 3.3. The packers' rate has only a slight decrease as more and more orders are batched. This decrease is due to walking time between bins or pack stations. 


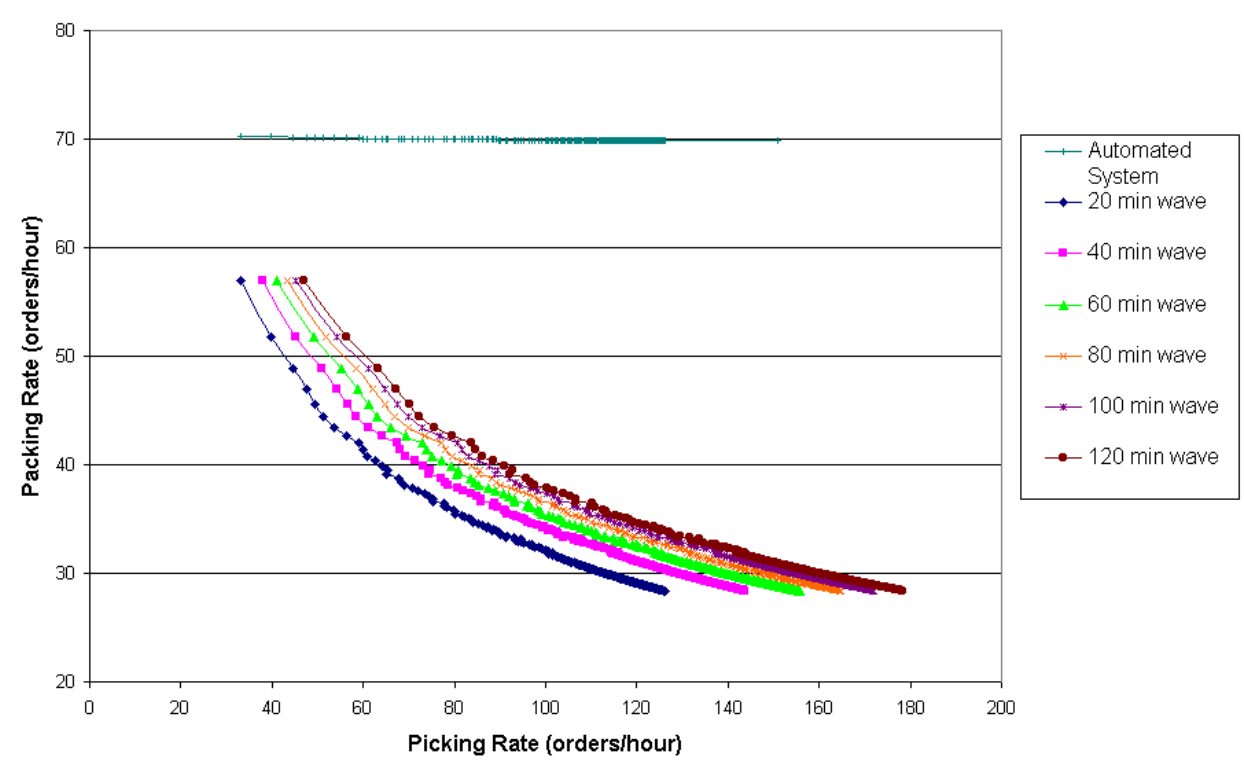

Figure 3.10: Actual Efficiency Curve with Complete Wave Overlap.

Figure 3.11 shows the system with waves that do not overlap. Notice the automated curve this figure has a dramatic decrease in packing efficiency as the picking efficiency increases. As discussed before, the decrease is due to the amount of time spent waiting for bins to become ready to pack and walking from bin to bin. In this situation, the packer may have to travel between several pack stations in order to reach the next bin that is ready to pack.

Figure 3.12 shows the packer efficiency decrease in the automated system due to walking times only. Notice the difference between the decrease from Figure 3.11, where waiting times are also included. Figure 3.12 illustrates how much time is spent walking if bins are packed randomly among the packer's assigned work stations. This graph would be similar to a system with partial overlap, where several bins are ready, but at different locations in the worker's area.

To summarize, the theoretical curve developed in Section 3.3 is a good estimate of the actual efficiency curve, especially in the case of wave overlap. Due to obvious benefits, and low marginal cost, we will proceed using the assumption that waves overlap in the remainder of this study. 


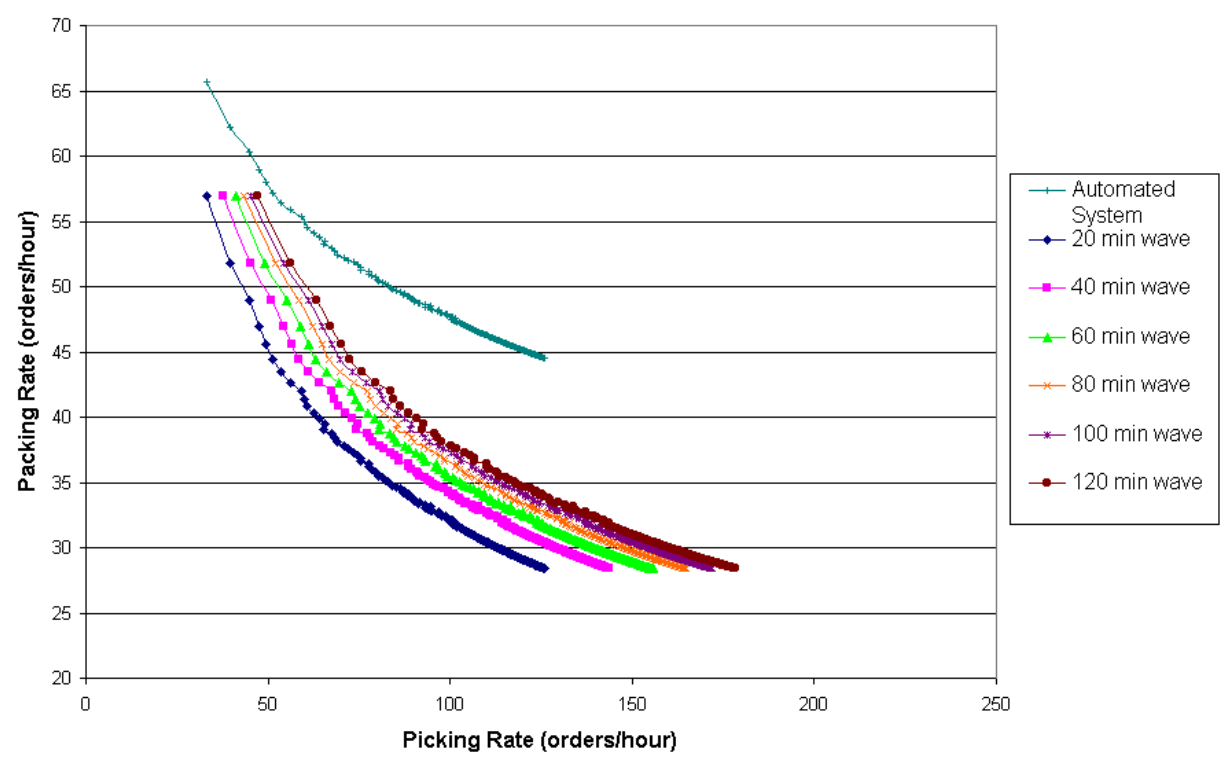

Figure 3.11: Actual Efficiency Curve with No Wave Overlap.

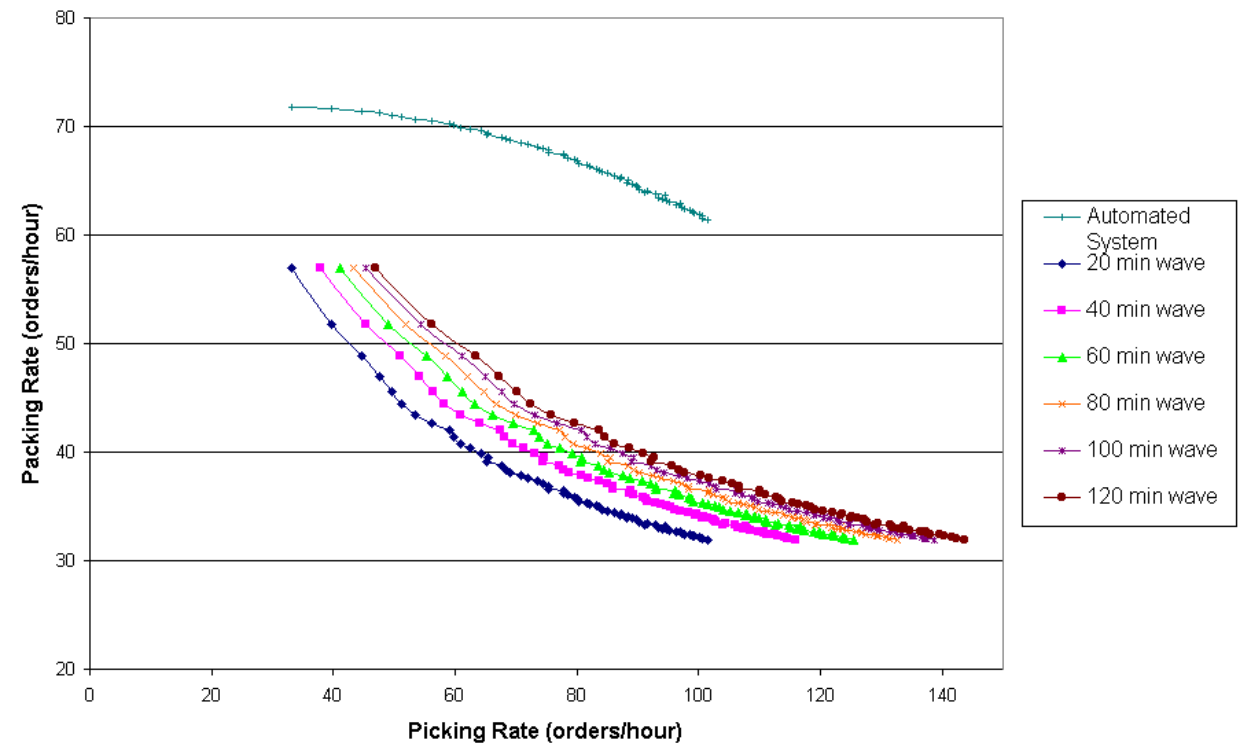

Figure 3.12: Actual Efficiency Curve with No Wave Overlap (Decrease due to Walking Times Only). 


\subsection{Company Data and Modeling}

The primary goal of this research is to develop a model that will be useful to companies needing to make a decision about their sortation system design. A company in this position would provide input data for a deterministic spreadsheet model, discussed thoroughly in Chapter 4. This input data includes the average hourly demand, the estimated costs of the sorters they are considering, as well as any hardware, scanners, and control systems required to accompany this sorter. Other input data includes the labor costs of pickers, packers, and inductors, induction rates, and maximum and minimum picking and packing rates. Economic input data such as the interest rate and number of years of use are input in order to determine the capital recovery $(\mathrm{A} / \mathrm{P}$ value $)$.

Once this data has been input into the spreadsheet model, the total annual cost will be calculated for a variety of configurations. The least expensive configuration will be recommended as the system to implement. The recommendation includes all output data, including if the system should have manual or automated sortation. More detailed recommendations include the wave length, picker and packer rates, number of pickers, packers, and pack stations. In addition, automated systems must include data on the number of inductors and induction stations required to meet demand.

Although this recommendation is based on verified relationships between each parameter and actual company input data, stochastic picker, packer, and inductor rates, and the random locations of the items in each order produce different results each time simulations are run. Random effects are present within a company from minute to minute, so the recommended system must overestimate to allow for these effects. Therefore, after recommending a system configuration, a system simulation that uses the recommended configuration as input would be run to determine if the subsystems (picking, sorting, and packing) will meet the desired throughput. If throughput is met, the company can proceed and implement the recommended configuration. However, if throughput is not met, the subsystem that has not met demand must be reanalyzed.

In Chapter 4 we discuss our deterministic model of this order fulfillment system. Following 
the development of the model, a sensitivity analysis is presented in Chapter 5 for key model parameters. Chapter 6 provides the details of our simulation methodology developed to test our deterministic model. 


\section{Chapter 4}

\section{Deterministic Model}

In order to understand fundamental relationships between each factor in a sortation system, a descriptive spreadsheet model was developed. This model not only shows relationships within both automated sortation systems and manual sortation systems, but also serves as a tool for comparison between the systems. A range of parameters are used as input to determine the effects on the system. Parameters include factors such as demand rates, labor rates, fixed and variable costs, order sizes, wave size, and sorter capacity. That is, based on this deterministic input, the number of employees, packing stations, and induction stations required to meet demand will be determined. For each set of parameters, a total annualized system cost will be determined.

This chapter discusses the fundamental relationships between each parameter and variable in the system, as well as all notation, assumptions, and calculations made. Although manual and automated sortation systems are very similar, there are several key differences broken down in the following sections.

Wave lengths ranging from 20 minutes to 2 hours were tested, along with a range of picking and packing rates for each wave length. For these combinations of wave length, picking rate, and packing rate, a total annual system cost is estimated for each type of system. Results from each were compared in order to determine which sortation method is more appealing based on cost and throughput. The model provides guidelines for determining the size of the 
system based on the number of packing stations and wave lengths. This tool will provide a starting point from which the decision to use automation can be made. Based on the current demand, labor rates and variable costs, and the fixed cost of a sorter, companies can use this guide to determine if automation is within the company's budget and space limitations.

We now present the descriptive model in more detail, explaining the fundamental relationships, presenting an example, and outlining our experiments.

\subsection{Fundamental Relationships}

There are several fundamental relationships that exist between the parameters and variables in the descriptive model. For instance, the numbers of required pickers, packers, and inductors are functions of the demand and the standard rate at which each type of worker performs. That is, the number of pack stations required is a function of the number of orders per wave, the number of orders per pack station, and the number of required packers. Likewise, the total annual labor cost is the sum of the labor costs for pickers, packers, and inductors, and the total annual cost of the system is the sum of the total annual labor cost plus the annualized costs of the sorter, pack stations, and induction stations. When examining a manual system, the total annual system cost is simply the cost of labor and the annualized cost of the pack stations. Costs associated with the picking area equipment are not considered.

Notation and mathematical expressions to illustrate these relationships are now presented.

\subsubsection{Notation and Illustrated Relationships}

\section{Organization}

- Labor costs for inductors, packers, and pickers, are denoted by the subscript $\ell$.

- Fixed costs are denoted by the superscript $C$. 
- Variable costs are denoted by the subscript $c$.

- Upper case variables denote system-wide variables.

- Lower case variables denote variables specific to manual or automated systems.

\section{Notation and Definitions}

The following notation and definitions are separated into parameters or variables used in both automated and manual system analysis and those used solely in manual or automated system analysis.

\section{Automated and Manual Systems}

- $D$ represents the demand, in orders per hour.

- $W$ represents the wave length, or the length of time (in minutes) over which a collection of orders are processed. This time is generally the same in all areas of the DC. The wave length is set at a certain length in order to balance activity between all areas of the DC.

- $N_{w}$ represents the number of orders per wave. This is determined by multiplying the number of orders per hour by the wave length:

$$
N_{w}=D *(W / 60)
$$

- $N_{o}$ represents the average number of items contained in each order, whether the items are unique or identical.

- $N_{q}$ represents the number of orders per pack station, or the capacity of the pack station. This is based on an average number of items per order, $N_{o}$.

- $p(n)$ represents the picking standard per hour, or the rate at which the workers pick items from cases when assigned $n$ orders worth of items to pick. 
- $q(m)$ represents the packing standard per hour, given in items per hour. This is the rate at which the workers pack items into orders when $m$ orders are delivered to the packing station. This rate includes the final sort into orders (not including the automated sortation into packing stations), constructing boxes, packing the box or bag with items, fliers, and catalogs, and attaching the shipping label. This standard is generally much higher than the picking standard.

- $r_{p}$ represents the number of required pickers, and is determined by the following:

$$
r_{p}=\lceil D / p(n)\rceil
$$

- $r_{q}$ represents the minimum number of required packers, and is determined by the following:

$$
r_{q}=\lceil D / q(m)\rceil
$$

- $n_{q}$ represents the minimum number of pack stations, and is determined by the following:

$$
n_{q}=\left\lceil N_{w} / N_{q}\right\rceil
$$

- $r_{a}$ represents both the actual number of required packers and the actual number of pack stations required. This is determined by the following:

$$
r_{a}=\operatorname{maximum}\left\{r_{q}, n_{q}\right\}
$$

- $\ell_{p}$ represents the annual labor cost per picker.

- $\ell_{q}$ represents the annual labor cost per packer.

- $i$ represents the interest rate used to calculate the capital recovery factor $(A / P)$.

- $n$ represents the number of years of service used to calculate the capital recovery factor $(A / P)$.

- $A$ represents the annualized cost of a capital expense, $P$ :

$$
A=P(A / P)=P\left[\frac{i(i+1)^{n}}{(1+i)^{n}-1}\right]
$$




\section{Manual Systems}

- $c_{q}^{m}$ represents the annualized cost per pack station. This is the variable cost associated with each pack station. This includes the packing tables and conveyors used in this area.

- $T_{\ell}^{m}$ represents the total annual labor cost. The following equation represents this cost for the manual system:

$$
T_{\ell}^{m}=\ell_{p} r_{p}+\ell_{q} r_{q}
$$

- $T^{m}$ represents the total annual cost of the system. The following equation represents this cost for the manual system:

$$
T^{m}=T_{\ell}^{m}+r_{q} c_{q}
$$

\section{Automated Systems}

- $c_{q}^{a}$ represents the annualized cost per pack station. This is the variable cost associated with each pack station. This cost includes the chutes or bins where parts are delivered, the pack station tables, and other associated equipment.

- $s$ represents the speed of the conveyor in trays or items per minute.

- $t$ represents the induction rate, or the rate at which workers place parts on the sorter in items per minute.

- $x$ represents the total required induction rate over all inductors, in number of items per minute:

$$
x=D\left(N_{o} / 60\right)
$$

- $r_{s}$ represents the number of required induction stations. As mentioned before, induction stations can be either side-by-side or split. The number of induction stations depends on the total induction rate required, $x$, and the conveyor speed, $s$. The following 
equation represents the number of required induction stations. The results from [31] are used, assuming each station represents a "fast" inductor (which could be one "fast" inductor or many slow inductors that represent one):

$$
r_{s}= \begin{cases}1 & \text { if } x \leq s \\ \lceil x /(2 s-x)\rceil & \text { if } x>s\end{cases}
$$

- $r_{t}$ represents the number of required inductors. The number of inductors is dependent on the number of induction stations, the induction rate of each inductor, and the speed of the conveyor. The following expression ignores side-by-side inductor blocking since it cannot easily be represented in closed-form if the number of inductors for each station is greater than two (this will be addressed in Chapter 6):

$$
r_{t}= \begin{cases}1 & \text { if } x \leq t \\ \lceil x / t\rceil & \text { if } t<x \leq s \\ r_{s} *\lceil s / t\rceil & \text { if } x>s\end{cases}
$$

- $c_{t}$ represents the annualized induction station cost. This is the variable cost associated with each induction station.

- $C^{s}$ represents the annualized fixed cost of the sorter. This is the base cost of the sorter. When added to the variable costs mentioned above, the total hardware cost of the automated sortation system is determined.

- $C_{t}^{s}$ is the total hardware cost of the automated sortation system.

$$
C^{s}+r_{s} c_{t}+r_{a} c_{q}
$$

- $\ell_{t}$ represents the annual labor cost per inductor.

- $T_{\ell}^{a}$ represents the total annual labor cost. The following equation represents this cost for the automated system: 


$$
T_{\ell}^{a}=\ell_{p} r_{p}+\ell_{q} r_{q}+\ell_{t} r_{t}
$$

- $T^{a}$ represents the total annual cost of the system. The following equation represents this cost for the automated system:

$$
T^{a}=T_{\ell}^{a}+C_{s}+r_{a} c_{q}+r_{s} c_{t}
$$

\subsubsection{Assumptions}

- Each model input is deterministic and time independent. Most sortation systems are designed for particular peaks in demand (e.g., yearly, daily, or hourly volumes).

- Each capital cost is annualized using the capital recovery factor, $A / P$, where $A$ is the annual cost given $P$, the present cost, with an interest rate of $i$, and $n$ interest periods. These interest rates typically vary between $10 \%$ and $20 \%$ and $n$ varies between 5 and 10 years.

- It is desired for the workload of pickers, packers, and inductors to be even.

- Wave lengths are the same in all areas of the distribution center (i.e., picking, sorting, and packing).

- The number of items per order is based on an average of 3 items per order. This can be changed, but represents the correct order of magnitude for many companies concerned with order fulfillment center design.

- For all sensitivity analyses and simulation data, we assume that there is overlap in waves. In other words, processing is completed on some or all of the orders in a wave before the next wave begins. However, we do discuss waves with no overlap briefly in the automated packing simulations in Chapter 5.

- Base levels of each parameter are presented in Table 4.1. The pack station cost for manual systems is much less than the cost for a pack station in the automated system. The sensitivity analysis varies each parameter from the base level and then varies combinations of these parameters to see the effects. 
Table 4.1: Sortation System Costs [4].

\begin{tabular}{lr} 
Cost Parameter & Cost $(\$)$ \\
\hline Annual Labor Cost per Picker $\left(\ell_{p}\right)$ & 30,000 \\
Annual Labor Cost per Packer $\left(\ell_{q}\right)$ & 30,000 \\
Annual Labor Cost per Inductor $\left(\ell_{t}\right)$ & 30,000 \\
Pack Station Cost per Station (Automated System) $\left(c_{q}^{a}\right)$ & 10,000 \\
Pack Station Cost per Station (Manual System) $\left(c_{q}^{m}\right)$ & 700 \\
Sorter Fixed Cost $\left(C_{t}^{s}\right)$ & 250,000 \\
Induction Station Cost per Station $\left(c_{t}\right)$ & 50,000 \\
\hline
\end{tabular}

- The maximum throughput in a system with split induction stations is represented by the following equation:

$$
\frac{2 r_{s}}{r_{s}+1} s
$$

\section{Relationship between Picking and Packing}

As mentioned earlier in Section 3.1, an important relationship exists between the picking standard and the packing standard. In manual systems, as the batch size being picked increases, the packing standard decreases. However, in automated systems, as the batch size being picked increases, the packing standard will remain approximately constant in most systems due to the nature of the orders as they arrive to the pack stations. As was shown previously in Figure 3.3, this relationship must be carefully reflected in the manual system model so as to make an accurate comparison with the automated system model.

In Figure 4.1 the relationship between picking and packing efficiencies is shown in two ways in the automated system curve. Realistically, if a picker retrieves more and more items, the packing efficiency will decrease slightly for the automated system. Although the sorter works at a constant rate, the packers will be required to move between packing stations, set up more shipping boxes and inserts, and organize more orders. This will cause the slight decrease in the packing efficiency. Decreasing efficiencies are more prevalent in systems without overlapping waves. The straight line represents a system with overlapping waves. In this system, the amount of time spent walking is insignificant. 


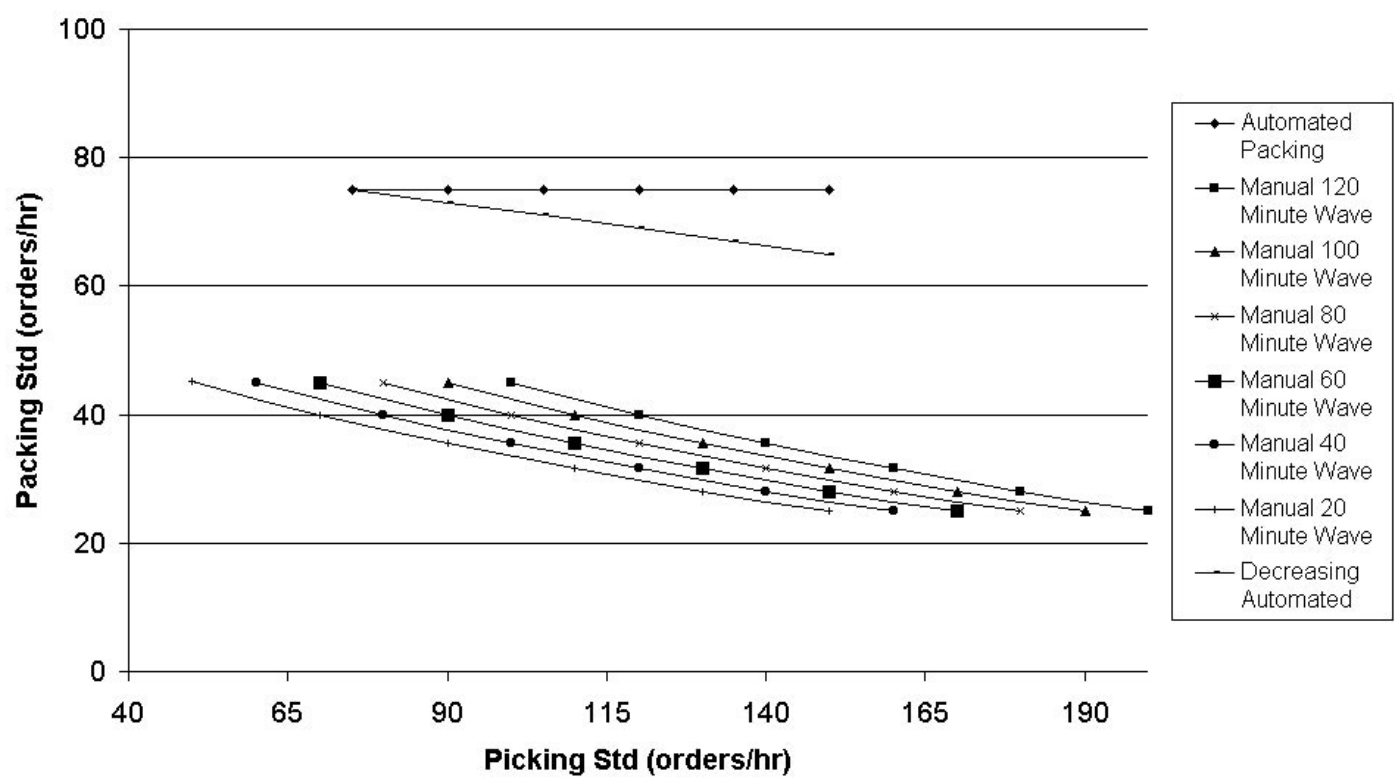

Figure 4.1: Decreasing Automated Picking Efficiency Curves.

Section 4.2 presents the relationships in the manual system. Characteristics and relationships in automated systems are discussed in Section 4.3. Example calculations for both a manual and an automated system configuration are presented in Section 4.4. Finally, a discussion of the experiments run for both systems is discussed in Section 4.5.

\subsection{Manual Sortation System}

A manual sortation system consists of all pickers, packers, and pack stations necessary to meet demand. Although automated systems also include pack stations, the manual pack stations are much less expensive. Manual pack stations include packing tables and all accessories, such as any shelfs, dunnage holders, and other items used to pack boxes. A simple conveyor system usually accompanies the entire packing area, which can also be included in the pack station cost.

The picking and packing relationship for the manual system is very different than the automated system, thus requiring more calculations to be made. This relationship, discussed in 
Section 3.3, requires a different range of picking and packing rates for each wave length. For instance, a 20-minute wave may only be able to pick from 75 to 125 orders per hour, while a 120-minute wave can pack between 135 and 185 orders per hour, based on the increase in efficiency due to intelligent order-batching. Therefore, for each wave length, the descriptive model tests a range of picking and packing efficiencies. The configuration in each wave that balances the number of pickers and packers in a way that results in the lowest total number of workers provides the best solution. Since the pack station cost in a manual system is so low, the number of pack stations tends to be irrelevant unless two picking and packing combinations have almost the same total number of employees.

\subsection{Automated Sortation System}

In the automated sortation system descriptive model, the input parameters include demand, picker, packer, and inductor rates, both fixed and variable capital costs, and the number of orders per pack station. The fixed cost includes the control system, scanner, and related hardware. Variable costs include the pack stations, induction stations, and trays or belts for the sorter. Pack station costs here include the chutes leading from the sorter to the pack station and all accessories, such as packing tables, conveyors, and shelving used at the pack tables. Automated systems must also consider the number of inductors and induction stations required to meet desired demand levels. Even though inductors usually work at a fast pace, the stations and additional trays or belts that must be purchased can be the additional cost that makes this system more expensive than a manual system.

Batch sizes increase as wave lengths increase with an automated sortation system. Since the sorter eliminates the effects of picking on the packing rates, the pickers can work at the highest rate possible. Packing rates remain fairly constant, with only a slight decrease in efficiency as the wave length or batch size increases. Also, since we are assuming that orders arrive to the pack station already individually sorted, the packer's rate will be much higher than a manual packer. The descriptive model takes all of these relationships into account, and calculates the total annual system cost for each of the common wave lengths (with only 
one picking and packing rate combination for each wave length tested). The combination of higher picking and packing rates results in fewer workers for the automated system over its manual counterpart.

\subsection{Example}

In order to illustrate the relationships developed in the descriptive model, a numerical example is presented. In this example, a point was selected from the picking efficiency curve to demonstrate the differences between the manual and automated sortation systems. The picking efficiency curve in this example is presented in Figure 4.2. The manual curve is valid for a 20-minute wave length, while on the automated curve, the point at $(75,75)$ is valid for the 20-minute wave length. The manual system has a different curve for each wave length, because as picking efficiency increases with a longer wave length (i.e., more items can be collected at one location as the number of orders per wave increases), the packing efficiency decreases. In the automated picking curve, the picking efficiency increases as each wave length increases, while packing efficiency remains constant regardless. Although we have considered only one point from the manual curve and one point from the automated curve, the spreadsheet model will perform the following calculations for each of the six combinations of picking and packing rates in the wave length for the manual system, and for each wave length for the automated system.

For this example, the parameters in Table 4.2 were used to calculate the labor requirements and costs, the amount of hardware or size of the sorter, and the total annual system cost. The table is separated into parameters used in both manual and automated systems, followed by parameters unique to manual systems, and finally parameters used only in automated systems. 
Table 4.2: Example Parameters.

\begin{tabular}{lcc} 
Parameter & Symbol & Value \\
\hline Demand & $D$ & 1100 orders $/ \mathrm{hr}$ \\
Items/order & $N_{o}$ & 3 items \\
Wave Length & $W$ & 20 minutes \\
Orders/Pack Station & $N_{q}$ & 25 orders \\
Labor Cost/Picker & $\ell_{p}$ & $\$ 30,000$ \\
Labor Cost/Packer & $\ell_{q}$ & $\$ 30,000$ \\
Interest Rate & $i$ & $20 \%$ \\
Years of Service & $y$ & 5 years \\
\hline Manual Picking Std & $p(n)$ & 50 orders/hr \\
Manual Packing Std & $q(m)$ & 45 orders/hr \\
Annualized Cost/Pack Station & $c_{q}^{m}$ & $\$ 234.08$ \\
\hline Conveyor Speed & $s$ & 100 items/minute \\
Induction Std & $t$ & 20 items $/ \mathrm{minute}$ \\
Picking Std (automated) & $p(n)$ & 75 orders/hr \\
Packing Std (automated) & $q(m)$ & 75 orders/hr \\
Labor Cost/Inductor & $\ell_{t}$ & $\$ 30,000$ \\
Annualized Cost/Induction Station & $c_{t}$ & $\$ 16,720$ \\
Annualized Cost/Pack Station & $c_{q}^{a}$ & $\$ 3,334$ \\
Annualized Fixed Cost of Sorter & $C^{s}$ & $\$ 83,600$ \\
\hline
\end{tabular}




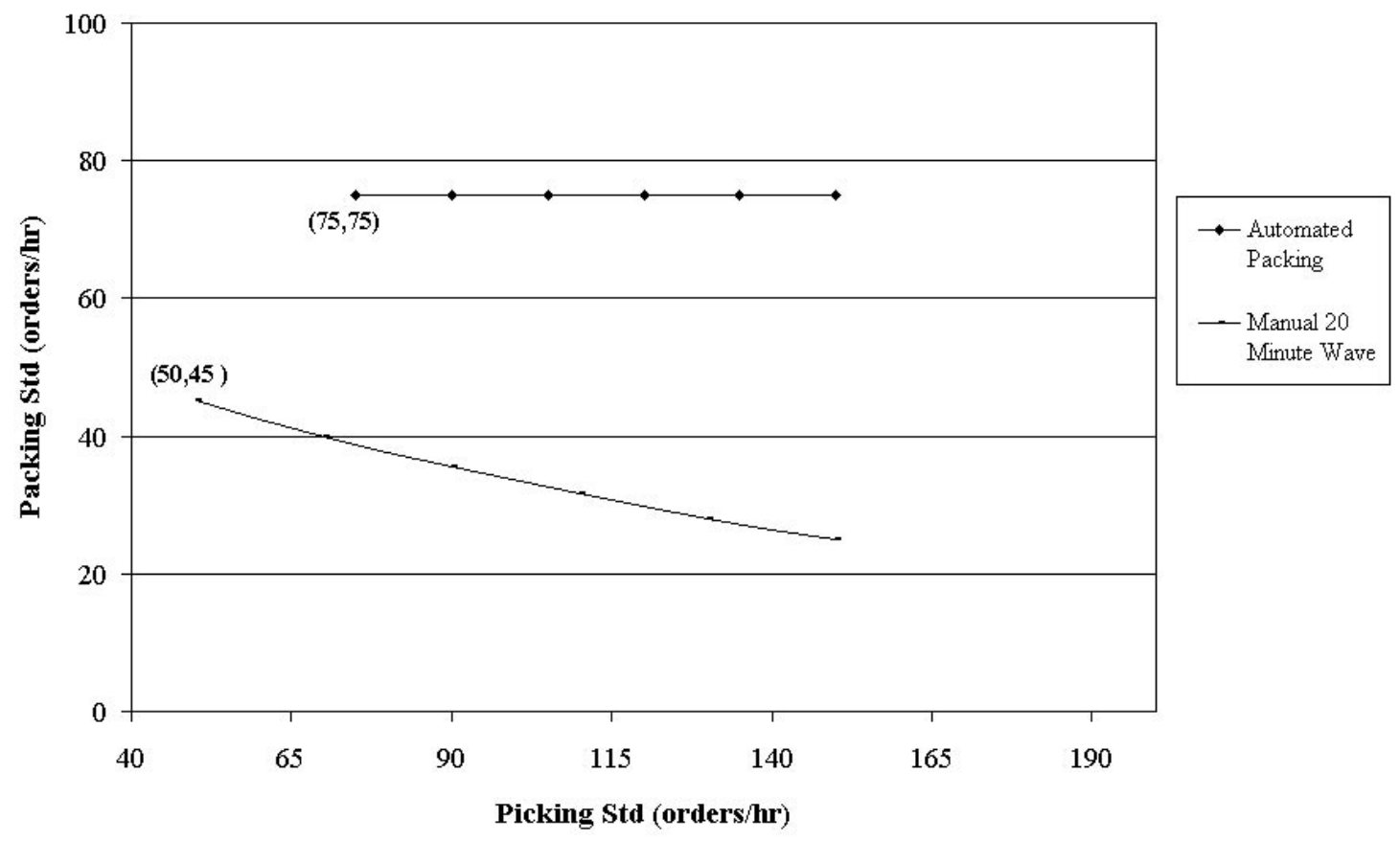

Figure 4.2: Example Picking vs. Packing Efficiency Curve.

\subsubsection{Calculations}

Sample calculations for a manual system with a 20-minute wave are presented in Table 4.3. Automated system calculations are presented in Table 4.4. Again, a 20-minute wave was used to make these calculations.

For the system, a basic tradeoff between the amount invested in automation and the amount of labor savings realized needs to be made. In this example, automation results in labor savings of $\$ 420,000$. However, the annual investment of the manual system is $\$ 144,628$ less than the automated system, mainly because of the expense of the pack stations, the sorter, and the accompanying hardware. Overall, this tradeoff suggests that an automated system should be selected, resulting in annual savings of approximately $\$ 275,372$.

Again, these calculations are repeated for all possible combinations. That is, for each combination of picking and packing rates within a wave for a manual system, calculations such as those in Table 4.3 are performed. This results in six identical sets of calculations for each 
Table 4.3: Example Calculations for the Manual System.

\begin{tabular}{|c|c|c|c|}
\hline Variable & Symbol & $\begin{array}{c}\text { Formula } \\
\text { Calculation }\end{array}$ & Value \\
\hline Items/Wave & $N_{w}$ & $\begin{array}{l}\left(D * N_{o} * W\right) / 60 \\
(1100 * 3 * 20) / 60\end{array}$ & 1100 \\
\hline Pickers & $r_{p}$ & $\begin{array}{c}\lceil D / p(n)\rceil \\
\lceil(1100 / 50)\rceil\end{array}$ & 22 \\
\hline $\begin{array}{l}\text { Minimum Required } \\
\text { Packers }\end{array}$ & $r_{q}$ & $\begin{array}{c}\lceil D / q(m)\rceil \\
\lceil(1100 / 45)\rceil\end{array}$ & 25 \\
\hline $\begin{array}{l}\text { Minimum Required } \\
\text { Pack Stations }\end{array}$ & $n_{q}$ & $\begin{array}{c}\left\lceil N_{w} / N_{q}\right\rceil \\
\lceil(1100 / 75)\rceil\end{array}$ & 15 \\
\hline $\begin{array}{l}\text { Actual Number of Packers } \\
\text { and Pack Stations }\end{array}$ & $r_{a}$ & $\begin{array}{l}\operatorname{maximum}\left\{r_{q}, n_{q}\right\} \\
\operatorname{maximum}\{25,15\}\end{array}$ & 25 \\
\hline $\begin{array}{l}\text { Total Annual } \\
\text { Labor Cost }\end{array}$ & $T_{\ell}^{m}$ & $\begin{array}{c}\ell_{p} r_{p}+\ell_{q} r_{q} \\
30000 *(22+25) \\
\end{array}$ & $\$ 1,410,000$ \\
\hline $\begin{array}{l}\text { Total Annual } \\
\text { Manual System Cost }\end{array}$ & $T^{m}$ & $\begin{array}{c}T_{\ell}^{m}+r_{q} c_{q} \\
1410000+25 * 234.08\end{array}$ & $\$ 1,415,852$ \\
\hline
\end{tabular}

of the wave lengths considered. This equates to thirty-six total sets of calculations when 20minute to 2-hour wave lengths are considered. The automated system calculations such as those in Table 4.4 are performed for each wave length considered in the model. This equates to six sets of calculations like those in Table 4.4 when 20-minute to 2-hour wave lengths are considered. The prescriptive deterministic model considers all possible combinations (e.g., forty-two total combinations when six batching levels and six wave lengths are considered) and uses the results from the descriptive model to determine the solution with the lowest annual cost. Results from experiments with a demand level of 1100 orders per hour are presented in Appendix $\mathrm{C}$ for both manual and automated systems. Notice that this example does not provide the best solution for either the manual or the automated system.

As we can see in the calculations in Table 4.4, although the capital investment of a sorter and more expensive pack stations is quite high, the labor savings are significant enough to make automation preferable. This will not always be the case. This example used a relatively low sorter fixed cost. In our following experiments with the model, we will consider many scenarios and cost ranges, as well as a variety of picking and packing efficiency curves. 
Table 4.4: Example Calculations for the Automated System.

\begin{tabular}{|c|c|c|c|}
\hline Variable & Symbol & $\begin{array}{c}\text { Formula } \\
\text { Calculation }\end{array}$ & Value \\
\hline Items/Wave & $N_{w}$ & $\begin{array}{l}\left(D * N_{o} * W\right) / 60 \\
(1100 * 3 * 20) / 60\end{array}$ & 1100 \\
\hline Pickers & $r_{p}$ & $\begin{array}{c}\lceil D / p(n)\rceil \\
\lceil(1100 / 75)\rceil\end{array}$ & 15 \\
\hline Total Induction Rate & $x$ & $\begin{array}{c}D\left(N_{o} / 60\right) \\
(1100 * 3) / 60\end{array}$ & 55 \\
\hline $\begin{array}{l}\text { Minimum Required } \\
\text { Packers }\end{array}$ & $r_{q}$ & $\begin{array}{c}\lceil D / q(m)\rceil \\
\lceil(1100 / 75)\rceil\end{array}$ & 15 \\
\hline $\begin{array}{l}\text { Minimum Required } \\
\text { Pack Stations }\end{array}$ & $n_{q}$ & $\begin{array}{c}\left\lceil N_{w} / N_{q}\right\rceil \\
\lceil(1100 / 75)\rceil \\
\end{array}$ & 15 \\
\hline $\begin{array}{l}\text { Actual Number of Packers } \\
\text { and Pack Stations }\end{array}$ & $r_{a}$ & $\begin{array}{l}\operatorname{maximum}\left\{r_{q}, n_{q}\right\} \\
\operatorname{maximum}\{15,15\}\end{array}$ & 15 \\
\hline $\begin{array}{l}\text { Induction } \\
\text { Stations }\end{array}$ & $r_{s}$ & $\begin{array}{c}1, \text { since } x<s \\
1\end{array}$ & 1 \\
\hline Inductors & $r_{t}$ & $\begin{array}{l}\lceil(x / t)\rceil \\
\lceil 55 / 20\rceil\end{array}$ & 3 \\
\hline $\begin{array}{l}\text { Total Annual } \\
\text { Labor Cost }\end{array}$ & $T_{\ell}^{a}$ & $\begin{array}{c}\ell_{p} r_{p}+\ell_{q} r_{q}+\ell_{i} r_{t} \\
30000 *(15+15+3)\end{array}$ & $\$ 990,000$ \\
\hline $\begin{array}{l}\text { Total Annual } \\
\text { Automated System Cost }\end{array}$ & $T^{a}$ & $\begin{array}{c}T_{\ell}^{a}+C_{s}+r_{a} c_{q}+r_{s} c_{t} \\
(990000+83600+15 * 3344+1 * 16720)\end{array}$ & $\$ 1,140,480$ \\
\hline
\end{tabular}

\subsection{Model Experiments}

Using the spreadsheet created to model this system, several experiments were tested. Different efficiency curves describing the relationship between picking and packing standards were input to determine the effect this curve has on the model. In addition, wave lengths of sizes varying from 20 minutes to two hours were tested. Each picking and packing standard was input for each wave length.

Additionally, for the automated system, two general relationships between picking and packing were investigated. First, picking standards were increased, while packing standards remained constant. This relationship assumes that the automated sorter adequately sorts the work to pack stations independently of the picking efficiency. Second, picking standards were increased, while packing standards slightly decreased. This relationship assumes that a decrease in packing efficiency would be attributed to the time spent by packers moving 
between pack stations for multiple orders. Packers must move between stations since the packer can pack more orders in a wave than one station's capacity. This decrease can also be attributed to the amount of time needed to prepare shipping boxes and additional items placed in the box with the order, such as packing slips, inserts (magazines, coupons), and dunnage. This type of relationship is found in a system with no overlap.

The sensitivity analysis in Chapter 5 provides insight into how the input parameters interact with one another in each combination of picking and packing rates for both automated and manual systems. Each time a parameter is changed, a full set of model experiments is run to determine changes in the recommended system design. 


\section{Chapter 5}

\section{Sensitivity Analysis}

In order to determine the effects of changes in each parameter, a sensitivity analysis was conducted. This analysis determined what parameter changes resulted in a shift to a different picking scheme and standard as well as the best overall solution for a wave length. This solution includes the type of system (automated or manual), the number of pickers, packers, and inductors (if an automated system is selected), the wave length that should be used, and the total annual cost for the system. Each parameter was increased and decreased until changes can be seen. In some cases, the amount of increase or decrease might be unrealistic, but still provides valuable information about the relationships within the model. Table 5.1 shows each factor that was changed and the anticipated effect it had on the decisions to be made.

Clearly, with as many factors and anticipated effects as are presented in Table 5.1, we cannot look at all possible combinations. Our methodology was to begin with the standard set of parameters for a problem and determine the solution. As mentioned above, the solution defines the type of system (automated or manual), the number of pickers, packers, and inductors (if automated), the wave length, and the picking methodology (as defined by the picking versus packing curves). We then changed a factor to determine the sensitivity of the solution to the factor. 
Table 5.1: Sensitivity Analysis.

\begin{tabular}{lc} 
Parameter Changed & Anticipated Effect on Decision \\
\hline Picker or Packer Cost & Wave Length, Automated vs Manual \\
Inductor Cost & Wave Length, Automated vs Manual (only if many inductors needed) \\
Automated Pack Station Costs & Wave Length, Automated vs Manual \\
Picker or Packer Std Rates & Number of Required Workers, Wave Length, Automated vs Manual \\
Inductor Rates & Number of Inductors, Wave Length, Automated vs Manual \\
Sorter Costs & Wave Length, Automated vs Manual \\
Demand & Everything \\
Wave Size & Everything \\
\hline
\end{tabular}

\subsection{Methodology}

The sensitivity analysis was conducted in the following manner. Five factors we deemed to be important were tested, including demand, sorter cost, labor costs, pack station costs (for the automated system), and induction rates. A full factorial experiment was completed, where each of the five factors was tested with each combination of the other factors. Table 5.2 shows the experiments run for a sorter cost of $\$ 250,000$. In this table, wave length is abbreviated WL and pack station is abbreviated PS. Similar test for sorter costs of $\$ 500,000$ and $\$ 1,000,000$ were conducted. A full table of experiments and results can be seen in Appendix D (Tables D.1, D.2, D.3, and D.4) for each of the five factors. Each experiment consists of using the experimental parameters as input data into the deterministic spreadsheet model, which then finds the best solution over the range of wave lengths and picking and packing efficiencies. The results from this experiment are the best automated and manual solutions over these ranges. From this information, we can see the effects on the solutions as well as the difference between automated and manual solutions. Also, we can make generalizations as to when automation is preferred over manual sortation, based on varying parameters. 
Table 5.2: Sensitivity Analysis Experiments for Sorter Cost of $\$ 250,000$.

\begin{tabular}{|c|c|c|c|c|c|c|c|c|}
\hline $\begin{array}{c}\text { Demand } \\
\text { Level } \\
\text { (orders/hr) }\end{array}$ & $\begin{array}{c}\text { Labor } \\
\text { Cost } \\
(\$) \\
\end{array}$ & $\begin{array}{c}\text { PS Cost } \\
\text { (Auto) } \\
(\$)\end{array}$ & $\begin{array}{c}\text { Induction } \\
\text { Rate } \\
\text { (items/min) }\end{array}$ & $\begin{array}{c}\text { Manual } \\
\text { Best } \\
(\$) \\
\end{array}$ & $\begin{array}{c}\text { WL } \\
\text { Manual } \\
\text { (min) }\end{array}$ & $\begin{array}{c}\text { Automated } \\
\text { Best } \\
(\$) \\
\end{array}$ & $\begin{array}{l}\text { WL } \\
\text { Auto } \\
\text { (min) }\end{array}$ & $\begin{array}{c}\text { Difference } \\
(\mathrm{M}-\mathrm{A}) \\
(\$)\end{array}$ \\
\hline 550 & 18000 & 2,500 & 20 & $\begin{array}{c}350,661 \\
\end{array}$ & 100 & 389,104 & 120 & $(38,443)$ \\
\hline 550 & 18000 & 2,500 & 60 & 350,661 & 100 & 371,104 & 120 & $(20,443)$ \\
\hline 550 & 18000 & 10,000 & 20 & 350,661 & 100 & 451,072 & 20 & $(100,411)$ \\
\hline 550 & 18000 & 10,000 & 60 & 350,661 & 100 & 433,072 & 20 & $(82,411)$ \\
\hline 550 & 18000 & 20,000 & 20 & 350,661 & 100 & 477,824 & 20 & $(127,163)$ \\
\hline 550 & 18000 & 20,000 & 60 & 350,661 & 100 & 459,824 & 20 & $(109,163)$ \\
\hline 550 & 30000 & 2,500 & 20 & 578,661 & 100 & 557,104 & 120 & 21,557 \\
\hline 550 & 30000 & 2,500 & 60 & 578,661 & 100 & 527,104 & 120 & 51,557 \\
\hline 550 & 30000 & 10,000 & 20 & 578,661 & 100 & 650,640 & 80 & $(71,979)$ \\
\hline 550 & 30000 & 10,000 & 60 & 578,661 & 100 & 620,640 & 80 & $(41,979)$ \\
\hline 550 & 30000 & 20,000 & 20 & 578,661 & 100 & 693,824 & 20 & $(115,163)$ \\
\hline 550 & 30000 & 20,000 & 60 & 578,661 & 100 & 663,824 & 20 & $(85,163)$ \\
\hline 1100 & 18000 & 2,500 & 20 & 668,599 & 120 & 641,888 & 120 & 26,711 \\
\hline 1100 & 18000 & 2,500 & 60 & 668,599 & 120 & 605,888 & 120 & 62,711 \\
\hline 1100 & 18000 & 10,000 & 20 & 668,599 & 120 & 744,480 & 20 & $(75,881)$ \\
\hline 1100 & 18000 & 10,000 & 60 & 668,599 & 120 & 708,480 & 20 & $(39,881)$ \\
\hline 1100 & 18000 & 20,000 & 20 & 668,599 & 120 & 794,640 & 20 & $(126,041)$ \\
\hline 1100 & 18000 & 20,000 & 60 & 668,599 & 120 & 758,640 & 20 & $(90,041)$ \\
\hline 1100 & 30000 & 2,500 & 20 & $1,100,599$ & 120 & 953,888 & 120 & 146,711 \\
\hline 1100 & 30000 & 2,500 & 60 & $1,100,599$ & 120 & 893,888 & 120 & 206,711 \\
\hline 1100 & 30000 & 10,000 & 20 & $1,100,599$ & 120 & $1,117,456$ & 60 & $(16,857)$ \\
\hline 1100 & 30000 & 10,000 & 60 & $1,100,599$ & 120 & $1,057,456$ & 60 & 43,143 \\
\hline 1100 & 30000 & 20,000 & 20 & $1,100,599$ & 120 & $1,190,640$ & 20 & $(90,041)$ \\
\hline 1100 & 30000 & 20,000 & 60 & $1,100,599$ & 120 & $1,130,640$ & 20 & $(30,041)$ \\
\hline 2200 & 18000 & 2,500 & 20 & $1,319,198$ & 120 & $1,254,176$ & 120 & 65,022 \\
\hline 2200 & 18000 & 2,500 & 60 & $1,319,198$ & 120 & $1,146,176$ & 120 & 173,022 \\
\hline 2200 & 18000 & 10,000 & 20 & $1,319,198$ & 120 & $1,477,360$ & 20 & $(158,162)$ \\
\hline 2200 & 18000 & 10,000 & 60 & $1,319,198$ & 120 & $1,369,360$ & 20 & $(50,162)$ \\
\hline 2200 & 18000 & 20,000 & 20 & $1,319,198$ & 120 & $1,577,680$ & 20 & $(258,482)$ \\
\hline 2200 & 18000 & 20,000 & 60 & $1,319,198$ & 120 & $1,469,680$ & 20 & $(150,482)$ \\
\hline 2200 & 30000 & 2,500 & 20 & $2,171,198$ & 120 & $1,914,176$ & 120 & 257,022 \\
\hline 2200 & 30000 & 2,500 & 60 & $2,171,198$ & 120 & $1,734,176$ & 120 & 437,022 \\
\hline 2200 & 30000 & 10,000 & 20 & $2,171,198$ & 120 & $2,241,312$ & 60 & $(70,114)$ \\
\hline 2200 & 30000 & 10,000 & 60 & $2,171,198$ & 120 & $2,061,312$ & 60 & 109,886 \\
\hline 2200 & 30000 & 20,000 & 20 & $2,171,198$ & 120 & $2,417,680$ & 20 & $(246,482)$ \\
\hline 2200 & 30000 & 20,000 & 60 & $2,171,198$ & 120 & $2,237,680$ & 20 & $(66,482)$ \\
\hline 3300 & 18000 & 2,500 & 20 & $1,987,797$ & 120 & $2,025,904$ & 120 & $(38,107)$ \\
\hline 3300 & 18000 & 2,500 & 60 & $1,987,797$ & 120 & $1,755,904$ & 120 & 231,893 \\
\hline 3300 & 18000 & 10,000 & 20 & $1,987,797$ & 120 & $2,348,336$ & 20 & $(360,539)$ \\
\hline 3300 & 18000 & 10,000 & 60 & $1,987,797$ & 120 & $2,078,336$ & 20 & $(90,539)$ \\
\hline 3300 & 18000 & 20,000 & 20 & $1,987,797$ & 120 & $2,495,472$ & 20 & $(507,675)$ \\
\hline 3300 & 18000 & 20,000 & 60 & $1,987,797$ & 120 & $2,225,472$ & 20 & $(237,675)$ \\
\hline 3300 & 30000 & 2,500 & 20 & $3,271,797$ & 120 & $3,117,904$ & 120 & 153,893 \\
\hline 3300 & 30000 & 2,500 & 60 & $3,271,797$ & 120 & $2,667,904$ & 120 & 603,893 \\
\hline 3300 & 30000 & 10,000 & 20 & $3,271,797$ & 120 & $3,638,608$ & 60 & $(366,811)$ \\
\hline 3300 & 30000 & 10,000 & 60 & $3,271,797$ & 120 & $3,188,608$ & 60 & 83,189 \\
\hline 3300 & 30000 & 20,000 & 20 & $3,271,797$ & 120 & $3,851,472$ & 20 & $(579,675)$ \\
\hline 3300 & 30000 & 20,000 & 60 & $3,271,797$ & 120 & $3,401,472$ & 20 & $(129,675)$ \\
\hline
\end{tabular}




\subsection{Results}

As mentioned before, five main input parameters were tested in the sensitivity analysis of the prescriptive spreadsheet model. Of the five input parameters that were varied in the deterministic model, only four proved to be significant in determining whether a system should have automated sortation or not. The parameter that tended to not be significant was the induction rate since the number of inductors required in a system remains fairly low, even for a system with high demands. However, in experiments where a manual system has a relatively close cost to the automated system, the induction rate may end up being the deciding factor. This just proves that every input factor is important in some cases where the decision is more difficult. Results from demand levels of 550 and 3300 orders per hour are presented in Figures D.1 and D.4, where pack station costs are abbreviated as "PS Cost," and labor costs are abbreviated as "L". In each of these figures, the interest rate and life used are $20 \%$ and 5 years, respectively.

In what follows, each input parameter will be discussed in detail. Then the effects of varying parameters simultaneously will be discussed. For each separate parameter, the base case will be tested. The base case considered is a demand level of 1100 orders per hour, a sorter cost of $\$ 250,000$, labor costs of $\$ 30,000$ per employee, pack station costs of $\$ 10,000$ per pack station for the automated system and $\$ 700$ for the manual system, and induction rates of 20 trays per minute (items per minute). The total annual system cost for the base case is $\$ 1,100,599$ for the best manual system. This configuration requires 11 pickers, 25 packers, and 88 pack stations. The automated system for the base case costs $\$ 1,117,456$ with 11 pickers, 15 packers, 44 pack stations, 3 inductors, and 1 induction station. The manual system is recommended here, mainly due to the high cost of pack stations in an automated system.

\subsubsection{Demand}

Demand levels have the largest effect on a recommended system configuration. Intuition leads to the conclusion that at very low demand levels, automation would not be feasible for 
a company. The cost of the sorter would add a significant amount to the total annual system cost, and greatly overshadows the cost of labor for a low demand. In contrast, at very high demand levels, the number of employees required to meet demand makes the total annual system cost for the manual system extremely high. Regardless of the sorter costs input, the labor costs remain a very high percentage of the total cost.

In addition to the base demand levels, three additional levels of demand were used to calculate the total annual system cost and determine labor, pack station, and induction station requirements. In general, increasing demand to 3300 orders per hour causes a large increase. With the same parameters, the manual system requires 33 pickers, 74 packers, and 264 pack stations at a cost of $\$ 3,271,797$. However, the automated system is still more expensive, at a cost of $\$ 3,305,168$. This configuration requires less workers (32 pickers, 44 packers, and 15 inductors), but requires 132 pack stations and 3 induction stations. The pack stations and induction stations cost approximately $\$ 311,000$ for the automated system, and the pack stations in the manual system are only $\$ 20,600$.

It would seem as though if demand were increased enough, labor requirements would be high enough to justify the capital costs involved in purchasing a sorter. However, the additional pack stations required make the automated system even less attractive. Induction stations would also need to be added to meet demand at high levels. Since it is very rare to have more than four induction stations, the requirement of more induction stations eventually requires another sorter due to size limitations.

Lowering demand to 550 orders per hour has a significant effect on the recommended system configuration. Large labor reductions are achieved when demand is lowered. The manual system requires only 5 pickers, 14 packers, and 37 pack stations in the recommended system at a cost of $\$ 578,661$. The automated system requires 5 pickers, 8 packers, 30 pack stations, and 1 induction station. Although this system reduces labor requirements by 5 workers, the $\$ 150,000$ saved does not make up for the cost of pack stations and the induction station.

By comparing Figures 5.1 and 5.2, we can see that demand has a large effect on the type of system recommended. In these figures, a negative (down) bar represents a manual system recommendation, while a positive (up) bar represents an automated system recommenda- 
tion. In the system with low demand, automated systems are rarely recommended, but in systems with high demand, automation is recommended quite often. Keep in mind that the combination of other parameters, which will be discussed in Section 5.2.6, has an impact on this decision as well.

\subsubsection{Labor Costs}

Labor costs are a high percentage of the total annual cost of an order fulfillment system. For example, in the base case discussed above, the manual system requires 36 employees and the automated system requires 29 employees. Labor costs for each represent over $77 \%$ of the total annual cost. Therefore, when labor costs are reduced, it makes a significant impact on the system configuration and costs.

When labor costs are lowered to $\$ 18,000$ a year per employee in the base case, the total annual cost for the manual system is reduced to $\$ 668,600$ and the automated system is reduced to $\$ 744,480$. The manual system is more likely to be recommended at lower labor costs since the labor costs will be a lower percentage of the total annual cost.

With a labor cost per employee of $\$ 33,000$ a year, the manual system has a slightly higher total annual system cost of $\$ 1,208,599$, which is compared to the automated system at $\$ 1,204,456$. Any labor cost higher than this with the base case recommends an automated system since the savings due to labor increase while the other costs remain constant.

Looking at Figure 5.1, we see that although manual systems are usually preferred over automated systems, the labor cost can be the deciding factor. For example, with a low sorter cost of $\$ 250,000$, and a low pack station cost of $\$ 2,500$, the higher labor costs of $\$ 30,000$ result in the automated system having a lower cost than the manual system.

\subsubsection{Pack Station Costs}

Surprisingly, pack station costs have a significant effect on the total annual system cost. Automated systems require more expensive pack stations that hold more than one order, 


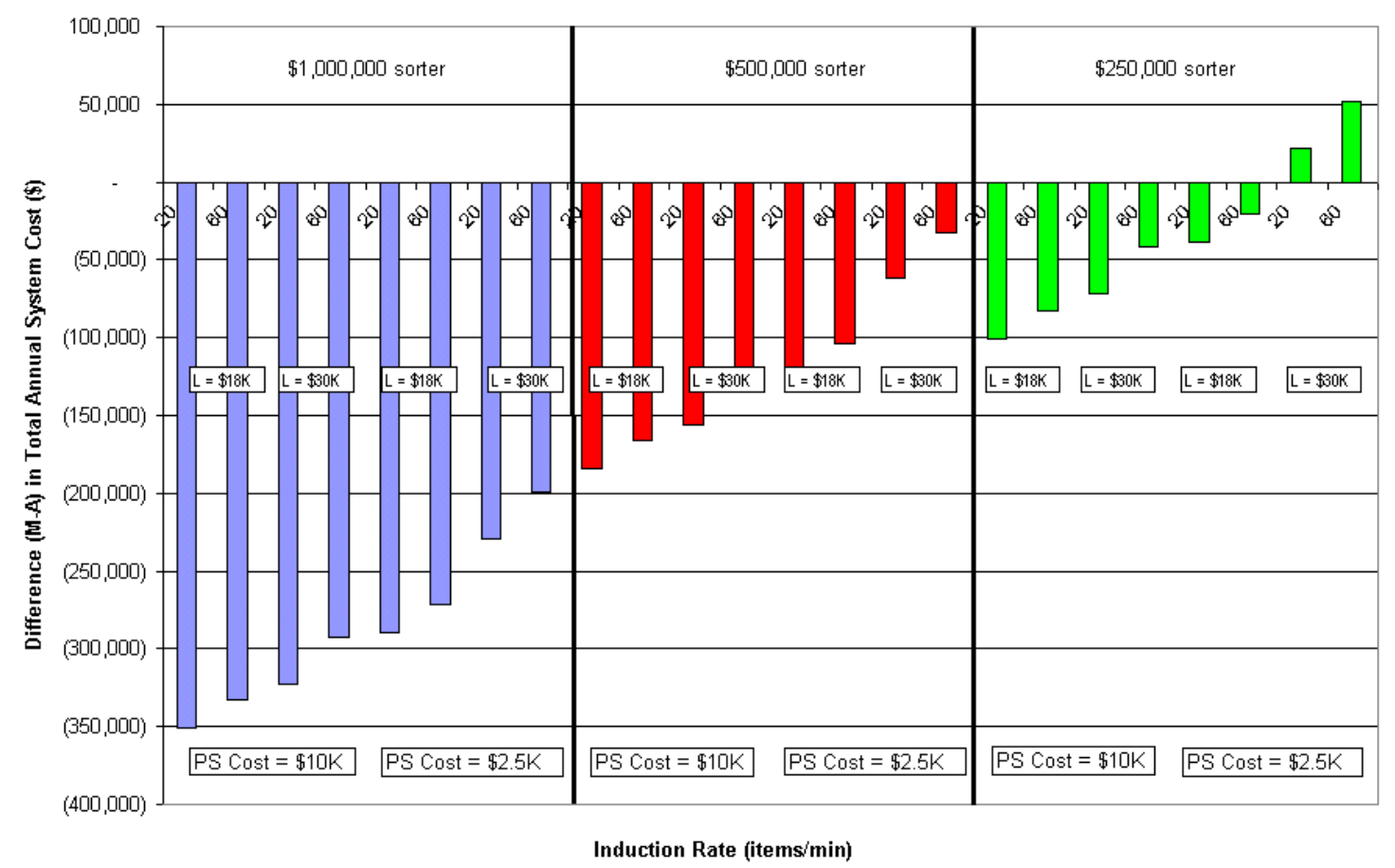

Figure 5.1: Sensitivity Data at Demand $=550$ Orders $/$ Hour.

usually containing several bins to hold the sorted orders. These stations are fairly expensive because of the equipment connecting the sorter to the bins. Manual systems require very simple pack stations, consisting of only a packing table and inexpensive accessories.

Since the manual pack stations are so inexpensive, the cost were not varied. However, three costs were considered for the automated system pack station costs. The base case of $\$ 10,000$ per pack station was raised to $\$ 20,000$ (not shown in Figure 5.1) and lowered to $\$ 2,500$ per pack station.

The base case requires 44 pack stations for the best automated system, at a total cost of $\$ 294,272$. A pack station cost of $\$ 20,000$ raises the total cost of pack stations to $\$ 588,544$. Lowering the pack station cost to $\$ 2,500$ per pack station lowers the total cost of pack stations to $\$ 73,568$, which results in the automated system being recommended. This automated system has a total annual system cost of $\$ 953,888$. At the highest pack station cost, none of the combinations result in a recommendation of automation. In fact, even with a $\$ 10,000$ 


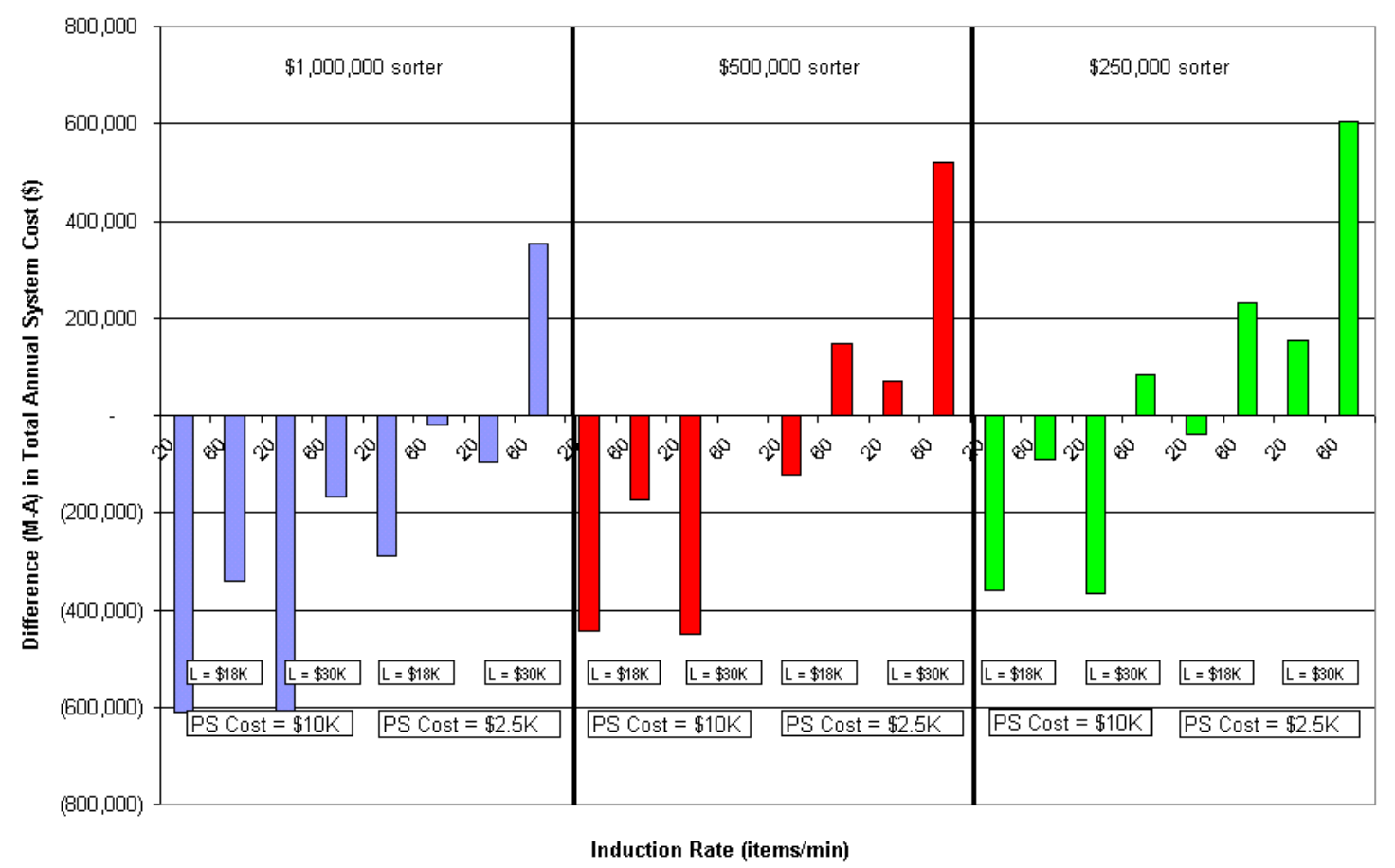

Figure 5.2: Sensitivity Data at Demand $=3300$ Orders/Hour.

cost per pack station, only the combination of a low sorter cost, high labor cost, and high induction rate results in the recommendation of an automated system.

\subsubsection{Sorter Costs}

At relatively low demand levels, such as the base level of 1100 orders per hour, the cost of labor outweighs the capital costs involved with purchasing a sorter only if the sorter cost is low. The base sorter cost of $\$ 250,000$ is not low enough to outweigh the labor costs of a manual system. However, lowering this cost to $\$ 199,500$ will result in an automated system with a total annual cost $\$ 30$ lower than the manual system. Of course, this sorter cost is unrealistically low.

The three levels of sorter cost were $\$ 250,000, \$ 500,000$, and $\$ 1,000,000$. Each cost is annualized by a capital recovery factor $(A / P)$ of 0.3344 . Each cost increase makes it harder for the automated system to outperform the manual system, regardless of the demand level. Other 
parameters must be adjusted to recommend the systems with higher sorter costs.

\subsubsection{Induction Rates}

At the base demand level of 1100 orders per hour and an induction rate of 20 items per minute, only 3 inductors are required, resulting in an automated system cost of $\$ 1,117,456$. With an induction rate increase to 60 items per minute, the system annual cost reduces to $\$ 1,057,456$, which is lower than the manual system cost of $\$ 1,100,599$. Although this parameter seems to make little difference in the system, it reduces the manpower requirements from 3 inductors to 1 inductor, for a savings of $\$ 60,000$. This is an example of how varying the induction rate can change the system recommendations when the two systems (automated and manual) have similar annual costs.

When demand levels are higher or lower, the two systems will have a larger gap in their total annual costs, making the induction rate parameter less significant.

\subsubsection{Combination of Parameters}

Sensitivity analyses showed that varying a single parameter may not change the recommended system configuration, but varying several parameters simultaneously has a significant effect on the system.

For example, at a demand level of 1100 orders per hour, a high sorter cost of $\$ 1,000,000$ and pack station costs of $\$ 20,000$ results in a manual system recommendation for every combination of other parameters tested. At high demand levels, with low sorter costs and low pack station costs ( $\$ 250,000$ and $\$ 2,500$, respectively), the automated sortation system is recommended for every combination of other parameters, except with low labor costs and low induction rates. However, at the same demand level, a medium pack station cost $(\$ 10,000)$ results in mixed recommendations that depend on the induction rate. A high induction rate results in an automated sortation system recommendation, while a low induction rate results in manual sortation. 
A high demand level with a high sorter cost $(\$ 1,000,000)$ and high or medium pack station costs always resulted in recommending the manual system. However, the same system with a low pack station cost varied based on the labor costs. Low labor costs resulted in a manual recommendation, while high labor costs resulted in an automated system recommendation.

At low demand levels, a high sorter cost results in a manual system in every experiment. A medium sorter cost $(\$ 500,000)$, also results in a manual recommendation. Only at low sorter costs with low pack station costs and high labor costs $(\$ 30,000)$ is the automated system recommended.

In general, we can see that although one parameter change might not be enough to cause the system recommendation to change, combinations may result in different configurations. A company can vary the costs to determine which would be more beneficial based on their needs.

\subsection{Results for Order-Batching in a Manual System}

In addition to the effects of changing parameter values, a sensitivity analysis was conducted to determine the effects of batching in both automated and manual systems. Order-batching has a significant impact on the resources needed to meet demand. This section describes the

effects of order-batching as well as lengthening the wave in a manual system. First, however, we describe an approximate model to determine both the picking and packing rates, therefore providing the optimal level of order-batching.

\subsubsection{Points Along a Curve}

As discussed earlier, points on an efficiency curve (see Figure 3.3) represent a change in order-batching for a picker. Each point is a different picking rate that reflects the number of orders picked in a batch. As the batch size increases, the picking rate increases as well since the picker may be able to reduce the number of aisles traversed. This in turn will reduce the number of pickers required to meet demand, but will also increase the number of packers 
required.

Because of this tradeoff, we experimented with different combinations of picking and packing rates for each wave length tested. The combinations varied from the picker's lowest rate to the highest, and then considered the packing rates associated with that batch size. From these combinations, we identified the lowest total annual system cost, and noted the tradeoffs that were made. The combinations represent the points on the curve for each wave length. For instance, at the base demand level of 1100 orders per hour, a picking rate of 50 orders per hour requires 22 pickers and 25 packers. On the other hand, increasing the picking standard to 110 orders per hour requires only 10 pickers, but increases the number of packers to 35 . In this case, increasing the picking rate (which results from a batch size increase) reduces the total annual system cost by $\$ 57,659$ since the increase in picking rate reduces the number of employees by 2 , saving $\$ 60,000$, but it also adds 10 pack stations at an annualized cost of $\$ 2,341$. The addition of pack stations is insignificant in this situation, but would not be the case in an automated system with high pack station costs.

Since the number of pickers decreases with a picking rate increase (or batch size increase), the number of packers increases. Therefore, the level of each that minimizes the total annual system cost may not be the largest batch size. In fact, this would never be the case unless the packing rate did not decrease as rapidly as the picking rate increased. The addition of pack stations would also be important if the pack station cost were higher. In this example, the recommendation (from the 20-minute wave) is a picking rate of 70 orders per hour, which requires 16 pickers and 28 packers at a cost of $\$ 1,326,554$. This solution requires one less employee than the picking rate of 110 orders per hour.

\section{Shifting Curves and Wave Length Increases}

As the wave length increases, the manual efficiency curves shift up and to the right. The packing rate will stay constant, because the packers will receive the same proportion of orders in a wave (i.e., twice as much in a wave twice as long). However, the pickers become more efficient because they have a better selection of orders from which to batch. 
For example, the 20-minute wave length has a picking standard of 50 orders per hour corresponding to the packing rate of 45 orders per hour. This level of batching is low since the pickers only have 20 minutes worth of orders to pick. This system requires 22 pickers and 25 packers at a cost of $\$ 1,415,852$. If the wave length increases to 60 minutes, the picking standard can increase because the pickers will batch in a way that will save multiple trips to one location or aisle. This level of batching has a picking rate of 70 orders per hour, with the packing rate remaining constant at 45 orders per hour. This level of batching requires only 16 pickers and 25 packers at a cost of $\$ 1,240,300$. By increasing the wave length by 40 minutes, the company would save $\$ 175,552$ annually. Again, when considering only the labor costs, the longer the wave length, the better the system cost.

\section{Relationship Between Each Combination}

Now that we have considered the two main relationships shown in the efficiency curves (i.e., shifting along the curve and shifting from curve to curve), we now consider the effects of the combined relationship. The shift along the curve represents batching levels, and a level which minimizes the total number of pickers and packers is the recommended combination of picking and packing rates at that wave length. Based on average picking and packing rates provided to us by distribution centers we have contacted, pickers can work at a much faster rate than packers. Reasons for this include the intelligent batching methods and pick rate increases due to improving order-picking technologies (e.g., pick to light) and the manual sorting required by packers. Also, the packers have more setup time and additional items to pack such as inserts or packing material.

Since pickers, in general, work at a faster rate than packers, the results show that a low or average picking rate is usually selected, since doing so will ensure the packing rate does not fall too drastically. Also, as the wave length increases, it appears that the batch size is typically reduced to maintain a high level of picking efficiency and improve the packing efficiency. 


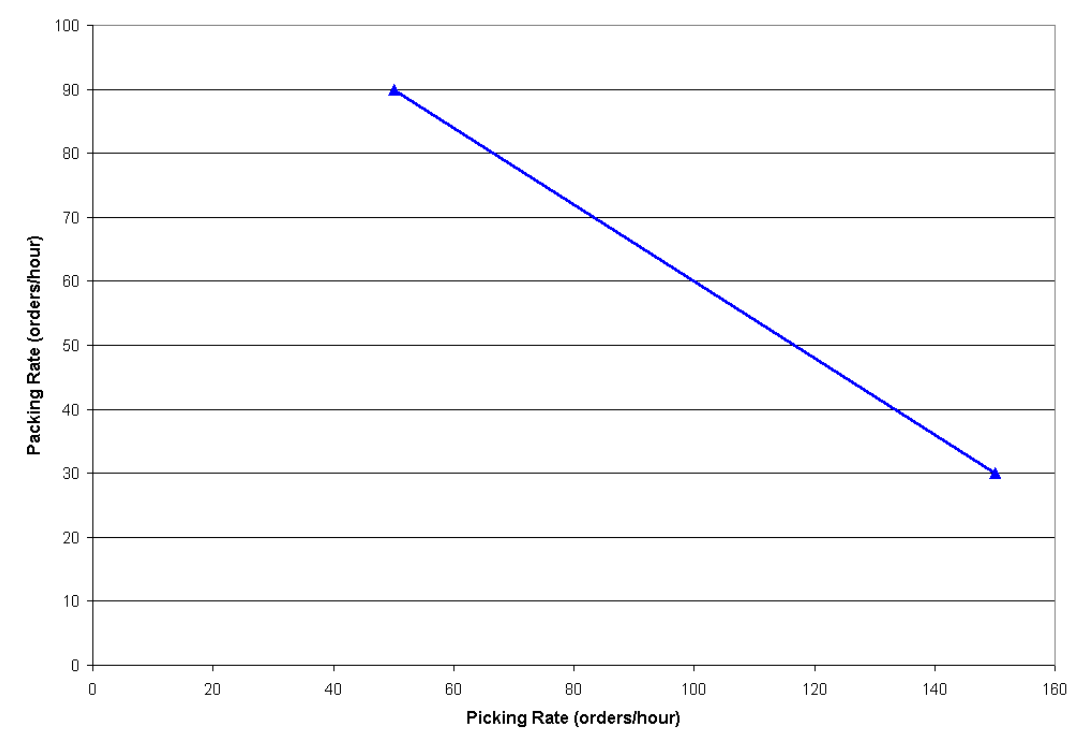

Figure 5.3: Estimated Picking vs. Packing Relationship.

\subsubsection{Approximate Model to Determine Optimal Order-Batching}

As discussed before, the biggest tradeoff exists between the picking and packing rates selected for the manual sortation system. Again, pack stations are the only other cost in this system, and their costs are low relative to the labor costs.

We have seen from simulation and verification of our theoretical efficiency curve that the relationship between picking and packing efficiencies is non-linear in general. However, the curve is nearly linear and can be estimated as a linear relationship as shown in Figure 5.3. From this relationship, we can predict the packing rate given a picking rate or vice versa. The following notation was used to estimate this relationship.

- $p_{1}$ is the picking rate in orders per hour.

- $p_{2}$ is the packing rate in orders per hour.

- $\bar{p}_{1}$ is the picking rate lower bound.

- $\hat{p}_{1}$ is the picking rate upper bound. 
- $\bar{p}_{2}$ is the packing rate lower bound.

- $\hat{p}_{2}$ is the packing rate upper bound.

- $D$ is the demand in orders per hour.

- $z$ is the objective function value corresponding to the total number of employees.

- $p_{1}^{*}$ is the picking rate that minimizes the objective function (unconstrained).

- $p_{2}^{*}$ is the packing rate that minimizes the objective function (unconstrained).

- $p_{1}^{\min }$ is the picking rate that minimizes the objective function (constrained).

- $p_{2}^{\min }$ is the packing rate that minimizes the objective function (constrained).

The linear relationship between $p_{1}$ and $p_{2}$ is defined in (5.1) below. The slope, $m$, and intercept, $b$, are determined in terms of the problem parameters as shown in (5.2) and (5.3), respectively.

$$
\begin{gathered}
p_{2}=\hat{p}_{2}-\left(p_{1}-\bar{p}_{1}\right)\left[\frac{\hat{p}_{2}-\bar{p}_{2}}{\hat{p}_{1}-\bar{p}_{1}}\right] \\
m=-\left[\frac{\hat{p}_{2}-\bar{p}_{2}}{\hat{p}_{1}-\bar{p}_{1}}\right] \\
b=\hat{p}_{2}-m \bar{p}_{1}
\end{gathered}
$$

Thus, (5.1) can be written as shown in (5.4):

$$
p_{2}=b+m p_{1} .
$$

Since this equation is linear, we know that it is a convex function. The objective function, $z$, where $p_{2}$ depends on the value of $p_{1}$, is shown in Figure 5.4 for the data illustrated in Figure 5.3 and described in general by (5.5): 


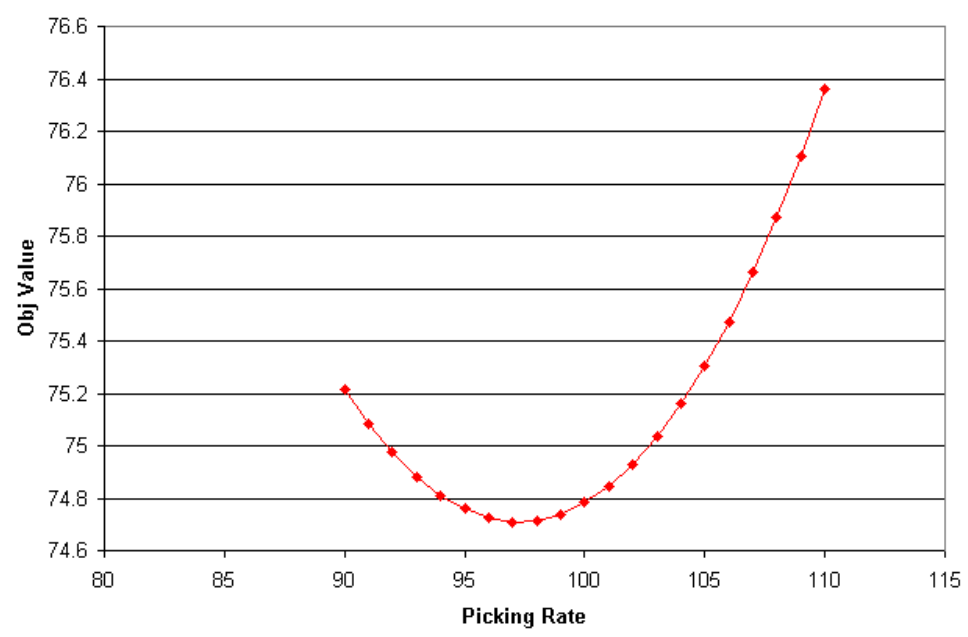

Figure 5.4: Picking Rate vs. Number of Employees.

$$
z=\frac{D}{p_{1}}+\frac{D}{p_{2}} .
$$

The objective function, (5.5), can be rewritten as follows by substituting for $p_{2}$ in (5.5) with (5.4). That is,

$$
z=\frac{D}{p_{1}}+\frac{D}{b+m p_{1}} .
$$

Note that we ignore the fact that the number of pickers and packers must be integer in (5.6). Examination of (5.6) leads to our first result.

Lemma 1 Our objective function, represented by (5.6), is a convex function.

Proof: A function $f(x)$ is convex if it is defined on a continuous interval and the second derivative $f^{\prime \prime}(x)>0$ for all $x$ on the interior of that interval. Therefore, we must solve for the second derivative of (5.6) as follows:

$$
z^{\prime}=\frac{-D}{p_{1}^{2}}+\frac{-D m}{\left(b+m p_{1}\right)^{2}} ; \text { and }
$$




$$
z^{\prime \prime}=\frac{2 D}{p_{1}^{3}}+\frac{2 m^{2} D}{\left(b+m p_{1}\right)^{3}}
$$

Now, we must determine if $z^{\prime \prime}>0$ for all $p_{1}$ on the interior of the interval. Looking at the first fraction of (5.8), we know that demand, $D$, is always positive, as well as the picking rate, $p_{1}$; therefore, the first fraction is always positive. The numerator of the second fraction is positive since demand is always positive and $m$ is squared. The denominator is less clear, but since $p_{2}=b+m p_{1}$, we know that it is always positive since $p_{2}$ is always positive (packing rates must always be positive values). Therefore, $z^{\prime \prime}>0$, which completes the proof.

Since the objective function is convex, we know that the minimum exists at the point where the derivative is set equal to zero and solved for $p_{1}$. Doing so and performing additional analysis leads to the following expression for $p_{1}^{*}$ :

$$
p_{1}^{*}= \begin{cases}\frac{-2 b m-\sqrt{4 b^{2} m^{2}-4 b^{2} m(m+1)}}{2 m(m+1)} & \text { if } m \neq-1, \\ b / 2 & \text { if } m=-1 .\end{cases}
$$

Result 1 The expression, (5.9), yields the optimal value for $p_{1}^{*}$ when $p_{1}$ and $p_{2}$ are only constrained to be greater than zero.

The proof of Result 1 is presented in Appendix E.

A surprising result that follows from Result 1 is that the demand level, $D$, does not play a factor in the optimal picking or packing rate for our system. We formally state this in our next result.

Result 2 The optimal picking and packing rates, $p_{1}^{*}$ and $p_{2}^{*}$, are not dependent on the demand level, $D$.

Proof: The result is clearly true based on an examination of (5.9) and noting that neither $b$ nor $m$ are dependent on $D$. 
Note that Result 1 is for the case where $p_{1}$ is only constrained to be greater than zero. However, we know that $p_{1}$ must also be between its lower and upper bound, $\overline{p_{1}}$ and $\hat{p_{1}}$. If $p_{1}^{*}$ is between the lower and upper bounds on the picking rate, then $p_{1}^{*}$ is the optimal picking rate, i.e., $p_{1}^{\min }=p_{1}^{*}$. However, if $p_{1}^{*}$ lies outside of its bounds, then the nearest boundary to $p_{1}^{*}$ is the optimal value for $p_{1}$. That is, we will move along the curve that represents the objective function until we are feasible. This results in a shift of $p_{1}$ to the right if $p_{1}^{*}<\overline{p_{1}}$ and to the left if $p_{1}^{*}>\hat{p_{1}}$. This is represented in the following expression and formally stated in Theorem 1:

$$
p_{1}^{\text {min }}= \begin{cases}p_{1}^{*} & \text { if } \bar{p}_{1} \leq p_{1}^{*} \leq \hat{p}_{1} \\ \hat{p}_{1} & \text { if } p_{1}^{*}>\hat{p}_{1}, \\ \bar{p}_{1} & \text { if } p_{1}^{*}<\bar{p}_{1} .\end{cases}
$$

Theorem 1 The expression (5.10) yields the optimal value for $p_{1}^{\text {min }}$ when $p_{1}$ is constrained to be within $\left(\bar{p}_{1}, \hat{p}_{1}\right)$.

Proof: Theorem 1 is illustrated by Figures 5.6, 5.5, and 5.7. Case 1 (Figure 5.5) represents a situation where the boundaries include the minimum, $p_{1}^{*}$, which is the optimal picking rate. Case 2 (Figure 5.6) is a situation where the picking rate boundaries are lower than the actual minimum of the objective function, resulting in an optimal picking rate of $\hat{p}_{1}$. The boundaries of Case 3 (Figure 5.7) are higher than the actual minimum, resulting in an optimal picking rate of $\bar{p}_{1}$.

In all cases, once $p_{1}^{\min }$ is determined, $p_{2}^{\min }$ is found by using (5.1). This analysis is helpful in estimating the optimal picking and packing rates if the workers' ranges were to increase or decrease in a manner where the slope, $m$, remains the same. For instance, consider a situation where the workers were at an optimal picking and packing rate and then the orderbatching methods were improved, therefore increasing the pickers' upper bound. Suppose that this improvement in picking reduces the packers' lower bound by the same amount, since some packers will not be able to keep up at this higher rate. If the current range includes the objective function minimum, found by (5.9), then this change has no effect. If 


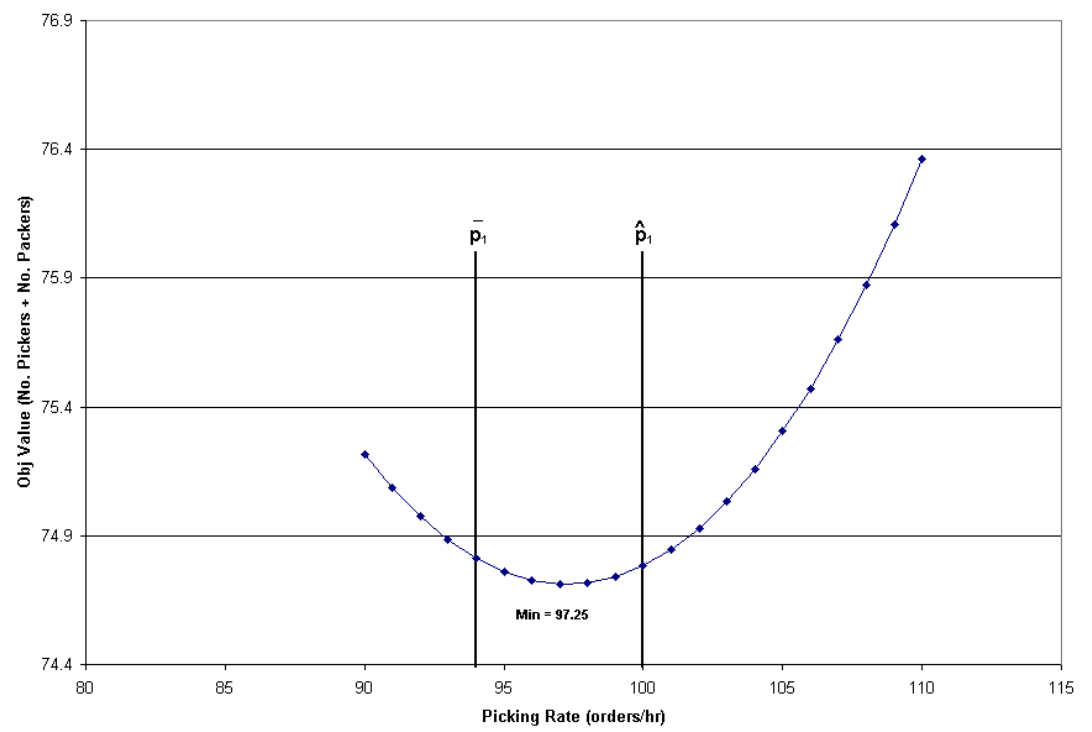

Figure 5.5: Case 1 - Minimum is Included in Picking Boundaries.

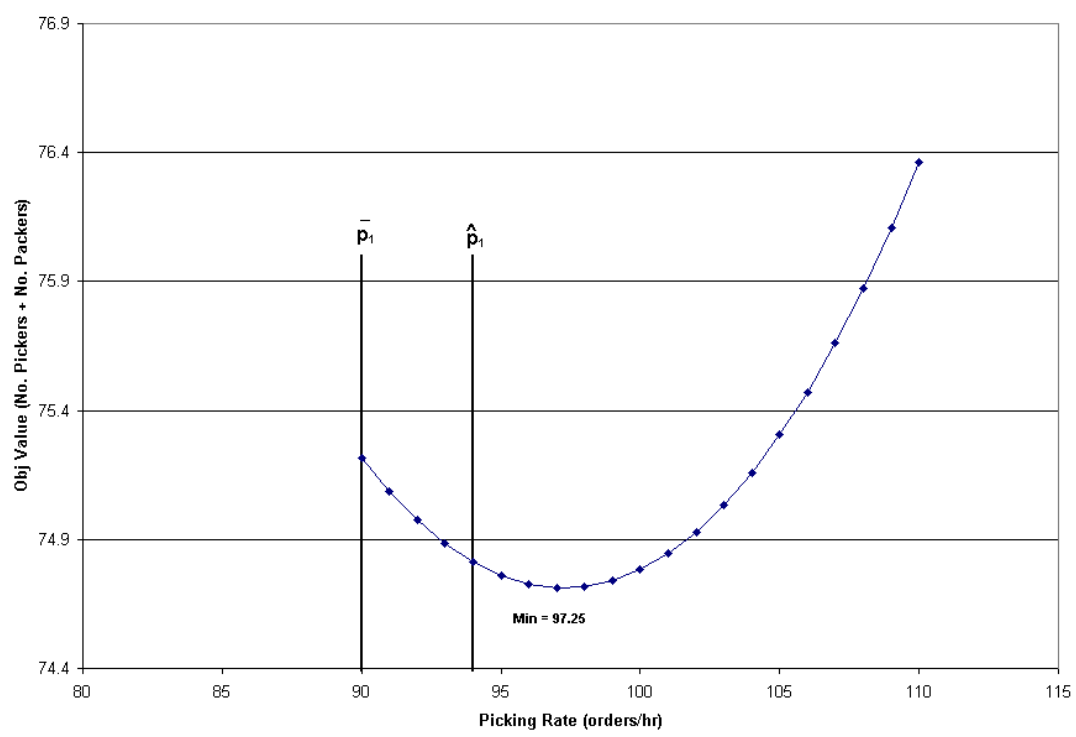

Figure 5.6: Case 2-Minimum is Lower than Picking Boundaries. 


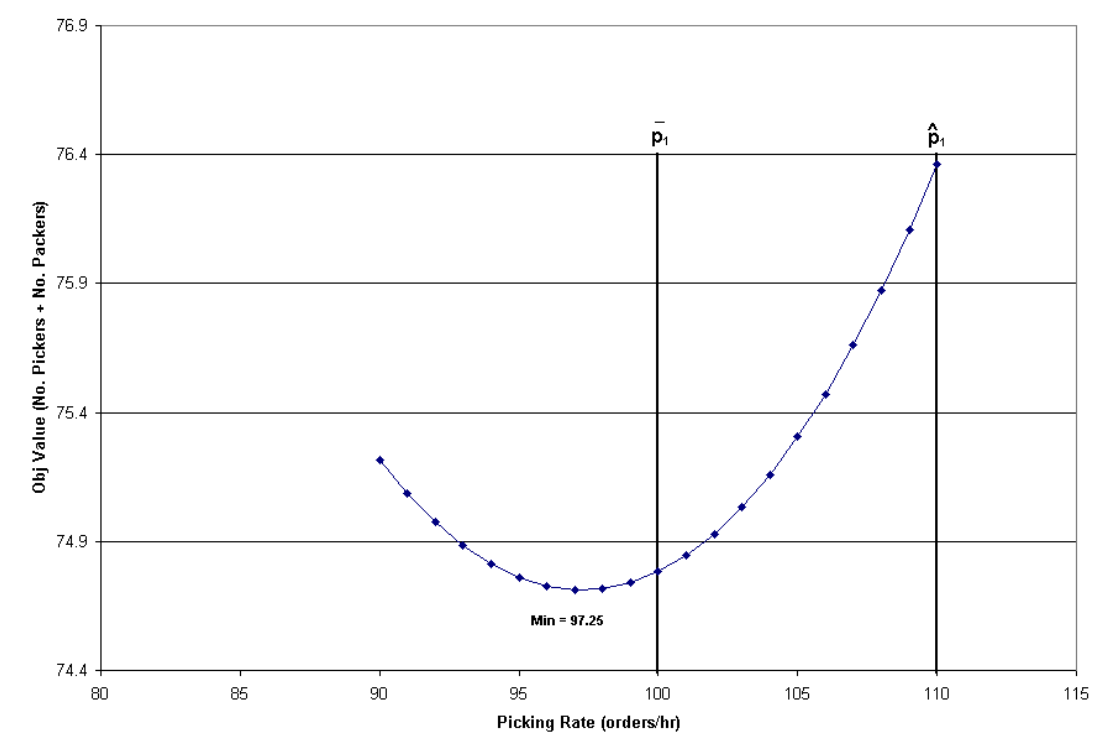

Figure 5.7: Case 3 - Minimum is Higher than Picking Boundaries.

the range is to the right of the minimum (i.e., picking rates exceed the actual minimum of the objective function), this shift in upper bound still has no effect on the optimal picking rate. However, if the range is to the left of the minimum, increasing the upper bound will shift the optimal picking rate to either the upper bound (if the minimum is still not included), or the actual minimum of the objective function. Similar results hold if the bounds decrease. The following example illustrates these results.

\section{Example}

To illustrate the behavior of optimal picking and packing rates, consider the following example. Table 5.3 provides several boundaries for picking and packing rates. Notice that $p_{1}^{*}$ remains the same for each set of boundaries, since the boundaries are still of equal size (i.e., slope of (5.6) does not change). Figure 5.8 illustrates how the boundaries move around $p_{1}^{*}$. Picking boundaries of 90 and 110 orders per hour do not include $p_{1}^{*}$, but 70 and 100 do. As shown in Table 5.3, this results in an optimal picking rate of 90 orders per hour for the $(90,110)$ boundaries, and 85 (the actual minimum) for the $(70,100)$ picking boundaries.

Similar results do not hold for systems where the rates increase or decrease in a manner 
Table 5.3: Example Picking and Packing Boundaries and Resulting Optimal Rates ( $m$ does not change).

\begin{tabular}{|cc|cc|cc|cc|c|}
\hline $\bar{p}_{1}$ & $\hat{p}_{1}$ & $p_{1}^{*}$ & $p_{1}^{\text {min }}$ & $\bar{p}_{2}$ & $\hat{p}_{2}$ & $p_{2}^{*}$ & $p_{2}^{\text {min }}$ & $z$ \\
\hline 90 & 110 & 85 & 90 & 60 & 80 & 85 & 80 & 94.44 \\
90 & 120 & 85 & 90 & 50 & 80 & 85 & 80 & 94.44 \\
80 & 110 & 85 & 85 & 60 & 90 & 85 & 85 & 94.12 \\
70 & 100 & 85 & 85 & 70 & 100 & 85 & 85 & 94.12 \\
\hline
\end{tabular}

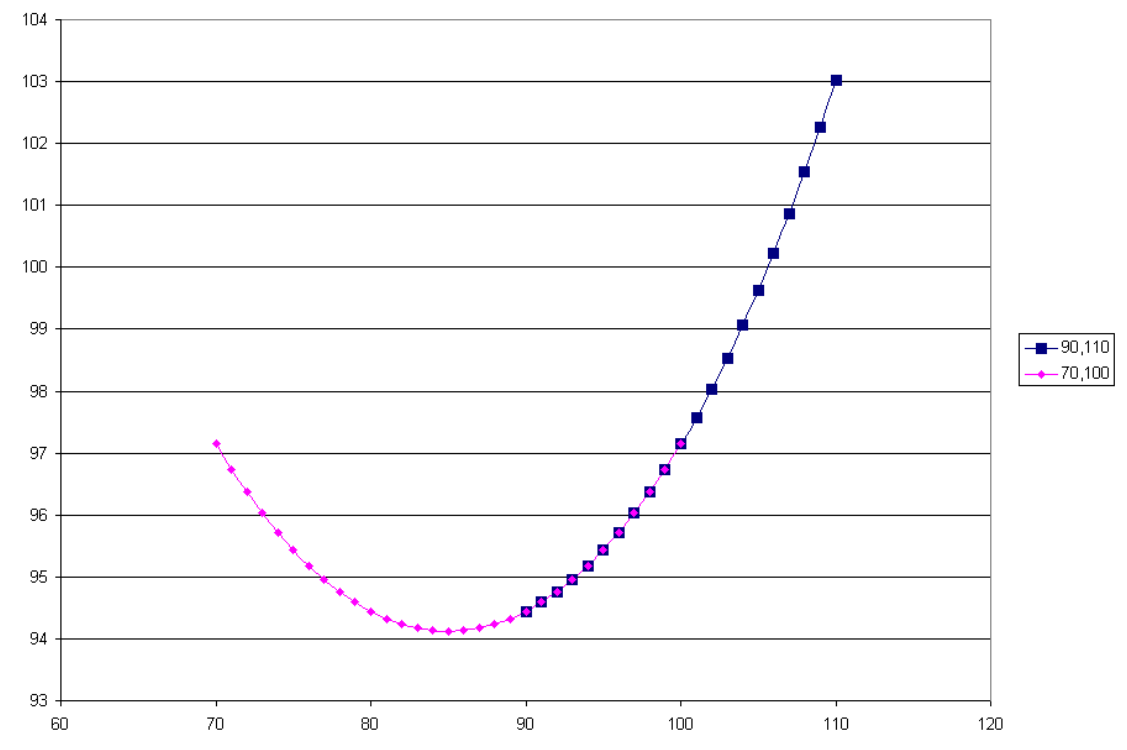

Figure 5.8: Example Objective Functions.

that changes the slope describing the linear relationship between picking and packing. It is difficult to predict the optimal picking rate for this type of increase or decrease. Using intuition, we can predict the reaction of a system with similar lower and upper bounds in much the same way as systems with identical slopes. However, when the boundaries do not overlap, this becomes more difficult. Consider the examples in Table 5.4. The first set of examples shows two similar boundaries, $(50,100)$ and $(50,100)$. Here, if we raise the picking lower bound while keeping the same packing boundaries, we expect that since the pickers are more efficient, we can allow the packers to move towards their upper bound, which does occur in this case. Lowering the picking lower bound would cause the optimal picking rate to increase, since the pickers are now less efficient. Using the same idea, however, by looking at the second set of examples in Table 5.4, the behavior of the picking and packing optimal 
rates is not predictable. In the case where $\bar{p}_{1}$ is decreased from 75 to 70 , the optimal picking rate should increase since the pickers are not as efficient. However, just the opposite happens. However, by increasing the packing rate lower bound in the last trial, the optimal packing rate does not increase as expected.

Table 5.4: Example Picking and Packing Boundaries and Resulting Optimal Rates ( $m$ changes).

\begin{tabular}{|cc|c|cc|c|c|}
\hline $\bar{p}_{1}$ & $\hat{p}_{1}$ & $p_{1}^{\text {min }}$ & $\bar{p}_{2}$ & $\hat{p}_{2}$ & $p_{2}^{\text {min }}$ & $z$ \\
\hline 50 & 100 & 75.0 & 50 & 100 & 75.0 & 106.67 \\
55 & 100 & 74.4 & 50 & 100 & 78.4 & 104.75 \\
45 & 100 & 75.7 & 50 & 100 & 72.1 & 108.33 \\
\hline 75 & 100 & 75.0 & 40 & 60 & 60.0 & 120.00 \\
70 & 100 & 71.9 & 40 & 60 & 58.7 & 123.74 \\
75 & 100 & 76.4 & 45 & 60 & 59.2 & 119.67 \\
\hline
\end{tabular}

In summary, our results with this approximate analytical model provide the foundation for evaluating changes in order-batching. In particular, a choice between increasing picking or packing efficiencies can be evaluated quickly with this model. Also, situations where increases in either or both do not lead to improvements in the system can be identified.

\subsection{Results for Order-Batching in an Automated Sys- tem}

In an automated system, as the amount of batching increases, the picking rate will increase as well. However, increasing the batching has little or no effect on the packing rate. The automated sorter ensures that each packer will receive the orders at a constant rate. As the wave length increases, the batch size increases, since the pickers have a longer period of time to retrieve items for orders. At a wave length of 20 minutes, the picking rate is 75 orders per hour, requiring 15 pickers, 15 packers, and 3 inductors. The total annual system cost at this level of batching is $\$ 1,140,480$. With the wave length increased to 80 minutes, the picking rate increases to 120 orders per hour, therefore requiring fewer pickers. This system 
requires 10 pickers, 15 packers, and 3 inductors at a cost of $\$ 1,137,616$. Savings of $\$ 150,000$ result from reducing the number of employees by 5 , but the increase in pack stations from 15 to 59 at $\$ 10,000$ per pack station adds an additional $\$ 147,136$. The total savings from batching is $\$ 2,864$.

The system discussed above considered a system where no time was spent walking from bin to bin or between pack stations, and no time was spent waiting for orders to become ready to pack. The packing rate remains constant over each increase in picking rate. Therefore, the best solution from the experiments will occur at the best combination of picking rate and number of pack stations required. The solution that provides the best cost from these two factors will be recommended. For instance, in the base case, the solution with a picking rate of 105 orders per hour, packing rate of 75 orders per hour, and a requirement of 44 pack stations has a total annual cost of $\$ 1,117,456$.

However, if we now consider a system where the packing rate deteriorates due to walking and waiting times, such as a system with no overlap, the recommendation will be somewhat different. Now, we must consider the solution that minimizes the costs associated with pickers, pack stations, and packers, since the packing rate is no longer constant. At the same picking rate as the best automated solution where packing rate remains constant, the total annual cost is now $\$ 1,147,456$. This system requires an extra packer at $\$ 30,000$ due to the decrease in packing efficiency cause by walking times. In fact, from the simulation results, this decrease in packing efficiency can become quite pronounced as the wave length increases (see Figure 3.11). 


\section{Chapter 6}

\section{Throughput Simulation Results}

After a company has provided input data such as their demand level, efficiency curves, capital costs, labor costs, and sorter speed, the deterministic spreadsheet model recommends a configuration. This recommendation consists of whether the system is manual or automated, the number of employees, the number of pack stations and induction stations, the picking and packing rates, and the wave length. This spreadsheet output then becomes the input for a throughput simulation. The throughput simulation determines if the demand can be met using the recommended configuration.

The throughput simulation is important for several reasons. First, any inaccuracies in the spreadsheet model can be discovered by running throughput simulations. The inaccuracies will manifest themselves as a system that does not meet demand. Second, the stochastic nature of the order fulfillment process can be modeled more accurately. Since stochastic factors degrade the performance of any system, our deterministic model is susceptible to the effects of stochastic factors. Third, the effects of rounding in the deterministic spreadsheet model can be quantified. Since many design parameters have been rounded to ensure sufficient capacity in the deterministic model, this additional capacity may or may not balance the negative impact of the stochastic factors.

The descriptive model provides a preliminary analysis of the system, ignoring all stochastic elements. This model defines a given situation with a particular set of parameters, such as 
those used in the example previously presented. In order to develop a more realistic model of the sortation system, stochastic elements must be introduced.

Variability is present in almost every parameter represented in this model. Demand may have peaks or fluctuations based on the time of day, day of the week, or even the month or season. Additionally, while labor standards are set by the engineers, each worker will actually work at different levels. Although these types of variability are important, we do not consider them. Rather, we consider a peak wave size and equivalent workers all working at a minimum labor standard. However, we do incorporate randomness into wave composition and unit processing times.

The goal of the simulation study is to refine the data for the descriptive model and to give insights into the critical tradeoffs. This model allows the system to be examined under different parameters with stochastic processing times, demands, and other model inputs.

Since waves are being used, it is not necessary to model the entire system. With a wave, only items included in the orders in that particular wave may be processed, and processing cannot begin on items from the next wave until the current wave is completed. Therefore, there is little interaction between picking, sorting, and packing for one particular wave. Thus, three smaller systems, picking, sorting, and packing, may be simulated separately -although not independently, as will be discussed next.

As stated previously, the three sub-systems (picking, sorting, and packing) may be considered separately. The goal is to determine the throughput rate of a particular design and its associated wave length, while considering the stochastic elements of the system. Note that since many of the components of the system are integer in nature, the system determined from the descriptive model for a particular throughput rate may actually have a predicted throughput capability that is higher. Of course, the stochastic elements modeled in the simulation model have a negative effect on the throughput capability. The simulation model is used to gauge this effect relative to the target throughput by using the simulation model to measure the average total time required to pick, pack, or induct all orders assigned to the worker for a wave. The average total time and the maximum time can then be compared to the wave length for which the system was designed. 
Each of the three sub-systems is somewhat different in the approach we have taken with it. In the picking sub-system we consider a rectangular picking area with an input/output point at one end of the area. Other researchers have considered such a layout (e.g., see [39]). In fact, fairly accurate analytical models exist to predict the time to pick a certain number of items. However, since we are concerned with the effects of variance, a simulation model is needed. Since each point on the picking efficiency curve is based on the throughput given a certain level of order-batching for the pickers, the analytical models are used to verify this point on the curve before beginning the simulation. In the simulation model, the items for orders are randomly generated and then batched for each picker based on the point on the curve of interest (i.e., how many orders' worth of items to pick in one batch). In many cases, more items are assigned to a picker than are necessary to meet throughput to make the data requirements more straightforward. Picking tours follow the traversal strategy.

The sorting sub-system only relates to the automated system, and only in terms of the induction process. The work by Meller and Johnson [31] provides an analytical model for inductors. The inductors can have different induction rates and can consider setup times if necessary. The total time to induct all items in a wave can be effectively estimated. For the most part, this analytical model has already been incorporated into our descriptive model (a modification to the material presented in Chapter 4 will be presented later in this chapter). Although comparing their work to simulation output proved this analytical model to be very accurate, it does not consider anything other than an average wave, and thus, simulation is needed.

The packing sub-system must consider both the manual and automated designs and is different from the other two sub-system simulations. The difference comes from the fact that there is not an analytical model to support the simulation model. However, this sub-system is also the simplest, with very few interactions to consider. That is, unlike picking or sorting where pickers and inductors interact with one another, for the most part packers at different stations do not interact - especially in the manual design. In the automated design, packers may interact with one another since two packers may, over different waves, pack at the same chute due to the stochastic nature of the system. However, we ignore the possibility in our 
simulation model and simulate each packer independently. By varying the packing rates and walking rates in the automated system, the total time to pack all orders can be effectively estimated and the appropriate statistics constructed.

\subsection{Methodology}

The throughput simulation is performed for each subsystem: picking, sorting, and packing. However, simulations were not performed for manual sortation and packing. The picking simulation was run for the recommended wave length, picker rate, and batch size over 100 replications. For each replication, the total time to pick all items was measured. We collected statistics on the average, minimum, and maximum of this time. The picker utilization was also determined by dividing the total time spent picking all items for the batch by the wave length. For instance, if a picker took 118 minutes to pick all orders in a 120-minute wave, the utilization is $118 / 120$, or 0.9833 (98.33\%). In addition, the number of waves where the items could not be picked within the wave was tabulated. Similarly, the packing simulation uses the recommended wave length, packing rate, and batch size as input, and calculates the amount of time spent packing the assigned orders for each replication. Again, various statistics over 100 replications were recorded. We assume that our manual sortation and packing estimates are valid, therefore only the picking subsystem is simulated for manual systems. The automated sortation is slightly different. Here, based on an induction rate, the effective rate for each inductor due to inductor blocking is calculated. Using this rate, the total number of items to be assigned to each inductor is determined. Again, statistics are collected for the induction subsystem over 100 replications.

\subsection{Data}

The tables in Section 6.2 present the data used in the throughput simulation runs. Data examples were generated in a manner that considers many different scenarios. For example, data that result in recommendations of manual and automated systems were generated. In 
Table 6.1: Input Data for Deterministic Spreadsheet Model.

\begin{tabular}{|c|c|c|c|c|c|c|c|c|c|c|c|}
\hline $\begin{array}{l}\text { Trial } \\
\text { No. }\end{array}$ & $\begin{array}{c}\text { Demand } \\
\text { Level } \\
\text { (orders/hour) }\end{array}$ & $\begin{array}{c}\text { Interest } \\
\text { Rate } \\
(\%) \\
\end{array}$ & $\begin{array}{c}\text { Years } \\
\text { of Service }\end{array}$ & $\mathrm{A} / \mathrm{P}$ & $\begin{array}{c}\text { Labor } \\
\text { Cost } \\
(\$) \\
\end{array}$ & $\begin{array}{c}\text { Pack Station } \\
\text { Cost } \\
(\$) \\
\end{array}$ & $\begin{array}{c}\text { Sorter } \\
\text { Cost } \\
(\$) \\
\end{array}$ & $\begin{array}{l}\text { Sorter } \\
\text { Speed } \\
(\mathrm{tpm}) \\
\end{array}$ & $\begin{array}{c}\text { Induction } \\
\text { Rate } \\
\text { (tpm) }\end{array}$ & $\begin{array}{l}\text { Picker } \\
\text { Rate }\end{array}$ & $\begin{array}{c}\text { Packer } \\
\text { Rate }\end{array}$ \\
\hline 1 & 3000 & 0.1 & 10 & 0.1627 & 30,000 & 2,500 & 250,000 & 200 & 120 & $\mathrm{H}$ & $\overline{\mathrm{H}}$ \\
\hline 2 & 3000 & 0.2 & 5 & 0.3344 & 18,000 & 20,000 & 250,000 & 200 & 120 & $\mathrm{~L}$ & L \\
\hline 3 & 3000 & 0.1 & 5 & 0.2638 & 18,000 & 10,000 & $1,000,000$ & 150 & 120 & M & M \\
\hline 4 & 3000 & 0.1 & 10 & 0.1627 & 18,000 & 20,000 & 250,000 & 200 & 60 & $\mathrm{H}$ & $\mathrm{H}$ \\
\hline 5 & 3000 & 0.2 & 5 & 0.3344 & 18,000 & 20,000 & $1,000,000$ & 100 & 20 & M & M \\
\hline 6 & 1100 & 0.1 & 10 & 0.1627 & 30,000 & 10,000 & 500,000 & 200 & 120 & $\mathrm{H}$ & L \\
\hline 7 & 1100 & 0.1 & 5 & 0.2638 & 30,000 & 2,500 & 500,000 & 100 & 120 & $\mathrm{~L}$ & L \\
\hline 8 & 1100 & 0.2 & 5 & 0.3344 & 18,000 & 20,000 & 500,000 & 100 & 120 & $\mathrm{~L}$ & $\mathrm{H}$ \\
\hline 9 & 1100 & 0.1 & 5 & 0.2638 & 18,000 & 20,000 & $1,000,000$ & 150 & 60 & $\mathrm{H}$ & $\mathrm{L}$ \\
\hline 10 & 1100 & 0.1 & 5 & 0.2638 & 18,000 & 10,000 & 250,000 & 200 & 20 & $\mathrm{~L}$ & $\mathrm{~L}$ \\
\hline 11 & 550 & 0.1 & 5 & 0.2638 & 30,000 & 2,500 & 250,000 & 200 & 120 & $\mathrm{H}$ & $\mathrm{H}$ \\
\hline 12 & 550 & 0.2 & 5 & 0.3344 & 30,000 & 10,000 & 500,000 & 200 & 60 & M & M \\
\hline 13 & 550 & 0.2 & 5 & 0.3344 & 30,000 & 10,000 & 250,000 & 150 & 60 & $\mathrm{H}$ & $\mathrm{H}$ \\
\hline 14 & 550 & 0.2 & 10 & 0.2385 & 30,000 & 10,000 & $1,000,000$ & 150 & 20 & $\mathrm{~L}$ & $\mathrm{H}$ \\
\hline 15 & 550 & 0.2 & 5 & 0.3344 & 18,000 & 20,000 & $1,000,000$ & 100 & 20 & M & M \\
\hline
\end{tabular}

addition, different demand levels were tested with high, medium, and low combinations of picking and packing rates. For each combination, a new efficiency curve and spreadsheet model were constructed from the simulated data. The construction of these curves and accompanying data is presented in Appendix F. In each table from this point on, the levels will be abbreviated as $\mathrm{H}$ for high, $\mathrm{M}$ for medium, and $\mathrm{L}$ for low. These scenarios ensure that the spreadsheet model is robust. Table 6.1 presents the input data for the spreadsheet model. Using the spreadsheet models adjusted by verification simulations, the recommendation for each set of input data is recorded in Tables 6.2 and 6.3. Tables 6.2 and 6.3 vary due to an assumption of whether or not overlapping or non-overlapping waves were used. The purpose of this distinction is to show the effects of overlapping on both the system configuration and the efficiency of the pickers and packers. All trials were performed for both types of waves.

Table 6.2 presents the recommendations provided by the spreadsheet model for waves that overlap. Table 6.3 presents the recommendations for non-overlapping waves. Notice that the manual system is recommended more often given the same set of input data with nonoverlapping waves. This is due to the fact that packing rates are lower in the automated system for non-overlapping waves because of the time spent waiting for bins to become ready to pack and walking time between bins that may be spread over a large distance. Systems with non-overlapping waves are not commonly used, but are presented here to illustrate the usefulness of the model in estimating throughput and picking and packing times for this type of system as well as systems with waves that overlap. In fact, systems with overlapping waves always result in a lower total annual system cost than systems without overlapping waves. 
Table 6.2: Output Data for Deterministic Spreadsheet Model with Overlapping Waves.

\begin{tabular}{|c|c|c|c|c|c|c|c|c|c|c|}
\hline $\begin{array}{l}\text { Trial } \\
\text { No. }\end{array}$ & $\begin{array}{c}\text { Wave } \\
\text { Length } \\
(\min )\end{array}$ & $\begin{array}{c}\text { Picker } \\
\text { Rate } \\
\text { (orders } / \text { hr) }\end{array}$ & $\begin{array}{c}\text { Packer } \\
\text { Rate } \\
\text { (orders } / \text { hr) }\end{array}$ & $\begin{array}{c}\text { Required } \\
\text { Pickers }\end{array}$ & $\begin{array}{c}\text { Required } \\
\text { Packers }\end{array}$ & $\begin{array}{c}\text { Manual } \\
\text { or } \\
\text { Automated }\end{array}$ & $\begin{array}{l}\text { Required } \\
\text { Inductors }\end{array}$ & $\begin{array}{c}\text { Required } \\
\text { Induction } \\
\text { Stations }\end{array}$ & $\begin{array}{l}\text { Required } \\
\text { Pack } \\
\text { Stations }\end{array}$ & $\begin{array}{l}\text { Total Annual } \\
\text { System Cost } \\
(\$)\end{array}$ \\
\hline 1 & 120 & 288.87 & 117.96 & 11 & 26 & Automated & $\overline{2}$ & 1 & 240 & $1,316,471$ \\
\hline 2 & 40 & 95.38 & 47.7 & 32 & 63 & Automated & 2 & 1 & 80 & $2,381,321$ \\
\hline 3 & 60 & 173.16 & 69.9 & 18 & 43 & Automated & 2 & 1 & 120 & $1,727,544$ \\
\hline 4 & 40 & 169.48 & 118.15 & 18 & 26 & Automated & 3 & 1 & 80 & $1,155,216$ \\
\hline 5 & 60 & 86.07 & 39.84 & 35 & 76 & Manual & $\mathrm{n} / \mathrm{a}$ & $\mathrm{n} / \mathrm{a}$ & 120 & $2,026,090$ \\
\hline 6 & 80 & 241.25 & 47.69 & 5 & 24 & Automated & 1 & 1 & 59 & $1,085,530$ \\
\hline 7 & 120 & 247.53 & 47.69 & 5 & 24 & Automated & 1 & 1 & 88 & $1,103,124$ \\
\hline 8 & 80 & 75.59 & 48.32 & 15 & 23 & Manual & $\mathrm{n} / \mathrm{a}$ & $\mathrm{n} / \mathrm{a}$ & 59 & 697,811 \\
\hline 9 & 60 & 126.16 & 29.87 & 9 & 37 & Manual & $\mathrm{n} / \mathrm{a}$ & $\mathrm{n} / \mathrm{a}$ & 44 & 836,125 \\
\hline 10 & 60 & 139.51 & 47.7 & 8 & 24 & Automated & 3 & 1 & 44 & 825,210 \\
\hline 11 & 120 & 288.87 & 117.96 & 2 & 5 & Automated & 1 & 1 & 44 & 348,157 \\
\hline 12 & 60 & 86.07 & 39.84 & 7 & 14 & Manual & $\mathrm{n} / \mathrm{a}$ & $\mathrm{n} / \mathrm{a}$ & 22 & 381,673 \\
\hline 13 & 60 & 209.78 & 118.07 & 3 & 5 & Automated & 1 & 1 & 22 & 394,032 \\
\hline 14 & 80 & 79.92 & 45.94 & 7 & 12 & Manual & $\mathrm{n} / \mathrm{a}$ & $\mathrm{n} / \mathrm{a}$ & 30 & 577,022 \\
\hline 15 & 60 & 86.07 & 39.84 & 7 & 14 & Manual & $\mathrm{n} / \mathrm{a}$ & $\mathrm{n} / \mathrm{a}$ & 22 & 381,673 \\
\hline
\end{tabular}

Table 6.3: Output Data for Deterministic Spreadsheet Model with Non-Overlapping Waves.

\begin{tabular}{|c|c|c|c|c|c|c|c|c|c|c|}
\hline $\begin{array}{l}\text { Trial } \\
\text { No. }\end{array}$ & $\begin{array}{c}\text { Wave } \\
\text { Length } \\
(\text { min })\end{array}$ & $\begin{array}{c}\text { Picker } \\
\text { Rate } \\
\text { (orders /hr) } \\
\end{array}$ & $\begin{array}{c}\text { Packer } \\
\text { Rate } \\
\text { (orders } / \text { hr) } \\
\end{array}$ & $\begin{array}{c}\text { Required } \\
\text { Pickers }\end{array}$ & $\begin{array}{c}\text { Required } \\
\text { Packers }\end{array}$ & $\begin{array}{c}\text { Manual } \\
\text { or } \\
\text { Automated } \\
\end{array}$ & $\begin{array}{l}\text { Required } \\
\text { Inductors }\end{array}$ & $\begin{array}{c}\text { Required } \\
\text { Induction } \\
\text { Stations } \\
\end{array}$ & $\begin{array}{c}\text { Required } \\
\text { Pack } \\
\text { Stations } \\
\end{array}$ & $\begin{array}{l}\text { Total Annual } \\
\text { System Cost } \\
(\$)\end{array}$ \\
\hline 1 & 40 & 169.48 & 83.45 & 18 & 36 & Automated & 2 & 1 & 80 & $1,761,373$ \\
\hline 2 & 100 & 91.43 & 28.99 & 33 & 104 & Manual & $\mathrm{n} / \mathrm{a}$ & $\mathrm{n} / \mathrm{a}$ & 200 & $2,512,816$ \\
\hline 3 & 60 & 86.07 & 39.84 & 35 & 76 & Manual & $\mathrm{n} / \mathrm{a}$ & $\mathrm{n} / \mathrm{a}$ & 120 & $2,020,159$ \\
\hline 4 & 20 & 113.45 & 93.21 & 27 & 33 & Automated & 3 & 1 & 40 & $1,313,020$ \\
\hline 5 & 60 & 86.07 & 39.84 & 35 & 76 & Manual & $\mathrm{n} / \mathrm{a}$ & $\mathrm{n} / \mathrm{a}$ & 120 & $2,026,090$ \\
\hline 6 & 40 & 169.48 & 33.85 & 7 & 33 & Automated & 1 & 1 & 30 & $1,368,334$ \\
\hline 7 & 120 & 247.53 & 30.62 & 5 & 36 & Automated & 1 & 1 & 88 & $1,463,124$ \\
\hline 8 & 80 & 75.59 & 48.32 & 15 & 23 & Manual & $\mathrm{n} / \mathrm{a}$ & $\mathrm{n} / \mathrm{a}$ & 59 & 697,811 \\
\hline 9 & 60 & 126.16 & 29.87 & 9 & 37 & Manual & $\mathrm{n} / \mathrm{a}$ & $\mathrm{n} / \mathrm{a}$ & 44 & 836,125 \\
\hline 10 & 100 & 91.43 & 28.99 & 13 & 38 & Manual & $\mathrm{n} / \mathrm{a}$ & $\mathrm{n} / \mathrm{a}$ & 74 & 931,665 \\
\hline 11 & 20 & 113.45 & 93.21 & 5 & 6 & Automated & 1 & 1 & 8 & 444,415 \\
\hline 12 & 60 & 86.07 & 39.84 & 7 & 14 & Manual & $\mathrm{n} / \mathrm{a}$ & $\mathrm{n} / \mathrm{a}$ & 22 & 381,673 \\
\hline 13 & 20 & 113.45 & 93.21 & 5 & 6 & Automated & 1 & 1 & 8 & 450,639 \\
\hline 14 & 80 & 79.92 & 45.94 & 7 & 12 & Manual & $\mathrm{n} / \mathrm{a}$ & $\mathrm{n} / \mathrm{a}$ & 30 & 577,022 \\
\hline 15 & 60 & 86.07 & 39.84 & 7 & 14 & Manual & $\mathrm{n} / \mathrm{a}$ & $\mathrm{n} / \mathrm{a}$ & 22 & 381,673 \\
\hline
\end{tabular}

Each system configuration was tested using simulation to determine if the desired throughput would be met. Although we have no way to simulate manual packing, the time study data should be sufficient for the manual system because of the method we used to determine the picking rate as discussed below.

In order to determine the picking and packing rates used in the manual system calculations, we had to decide which subsystem's rate, picking or packing, should be set first. By examining the efficiency curve in Figure 3.3, we see that picking and packing rates are dependent upon one another. Therefore, an equal batch size must be given to each subsystem in order to balance the workload within a wave. Because the picking rates are generally higher than manual packing rates, we determined these rates first.

Based on simulated picking times for different batch sizes, the largest batch that can be picked within the wave length was first determined. For example, consider a system with high picking rates and low packing rates, such as the system described in Trial 9 of Table 6.1. The recommended wave length for this set of parameters is 60 minutes. Using these input 
parameters, the highest picking rate considered corresponds to a batch size of 210 orders. According to simulation data, this batch size can be completed in approximately 60 minutes. Batch sizes for each of the six combinations of picking and packing rates considered for a wave are set in much the same way. However, the time to pick all orders is divided by $6,5,4$, 3 , and 2 to determine the corresponding picking rate. Then, the picker is assigned $6,5,4,3$, 2 , or 1 appropriately-sized batches depending upon the rate. The final batch size is chosen so that the total time to pick all the assigned batches is approximately equal to the wave length. For example, the first combination requires a batch size of 21 orders, and according to simulation data, this batch size can be completed on average in 9.99 minutes. Therefore, 6 batches of 21 orders should be able to be picked within a 60 -minute wave. Similarly, the third combination requires a batch size of 36 orders, each being completed in 14.96 minutes. This means that 4 batches can be completed within 60 minutes. Six batches were considered in order to consider a wide range of picking and packing rates and numbers of employees. This method of batching orders determines the maximum batch size that a picker or packer can complete. The spreadsheet model then determines the best solution from this set of six combinations based on the total annual system cost.

In order to illustrate the excess throughput that can result from batching orders, we have calculated the required number of orders per picker and packer for each example trial, as well as the actual number of orders picked or packed as a result of order-batching. Table 6.4 presents this data for each trial, where the first fifteen trials are for systems with wave overlap, and the last fifteen are for systems without wave overlap.

The following example illustrates how setting either the picking or packing subsystem's batch size first affects the other subsystem. Consider the example summarized in Table 6.5. For Trial 2 with a system without overlapping waves, the best system cost occurs at a 100-minute wave, at the picking and packing rate combination which corresponds to a batch size of 26 orders. This batch size is taken from the efficiency curve and associated data developed from simulation experiments. Based on the picking time associated with this batch size, 17.06 minutes, the picker should be able to pick 5.86 batches within the allotted 100 minutes. However, we are not considering split batches, so the picker must be assigned an integer 
Table 6.4: Effects of Order-Batching on Subsystem Throughput.

\begin{tabular}{|c|c|c|c|c|c|c|c|c|c|c|}
\hline $\begin{array}{l}\text { Trial } \\
\text { No. }\end{array}$ & $\begin{array}{c}\text { Orders } \\
\text { Required } \\
\text { per Picker }\end{array}$ & $\begin{array}{c}\text { Batch } \\
\text { Size per } \\
\text { Picker }\end{array}$ & $\begin{array}{c}\text { Batches } \\
\text { per } \\
\text { Picker }\end{array}$ & $\begin{array}{c}\text { Total } \\
\text { Orders } \\
\text { per Picker }\end{array}$ & $\begin{array}{c}\text { Orders } \\
\text { Required } \\
\text { per Packer }\end{array}$ & $\begin{array}{c}\text { Batch } \\
\text { Size per } \\
\text { Packer }\end{array}$ & $\begin{array}{l}\text { Batches } \\
\text { per } \\
\text { Packer }\end{array}$ & $\begin{array}{l}\text { Total } \\
\text { Orders } \\
\text { Picked }\end{array}$ & $\begin{array}{c}\text { Total } \\
\text { Orders } \\
\text { Packed }\end{array}$ & $\begin{array}{c}\text { Orders } \\
\text { Needed to } \\
\text { Meet Demand }\end{array}$ \\
\hline 1 & 546 & 546 & 1 & 546 & 231 & 231 & 1 & 6006 & 6006 & 6000 \\
\hline 2 & 63 & 63 & 1 & 63 & 32 & 32 & 1 & 2016 & 2016 & 2000 \\
\hline 3 & 167 & 167 & 1 & 167 & 70 & 70 & 1 & 3006 & 3010 & 3000 \\
\hline 4 & 112 & 112 & 1 & 112 & 77 & 77 & 1 & 2016 & 2002 & 2000 \\
\hline 5 & 86 & 15 & 6 & 90 & 40 & 15 & 3 & 3150 & 3420 & 3000 \\
\hline 6 & 294 & 294 & 1 & 294 & 62 & 62 & 1 & 1470 & 1488 & 1467 \\
\hline 7 & 440 & 440 & 1 & 440 & 92 & 92 & 1 & 2200 & 2208 & 2200 \\
\hline 8 & 98 & 17 & 6 & 102 & 64 & 17 & 4 & 1530 & 1564 & 1467 \\
\hline 9 & 123 & 21 & 6 & 126 & 30 & 21 & 2 & 1134 & 1554 & 1100 \\
\hline 10 & 138 & 138 & 1 & 138 & 46 & 46 & 1 & 1104 & 1104 & 1100 \\
\hline 11 & 550 & 550 & 1 & 550 & 220 & 220 & 1 & 1100 & 1100 & 1100 \\
\hline 12 & 79 & 15 & 6 & 90 & 40 & 15 & 3 & 630 & 630 & 550 \\
\hline 13 & 184 & 184 & 1 & 184 & 110 & 110 & 1 & 552 & 550 & 550 \\
\hline 14 & 105 & 22 & 5 & 110 & 62 & 22 & 3 & 770 & 792 & 733 \\
\hline 15 & 79 & 15 & 6 & 90 & 40 & 15 & 3 & 630 & 630 & 550 \\
\hline 1 & 112 & 112 & 1 & 112 & 56 & 59 & 1 & 2016 & 2124 & 2000 \\
\hline 2 & 152 & 26 & 6 & 156 & 49 & 26 & 2 & 5148 & 5408 & 5000 \\
\hline 3 & 86 & 15 & 6 & 90 & 40 & 15 & 3 & 3150 & 3420 & 3000 \\
\hline 4 & 38 & 38 & 1 & 38 & 31 & 31 & 1 & 1026 & 1023 & 1000 \\
\hline 5 & 86 & 15 & 6 & 90 & 40 & 15 & 3 & 3150 & 3420 & 3000 \\
\hline 6 & 105 & 105 & 1 & 105 & 23 & 23 & 1 & 735 & 759 & 733 \\
\hline 7 & 440 & 440 & 1 & 440 & 62 & 62 & 1 & 2200 & 2232 & 2200 \\
\hline 8 & 98 & 17 & 6 & 102 & 64 & 17 & 4 & 1530 & 1564 & 1467 \\
\hline 9 & 123 & 21 & 6 & 126 & 30 & 21 & 2 & 1134 & 1554 & 1100 \\
\hline 10 & 142 & 26 & 6 & 156 & 49 & 26 & 2 & 2028 & 1976 & 1833 \\
\hline 11 & 37 & 37 & 1 & 37 & 31 & 31 & 1 & 185 & 186 & 183 \\
\hline 12 & 79 & 15 & 6 & 90 & 40 & 15 & 3 & 630 & 630 & 550 \\
\hline 13 & 37 & 37 & 1 & 37 & 31 & 31 & 1 & 185 & 186 & 183 \\
\hline 14 & 105 & 22 & 5 & 110 & 62 & 22 & 3 & 770 & 792 & 733 \\
\hline 15 & 79 & 15 & 6 & 90 & 40 & 15 & 3 & 630 & 630 & 550 \\
\hline
\end{tabular}

number of batches. Assigning the picker five batches result in 130 orders per picker, while six batches result in 156 orders. The demand level for this trial is 3,000 orders per hour, therefore in 100 minutes, 5,000 orders must be processed. The picking rate associated with an order batch size of 26 results in the need for 33 pickers, therefore each picker must be able to process 152 orders $(\lceil 5000 / 33\rceil)$.

Since 152 orders are needed to meet the required throughput, the picker is assigned six batches $(\lceil 152 / 26\rceil)$. The result of rounding the number of pickers and the number of batches assigned to each picker is an excess of 4 orders per picker and an expected throughput time equal to 102.4 minutes $\left(17.06^{*} 6\right)$, which is also slightly over the wave length. The excess throughput over all pickers is then 132 orders when rounding is considered. That is, with 152 orders per picker and 33 pickers, the total throughput is 5,016 orders. However, the actual number of orders assigned to each picker, 156, results in a total throughput of 5,148 orders. The total excess throughput, without considering the effects of rounding the number of pickers required is 148 orders.

The packer is then assigned the same batch size of 26 orders, but since the packing rate is lower than the picking rate (time per batch equals 46.66 minutes with a low packing rate), the packer can only process 2 batches within the wave. Therefore, each packer is assigned 
52 orders. The packing rate associated with a 26 -order batch size results in the need for 104 packers. Again, this leads to a slightly higher throughput than the 49 orders required to meet demand $(\lceil 5000 / 104\rceil)$. The total throughput with this size batch is 5,408 orders, while the throughput required to meet demand is 5,096 (49*103). Notice that because of rounding this number is higher than the actual demand of 5,000 orders in the 100-minute wave. The excess when considering rounding is then 312 orders, while the total excess throughput is 408 orders.

Since the picking rates were set to be tighter, that is, to result in a total throughput closer to the actual throughput required, the packers pack much more than needed. This result is reassuring since we are not able to simulate manual packing. Therefore, even without simulation, we can confidently conclude that packers will meet demand.

We could reverse the order in which we set the rates, setting the packing rates first. Using the same input parameters, the spreadsheet model recommends a wave length for the manual system is 80 minutes. The demand level of 3,000 orders per hour requires that 4,000 orders be completed within the 80-minute wave. This system also requires 47 pickers and 89 packers. Since the packers are set first, their recommended rate corresponds to a batch size of 8 orders. This batch size requires each packer to process 6 batches, or 48 orders total within 80 minutes, since this picking and packing rate combination is the first of the six points on the efficiency curve that are considered. In order to meet demand, each packer must pack 45 orders $(\lceil 4000 / 89\rceil)$. The total throughput achieved by the packer is then 4,272 orders. The batch size of 8 orders assigned to each packer then results in an excess of 267 orders total when rounding is considered. Without considering the effects of rounding, the number of packers needed to meet demand results in an excess throughput of 272 orders more than the necessary 4,000 orders.

The pickers are assigned 11 batches of 8 orders ( 88 orders per picker), resulting in an excess of 2 orders per picker when considering the effects of rounding on the number of pickers needed $(\lceil 4000 / 47\rceil)$. The total excess with 47 pickers is 94 orders with rounding, and 136 orders without rounding the number of pickers needed. Again, this approach would virtually eliminate the need for picking simulations, since we are confident that throughput could be 
Table 6.5: Example Effects of the Order of Setting Subsystem Rates for Example Trial 2.

\begin{tabular}{lcc}
\hline & \multicolumn{2}{c}{ Subsystem Set First } \\
\cline { 2 - 3 } Variable & Picking & Packing \\
\hline Wave Length (min) & 100 & 80 \\
Number of Pickers & 33 & 47 \\
Number of Packers & 104 & 89 \\
Batch Size (orders) & 26 & 8 \\
Batches per Picker & 6 & 11 \\
Batches per Packer & 2 & 6 \\
Total Picked (orders) & 5148 & 4136 \\
Total Packed (orders) & 5408 & 4272 \\
Required Picking Throughput (with rounding) & 5016 & 4042 \\
Required Packing Throughput (with rounding) & 5096 & 4005 \\
Required Throughput (orders) & 5000 & 6000 \\
\hline
\end{tabular}

met within the wave length, even though the entire assignment for that picker may not be feasible. Table 6.5 summarizes the results discussed above.

For the inductor simulation, our preliminary analysis indicated that throughput was not being met with the recommended system configuration. With experiments we were able to show that this was due to the fact that we ignored inductor blocking in our analysis. That is, if our system required 40 items per minute to be inducted and our inductors worked at a rate of 20 items per minute, our model would recommend two inductors. However, the model developed by Meller and Johnson [31] indicates that such a system could only achieve a throughput of 39.2 items per minute $(20+19.2)$ due to the first inductor blocking the second inductor when the conveyor speed equals 100 trays per minute. We then decided to adjust our deterministic model to take inductor blocking into account. The adjusted model is presented in full in Appendix G and was used for all output data presented hereafter.

Finally, once the level of batching has been set appropriately, we are ready to simulate the system. The throughput simulation models provide the following output data, as shown in Tables 6.6 and 6.7. The output data for each replication is presented in Appendix F. Tables 6.8 and 6.9 provide information about the percentage of times that a subsystem completed the assigned work within the wave length allotted and within the wave length 
plus 5 and 10 percent. In addition, the percentage of time spent idle is used to calculate worker utilization.

The inductors have unusually low utilizations in some cases due to the fact that the addition of one inductor adds a large amount of capacity since inductors work at such a high rate. For instance, one inductor may be capable of inducting 6000 items per hour, but might only be required to induct 2000 items in an hour based on demand. In Table 6.6, notice that Trials 6,11 , and 13 have extremely low induction times compared to the wave length. The reason for this is that only one inductor is assigned to the station, but the inductor can work at a rate that would provide much more throughput than actually needed. In fact, even with a higher coefficient of variation $($ c.v. $=0.4)$, the inductors meet demand in almost every case. Tables 6.10 and 6.11 compare the induction results previously shown with a low inductor coefficient of variation (0.2), to results with high inductor variation (0.4). Tables F.23 through F.24 present the breakdown of demand by inductor and induction station for both high and low coefficients of variation.

In each of the above fifteen examples, the recommended system configuration calls for only one induction station. In order to show that the induction model is appropriate for systems with more than one station, three examples were simulated with the results from the prescriptive model. These results are presented in Appendix G. Results shown here provide confidence that the induction model is accurate in predicting the number of inductors needed for sorters with higher capacity as well, since none of the total times to induct the required number of items exceeds the wave length.

Note that there are many cases in Tables 6.8 and 6.9 that appear to indicate the pickers or packers are not meeting throughput (since there is a high percentage of throughput times that exceed the wave length). However, by examining Table 6.4 we see that this throughput time is based on a number of orders much higher than the required throughput. These results constitute a worst-case scenario for the system. That observation leads us to also construct a best-case scenario as explained next. 
Table 6.6: Output Data from Throughput Simulation Model (Overlapping Waves).

\begin{tabular}{|c|c|c|c|c|c|c|c|c|c|c|}
\hline \multirow{2}{*}{$\begin{array}{l}\text { Trial } \\
\text { No. }\end{array}$} & \multirow{2}{*}{$\begin{array}{c}\text { Wave } \\
\text { Length } \\
\text { (min) }\end{array}$} & \multicolumn{3}{|c|}{ Picking } & \multicolumn{3}{|c|}{ Packing } & \multicolumn{3}{|c|}{ Induction } \\
\hline & & $\begin{array}{l}\text { Avg } \\
(\min )\end{array}$ & $\begin{array}{l}\text { Min } \\
(\min )\end{array}$ & $\begin{array}{c}\operatorname{Max} \\
(\min )\end{array}$ & $\begin{array}{c}\text { Avg } \\
(\min )\end{array}$ & $\begin{array}{l}\text { Min } \\
(\min )\end{array}$ & $\begin{array}{c}\operatorname{Max} \\
(\min )\end{array}$ & $\begin{array}{c}\text { Avg } \\
(\min )\end{array}$ & $\begin{array}{l}\text { Min } \\
(\min )\end{array}$ & $\begin{array}{c}\text { Max } \\
(\min )\end{array}$ \\
\hline 1 & 120 & 113.87 & 113.32 & 114.40 & 117.43 & 114.97 & 119.51 & 90.00 & 90.00 & 90.01 \\
\hline 2 & 40 & 39.32 & 33.65 & 43.55 & 40.26 & 38.57 & 42.79 & 30.00 & 30.00 & 30.01 \\
\hline 3 & 60 & 58.33 & 56.32 & 60.28 & 60.08 & 59.51 & 60.85 & 60.00 & 59.99 & 60.01 \\
\hline 4 & 40 & 39.80 & 38.36 & 41.01 & 39.19 & 37.97 & 40.30 & 36.43 & 36.24 & 36.59 \\
\hline 5 & 60 & 63.05 & 45.56 & 79.04 & Manual & $\mathrm{n} / \mathrm{a}$ & $\mathrm{n} / \mathrm{a}$ & Manual & $\mathrm{n} / \mathrm{a}$ & $\mathrm{n} / \mathrm{a}$ \\
\hline 6 & 80 & 73.98 & 73.18 & 74.53 & 78.06 & 73.43 & 81.15 & 37.65 & 37.35 & 37.94 \\
\hline 7 & 120 & 109.42 & 108.47 & 110.01 & 115.89 & 112.64 & 119.92 & 66.00 & 66.00 & 66.00 \\
\hline 8 & 80 & 80.88 & 62.04 & 101.28 & Manual & $\mathrm{n} / \mathrm{a}$ & $\mathrm{n} / \mathrm{a}$ & Manual & $\mathrm{n} / \mathrm{a}$ & $\mathrm{n} / \mathrm{a}$ \\
\hline 9 & 60 & 60.43 & 51.73 & 68.71 & Manual & $\mathrm{n} / \mathrm{a}$ & $\mathrm{n} / \mathrm{a}$ & Manual & $\mathrm{n} / \mathrm{a}$ & $\mathrm{n} / \mathrm{a}$ \\
\hline 10 & 60 & 59.26 & 56.29 & 62.03 & 57.95 & 55.16 & 60.88 & 55.65 & 54.88 & 56.23 \\
\hline 11 & 120 & 114.68 & 114.14 & 115.20 & 111.82 & 109.50 & 113.94 & 28.25 & 28.01 & 28.54 \\
\hline 12 & 60 & 63.46 & 50.43 & 77.08 & Manual & $\mathrm{n} / \mathrm{a}$ & $\mathrm{n} / \mathrm{a}$ & Manual & $\mathrm{n} / \mathrm{a}$ & $\mathrm{n} / \mathrm{a}$ \\
\hline 13 & 60 & 53.81 & 52.73 & 54.56 & 56.06 & 54.05 & 57.56 & 27.48 & 27.19 & 27.86 \\
\hline 14 & 80 & 81.26 & 63.38 & 97.33 & Manual & $\mathrm{n} / \mathrm{a}$ & $\mathrm{n} / \mathrm{a}$ & Manual & $\mathrm{n} / \mathrm{a}$ & $\mathrm{n} / \mathrm{a}$ \\
\hline 15 & 60 & 62.63 & 46.09 & 81.02 & Manual & $\mathrm{n} / \mathrm{a}$ & $\mathrm{n} / \mathrm{a}$ & Manual & $\mathrm{n} / \mathrm{a}$ & $\mathrm{n} / \mathrm{a}$ \\
\hline
\end{tabular}

Table 6.7: Output Data from Throughput Simulation Model (Non-overlapping Waves).

\begin{tabular}{|c|c|c|c|c|c|c|c|c|c|c|}
\hline \multirow{2}{*}{$\begin{array}{l}\text { Trial } \\
\text { No. }\end{array}$} & \multirow{2}{*}{$\begin{array}{c}\text { Wave } \\
\text { Length } \\
(\mathrm{min})\end{array}$} & \multicolumn{3}{|c|}{ Picking } & \multicolumn{3}{|c|}{ Packing } & \multicolumn{3}{|c|}{ Induction } \\
\hline & & $\begin{array}{c}\text { Avg } \\
(\min )\end{array}$ & $\begin{array}{l}\text { Min } \\
(\min )\end{array}$ & $\begin{array}{c}\operatorname{Max} \\
(\min )\end{array}$ & $\begin{array}{c}\text { Avg } \\
(\min )\end{array}$ & $\begin{array}{c}\text { Min } \\
(\min )\end{array}$ & $\begin{array}{c}\operatorname{Max} \\
(\min )\end{array}$ & $\begin{array}{c}\text { Avg } \\
(\min )\end{array}$ & $\begin{array}{c}\text { Min } \\
(\min )\end{array}$ & $\begin{array}{c}\operatorname{Max} \\
(\min )\end{array}$ \\
\hline 1 & 40 & 39.66 & 38.45 & 40.81 & 40.88 & 38.10 & 43.70 & 30.00 & 30.00 & 30.01 \\
\hline 2 & 100 & 102.55 & 75.10 & 121.92 & Manual & $\mathrm{n} / \mathrm{a}$ & $\mathrm{n} / \mathrm{a}$ & Manual & $\mathrm{n} / \mathrm{a}$ & $\mathrm{n} / \mathrm{a}$ \\
\hline 3 & 60 & 63.37 & 49.37 & 76.44 & Manual & $\mathrm{n} / \mathrm{a}$ & $\mathrm{n} / \mathrm{a}$ & Manual & $\mathrm{n} / \mathrm{a}$ & $\mathrm{n} / \mathrm{a}$ \\
\hline 4 & 20 & 19.89 & 16.82 & 21.65 & 20.66 & 18.54 & 22.48 & 18.23 & 18.09 & 18.39 \\
\hline 5 & 60 & 63.37 & 47.12 & 80.23 & Manual & $\mathrm{n} / \mathrm{a}$ & $\mathrm{n} / \mathrm{a}$ & Manual & $\mathrm{n} / \mathrm{a}$ & $\mathrm{n} / \mathrm{a}$ \\
\hline 6 & 40 & 37.71 & 36.29 & 39.24 & 40.86 & 35.46 & 45.16 & 18.80 & 18.52 & 19.13 \\
\hline 7 & 120 & 109.42 & 108.47 & 110.01 & 120.64 & 109.78 & 127.49 & 66.00 & 66.00 & 66.00 \\
\hline 8 & 80 & 79.40 & 54.63 & 96.46 & Manual & $\mathrm{n} / \mathrm{a}$ & $\mathrm{n} / \mathrm{a}$ & Manual & $\mathrm{n} / \mathrm{a}$ & $\mathrm{n} / \mathrm{a}$ \\
\hline 9 & 60 & 59.40 & 50.41 & 70.55 & Manual & $\mathrm{n} / \mathrm{a}$ & $\mathrm{n} / \mathrm{a}$ & Manual & $\mathrm{n} / \mathrm{a}$ & $\mathrm{n} / \mathrm{a}$ \\
\hline 10 & 100 & 103.25 & 62.26 & 120.64 & Manual & $\mathrm{n} / \mathrm{a}$ & $\mathrm{n} / \mathrm{a}$ & Manual & $\mathrm{n} / \mathrm{a}$ & $\mathrm{n} / \mathrm{a}$ \\
\hline 11 & 20 & 19.53 & 17.29 & 22.20 & 20.85 & 18.80 & 23.46 & 4.70 & 4.55 & 4.80 \\
\hline 12 & 60 & 62.35 & 48.06 & 79.60 & Manual & $\mathrm{n} / \mathrm{a}$ & $\mathrm{n} / \mathrm{a}$ & Manual & $\mathrm{n} / \mathrm{a}$ & $\mathrm{n} / \mathrm{a}$ \\
\hline 13 & 20 & 19.58 & 17.88 & 22.04 & 20.76 & 18.03 & 22.81 & 9.16 & 8.95 & 9.35 \\
\hline 14 & 80 & 80.40 & 62.49 & 94.39 & Manual & $\mathrm{n} / \mathrm{a}$ & $\mathrm{n} / \mathrm{a}$ & Manual & $\mathrm{n} / \mathrm{a}$ & $\mathrm{n} / \mathrm{a}$ \\
\hline 15 & 60 & 61.93 & 47.31 & 84.98 & Manual & $\mathrm{n} / \mathrm{a}$ & $\mathrm{n} / \mathrm{a}$ & Manual & $\mathrm{n} / \mathrm{a}$ & $\mathrm{n} / \mathrm{a}$ \\
\hline
\end{tabular}


Table 6.8: Results from Throughput Simulation Model (Overlapping Waves).

\begin{tabular}{|c|c|c|c|c|c|c|c|c|c|c|c|c|}
\hline \multirow{3}{*}{$\begin{array}{l}\text { Trial } \\
\text { No. }\end{array}$} & \multicolumn{4}{|c|}{ Picking } & \multicolumn{4}{|c|}{ Packing } & \multicolumn{4}{|c|}{ Induction } \\
\hline & \multirow[t]{2}{*}{ Util. } & \multicolumn{3}{|c|}{$\%$ Buffer } & \multirow[t]{2}{*}{ Util. } & \multicolumn{3}{|c|}{$\%$ Buffer } & \multirow[t]{2}{*}{ Util. } & \multicolumn{3}{|c|}{$\%$ Buffer } \\
\hline & & None & 5 & 10 & & None & 5 & 10 & & None & 5 & 10 \\
\hline 1 & 0.9489 & 0 & 0 & 0 & 0.9786 & 0 & 0 & 0 & 0.7500 & 0 & 0 & 0 \\
\hline 2 & 0.9830 & 42 & 11 & 0 & 1.0066 & 56 & 2 & 0 & 0.7500 & 0 & 0 & 0 \\
\hline 3 & 0.9721 & 1 & 0 & 0 & 1.0014 & 63 & 0 & 0 & 0.9999 & 4 & 0 & 0 \\
\hline 4 & 0.9950 & 37 & 0 & 0 & 0.9799 & 3 & 0 & 0 & 0.9108 & 0 & 0 & 0 \\
\hline 5 & 1.0508 & 69 & 53 & 36 & Manual & $\mathrm{n} / \mathrm{a}$ & $\mathrm{n} / \mathrm{a}$ & $\mathrm{n} / \mathrm{a}$ & Manual & $\mathrm{n} / \mathrm{a}$ & $\mathrm{n} / \mathrm{a}$ & $\mathrm{n} / \mathrm{a}$ \\
\hline 6 & 0.9248 & 0 & 0 & 0 & 0.9758 & 6 & 0 & 0 & 0.4706 & 0 & 0 & 0 \\
\hline 7 & 0.9118 & 0 & 0 & 0 & 0.9657 & 0 & 0 & 0 & 0.5500 & 0 & 0 & 0 \\
\hline 8 & 1.0110 & 55 & 34 & 21 & Manual & $\mathrm{n} / \mathrm{a}$ & $\mathrm{n} / \mathrm{a}$ & $\mathrm{n} / \mathrm{a}$ & Manual & $\mathrm{n} / \mathrm{a}$ & $\mathrm{n} / \mathrm{a}$ & $\mathrm{n} / \mathrm{a}$ \\
\hline 9 & 1.0071 & 55 & 23 & 4 & Manual & $\mathrm{n} / \mathrm{a}$ & $\mathrm{n} / \mathrm{a}$ & $\mathrm{n} / \mathrm{a}$ & Manual & $\mathrm{n} / \mathrm{a}$ & $\mathrm{n} / \mathrm{a}$ & $\mathrm{n} / \mathrm{a}$ \\
\hline 10 & 0.9876 & 27 & 0 & 0 & 0.9659 & 1 & 0 & 0 & 0.9276 & 0 & 0 & 0 \\
\hline 11 & 0.9557 & 0 & 0 & 0 & 0.9318 & 0 & 0 & 0 & 0.2354 & 0 & 0 & 0 \\
\hline 12 & 1.0577 & 69 & 57 & 36 & Manual & $\mathrm{n} / \mathrm{a}$ & $\mathrm{n} / \mathrm{a}$ & $\mathrm{n} / \mathrm{a}$ & Manual & $\mathrm{n} / \mathrm{a}$ & $\mathrm{n} / \mathrm{a}$ & $\mathrm{n} / \mathrm{a}$ \\
\hline 13 & 0.8968 & 0 & 0 & 0 & 0.9343 & 0 & 0 & 0 & 0.4580 & 0 & 0 & 0 \\
\hline 14 & 1.0158 & 53 & 41 & 28 & Manual & $\mathrm{n} / \mathrm{a}$ & $\mathrm{n} / \mathrm{a}$ & $\mathrm{n} / \mathrm{a}$ & Manual & $\mathrm{n} / \mathrm{a}$ & $\mathrm{n} / \mathrm{a}$ & $\mathrm{n} / \mathrm{a}$ \\
\hline 15 & 1.0438 & 59 & 45 & 32 & Manual & $\mathrm{n} / \mathrm{a}$ & $\mathrm{n} / \mathrm{a}$ & $\mathrm{n} / \mathrm{a}$ & Manual & $\mathrm{n} / \mathrm{a}$ & $\mathrm{n} / \mathrm{a}$ & $\mathrm{n} / \mathrm{a}$ \\
\hline
\end{tabular}

Table 6.9: Results from Throughput Simulation Model (Non-overlapping Waves).

\begin{tabular}{|c|c|c|c|c|c|c|c|c|c|c|c|c|}
\hline \multirow{3}{*}{$\begin{array}{c}\text { Trial } \\
\text { No. }\end{array}$} & \multicolumn{4}{|c|}{ Picking } & \multicolumn{4}{|c|}{ Packing } & \multicolumn{4}{|c|}{ Induction } \\
\hline & \multirow[t]{2}{*}{ Util. } & \multicolumn{3}{|c|}{$\%$ Buffer } & \multirow[t]{2}{*}{ Util. } & \multicolumn{3}{|c|}{$\%$ Buffer } & \multirow[t]{2}{*}{ Util. } & \multicolumn{3}{|c|}{$\%$ Buffer } \\
\hline & & None & 5 & & & None & 5 & 10 & & None & 5 & 10 \\
\hline 1 & 0.9915 & 0 & 0 & 0 & 0.9970 & 87 & 11 & 0 & 0.7500 & $\overline{0}$ & 0 & 0 \\
\hline 2 & 1.0255 & 55 & 40 & 23 & Manual & $\mathrm{n} / \mathrm{a}$ & $\mathrm{n} / \mathrm{a}$ & $\mathrm{n} / \mathrm{a}$ & Manual & $\mathrm{n} / \mathrm{a}$ & $\mathrm{n} / \mathrm{a}$ & $\mathrm{n} / \mathrm{a}$ \\
\hline 3 & 1.0562 & 62 & 49 & 33 & Manual & $\mathrm{n} / \mathrm{a}$ & $\mathrm{n} / \mathrm{a}$ & $\mathrm{n} / \mathrm{a}$ & Manual & $\mathrm{n} / \mathrm{a}$ & $\mathrm{n} / \mathrm{a}$ & $\mathrm{n} / \mathrm{a}$ \\
\hline 4 & 0.9947 & 9 & 9 & 0 & 0.9837 & 92 & 29 & 2 & 0.9114 & 0 & 0 & 0 \\
\hline 5 & 1.0561 & 63 & 51 & 37 & Manual & $\mathrm{n} / \mathrm{a}$ & $\mathrm{n} / \mathrm{a}$ & $\mathrm{n} / \mathrm{a}$ & Manual & $\mathrm{n} / \mathrm{a}$ & $\mathrm{n} / \mathrm{a}$ & $\mathrm{n} / \mathrm{a}$ \\
\hline 6 & 0.9427 & 0 & 0 & 0 & 0.9965 & 70 & 25 & 5 & 0.4700 & 0 & 0 & 0 \\
\hline 7 & 0.9118 & 0 & 0 & 0 & 0.9970 & 70 & 2 & 0 & 0.5500 & 0 & 0 & 0 \\
\hline 8 & 0.9925 & 48 & 29 & 18 & Manual & $\mathrm{n} / \mathrm{a}$ & $\mathrm{n} / \mathrm{a}$ & $\mathrm{n} / \mathrm{a}$ & Manual & $\mathrm{n} / \mathrm{a}$ & $\mathrm{n} / \mathrm{a}$ & $\mathrm{n} / \mathrm{a}$ \\
\hline 9 & 0.9900 & 29 & 18 & 6 & Manual & $\mathrm{n} / \mathrm{a}$ & $\mathrm{n} / \mathrm{a}$ & $\mathrm{n} / \mathrm{a}$ & Manual & $\mathrm{n} / \mathrm{a}$ & $\mathrm{n} / \mathrm{a}$ & $\mathrm{n} / \mathrm{a}$ \\
\hline 10 & 1.0325 & 61 & 41 & 25 & Manual & $\mathrm{n} / \mathrm{a}$ & $\mathrm{n} / \mathrm{a}$ & $\mathrm{n} / \mathrm{a}$ & Manual & $\mathrm{n} / \mathrm{a}$ & $\mathrm{n} / \mathrm{a}$ & $\mathrm{n} / \mathrm{a}$ \\
\hline 11 & 0.9763 & 4 & 4 & 1 & 0.9927 & 81 & 36 & 15 & 0.2348 & 0 & 0 & 0 \\
\hline 12 & 1.0391 & 54 & 42 & 30 & Manual & $\mathrm{n} / \mathrm{a}$ & $\mathrm{n} / \mathrm{a}$ & $\mathrm{n} / \mathrm{a}$ & Manual & $\mathrm{n} / \mathrm{a}$ & $\mathrm{n} / \mathrm{a}$ & $\mathrm{n} / \mathrm{a}$ \\
\hline 13 & 0.9790 & 9 & 9 & 1 & 0.9884 & 81 & 41 & 7 & 0.4579 & 0 & 0 & 0 \\
\hline 14 & 1.0050 & 51 & 32 & 15 & Manual & $\mathrm{n} / \mathrm{a}$ & $\mathrm{n} / \mathrm{a}$ & $\mathrm{n} / \mathrm{a}$ & Manual & $\mathrm{n} / \mathrm{a}$ & $\mathrm{n} / \mathrm{a}$ & $\mathrm{n} / \mathrm{a}$ \\
\hline 15 & 1.0322 & 54 & 43 & 25 & Manual & $\mathrm{n} / \mathrm{a}$ & $\mathrm{n} / \mathrm{a}$ & $\mathrm{n} / \mathrm{a}$ & Manual & $\mathrm{n} / \mathrm{a}$ & $\mathrm{n} / \mathrm{a}$ & $\mathrm{n} / \mathrm{a}$ \\
\hline
\end{tabular}


Table 6.10: Comparison of Induction Results with High and Low Variation (Overlapping Waves).

\begin{tabular}{r|rccc|cccc}
\hline & \multicolumn{4}{|c|}{ c.v. $=0.2$} & \multicolumn{4}{c}{ c.v. $=0.4$} \\
\hline $\begin{array}{r}\text { Trial } \\
\text { No. }\end{array}$ & Util. & $\begin{array}{c}\text { Avg. } \\
\text { (min.) }\end{array}$ & $\begin{array}{c}\text { Min. } \\
(\text { min. })\end{array}$ & $\begin{array}{c}\text { Max. } \\
(\text { min. })\end{array}$ & Util. & $\begin{array}{c}\text { Avg. } \\
(\text { min. })\end{array}$ & $\begin{array}{c}\text { Min. } \\
(\text { min. })\end{array}$ & $\begin{array}{c}\text { Max. } \\
\text { (min.) }\end{array}$ \\
\hline 1 & 0.7500 & 90.00 & 90.01 & 90.00 & 0.7500 & 90.00 & 90.01 & 90.00 \\
2 & 0.7500 & 30.00 & 30.01 & 30.00 & 0.7500 & 30.00 & 30.01 & 30.00 \\
3 & 0.9999 & 60.00 & 60.01 & 59.99 & 1.0000 & 60.00 & 60.01 & 59.99 \\
4 & 0.9108 & 36.43 & 36.59 & 36.24 & 0.9344 & 37.38 & 37.83 & 37.07 \\
5 & Man. & Man. & Man. & Man. & Man. & Man. & Man. & Man. \\
6 & 0.4706 & 37.65 & 37.94 & 37.35 & 0.4418 & 35.34 & 35.77 & 34.91 \\
7 & 0.5500 & 66.00 & 66.00 & 66.00 & 0.5500 & 66.00 & 66.00 & 66.00 \\
8 & Man. & Man. & Man. & Man. & Man. & Man. & Man. & Man. \\
9 & Man. & Man. & Man. & Man. & Man. & Man. & Man. & Man. \\
10 & 0.9276 & 55.65 & 56.23 & 54.88 & 0.9282 & 55.69 & 56.42 & 54.67 \\
11 & 0.2354 & 28.25 & 28.54 & 28.01 & 0.2208 & 26.50 & 26.77 & 26.11 \\
12 & Man. & Man. & Man. & Man. & Man. & Man. & Man. & Man. \\
13 & 0.4580 & 27.48 & 27.86 & 27.19 & 0.4578 & 27.47 & 27.96 & 26.89 \\
14 & Man. & Man. & Man. & Man. & Man. & Man. & Man. & Man. \\
15 & Man. & Man. & Man. & Man. & Man. & Man. & Man. & Man. \\
\hline
\end{tabular}

Table 6.11: Comparison of Induction Results with High and Low Variation (Non-overlapping Waves).

\begin{tabular}{r|rrrr|rrrr}
\hline & \multicolumn{4}{|c|}{ c.v. $=0.2$} & \multicolumn{4}{c}{ c.v. $=0.4$} \\
\hline $\begin{array}{r}\text { Trial } \\
\text { No. }\end{array}$ & Util. & $\begin{array}{c}\text { Avg. } \\
(\text { min. })\end{array}$ & $\begin{array}{c}\text { Min. } \\
(\text { min. })\end{array}$ & $\begin{array}{c}\text { Max. } \\
(\text { min. })\end{array}$ & Util. & $\begin{array}{c}\text { Avg. } \\
(\text { min. })\end{array}$ & $\begin{array}{c}\text { Min. } \\
(\text { min. })\end{array}$ & $\begin{array}{c}\text { Max. } \\
(\text { min. })\end{array}$ \\
\hline 1 & 0.7500 & 30.00 & 30.01 & 30.00 & 0.7500 & 30.00 & 30.01 & 30.00 \\
2 & Man. & Man. & Man. & Man. & Man. & Man. & Man. & Man. \\
3 & Man. & Man. & Man. & Man. & Man. & Man. & Man. & Man. \\
4 & 0.9114 & 18.23 & 18.39 & 18.09 & 0.9350 & 18.70 & 18.96 & 18.50 \\
5 & Man. & Man. & Man. & Man. & Man. & Man. & Man. & Man. \\
6 & 0.4700 & 18.80 & 19.13 & 18.52 & 0.4411 & 17.64 & 17.95 & 17.38 \\
7 & 0.5500 & 66.00 & 66.00 & 66.00 & 0.5500 & 66.00 & 66.00 & 66.00 \\
8 & Man. & Man. & Man. & Man. & Man. & Man. & Man. & Man. \\
9 & Man. & Man. & Man. & Man. & Man. & Man. & Man. & Man. \\
10 & Man. & Man. & Man. & Man. & Man. & Man. & Man. & Man. \\
11 & 0.2348 & 4.70 & 4.80 & 4.55 & 0.2204 & 4.41 & 4.54 & 4.28 \\
12 & Man. & Man. & Man. & Man. & Man. & Man. & Man. & Man. \\
13 & 0.4579 & 9.16 & 9.35 & 8.95 & 0.4581 & 9.16 & 9.55 & 8.84 \\
14 & Man. & Man. & Man. & Man. & Man. & Man. & Man. & Man. \\
15 & Man. & Man. & Man. & Man. & Man. & Man. & Man. & Man. \\
\hline
\end{tabular}


Table 6.12: Output Data — Best Case Scenario (Overlapping Waves).

\begin{tabular}{|c|c|c|c|c|c|c|c|c|c|}
\hline \multirow{2}{*}{$\begin{array}{l}\text { Trial } \\
\text { No. }\end{array}$} & \multirow{2}{*}{$\begin{array}{c}\text { Wave } \\
\text { Length } \\
(\mathrm{min})\end{array}$} & \multicolumn{4}{|c|}{ Picking } & \multicolumn{4}{|c|}{ Packing } \\
\hline & & Ratio & $\begin{array}{c}\text { Avg } \\
(\min )\end{array}$ & $\begin{array}{l}\text { Min } \\
(\min )\end{array}$ & $\begin{array}{l}\operatorname{Max} \\
(\min )\end{array}$ & Ratio & $\begin{array}{c}\text { Avg } \\
(\min )\end{array}$ & $\begin{array}{l}\text { Min } \\
(\min )\end{array}$ & $\begin{array}{c}\operatorname{Max} \\
(\min )\end{array}$ \\
\hline 1 & 20 & 0.9990 & 113.76 & 113.21 & 114.28 & 0.9990 & 117.32 & 114.86 & 119.39 \\
\hline 2 & 40 & 0.9921 & 39.01 & 33.38 & 43.21 & .9921 & 39.94 & 38.27 & 42.45 \\
\hline 3 & 60 & 0.9980 & 58.21 & 56.20 & 60.16 & 0.9967 & 59.88 & 59.31 & 60.65 \\
\hline 4 & 40 & 0.9921 & 39.48 & 38.05 & 40.69 & 0.9990 & 39.16 & 37.93 & 40.26 \\
\hline 5 & 60 & 0.9524 & 60.05 & 43.39 & 75.27 & Manual & Manual & Manual & Manual \\
\hline 6 & 80 & 0.9977 & 73.82 & 73.02 & 74.36 & 0.9857 & 76.95 & 72.38 & 79.99 \\
\hline 7 & 120 & 1.0000 & 109.42 & 108.47 & 110.01 & 0.9964 & 115.47 & 112.23 & 119.49 \\
\hline 8 & 80 & 0.9586 & 77.53 & 59.47 & 97.09 & Manual & Manual & Manual & Manual \\
\hline 9 & 60 & 0.9700 & 58.62 & 50.18 & 66.65 & Manual & Manual & Manual & Manual \\
\hline 10 & 60 & 0.9964 & 59.04 & 56.08 & 61.81 & 0.9964 & 57.74 & 54.96 & 60.66 \\
\hline 11 & 120 & 1.0000 & 114.68 & 114.14 & 115 & 1.0000 & 111.82 & 109.50 & 113.94 \\
\hline 12 & 60 & 0.8730 & 55.40 & 44.03 & 67.29 & Manual & Manual & Manual & Manual \\
\hline 13 & 60 & 0.9964 & 53.61 & 52.54 & 54.36 & 1.0000 & 56.06 & 54.05 & 57.56 \\
\hline 14 & 80 & 0.952 & 77.39 & 60.36 & 92.69 & Manual & Manual & Manual & Manual \\
\hline 15 & 60 & 0.8730 & 54.67 & 40.24 & 70.74 & Manual & Manual & Manual & Manual \\
\hline
\end{tabular}

In order to show the effects of order-batching on the system throughput, we have developed similar results with a best-case scenario. That is, we have multiplied each picking and packing time by a ratio of the number of orders required to meet demand to the number of orders actually picked. For instance, Trial 5 in Table 6.6 requires a 60 -minute wave. The total pick time for 90 orders is 63.05 minutes, but only 86 orders were actually required. Therefore, we multiply 63.05 by $(86 / 90)$, or 0.9524 , in order to obtain the best-case picking time of 60.05 minutes. Tables 6.12 and 6.13 present the output data based on this method, with the ratio used to determine the best-case time presented for each subsystem. Tables 6.14 and 6.15 present the results for this system in terms of the percentage of waves where the picking time exceeds the wave length. The induction data is not presented for the best-case scenario. Since inductors work in terms of items instead of orders, batching does not affect their throughput capabilities.

The simulations performed verified that the descriptive spreadsheet model is a good approximation in terms of the numbers of pickers and packers required to meet demand, as well 
Table 6.13: Output Data - Best Case Scenario (Non-overlapping Waves).

\begin{tabular}{|c|c|c|c|c|c|c|c|c|c|}
\hline \multirow{2}{*}{$\begin{array}{c}\text { Trial } \\
\text { No. }\end{array}$} & \multirow{2}{*}{$\begin{array}{c}\text { Wave } \\
\text { Length } \\
(\min )\end{array}$} & \multicolumn{4}{|c|}{ Picking } & \multicolumn{4}{|c|}{ Packing } \\
\hline & & Ratio & $\begin{array}{c}\text { Avg } \\
(\min )\end{array}$ & $\begin{array}{l}\text { Min } \\
(\min )\end{array}$ & $\begin{array}{l}\operatorname{Max} \\
(\min )\end{array}$ & Ratio & $\begin{array}{c}\text { Avg } \\
(\min )\end{array}$ & $\begin{array}{c}\text { Min } \\
(\min )\end{array}$ & $\begin{array}{l}\operatorname{Max} \\
(\min )\end{array}$ \\
\hline 1 & 40 & 0.9921 & 39.35 & 38.15 & 40.49 & 0.9416 & 38.49 & 35.87 & 41.15 \\
\hline 2 & 100 & 0.9713 & 99.60 & 72.94 & 118.41 & Manual & Manual & Manual & Manual \\
\hline 3 & 60 & 0.9524 & 60.35 & 47.02 & 72.80 & Manual & Manual & Manual & Manual \\
\hline 4 & 20 & 0.9747 & 19.39 & 16.39 & 21.10 & 0.9775 & 20.19 & 18.12 & 21.97 \\
\hline 5 & 60 & 0.9524 & 60.35 & 44.88 & 76.41 & Manual & Manual & Manual & Manual \\
\hline 6 & 40 & 0.9977 & 37.62 & 36.20 & 39.15 & 0.9662 & 39.48 & 34.26 & 43.63 \\
\hline 7 & 120 & 1.0000 & 109.42 & 108.47 & 110.01 & 0.9857 & 118.91 & 108.21 & 125.66 \\
\hline 8 & 80 & 0.9586 & 76.12 & 52.37 & 92.47 & Manual & Manual & Manual & Manual \\
\hline 9 & 60 & 0.9700 & 57.62 & 48.90 & 68.44 & Manual & Manual & Manual & Manual \\
\hline 10 & 100 & 0.9040 & 93.34 & 56.29 & 109.06 & Manual & Manual & Manual & Manual \\
\hline 11 & 20 & 0.9910 & 19.35 & 17.13 & 22.00 & 0.9857 & 20.55 & 18.53 & 23.12 \\
\hline 12 & 60 & 0.8730 & 54.43 & 41.96 & 69.49 & Manual & Manual & Manual & Manual \\
\hline 13 & 20 & 0.9910 & 19.40 & 17.72 & 21.85 & 0.9857 & 20.46 & 17.78 & 22.48 \\
\hline 14 & 80 & 0.9524 & 76.57 & 59.51 & 89.89 & Manual & Manual & Manual & Manual \\
\hline 15 & 60 & 0.8730 & 54.07 & 41.31 & 74.19 & Manual & Manual & Manual & Manual \\
\hline
\end{tabular}

Table 6.14: Results - Best Case Scenario (Overlapping Waves).

\begin{tabular}{r|c|rrr|r|rrr}
\hline Trial & \multicolumn{4}{|c|}{ Picking } & \multicolumn{4}{|c}{ Packing } \\
\cline { 2 - 8 } No. & Util. & \multicolumn{3}{|c|}{$\%$ Buffer } & Util. & \multicolumn{3}{|c}{ Buffer } \\
\cline { 2 - 8 } & & None & 5 & 10 & & None & 5 & 10 \\
\hline 1 & 0.9480 & 0 & 0 & 0 & 0.9776 & 0 & 0 & 0 \\
2 & 0.9752 & 33 & 6 & 0 & 0.9986 & 42 & 1 & 0 \\
3 & 0.9702 & 1 & 0 & 0 & 0.9981 & 29 & 0 & 0 \\
4 & 0.9871 & 22 & 0 & 0 & 0.9789 & 2 & 0 & 0 \\
5 & 1.0008 & 53 & 35 & 16 & Manual & $\mathrm{n} / \mathrm{a}$ & $\mathrm{n} / \mathrm{a}$ & $\mathrm{n} / \mathrm{a}$ \\
6 & 0.9227 & 0 & 0 & 0 & 0.9618 & 0 & 0 & 0 \\
7 & 0.9118 & 0 & 0 & 0 & 0.9622 & 0 & 0 & 0 \\
8 & 0.9691 & 36 & 23 & 12 & Manual & $\mathrm{n} / \mathrm{a}$ & $\mathrm{n} / \mathrm{a}$ & $\mathrm{n} / \mathrm{a}$ \\
9 & 0.9769 & 36 & 9 & 1 & Manual & $\mathrm{n} / \mathrm{a}$ & $\mathrm{n} / \mathrm{a}$ & $\mathrm{n} / \mathrm{a}$ \\
10 & 0.9841 & 27 & 0 & 0 & 0.9624 & 1 & 0 & 0 \\
11 & 0.9557 & 0 & 0 & 0 & 0.9318 & 0 & 0 & 0 \\
12 & 0.9234 & 24 & 7 & 1 & Manual & $\mathrm{n} / \mathrm{a}$ & $\mathrm{n} / \mathrm{a}$ & $\mathrm{n} / \mathrm{a}$ \\
13 & 0.8936 & 0 & 0 & 0 & 0.9343 & 0 & 0 & 0 \\
14 & 0.9674 & 41 & 27 & 5 & Manual & $\mathrm{n} / \mathrm{a}$ & $\mathrm{n} / \mathrm{a}$ & $\mathrm{n} / \mathrm{a}$ \\
15 & 0.9112 & 17 & 8 & 4 & Manual & $\mathrm{n} / \mathrm{a}$ & $\mathrm{n} / \mathrm{a}$ & $\mathrm{n} / \mathrm{a}$ \\
\hline
\end{tabular}


Table 6.15: Results — Best Case Scenario (Non-overlapping Waves).

\begin{tabular}{|c|c|c|c|c|c|c|c|c|}
\hline \multirow{3}{*}{$\begin{array}{l}\text { Trial } \\
\text { No. }\end{array}$} & \multicolumn{4}{|c|}{ Picking } & \multicolumn{4}{|c|}{ Packing } \\
\hline & \multirow[t]{2}{*}{ Util. } & \multicolumn{3}{|c|}{$\%$ Buffer } & \multirow[t]{2}{*}{ Util. } & \multicolumn{3}{|c|}{$\%$ Buffer } \\
\hline & & None & 5 & 10 & & None & 5 & 10 \\
\hline 1 & 0.9836 & 0 & 0 & 0 & 0.9388 & 4 & 0 & 0 \\
\hline 2 & 0.9960 & 47 & 26 & 12 & Manual & $\mathrm{n} / \mathrm{a}$ & $\mathrm{n} / \mathrm{a}$ & $\mathrm{n} / \mathrm{a}$ \\
\hline 3 & 1.0059 & 43 & 33 & 19 & Manual & $\mathrm{n} / \mathrm{a}$ & $\mathrm{n} / \mathrm{a}$ & $\mathrm{n} / \mathrm{a}$ \\
\hline 4 & 0.9695 & 2 & 2 & 0 & 0.9615 & 63 & 9 & 0 \\
\hline 5 & 1.0058 & 46 & 36 & 18 & Manual & $\mathrm{n} / \mathrm{a}$ & $\mathrm{n} / \mathrm{a}$ & $\mathrm{n} / \mathrm{a}$ \\
\hline 6 & 0.9406 & 0 & 0 & 0 & 0.9628 & 34 & 8 & 0 \\
\hline 7 & 0.9118 & 0 & 0 & 0 & 0.9827 & 29 & 0 & 0 \\
\hline 8 & 0.9515 & 28 & 18 & 5 & Manual & $\mathrm{n} / \mathrm{a}$ & $\mathrm{n} / \mathrm{a}$ & $\mathrm{n} / \mathrm{a}$ \\
\hline 9 & 0.9603 & 19 & 7 & 3 & Manual & $\mathrm{n} / \mathrm{a}$ & $\mathrm{n} / \mathrm{a}$ & $\mathrm{n} / \mathrm{a}$ \\
\hline 10 & 0.9334 & 16 & 8 & 0 & Manual & $\mathrm{n} / \mathrm{a}$ & $\mathrm{n} / \mathrm{a}$ & $\mathrm{n} / \mathrm{a}$ \\
\hline 11 & 0.9675 & 4 & 4 & 1 & 0.9785 & 70 & 26 & 7 \\
\hline 12 & 0.9072 & 14 & 7 & 3 & Manual & $\mathrm{n} / \mathrm{a}$ & $\mathrm{n} / \mathrm{a}$ & $\mathrm{n} / \mathrm{a}$ \\
\hline 13 & 0.9702 & 3 & 3 & 0 & 0.9742 & 68 & 25 & 5 \\
\hline 14 & 0.9571 & 31 & 13 & 5 & Manual & $\mathrm{n} / \mathrm{a}$ & $\mathrm{n} / \mathrm{a}$ & $\mathrm{n} / \mathrm{a}$ \\
\hline 15 & 0.9011 & 12 & 9 & 5 & Manual & $\mathrm{n} / \mathrm{a}$ & $\mathrm{n} / \mathrm{a}$ & $\mathrm{n} / \mathrm{a}$ \\
\hline
\end{tabular}

as their level of batching and corresponding picking and packing rates. Although the excess throughput resulting from batching causes some processing times to exceed the wave length, the order in which we set the picking and packing rates can reduce this to a minimum for one system. The method used here, setting the picking rates first, was useful since our packing times are estimated from time study data. The excess throughput in the packing system provides us with a buffer that increases the likelihood of meeting demand within the wave.

From the results shown in this chapter, we can see that rounding the number of pickers, packers, and orders in a batch has a significant effect on the system. Therefore, when performing this type of analysis for an actual company, the amount of rounding can be taken into consideration, and the company can decide if batch sizes are constant over the wave or whether they would be varied to meet throughput. This would not only allow matching the number of orders picked or packed to match the required throughput, but would provide the workers with a reasonable buffer within the implemented wave length.

In addition, we notice that pickers and packers working in shorter waves requiring smaller batches are susceptible to exceeding the wave length when picking or packing their assigned 
batches. This is due to larger variance associated with a small batch. For instance, a picker with a batch size of 8 orders may have to travel into aisles spread throughout the picking area or may only have to travel through a few aisles relatively close together. This could be the difference between up to 24 aisles, or as few as one aisle. With a large batch size, however, the variance becomes much smaller since it is very likely the picker will visit a large number of aisles. This is true for both manual and automated systems, and, in general, does not make one system more attractive than the other. In such a case the batch size might have to be increased in order to lower the variance, which would increase the number of packers in a manual system. Another solution would be to assign more workers or provide a buffer within the wave length. Of course, since a manual system typically utilizes more pickers, the potential increase in pickers required for a manual system could be higher than for an automated system. Therefore, the simulation results suggest that the deterministic model appears to be conservative with respect to the benefits it calculates for automated systems in comparison with manual systems.

Finally, the simulation results verified that the adjusted model is very accurate in determining how many inductors are needed to meet throughput. This is true for both high and low coefficients of variation on the induction times. 


\section{Chapter 7}

\section{Conclusions and Future Research}

\subsection{Conclusions}

Changes in the way companies do business have led to a need for more efficient order ful-

fillment systems. Again, automated sortation is one way to improve the efficiency of a distribution center, if designed correctly. Tradeoffs between picking and packing efficiencies must be clearly understood. In addition, the larger tradeoff between the capital costs involved with implementing an automated sorter and the labor savings it will reap must be carefully weighed. Other system parameters such as the wave length, size of the system (in terms of packing and induction stations), and design of the sorter itself (speed of the conveyor, capacity of each pack station, etc.) must be factored into the decision to implement a sorter as well.

The complexity of the relationships and combinations of parameters leads to the need of a guide to help a company determine if this investment is wise. Our research is an attempt to provide such a guide. Although some relationships are simplified, the prescriptive model developed provides cost estimates over a wide variety of parameters. This model provides the company with many feasible combinations, with the recommended system design being the one with the lowest total annual cost. However, the company can see how changing the wave length or picking and packing rates will effect the cost of the system. In addition, the 
model illustrates the differences between manual and automated systems. For example, a manager could look at each combination of parameters' total annual cost for both manual and automated systems and determine very quickly if an automated system should be considered at all if every manual combination provides a much lower annual cost than the automated system.

Sensitivity analyses determined the effects of changing each parameter separately as well as in combination with one another. These analyses also provide insights into situations where one system would never be considered over another. For example, a system with very low demand would not implement an automated system design in most cases. We have also provided an analytical model to determine the optimal picking and packing rates for a manual system given a set of lower and upper bounds for each.

The relationships in the spreadsheet model were verified using simulation and time study data. This provides us with confidence that our model is accurate enough to make the decision as to whether manual or automated sortation should be used as well as an appropriate initial system design. However, systems with high variance may require adjustments to the output from the model.

Although we believe our model represents the most critical tradeoffs in the design of order fulfillment centers, we acknowledge the need for future research and discuss possible alternatives in the next section.

\subsection{Future Research}

Our model determines the best system configuration given a set of parameters. These parameters are all cost or throughput related. However, we should also consider parameters or constraints concerning the physical size of the sortation system. Most companies have a limited amount of square footage in which to put a sorter, so this constraint can be very important. For other companies, size is not an issue. This constraint should be taken into consideration when designing the sortation system. Some sorters have two levels, which 
might add to the sorter cost, but keep the sorter within the space restrictions. Also, the cost of the floor space square footage used by the sorter should be taken into account in the objective function.

Another issue not addressed by our model is demand fluctuations. This tends to be a seasonal problem for most companies, such as clothing and catalog sales. For instance, if a new clothing catalog comes out, there might be a peak in demand for a few weeks. Another good example is the Christmas season. We have considered a system designed for peak demand, but there are also many lulls in most businesses. Therefore, the decision must be made as to whether to design for peak or average demand. A tradeoff can be made between the lost efficiency during the lulls with a sorter designed for peak demand and the overutilization of the sorter during peak demands for a smaller sorter design. However, some sort of compromise in the system design may be warranted for a system with only a few peaks and many lulls. For example, a company could design for the average or typical demand levels, and then hire temporary workers, set up additional manual packing stations, and possibly increase the sorter speed or utilize additional induction stations during the peak times.

Another possible alternative that we observed in industry is pre-sorting the items for packing stations in an automated system. Without pre-sorting, the maximum throughput in a twoinduction station system is 1.33 times the speed of the sorting conveyor. In general, without pre-sorting, as shown previously in (4.1), the maximum throughput in a system with $r_{s}$ induction stations is $\left(\frac{2 r_{s}}{r_{s}+1}\right) s$, where $s$ is equal to the speed of the conveyor [31]. To increase throughput, pre-sorting is performed so that the items that belong in the bins before the next induction station are inducted at that station (to free all trays before the next induction station), allowing the system to achieve twice as much throughput in a two-induction station system, three times as much throughput in a three-station system, etc. However, the throughput increase achieved with pre-sorting must be balanced with its negative impact on picking since the possible picking batch combinations are reduced with pre-sorting.

Another limitation of our model is the method we used for order-batching in the manual system. We assigned each worker an equal-size batch, therefore sometimes exceeding the re- 
quired demand level. This results in pickers and packers who cannot complete their assigned orders within the allotted wave length. However, we should consider alternative methods of batching. For instance, each picker could be assigned full batches until the last batch, when the batch size could be the remainder of the orders needed to meet demand divided by the number of pickers. Another solution would be to assign faster pickers more work in order to make the batches adequately sized for each worker.

Another limitation in our model is the estimation of the picking efficiency increase between wave lengths. This estimation was made based on our time study data. The picking simulation model should be modified to include intelligent order-batching methods such as those described in Section 2.3.5. This would more accurately reflect the benefits of increasing the wave length on picking efficiencies.

As one can see, there are many possible alternatives for future research on this topic. Addressing such research issues would likely result in a model that is more widely applicable and of more value to engineers in industry designing order fulfillment centers. 


\section{Bibliography}

[1] "Cleco Systems: Sortation: Tomorrow's Technology Today!," http://www.cleco.nl/objects/images/sortation.jpg (2000).

[2] "Hanover Direct," (2000).

[3] "Rapistan Systems: Sortation Systems," http://www.rapistan.com/marketing/products/sorters/ (2000).

[4] "Gross \& Associates Rules of Thumb for Warehousing \& Distribution Equipment Costs," (2001), 7th Edition.

[5] Anonymous, "Throughput Soars with Sorter," Modern Materials Handling, pp. 69-70 (October 1999).

[6] Bozer, Y. A., and White, J. A., "A Generalized Design and Performance Analysis Model for End-of-Aisle Order-Picking Systems," IIE Transactions, 28, 4, 271-280 (1996).

[7] Bozer, Y. A., Quiroz, M. A., and Sharp, G. P., "An Evaluation of Alternative Control Strategies and Design Issues for Automated Order Accumulation and Sortation Systems," Material Flow, 4, 265-282 (1988).

[8] Bozer, Y. A., and Sharp, G. P., "An Empirical Evaluation of a General Purpose Automated Order Accumulation and Sortation System used in Batch Picking," Material Flow, 2, 111-131 (1985).

[9] Caron, F., Marchet, G., and Perego, A., "Routing Policies and COI-Based Storage Policies in Picker-to-Part Systems," International Journal of Production Research, 36, 3, 713-732 (1998).

[10] Cormier, G., and Gunn, E., "Simple Models and Insights for Warehouse Sizing," Journal of the Operational Research Society, 47, 5, 690-696 (1996).

[11] Cormier, G., and Gunn, E. A., "A Review of Warehouse Models," European Journal of Operational Research, 58, 3-13 (1992).

[12] Coyle, J., Bardi, E., and Langley, C., The Management of Business Logistics, West, St. Paul, MN (1996). 
[13] Daniels, R. L., Rummel, J. L., and Schantz, R., "A Model for Warehouse Order Picking," European Journal of Operational Research, 105, 1, 1-17 (1998).

[14] de Koster, R., and van der Poort, E., "Routing Orderpickers in a Warehouse: A Comparison Between Optimal and Heuristic Solutions," IIE Transactions, 30, 5, 469480 (1998).

[15] de Koster, R., van der Poort, E., and Wolters, M., "Efficient Orderbatching Methods in Warehouses," International Journal of Production Research, 37, 7, 1479-1504 (1999).

[16] Drury, J., "Towards More Efficient Orderpicking," In IMM Monographs 1, Cranfield, UK (1988). Institute of Materials Management.

[17] El Sayed, E. A., "Algorithms for Optimal Material Handling in Automatic Warehousing Systems," International Journal of Production Research, 19, 525-535 (1981).

[18] El Sayed, E. S., Proctor, C. L., and Elayat, H. A., "Analysis of Closed-Loop Conveyor Systems with Mutiple Poisson Inputs and Outputs," International Journal of Production Research, 14, 1, 99-109 (1976).

[19] Erlebacher, S., and Meller, R., "The Interaction of Location and Inventory in Designing Distribution Systems," IIE Transactions, 32, 2, 155-166 (2000).

[20] Forger, G., "How Fingerhut Plans to Ride the Growth Curve," Modern Materials Handling, pp. 48-51 (June 1993).

[21] Gibson, D. R., and Sharp, G. P., "Order Batching Procedures," European Journal of Operational Research, 58, 57-67 (1992).

[22] Goetschalckx, M., and Ratliff, H., "Order Picking in an Aisle," IIE Transactions, 20, 1, 53-62 (1988).

[23] Gould, L., "Increase Productivity by Choosing the Right Sortation Conveyor," Modern Materials Handling, pp. 54-56 (June 1991).

[24] Gray, A. E., Karmarkar, U. S., and Seidmann, A., "Design and Operation of an OrderConsolidation Warehouse: Models and Application," European Journal of Operational Research, 58, 14-34 (1992).

[25] Hausman, W. H., Schwarz, L. B., and Graves, S. C., "Optimal Storage Assignment in Automated Warehousing Systems," Management Science, 22, 6, 625-638 (1976).

[26] Jarvis, J. M., and McDowell, E. D., "Optimal Product Layout in an Order Picking Warehouse," IIE Transactions, 23, 1, 93-102 (1991).

[27] Johnson, M. E., "The Impact of Sorting Strategies on Automated Sortation System Performance," IIE Transactions, 30, 1, 67-77 (1998).

[28] Larson, T., March, H., and Kusiak, A., "A Heuristic Approach to Warehouse Layout with Class-Based Storage," IIE Transactions, 29, 4, 337-348 (1997). 
[29] Malmborg, C. J., "Storage Assignment Policy Tradeoffs," International Journal of Production Research, 34, 2, 363-378 (1996).

[30] Meller, R. D., "Optimal Order-to-Lane Assignments in an Order Accumulation/Sortation System," IIE Transactions, 29, 4, 293-301 (1997).

[31] Meller, R. D., and Johnson, E. M., "Sortation Results for Split-Case Batch-Picking Systems," Technical report, Department of Industrial Engineering, Auburn University (1999).

[32] Meller, R. D., and Johnson, M. E., "Design of Split-Case Sortation Systems," In 6th International Colloquium on Material Handling Research Proceedings, York, Pennsylvania (2000).

[33] Meller, R. D., and Mungwattana, A., "Multi-Shuttle Automated Storage/Retrieval Systems," IIE Transactions, 29, 925-938 (1997).

[34] Muth, E. J., "Analysis of Closed-Loop Conveyor Systems," AIIE Transactions, 4, 2, 134-143 (1972).

[35] Muth, E. J., "Analysis of Closed-Loop Conveyor Systems, the Discrete Flow Case," AIIE Transactions, 6, 1, 73-83 (1974).

[36] Muth, E. J., "A Model of a Closed-Loop Conveyor System with Random Material Flow," AIIE Transactions, 9, 4, 345-351 (1977).

[37] Proctor, C. L., El Sayed, E. S., and Elayat, H. A., "A Conveyor System with Homogeneous and Heterogeneous Servers with Dual Input," International Journal of Production Research, 15, 1, 73-85 (1977).

[38] Quick, R., "The Lessons Learned: The Rush to the Web Last Holiday Season Left Many Shoppers Fuming - And Many Web Sites Scrambling to Come Up With a Better Plan," The Asian Wall Street Journal, pg. T9 (April 24 2000).

[39] Ratliff, H. D., and Rosenthal, A. S., "Order-Picking in a Rectangular Warehouse: A Solvable Case of the Traveling Salesman Problem," Operations Research, 31, 3, 507-521 (1983).

[40] Tompkins, J. A., and White, J. A., Facilities Planning, John Wiley \& Sons, New York, New York (1984).

[41] van den Berg, J. P., "A Literature Survey on Planning and Control of Warehousing Systems," IIE Transactions on Scheduling \& Logistics, 31, 8, 751-762 (1999). 


\section{Appendix A}

\section{Time Study Data and Results}

All time study data collected for picking and packing rates are presented in this appendix. Tables A.1 through A.4 present data for the zone picking time study. Each table represents a different level of order-batching. Table A.5 summarizes the results from this time study. In each table, SKU stands for stock-keeping unit. The letters A, B, C, and D represent different zones within the picking area. For each level of batching, the batches were separated into zones and the time to pick all items within that zone was recorded. The average time to pick each batch size was then determined and used to estimate the effects of batching from one wave length to the next (see Table 3.2).

Packing time study results are also presented in this appendix. In Tables A.6 through A.10, times are presented for several batch sizes. A set of orders comprising a batch were sorted and packed. This was completed 10 times per batch size, and the average time to pack each batch size was determined. Table A.11 presents the summarized data for this time study, including packing times, sorting times, and packing rates for each level of order-batching tested. 
Table A.1: Zone Picking Time Study Data (Batch Size = 20).

\begin{tabular}{cccc}
\hline $\begin{array}{c}\text { Batch Size } \\
\text { (no. orders) }\end{array}$ & Zone & $\begin{array}{c}\text { Time } \\
\text { (minutes) }\end{array}$ & $\begin{array}{c}\text { Number } \\
\text { of SKUs }\end{array}$ \\
\hline 20 & $\mathrm{~A}$ & 9.43 & 82 \\
20 & $\mathrm{~B}$ & 6.34 & 26 \\
20 & $\mathrm{C}$ & 12.72 & 113 \\
20 & $\mathrm{D}$ & 9.86 & 79 \\
\hline 20 & Total & 38.35 & 300 \\
\hline
\end{tabular}

Table A.2: Zone Picking Time Study Data (Batch Size = 10).

\begin{tabular}{cccc}
\hline $\begin{array}{c}\text { Batch Size } \\
\text { (no. orders) }\end{array}$ & Zone & $\begin{array}{c}\text { Time } \\
\text { (minutes) }\end{array}$ & $\begin{array}{c}\text { Number } \\
\text { of SKUs }\end{array}$ \\
\hline $1-10$ & $\mathrm{~A}$ & 6.98 & 46 \\
$1-10$ & $\mathrm{~B}$ & 3.15 & 11 \\
$1-10$ & $\mathrm{C}$ & 8.62 & 65 \\
$1-10$ & $\mathrm{D}$ & 7.96 & 50 \\
\hline $1-10$ & Total & 26.71 & 172 \\
\hline $11-20$ & $\mathrm{~A}$ & 5.38 & 40 \\
$11-20$ & $\mathrm{~B}$ & 4.35 & 19 \\
$11-20$ & $\mathrm{C}$ & 10.21 & 75 \\
$11-20$ & $\mathrm{D}$ & 8.17 & 48 \\
\hline $11-20$ & Total & 28.11 & 182 \\
\hline 10 orders & Average & 27.41 & 354 \\
\hline & Total & 54.82 & 354 \\
\hline
\end{tabular}

Table A.3: Zone Picking Time Study Data (Batch Size $=5)$.

\begin{tabular}{cccc}
\hline $\begin{array}{c}\text { Batch Size } \\
\text { (no. orders) }\end{array}$ & Zone & $\begin{array}{c}\text { Time } \\
\text { (minutes) }\end{array}$ & $\begin{array}{c}\text { Number } \\
\text { of SKUs }\end{array}$ \\
\hline $1-5$ & $\mathrm{~A}$ & 6.19 & 38 \\
$1-5$ & $\mathrm{~B}$ & 2.50 & 11 \\
$1-5$ & $\mathrm{C}$ & 5.35 & 31 \\
$1-5$ & $\mathrm{D}$ & 4.89 & 31 \\
\hline $1-5$ & Total & 18.93 & 111 \\
\hline $6-10$ & $\mathrm{~A}$ & 3.57 & 10 \\
$6-10$ & $\mathrm{~B}$ & 0.33 & 2 \\
$6-10$ & $\mathrm{C}$ & 4.79 & 40 \\
$6-10$ & $\mathrm{D}$ & 5.85 & 35 \\
\hline $6-10$ & Total & 14.54 & 87 \\
\hline $11-15$ & $\mathrm{~A}$ & 3.22 & 18 \\
$11-15$ & $\mathrm{~B}$ & 2.15 & 11 \\
$11-15$ & $\mathrm{C}$ & 7.43 & 41 \\
$11-15$ & $\mathrm{D}$ & 3.53 & 21 \\
\hline $11-15$ & Total & 16.33 & 91 \\
\hline $16-20$ & $\mathrm{~A}$ & 3.51 & 24 \\
$16-20$ & $\mathrm{~B}$ & 2.99 & 10 \\
$16-20$ & $\mathrm{C}$ & 6.27 & 43 \\
$16-20$ & $\mathrm{D}$ & 4.78 & 27 \\
\hline $16-20$ & Total & 17.55 & 104 \\
\hline 5 orders & Average & 16.84 & \\
& Total & 67.35 & 393 \\
\hline
\end{tabular}


Table A.4: Zone Picking Time Study Data (Batch Size = 1).

\begin{tabular}{cccc}
\hline $\begin{array}{c}\text { Batch Size } \\
\text { (no. orders) }\end{array}$ & Zone & $\begin{array}{c}\text { Time } \\
\text { (minutes) }\end{array}$ & $\begin{array}{c}\text { Number } \\
\text { of SKUs }\end{array}$ \\
\hline 1 & All & 8.50 & 49 \\
2 & All & 4.09 & 41 \\
3 & All & 2.61 & 26 \\
4 & All & 2.73 & 6 \\
5 & All & 3.60 & 11 \\
6 & All & 4.52 & 21 \\
7 & All & 5.75 & 13 \\
8 & All & 4.01 & 36 \\
9 & All & 4.67 & 20 \\
10 & All & 3.97 & 11 \\
11 & All & 9.04 & 19 \\
12 & All & 6.65 & 14 \\
13 & All & 5.47 & 16 \\
14 & All & 4.29 & 18 \\
15 & All & 5.68 & 24 \\
16 & All & 3.30 & 14 \\
17 & All & 5.19 & 28 \\
18 & All & 4.82 & 18 \\
19 & All & 6.09 & 30 \\
20 & All & 4.46 & 22 \\
\hline & Total & 99.44 & 437 \\
\hline
\end{tabular}

Table A.5: Picking Time Study Summary.

\begin{tabular}{ccc}
\hline \multicolumn{3}{c}{ Zone A } \\
\hline Batch Size & Time $(\mathrm{min})$ & Total $(\mathrm{min})$ \\
\hline 20 & 9.43 & 9.43 \\
10 & 6.18 & 12.36 \\
5 & 4.12 & 16.49 \\
\hline \multicolumn{3}{c}{ Zone B } \\
\hline Batch Size & Time (min) & Total (min) \\
\hline 20 & 6.34 & 6.34 \\
10 & 3.75 & 7.50 \\
5 & 1.99 & 7.97 \\
\hline \multicolumn{3}{c}{} \\
\hline Batch Size & Time $(\mathrm{min})$ & Total $(\mathrm{min})$ \\
\hline 20 & 12.72 & 12.72 \\
10 & 9.42 & 18.83 \\
5 & 5.96 & 23.84 \\
\hline \multicolumn{3}{c}{}
\end{tabular}


Table A.6: Packing Time Study Data (Batch Size $=20$ Orders).

\begin{tabular}{ccccc}
\hline Batch Size & Orders Packed & No. Items & Sort Time & ST/Order \\
\hline 20 & ALL & 61 & $18: 41.56$ & \\
20 & ALL & 61 & $16: 35.37$ & \\
20 & ALL & 61 & $12: 28.78$ & \\
\hline & Average & 61 & $15: 55.24$ & $00: 47.76$ \\
\hline
\end{tabular}

Table A.7: Packing Time Study Data (Batch Size $=10$ Orders).

\begin{tabular}{|c|c|c|c|c|}
\hline Batch Size & Orders Packed & No. Items & Sort Time & ST/Order \\
\hline 10 & 11 through 20 & 29 & $06: 51.72$ & \\
\hline 10 & 1 through 10 & 32 & $06: 10.93$ & \\
\hline 10 & odds & 27 & $05: 48.62$ & \\
\hline 10 & evens & 30 & $05: 46.94$ & \\
\hline 10 & $1,20,2,19,3,18,4,17,5,16$ & 28 & 05:08.97 & \\
\hline 10 & $6,15,7,14,8,13,9,12,10,11$ & 33 & $06: 20.90$ & \\
\hline 10 & $1,3,4,7,18,19,10,12,9,13$ & 25 & $03: 50.57$ & \\
\hline 10 & $2,5,16,17,20,11,8,6,15,14$ & 36 & 07:11.09 & \\
\hline 10 & 1 through 10 & 29 & 03:51.13 & \\
\hline \multirow[t]{2}{*}{10} & 11 through 20 & 32 & 04:31.41 & \\
\hline & Average & 30.1 & $05: 33.23$ & 00:33.32 \\
\hline
\end{tabular}

Table A.8: Packing Time Study Data (Batch Size $=5$ Orders).

\begin{tabular}{|c|c|c|c|c|}
\hline Batch Size & Orders Packed & No. Items & Sort Time & ST/Order \\
\hline 5 & $1,2,3,4,5$ & 14 & $02: 13.25$ & \\
\hline 5 & $6,7,8,9,10$ & 18 & 04:04.44 & \\
\hline 5 & 1 through 5 & 14 & $02: 46.44$ & \\
\hline 5 & 5 through 9 & 18 & 03:39.41 & \\
\hline 5 & 6 through 10 & 18 & $03: 46.38$ & \\
\hline 5 & 11 through 15 & 15 & $01: 59.56$ & \\
\hline 5 & 16 through 20 & 14 & $01: 32.32$ & \\
\hline 5 & $2,4,6,8,10$ & 17 & 03:04.00 & \\
\hline 5 & $12,14,16,18,20$ & 13 & $01: 57.72$ & \\
\hline \multirow[t]{2}{*}{5} & 15 through 19 & 13 & $01: 26.58$ & \\
\hline & Average & 15.4 & $02: 39.01$ & $00: 31.80$ \\
\hline
\end{tabular}

Table A.9: Packing Time Study Data (Batch Size $=4$ Orders).

\begin{tabular}{|c|c|c|c|c|}
\hline Batch Size & Orders Packed & No. Items & Sort Time & ST/Order \\
\hline 4 & 1 through 4 & 12 & $02: 10.13$ & \\
\hline 4 & 11 through 14 & 12 & $01: 23.18$ & \\
\hline 4 & 16 through 19 & 10 & 01:06.41 & \\
\hline 4 & $2,6,10,14$ & 13 & $01: 59.97$ & \\
\hline 4 & $8,12,16,20$ & 13 & $01: 44.13$ & \\
\hline 4 & $5,7,9,11$ & 13 & $01: 56.47$ & \\
\hline 4 & $13,15,17,19$ & 11 & $01: 29.21$ & \\
\hline 4 & $17,3,19,4$ & 11 & 01:30.07 & \\
\hline 4 & $8,2,12,16$ & 12 & $01: 30.81$ & \\
\hline 4 & $3,9,14,20$ & 15 & 01:49.94 & \\
\hline \multirow[t]{2}{*}{4} & $18,6,13,9$ & 16 & $02: 21.10$ & \\
\hline & Average & 12.5 & $01: 43.77$ & $00: 25.94$ \\
\hline
\end{tabular}


Table A.10: Packing Time Study Data (Batch Size $=2$ Orders).

\begin{tabular}{|c|c|c|c|c|}
\hline Batch Size & Orders Packed & No. Items & Sort Time & ST/Order \\
\hline 2 & 1,3 & 7 & $00: 45.32$ & \\
\hline 2 & 5,7 & 4 & $00: 23.71$ & \\
\hline 2 & 9,11 & 9 & 00:50.03 & \\
\hline 2 & 13,15 & 7 & $00: 39.34$ & \\
\hline 2 & 17,19 & 4 & $00: 26.22$ & \\
\hline 2 & 2,4 & 5 & $00: 32.72$ & \\
\hline 2 & 6,8 & 10 & $01: 34.12$ & \\
\hline 2 & 10,12 & 3 & $00: 18.78$ & \\
\hline 2 & 14,16 & 6 & $00: 34.75$ & \\
\hline \multirow[t]{2}{*}{2} & 18,20 & 6 & $00: 24.57$ & \\
\hline & Average & 6.1 & $00: 38.96$ & $00: 19.48$ \\
\hline
\end{tabular}

Table A.11: Packing Time Study Summary.

\begin{tabular}{ccccccccc}
\hline $\begin{array}{c}\text { Sort Time } \\
\text { per Order }\end{array}$ & $\begin{array}{c}\text { Number } \\
\text { of Boxes }\end{array}$ & $\begin{array}{c}\text { Total Sort } \\
\text { Time }(\mathrm{min})\end{array}$ & $\begin{array}{c}\text { Sort Time } \\
(\mathrm{min})\end{array}$ & $\begin{array}{c}\text { Total Pack } \\
\text { Time }(\mathrm{min})\end{array}$ & $\begin{array}{c}\text { Pack Time } \\
\text { per Order }\end{array}$ & $\begin{array}{c}\text { Hour } \\
\text { per Order }\end{array}$ & $\begin{array}{c}\text { Packing } \\
\text { Rate }(\mathrm{o} / \mathrm{h})\end{array}$ & $\begin{array}{c}\text { Picking } \\
\text { Rate }(\mathrm{o} / \mathrm{h})\end{array}$ \\
\hline $00: 47.76$ & 20 & $15: 55.24$ & 15.92 & 32.59 & 1.63 & 0.0272 & 36.82 & 146.28 \\
$00: 33.32$ & 10 & $05: 33.23$ & 5.55 & 13.89 & 1.39 & 0.0231 & 43.21 & 120.08 \\
$00: 31.80$ & 5 & $02: 39.01$ & 2.65 & 6.82 & 1.36 & 0.0227 & 44.01 & 101.49 \\
$00: 25.94$ & 4 & $01: 43.77$ & 1.73 & 5.06 & 1.27 & 0.0211 & 47.40 & 93.63 \\
$00: 19.48$ & 2 & $00: 38.96$ & 0.65 & 2.32 & 1.16 & 0.0193 & 51.81 & 76.13 \\
\hline
\end{tabular}




\section{Appendix B}

\section{Verification Simulation Results}

Simulation data for picking and packing simulations are presented in this appendix. These simulations served to verify the relationships between order-batching and picking and packing times.

Picking simulations were completed for batch sizes ranging from one order to 750 orders. 100 replications were run for each batch size and wave length. The tables in this appendix represent the resulting average time to complete each batch size, and the corresponding picking rate in orders per hour. This was completed for high, medium, and low picking rates. In the model, this was represented as the time it takes to travel down an aisle. For the high level, 0.25 minutes was used for this time. The medium and low levels were 0.50 and 0.75 minutes, respectively. The picking area consisted of 50 aisles with 40 locations in each aisle, or 2000 total locations.

Packing simulation results are also presented in this appendix. The packing simulations were completed for the automated system only, but systems with wave overlap and no wave overlap were examined. Again, high, medium, and low levels of packing rates were considered. These values were represented as uniform distributions with the following upper and lower bounds in minutes per order: $(0.4,0.6)$ for high rates, $(0.8,0.9)$ for medium rates, and $(1.0,1.5)$ for low rates. The corresponding packing rates are $(100,150),(66.7,75)$, and $(40,60)$ orders per hour, respectively. 
Manual packing results are also presented in this appendix. Table B.7 presents orderbatching estimates based on extrapolated data from time studies presented in Tables A.6 through A.11. Although this table does not present simulation data, this data is used in the development of the efficiency curves in Appendix F.

Again, 100 replications were run, and a batch size and wave length were provided as input data. The resulting time to pack all orders assigned to a packer was recorded for both overlapping and non-overlapping waves.

Using the data collected in the simulation runs, the actual efficiency curves were developed for the following combinations of picking and packing rates: high picking and low packing, low picking and high packing, low levels of both, medium levels of both, and high levels of both. The resulting efficiency curves are used in the spreadsheet models which are presented in Appendix F.

Table B.1: Simulation Data for Picking Times (Medium Rate).

\begin{tabular}{|c|c|c|c|c|c|c|c|c|c|c|c|c|}
\hline \multirow{2}{*}{$\begin{array}{r}\text { Batch } \\
\text { Size }\end{array}$} & \multicolumn{6}{|c|}{ Picking Time (minutes) } & \multicolumn{6}{|c|}{ Picking Rate (orders/hr) } \\
\hline & 20 & 40 & 60 & 80 & 100 & 120 & 20 & 40 & 60 & 80 & 100 & 120 \\
\hline 1 & 1.74 & 1.50 & 1.35 & 1.25 & 1.17 & 1.11 & 34.47 & 40.10 & 44.33 & 47.93 & 51.14 & 54.10 \\
\hline 2 & 2.97 & 2.55 & 2.31 & 2.13 & 2.00 & 1.89 & 40.47 & 47.08 & 52.05 & 56.26 & 60.03 & 63.51 \\
\hline 3 & 4.23 & 3.64 & 3.29 & 3.05 & 2.85 & 2.70 & 42.51 & 49.45 & 54.67 & 59.10 & 63.06 & 66.72 \\
\hline 4 & 4.98 & 4.28 & 3.87 & 3.58 & 3.36 & 3.17 & 48.20 & 56.07 & 61.99 & 67.01 & 71.51 & 75.65 \\
\hline 5 & 6.02 & 5.18 & 4.68 & 4.33 & 4.06 & 3.84 & 49.80 & 57.93 & 64.05 & 69.24 & 73.88 & 78.16 \\
\hline 6 & 7.01 & 6.03 & 5.45 & 5.04 & 4.73 & 4.47 & 51.34 & 59.72 & 66.02 & 71.37 & 76.15 & 80.57 \\
\hline 7 & 7.53 & 6.47 & 5.86 & 5.42 & 5.08 & 4.80 & 55.77 & 64.88 & 71.73 & 77.54 & 82.74 & 87.53 \\
\hline 8 & 8.35 & 7.18 & 6.49 & 6.01 & 5.63 & 5.32 & 57.47 & 66.85 & 73.91 & 79.89 & 85.25 & 90.19 \\
\hline 9 & 9.44 & 8.12 & 7.34 & 6.79 & 6.37 & 6.02 & 57.17 & 66.51 & 73.53 & 79.49 & 84.82 & 89.73 \\
\hline 10 & 10.07 & 8.65 & 7.83 & 7.24 & 6.79 & 6.41 & 59.60 & 69.33 & 76.65 & 82.85 & 88.41 & 93.53 \\
\hline 11 & 10.81 & 9.29 & 8.41 & 7.78 & 7.29 & 6.89 & 61.05 & 71.02 & 78.52 & 84.88 & 90.56 & 95.81 \\
\hline 12 & 11.80 & 10.14 & 9.18 & 8.49 & 7.95 & 7.52 & 61.01 & 70.98 & 78.47 & 84.83 & 90.51 & 95.76 \\
\hline 13 & 12.08 & 10.39 & 9.39 & 8.69 & 8.14 & 7.70 & 64.56 & 75.10 & 83.03 & 89.75 & 95.77 & 101.32 \\
\hline 14 & 12.79 & 10.99 & 9.94 & 9.20 & 8.62 & 8.15 & 65.69 & 76.42 & 84.49 & 91.33 & 97.45 & 103.10 \\
\hline 15 & 13.45 & 11.56 & 10.46 & 9.67 & 9.07 & 8.57 & 66.92 & 77.85 & 86.07 & 93.04 & 99.28 & 105.03 \\
\hline 16 & 14.37 & 12.35 & 11.17 & 10.33 & 9.68 & 9.15 & 66.82 & 77.73 & 85.94 & 92.90 & 99.13 & 104.88 \\
\hline 17 & 14.85 & 12.77 & 11.55 & 10.68 & 10.01 & 9.46 & 68.67 & 79.88 & 88.32 & 95.47 & 101.87 & 107.77 \\
\hline 18 & 15.54 & 13.36 & 12.08 & 11.17 & 10.47 & 9.90 & 69.51 & 80.87 & 89.40 & 96.65 & 103.12 & 109.10 \\
\hline 19 & 16.18 & 13.91 & 12.58 & 11.64 & 10.91 & 10.31 & 70.44 & 81.95 & 90.60 & 97.94 & 104.50 & 110.56 \\
\hline 20 & 16.69 & 14.35 & 12.98 & 12.01 & 11.25 & 10.64 & 71.88 & 83.62 & 92.45 & 99.94 & 106.64 & 112.81 \\
\hline 21 & 17.45 & 15.00 & 13.57 & 12.55 & 11.76 & 11.12 & 72.20 & 83.99 & 92.86 & 100.38 & 107.11 & 113.32 \\
\hline 22 & 17.82 & 15.32 & 13.85 & 12.82 & 12.01 & 11.35 & 74.09 & 86.19 & 95.29 & 103.00 & 109.91 & 116.28 \\
\hline
\end{tabular}


Table B.1: Simulation Data for Picking Times (Medium Rate) - continued.

\begin{tabular}{|c|c|c|c|c|c|c|c|c|c|c|c|c|}
\hline \multirow{2}{*}{$\begin{array}{r}\text { Batch } \\
\text { Size }\end{array}$} & \multicolumn{6}{|c|}{ Picking Time (minutes) } & \multicolumn{6}{|c|}{ Picking Rate (orders/hr) } \\
\hline & 20 & 40 & 60 & 80 & 100 & 120 & 20 & 40 & 60 & 80 & 100 & 120 \\
\hline 23 & 18.38 & 15.80 & 14.29 & 13.22 & 12.39 & 11.71 & 75.07 & 87.33 & 96.55 & 104.37 & 111.36 & 117.82 \\
\hline 24 & 19.03 & 16.36 & 14.80 & 13.69 & 12.83 & 12.13 & 75.66 & 88.02 & 97.31 & 105.19 & 112.25 & 118.75 \\
\hline 25 & 19.62 & 16.86 & 15.25 & 14.11 & 13.22 & 12.50 & 76.47 & 88.95 & 98.35 & 106.31 & 113.44 & 120.01 \\
\hline 26 & 19.94 & 17.14 & 15.50 & 14.34 & 13.44 & 12.71 & 78.23 & 91.00 & 100.61 & 108.76 & 116.05 & 122.78 \\
\hline 27 & 20.71 & 17.81 & 16.11 & 14.90 & 13.96 & 13.20 & 78.21 & 90.98 & 100.59 & 108.73 & 116.02 & 122.75 \\
\hline 28 & 21.33 & 18.34 & 16.58 & 15.34 & 14.38 & 13.59 & 78.76 & 91.62 & 101.30 & 109.50 & 116.84 & 123.61 \\
\hline 29 & 21.74 & 18.69 & 16.90 & 15.64 & 14.65 & 13.85 & 80.04 & 93.11 & 102.95 & 111.28 & 118.74 & 125.62 \\
\hline 30 & 22.25 & 19.13 & 17.30 & 16.00 & 15.00 & 14.18 & 80.90 & 94.10 & 104.04 & 112.47 & 120.01 & 126.96 \\
\hline 31 & 22.89 & 19.67 & 17.79 & 16.46 & 15.43 & 14.58 & 81.28 & 94.55 & 104.53 & 113.00 & 120.57 & 127.56 \\
\hline 32 & 23.41 & 20.12 & 18.20 & 16.83 & 15.78 & 14.91 & 82.03 & 95.43 & 105.51 & 114.05 & 121.70 & 128.75 \\
\hline 33 & 23.90 & 20.55 & 18.58 & 17.19 & 16.11 & 15.23 & 82.84 & 96.37 & 106.54 & 115.17 & 122.89 & 130.01 \\
\hline 34 & 24.45 & 21.02 & 19.01 & 17.59 & 16.48 & 15.58 & 83.43 & 97.06 & 107.31 & 116.00 & 123.77 & 130.95 \\
\hline 35 & 24.87 & 21.38 & 19.34 & 17.89 & 16.76 & 15.85 & 84.44 & 98.23 & 108.60 & 117.40 & 125.26 & 132.52 \\
\hline 36 & 25.20 & 21.67 & 19.60 & 18.13 & 16.99 & 16.06 & 85.70 & 99.70 & 110.22 & 119.15 & 127.14 & 134.51 \\
\hline 37 & 25.83 & 22.20 & 20.08 & 18.58 & 17.41 & 16.46 & 85.95 & 99.98 & 110.54 & 119.49 & 127.50 & 134.89 \\
\hline 38 & 26.42 & 22.71 & 20.54 & 19.00 & 17.81 & 16.83 & 86.31 & 100.40 & 111.00 & 119.99 & 128.04 & 135.46 \\
\hline 39 & 26.75 & 23.00 & 20.80 & 19.24 & 18.03 & 17.05 & 87.47 & 101.75 & 112.50 & 121.61 & 129.76 & 137.28 \\
\hline 40 & 27.40 & 23.55 & 21.30 & 19.71 & 18.47 & 17.46 & 87.59 & 101.89 & 112.65 & 121.78 & 129.94 & 137.47 \\
\hline 41 & 27.89 & 23.98 & 21.69 & 20.06 & 18.80 & 17.77 & 88.19 & 102.59 & 113.43 & 122.62 & 130.83 & 138.42 \\
\hline 42 & 28.46 & 24.46 & 22.13 & 20.47 & 19.18 & 18.13 & 88.55 & 103.01 & 113.88 & 123.11 & 131.36 & 138.97 \\
\hline 43 & 28.76 & 24.72 & 22.36 & 20.68 & 19.39 & 18.32 & 89.72 & 104.36 & 115.39 & 124.73 & 133.09 & 140.80 \\
\hline 44 & 28.91 & 24.85 & 22.48 & 20.80 & 19.49 & 18.42 & 91.31 & 106.22 & 117.44 & 126.95 & 135.46 & 143.31 \\
\hline 45 & 29.71 & 25.54 & 23.10 & 21.37 & 20.03 & 18.93 & 90.87 & 105.71 & 116.87 & 126.33 & 134.80 & 142.61 \\
\hline 46 & 29.95 & 25.75 & 23.29 & 21.54 & 20.19 & 19.08 & 92.15 & 107.20 & 118.52 & 128.12 & 136.70 & 144.63 \\
\hline 47 & 30.61 & 26.32 & 23.80 & 22.02 & 20.64 & 19.51 & 92.11 & 107.15 & 118.47 & 128.06 & 136.65 & 144.57 \\
\hline 48 & 30.88 & 26.55 & 24.01 & 22.21 & 20.82 & 19.68 & 93.27 & 108.49 & 119.95 & 129.67 & 138.36 & 146.38 \\
\hline 49 & 31.22 & 26.84 & 24.27 & 22.45 & 21.04 & 19.89 & 94.17 & 109.55 & 121.12 & 130.93 & 139.71 & 147.80 \\
\hline 50 & 31.87 & 27.40 & 24.78 & 22.92 & 21.48 & 20.31 & 94.13 & 109.50 & 121.07 & 130.87 & 139.65 & 147.74 \\
\hline 51 & 32.47 & 27.92 & 25.25 & 23.36 & 21.89 & 20.69 & 94.23 & 109.62 & 121.19 & 131.01 & 139.79 & 147.89 \\
\hline 52 & 32.83 & 28.22 & 25.53 & 23.62 & 22.13 & 20.92 & 95.03 & 110.55 & 122.22 & 132.12 & 140.97 & 149.14 \\
\hline 53 & 33.41 & 28.72 & 25.97 & 24.03 & 22.52 & 21.29 & 95.19 & 110.73 & 122.43 & 132.34 & 141.21 & 149.39 \\
\hline 54 & 33.79 & 29.04 & 26.27 & 24.30 & 22.77 & 21.53 & 95.90 & 111.56 & 123.34 & 133.33 & 142.26 & 150.51 \\
\hline 55 & 34.50 & 29.66 & 26.83 & 24.82 & 23.26 & 21.98 & 95.65 & 111.27 & 123.02 & 132.98 & 141.90 & 150.12 \\
\hline 56 & 34.81 & 29.93 & 27.07 & 25.04 & 23.47 & 22.18 & 96.52 & 112.28 & 124.13 & 134.19 & 143.18 & 151.48 \\
\hline 57 & 35.24 & 30.29 & 27.40 & 25.35 & 23.75 & 22.45 & 97.05 & 112.90 & 124.82 & 134.93 & 143.98 & 152.32 \\
\hline 58 & 35.61 & 30.61 & 27.69 & 25.61 & 24.00 & 22.69 & 97.72 & 113.68 & 125.69 & 135.87 & 144.97 & 153.37 \\
\hline 59 & 35.57 & 30.58 & 27.66 & 25.58 & 23.98 & 22.66 & 99.52 & 115.77 & 128.00 & 138.36 & 147.64 & 156.19 \\
\hline 60 & 36.41 & 31.30 & 28.31 & 26.19 & 24.55 & 23.20 & 98.86 & 115.00 & 127.15 & 137.45 & 146.66 & 155.16 \\
\hline 61 & 36.70 & 31.55 & 28.54 & 26.40 & 24.74 & 23.39 & 99.72 & 116.00 & 128.25 & 138.64 & 147.93 & 156.50 \\
\hline 62 & 37.39 & 32.14 & 29.07 & 26.90 & 25.21 & 23.83 & 99.48 & 115.73 & 127.95 & 138.31 & 147.58 & 156.13 \\
\hline 63 & 37.74 & 32.44 & 29.34 & 27.14 & 25.44 & 24.05 & 100.16 & 116.52 & 128.82 & 139.26 & 148.59 & 157.20 \\
\hline 64 & 38.09 & 32.74 & 29.61 & 27.39 & 25.67 & 24.27 & 100.83 & 117.29 & 129.67 & 140.18 & 149.57 & 158.24 \\
\hline
\end{tabular}


Table B.1: Simulation Data for Picking Times (Medium Rate) - continued.

\begin{tabular}{|c|c|c|c|c|c|c|c|c|c|c|c|c|}
\hline \multirow{2}{*}{$\begin{array}{r}\text { Batch } \\
\text { Size }\end{array}$} & \multicolumn{6}{|c|}{ Picking Time (minutes) } & \multicolumn{6}{|c|}{ Picking Rate (orders/hr) } \\
\hline & 20 & 40 & 60 & 80 & 100 & 120 & 20 & 40 & 60 & 80 & 100 & 120 \\
\hline 65 & 38.33 & 32.95 & 29.80 & 27.57 & 25.84 & 24.42 & 101.75 & 118.37 & 130.87 & 141.47 & 150.95 & 159.69 \\
\hline 66 & 38.95 & 33.48 & 30.29 & 28.02 & 26.26 & 24.82 & 101.66 & 118.26 & 130.75 & 141.34 & 150.82 & 159.56 \\
\hline 67 & 39.14 & 33.65 & 30.44 & 28.15 & 26.39 & 24.94 & 102.70 & 119.47 & 132.08 & 142.78 & 152.35 & 161.18 \\
\hline 68 & 39.84 & 34.25 & 30.98 & 28.66 & 26.86 & 25.39 & 102.41 & 119.13 & 131.71 & 142.38 & 151.92 & 160.72 \\
\hline 69 & 40.03 & 34.41 & 31.12 & 28.79 & 26.98 & 25.50 & 103.43 & 120.32 & 133.02 & 143.80 & 153.44 & 162.33 \\
\hline 70 & 40.81 & 35.08 & 31.73 & 29.35 & 27.51 & 26.00 & 102.92 & 119.73 & 132.37 & 143.09 & 152.69 & 161.53 \\
\hline 71 & 40.72 & 35.01 & 31.66 & 29.29 & 27.45 & 25.95 & 104.61 & 121.69 & 134.54 & 145.44 & 155.19 & 164.18 \\
\hline 72 & 41.15 & 35.38 & 32.00 & 29.60 & 27.74 & 26.22 & 104.98 & 122.12 & 135.01 & 145.95 & 155.73 & 164.76 \\
\hline 73 & 41.63 & 35.79 & 32.37 & 29.94 & 28.06 & 26.53 & 105.21 & 122.39 & 135.32 & 146.27 & 156.08 & 165.12 \\
\hline 74 & 42.07 & 36.16 & 32.71 & 30.26 & 28.36 & 26.81 & 105.54 & 122.77 & 135.74 & 146.73 & 156.57 & 165.64 \\
\hline 75 & 42.65 & 36.66 & 33.16 & 30.68 & 28.75 & 27.18 & 105.51 & 122.73 & 135.69 & 146.68 & 156.52 & 165.59 \\
\hline 76 & 42.89 & 36.87 & 33.35 & 30.85 & 28.91 & 27.33 & 106.32 & 123.69 & 136.75 & 147.82 & 157.73 & 166.87 \\
\hline 77 & 42.92 & 36.89 & 33.37 & 30.87 & 28.93 & 27.34 & 107.65 & 125.23 & 138.46 & 149.67 & 159.70 & 168.96 \\
\hline 78 & 43.84 & 37.69 & 34.09 & 31.53 & 29.55 & 27.93 & 106.75 & 124.18 & 137.29 & 148.41 & 158.36 & 167.53 \\
\hline 79 & 44.29 & 38.07 & 34.44 & 31.86 & 29.86 & 28.22 & 107.02 & 124.49 & 137.64 & 148.79 & 158.76 & 167.96 \\
\hline 80 & 44.49 & 38.25 & 34.59 & 32.00 & 29.99 & 28.35 & 107.89 & 125.50 & 138.75 & 149.99 & 160.05 & 169.32 \\
\hline 81 & 44.75 & 38.47 & 34.79 & 32.19 & 30.16 & 28.51 & 108.61 & 126.34 & 139.68 & 151.00 & 161.12 & 170.46 \\
\hline 82 & 45.37 & 39.00 & 35.28 & 32.63 & 30.58 & 28.91 & 108.44 & 126.14 & 139.46 & 150.76 & 160.87 & 170.19 \\
\hline 83 & 45.79 & 39.37 & 35.61 & 32.94 & 30.87 & 29.18 & 108.75 & 126.50 & 139.86 & 151.19 & 161.33 & 170.67 \\
\hline 84 & 46.17 & 39.69 & 35.90 & 33.21 & 31.12 & 29.42 & 109.16 & 126.98 & 140.39 & 151.76 & 161.93 & 171.31 \\
\hline 85 & 46.47 & 39.95 & 36.13 & 33.42 & 31.32 & 29.61 & 109.75 & 127.67 & 141.16 & 152.59 & 162.82 & 172.25 \\
\hline 86 & 46.72 & 40.17 & 36.33 & 33.61 & 31.50 & 29.77 & 110.43 & 128.47 & 142.03 & 153.53 & 163.83 & 173.32 \\
\hline 87 & 47.24 & 40.61 & 36.73 & 33.98 & 31.84 & 30.10 & 110.50 & 128.55 & 142.12 & 153.63 & 163.93 & 173.43 \\
\hline 88 & 47.46 & 40.80 & 36.90 & 34.14 & 31.99 & 30.24 & 111.25 & 129.42 & 143.09 & 154.68 & 165.04 & 174.61 \\
\hline 89 & 48.07 & 41.32 & 37.37 & 34.57 & 32.40 & 30.63 & 111.09 & 129.23 & 142.88 & 154.45 & 164.80 & 174.35 \\
\hline 90 & 48.36 & 41.57 & 37.60 & 34.79 & 32.60 & 30.82 & 111.66 & 129.89 & 143.60 & 155.23 & 165.64 & 175.24 \\
\hline 91 & 48.63 & 41.80 & 37.81 & 34.98 & 32.78 & 30.98 & 112.28 & 130.62 & 144.41 & 156.11 & 166.57 & 176.22 \\
\hline 92 & 49.16 & 42.26 & 38.23 & 35.36 & 33.14 & 31.33 & 112.28 & 130.61 & 144.40 & 156.10 & 166.56 & 176.21 \\
\hline 93 & 49.60 & 42.63 & 38.56 & 35.67 & 33.43 & 31.60 & 112.51 & 130.88 & 144.70 & 156.42 & 166.91 & 176.58 \\
\hline 94 & 49.86 & 42.86 & 38.77 & 35.86 & 33.61 & 31.77 & 113.12 & 131.58 & 145.48 & 157.26 & 167.80 & 177.53 \\
\hline 95 & 50.38 & 43.30 & 39.17 & 36.23 & 33.96 & 32.10 & 113.15 & 131.62 & 145.52 & 157.31 & 167.86 & 177.58 \\
\hline 96 & 50.53 & 43.44 & 39.29 & 36.34 & 34.06 & 32.19 & 114.00 & 132.61 & 146.61 & 158.49 & 169.11 & 178.91 \\
\hline 97 & 50.98 & 43.82 & 39.64 & 36.67 & 34.36 & 32.48 & 114.17 & 132.81 & 146.84 & 158.73 & 169.37 & 179.18 \\
\hline 98 & 51.38 & 44.17 & 39.95 & 36.96 & 34.64 & 32.74 & 114.43 & 133.12 & 147.17 & 159.09 & 169.76 & 179.60 \\
\hline 99 & 51.83 & 44.56 & 40.30 & 37.28 & 34.94 & 33.02 & 114.60 & 133.32 & 147.40 & 159.33 & 170.01 & 179.87 \\
\hline 100 & 52.15 & 44.83 & 40.55 & 37.51 & 35.16 & 33.23 & 115.05 & 133.83 & 147.96 & 159.95 & 170.67 & 180.56 \\
\hline 101 & 52.55 & 45.17 & 40.86 & 37.80 & 35.42 & 33.48 & 115.33 & 134.16 & 148.32 & 160.34 & 171.09 & 181.00 \\
\hline 102 & 52.78 & 45.37 & 41.04 & 37.96 & 35.58 & 33.63 & 115.96 & 134.89 & 149.13 & 161.21 & 172.02 & 181.99 \\
\hline 103 & 53.16 & 45.69 & 41.33 & 38.23 & 35.83 & 33.87 & 116.26 & 135.25 & 149.53 & 161.64 & 172.47 & 182.47 \\
\hline 104 & 53.51 & 46.00 & 41.60 & 38.49 & 36.07 & 34.09 & 116.62 & 135.66 & 149.99 & 162.13 & 173.00 & 183.03 \\
\hline 105 & 53.95 & 46.37 & 41.94 & 38.80 & 36.36 & 34.37 & 116.78 & 135.85 & 150.20 & 162.36 & 173.25 & 183.29 \\
\hline 106 & 54.10 & 46.51 & 42.07 & 38.92 & 36.47 & 34.47 & 117.55 & 136.74 & 151.18 & 163.43 & 174.38 & 184.49 \\
\hline
\end{tabular}


Table B.1: Simulation Data for Picking Times (Medium Rate) - continued.

\begin{tabular}{|c|c|c|c|c|c|c|c|c|c|c|c|c|}
\hline \multirow{2}{*}{$\begin{array}{r}\text { Batch } \\
\text { Size }\end{array}$} & \multicolumn{6}{|c|}{ Picking Time (minutes) } & \multicolumn{6}{|c|}{ Picking Rate (orders/hr) } \\
\hline & 20 & 40 & 60 & 80 & 100 & 120 & 20 & 40 & 60 & 80 & 100 & 120 \\
\hline 107 & 54.46 & 46.82 & 42.34 & 39.17 & 36.71 & 34.70 & 117.89 & 137.14 & 151.62 & 163.90 & 174.88 & 185.02 \\
\hline 108 & 54.98 & 47.26 & 42.75 & 39.55 & 37.06 & 35.03 & 117.86 & 137.10 & 151.58 & 163.85 & 174.84 & 184.97 \\
\hline 109 & 55.27 & 47.52 & 42.98 & 39.76 & 37.26 & 35.22 & 118.32 & 137.64 & 152.17 & 164.50 & 175.53 & 185.70 \\
\hline 110 & 55.88 & 48.04 & 43.45 & 40.19 & 37.67 & 35.61 & 118.11 & 137.39 & 151.90 & 164.20 & 175.21 & 185.36 \\
\hline 111 & 56.04 & 48.18 & 43.57 & 40.31 & 37.78 & 35.71 & 118.84 & 138.25 & 152.84 & 165.22 & 176.30 & 186.51 \\
\hline 112 & 56.29 & 48.39 & 43.77 & 40.49 & 37.94 & 35.87 & 119.38 & 138.87 & 153.54 & 165.98 & 177.10 & 187.36 \\
\hline 113 & 56.80 & 48.83 & 44.17 & 40.86 & 38.29 & 36.19 & 119.36 & 138.85 & 153.51 & 165.95 & 177.07 & 187.33 \\
\hline 114 & 57.14 & 49.12 & 44.42 & 41.10 & 38.51 & 36.40 & 119.72 & 139.26 & 153.97 & 166.44 & 177.60 & 187.89 \\
\hline 115 & 57.20 & 49.17 & 44.48 & 41.14 & 38.56 & 36.45 & 120.62 & 140.32 & 155.14 & 167.70 & 178.95 & 189.31 \\
\hline 116 & 58.14 & 49.98 & 45.20 & 41.82 & 39.19 & 37.04 & 119.72 & 139.27 & 153.97 & 166.44 & 177.60 & 187.89 \\
\hline 117 & 58.29 & 50.11 & 45.32 & 41.93 & 39.29 & 37.14 & 120.43 & 140.09 & 154.88 & 167.43 & 178.65 & 189.00 \\
\hline 118 & 58.69 & 50.45 & 45.63 & 42.21 & 39.56 & 37.39 & 120.64 & 140.33 & 155.15 & 167.72 & 178.96 & 189.33 \\
\hline 119 & 58.73 & 50.49 & 45.67 & 42.24 & 39.59 & 37.42 & 121.57 & 141.42 & 156.35 & 169.01 & 180.34 & 190.79 \\
\hline 120 & 59.32 & 51.00 & 46.13 & 42.67 & 39.99 & 37.80 & 121.37 & 141.18 & 156.09 & 168.73 & 180.04 & 190.48 \\
\hline 121 & 59.72 & 51.33 & 46.43 & 42.95 & 40.25 & 38.05 & 121.58 & 141.43 & 156.36 & 169.03 & 180.36 & 190.81 \\
\hline 122 & 59.71 & 51.33 & 46.43 & 42.95 & 40.25 & 38.05 & 122.59 & 142.61 & 157.67 & 170.43 & 181.86 & 192.40 \\
\hline 123 & 60.42 & 51.94 & 46.98 & 43.46 & 40.73 & 38.50 & 122.14 & 142.08 & 157.08 & 169.81 & 181.19 & 191.69 \\
\hline 124 & 60.63 & 52.12 & 47.14 & 43.61 & 40.87 & 38.63 & 122.71 & 142.74 & 157.82 & 170.60 & 182.04 & 192.58 \\
\hline 125 & 60.67 & 52.16 & 47.18 & 43.64 & 40.90 & 38.66 & 123.61 & 143.79 & 158.98 & 171.85 & 183.37 & 194.00 \\
\hline 126 & 61.29 & 52.68 & 47.65 & 44.08 & 41.31 & 39.05 & 123.36 & 143.50 & 158.65 & 171.50 & 183.00 & 193.60 \\
\hline 127 & 61.51 & 52.88 & 47.83 & 44.24 & 41.46 & 39.19 & 123.88 & 144.11 & 159.33 & 172.24 & 183.78 & 194.43 \\
\hline 128 & 62.12 & 53.40 & 48.30 & 44.68 & 41.87 & 39.58 & 123.64 & 143.83 & 159.02 & 171.89 & 183.42 & 194.05 \\
\hline 129 & 62.61 & 53.82 & 48.68 & 45.04 & 42.21 & 39.89 & 123.62 & 143.80 & 158.99 & 171.87 & 183.39 & 194.01 \\
\hline 130 & 62.77 & 53.96 & 48.80 & 45.15 & 42.31 & 39.99 & 124.27 & 144.56 & 159.83 & 172.77 & 184.35 & 195.03 \\
\hline 131 & 63.10 & 54.24 & 49.06 & 45.38 & 42.53 & 40.20 & 124.57 & 144.91 & 160.21 & 173.19 & 184.79 & 195.50 \\
\hline 132 & 63.42 & 54.52 & 49.31 & 45.62 & 42.75 & 40.41 & 124.88 & 145.26 & 160.60 & 173.61 & 185.25 & 195.98 \\
\hline 133 & 63.88 & 54.91 & 49.67 & 45.95 & 43.06 & 40.70 & 124.92 & 145.32 & 160.66 & 173.68 & 185.32 & 196.06 \\
\hline 134 & 64.16 & 55.16 & 49.89 & 46.15 & 43.25 & 40.88 & 125.31 & 145.77 & 161.16 & 174.21 & 185.89 & 196.66 \\
\hline 135 & 64.32 & 55.29 & 50.01 & 46.26 & 43.36 & 40.98 & 125.94 & 146.50 & 161.97 & 175.09 & 186.83 & 197.65 \\
\hline 136 & 64.82 & 55.72 & 50.40 & 46.62 & 43.69 & 41.30 & 125.89 & 146.45 & 161.91 & 175.02 & 186.76 & 197.58 \\
\hline 137 & 65.08 & 55.95 & 50.60 & 46.81 & 43.87 & 41.47 & 126.30 & 146.93 & 162.44 & 175.60 & 187.37 & 198.23 \\
\hline 138 & 65.30 & 56.14 & 50.78 & 46.97 & 44.02 & 41.61 & 126.79 & 147.49 & 163.07 & 176.28 & 188.09 & 198.99 \\
\hline 139 & 65.88 & 56.63 & 51.22 & 47.38 & 44.41 & 41.97 & 126.60 & 147.27 & 162.82 & 176.01 & 187.81 & 198.69 \\
\hline 140 & 66.23 & 56.93 & 51.49 & 47.64 & 44.64 & 42.20 & 126.84 & 147.55 & 163.13 & 176.34 & 188.16 & 199.06 \\
\hline 141 & 66.51 & 57.17 & 51.71 & 47.84 & 44.83 & 42.38 & 127.21 & 147.98 & 163.60 & 176.85 & 188.71 & 199.64 \\
\hline 142 & 66.91 & 57.52 & 52.02 & 48.12 & 45.10 & 42.63 & 127.34 & 148.13 & 163.78 & 177.04 & 188.91 & 199.86 \\
\hline 143 & 67.07 & 57.66 & 52.15 & 48.24 & 45.21 & 42.74 & 127.92 & 148.81 & 164.52 & 177.84 & 189.77 & 200.76 \\
\hline 144 & 67.69 & 58.18 & 52.63 & 48.68 & 45.63 & 43.13 & 127.65 & 148.49 & 164.17 & 177.47 & 189.37 & 200.34 \\
\hline 145 & 68.02 & 58.47 & 52.89 & 48.92 & 45.85 & 43.34 & 127.91 & 148.79 & 164.50 & 177.83 & 189.75 & 200.74 \\
\hline 146 & 68.17 & 58.60 & 53.01 & 49.03 & 45.95 & 43.44 & 128.50 & 149.48 & 165.26 & 178.65 & 190.62 & 201.67 \\
\hline 147 & 68.44 & 58.83 & 53.21 & 49.23 & 46.13 & 43.61 & 128.87 & 149.92 & 165.75 & 179.17 & 191.18 & 202.26 \\
\hline 148 & 68.85 & 59.18 & 53.53 & 49.52 & 46.41 & 43.87 & 128.99 & 150.05 & 165.89 & 179.33 & 191.35 & 202.43 \\
\hline
\end{tabular}


Table B.1: Simulation Data for Picking Times (Medium Rate) - continued.

\begin{tabular}{|c|c|c|c|c|c|c|c|c|c|c|c|c|}
\hline \multirow{2}{*}{$\begin{array}{r}\text { Batch } \\
\text { Size }\end{array}$} & \multicolumn{6}{|c|}{ Picking Time (minutes) } & \multicolumn{6}{|c|}{ Picking Rate (orders/hr) } \\
\hline & 20 & 40 & 60 & 80 & 100 & 120 & 20 & 40 & 60 & 80 & 100 & 120 \\
\hline 149 & 69.11 & 59.41 & 53.74 & 49.71 & 46.59 & 44.04 & 129.35 & 150.47 & 166.36 & 179.84 & 191.89 & 203.01 \\
\hline 150 & 69.42 & 59.67 & 53.97 & 49.93 & 46.79 & 44.23 & 129.65 & 150.82 & 166.75 & 180.26 & 192.34 & 203.48 \\
\hline 151 & 69.92 & 60.11 & 54.37 & 50.29 & 47.14 & 44.55 & 129.57 & 150.72 & 166.64 & 180.14 & 192.21 & 203.35 \\
\hline 152 & 70.28 & 60.42 & 54.65 & 50.55 & 47.38 & 44.78 & 129.77 & 150.95 & 166.90 & 180.41 & 192.51 & 203.66 \\
\hline 153 & 70.41 & 60.53 & 54.75 & 50.64 & 47.46 & 44.86 & 130.38 & 151.67 & 167.68 & 181.26 & 193.41 & 204.62 \\
\hline 154 & 70.77 & 60.83 & 55.02 & 50.90 & 47.70 & 45.09 & 130.57 & 151.89 & 167.93 & 181.53 & 193.70 & 204.92 \\
\hline 155 & 71.24 & 61.24 & 55.39 & 51.24 & 48.02 & 45.39 & 130.55 & 151.87 & 167.91 & 181.51 & 193.67 & 204.90 \\
\hline 156 & 71.51 & 61.47 & 55.60 & 51.43 & 48.20 & 45.56 & 130.90 & 152.27 & 168.35 & 181.99 & 194.19 & 205.44 \\
\hline 157 & 71.83 & 61.74 & 55.85 & 51.66 & 48.42 & 45.77 & 131.15 & 152.57 & 168.68 & 182.34 & 194.56 & 205.83 \\
\hline 158 & 72.11 & 61.99 & 56.07 & 51.87 & 48.61 & 45.95 & 131.47 & 152.93 & 169.08 & 182.78 & 195.03 & 206.33 \\
\hline 159 & 72.55 & 62.37 & 56.41 & 52.19 & 48.91 & 46.23 & 131.49 & 152.96 & 169.11 & 182.81 & 195.06 & 206.36 \\
\hline 160 & 72.84 & 62.61 & 56.63 & 52.39 & 49.10 & 46.41 & 131.80 & 153.33 & 169.52 & 183.25 & 195.53 & 206.86 \\
\hline 161 & 73.18 & 62.91 & 56.90 & 52.64 & 49.33 & 46.63 & 132.00 & 153.56 & 169.77 & 183.52 & 195.83 & 207.17 \\
\hline 162 & 73.39 & 63.09 & 57.07 & 52.79 & 49.47 & 46.76 & 132.43 & 154.06 & 170.33 & 184.12 & 196.46 & 207.85 \\
\hline 163 & 73.83 & 63.46 & 57.40 & 53.10 & 49.76 & 47.04 & 132.47 & 154.11 & 170.38 & 184.18 & 196.52 & 207.91 \\
\hline 164 & 74.16 & 63.75 & 57.67 & 53.34 & 49.99 & 47.26 & 132.68 & 154.34 & 170.64 & 184.46 & 196.83 & 208.23 \\
\hline 165 & 74.38 & 63.94 & 57.84 & 53.50 & 50.14 & 47.40 & 133.09 & 154.82 & 171.17 & 185.04 & 197.44 & 208.88 \\
\hline 166 & 74.92 & 64.40 & 58.25 & 53.89 & 50.50 & 47.74 & 132.95 & 154.65 & 170.98 & 184.83 & 197.22 & 208.65 \\
\hline 167 & 74.99 & 64.47 & 58.31 & 53.94 & 50.55 & 47.78 & 133.62 & 155.43 & 171.85 & 185.76 & 198.22 & 209.70 \\
\hline 168 & 75.46 & 64.86 & 58.67 & 54.27 & 50.86 & 48.08 & 133.59 & 155.40 & 171.81 & 185.73 & 198.18 & 209.66 \\
\hline 169 & 75.77 & 65.13 & 58.91 & 54.50 & 51.07 & 48.28 & 133.83 & 155.68 & 172.12 & 186.06 & 198.54 & 210.04 \\
\hline 170 & 76.24 & 65.54 & 59.28 & 54.84 & 51.39 & 48.58 & 133.79 & 155.64 & 172.07 & 186.01 & 198.48 & 209.98 \\
\hline 171 & 76.40 & 65.68 & 59.40 & 54.95 & 51.50 & 48.68 & 134.29 & 156.22 & 172.72 & 186.70 & 199.22 & 210.76 \\
\hline 172 & 76.79 & 66.01 & 59.70 & 55.23 & 51.76 & 48.93 & 134.40 & 156.34 & 172.85 & 186.85 & 199.38 & 210.93 \\
\hline 173 & 77.10 & 66.28 & 59.95 & 55.45 & 51.97 & 49.12 & 134.63 & 156.62 & 173.16 & 187.18 & 199.73 & 211.30 \\
\hline 174 & 77.36 & 66.50 & 60.15 & 55.64 & 52.14 & 49.29 & 134.96 & 157.00 & 173.58 & 187.63 & 200.21 & 211.81 \\
\hline 175 & 77.63 & 66.73 & 60.36 & 55.84 & 52.33 & 49.46 & 135.26 & 157.34 & 173.96 & 188.05 & 200.66 & 212.28 \\
\hline 176 & 78.10 & 67.13 & 60.72 & 56.17 & 52.64 & 49.76 & 135.22 & 157.30 & 173.91 & 187.99 & 200.59 & 212.22 \\
\hline 177 & 78.39 & 67.38 & 60.95 & 56.38 & 52.84 & 49.94 & 135.48 & 157.61 & 174.25 & 188.36 & 200.99 & 212.63 \\
\hline 178 & 78.94 & 67.86 & 61.38 & 56.78 & 53.21 & 50.30 & 135.30 & 157.39 & 174.01 & 188.11 & 200.71 & 212.34 \\
\hline 179 & 79.13 & 68.02 & 61.52 & 56.92 & 53.34 & 50.42 & 135.73 & 157.89 & 174.56 & 188.70 & 201.35 & 213.02 \\
\hline 180 & 79.34 & 68.20 & 61.69 & 57.06 & 53.48 & 50.55 & 136.13 & 158.36 & 175.08 & 189.26 & 201.94 & 213.65 \\
\hline 181 & 79.71 & 68.52 & 61.98 & 57.33 & 53.73 & 50.79 & 136.24 & 158.49 & 175.22 & 189.41 & 202.11 & 213.82 \\
\hline 182 & 80.12 & 68.87 & 62.29 & 57.63 & 54.01 & 51.05 & 136.30 & 158.56 & 175.30 & 189.50 & 202.20 & 213.92 \\
\hline 183 & 80.28 & 69.01 & 62.42 & 57.75 & 54.12 & 51.15 & 136.77 & 159.10 & 175.90 & 190.14 & 202.89 & 214.65 \\
\hline 184 & 80.65 & 69.33 & 62.71 & 58.01 & 54.37 & 51.39 & 136.89 & 159.24 & 176.05 & 190.31 & 203.07 & 214.84 \\
\hline 185 & 80.91 & 69.55 & 62.91 & 58.20 & 54.54 & 51.55 & 137.19 & 159.59 & 176.44 & 190.73 & 203.52 & 215.31 \\
\hline 186 & 81.34 & 69.92 & 63.24 & 58.51 & 54.83 & 51.83 & 137.20 & 159.61 & 176.46 & 190.75 & 203.54 & 215.33 \\
\hline 187 & 81.72 & 70.25 & 63.54 & 58.78 & 55.09 & 52.07 & 137.30 & 159.72 & 176.58 & 190.89 & 203.68 & 215.48 \\
\hline 188 & 81.99 & 70.48 & 63.75 & 58.97 & 55.27 & 52.24 & 137.58 & 160.04 & 176.94 & 191.27 & 204.09 & 215.92 \\
\hline 189 & 82.38 & 70.82 & 64.05 & 59.26 & 55.53 & 52.49 & 137.65 & 160.13 & 177.04 & 191.38 & 204.20 & 216.04 \\
\hline 190 & 82.57 & 70.98 & 64.20 & 59.39 & 55.66 & 52.61 & 138.06 & 160.60 & 177.56 & 191.94 & 204.81 & 216.68 \\
\hline
\end{tabular}


Table B.1: Simulation Data for Picking Times (Medium Rate) - continued.

\begin{tabular}{|c|c|c|c|c|c|c|c|c|c|c|c|c|}
\hline \multirow{2}{*}{$\begin{array}{r}\text { Batch } \\
\text { Size }\end{array}$} & \multicolumn{6}{|c|}{ Picking Time (minutes) } & \multicolumn{6}{|c|}{ Picking Rate (orders/hr) } \\
\hline & 20 & 40 & 60 & 80 & 100 & 120 & 20 & 40 & 60 & 80 & 100 & 120 \\
\hline 191 & 83.03 & 71.38 & 64.56 & 59.72 & 55.97 & 52.91 & 138.02 & 160.56 & 177.51 & 191.89 & 204.75 & 216.61 \\
\hline 192 & 83.25 & 71.56 & 64.73 & 59.88 & 56.12 & 53.04 & 138.38 & 160.98 & 177.98 & 192.39 & 205.29 & 217.18 \\
\hline 193 & 83.61 & 71.87 & 65.01 & 60.14 & 56.36 & 53.27 & 138.51 & 161.12 & 178.14 & 192.56 & 205.47 & 217.38 \\
\hline 194 & 83.80 & 72.04 & 65.16 & 60.28 & 56.49 & 53.40 & 138.90 & 161.58 & 178.64 & 193.11 & 206.06 & 218.00 \\
\hline 195 & 84.31 & 72.48 & 65.56 & 60.64 & 56.83 & 53.72 & 138.77 & 161.43 & 178.47 & 192.93 & 205.86 & 217.79 \\
\hline 196 & 84.50 & 72.64 & 65.70 & 60.78 & 56.96 & 53.84 & 139.18 & 161.90 & 179.00 & 193.50 & 206.47 & 218.43 \\
\hline 197 & 84.67 & 72.79 & 65.84 & 60.90 & 57.08 & 53.95 & 139.59 & 162.39 & 179.54 & 194.08 & 207.09 & 219.09 \\
\hline 198 & 85.10 & 73.16 & 66.17 & 61.21 & 57.36 & 54.22 & 139.60 & 162.39 & 179.54 & 194.08 & 207.10 & 219.09 \\
\hline 199 & 85.56 & 73.55 & 66.52 & 61.54 & 57.67 & 54.51 & 139.56 & 162.34 & 179.48 & 194.02 & 207.03 & 219.02 \\
\hline 200 & 85.88 & 73.83 & 66.77 & 61.77 & 57.89 & 54.72 & 139.73 & 162.54 & 179.71 & 194.26 & 207.29 & 219.30 \\
\hline 201 & 86.00 & 73.93 & 66.87 & 61.86 & 57.97 & 54.80 & 140.23 & 163.12 & 180.35 & 194.96 & 208.03 & 220.08 \\
\hline 202 & 86.36 & 74.24 & 67.15 & 62.12 & 58.21 & 55.02 & 140.35 & 163.26 & 180.50 & 195.12 & 208.20 & 220.26 \\
\hline 203 & 86.64 & 74.48 & 67.36 & 62.32 & 58.40 & 55.20 & 140.59 & 163.54 & 180.81 & 195.46 & 208.56 & 220.64 \\
\hline 204 & 87.07 & 74.85 & 67.70 & 62.63 & 58.69 & 55.48 & 140.57 & 163.53 & 180.80 & 195.44 & 208.54 & 220.62 \\
\hline 205 & 87.26 & 75.01 & 67.84 & 62.76 & 58.82 & 55.60 & 140.96 & 163.98 & 181.30 & 195.98 & 209.12 & 221.24 \\
\hline 206 & 87.73 & 75.41 & 68.21 & 63.10 & 59.13 & 55.90 & 140.89 & 163.90 & 181.21 & 195.88 & 209.01 & 221.13 \\
\hline 207 & 88.14 & 75.77 & 68.53 & 63.40 & 59.41 & 56.16 & 140.91 & 163.92 & 181.23 & 195.91 & 209.04 & 221.16 \\
\hline 208 & 88.28 & 75.89 & 68.64 & 63.50 & 59.51 & 56.25 & 141.36 & 164.44 & 181.81 & 196.53 & 209.71 & 221.86 \\
\hline 209 & 88.53 & 76.11 & 68.84 & 63.68 & 59.68 & 56.41 & 141.64 & 164.77 & 182.17 & 196.92 & 210.12 & 222.30 \\
\hline 210 & 88.88 & 76.40 & 69.11 & 63.93 & 59.91 & 56.63 & 141.77 & 164.91 & 182.33 & 197.09 & 210.31 & 222.49 \\
\hline 211 & 89.22 & 76.70 & 69.37 & 64.18 & 60.15 & 56.85 & 141.89 & 165.06 & 182.49 & 197.27 & 210.49 & 222.69 \\
\hline 212 & 89.57 & 77.00 & 69.64 & 64.42 & 60.38 & 57.07 & 142.02 & 165.20 & 182.65 & 197.44 & 210.68 & 222.89 \\
\hline 213 & 89.80 & 77.20 & 69.82 & 64.59 & 60.53 & 57.22 & 142.31 & 165.55 & 183.03 & 197.86 & 211.12 & 223.35 \\
\hline 214 & 90.15 & 77.49 & 70.09 & 64.84 & 60.77 & 57.44 & 142.43 & 165.69 & 183.19 & 198.02 & 211.30 & 223.54 \\
\hline 215 & 90.63 & 77.91 & 70.47 & 65.19 & 61.09 & 57.74 & 142.34 & 165.58 & 183.07 & 197.90 & 211.16 & 223.40 \\
\hline 216 & 90.90 & 78.14 & 70.68 & 65.38 & 61.28 & 57.92 & 142.57 & 165.85 & 183.36 & 198.21 & 211.50 & 223.75 \\
\hline 217 & 91.04 & 78.26 & 70.79 & 65.49 & 61.37 & 58.01 & 143.01 & 166.36 & 183.93 & 198.82 & 212.15 & 224.44 \\
\hline 218 & 91.52 & 78.67 & 71.16 & 65.83 & 61.69 & 58.31 & 142.92 & 166.26 & 183.81 & 198.70 & 212.02 & 224.30 \\
\hline 219 & 91.95 & 79.04 & 71.49 & 66.14 & 61.98 & 58.59 & 142.90 & 166.24 & 183.79 & 198.68 & 211.99 & 224.28 \\
\hline 220 & 92.15 & 79.32 & 71.74 & 66.32 & 62.16 & 58.73 & 143.12 & 166.42 & 183.99 & 199.02 & 212.34 & 224.75 \\
\hline 221 & 92.49 & 79.51 & 71.92 & 66.53 & 62.35 & 58.93 & 143.36 & 166.77 & 184.38 & 199.31 & 212.68 & 225.00 \\
\hline 222 & 92.74 & 79.80 & 72.09 & 66.73 & 62.59 & 59.12 & 143.62 & 166.92 & 184.78 & 199.62 & 212.83 & 225.29 \\
\hline 223 & 92.99 & 80.09 & 72.32 & 66.95 & 62.80 & 59.36 & 143.89 & 167.06 & 185.01 & 199.85 & 213.07 & 225.42 \\
\hline 224 & 93.34 & 80.31 & 72.58 & 67.15 & 62.94 & 59.48 & 143.99 & 167.35 & 185.17 & 200.15 & 213.52 & 225.97 \\
\hline 225 & 93.70 & 80.55 & 72.86 & 67.40 & 63.16 & 59.70 & 144.08 & 167.60 & 185.30 & 200.31 & 213.73 & 226.12 \\
\hline 226 & 94.00 & 80.81 & 73.09 & 67.61 & 63.37 & 59.90 & 144.25 & 167.80 & 185.52 & 200.55 & 213.99 & 226.39 \\
\hline 227 & 94.22 & 80.99 & 73.26 & 67.77 & 63.51 & 60.03 & 144.56 & 168.16 & 185.92 & 200.98 & 214.45 & 226.88 \\
\hline 228 & 94.51 & 81.25 & 73.49 & 67.98 & 63.71 & 60.22 & 144.74 & 168.37 & 186.15 & 201.23 & 214.72 & 227.16 \\
\hline 229 & 95.06 & 81.71 & 73.91 & 68.37 & 64.08 & 60.57 & 144.55 & 168.15 & 185.90 & 200.96 & 214.43 & 226.86 \\
\hline 230 & 95.18 & 81.82 & 74.01 & 68.46 & 64.16 & 60.65 & 144.99 & 168.66 & 186.47 & 201.57 & 215.09 & 227.55 \\
\hline 231 & 95.51 & 82.11 & 74.26 & 68.70 & 64.38 & 60.86 & 145.11 & 168.80 & 186.63 & 201.74 & 215.27 & 227.74 \\
\hline 232 & 96.01 & 82.54 & 74.65 & 69.06 & 64.72 & 61.18 & 144.98 & 168.65 & 186.46 & 201.56 & 215.07 & 227.54 \\
\hline
\end{tabular}


Table B.1: Simulation Data for Picking Times (Medium Rate) - continued.

\begin{tabular}{|c|c|c|c|c|c|c|c|c|c|c|c|c|}
\hline \multirow{2}{*}{$\begin{array}{r}\text { Batch } \\
\text { Size }\end{array}$} & \multicolumn{6}{|c|}{ Picking Time (minutes) } & \multicolumn{6}{|c|}{ Picking Rate (orders/hr) } \\
\hline & 20 & 40 & 60 & 80 & 100 & 120 & 20 & 40 & 60 & 80 & 100 & 120 \\
\hline 233 & 96.14 & 82.64 & 74.75 & 69.15 & 64.80 & 61.25 & 145.42 & 169.16 & 187.03 & 202.18 & 215.73 & 228.23 \\
\hline 234 & 96.53 & 82.98 & 75.05 & 69.43 & 65.07 & 61.50 & 145.45 & 169.20 & 187.07 & 202.22 & 215.78 & 228.28 \\
\hline 235 & 96.82 & 83.23 & 75.28 & 69.64 & 65.26 & 61.69 & 145.63 & 169.41 & 187.30 & 202.47 & 216.05 & 228.57 \\
\hline 236 & 97.19 & 83.55 & 75.57 & 69.91 & 65.51 & 61.93 & 145.70 & 169.48 & 187.38 & 202.56 & 216.14 & 228.66 \\
\hline 237 & 97.49 & 83.81 & 75.80 & 70.12 & 65.72 & 62.12 & 145.86 & 169.67 & 187.59 & 202.78 & 216.37 & 228.91 \\
\hline 238 & 97.68 & 83.97 & 75.95 & 70.26 & 65.85 & 62.24 & 146.19 & 170.06 & 188.02 & 203.24 & 216.87 & 229.43 \\
\hline 239 & 98.02 & 84.26 & 76.22 & 70.51 & 66.08 & 62.46 & 146.29 & 170.18 & 188.15 & 203.39 & 217.02 & 229.60 \\
\hline 240 & 98.33 & 84.53 & 76.45 & 70.73 & 66.28 & 62.65 & 146.45 & 170.36 & 188.35 & 203.60 & 217.25 & 229.84 \\
\hline 241 & 98.70 & 84.85 & 76.74 & 70.99 & 66.53 & 62.89 & 146.50 & 170.42 & 188.42 & 203.68 & 217.33 & 229.92 \\
\hline 242 & 99.07 & 85.17 & 77.03 & 71.26 & 66.78 & 63.13 & 146.56 & 170.49 & 188.49 & 203.76 & 217.42 & 230.02 \\
\hline 243 & 99.24 & 85.31 & 77.16 & 71.38 & 66.90 & 63.23 & 146.91 & 170.90 & 188.95 & 204.25 & 217.94 & 230.57 \\
\hline 244 & 99.58 & 85.60 & 77.43 & 71.63 & 67.13 & 63.45 & 147.01 & 171.02 & 189.08 & 204.39 & 218.09 & 230.73 \\
\hline 245 & 99.78 & 85.78 & 77.59 & 71.77 & 67.26 & 63.58 & 147.32 & 171.37 & 189.47 & 204.81 & 218.54 & 231.21 \\
\hline 246 & 100.35 & 86.27 & 78.03 & 72.18 & 67.65 & 63.94 & 147.08 & 171.10 & 189.17 & 204.49 & 218.19 & 230.84 \\
\hline 247 & 100.61 & 86.49 & 78.23 & 72.37 & 67.82 & 64.11 & 147.30 & 171.35 & 189.44 & 204.79 & 218.51 & 231.17 \\
\hline 248 & 101.01 & 86.83 & 78.54 & 72.65 & 68.09 & 64.36 & 147.32 & 171.37 & 189.47 & 204.81 & 218.54 & 231.21 \\
\hline 249 & 101.19 & 86.98 & 78.68 & 72.78 & 68.21 & 64.47 & 147.65 & 171.76 & 189.89 & 205.27 & 219.03 & 231.73 \\
\hline 250 & 101.55 & 87.29 & 78.96 & 73.04 & 68.45 & 64.70 & 147.71 & 171.83 & 189.98 & 205.37 & 219.13 & 231.83 \\
\hline 251 & 101.81 & 87.52 & 79.16 & 73.23 & 68.63 & 64.87 & 147.93 & 172.08 & 190.25 & 205.66 & 219.45 & 232.16 \\
\hline 252 & 102.07 & 87.74 & 79.36 & 73.42 & 68.80 & 65.04 & 148.13 & 172.32 & 190.52 & 205.95 & 219.75 & 232.49 \\
\hline 253 & 102.40 & 88.03 & 79.62 & 73.65 & 69.03 & 65.24 & 148.25 & 172.45 & 190.66 & 206.10 & 219.92 & 232.66 \\
\hline 254 & 102.74 & 88.32 & 79.88 & 73.90 & 69.25 & 65.46 & 148.34 & 172.56 & 190.78 & 206.24 & 220.06 & 232.81 \\
\hline 255 & 103.06 & 88.60 & 80.13 & 74.13 & 69.47 & 65.67 & 148.45 & 172.69 & 190.93 & 206.40 & 220.23 & 232.99 \\
\hline 256 & 103.37 & 88.86 & 80.37 & 74.35 & 69.68 & 65.86 & 148.60 & 172.86 & 191.12 & 206.60 & 220.44 & 233.22 \\
\hline 257 & 103.59 & 89.05 & 80.54 & 74.51 & 69.83 & 66.00 & 148.86 & 173.17 & 191.46 & 206.96 & 220.83 & 233.63 \\
\hline 258 & 103.89 & 89.31 & 80.78 & 74.73 & 70.03 & 66.20 & 149.00 & 173.33 & 191.63 & 207.15 & 221.04 & 233.85 \\
\hline 259 & 104.33 & 89.69 & 81.12 & 75.04 & 70.33 & 66.48 & 148.95 & 173.27 & 191.57 & 207.08 & 220.96 & 233.77 \\
\hline 260 & 104.58 & 89.90 & 81.31 & 75.22 & 70.49 & 66.63 & 149.17 & 173.53 & 191.86 & 207.39 & 221.30 & 234.12 \\
\hline 261 & 104.89 & 90.17 & 81.56 & 75.45 & 70.71 & 66.83 & 149.29 & 173.67 & 192.01 & 207.56 & 221.48 & 234.31 \\
\hline 262 & 105.16 & 90.40 & 81.77 & 75.64 & 70.89 & 67.01 & 149.49 & 173.89 & 192.26 & 207.83 & 221.76 & 234.61 \\
\hline 263 & 105.61 & 90.79 & 82.12 & 75.96 & 71.19 & 67.29 & 149.41 & 173.81 & 192.17 & 207.73 & 221.65 & 234.50 \\
\hline 264 & 105.88 & 91.02 & 82.33 & 76.16 & 71.37 & 67.46 & 149.60 & 174.03 & 192.41 & 207.99 & 221.93 & 234.79 \\
\hline 265 & 106.05 & 91.17 & 82.46 & 76.28 & 71.49 & 67.57 & 149.93 & 174.40 & 192.82 & 208.44 & 222.41 & 235.30 \\
\hline 266 & 106.43 & 91.49 & 82.75 & 76.55 & 71.74 & 67.81 & 149.96 & 174.44 & 192.87 & 208.49 & 222.46 & 235.35 \\
\hline 267 & 106.64 & 91.67 & 82.92 & 76.71 & 71.89 & 67.95 & 150.22 & 174.75 & 193.20 & 208.85 & 222.85 & 235.76 \\
\hline 268 & 107.10 & 92.06 & 83.27 & 77.03 & 72.19 & 68.24 & 150.15 & 174.66 & 193.11 & 208.75 & 222.74 & 235.65 \\
\hline 269 & 107.37 & 92.30 & 83.48 & 77.23 & 72.37 & 68.41 & 150.33 & 174.87 & 193.34 & 209.00 & 223.01 & 235.93 \\
\hline 270 & 107.69 & 92.58 & 83.73 & 77.46 & 72.59 & 68.62 & 150.43 & 174.99 & 193.47 & 209.14 & 223.16 & 236.09 \\
\hline 271 & 107.92 & 92.77 & 83.91 & 77.63 & 72.75 & 68.76 & 150.66 & 175.27 & 193.77 & 209.47 & 223.51 & 236.46 \\
\hline 272 & 108.29 & 93.09 & 84.20 & 77.89 & 73.00 & 69.00 & 150.71 & 175.31 & 193.83 & 209.53 & 223.57 & 236.53 \\
\hline 273 & 108.68 & 93.42 & 84.50 & 78.17 & 73.26 & 69.24 & 150.72 & 175.33 & 193.85 & 209.55 & 223.60 & 236.55 \\
\hline 274 & 109.01 & 93.71 & 84.76 & 78.41 & 73.48 & 69.46 & 150.81 & 175.44 & 193.96 & 209.67 & 223.73 & 236.69 \\
\hline
\end{tabular}


Table B.1: Simulation Data for Picking Times (Medium Rate) - continued.

\begin{tabular}{|c|c|c|c|c|c|c|c|c|c|c|c|c|}
\hline \multirow{2}{*}{$\begin{array}{r}\text { Batch } \\
\text { Size }\end{array}$} & \multicolumn{6}{|c|}{ Picking Time (minutes) } & \multicolumn{6}{|c|}{ Picking Rate (orders/hr) } \\
\hline & 20 & 40 & 60 & 80 & 100 & 120 & 20 & 40 & 60 & 80 & 100 & 120 \\
\hline 275 & 109.18 & 93.85 & 84.89 & 78.53 & 73.60 & 69.56 & 151.13 & 175.81 & 194.37 & 210.11 & 224.20 & 237.19 \\
\hline 276 & 109.51 & 94.14 & 85.15 & 78.77 & 73.82 & 69.78 & 151.22 & 175.91 & 194.48 & 210.24 & 224.33 & 237.33 \\
\hline 277 & 109.78 & 94.37 & 85.36 & 78.96 & 74.00 & 69.95 & 151.39 & 176.11 & 194.71 & 210.48 & 224.59 & 237.60 \\
\hline 278 & 110.08 & 94.63 & 85.59 & 79.18 & 74.21 & 70.14 & 151.52 & 176.26 & 194.87 & 210.66 & 224.78 & 237.80 \\
\hline 279 & 110.32 & 94.84 & 85.78 & 79.35 & 74.37 & 70.29 & 151.74 & 176.51 & 195.15 & 210.96 & 225.10 & 238.14 \\
\hline 280 & 110.73 & 95.19 & 86.10 & 79.65 & 74.65 & 70.56 & 151.71 & 176.49 & 195.12 & 210.93 & 225.07 & 238.11 \\
\hline 281 & 110.99 & 95.41 & 86.30 & 79.83 & 74.82 & 70.72 & 151.90 & 176.71 & 195.37 & 211.19 & 225.35 & 238.41 \\
\hline 282 & 111.30 & 95.68 & 86.54 & 80.06 & 75.03 & 70.92 & 152.02 & 176.84 & 195.51 & 211.35 & 225.52 & 238.58 \\
\hline 283 & 111.85 & 96.15 & 86.97 & 80.45 & 75.40 & 71.27 & 151.81 & 176.60 & 195.25 & 211.06 & 225.21 & 238.26 \\
\hline 284 & 112.09 & 96.36 & 87.15 & 80.62 & 75.56 & 71.42 & 152.02 & 176.84 & 195.52 & 211.35 & 225.52 & 238.59 \\
\hline 285 & 112.32 & 96.55 & 87.33 & 80.79 & 75.71 & 71.56 & 152.25 & 177.11 & 195.81 & 211.67 & 225.86 & 238.94 \\
\hline 286 & 112.69 & 96.87 & 87.62 & 81.05 & 75.96 & 71.80 & 152.28 & 177.14 & 195.85 & 211.71 & 225.90 & 238.99 \\
\hline 287 & 112.93 & 97.08 & 87.81 & 81.23 & 76.12 & 71.95 & 152.49 & 177.38 & 196.11 & 212.00 & 226.21 & 239.32 \\
\hline 288 & 113.17 & 97.28 & 87.99 & 81.40 & 76.29 & 72.11 & 152.69 & 177.62 & 196.38 & 212.28 & 226.51 & 239.64 \\
\hline 289 & 113.39 & 97.47 & 88.16 & 81.56 & 76.43 & 72.25 & 152.93 & 177.90 & 196.68 & 212.61 & 226.86 & 240.01 \\
\hline 290 & 113.85 & 97.87 & 88.52 & 81.89 & 76.74 & 72.54 & 152.84 & 177.79 & 196.57 & 212.49 & 226.73 & 239.87 \\
\hline 291 & 114.16 & 98.14 & 88.77 & 82.11 & 76.96 & 72.74 & 152.94 & 177.91 & 196.70 & 212.63 & 226.88 & 240.03 \\
\hline 292 & 114.55 & 98.47 & 89.07 & 82.39 & 77.22 & 72.99 & 152.95 & 177.92 & 196.71 & 212.64 & 226.89 & 240.04 \\
\hline 293 & 114.83 & 98.71 & 89.28 & 82.59 & 77.40 & 73.16 & 153.10 & 178.10 & 196.91 & 212.85 & 227.12 & 240.28 \\
\hline 294 & 115.14 & 98.97 & 89.52 & 82.81 & 77.61 & 73.36 & 153.21 & 178.23 & 197.05 & 213.01 & 227.29 & 240.46 \\
\hline 295 & 115.37 & 99.18 & 89.71 & 82.99 & 77.77 & 73.51 & 153.42 & 178.46 & 197.31 & 213.29 & 227.59 & 240.78 \\
\hline 296 & 115.72 & 99.48 & 89.98 & 83.24 & 78.01 & 73.74 & 153.47 & 178.53 & 197.38 & 213.36 & 227.67 & 240.86 \\
\hline 297 & 116.04 & 99.75 & 90.22 & 83.46 & 78.22 & 73.94 & 153.57 & 178.64 & 197.51 & 213.50 & 227.82 & 241.02 \\
\hline 298 & 116.20 & 99.89 & 90.35 & 83.58 & 78.33 & 74.04 & 153.87 & 178.99 & 197.89 & 213.92 & 228.26 & 241.49 \\
\hline 299 & 116.64 & 100.27 & 90.69 & 83.90 & 78.63 & 74.32 & 153.81 & 178.92 & 197.82 & 213.84 & 228.17 & 241.39 \\
\hline 300 & 116.94 & 100.52 & 90.92 & 84.11 & 78.83 & 74.51 & 153.93 & 179.06 & 197.97 & 214.01 & 228.35 & 241.59 \\
\hline 301 & 117.24 & 100.78 & 91.16 & 84.33 & 79.03 & 74.70 & 154.04 & 179.20 & 198.12 & 214.17 & 228.52 & 241.76 \\
\hline 302 & 117.51 & 101.01 & 91.37 & 84.52 & 79.21 & 74.87 & 154.20 & 179.38 & 198.32 & 214.39 & 228.76 & 242.01 \\
\hline 303 & 117.76 & 101.23 & 91.56 & 84.70 & 79.38 & 75.03 & 154.38 & 179.59 & 198.55 & 214.63 & 229.02 & 242.29 \\
\hline 304 & 118.13 & 101.55 & 91.85 & 84.97 & 79.63 & 75.27 & 154.40 & 179.62 & 198.58 & 214.67 & 229.06 & 242.33 \\
\hline 305 & 118.47 & 101.84 & 92.11 & 85.21 & 79.86 & 75.48 & 154.47 & 179.70 & 198.67 & 214.76 & 229.16 & 242.44 \\
\hline 306 & 118.70 & 102.04 & 92.29 & 85.38 & 80.02 & 75.63 & 154.67 & 179.93 & & 215.04 & 229.46 & 242.75 \\
\hline 307 & 118.98 & 102.28 & 92.51 & 85.58 & 80.20 & 75.81 & 154.81 & 180.09 & 199.11 & 215.24 & 229.66 & 242.97 \\
\hline 308 & 119.24 & 102.51 & 92.72 & 85.77 & 80.38 & 75.98 & 154.98 & 180.28 & 199.32 & 215.46 & 229.90 & 243.23 \\
\hline 309 & 119.59 & 102.81 & 92.99 & 86.02 & 80.62 & 76.20 & 155.03 & 180.34 & 199.38 & 215.53 & 229.98 & 243.31 \\
\hline 310 & 119.87 & 103.05 & 93.21 & 86.22 & 80.81 & 76.38 & 155.16 & 180.50 & 199.56 & 215.72 & 230.18 & 243.52 \\
\hline 311 & 120.36 & 103.46 & 93.58 & 86.57 & 81.13 & 76.69 & 155.04 & 180.36 & 199.40 & 215.55 & 230.00 & 243.33 \\
\hline 312 & 120.57 & 103.64 & 93.74 & 86.72 & 81.27 & 76.82 & 155.27 & 180.62 & 199.69 & 215.87 & 230.34 & 243.68 \\
\hline 313 & 120.90 & 103.93 & 94.01 & 86.96 & 81.50 & 77.04 & 155.33 & 180.69 & 199.77 & 215.95 & 230.43 & 243.78 \\
\hline 314 & 121.22 & 104.21 & 94.26 & 87.19 & 81.72 & 77.24 & 155.41 & 180.79 & 199.88 & 216.07 & 230.55 & 243.91 \\
\hline 315 & 121.50 & 104.44 & 94.47 & 87.39 & 81.90 & 77.42 & 155.56 & 180.96 & 200.07 & 216.27 & 230.77 & 244.14 \\
\hline 316 & 121.71 & 104.63 & 94.64 & 87.54 & 82.04 & 77.55 & 155.78 & 181.21 & 200.35 & 216.58 & 231.09 & 244.48 \\
\hline
\end{tabular}


Table B.1: Simulation Data for Picking Times (Medium Rate) - continued.

\begin{tabular}{|c|c|c|c|c|c|c|c|c|c|c|c|c|}
\hline \multirow{2}{*}{$\begin{array}{r}\text { Batch } \\
\text { Size }\end{array}$} & \multicolumn{6}{|c|}{ Picking Time (minutes) } & \multicolumn{6}{|c|}{ Picking Rate (orders/hr) } \\
\hline & 20 & 40 & 60 & 80 & 100 & 120 & 20 & 40 & 60 & 80 & 100 & 120 \\
\hline 317 & 122.17 & 105.02 & 94.99 & 87.87 & 82.35 & 77.84 & 155.69 & 181.11 & 200.23 & 216.45 & 230.96 & 244.34 \\
\hline 318 & 122.38 & 105.20 & 95.15 & 88.02 & 82.49 & 77.97 & 155.91 & 181.37 & 200.52 & 216.76 & 231.30 & 244.70 \\
\hline 319 & 122.65 & 105.44 & 95.37 & 88.22 & 82.68 & 78.15 & 156.05 & 181.53 & 200.70 & 216.96 & 231.50 & 244.91 \\
\hline 320 & 123.06 & 105.78 & 95.68 & 88.51 & 82.95 & 78.41 & 156.03 & 181.50 & 200.67 & 216.92 & 231.46 & 244.87 \\
\hline 321 & 123.17 & 105.88 & 95.76 & 88.59 & 83.02 & 78.48 & 156.38 & 181.91 & 201.12 & 217.41 & 231.98 & 245.42 \\
\hline 322 & 123.66 & 106.31 & 96.15 & 88.95 & 83.36 & 78.79 & 156.23 & 181.74 & 200.93 & 217.21 & 231.77 & 245.20 \\
\hline 323 & 123.86 & 106.47 & 96.30 & 89.09 & 83.49 & 78.92 & 156.47 & 182.02 & 201.24 & 217.54 & 232.12 & 245.57 \\
\hline 324 & 124.21 & 106.77 & 96.58 & 89.34 & 83.73 & 79.14 & 156.51 & 182.07 & 201.29 & 217.60 & 232.18 & 245.64 \\
\hline 325 & 124.47 & 107.00 & 96.78 & 89.53 & 83.90 & 79.31 & 156.66 & 182.24 & 201.49 & 217.81 & 232.41 & 245.87 \\
\hline 326 & 124.72 & 107.22 & 96.98 & 89.71 & 84.07 & 79.47 & 156.83 & 182.43 & 201.70 & 218.04 & 232.65 & 246.13 \\
\hline 327 & 125.14 & 107.58 & 97.30 & 90.01 & 84.36 & 79.74 & 156.78 & 182.38 & 201.64 & 217.98 & 232.59 & 246.06 \\
\hline 328 & 125.40 & 107.80 & 97.50 & 90.20 & 84.53 & 79.90 & 156.94 & 182.56 & 201.84 & 218.19 & 232.81 & 246.30 \\
\hline 329 & 125.81 & 108.15 & 97.82 & 90.49 & 84.81 & 80.16 & 156.90 & 182.52 & 201.80 & 218.14 & 232.77 & 246.25 \\
\hline 330 & 126.11 & 108.40 & 98.05 & 90.70 & 85.01 & 80.35 & 157.01 & 182.65 & 201.94 & 218.29 & 232.92 & 246.42 \\
\hline 331 & 126.27 & 108.55 & 98.18 & 90.83 & 85.12 & 80.46 & 157.28 & 182.96 & 202.28 & 218.66 & 233.32 & 246.84 \\
\hline 332 & 126.65 & 108.87 & 98.47 & 91.09 & 85.37 & 80.70 & 157.29 & 182.97 & 202.29 & 218.68 & 233.33 & 246.85 \\
\hline 333 & 126.85 & 109.05 & 98.63 & 91.24 & 85.51 & 80.82 & 157.51 & 183.23 & 202.58 & 218.98 & 233.66 & 247.20 \\
\hline 334 & 127.38 & 109.50 & 99.04 & 91.62 & 85.87 & 81.16 & 157.32 & 183.01 & 202.34 & 218.72 & 233.39 & 246.91 \\
\hline 335 & 127.64 & 109.73 & 99.25 & 91.81 & 86.04 & 81.33 & 157.47 & 183.18 & 202.53 & 218.93 & 233.60 & 247.14 \\
\hline 336 & 127.89 & 109.94 & 99.44 & 91.99 & 86.21 & 81.49 & 157.63 & 183.37 & 202.74 & 219.15 & 233.85 & 247.40 \\
\hline 337 & 128.18 & 110.19 & 99.66 & 92.20 & 86.40 & 81.67 & 157.75 & 183.51 & 202.88 & 219.32 & 234.02 & 247.58 \\
\hline 338 & 128.65 & 110.59 & 100.03 & 92.54 & 86.72 & 81.97 & 157.63 & 183.37 & 202.74 & 219.16 & 233.85 & 247.40 \\
\hline 339 & 128.73 & 110.66 & 100.09 & 92.59 & 86.77 & 82.02 & 158.01 & 183.81 & 203.22 & 219.68 & 234.40 & 247.99 \\
\hline 340 & 129.11 & 110.99 & 100.39 & 92.86 & 87.03 & 82.26 & 158.01 & 183.81 & 203.22 & & 234.40 & 247.98 \\
\hline 341 & 129.32 & 111.17 & 100.55 & 93.02 & 87.17 & 82.40 & 158.21 & 184.05 & 203.48 & 219.96 & 234.71 & 248.31 \\
\hline 342 & 129.67 & 111.47 & 100.82 & 93.27 & 87.41 & 82.62 & 158.25 & 184.09 & 203.52 & 220.01 & 234.76 & 248.36 \\
\hline 343 & 130.10 & 111.84 & 101.16 & 93.58 & 87.70 & 82.90 & 158.18 & 184.01 & 203.44 & 219.92 & 234.66 & 248.26 \\
\hline 344 & 130.38 & 112.08 & 101.37 & 93.78 & 87.89 & 83.07 & 158.31 & 184.16 & 203.61 & 220.10 & 234.85 & 248.46 \\
\hline 345 & 130.68 & 112.34 & 101.61 & 94.00 & 88.09 & 83.27 & 158.40 & 184.26 & 203.72 & 220.22 & 234.98 & 248.60 \\
\hline 346 & 130.92 & 112.55 & 101.80 & 94.17 & 88.25 & 83.42 & 158.57 & 184.46 & 203.94 & 220.45 & 235.23 & 248.86 \\
\hline 347 & 131.24 & 112.82 & 102.04 & 94.40 & 88.47 & 83.62 & 158.64 & 184.54 & 204.03 & 220.55 & 235.34 & 248.98 \\
\hline 348 & 131.53 & 113.07 & 102.27 & 94.61 & 88.66 & 83.81 & 158.75 & 184.67 & 204.17 & 220.71 & 235.50 & 249.15 \\
\hline 349 & 131.83 & 113.32 & 102.50 & 94.82 & 88.86 & 84.00 & 158.84 & 184.78 & 204.29 & 220.84 & 235.64 & 249.30 \\
\hline 350 & 132.12 & 113.57 & 102.73 & & & & & 184.90 & 204.43 & & 235.80 & 249.46 \\
\hline 351 & 132.43 & 113.84 & 102.97 & 95.25 & 89.27 & 84.38 & 159.03 & 185.00 & 204.53 & 221.10 & 235.92 & 249.59 \\
\hline 352 & 132.66 & 114.04 & 103.15 & 95.42 & 89.43 & 84.53 & 159.20 & 185.20 & 204.75 & 221.34 & 236.18 & 249.86 \\
\hline 353 & 133.02 & 114.35 & 103.43 & 95.68 & 89.67 & 84.76 & 159.23 & 185.22 & 204.78 & 221.37 & 236.21 & 249.90 \\
\hline 354 & 133.31 & 114.60 & 103.65 & 95.89 & 89.86 & 84.94 & 159.33 & 185.34 & 204.91 & 221.51 & 236.36 & 250.05 \\
\hline 355 & 133.65 & 114.89 & 103.92 & 96.13 & 90.09 & 85.16 & 159.37 & 185.39 & 204.97 & 221.57 & 236.42 & 250.12 \\
\hline 356 & 133.91 & 115.12 & 104.12 & 96.32 & 90.27 & 85.33 & 159.51 & 185.55 & 205.14 & 221.76 & 236.62 & 250.33 \\
\hline 357 & 134.28 & 115.43 & 104.41 & 96.59 & 90.52 & 85.56 & 159.51 & 185.56 & 205.15 & 221.77 & 236.64 & 250.35 \\
\hline 358 & 134.57 & 115.68 & 104.63 & 96.79 & 90.71 & 85.74 & 159.62 & 185.69 & 205.29 & 221.92 & 236.80 & 250.52 \\
\hline
\end{tabular}


Table B.1: Simulation Data for Picking Times (Medium Rate) - continued.

\begin{tabular}{|c|c|c|c|c|c|c|c|c|c|c|c|c|}
\hline \multirow{2}{*}{$\begin{array}{r}\text { Batch } \\
\text { Size }\end{array}$} & \multicolumn{6}{|c|}{ Picking Time (minutes) } & \multicolumn{6}{|c|}{ Picking Rate (orders/hr) } \\
\hline & 20 & 40 & 60 & 80 & 100 & 120 & 20 & 40 & 60 & 80 & 100 & 120 \\
\hline 359 & 134.87 & 115.94 & 104.87 & 97.01 & 90.91 & 85.94 & 159.71 & 185.79 & 205.40 & 222.04 & 236.92 & 250.65 \\
\hline 360 & 135.14 & 116.17 & 105.08 & 97.21 & 91.10 & 86.11 & 159.83 & 185.93 & 205.56 & 222.21 & 237.11 & 250.85 \\
\hline 361 & 135.48 & 116.46 & 105.34 & 97.45 & 91.32 & 86.32 & 159.88 & 185.98 & 205.62 & 222.28 & 237.18 & 250.92 \\
\hline 362 & 135.80 & 116.74 & 105.59 & 97.68 & 91.54 & 86.53 & 159.94 & 186.06 & 205.71 & 222.37 & 237.28 & 251.02 \\
\hline 363 & 136.00 & 116.91 & 105.74 & 97.82 & 91.67 & 86.65 & 160.15 & 186.30 & 205.97 & 222.66 & 237.58 & 251.35 \\
\hline 364 & 136.36 & 117.22 & 106.03 & 98.08 & 91.92 & 86.89 & 160.16 & 186.31 & 205.99 & 222.67 & 237.60 & 251.36 \\
\hline 365 & 136.70 & 117.51 & 106.29 & 98.32 & 92.14 & 87.10 & 160.21 & 186.37 & 206.05 & 222.74 & 237.67 & 251.44 \\
\hline 366 & 136.95 & 117.73 & 106.48 & 98.50 & 92.32 & 87.26 & 160.35 & 186.53 & 206.23 & 222.93 & 237.88 & 251.66 \\
\hline 367 & 137.25 & 117.98 & 106.72 & 98.72 & 92.52 & 87.45 & 160.44 & 186.64 & 206.34 & 223.06 & 238.01 & 251.80 \\
\hline 368 & 137.65 & 118.33 & 107.03 & 99.01 & 92.79 & 87.71 & 160.41 & 186.60 & 206.30 & 223.01 & 237.96 & 251.75 \\
\hline 369 & 137.85 & 118.50 & 107.19 & 99.15 & 92.93 & 87.84 & 160.61 & 186.83 & 206.56 & 223.29 & 238.26 & 252.06 \\
\hline 370 & 138.16 & 118.77 & 107.42 & 99.37 & 93.13 & 88.03 & 160.68 & 186.92 & 206.66 & 223.40 & 238.37 & 252.19 \\
\hline 371 & 138.50 & 119.06 & 107.69 & 99.62 & 93.36 & 88.25 & 160.72 & 186.96 & 206.70 & 223.44 & 238.42 & 252.24 \\
\hline 372 & 138.84 & 119.35 & 107.95 & 99.86 & 93.59 & 88.46 & 160.77 & 187.02 & 206.76 & 223.51 & 238.49 & 252.31 \\
\hline 373 & 139.05 & 119.53 & 108.12 & 100.02 & 93.73 & 88.60 & 160.95 & 187.23 & 207.00 & 223.77 & 238.77 & 252.60 \\
\hline 374 & 139.38 & 119.82 & 108.37 & 100.25 & 93.95 & 88.81 & 161.00 & 187.29 & 207.07 & 223.84 & 238.84 & 252.68 \\
\hline 375 & 139.76 & 120.15 & 108.67 & 100.53 & 94.21 & 89.05 & 160.99 & 187.27 & 207.05 & 223.82 & 238.82 & 252.66 \\
\hline 376 & 140.12 & 120.46 & 108.95 & 100.79 & 94.46 & 89.28 & 161.00 & 187.29 & 207.06 & 223.84 & 238.84 & 252.68 \\
\hline 377 & 140.39 & 120.69 & 109.16 & 100.98 & 94.64 & 89.45 & 161.12 & 187.43 & 207.22 & 224.01 & 239.02 & 252.87 \\
\hline 378 & 140.71 & 120.96 & 109.40 & 101.21 & 94.85 & 89.65 & 161.19 & 187.51 & 207.31 & 224.10 & 239.12 & 252.97 \\
\hline 379 & 141.00 & 121.21 & 109.63 & 101.42 & 95.04 & 89.84 & 161.28 & 187.61 & 207.43 & 224.23 & 239.26 & 253.12 \\
\hline 380 & 141.22 & 121.40 & 109.80 & 101.58 & 95.19 & 89.98 & 161.45 & 187.81 & 207.65 & 224.46 & 239.51 & 253.39 \\
\hline 381 & 141.48 & 121.62 & 110.00 & 101.76 & 95.37 & 90.15 & 161.58 & 187.96 & 207.81 & 224.64 & 239.70 & 253.59 \\
\hline 382 & 141.83 & 121.92 & 110.27 & 102.01 & 95.60 & 90.37 & 161.61 & 187.99 & 207.84 & 224.68 & 239.74 & 253.63 \\
\hline 383 & 142.14 & 122.19 & 110.52 & 102.24 & 95.82 & 90.57 & 161.67 & 188.07 & 207.93 & 224.77 & 239.83 & 253.73 \\
\hline 384 & 142.38 & 122.39 & 110.70 & 102.41 & 95.98 & 90.72 & 161.82 & 188.24 & 208.12 & 224.98 & 240.06 & 253.97 \\
\hline 385 & 142.74 & 122.70 & 110.98 & 102.67 & 96.22 & 90.95 & 161.84 & 188.26 & 208.14 & 225.00 & 240.08 & 253.99 \\
\hline 386 & 143.04 & 122.96 & 111.22 & 102.88 & 96.42 & 91.14 & 161.92 & 188.35 & 208.24 & 225.11 & 240.20 & 254.12 \\
\hline 387 & 143.40 & 123.27 & 111.49 & 103.14 & 96.66 & 91.37 & 161.93 & 188.37 & 208.26 & 225.13 & 240.22 & 254.14 \\
\hline 388 & 143.71 & 123.54 & 111.74 & 103.37 & 96.87 & 91.57 & 161.99 & 188.44 & 208.34 & 225.21 & 240.31 & 254.24 \\
\hline 389 & 143.96 & 123.76 & 111.94 & 103.55 & 97.04 & 91.73 & 162.13 & 188.60 & 208.51 & 225.40 & 240.51 & 254.45 \\
\hline 390 & 144.29 & 124.03 & 112.19 & 103.78 & 97.26 & 91.94 & 162.18 & 188.66 & 208.58 & 225.47 & 240.59 & 254.53 \\
\hline 391 & 144.51 & 124.23 & 112.36 & 103.94 & 97.41 & 92.08 & 162.34 & 188.84 & 208.79 & 225.70 & 240.83 & 254.78 \\
\hline 392 & 144.75 & 124.44 & 112.55 & 104.12 & 97.58 & 92.23 & 162.48 & 189.01 & 208.97 & 225.90 & 241.04 & 255.01 \\
\hline 393 & 145.23 & 124.84 & 112.92 & 104.46 & 97.90 & 92.53 & 162.37 & 188.88 & 208.82 & 225.74 & 240.87 & 254.82 \\
\hline 394 & 145.36 & 124.95 & 113.02 & 104.55 & 97.98 & 92.62 & 162.64 & 189.19 & 209.17 & 226.11 & 241.27 & 255.25 \\
\hline 395 & 145.80 & 125.34 & 113.37 & 104.87 & 98.28 & 92.90 & 162.55 & 189.09 & 209.06 & 225.99 & 241.14 & 255.11 \\
\hline 396 & 146.12 & 125.61 & 113.61 & 105.10 & 98.50 & 93.10 & 162.60 & 189.15 & 209.13 & 226.07 & 241.22 & 255.20 \\
\hline 397 & 146.27 & 125.74 & 113.73 & 105.21 & 98.60 & 93.20 & 162.85 & 189.44 & 209.45 & 226.41 & 241.59 & 255.59 \\
\hline 398 & 146.74 & 126.14 & 114.09 & 105.54 & 98.91 & 93.50 & 162.74 & 189.31 & 209.30 & 226.26 & 241.42 & 255.41 \\
\hline 399 & 147.04 & 126.40 & 114.33 & 105.76 & 99.12 & 93.69 & 162.82 & 189.40 & 209.40 & 226.36 & 241.53 & 255.53 \\
\hline 400 & 147.29 & 126.62 & 114.52 & 105.94 & 99.29 & 93.85 & 162.94 & 189.55 & 209.57 & 226.54 & 241.73 & 255.73 \\
\hline
\end{tabular}


Table B.1: Simulation Data for Picking Times (Medium Rate) - continued.

\begin{tabular}{|c|c|c|c|c|c|c|c|c|c|c|c|c|}
\hline \multirow{2}{*}{$\begin{array}{r}\text { Batch } \\
\text { Size }\end{array}$} & \multicolumn{6}{|c|}{ Picking Time (minutes) } & \multicolumn{6}{|c|}{ Picking Rate (orders/hr) } \\
\hline & 20 & 40 & 60 & 80 & 100 & 120 & 20 & 40 & 60 & 80 & 100 & 120 \\
\hline 401 & 147.58 & 126.86 & 114.75 & 106.15 & 99.48 & 94.03 & 163.03 & 189.65 & 209.68 & 226.66 & 241.85 & 255.87 \\
\hline 402 & 147.89 & 127.13 & 114.99 & 106.38 & 99.69 & 94.23 & 163.09 & 189.72 & 209.76 & 226.74 & 241.94 & 255.96 \\
\hline 403 & 8.13 & 127.34 & 115.17 & 106.54 & 99.85 & 94.38 & 163.24 & 189.89 & 209.94 & 226.95 & 242.16 & 256.19 \\
\hline 404 & 148.48 & 127.64 & 115.44 & 106.79 & 100.09 & 94.60 & 163.26 & 189.92 & 209.97 & 226.98 & 242.19 & 256.23 \\
\hline 405 & 148.77 & 127.89 & 115.68 & 107.01 & 100.29 & 94.79 & 163.34 & 190.01 & 210.07 & 227.09 & 242.31 & 256.35 \\
\hline 406 & 149.10 & 128.17 & 115.93 & 107.24 & 100.50 & 95.00 & 163.38 & 190.06 & 210.13 & 227.15 & 242.38 & 256.42 \\
\hline 407 & 149.37 & 128.40 & 116.14 & 107.44 & 100.69 & 95.17 & 163.49 & 190.18 & 210.26 & 227.29 & 242.53 & 256.58 \\
\hline 408 & 149.66 & 128.65 & 116.36 & 107.64 & 100.88 & 95.36 & 163.58 & 190.28 & 210.38 & 227.42 & 242.66 & 256.72 \\
\hline 409 & 149.90 & 128.86 & 116.55 & 107.82 & 101.04 & 95.51 & 163.71 & 190.44 & 210.55 & 227.61 & 242.87 & 256.94 \\
\hline 410 & 150.34 & 129.24 & 116.89 & 108.14 & 101.34 & 95.79 & 163.63 & 190.35 & 210.45 & 227.49 & 242.74 & 256.81 \\
\hline 411 & 150.51 & 129.38 & 117.02 & 108.25 & 101.45 & 95.90 & 163.85 & 190.60 & 210.73 & 227.80 & 243.07 & 257.15 \\
\hline 412 & 150.79 & 129.62 & 117.24 & 108.46 & 101.65 & 96.08 & 163.94 & 190.71 & 210.84 & 227.92 & 243.20 & 257.29 \\
\hline 413 & 151.25 & 130.02 & 117.60 & 108.79 & 101.95 & 96.37 & 163.84 & 190.59 & 210.71 & 227.78 & 243.05 & 257.13 \\
\hline 414 & 151.50 & 130.23 & 117.79 & 108.97 & 102.12 & 96.53 & 163.96 & 190.74 & 210.88 & 227.96 & 243.24 & 257.33 \\
\hline 415 & 151.82 & 130.51 & 118.04 & 109.20 & 102.34 & 96.73 & 164.01 & 190.79 & 210.94 & 228.03 & 243.31 & 257.41 \\
\hline 416 & 152.17 & 130.81 & 118.32 & 109.45 & 102.57 & 96.96 & 164.03 & 190.81 & 210.96 & 228.05 & 243.33 & 257.43 \\
\hline 417 & 152.43 & 131.04 & 118.52 & 109.64 & 102.75 & 97.13 & 164.14 & 190.94 & 211.10 & 228.20 & 243.49 & 257.60 \\
\hline 418 & 152.65 & 131.23 & 118.69 & 109.80 & 102.90 & 97.27 & 164.29 & 191.12 & 211.30 & 228.41 & 243.72 & 257.85 \\
\hline 419 & 153.00 & 131.53 & 118.97 & 110.05 & 103.14 & 97.49 & 164.31 & 191.14 & 211.32 & 228.44 & 243.75 & 257.87 \\
\hline 420 & 153.30 & 131.78 & 119.20 & 110.27 & 103.34 & 97.68 & 164.38 & 191.22 & 211.42 & 228.54 & 243.86 & 257.99 \\
\hline 421 & 153.58 & 132.02 & 119.41 & 110.46 & 103.52 & 97.85 & 164.48 & 191.33 & 211.54 & 228.67 & 244.00 & 258.14 \\
\hline 422 & 153.91 & 132.30 & 119.67 & 110.70 & 103.75 & 98.06 & 164.52 & 191.38 & 211.59 & 228.72 & 244.06 & 258.20 \\
\hline 423 & 154.20 & 132.56 & 119.90 & 110.91 & 103.95 & 0825 & 164.59 & 191.46 & 211.68 & & 244.16 & \\
\hline 424 & 154.52 & 132.83 & 120.14 & 111.14 & 104.16 & 98.46 & 164.64 & 191.52 & 211.75 & 228.90 & 244.24 & 258.39 \\
\hline 425 & 154.78 & 133.05 & 120.35 & 111.33 & 104.34 & 98.62 & 164.75 & 191.65 & 211.89 & 229.05 & 244.40 & 258.57 \\
\hline 426 & 155.15 & 133.37 & 120.63 & 111.60 & 104.59 & 98.86 & 164.74 & 191.64 & 211.88 & 229.04 & 244.39 & 258.55 \\
\hline 427 & 155.38 & 133.57 & 120.81 & 111.76 & 104.74 & 99.00 & 164.89 & 191.81 & 212.07 & 229.24 & 244.61 & 258.79 \\
\hline 428 & 155.75 & 133.88 & 121.10 & 112.02 & 104.99 & 99.24 & 164.88 & 191.81 & 212.06 & & 244.60 & 258.78 \\
\hline 429 & 156.10 & 134.19 & 121.37 & 112.28 & 105.23 & 99.46 & 164.89 & 191.82 & 212.07 & 229.25 & 244.62 & 258.79 \\
\hline 430 & 156.35 & 134.40 & 121.56 & & & & 165.02 & 191.96 & 212.23 & & 244.80 & 258.99 \\
\hline 431 & 156.53 & 134.56 & 121.71 & 112.59 & 105.52 & 99.74 & 165.21 & 192.18 & 212.48 & 229.68 & 245.08 & 259.28 \\
\hline 432 & 156.94 & 134.91 & 122.03 & 112.88 & 105.79 & 100.00 & 165.16 & 192.13 & 212.41 & & & 259.21 \\
\hline 433 & 157.25 & 135.18 & 122.27 & 113.11 & 106.00 & 100.20 & 165.21 & 192.19 & 212.48 & 229.69 & 245.09 & 259.29 \\
\hline 434 & 157.64 & 135.51 & 122.57 & 113.39 & 106.26 & 100.44 & 165.19 & 192.16 & 212.45 & 229.66 & 245.05 & 259.25 \\
\hline 435 & 157.85 & 135.69 & 122.73 & 113.54 & 106.41 & 100.58 & 165.35 & 192.34 & 212.65 & 229.88 & 245.29 & 259.50 \\
\hline 436 & 158.11 & 135.92 & 122.94 & 113.72 & 106.58 & 100.74 & 165.45 & 192.47 & 212.79 & 230.03 & 245.45 & 259.67 \\
\hline 437 & 158.39 & 136.16 & 123.16 & 113.93 & 106.77 & 100.92 & 165.54 & 192.57 & 212.90 & 230.14 & 245.57 & 259.80 \\
\hline 438 & 158.73 & 136.45 & 123.42 & 114.17 & 107.00 & 101.14 & 165.56 & 192.60 & 212.93 & 230.18 & 245.61 & 259.84 \\
\hline 439 & 159.09 & 136.76 & 123.70 & 114.43 & 107.24 & 101.37 & 165.57 & 192.60 & 212.94 & 230.18 & 245.61 & 259.85 \\
\hline 440 & 159.42 & 137.04 & 123.95 & 114.67 & 107.46 & 101.58 & 165.60 & 192.64 & 212.98 & 230.23 & 245.67 & 259.90 \\
\hline 441 & 159.69 & 137.27 & 124.16 & 114.86 & 107.64 & 101.75 & 165.70 & 192.75 & 213.11 & 230.37 & 245.81 & 260.05 \\
\hline 442 & 159.98 & 137.52 & 124.39 & 115.07 & 107.84 & 101.93 & 165.77 & 192.84 & 213.20 & 230.47 & 245.92 & 260.17 \\
\hline
\end{tabular}


Table B.1: Simulation Data for Picking Times (Medium Rate) - continued.

\begin{tabular}{|c|c|c|c|c|c|c|c|c|c|c|c|c|}
\hline \multirow{2}{*}{$\begin{array}{r}\text { Batch } \\
\text { Size }\end{array}$} & \multicolumn{6}{|c|}{ Picking Time (minutes) } & \multicolumn{6}{|c|}{ Picking Rate (orders/hr) } \\
\hline & 20 & 40 & 60 & 80 & 100 & 120 & 20 & 40 & 60 & 80 & 100 & 120 \\
\hline 443 & 160.27 & 137.77 & 124.61 & 115.28 & 108.03 & 102.12 & 165.85 & 192.93 & 213.30 & 230.58 & 246.04 & 260.29 \\
\hline 444 & 160.59 & 138.05 & 124.87 & 115.51 & 108.25 & 102.33 & 165.88 & 192.97 & 213.35 & 230.63 & 46.09 & 260.35 \\
\hline 445 & 160.79 & 138.22 & 125.02 & 115.65 & 108.39 & 102.45 & 166.05 & 193.17 & 213.57 & 230.86 & 246.34 & 260.61 \\
\hline 446 & & 138.51 & 125 & 115.89 & & & 6.09 & 193.20 & 213.61 & 30.91 & 246.39 & 60.66 \\
\hline 447 & 161.51 & 138.84 & 125.58 & 116.17 & 108.87 & 102.91 & 166.06 & 193.17 & 213.57 & 230.87 & 246.35 & 260.62 \\
\hline 448 & 161.79 & 139.08 & 125.79 & 116.37 & 109.06 & 103.09 & 166.14 & 193.27 & 213.68 & 230.99 & 246.47 & 260.75 \\
\hline 449 & 162.10 & 139.35 & 126.04 & 116.60 & 109.27 & 103.29 & 166.19 & 193.33 & 213.74 & 231.05 & 246.54 & 260.83 \\
\hline 450 & 162.43 & 139.63 & 126.30 & 116.83 & 109.50 & 103.50 & 166.22 & 193.36 & 213.78 & 231.10 & 246.59 & 260.87 \\
\hline 451 & 162.80 & 139.95 & 126.58 & 117.10 & 109.74 & 103.73 & 6.22 & 193.35 & 213.77 & 231.09 & 46.58 & 260.87 \\
\hline 452 & 162.92 & 140.05 & 126.67 & 117.18 & 109.82 & 103.81 & 166.47 & 193.65 & 214.09 & 231.43 & 246.95 & 261.26 \\
\hline 453 & 163.32 & 140.40 & 126.99 & 117.47 & 110.09 & 104.06 & 166.42 & 193.59 & 214.03 & 231.37 & 246.88 & 261.18 \\
\hline 454 & 163.61 & 140.65 & 127.21 & 117.68 & 110.29 & 104.25 & 166.49 & 193.68 & 214.13 & 231.47 & 246.99 & 261.30 \\
\hline 455 & 163.85 & 140.85 & 127.40 & 117.85 & 110.45 & 104.40 & 166.62 & 193.82 & 214.29 & 231.65 & 247.18 & 261.50 \\
\hline 456 & 164.21 & 141.16 & 127.68 & 118.11 & 110.69 & 104.63 & 166.62 & 193.82 & 214.29 & 231.64 & 247.17 & 261.49 \\
\hline 457 & 164.46 & 141.37 & 127.87 & 118.29 & 110.86 & 104.79 & 166.73 & 193.95 & 214.44 & & & 261.67 \\
\hline 458 & 164.72 & 141.60 & 128.08 & 118.48 & 111.04 & 104.96 & 166.83 & 194.06 & 214.56 & 231.94 & 247.48 & 261.82 \\
\hline 459 & 165.06 & 141.89 & 128.34 & 118.72 & & 105.17 & & 194.09 & 214.59 & 231.97 & & 261.86 \\
\hline 460 & 165.36 & 142.15 & 128.57 & 118.94 & 111.47 & 105.36 & 166.91 & 194.16 & 214.66 & 232.05 & 247.60 & 261.95 \\
\hline 461 & 165.72 & 142.46 & 128.85 & 119.20 & 111.71 & 105.59 & 166.91 & 194.16 & 214.67 & 232.05 & 247.61 & 261.95 \\
\hline 462 & 166.00 & 142.70 & 129.07 & 119.40 & 111.90 & & 166.99 & 194.26 & 214.77 & 232.17 & 247.73 & 262.08 \\
\hline 463 & 166.23 & 142.90 & 129.25 & 119.57 & 112.06 & 105.92 & 167.11 & 194.40 & 214.93 & 232.34 & 247.91 & 262.27 \\
\hline 464 & 166.51 & 143.14 & 129.46 & 119.76 & & 106.09 & 167.20 & 194.50 & 215.04 & 232.46 & 248.04 & 262.41 \\
\hline 465 & 166.88 & 143.45 & 129.75 & 120.03 & 112.49 & 106.33 & 167.19 & 194.49 & 215.02 & 232.44 & 248.02 & 262.39 \\
\hline 466 & 167.16 & 143.70 & 129.97 & 120.23 & 112.68 & 106.51 & 167.27 & 194.58 & 215.12 & 232.55 & 248.14 & 262.51 \\
\hline 467 & 167.44 & 143.94 & 130.19 & 120.44 & 112.87 & 106.69 & 167.34 & 194.66 & 215.22 & 232.65 & 248.25 & 262.63 \\
\hline 468 & 167.82 & 144.26 & 130.48 & 120.71 & 113.12 & 106.93 & 167.33 & 194.65 & 215.20 & 232.63 & 248.23 & 262.61 \\
\hline 469 & 168.13 & 144.53 & 130.72 & 120.93 & 113.33 & & 167.37 & 194.70 & 215.26 & 232.70 & 248.30 & 262.68 \\
\hline 470 & 168.33 & 144.70 & 130.88 & 121.07 & 113.47 & 107.25 & 167.53 & 194.89 & 215.47 & 232.92 & 248.53 & 262.93 \\
\hline 471 & 168.73 & 145.05 & 131.20 & & & & 107.48 & 194.83 & & & & 262.85 \\
\hline 472 & 168.92 & 145.21 & 131.34 & 121.50 & 113.87 & 107.63 & 167.65 & 195.02 & 215.62 & 233.08 & 248.70 & 263.11 \\
\hline 473 & & & & & & & & & & & & \\
\hline 474 & & & & & & & & & & & & \\
\hline 475 & 169.87 & 146.03 & 132.08 & 122.18 & 114.51 & 108.24 & 167.78 & 195.17 & 215.78 & 233.26 & 248.89 & 263.32 \\
\hline 476 & 170.17 & 146.29 & 132.31 & 122.40 & 114.71 & & 167.83 & 195.24 & 215.85 & 233.33 & & 263.40 \\
\hline 477 & 170.46 & 146.53 & 132.54 & 122.61 & 114.90 & 108.61 & 167.90 & 195.32 & 215.94 & 233.43 & 249.08 & 263.51 \\
\hline 478 & 170.80 & 146.82 & 132.80 & 122.85 & 115.13 & & 167.92 & 195.34 & 215.96 & 233.46 & 249.10 & 263.54 \\
\hline 479 & 171.11 & 147.09 & 133.04 & 123.08 & 115.3 & 109.03 & 167.96 & 195.38 & 216.02 & 233.51 & 249.17 & 263.60 \\
\hline 480 & 171.39 & 147.33 & 133.26 & 123.27 & 115.53 & 109.20 & 168.04 & 195.48 & 216.12 & 233.63 & 249.29 & 263.73 \\
\hline 481 & 171.80 & 147.69 & 133.58 & 123.57 & 115.8 & 109.47 & 167.99 & 195.42 & 216.05 & 233.55 & 249.21 & 263.65 \\
\hline 482 & 171.93 & 147.80 & 133.68 & 123.67 & 115.90 & 109.55 & 168.20 & 195.67 & 216.33 & 233.85 & 249.53 & 263.99 \\
\hline 483 & 172.32 & 148.13 & 133.98 & 123.94 & 116.16 & 109.79 & 168.18 & 195.64 & 216.30 & 233.82 & 249.49 & 263.95 \\
\hline 484 & 172.63 & 148.40 & 134.23 & 124.17 & 116.37 & 109.99 & 168.22 & 195.69 & 216.35 & 233.87 & 249.55 & 264.01 \\
\hline
\end{tabular}


Table B.1: Simulation Data for Picking Times (Medium Rate) - continued.

\begin{tabular}{|c|c|c|c|c|c|c|c|c|c|c|c|c|}
\hline \multirow{2}{*}{$\begin{array}{r}\text { Batch } \\
\text { Size }\end{array}$} & \multicolumn{6}{|c|}{ Picking Time (minutes) } & \multicolumn{6}{|c|}{ Picking Rate (orders/hr) } \\
\hline & 20 & 40 & 60 & 80 & 100 & 120 & 20 & 40 & 60 & 80 & 100 & 120 \\
\hline 485 & 172.94 & 148.67 & 134.47 & 124.39 & 116.58 & 110.19 & 168.27 & 195.74 & 216.41 & 233.94 & 249.62 & 264.08 \\
\hline 486 & 173.28 & 148.96 & 134.73 & 124.64 & 116.81 & 110.41 & 168.28 & 195.75 & 216.43 & 233.96 & 249.64 & 264.10 \\
\hline 487 & 173.49 & 149.14 & 134.90 & 124.79 & 116.95 & 110.55 & 168.42 & 195.92 & 216.61 & 234.15 & 249.85 & 264.33 \\
\hline 488 & 17 & 149. & 135. & 125.00 & 117. & & & 195.99 & 216.68 & 234.23 & 249.93 & 64.42 \\
\hline 489 & 174.14 & 149.70 & 135.40 & 125.25 & 117.38 & 110.96 & 168.49 & 196.00 & 216.69 & 234.24 & 249.95 & 264.43 \\
\hline 490 & 174.40 & 149.92 & 135.60 & 125.44 & 117.56 & 111.12 & 168.58 & 196.10 & 216.81 & 234.37 & 250.08 & 264.57 \\
\hline 491 & 174.69 & 150.17 & 135.82 & 125.65 & 117.75 & 111.30 & 168.65 & 196.18 & 216.90 & 234.47 & 250.18 & 264.68 \\
\hline 492 & 175.03 & 150.46 & 136.09 & 125.89 & 117.98 & 111.52 & 168.66 & 196.20 & 216.92 & 234.49 & 250.21 & 264.70 \\
\hline 493 & 175.29 & 150.69 & 136.30 & 126.09 & 118.16 & & & 196.30 & 217.03 & 234.60 & 250.33 & 264.83 \\
\hline 494 & 175.63 & 150.98 & 136.55 & 126.32 & 118.39 & 111.90 & 168.77 & 196.32 & 217.06 & 234.64 & 250.36 & 264.87 \\
\hline 495 & 175.87 & 151.19 & 136.75 & 126.50 & 118.56 & 112.06 & 168.87 & 196.44 & 217.19 & 234.78 & 250.52 & 265.03 \\
\hline 496 & 176.23 & 151.49 & 137.02 & 126.76 & 118.79 & 112.29 & 168.87 & 196.44 & 217.19 & 234.78 & 250.52 & 265.03 \\
\hline 497 & 176.45 & 151.68 & 137.19 & 126.92 & 118.94 & 112.43 & 169.00 & 196.60 & 217.36 & 234.96 & 250.71 & 265.24 \\
\hline 498 & 176.72 & 151.92 & 137.41 & 127.11 & 119. & 112.60 & 169.08 & 196.69 & 217.46 & 235.07 & 250.83 & 265.36 \\
\hline 499 & 177.22 & 152.34 & 137.79 & 127.47 & & 112.92 & 168.95 & 196.53 & 217.29 & 234.88 & & 265.15 \\
\hline 500 & 177.41 & 152.51 & 137.94 & 127.61 & 119.59 & 113.04 & 169.10 & 196.71 & 217.48 & 235.09 & 250.85 & 265.39 \\
\hline 501 & 177.65 & 152.72 & & 127.78 & 119.75 & 113.19 & 169.21 & 196.84 & & & 251.02 & 265.56 \\
\hline 502 & 178.06 & 153.07 & 138.45 & 128.08 & 120.03 & 113.46 & 169.16 & 196.78 & 217.55 & 235.17 & 250.94 & 265.48 \\
\hline 503 & 178.31 & 153.28 & 138.64 & 128.26 & 120.20 & 113.61 & 169.25 & 196.89 & 217.68 & 235.31 & 251.09 & 265.63 \\
\hline 504 & 178.64 & 153.57 & 138.90 & 128.49 & 120.42 & 113.83 & 169.28 & 196.92 & 217.71 & 235.34 & 251.12 & 265.67 \\
\hline 505 & 178.88 & 153.77 & 139.08 & 128.66 & 120.58 & 113.98 & 169.39 & 197.05 & 217.86 & 235.50 & 251.29 & 265.85 \\
\hline 506 & 179.19 & 154.04 & 139.33 & 128.89 & 120.79 & & 169.43 & 197.09 & 217.90 & 235.55 & 251.34 & 265.90 \\
\hline 507 & 179.53 & 154.33 & 139.59 & 129.13 & 121.02 & 114.39 & 169.44 & 197.11 & 217.92 & 235.57 & 251.36 & 265.93 \\
\hline 508 & 179.73 & 154.50 & 139.75 & 129.28 & 121.15 & 114.52 & 169.59 & 197.28 & 218.11 & 235.78 & 251.58 & 266.16 \\
\hline 509 & 180.06 & 154.79 & 140.00 & 129.51 & 121.38 & 114.73 & 169.61 & 197.30 & 218.14 & 235.80 & 251.61 & 266.19 \\
\hline 510 & 180.52 & 155.18 & 140.36 & 129.84 & 121.69 & 115.02 & 16051 & 197.19 & 218.01 & & 251.46 & 266.04 \\
\hline 511 & 180.69 & 155.33 & 140.50 & 129.97 & 121.80 & & 169.68 & 197.38 & 218.23 & 235.90 & 251.72 & 266.30 \\
\hline 512 & 180.95 & 155.55 & 140.69 & 130.15 & 121.98 & 115.30 & 169.77 & 197.49 & 218.35 & 236.03 & 251.85 & 266.45 \\
\hline 513 & & 155.85 & & & & & & & & & 251.86 & 266.45 \\
\hline 514 & 181.61 & 156.12 & 141.21 & 130.63 & 122.42 & 115.72 & 169.81 & 197.54 & 218.40 & 236.09 & 251.91 & 266.51 \\
\hline 515 & & & & & & & & & & & & \\
\hline 516 & & & & & & & & & & & & \\
\hline 517 & 182.52 & 156.90 & 141.92 & 131.29 & 123.04 & 116.30 & 169.95 & 197.70 & 218.58 & 236.28 & 252.12 & 266.73 \\
\hline 518 & 182.85 & 157.18 & 142.17 & 131.52 & 123.26 & & 169.98 & 197.73 & 218.61 & 236.32 & 252.16 & 266.77 \\
\hline 519 & 183.10 & 157.40 & 142.37 & 131.70 & 123.43 & 116.67 & 170.07 & 197.84 & 218.73 & 236.45 & 252.30 & 266.91 \\
\hline 520 & 183.36 & 157.62 & 142.57 & 131.88 & 123.60 & 116.83 & 170.16 & 197.94 & 218.85 & 236.57 & 252.43 & 267.06 \\
\hline 521 & 183.77 & 157.98 & 142.89 & 132.18 & 123. & 117.09 & 170.10 & 197.88 & 218.77 & 236.49 & 252.35 & 266.97 \\
\hline 522 & 184.02 & 158.19 & 143.09 & 132.36 & 124.05 & 117.25 & 170.19 & 197.98 & 218.89 & 236.62 & 252.48 & 267.11 \\
\hline 523 & 184.26 & 158.40 & 143.27 & 132.54 & 124.21 & 117.41 & 170.30 & 198.10 & 219.02 & 236.76 & 252.64 & 267.27 \\
\hline 524 & 184.59 & 158.68 & 143.53 & 132.77 & 124.43 & 117.62 & 170.32 & 198.13 & 219.05 & 236.80 & 252.67 & 267.31 \\
\hline 525 & 184.86 & 158.91 & 143.73 & 132.96 & 124.61 & 117.78 & 170.40 & 198.23 & 219.16 & 236.91 & 252.79 & 267.44 \\
\hline 526 & 185.18 & 159.18 & 143.98 & 133.19 & 124.83 & 117.99 & 170.43 & 198.26 & 219.20 & 236.95 & 252.83 & 267.48 \\
\hline
\end{tabular}


Table B.1: Simulation Data for Picking Times (Medium Rate) - continued.

\begin{tabular}{|c|c|c|c|c|c|c|c|c|c|c|c|c|}
\hline \multirow{2}{*}{$\begin{array}{r}\text { Batch } \\
\text { Size }\end{array}$} & \multicolumn{6}{|c|}{ Picking Time (minutes) } & \multicolumn{6}{|c|}{ Picking Rate (orders/hr) } \\
\hline & 20 & 40 & 60 & 80 & 100 & 120 & 20 & 40 & 60 & 80 & 100 & 120 \\
\hline 527 & 185.64 & 159.58 & 144.34 & 133.53 & 125.14 & 118.28 & 170.33 & 198.14 & 219.06 & 236.81 & 252.68 & 267.32 \\
\hline 528 & 185.74 & 159.67 & 144.42 & 133.60 & 125.21 & 118.35 & 170.56 & 198.41 & 219.36 & 237.13 & 253.02 & 267.68 \\
\hline 529 & 186.18 & 160.04 & 144.76 & 133.91 & 125.50 & 118.63 & 170.48 & 198.32 & 219.26 & 237.02 & 252.91 & 267.56 \\
\hline 530 & 1 & 160.23 & 144. & 134.07 & & & & & 219.42 & 237.19 & 53.09 & 267.76 \\
\hline 531 & 186.73 & 160.52 & 145.19 & 134.31 & 125.87 & 118.98 & 170.62 & 198.48 & 219.44 & 237.22 & 253.12 & 267.78 \\
\hline 532 & 187.01 & 160.76 & 145.41 & 134.51 & 126.06 & 119.16 & 170.68 & 198.55 & 219.52 & 237.30 & 253.20 & 267.88 \\
\hline 533 & 187.32 & 161.02 & 145.64 & 134.73 & 126.27 & 119.35 & 170.73 & 198.60 & 219.58 & 237.36 & 253.27 & 267.95 \\
\hline 534 & 187.64 & 161.30 & 145.89 & 134.96 & 126.48 & 119.56 & 170.75 & 198.64 & 219.61 & 237.40 & 253.31 & 267.99 \\
\hline 535 & 37.92 & 161.55 & 146. & 135.17 & 126.68 & 19.74 & 170 & 198.70 & 219.69 & 237.48 & 253.40 & 68.08 \\
\hline 536 & 188.29 & 161.86 & 146.40 & 135.43 & 126.92 & 119.97 & 170.80 & 198.69 & 219.67 & 237.46 & 253.38 & 268.06 \\
\hline 537 & 188.52 & 162.06 & 146.58 & 135.60 & 127.08 & 120.12 & 170.91 & 198.81 & 219.81 & 237.61 & 253.54 & 268.23 \\
\hline 538 & 188.88 & 162.36 & 146.86 & 135.85 & 127.32 & 120.35 & 170.91 & 198.81 & 219.81 & 237.61 & 253.54 & 268.23 \\
\hline 539 & 189.17 & 162.62 & 147.09 & 136.07 & 127.52 & 120.53 & 170.96 & 198.87 & 219.87 & 237.68 & 253.61 & 268.31 \\
\hline 540 & 189.53 & 162.93 & 147.37 & 136.33 & 127.76 & 120.76 & 170. & 198.86 & 219.86 & 237.67 & 253.60 & 268.29 \\
\hline 541 & 189.62 & 163.01 & 147.44 & 136.39 & 127.82 & 120.82 & & 199.13 & & & 253.95 & 268.66 \\
\hline 542 & 190.04 & 163.37 & 147.76 & 136.69 & 128.10 & 121.09 & 171.12 & 199.06 & 220.08 & 237.91 & 253.86 & 268.57 \\
\hline 543 & 190.00 & 163.66 & 148.03 & 136.94 & 128.33 & 121.31 & & 199.07 & 220.09 & & & 268.58 \\
\hline 544 & 190.71 & 163.94 & 148.28 & 137.17 & 128.56 & 121.52 & 171.15 & 199.09 & 220.12 & 237.95 & 253.90 & 268.61 \\
\hline 545 & 190.98 & 164.17 & 148.49 & 137.37 & 128.74 & 121.69 & 171.22 & 199.18 & 220.21 & 238.05 & 254.00 & 268.72 \\
\hline 546 & 191.26 & 164.41 & 148.71 & 137.57 & 128.93 & 121.86 & 171.29 & 199.25 & 220.29 & 238.14 & 254.10 & 268.82 \\
\hline 547 & 191.52 & 164.63 & 148.91 & 137.75 & 129.10 & 122.03 & 171.37 & 199.35 & 220.40 & 238.25 & 254.23 & 268.96 \\
\hline 548 & 191.93 & 164.99 & 149.23 & 138.05 & 129.38 & 122.29 & 171.31 & 199.28 & 220.33 & 238.17 & 254.14 & 268.86 \\
\hline 549 & 192.23 & 165.24 & 149.46 & 138.26 & 129.58 & 122.48 & 171.36 & 199.34 & 220.39 & 238.24 & 254.21 & 268.94 \\
\hline 550 & 192.44 & 165.43 & 149.63 & 138.42 & 129.72 & 122.62 & 171.48 & & 220.55 & & 254.39 & 269.13 \\
\hline 551 & 192.83 & 165.76 & 149.93 & 138.69 & 129.98 & 122.86 & 171.45 & 199.44 & 220.51 & 238.36 & 254.34 & 269.08 \\
\hline 552 & 193.02 & 165.92 & 150.08 & 138.83 & 130.11 & 122.98 & 171.59 & 199.61 & 220.69 & 238.56 & 254.55 & 269.30 \\
\hline 553 & 193.28 & 166.15 & 150.28 & 139.02 & 130.29 & 123.15 & 171.67 & 199.70 & 220.79 & 238.67 & 254.67 & 269.43 \\
\hline 554 & 193.66 & 166.47 & 150.57 & 139.29 & 130.54 & 123.39 & 171.64 & 199.67 & 220.75 & 238.63 & 254.63 & 269.38 \\
\hline 555 & 193.96 & 166.73 & 150.81 & & & & & & & & & 269.45 \\
\hline 556 & 194.22 & 166.96 & 151.01 & 139.70 & 130.92 & 123.75 & 171.76 & 199.81 & 220.91 & 238.80 & 254.80 & 269.57 \\
\hline 557 & & & & & 131.13 & & & & & & & \\
\hline 558 & & & & & & & & & & & & \\
\hline 559 & 195.18 & 167.78 & 151.76 & 140.39 & 131.57 & 124.36 & 171.84 & 199.90 & 221.01 & 238.91 & 254.93 & 269.70 \\
\hline 560 & 195.40 & 167.97 & 151.93 & 140.55 & 131.72 & 124.50 & 171.96 & 200.03 & 221.16 & 239.07 & 255.09 & 269.87 \\
\hline 561 & 195.76 & 168.28 & 152.21 & 140.80 & 131.96 & 124.73 & 171.95 & 200.02 & 221.15 & 239.06 & 255.08 & 269.86 \\
\hline 562 & 196.03 & 168.52 & 152.42 & 141.00 & 132.14 & 124.91 & 172.01 & 200.10 & 221.23 & 239.15 & 255.18 & 269.96 \\
\hline 563 & 196.36 & 168.80 & 152.68 & 141.24 & 132.37 & 125.12 & 172.03 & 200.12 & 221.25 & 239.17 & 255.20 & 269.99 \\
\hline 564 & 196.60 & 169.00 & 152.86 & 141.41 & 132.52 & 125.26 & 172.13 & 200.24 & 221.38 & 239.31 & 255.35 & 270.15 \\
\hline 565 & 197.00 & 169.35 & 153.18 & 141.70 & 132.80 & 125.52 & 172.08 & 200.18 & 221.31 & 239.24 & 255.28 & 270.07 \\
\hline 566 & 197.23 & 169.54 & 153.35 & 141.86 & 132.95 & 125.67 & 172.19 & 200.30 & 221.46 & 239.39 & 255.44 & 270.24 \\
\hline 567 & 197.55 & 169.82 & 153.60 & 142.09 & 133.17 & 125.87 & 172.21 & 200.33 & 221.48 & 239.42 & 255.47 & 270.27 \\
\hline 568 & 197.87 & 170.10 & 153.85 & 142.33 & 133.38 & 126.08 & 172.23 & 200.35 & 221.51 & 239.45 & 255.50 & 270.31 \\
\hline
\end{tabular}


Table B.1: Simulation Data for Picking Times (Medium Rate) - continued.

\begin{tabular}{|c|c|c|c|c|c|c|c|c|c|c|c|c|}
\hline \multirow{2}{*}{$\begin{array}{r}\text { Batch } \\
\text { Size }\end{array}$} & \multicolumn{6}{|c|}{ Picking Time (minutes) } & \multicolumn{6}{|c|}{ Picking Rate (orders/hr) } \\
\hline & 20 & 40 & 60 & 80 & 100 & 120 & 20 & 40 & 60 & 80 & 100 & 120 \\
\hline 569 & 198.12 & 170.31 & 154.04 & 142.50 & 133.55 & 126.24 & 172.32 & 200.46 & 221.63 & 239.58 & 255.63 & 270.45 \\
\hline 570 & 198.47 & 170.61 & 154.32 & 142.76 & 133.79 & 126.46 & 172.32 & 200.45 & 221.62 & 239.57 & 255.63 & 270.44 \\
\hline 571 & 198.70 & 170.81 & 154.49 & 142.92 & 133.94 & 126.60 & 172.42 & 200.58 & 221.76 & 239.72 & 255.79 & 270.61 \\
\hline 572 & 199.03 & 171.09 & 154.75 & 143.16 & 134.16 & 126.82 & 172.44 & 200.59 & 221.77 & 239.74 & 255.81 & 270.63 \\
\hline 573 & 199.41 & 171.42 & 155.05 & 143.43 & 134.42 & 127.06 & 172.41 & 200.56 & 221.74 & 239.70 & 255.77 & 270.59 \\
\hline 574 & 199.64 & 171.62 & 155.23 & 143.60 & 134.58 & 127.21 & 172.51 & 200.67 & 221.87 & 239.84 & 255.91 & 270.74 \\
\hline 575 & 199.96 & 171.90 & 155.48 & 143.83 & 134.79 & 127.41 & 172.53 & 200.70 & 221.90 & 239.87 & 255.95 & 270.78 \\
\hline 576 & 200.27 & 172.16 & 155.72 & 144.05 & 135.00 & 127.61 & 172.56 & 200.74 & 221.94 & 239.91 & 256.00 & 270.83 \\
\hline 577 & 200.63 & 172.47 & 156.00 & 144.31 & 135.25 & 127.84 & 172.55 & 200.73 & 221.92 & 239.90 & 255.98 & 270.81 \\
\hline 578 & 200.98 & 172.77 & 156.27 & 144.56 & 135.48 & 128.06 & 172.56 & 200.73 & 221.93 & 239.91 & 255.99 & 270.82 \\
\hline 579 & 201.15 & 172.92 & 156.40 & 144.68 & 135.59 & 128.17 & 172.71 & 200.90 & 222.12 & 240.11 & 256.21 & 271.05 \\
\hline 580 & 201.36 & 173.10 & 156.57 & 144.84 & 135.74 & 128.30 & 172.82 & 201.04 & 222.27 & 240.27 & 256.38 & 271.23 \\
\hline 581 & 201.73 & 173.42 & 156.85 & 145.10 & 135.99 & 128.54 & 172.80 & 201.02 & 222.25 & 240.25 & 256.35 & 271.20 \\
\hline 582 & 202.07 & 173.71 & 157.11 & 145.34 & 136.21 & 128.75 & 172.81 & 201.03 & 222.26 & 240.26 & 256.36 & 271.22 \\
\hline 583 & 202.39 & 173.98 & 157.37 & 145.58 & 136.43 & 128.96 & 172.83 & 201.05 & 222.28 & 240.29 & 256.39 & 271.25 \\
\hline 584 & 202.63 & 174.19 & 157.55 & 145.75 & 136.59 & 129.11 & 172.92 & 201.16 & 222.40 & 240.41 & 256.53 & 271.40 \\
\hline 585 & 202.90 & 174.42 & 157.76 & 145.94 & 136.77 & 129.28 & 172.99 & 201.24 & 222.49 & 240.51 & 256.63 & 271.50 \\
\hline 586 & 203.26 & 174.73 & 158.04 & 146.20 & 137.01 & 129.51 & 172.98 & 201.23 & 222.48 & 240.50 & 256.62 & 271.49 \\
\hline 587 & 203.61 & 175.03 & 158.31 & 146.45 & 137.25 & 129.73 & 172.98 & 201.23 & 222.48 & 240.49 & 256.61 & 271.48 \\
\hline 588 & 203.79 & 175.19 & 158.46 & 146.58 & 137.38 & 129.85 & 173.12 & 201.38 & 222.65 & 240.68 & 256.81 & 271.69 \\
\hline 589 & 204.13 & 175.48 & 158.72 & 146.83 & 137.60 & 130.07 & 173.12 & 201.39 & 222.66 & 240.69 & 256.82 & 271.70 \\
\hline 590 & 204.52 & 175.82 & 159.02 & 147.11 & 137.87 & 130.32 & 173.08 & 201.35 & 222.61 & 240.64 & 256.77 & 271.65 \\
\hline 591 & 204.68 & 175.95 & 159.15 & 147.22 & & 130.42 & & 201.53 & 222.81 & & & 271.90 \\
\hline 592 & 205.13 & 176.34 & 159.50 & 147.55 & 138.28 & 130.70 & 173.16 & 201.43 & 222.70 & 240.74 & 256.88 & 271.76 \\
\hline 593 & 205.42 & 176.59 & 159.72 & 147.75 & 138.47 & 130.89 & 173.21 & 201.49 & 222.76 & 240.81 & 256.95 & 271.84 \\
\hline 594 & 205.61 & 176.75 & 159.87 & 147.89 & 138.60 & 131.01 & 173.34 & 201.64 & 222.93 & 240.99 & 257.14 & 272.04 \\
\hline 595 & 205.95 & 177.04 & 160.13 & 148.13 & 138.83 & 131.22 & 173.35 & 201.65 & 222.94 & 241.00 & 257.16 & 272.06 \\
\hline 596 & 206.20 & 177.26 & 160.33 & 148.32 & 139.00 & 131.39 & 173.42 & 201.74 & 223.04 & 241.11 & 257.27 & 272.18 \\
\hline 597 & 206.50 & 177.51 & 160.56 & 148.53 & 139.20 & 131.57 & 173.46 & 201.79 & 223.10 & 241.17 & 257.33 & 272.24 \\
\hline 598 & 206.89 & 177.85 & 160.86 & & & & 173.42 & & 223.05 & & & 272.18 \\
\hline 599 & 207.18 & 178.10 & 161.09 & 149.02 & 139.66 & 132.01 & 173.47 & 201.80 & 223.11 & 241.18 & 257.35 & 272.26 \\
\hline 600 & 207.59 & 178.46 & 161.41 & & 139.94 & & 173.42 & 201.73 & & & & 272.17 \\
\hline 601 & 207.77 & 178.60 & 161.54 & 149.44 & 140.05 & 132.38 & 173.56 & 201.90 & 223.22 & 241.30 & 257.47 & 272.39 \\
\hline 602 & 208.12 & 178.91 & 161.82 & 149.70 & 140.29 & 132.61 & 173.55 & 201.89 & 223.21 & 241.28 & 257.46 & 272.38 \\
\hline 603 & 208.28 & 179.04 & 161.94 & 149.81 & 140.40 & 132.71 & 173.71 & 202.08 & 223.41 & 241.51 & 257.70 & 272.63 \\
\hline 604 & 208.73 & 179.43 & 162.29 & 150.13 & 140.70 & 132.99 & 173.63 & 201.97 & 223.30 & 241.39 & 257.57 & 272.50 \\
\hline 605 & 209.00 & 179.66 & 162.50 & 150.33 & 140.88 & 133.17 & 173.69 & 202.05 & 223.38 & 241.47 & 257.66 & 272.59 \\
\hline 606 & 209.34 & 179.96 & 162.77 & 150.57 & 141.11 & 133.39 & 173.69 & 202.05 & 223.38 & 241.48 & 257.66 & 272.59 \\
\hline 607 & 209.59 & 180.17 & 162.96 & 150.75 & 141.28 & 133.54 & 173.77 & 202.14 & 223.49 & 241.59 & 257.78 & 272.72 \\
\hline 608 & 209.82 & 180.37 & 163.14 & 150.92 & 141.44 & 133.69 & 173.86 & 202.25 & 223.61 & 241.72 & 257.93 & 272.87 \\
\hline 609 & 210.12 & 180.62 & 163.37 & 151.13 & 141.64 & 133.88 & 173.90 & 202.30 & 223.66 & 241.78 & 257.98 & 272.93 \\
\hline 610 & 210.52 & 180.97 & 163.69 & 151.42 & 141.91 & 134.14 & 173.85 & 202.24 & 223.60 & 241.71 & 257.91 & 272.85 \\
\hline
\end{tabular}


Table B.1: Simulation Data for Picking Times (Medium Rate) - continued.

\begin{tabular}{|c|c|c|c|c|c|c|c|c|c|c|c|c|}
\hline \multirow{2}{*}{$\begin{array}{r}\text { Batch } \\
\text { Size }\end{array}$} & \multicolumn{6}{|c|}{ Picking Time (minutes) } & \multicolumn{6}{|c|}{ Picking Rate (orders/hr) } \\
\hline & 20 & 40 & 60 & 80 & 100 & 120 & 20 & 40 & 60 & 80 & 100 & 120 \\
\hline 611 & 210.73 & 181.15 & 163.85 & 151.57 & 142.05 & 134.27 & 173.97 & 202.38 & 223.75 & 241.87 & 258.08 & 273.03 \\
\hline 612 & 0.97 & 181.36 & 84.04 & 151.75 & 142.21 & 134.42 & 174.05 & 202.47 & 223.85 & 241.98 & 58.20 & 273.16 \\
\hline 613 & 211.34 & 181.67 & 164.32 & 152.01 & 142.46 & 134.66 & 174.03 & 202.45 & 223.83 & 241.96 & 258.18 & 273.14 \\
\hline 614 & & 181 & 164. & 152.23 & 142.66 & & 174 & 202.49 & 223.87 & 242.01 & 68.23 & 273.19 \\
\hline 615 & 212.08 & 182.32 & 164.90 & 152.55 & 142.96 & 135.13 & 173.99 & 202.40 & 223.77 & 241.89 & 258.11 & 273.06 \\
\hline 616 & 212.32 & 182.52 & 165.09 & 152.72 & 143.12 & 135.29 & 174.07 & 202.50 & 223.88 & 242.01 & 258.24 & 273.20 \\
\hline 617 & 212.53 & 182.70 & 165.25 & 152.87 & 143.26 & 135.42 & 174.19 & 202.63 & 224.03 & 242.17 & 258.41 & 273.38 \\
\hline 618 & 212.86 & 182.98 & 165.50 & 153.10 & 143.49 & 135.63 & 174.20 & 202.64 & 224.04 & 242.19 & 258.42 & 273.40 \\
\hline 619 & 213.00 & 183.10 & 165. & 153.21 & 143.58 & & 174.37 & 202.84 & 224.26 & 242.42 & 258.67 & 273.66 \\
\hline 620 & 213.44 & 183.48 & 165.95 & 153.52 & 143.88 & 135.99 & 174.29 & 202.75 & 224.16 & 242.31 & 258.56 & 273.54 \\
\hline 621 & 213.75 & 183.75 & 166.20 & 153.75 & 144.09 & 136.20 & 174.31 & 202.78 & 224.19 & 242.35 & 258.59 & 273.58 \\
\hline 622 & 214.11 & 184.06 & 166.48 & 154.00 & 144.33 & 136.42 & 174.30 & 202.76 & 224.18 & 242.33 & 258.58 & 273.56 \\
\hline 623 & 214.32 & 184.24 & 166.64 & 154.16 & 144.47 & 136.56 & 174.41 & 202.89 & 224.31 & 242.48 & 258.73 & 273.73 \\
\hline 624 & 214.60 & 184.48 & 166.86 & 154.36 & 144.66 & 136.74 & 174.46 & 202.95 & 224.38 & 242.56 & 258.82 & 273.81 \\
\hline 625 & 215.01 & 184.83 & 167.17 & 154.65 & 144.93 & 136.99 & 174.41 & 202.89 & 224.32 & & & 273.73 \\
\hline 626 & 215.20 & 185.00 & 167.33 & 154.79 & 145.07 & 137.12 & 174.53 & 203.03 & 224.47 & 242.65 & 258.92 & 273.92 \\
\hline 627 & 215.58 & 185.32 & 167.62 & 155.06 & 145.32 & & 174.51 & 203.00 & 224.44 & 242.61 & & 273.88 \\
\hline 628 & 215.89 & 185.58 & 167.86 & 155.28 & 145.53 & 137.56 & 174.54 & 203.03 & 224.47 & 242.66 & 258.92 & 273.92 \\
\hline 629 & 216.30 & 185.94 & 168.18 & 155.58 & 145.81 & 137.82 & 174.48 & 202.97 & 224.40 & 242.57 & 258.83 & 273.83 \\
\hline 630 & 216.45 & 186.07 & 168.29 & 155.68 & & 137.91 & 174.64 & 203.15 & 224.61 & 242.80 & 259.07 & 274.09 \\
\hline 631 & 216.73 & 186.31 & 168.52 & 155.89 & 146.10 & 138.09 & 174.69 & 203.21 & 224.67 & 242.86 & 259.14 & 274.16 \\
\hline 632 & 216.99 & 186.53 & 168.71 & 156.07 & 146.27 & 138.26 & 174.76 & 203.29 & 224.76 & 242.96 & 259.25 & 274.27 \\
\hline 633 & 217.33 & 186.83 & 168.23 & 156.32 & 146.51 & 138.48 & 174.76 & 203.29 & 225.76 & 242.96 & 259.24 & 274.26 \\
\hline 634 & 217.67 & 187.12 & 169.25 & 156.56 & 146.73 & 138.69 & 174.76 & 203.29 & 224.76 & 242.97 & 259.25 & 274.28 \\
\hline 635 & 218.02 & 187.41 & 169.51 & 156.81 & 146.96 & & 174.76 & 203.29 & 224.76 & 242.96 & 259.25 & 274.27 \\
\hline 636 & 218.21 & 187.59 & 169.67 & 156.96 & 147.10 & 139.04 & 174.87 & 203.43 & 224.91 & 243.12 & 259.42 & 274.45 \\
\hline 637 & 218.48 & 187.81 & 169.87 & 157.14 & 147.27 & & 174.94 & 203.50 & 224.99 & & & 274.56 \\
\hline 638 & 218.84 & 188.12 & 170.16 & 157.41 & 147.52 & 139.44 & 174.92 & 203.48 & 224.97 & 243.19 & 259.49 & 274.53 \\
\hline 639 & 219.15 & & 170.39 & 157.63 & & & 174.95 & & & & & 274.58 \\
\hline 640 & 219.48 & 188.68 & 170.65 & 157.87 & 147.95 & 139.85 & 174.96 & 203.52 & 225.02 & 243.24 & 259.55 & 274.58 \\
\hline 641 & & & & & & & & & & & & \\
\hline 642 & 220.00 & & & & & & & & & & & \\
\hline 643 & 220.36 & 189.43 & 171.34 & 158.50 & 148.54 & 140.41 & 175.08 & 203.66 & 225.17 & 243.41 & 259.72 & 274.77 \\
\hline 644 & 220.70 & 189.73 & 171.60 & 158.75 & 148.77 & & 175.08 & 203.66 & 225.17 & 243.41 & 259.72 & 274.77 \\
\hline 645 & 220.98 & 189.96 & 171.82 & 158.94 & 148.96 & 140.80 & 175.13 & 203.73 & 225.24 & 243.48 & 259.80 & 274.86 \\
\hline 646 & 221.16 & 190.12 & 171.96 & 159.08 & 149.08 & & 175.26 & 203.87 & 225.40 & 243.66 & 259.99 & 275.05 \\
\hline 647 & 221.54 & 190.44 & 172.25 & 159.35 & 149.34 & 141.16 & 175.23 & 203.84 & 225.37 & 243.62 & 259.95 & 275.01 \\
\hline 648 & 221.97 & 190.81 & 172.59 & 159.66 & 149.63 & 141.43 & 175.16 & 203.76 & 225.28 & 243.52 & 259.85 & 274.91 \\
\hline 649 & 222.13 & 190.95 & 172.71 & 159.77 & 149.74 & 141.53 & 175.30 & 203.93 & 225.46 & 243.72 & 260.06 & 275.13 \\
\hline 650 & 222.55 & 191.31 & 173.04 & 160.08 & 150.02 & 141.80 & 175.24 & 203.85 & 225.38 & 243.64 & 259.97 & 275.03 \\
\hline 651 & 222.70 & 191.44 & 173.16 & 160.18 & 150.12 & 141.90 & 175.39 & 204.03 & 225.58 & 243.85 & 260.19 & 275.27 \\
\hline 652 & 223.14 & 191.82 & 173.50 & 160.50 & 150.42 & 142.18 & 175.31 & 203.94 & 225.48 & 243.74 & 260.08 & 275.15 \\
\hline
\end{tabular}


Table B.1: Simulation Data for Picking Times (Medium Rate) - continued.

\begin{tabular}{|c|c|c|c|c|c|c|c|c|c|c|c|c|}
\hline \multirow{2}{*}{$\begin{array}{r}\text { Batch } \\
\text { Size }\end{array}$} & \multicolumn{6}{|c|}{ Picking Time (minutes) } & \multicolumn{6}{|c|}{ Picking Rate (orders/hr) } \\
\hline & 20 & 40 & 60 & 80 & 100 & 120 & 20 & 40 & 60 & 80 & 100 & 120 \\
\hline 653 & 223.42 & 192.06 & 173.72 & 160.70 & 150.61 & 142.36 & 175.36 & 204.00 & 225.54 & 243.81 & 260.15 & 275.22 \\
\hline 654 & 223.78 & 192.37 & 174.00 & 160.96 & 150.85 & 142.59 & 175.35 & 203.98 & 225.52 & 243.78 & 260.13 & 275.20 \\
\hline 655 & 224.07 & 192.62 & 174.23 & 161.17 & 151.05 & 142.77 & 175.39 & 204.03 & 225.57 & 243.84 & 260.18 & 275.26 \\
\hline 656 & 224.34 & 192.85 & 174.43 & 161.36 & 151.22 & 142.94 & 175.45 & 204.10 & 225.65 & 243.92 & 260.27 & 275.36 \\
\hline 657 & 224.53 & 193.01 & 174.58 & 161.50 & 151.35 & 143.06 & 175.57 & 204.23 & 225.80 & 244.09 & 260.45 & 275.54 \\
\hline 658 & 224.82 & 193.26 & 174.80 & 161.71 & 151.55 & 143.25 & 175.61 & 204.28 & 225.85 & 244.15 & 260.51 & 275.61 \\
\hline 659 & 225.12 & 193.52 & 175.04 & 161.92 & 151.75 & 143.44 & 175.64 & 204.32 & 225.89 & 244.19 & 260.56 & 275.65 \\
\hline 660 & 225.58 & 193.92 & 175.39 & 162.25 & 152.06 & 143.73 & 175.55 & 204.21 & 225.78 & 244.06 & 260.42 & 275.51 \\
\hline 661 & 225.77 & 194.08 & 175.54 & 162.39 & 152.19 & 143.85 & 175.67 & 204.35 & 225.93 & 244.23 & 260.60 & 275.70 \\
\hline 662 & 226.08 & 194.35 & 175.79 & 162.62 & 152.40 & 144.05 & 175.69 & 204.38 & 225.96 & 244.26 & 260.63 & 275.73 \\
\hline 663 & 226.49 & 194.70 & 176.10 & 162.91 & 152.68 & 144.31 & 175.64 & 204.31 & 225.89 & 244.18 & 260.55 & 275.65 \\
\hline 664 & 226.72 & 194.90 & 176.29 & 163.08 & 152.83 & 144.46 & 175.72 & 204.41 & 226.00 & 244.30 & 260.68 & 275.78 \\
\hline 665 & 226.92 & 195.07 & 176.44 & 163.22 & 152.96 & 144.58 & 175.84 & 204.55 & 226.15 & 244.46 & 260.85 & 275.96 \\
\hline 666 & 227.15 & 195.27 & 176.62 & 163.38 & 153.12 & 144.73 & 175.92 & 204.64 & 226.25 & 244.58 & 260.97 & 276.09 \\
\hline 667 & 227.48 & 195.54 & 176.84 & 163.61 & 153.35 & 144.94 & 175.93 & 204.66 & 226.30 & 244.60 & 260.98 & 276.11 \\
\hline 668 & 227.78 & 195.82 & 177.10 & 163.85 & 153.57 & 145.13 & 175.96 & 204.68 & 226.31 & 244.62 & 260.99 & 276.16 \\
\hline 669 & 228.13 & 196.09 & 177.38 & 164.08 & 153.81 & 145.36 & 175.95 & 204.70 & 226.29 & 244.63 & 260.97 & 276.15 \\
\hline 670 & 228.51 & 196.40 & 177.60 & 164.32 & 154.01 & 145.58 & 175.92 & 204.68 & 226.35 & 244.65 & 261.02 & 276.13 \\
\hline 671 & 228.79 & 196.68 & 177.89 & 164.57 & 154.23 & 145.78 & 175.97 & 204.70 & 226.31 & 244.64 & 261.04 & 276.17 \\
\hline 672 & 229.19 & 197.02 & 178.21 & 164.85 & 154.50 & 146.04 & 175.92 & 204.65 & 226.26 & 244.58 & 260.98 & 276.10 \\
\hline 673 & 229.30 & 197.11 & 178.29 & 164.93 & 154.57 & 146.10 & 176.10 & 204.86 & 226.49 & 244.83 & 261.25 & 276.38 \\
\hline 674 & 229.64 & 197.40 & 178.55 & 165.17 & 154.80 & 146.32 & 176.10 & 204.86 & 226.49 & 244.84 & 261.25 & 276.39 \\
\hline 675 & 230.00 & 197.71 & 178.83 & 165.43 & 155.04 & 146.55 & 176.09 & 204.84 & 226.47 & & 261.23 & 276.36 \\
\hline 676 & 230.33 & 198.00 & 179.09 & 165.67 & 155.26 & 146.76 & 176.10 & 204.85 & 226.48 & 244.82 & 261.24 & 276.37 \\
\hline 677 & 230.63 & 198.25 & 179.32 & 165.88 & 155.46 & 146.95 & 176.13 & 204.89 & 226.52 & 244.87 & 261.29 & 276.43 \\
\hline 678 & 230.85 & 198.45 & 179.49 & 166.04 & 155.61 & 147.09 & 176.22 & 204.99 & 226.64 & 245.00 & 261.42 & 276.57 \\
\hline 679 & 231.21 & 198.76 & 179.77 & 166.30 & 155.86 & 147.32 & 176.20 & 204.97 & 226.62 & 244.97 & 261.39 & 276.54 \\
\hline 680 & 231.52 & 199.02 & 180.01 & 166.52 & 156.06 & 147.52 & 176.23 & 205.00 & 226.65 & 245.01 & 261.43 & 276.58 \\
\hline 681 & 231.75 & 199.22 & 180.19 & 166.69 & 156.22 & 147.66 & 176.31 & 205.10 & 226.76 & 245.12 & 261.55 & 276.71 \\
\hline 682 & 232.09 & 199.52 & 180.46 & & & & 176.31 & & 226.75 & & & \\
\hline 683 & 232.27 & 199.67 & 180.60 & 167.07 & 156.57 & 148.00 & 176.43 & 205.24 & 226.91 & 245.29 & 261.73 & 276.90 \\
\hline 684 & 232.81 & 200.13 & 181.02 & 167.45 & 156.93 & & 176.28 & & 226.72 & & 261.51 & 276.67 \\
\hline 685 & 232.97 & 200.27 & 181.14 & 167.57 & 157.04 & 148.44 & 176.42 & 205.22 & 226.89 & 245.27 & 261.71 & 276.87 \\
\hline 686 & 233.28 & 200.54 & 181.38 & 167.79 & 157.25 & 148.64 & 176.44 & 205.25 & 226.92 & 245.30 & 261.75 & 276.91 \\
\hline 687 & 233.61 & 200.82 & 181.64 & 168.03 & 157.47 & 148.85 & 176.45 & 205.26 & 226.94 & 245.32 & 261.76 & 276.93 \\
\hline 688 & 233.84 & 201.02 & 181.82 & 168.20 & 157.63 & 149.00 & 176.53 & 205.35 & 227.04 & 245.42 & 261.88 & 277.05 \\
\hline 689 & 234.22 & 201.34 & 182.11 & 168.47 & 157.88 & 149.24 & 176.50 & 205.32 & 227.00 & 245.39 & 261.84 & 277.01 \\
\hline 690 & 234.48 & 201.57 & 182.32 & 168.66 & 158.06 & 149.41 & 176.56 & 205.39 & 227.08 & 245.47 & 261.92 & 277.10 \\
\hline 691 & 234.86 & 201.90 & 182.61 & 168.93 & 158.32 & 149.65 & 176.53 & 205.35 & 227.04 & 245.43 & 261.88 & 277.05 \\
\hline 692 & 235.07 & 202.08 & 182.78 & 169.08 & 158.46 & 149.78 & 176.63 & 205.46 & 227.16 & 245.56 & 262.02 & 277.20 \\
\hline 693 & 235.33 & 202.30 & 182.98 & 169.27 & 158.64 & 149.95 & 176.69 & 205.53 & 227.24 & 245.64 & 262.11 & 277.30 \\
\hline 694 & 235.68 & 202.60 & 183.25 & 169.52 & 158.87 & 150.17 & 176.68 & 205.53 & 227.23 & 245.63 & 262.10 & 277.29 \\
\hline
\end{tabular}


Table B.1: Simulation Data for Picking Times (Medium Rate) - continued.

\begin{tabular}{|c|c|c|c|c|c|c|c|c|c|c|c|c|}
\hline \multirow{2}{*}{$\begin{array}{r}\text { Batch } \\
\text { Size }\end{array}$} & \multicolumn{6}{|c|}{ Picking Time (minutes) } & \multicolumn{6}{|c|}{ Picking Rate (orders/hr) } \\
\hline & 20 & 40 & 60 & 80 & 100 & 120 & 20 & 40 & 60 & 80 & 100 & 120 \\
\hline 695 & 236.04 & 202.91 & 183.53 & 169.78 & 159.11 & 150.40 & 176.67 & 205.51 & 227.22 & 245.62 & 262.08 & 277.27 \\
\hline 696 & 236.29 & 203.12 & 183.72 & 169.95 & 159.28 & 150.55 & 176.74 & 205.59 & 227.30 & 245.71 & 262.18 & 277.38 \\
\hline 697 & 236.64 & 203.42 & 183.99 & 170.21 & 159.51 & 150.78 & 176.73 & 205.58 & 227.29 & 245.70 & 262.17 & 277.36 \\
\hline 698 & 236.83 & 203.59 & 184.15 & 170.35 & 159.65 & 150.90 & 176.83 & 205.71 & 227.43 & 245.85 & 262.33 & 277.53 \\
\hline 699 & 237.12 & 203.83 & 184.37 & 170.55 & 159.84 & 151.08 & 176.87 & 205.76 & 227.48 & 245.91 & 262.39 & 277.60 \\
\hline 700 & 237.46 & 204.13 & 184.63 & 170.80 & 160.07 & 151.30 & 176.87 & 205.75 & 227.48 & 245.90 & 262.38 & 277.59 \\
\hline 701 & 237.87 & 204.48 & 184.95 & 171.09 & 160.34 & 151.56 & 176.82 & 205.69 & 227.41 & 245.83 & 262.31 & 277.51 \\
\hline 702 & 238.19 & 204.76 & 185.20 & 171.33 & 160.56 & 151.77 & 176.83 & 205.71 & 227.43 & 245.85 & 262.33 & 277.53 \\
\hline 703 & 238.42 & 204.96 & 185.38 & 171.49 & 160.72 & 151.92 & 176.91 & 205.80 & 227.53 & 245.96 & 262.45 & 277.65 \\
\hline 704 & 238.60 & 205.11 & 185.52 & 171.62 & 160.83 & 152.03 & 177.04 & 205.94 & 227.69 & 246.13 & 262.63 & 277.85 \\
\hline 705 & 238.89 & 205.36 & 185.75 & 171.83 & 161.03 & 152.21 & 177.07 & 205.98 & 227.73 & 246.18 & 262.68 & 277.90 \\
\hline 706 & 239.42 & 205.82 & 186.16 & 172.21 & 161.39 & 152.55 & 176.92 & 205.81 & 227.55 & 245.98 & 262.46 & 277.67 \\
\hline 707 & 239.48 & 205.87 & 186.21 & 172.25 & 161.43 & 152.59 & 177.13 & 206.05 & 227.81 & 246.26 & 262.77 & 278.00 \\
\hline 708 & 239.93 & 206.25 & 186.55 & 172.57 & 161.73 & 152.87 & 177.05 & 205.96 & 227.71 & 246.16 & 262.66 & 277.88 \\
\hline 709 & 240.10 & 206.40 & 186.69 & 172.70 & 161.85 & 152.98 & 177.18 & 206.11 & 227.87 & 246.33 & 262.84 & 278.07 \\
\hline 710 & 240.54 & 206.78 & 187.03 & 173.01 & 162.14 & 153.26 & 177.10 & 206.02 & 227.78 & 246.22 & 262.73 & 277.95 \\
\hline 711 & 240.71 & 206.92 & 187.16 & 173.13 & 162.26 & 153.37 & 177.23 & 206.17 & 227.94 & 246.40 & 262.92 & 278.15 \\
\hline 712 & 241.20 & 207.34 & 187.54 & 173.49 & 162.59 & 153.69 & 177.11 & 206.03 & 227.79 & 246.24 & 262.75 & 277.97 \\
\hline 713 & 241.38 & 207.50 & 187.68 & 173.62 & 162.71 & 153.80 & 177.23 & 206.17 & 227.94 & 246.41 & 262.92 & 278.16 \\
\hline 714 & 241.67 & 207.74 & 187.90 & 173.82 & 162.90 & 153.98 & 177.27 & 206.21 & 227.99 & 246.46 & 262.98 & 278.21 \\
\hline 715 & 241.87 & 207.92 & 188.06 & 173.97 & 163.04 & 154.11 & 177.37 & 206.33 & 228.11 & 246.59 & 263.12 & 278.36 \\
\hline 716 & 242.25 & 208.25 & 188.36 & 174.25 & 163.30 & 154.36 & 177.33 & 206.29 & 228.07 & 246.55 & 263.07 & 278.32 \\
\hline 717 & 242.72 & 208.65 & 188.72 & 174.58 & 163.62 & 154.65 & 177.24 & 206.18 & 227.95 & & & \\
\hline 718 & 242.93 & 208.84 & 188.89 & 174.74 & 163.76 & 154.79 & 177.33 & 206.29 & 228.07 & 246.54 & 263.07 & 278.31 \\
\hline 719 & 243.16 & 209.03 & 189.06 & 174.90 & 163.91 & 154.93 & 177.41 & 206.38 & 228.18 & 246.66 & 263.19 & 278.44 \\
\hline 720 & 243.56 & 209.37 & 189.37 & 175.19 & 164.18 & 155.19 & 177.37 & 206.33 & 228.12 & 246.60 & 263.13 & 278.37 \\
\hline 721 & 243.89 & 209.66 & 189.63 & 175.43 & 164.40 & 155.40 & 177.37 & 206.34 & 228.12 & 246.60 & 263.13 & 278.38 \\
\hline 722 & 244.22 & 209.94 & 189.89 & 175.66 & 164.63 & 155.61 & 177.38 & 206.34 & 228.13 & & 263.14 & \\
\hline 723 & 244.49 & 210.17 & 190.10 & 175.85 & 164.81 & 155.78 & 177.43 & 206.40 & 228.20 & 246.68 & 263.22 & 278.47 \\
\hline 724 & 244.66 & & & 175.98 & & & 177.55 & & 228.36 & & & 278.66 \\
\hline 725 & 245.04 & 210.65 & 190.53 & 176.25 & 165.18 & 156.13 & 177.52 & 206.51 & 228.31 & 246.81 & 263.35 & 278.61 \\
\hline 726 & 245.20 & & & & & & 177.65 & 206.66 & & & & \\
\hline 727 & 245.62 & 211.15 & 190.98 & 176.67 & 165.57 & 156.50 & 177.59 & 206.59 & 228.40 & 246.90 & 263.45 & 278.72 \\
\hline 728 & 245.88 & 211.37 & 191.18 & 176.85 & 165.74 & 156.67 & 177.65 & 206.66 & 228.48 & 246.98 & 263.54 & 278.81 \\
\hline 729 & 246.24 & 211.67 & 191.46 & 177.11 & 165.99 & 156.89 & 177.63 & 206.64 & 228.46 & 246.96 & 263.52 & 278.79 \\
\hline 730 & 246.46 & 211.86 & 191.63 & 177.27 & 166.13 & 157.03 & 177.72 & 206.74 & 228.57 & 247.08 & 263.64 & 278.92 \\
\hline 731 & 246.76 & 212.13 & 191.87 & 177.49 & 166.34 & 157.23 & 177.74 & 206.76 & 228.60 & 247.11 & 263.68 & 278.96 \\
\hline 732 & 247.13 & 212.45 & 192.15 & 177.76 & 166.59 & 157.47 & 177.72 & 206.74 & 228.57 & 247.08 & 263.64 & 278.92 \\
\hline 733 & 247.50 & 212.76 & 192.44 & 178.02 & 166.84 & 157.70 & 177.69 & 206.71 & 228.54 & 247.05 & 263.61 & 278.88 \\
\hline 734 & 247.69 & 212.93 & 192.59 & 178.16 & 166.97 & 157.82 & 177.80 & 206.83 & 228.67 & 247.19 & 263.77 & 279.05 \\
\hline 735 & 248.07 & 213.25 & 192.88 & 178.43 & 167.22 & 158.06 & 177.77 & 206.80 & 228.63 & 247.15 & 263.72 & 279.00 \\
\hline 736 & 248.33 & 213.48 & 193.09 & 178.62 & 167.40 & 158.23 & 177.83 & 206.86 & 228.71 & 247.23 & 263.80 & 279.09 \\
\hline
\end{tabular}


Table B.1: Simulation Data for Picking Times (Medium Rate) - continued.

\begin{tabular}{r|rrrrrr|rrrrrr}
\hline Batch & \multicolumn{7}{|c|}{ Picking Time (minutes) } & \multicolumn{5}{c}{ Picking Rate (orders/hr) } \\
\cline { 2 - 14 } Size & 20 & 40 & 60 & 80 & 100 & 120 & 20 & 40 & 60 & 80 & 100 & 120 \\
\hline 737 & 248.59 & 213.70 & 193.29 & 178.80 & 167.57 & 158.39 & 177.88 & 206.93 & 228.78 & 247.31 & 263.89 & 279.18 \\
738 & 248.84 & 213.91 & 193.48 & 178.99 & 167.74 & 158.55 & 177.94 & 207.00 & 228.86 & 247.39 & 263.98 & 279.27 \\
739 & 249.27 & 214.28 & 193.81 & 179.29 & 168.03 & 158.83 & 177.88 & 206.93 & 228.78 & 247.31 & 263.88 & 279.17 \\
740 & 249.45 & 214.44 & 193.96 & 179.43 & 168.15 & 158.94 & 177.99 & 207.05 & 228.92 & 247.46 & 264.04 & 279.34 \\
741 & 249.81 & 214.74 & 194.23 & 179.68 & 168.39 & 159.17 & 177.98 & 207.04 & 228.90 & 247.44 & 264.03 & 279.32 \\
742 & 249.96 & 214.87 & 194.35 & 179.79 & 168.49 & 159.27 & 178.11 & 207.19 & 229.07 & 247.62 & 264.22 & 279.53 \\
743 & 250.36 & 215.22 & 194.66 & 180.08 & 168.77 & 159.52 & 178.06 & 207.14 & 229.01 & 247.56 & 264.15 & 279.46 \\
744 & 250.65 & 215.47 & 194.89 & 180.28 & 168.96 & 159.70 & 178.10 & 207.18 & 229.06 & 247.61 & 264.21 & 279.52 \\
745 & 251.00 & 215.77 & 195.16 & 180.54 & 169.20 & 159.93 & 178.09 & 207.17 & 229.04 & 247.59 & 264.19 & 279.50 \\
746 & 251.28 & 216.01 & 195.38 & 180.74 & 169.38 & 160.11 & 178.13 & 207.22 & 229.10 & 247.65 & 264.25 & 279.56 \\
747 & 251.50 & 216.20 & 195.55 & 180.90 & 169.54 & 160.25 & 178.21 & 207.31 & 229.20 & 247.76 & 264.37 & 279.69 \\
748 & 251.92 & 216.56 & 195.87 & 181.20 & 169.82 & 160.51 & 178.15 & 207.24 & 229.13 & 247.68 & 264.29 & 279.60 \\
749 & 252.16 & 216.76 & 196.06 & 181.37 & 169.98 & 160.67 & 178.22 & 207.32 & 229.22 & 247.78 & 264.39 & 279.71 \\
750 & 252.48 & 217.04 & 196.31 & 181.60 & 170.20 & 160.87 & 178.23 & 207.33 & 229.23 & 247.79 & 264.40 & 279.72 \\
\hline
\end{tabular}

Table B.2: Simulation Data for Picking Times (High Rate).

\begin{tabular}{|c|c|c|c|c|c|c|c|c|c|c|c|c|}
\hline \multirow{2}{*}{$\begin{array}{c}\text { Batch } \\
\text { Size }\end{array}$} & \multicolumn{6}{|c|}{ Picking Time (minutes) } & \multicolumn{6}{|c|}{ Picking Rate (orders/hr) } \\
\hline & 20 & 40 & 60 & 80 & 100 & 120 & 20 & 40 & 60 & 80 & 100 & 120 \\
\hline 1 & 1.29 & 1.11 & 1.01 & 0.93 & 0.87 & 0.82 & 46.35 & 53.92 & 59.61 & 64.44 & 68.76 & 72.74 \\
\hline 2 & 2.27 & 1.95 & 1.77 & 1.63 & 1.53 & 1.45 & 52.81 & 61.43 & 67.92 & 73.42 & 78.34 & 82.88 \\
\hline 3 & 2.92 & 2.51 & 2.27 & 2.10 & 1.97 & 1.86 & 61.65 & 71.71 & 79.29 & 85.71 & 91.45 & 96.75 \\
\hline 4 & 3.79 & 3.26 & 2.95 & 2.73 & 2.55 & 2.41 & 63.33 & 73.67 & 81.45 & 88.05 & 93.95 & 99.39 \\
\hline 5 & 4.37 & 3.75 & 3.40 & 3.14 & 2.94 & 2.78 & 68.69 & 79.91 & 88.34 & 95.50 & 101.90 & 107.81 \\
\hline 6 & 5.11 & 4.39 & 3.97 & 3.68 & 3.44 & 3.26 & 70.45 & 81.96 & 90.61 & 97.95 & 104.52 & 110.57 \\
\hline 7 & 5.57 & 4.79 & 4.33 & 4.01 & 3.75 & 3.55 & 75.40 & 87.71 & 96.97 & 104.83 & 111.86 & 118.34 \\
\hline 8 & 6.30 & 5.41 & 4.89 & 4.53 & 4.24 & 4.01 & 76.25 & 88.70 & 98.07 & 106.01 & 113.12 & 119.67 \\
\hline 9 & 6.94 & 5.97 & 5.40 & 4.99 & 4.68 & 4.42 & 77.82 & 90.52 & 100.08 & 108.19 & 115.44 & 122.13 \\
\hline 10 & 7.38 & 6.35 & 5.74 & 5.31 & 4.98 & 4.70 & 81.27 & 94.54 & 104.52 & 112.99 & 120.56 & 127.55 \\
\hline 11 & 7.86 & 6.76 & 6.11 & 5.66 & 5.30 & 5.01 & 83.94 & 97.65 & 107.96 & 116.70 & 124.52 & 131.74 \\
\hline 12 & 8.25 & 7.09 & 6.41 & 5.93 & 5.56 & 5.25 & 87.32 & 101.57 & 112.30 & 121.40 & 129.53 & 137.04 \\
\hline 13 & 9.02 & 7.75 & 7.01 & 6.49 & 6.08 & 5.75 & 86.51 & 100.63 & 111.26 & 120.27 & 128.33 & 135.77 \\
\hline 14 & 9.40 & 8.08 & 7.31 & 6.76 & 6.34 & 5.99 & 89.36 & 103.95 & 114.93 & 124.23 & 132.56 & 140.24 \\
\hline 15 & 9.96 & 8.57 & 7.75 & 7.17 & 6.72 & 6.35 & 90.32 & 105.07 & 116.16 & 125.57 & 133.99 & 141.75 \\
\hline 16 & 10.50 & 9.02 & 8.16 & 7.55 & 7.08 & 6.69 & 91.45 & 106.38 & 117.62 & 127.14 & 135.66 & 143.52 \\
\hline 17 & 10.95 & 9.41 & 8.51 & 7.87 & 7.38 & 6.98 & 93.17 & 108.39 & 119.83 & 129.54 & 138.22 & 146.23 \\
\hline 18 & 11.52 & 9.90 & 8.95 & 8.28 & 7.76 & 7.34 & 93.78 & 109.09 & 120.61 & 130.38 & 139.12 & 147.18 \\
\hline 19 & 11.94 & 10.27 & 9.29 & 8.59 & 8.05 & 7.61 & 95.46 & 111.04 & 122.77 & 132.71 & 141.61 & 149.81 \\
\hline 20 & 12.51 & 10.75 & 9.73 & 9.00 & 8.43 & 7.97 & 95.94 & 111.61 & 123.39 & 133.38 & 142.33 & 150.57 \\
\hline 21 & 12.85 & 11.04 & 9.99 & 9.24 & 8.66 & 8.18 & 98.09 & 114.11 & 126.16 & 136.37 & 145.52 & 153.95 \\
\hline 22 & 13.28 & 11.42 & 10.33 & 9.55 & 8.95 & 8.46 & 99.40 & 115.63 & 127.84 & 138.19 & 147.46 & 156.00 \\
\hline
\end{tabular}


Table B.2: Simulation Data for Picking Times (High Rate)—continued.

\begin{tabular}{|c|c|c|c|c|c|c|c|c|c|c|c|c|}
\hline \multirow{2}{*}{$\begin{array}{c}\text { Batch } \\
\text { Size }\end{array}$} & \multicolumn{6}{|c|}{ Picking Time (minutes) } & \multicolumn{6}{|c|}{ Picking Rate (orders/hr) } \\
\hline & 20 & 40 & 60 & 80 & 100 & 120 & 20 & 40 & 60 & 80 & 100 & 120 \\
\hline 23 & 13.57 & 11.67 & 10.55 & 9.76 & 9.15 & 8.65 & 101.68 & 118.28 & 130.77 & 141.36 & 150.84 & 159.58 \\
\hline 24 & 14.22 & 12.22 & 11.05 & 10.22 & 9.58 & 9.06 & 101.30 & 117.84 & 130.28 & 140.83 & 150.27 & 158.98 \\
\hline 25 & 14.65 & 12.59 & 11.39 & 10.54 & 9.88 & 9.33 & 102.39 & 119.11 & 131.69 & 142.35 & 151.90 & 160.70 \\
\hline 26 & 15.03 & 12.92 & 11.69 & 10.81 & 10.13 & 9.58 & 103.76 & 120.70 & 133.45 & 144.25 & 153.92 & 162.84 \\
\hline 27 & 15.46 & 13.29 & 12.02 & 11.12 & 10.42 & 9.85 & 104.78 & 121.89 & 134.76 & 145.68 & 155.44 & 164.45 \\
\hline 28 & 15.82 & 13.60 & 12.30 & 11.38 & 10.66 & 10.08 & 106.23 & 123.57 & 136.62 & 147.69 & 157.59 & 166.72 \\
\hline 29 & 16.45 & 14.14 & 12.79 & 11.83 & 11.09 & 10.48 & 105.77 & 123.04 & 136.03 & 147.05 & 156.91 & 166.00 \\
\hline 30 & 16.89 & 14.52 & 13.14 & 12.15 & 11.39 & 10.76 & 106.54 & 123.94 & 137.03 & 148.13 & 158.05 & 167.21 \\
\hline 31 & 17.13 & 14.72 & 13.32 & 12.32 & 11.55 & 10.91 & 108.60 & 126.33 & 139.67 & 150.99 & 161.11 & 170.44 \\
\hline 32 & 17.51 & 15.05 & 13.62 & 12.60 & 11.81 & 11.16 & 109.63 & 127.53 & 141.00 & 152.42 & 162.64 & 172.06 \\
\hline 33 & 17.95 & 15.43 & 13.96 & 12.91 & 12.10 & 11.44 & 110.31 & 128.32 & 141.87 & 153.36 & 163.64 & 173.12 \\
\hline 34 & 18.34 & 15.77 & 14.26 & 13.19 & 12.37 & 11.69 & 111.21 & 129.36 & 143.02 & 154.61 & 164.97 & 174.53 \\
\hline 35 & 18.80 & 16.16 & 14.61 & 13.52 & 12.67 & 11.98 & 111.72 & 129.96 & 143.69 & 155.33 & 165.74 & 175.34 \\
\hline 36 & 19.24 & 16.54 & 14.96 & 13.84 & 12.97 & 12.26 & 112.27 & 130.61 & 144.40 & 156.09 & 166.56 & 176.21 \\
\hline 37 & 19.57 & 16.82 & 15.21 & 14.07 & 13.19 & 12.47 & 113.45 & 131.98 & 145.92 & 157.73 & 168.31 & 178.06 \\
\hline 38 & 20.16 & 17.33 & 15.67 & 14.50 & 13.59 & 12.84 & 113.12 & 131.59 & 145.49 & 157.27 & 167.81 & 177.53 \\
\hline 39 & 20.38 & 17.52 & 15.85 & 14.66 & 13.74 & 12.99 & 114.79 & 133.54 & 147.64 & 159.59 & 170.29 & 180.16 \\
\hline 40 & 20.73 & 17.82 & 16.12 & 14.91 & 13.98 & 13.21 & 115.75 & 134.65 & 148.87 & 160.92 & 171.71 & 181.66 \\
\hline 41 & 21.18 & 18.20 & 16.47 & 15.23 & 14.27 & 13.49 & 116.17 & 135.14 & 149.41 & 161.51 & 172.33 & 182.32 \\
\hline 42 & 21.57 & 18.54 & 16.77 & 15.52 & 14.54 & 13.75 & 116.82 & 135.89 & 150.24 & 162.41 & 173.30 & 183.34 \\
\hline 43 & 21.91 & 18.83 & 17.03 & 15.76 & 14.77 & 13.96 & 117.77 & 137.00 & 151.46 & 163.73 & 174.71 & 184.83 \\
\hline 44 & 22.20 & 19.08 & 17.26 & 15.97 & 14.96 & 14.14 & 118.93 & 138.34 & 152.95 & 165.34 & 176.43 & 186.65 \\
\hline 45 & 22.79 & 19.59 & 17.72 & 16.39 & 15.36 & 14.52 & 118.46 & 137.80 & 152.35 & 164.69 & 175.73 & 185.91 \\
\hline 46 & 23.18 & 19.92 & 18.02 & 16.67 & 15.62 & 14.77 & 119.09 & 138.54 & 153.17 & 165.57 & 176.67 & 186.91 \\
\hline 47 & 23.47 & 20.17 & 18.25 & 16.88 & 15.82 & 14.95 & 120.17 & 139.80 & 154.56 & 167.08 & 178.28 & 188.61 \\
\hline 48 & 23.91 & 20.55 & 18.59 & 17.20 & 16.12 & 15.24 & 120.45 & 140.12 & 154.91 & 167.46 & 178.68 & 189.04 \\
\hline 49 & 24.34 & 20.93 & 18.93 & 17.51 & 16.41 & 15.51 & 120.77 & 140.49 & 155.32 & 167.91 & 179.16 & 189.54 \\
\hline 50 & 24.62 & 21.16 & 19.14 & 17.70 & 16.59 & 15.68 & 121.88 & 141.78 & 156.75 & 169.44 & 180.80 & 191.28 \\
\hline 51 & 25.07 & 21.55 & 19.49 & 18.03 & 16.90 & 15.97 & 122.06 & 141.99 & 156.98 & 169.70 & 181.07 & 191.57 \\
\hline 52 & 25.39 & 21.83 & 19.74 & 18.26 & 17.12 & 16.18 & 122.88 & 142.94 & 158.04 & 170.84 & 182.29 & 192.85 \\
\hline 53 & 25.86 & 22.23 & 20.11 & 18.60 & 17.43 & 16.48 & 122.96 & 143.04 & 158.14 & 170.95 & 182.41 & 192.98 \\
\hline 54 & 26.19 & 22.51 & 20.36 & 18.84 & 17.65 & 16.69 & 123.73 & 143.93 & 159.13 & 172.02 & 183.55 & 194.18 \\
\hline 55 & 26.49 & 22.77 & 20.59 & 19.05 & 17.85 & 16.88 & 124.59 & 144.93 & 160.23 & 173.21 & 184.82 & 195.53 \\
\hline 56 & 26.97 & 23.18 & 20.97 & 19.40 & 18.18 & 17.18 & 124.58 & 144.92 & 160.23 & 173.20 & 184.81 & 195.52 \\
\hline 57 & 27.27 & 23.44 & 21.20 & 19.62 & 18.38 & 17.38 & 125.40 & 145.88 & 161.29 & 174.35 & 186.04 & 196.82 \\
\hline 58 & 27.49 & 23.63 & 21.38 & 19.77 & 18.53 & 17.52 & 126.59 & 147.25 & 162.80 & 175.99 & 187.79 & 198.67 \\
\hline 59 & 27.78 & 23.88 & 21.60 & 19.98 & 18.73 & 17.70 & 127.42 & 148.22 & 163.87 & 177.15 & 189.02 & 199.97 \\
\hline 60 & 28.36 & 24.38 & 22.05 & 20.40 & 19.12 & 18.07 & 126.95 & 147.68 & 163.28 & 176.50 & 188.33 & 199.25 \\
\hline 61 & 28.80 & 24.76 & 22.40 & 20.72 & 19.42 & 18.35 & 127.06 & 147.81 & 163.42 & 176.66 & 188.50 & 199.42 \\
\hline 62 & 29.13 & 25.04 & 22.65 & 20.95 & 19.64 & 18.56 & 127.69 & 148.54 & 164.23 & 177.53 & 189.43 & 200.41 \\
\hline 63 & 29.49 & 25.35 & 22.93 & 21.21 & 19.88 & 18.79 & 128.20 & 149.13 & 164.88 & 178.23 & 190.18 & 201.20 \\
\hline 64 & 29.82 & 25.64 & 23.19 & 21.45 & 20.10 & 19.00 & 128.76 & 149.78 & 165.60 & 179.01 & 191.01 & 202.08 \\
\hline
\end{tabular}


Table B.2: Simulation Data for Picking Times (High Rate)—continued.

\begin{tabular}{|c|c|c|c|c|c|c|c|c|c|c|c|c|}
\hline \multirow{2}{*}{$\begin{array}{c}\text { Batch } \\
\text { Size }\end{array}$} & \multicolumn{6}{|c|}{ Picking Time (minutes) } & \multicolumn{6}{|c|}{ Picking Rate (orders/hr) } \\
\hline & 20 & 40 & 60 & 80 & 100 & 120 & 20 & 40 & 60 & 80 & 100 & 120 \\
\hline 65 & 30.13 & 25.90 & 23.43 & 21.67 & 20.31 & 19.20 & 129.43 & 150.56 & 166.46 & 179.94 & 192.00 & 203.13 \\
\hline 66 & 30.52 & 26.23 & 23.73 & 21.95 & 20.57 & 19.45 & 129.76 & 150.94 & 166.88 & 180.40 & 192.49 & 203.65 \\
\hline 67 & 30.90 & 26.56 & 24.03 & 22.23 & 20.83 & 19.69 & 130.10 & 151.34 & 167.32 & 180.87 & 193.00 & 204.18 \\
\hline 68 & 31.25 & 26.87 & 24.30 & 22.48 & 21.07 & 19.91 & 130.55 & 151.87 & 167.91 & 181.50 & 193.67 & 204.89 \\
\hline 69 & 31.61 & 27.17 & 24.58 & 22.74 & 21.31 & 20.14 & 130.97 & 152.35 & 168.44 & 182.08 & 194.29 & 205.54 \\
\hline 70 & 32.12 & 27.61 & 24.97 & 23.10 & 21.65 & 20.47 & 130.76 & 152.11 & 168.18 & 181.80 & 193.99 & 205.23 \\
\hline 71 & 32.34 & 27.80 & 25.14 & 23.26 & 21.80 & 20.60 & 131.74 & 153.25 & 169.43 & 183.15 & 195.43 & 206.75 \\
\hline 72 & 32.60 & 28.03 & 25.35 & 23.45 & 21.98 & 20.77 & 132.50 & 154.14 & 170.41 & 184.21 & 196.56 & 207.95 \\
\hline 73 & 32.98 & 28.35 & 25.64 & 23.72 & 22.23 & 21.01 & 132.82 & 154.51 & 170.83 & 184.66 & 197.04 & 208.46 \\
\hline 74 & 33.31 & 28.63 & 25.90 & 23.96 & 22.45 & 21.22 & 133.30 & 155.06 & 171.44 & 185.32 & 197.75 & 209.21 \\
\hline 75 & 33.55 & 28.84 & 26.09 & 24.13 & 22.62 & 21.38 & 134.11 & 156.01 & 172.49 & 186.46 & 198.95 & 210.48 \\
\hline 76 & 34.02 & 29.24 & 26.45 & 24.47 & 22.93 & 21.67 & 134.05 & 155.94 & 172.41 & 186.37 & 198.87 & 210.39 \\
\hline 77 & 34.29 & 29.48 & 26.67 & 24.67 & 23.12 & 21.85 & 134.71 & 156.71 & 173.26 & 187.29 & 199.85 & 211.43 \\
\hline 78 & 34.74 & 29.87 & 27.01 & 24.99 & 23.42 & 22.14 & 134.70 & 156.70 & 173.24 & 187.27 & 199.83 & 211.41 \\
\hline 79 & 35.11 & 30.18 & 27.30 & 25.25 & 23.67 & 22.37 & 135.01 & 157.06 & 173.65 & 187.71 & 200.29 & 211.90 \\
\hline 80 & 35.55 & 30.56 & 27.64 & 25.57 & 23.97 & 22.65 & 135.01 & 157.06 & 173.64 & 187.71 & 200.29 & 211.90 \\
\hline 81 & 35.74 & 30.72 & 27.79 & 25.71 & 24.09 & 22.77 & 135.98 & 158.19 & 174.89 & 189.06 & 201.73 & 213.42 \\
\hline 82 & 36.08 & 31.02 & 28.06 & 25.95 & 24.32 & 22.99 & 136.35 & 158.62 & 175.37 & 189.57 & 202.28 & 214.00 \\
\hline 83 & 36.46 & 31.34 & 28.35 & 26.22 & 24.57 & 23.23 & 136.60 & 158.91 & 175.69 & 189.92 & 202.65 & 214.39 \\
\hline 84 & 36.85 & 31.68 & 28.65 & 26.50 & 24.84 & 23.48 & 136.78 & 159.11 & 175.91 & 190.16 & 202.90 & 214.66 \\
\hline 85 & 37.28 & 32.04 & 28.98 & 26.81 & 25.13 & 23.75 & 136.81 & 159.15 & 175.96 & 190.21 & 202.96 & 214.72 \\
\hline 86 & 37.51 & 32.24 & 29.16 & 26.98 & 25.28 & 23.90 & 137.57 & 160.03 & 176.93 & 191.26 & 204.08 & 215.91 \\
\hline 87 & 37.93 & 32.61 & 29.49 & 27.28 & 25.57 & 24.17 & 137.63 & 160.10 & 177.00 & 191.34 & 204.17 & 216.00 \\
\hline 88 & 38.21 & 32.85 & 29.71 & 27.48 & 25.76 & 24.35 & 138.18 & 160.74 & 177.71 & 192.11 & 204.99 & 216.86 \\
\hline 89 & 38.55 & 33.14 & 29.97 & 27.73 & 25.99 & 24.56 & 138.52 & 161.14 & 178.15 & 192.58 & 205.49 & 217.40 \\
\hline 90 & 38.84 & 33.39 & 30.20 & 27.94 & 26.18 & 24.75 & 139.03 & 161.73 & 178.81 & 193.29 & 206.25 & 218.20 \\
\hline 91 & 39.19 & 33.69 & 30.47 & 28.19 & 26.42 & 24.97 & 139.33 & 162.08 & 179.19 & 193.71 & 206.69 & 218.67 \\
\hline 92 & 39.52 & 33.98 & 30.73 & 28.43 & 26.64 & 25.18 & 139.66 & 162.47 & 179.62 & 194.17 & 207.19 & 219.19 \\
\hline 93 & 39.90 & 34.30 & 31.02 & 28.70 & 26.89 & 25.42 & 139.86 & 162.69 & 179.87 & 194.44 & 207.47 & 219.50 \\
\hline 94 & 40.17 & 34.53 & 31.23 & 28.89 & 27.08 & 25.59 & 140.42 & 163.34 & 180.59 & 195.22 & 208.31 & 220.38 \\
\hline 95 & 40.67 & 34.96 & 31.62 & 29.25 & 27.42 & 25.91 & 140.15 & 163.03 & 180.25 & 194.85 & 207.91 & 219.96 \\
\hline 96 & 40.88 & 35.14 & 31.78 & 29.40 & 27.56 & 26.05 & 140.91 & 163.91 & 181.22 & 195.90 & 209.03 & 221.14 \\
\hline 97 & 41.34 & 35.54 & 32.14 & 29.73 & 27.87 & 26.34 & 140.79 & 163.78 & 181.07 & 195.74 & 208.86 & 220.96 \\
\hline 98 & 41.50 & 35.68 & 32.27 & 29.85 & 27.97 & 26.44 & 141.69 & 164.82 & 182.23 & 196.98 & 210.19 & 222.37 \\
\hline 99 & 41.93 & 36.05 & 32.60 & 30.16 & 28.27 & 26.72 & 141.66 & 164.79 & 182.19 & 196.95 & 210.15 & 222.33 \\
\hline 100 & 42.34 & 36.40 & 32.92 & 30.46 & 28.54 & 26.98 & 141.70 & 164.84 & 182.24 & 197.01 & 210.21 & 222.39 \\
\hline 101 & 42.46 & 36.50 & 33.01 & 30.54 & 28.62 & 27.05 & 142.74 & 166.05 & 183.58 & 198.45 & 211.75 & 224.02 \\
\hline 102 & 43.04 & 37.00 & 33.47 & 30.96 & 29.01 & 27.43 & 142.19 & 165.40 & 182.87 & 197.68 & 210.93 & 223.15 \\
\hline 103 & 43.20 & 37.14 & 33.59 & 31.08 & 29.12 & 27.53 & 143.04 & 166.40 & 183.97 & 198.87 & 212.20 & 224.50 \\
\hline 104 & 43.48 & 37.38 & 33.81 & 31.28 & 29.31 & 27.71 & 143.51 & 166.94 & 184.57 & 199.52 & 212.89 & 225.23 \\
\hline 105 & 43.92 & 37.76 & 34.15 & 31.59 & 29.61 & 27.99 & 143.44 & 166.86 & 184.48 & 199.42 & 212.79 & 225.12 \\
\hline 106 & 44.14 & 37.94 & 34.32 & 31.75 & 29.75 & 28.12 & 144.10 & 167.63 & 185.33 & 200.34 & 213.77 & 226.16 \\
\hline
\end{tabular}


Table B.2: Simulation Data for Picking Times (High Rate)—continued.

\begin{tabular}{|c|c|c|c|c|c|c|c|c|c|c|c|c|}
\hline \multirow{2}{*}{$\begin{array}{c}\text { Batch } \\
\text { Size }\end{array}$} & \multicolumn{6}{|c|}{ Picking Time (minutes) } & \multicolumn{6}{|c|}{ Picking Rate (orders/hr) } \\
\hline & 20 & 40 & 60 & 80 & 100 & 120 & 20 & 40 & 60 & 80 & 100 & 120 \\
\hline 107 & 44.56 & 38.30 & 34.64 & 32.05 & 30.04 & 28.39 & 144.08 & 167.61 & 185.31 & 200.32 & 213.74 & 226.13 \\
\hline 108 & 44.85 & 38.55 & 34.87 & 32.26 & 30.23 & 28.57 & 144.50 & 168.09 & 185.84 & 200.89 & 214.36 & 226.78 \\
\hline 109 & 45.21 & 38.86 & 35.15 & 32.52 & 30.47 & 28.81 & 144.66 & 168.28 & 186.06 & 201.12 & 214.61 & 227.04 \\
\hline 110 & 45.64 & 39.24 & 35.49 & 32.83 & 30.77 & 29.08 & 144.60 & 168.21 & 185.98 & 201.04 & 214.52 & 226.95 \\
\hline 111 & 45.92 & 39.47 & 35.70 & 33.03 & 30.95 & 29.26 & 145.04 & 168.72 & 186.54 & 201.65 & 215.16 & 227.63 \\
\hline 112 & 46.12 & 39.65 & 35.86 & 33.18 & 31.09 & 29.39 & 145.69 & 169.48 & 187.38 & 202.56 & 216.13 & 228.66 \\
\hline 113 & 46.65 & 40.11 & 36.28 & 33.56 & 31.45 & 29.73 & 145.32 & 169.05 & 186.91 & 202.04 & 215.59 & 228.08 \\
\hline 114 & 46.92 & 40.34 & 36.48 & 33.75 & 31.63 & 29.90 & 145.77 & 169.57 & 187.48 & 202.66 & 216.25 & 228.78 \\
\hline 115 & 47.16 & 40.54 & 36.67 & 33.92 & 31.79 & 30.05 & 146.32 & 170.21 & 188.19 & 203.43 & 217.07 & 229.64 \\
\hline 116 & 47.60 & 40.92 & 37.01 & 34.24 & 32.09 & 30.33 & 146.23 & 170.10 & 188.06 & 203.30 & 216.92 & 229.49 \\
\hline 117 & 47.95 & 41.22 & 37.29 & 34.49 & 32.33 & 30.55 & 146.39 & 170.29 & 188.28 & 203.52 & 217.17 & 229.75 \\
\hline 118 & 48.20 & 41.43 & 37.48 & 34.67 & 32.49 & 30.71 & 146.89 & 170.88 & 188.92 & 204.23 & 217.92 & 230.54 \\
\hline 119 & 48.53 & 41.72 & 37.73 & 34.91 & 32.71 & 30.92 & 147.12 & 171.15 & 189.22 & 204.54 & 218.26 & 230.90 \\
\hline 120 & 48.71 & 41.88 & 37.88 & 35.04 & 32.84 & 31.04 & 147.80 & 171.93 & 190.09 & 205.48 & 219.26 & 231.96 \\
\hline 121 & 49.18 & 42.28 & 38.24 & 35.38 & 33.15 & 31.34 & 147.61 & 171.71 & 189.84 & 205.22 & 218.98 & 231.67 \\
\hline 122 & 49.56 & 42.60 & 38.53 & 35.65 & 33.41 & 31.58 & 147.71 & 171.82 & 189.97 & 205.35 & 219.12 & 231.82 \\
\hline 123 & 49.88 & 42.88 & 38.78 & 35.88 & 33.62 & 31.78 & 147.95 & 172.11 & 190.29 & 205.70 & 219.49 & 232.20 \\
\hline 124 & 50.04 & 43.02 & 38.91 & 35.99 & 33.73 & 31.89 & 148.67 & 172.95 & 191.21 & 206.70 & 220.56 & 233.34 \\
\hline 125 & 50.47 & 43.39 & 39.24 & 36.30 & 34.02 & 32.16 & 148.59 & 172.85 & 191.11 & 206.59 & 220.43 & 233.21 \\
\hline 126 & 50.81 & 43.68 & 39.51 & 36.55 & 34.25 & 32.38 & 148.78 & 173.07 & 191.34 & 206.84 & 220.71 & 233.49 \\
\hline 127 & 51.07 & 43.90 & 39.71 & 36.74 & 34.43 & 32.54 & 149.20 & 173.56 & 191.89 & 207.43 & 221.33 & 234.16 \\
\hline 128 & 51.36 & 44.15 & 39.94 & 36.94 & 34.62 & 32.73 & 149.52 & 173.94 & 192.31 & 207.88 & 221.82 & 234.67 \\
\hline 129 & 51.78 & 44.51 & 40.26 & 37.24 & 34.90 & 32.99 & 149.48 & 173.89 & 192.26 & 207.83 & 221.76 & 234.61 \\
\hline 130 & 52.03 & 44.73 & 40.45 & 37.42 & 35.07 & 33.15 & 149.92 & 174.40 & 192.81 & 208.43 & 222.40 & 235.29 \\
\hline 131 & 52.51 & 45.14 & 40.83 & 37.77 & 35.39 & 33.46 & 149.70 & 174.14 & 192.53 & 208.12 & 222.07 & 234.94 \\
\hline 132 & 52.68 & 45.28 & 40.96 & 37.89 & 35.51 & 33.57 & 150.35 & 174.89 & 193.36 & 209.02 & 223.03 & 235.96 \\
\hline 133 & 53.14 & 45.68 & 41.31 & 38.22 & 35.82 & 33.86 & 150.18 & 174.70 & 193.15 & 208.80 & 222.79 & 235.70 \\
\hline 134 & 53.29 & 45.81 & 41.44 & 38.33 & 35.92 & 33.96 & 150.87 & 175.50 & 194.03 & 209.75 & 223.81 & 236.78 \\
\hline 135 & 53.69 & 46.16 & 41.75 & 38.62 & 36.20 & 34.21 & 150.85 & 175.48 & 194.02 & 209.73 & 223.79 & 236.75 \\
\hline 136 & 54.03 & 46.44 & 42.01 & 38.86 & 36.42 & 34.42 & 151.04 & 175.70 & 194.25 & 209.98 & 224.06 & 237.04 \\
\hline 137 & 54.29 & 46.67 & 42.21 & 39.05 & 36.59 & 34.59 & 151.42 & 176.14 & 194.74 & 210.52 & 224.63 & 237.64 \\
\hline 138 & 54.72 & 47.04 & 42.55 & 39.36 & 36.89 & 34.87 & 151.30 & 176.01 & 194.59 & 210.36 & 224.46 & 237.46 \\
\hline 139 & 54.93 & 47.22 & 42.71 & 39.51 & 37.03 & 35.00 & 151.83 & 176.62 & 195.27 & 211.08 & 225.23 & 238.28 \\
\hline 140 & 55.32 & 47.55 & 43.01 & 39.79 & 37.29 & 35.25 & 151.85 & 176.64 & 195.30 & 211.11 & 225.27 & 238.32 \\
\hline 141 & 55.47 & 47.68 & 43.13 & 39.90 & 37.39 & 35.34 & 152.52 & 177.43 & 196.16 & 212.05 & 226.27 & 239.38 \\
\hline 142 & 55.85 & 48.01 & 43.42 & 40.17 & 37.65 & 35.59 & 152.55 & 177.46 & 196.20 & 212.09 & 226.31 & 239.43 \\
\hline 143 & 56.25 & 48.35 & 43.73 & 40.46 & 37.92 & 35.84 & 152.54 & 177.45 & 196.19 & 212.08 & 226.29 & 239.41 \\
\hline 144 & 56.53 & 48.60 & 43.95 & 40.66 & 38.11 & 36.02 & 152.84 & 177.79 & 196.57 & 212.49 & 226.73 & 239.87 \\
\hline 145 & 56.92 & 48.93 & 44.26 & 40.94 & 38.37 & 36.27 & 152.85 & 177.81 & 196.59 & 212.51 & 226.75 & 239.89 \\
\hline 146 & 57.22 & 49.19 & 44.49 & 41.16 & 38.57 & 36.46 & 153.08 & 178.08 & 196.88 & 212.83 & 227.10 & 240.25 \\
\hline 147 & 57.51 & 49.44 & 44.72 & 41.37 & 38.77 & 36.64 & 153.36 & 178.40 & 197.24 & 213.22 & 227.51 & 240.69 \\
\hline 148 & 57.81 & 49.70 & 44.95 & 41.58 & 38.97 & 36.83 & 153.61 & 178.69 & 197.56 & 213.56 & 227.88 & 241.08 \\
\hline
\end{tabular}


Table B.2: Simulation Data for Picking Times (High Rate)—continued.

\begin{tabular}{|c|c|c|c|c|c|c|c|c|c|c|c|c|}
\hline \multirow{2}{*}{$\begin{array}{c}\text { Batch } \\
\text { Size }\end{array}$} & \multicolumn{6}{|c|}{ Picking Time (minutes) } & \multicolumn{6}{|c|}{ Picking Rate (orders/hr) } \\
\hline & 20 & 40 & 60 & 80 & 100 & 120 & 20 & 40 & 60 & 80 & 100 & 120 \\
\hline 149 & 58.16 & 50.00 & 45.22 & 41.83 & 39.21 & 37.06 & 153.71 & 178.81 & 197.69 & 213.71 & 228.03 & 241.24 \\
\hline 150 & 58.36 & 50.17 & 45.38 & 41.98 & 39.34 & 37.18 & 154.22 & 179.40 & 198.35 & 214.41 & 228.78 & 242.04 \\
\hline 151 & 58.79 & 50.53 & 45.71 & 42.28 & 39.63 & 37.46 & 154.12 & 179.28 & 198.21 & 214.27 & 228.63 & 241.88 \\
\hline 152 & 59.16 & 50.86 & 46.00 & 42.56 & 39.88 & 37.70 & 154.15 & 179.32 & 198.25 & 214.31 & 228.67 & 241.92 \\
\hline 153 & 59.52 & 51.16 & 46.28 & 42.81 & 40.12 & 37.92 & 154.24 & 179.43 & 198.38 & 214.44 & 228.82 & 242.08 \\
\hline 154 & 59.80 & 51.41 & 46.50 & 43.02 & 40.31 & 38.11 & 154.50 & 179.73 & 198.71 & 214.81 & 229.20 & 242.49 \\
\hline 155 & 59.95 & 51.53 & 46.61 & 43.12 & 40.41 & 38.20 & 155.14 & 180.47 & 199.53 & 215.69 & 230.14 & 243.48 \\
\hline 156 & 60.35 & 51.88 & 46.92 & 43.41 & 40.68 & 38.45 & 155.10 & 180.43 & 199.48 & 215.64 & 230.09 & 243.42 \\
\hline 157 & 60.61 & 52.10 & 47.12 & 43.59 & 40.85 & 38.62 & 155.43 & 180.81 & 199.90 & 216.09 & 230.58 & 243.94 \\
\hline 158 & 61.03 & 52.46 & 47.45 & 43.90 & 41.14 & 38.88 & 155.34 & 180.71 & 199.79 & 215.97 & 230.45 & 243.80 \\
\hline 159 & 61.26 & 52.66 & 47.63 & 44.06 & 41.29 & 39.03 & 155.73 & 181.16 & 200.29 & 216.51 & 231.02 & 244.41 \\
\hline 160 & 61.71 & 53.05 & 47.98 & 44.39 & 41.60 & 39.32 & 155.56 & 180.96 & 200.07 & 216.27 & 230.77 & 244.14 \\
\hline 161 & 61.95 & 53.25 & 48.17 & 44.56 & 41.76 & 39.47 & 155.93 & 181.39 & 200.55 & 216.79 & 231.33 & 244.73 \\
\hline 162 & 62.26 & 53.52 & 48.41 & 44.78 & 41.97 & 39.67 & 156.13 & 181.62 & 200.80 & 217.06 & 231.61 & 245.03 \\
\hline 163 & 62.61 & 53.82 & 48.68 & 45.03 & 42.20 & 39.89 & 156.21 & 181.71 & 200.90 & 217.17 & 231.73 & 245.16 \\
\hline 164 & 63.01 & 54.17 & 48.99 & 45.32 & 42.48 & 40.15 & 156.16 & 181.65 & 200.84 & 217.10 & 231.66 & 245.08 \\
\hline 165 & 63.25 & 54.37 & 49.18 & 45.50 & 42.64 & 40.30 & 156.52 & 182.07 & 201.30 & 217.60 & 232.19 & 245.65 \\
\hline 166 & 63.61 & 54.68 & 49.46 & 45.75 & 42.88 & 40.53 & 156.59 & 182.15 & 201.39 & 217.70 & 232.29 & 245.75 \\
\hline 167 & 63.79 & 54.84 & 49.60 & 45.88 & 43.00 & 40.65 & 157.08 & 182.72 & 202.02 & 218.38 & 233.02 & 246.52 \\
\hline 168 & 64.16 & 55.16 & 49.89 & 46.15 & 43.25 & 40.88 & 157.10 & 182.76 & 202.06 & 218.42 & 233.06 & 246.57 \\
\hline 169 & 64.40 & 55.36 & 50.07 & 46.32 & 43.41 & 41.04 & 157.45 & 183.16 & 202.50 & 218.90 & 233.57 & 247.11 \\
\hline 170 & 64.85 & 55.75 & 50.43 & 46.65 & 43.72 & 41.32 & 157.28 & 182.96 & 202.28 & 218.66 & 233.32 & 246.84 \\
\hline 171 & 65.09 & 55.95 & 50.61 & 46.82 & 43.87 & 41.47 & 157.63 & 183.37 & 202.74 & 219.16 & 233.85 & 247.40 \\
\hline 172 & 65.41 & 56.23 & 50.86 & 47.05 & 44.10 & 41.68 & 157.76 & 183.52 & 202.90 & 219.33 & 234.04 & 247.60 \\
\hline 173 & 65.71 & 56.49 & 51.09 & 47.27 & 44.30 & 41.87 & 157.96 & 183.75 & 203.16 & 219.61 & 234.33 & 247.91 \\
\hline 174 & 66.05 & 56.78 & 51.36 & 47.51 & 44.53 & 42.09 & 158.05 & 183.86 & 203.27 & 219.74 & 234.47 & 248.05 \\
\hline 175 & 66.35 & 57.04 & 51.59 & 47.72 & 44.73 & 42.28 & 158.25 & 184.09 & 203.53 & 220.01 & 234.76 & 248.36 \\
\hline 176 & 66.64 & 57.29 & 51.82 & 47.94 & 44.92 & 42.46 & 158.45 & 184.33 & 203.79 & 220.30 & 235.06 & 248.68 \\
\hline 177 & 66.99 & 57.59 & 52.09 & 48.18 & 45.16 & 42.68 & 158.53 & 184.42 & 203.89 & 220.40 & 235.18 & 248.81 \\
\hline 178 & 67.32 & 57.87 & 52.34 & 48.42 & 45.38 & 42.89 & 158.65 & 184.55 & 204.04 & 220.57 & 235.35 & 248.99 \\
\hline 179 & 67.60 & 58.11 & 52.56 & 48.62 & 45.57 & 43.07 & 158.88 & 184.82 & 204.34 & 220.89 & 235.70 & 249.35 \\
\hline 180 & 67.90 & 58.37 & 52.79 & 48.84 & 45.77 & 43.26 & 159.06 & 185.03 & 204.57 & 221.13 & 235.96 & 249.63 \\
\hline 181 & 68.23 & 58.65 & 53.05 & 49.08 & 45.99 & 43.47 & 159.17 & 185.15 & 204.71 & 221.29 & 236.12 & 249.80 \\
\hline 182 & 68.62 & 58.99 & 53.35 & 49.36 & 46.26 & 43.72 & 159.14 & 185.12 & 204.67 & 221.25 & 236.08 & 249.76 \\
\hline 183 & 68.83 & 59.17 & 53.52 & 49.51 & 46.40 & 43.85 & 159.53 & 185.58 & 205.17 & 221.79 & 236.66 & 250.37 \\
\hline 184 & 69.27 & 59.55 & 53.86 & 49.82 & 46.69 & 44.14 & 159.38 & 185.40 & 204.98 & 221.58 & 236.43 & 250.13 \\
\hline 185 & 69.42 & 59.68 & 53.98 & 49.93 & 46.80 & 44.23 & 159.89 & 186.00 & 205.64 & 222.29 & 237.19 & 250.94 \\
\hline 186 & 69.78 & 59.98 & 54.25 & 50.19 & 47.04 & 44.46 & 159.94 & 186.05 & 205.70 & 222.36 & 237.26 & 251.01 \\
\hline 187 & 70.12 & 60.28 & 54.52 & 50.44 & 47.27 & 44.68 & 160.01 & 186.14 & 205.80 & 222.46 & 237.38 & 251.13 \\
\hline 188 & 70.45 & 60.56 & 54.78 & 50.67 & 47.49 & 44.89 & 160.12 & 186.26 & 205.93 & 222.61 & 237.53 & 251.29 \\
\hline 189 & 70.69 & 60.76 & 54.96 & 50.84 & 47.65 & 45.04 & 160.43 & 186.62 & 206.33 & 223.04 & 237.99 & 251.78 \\
\hline 190 & 70.98 & 61.02 & 55.19 & 51.06 & 47.85 & 45.23 & 160.60 & 186.82 & 206.55 & 223.28 & 238.24 & 252.05 \\
\hline
\end{tabular}


Table B.2: Simulation Data for Picking Times (High Rate)—continued.

\begin{tabular}{|c|c|c|c|c|c|c|c|c|c|c|c|c|}
\hline \multirow{2}{*}{$\begin{array}{c}\text { Batch } \\
\text { Size }\end{array}$} & \multicolumn{6}{|c|}{ Picking Time (minutes) } & \multicolumn{6}{|c|}{ Picking Rate (orders/hr) } \\
\hline & 20 & 40 & 60 & 80 & 100 & 120 & 20 & 40 & 60 & 80 & 100 & 120 \\
\hline 191 & 71.35 & 61.33 & 55.48 & 51.32 & 48.10 & 45.46 & 160.62 & 186.84 & 206.57 & 223.30 & 238.27 & 252.08 \\
\hline 192 & 71.63 & 61.57 & 55.69 & 51.52 & 48.28 & 45.64 & 160.83 & 187.09 & 206.85 & 223.60 & 238.59 & 252.42 \\
\hline 193 & 71.92 & 61.83 & 55.92 & 51.73 & 48.48 & 45.83 & 161.01 & 187.29 & 207.07 & 223.84 & 238.85 & 252.69 \\
\hline 194 & 72.25 & 62.11 & 56.18 & 51.97 & 48.70 & 46.03 & 161.11 & 187.42 & 207.21 & 223.99 & 239.00 & 252.85 \\
\hline 195 & 72.48 & 62.30 & 56.35 & 52.13 & 48.86 & 46.18 & 161.43 & 187.79 & 207.62 & 224.44 & 239.48 & 253.36 \\
\hline 196 & 72.90 & 62.66 & 56.68 & 52.43 & 49.14 & 46.45 & 161.33 & 187.67 & 207.48 & 224.29 & 239.32 & 253.19 \\
\hline 197 & 73.18 & 62.91 & 56.90 & 52.64 & 49.33 & 46.63 & 161.51 & 187.88 & 207.72 & 224.54 & 239.60 & 253.48 \\
\hline 198 & 73.57 & 63.24 & 57.20 & 52.92 & 49.59 & 46.88 & 161.48 & 187.85 & 207.68 & 224.50 & 239.55 & 253.43 \\
\hline 199 & 73.78 & 63.42 & 57.37 & 53.07 & 49.73 & 47.01 & 161.83 & 188.26 & 208.13 & 224.99 & 240.07 & 253.98 \\
\hline 200 & 74.21 & 63.79 & 57.70 & 53.38 & 50.02 & 47.28 & 161.70 & 188.10 & 207.97 & 224.81 & 239.88 & 253.78 \\
\hline 201 & 74.41 & 63.97 & 57.86 & 53.52 & 50.16 & 47.41 & 162.07 & 188.53 & 208.44 & 225.32 & 240.43 & 254.36 \\
\hline 202 & 74.68 & 64.20 & 58.07 & 53.72 & 50.34 & 47.58 & 162.29 & 188.79 & 208.73 & 225.63 & 240.76 & 254.71 \\
\hline 203 & 75.10 & 64.56 & 58.39 & 54.02 & 50.62 & 47.85 & 162.19 & 188.67 & 208.60 & 225.49 & 240.61 & 254.55 \\
\hline 204 & 75.45 & 64.86 & 58.66 & 54.27 & 50.86 & 48.07 & 162.23 & 188.71 & 208.64 & 225.54 & 240.66 & 254.60 \\
\hline 205 & 75.65 & 65.03 & 58.82 & 54.42 & 51.00 & 48.20 & 162.58 & 189.13 & 209.10 & 226.04 & 241.19 & 255.17 \\
\hline 206 & 75.99 & 65.33 & 59.09 & 54.66 & 51.23 & 48.42 & 162.64 & 189.20 & 209.18 & 226.12 & 241.28 & 255.26 \\
\hline 207 & 76.26 & 65.56 & 59.29 & 54.85 & 51.41 & 48.59 & 162.86 & 189.46 & 209.46 & 226.43 & 241.61 & 255.61 \\
\hline 208 & 76.62 & 65.87 & 59.58 & 55.11 & 51.65 & 48.82 & 162.87 & 189.47 & 209.47 & 226.44 & 241.62 & 255.62 \\
\hline 209 & 76.88 & 66.09 & 59.78 & 55.30 & 51.82 & 48.99 & 163.11 & 189.74 & 209.78 & 226.77 & 241.97 & 255.99 \\
\hline 210 & 77.24 & 66.39 & 60.05 & 55.55 & 52.06 & 49.21 & 163.14 & 189.77 & 209.81 & 226.81 & 242.01 & 256.04 \\
\hline 211 & 77.54 & 66.66 & 60.29 & 55.77 & 52.27 & 49.41 & 163.27 & 189.93 & 209.99 & 226.99 & 242.21 & 256.24 \\
\hline 212 & 77.84 & 66.92 & 60.52 & 55.99 & 52.47 & 49.60 & 163.41 & 190.09 & 210.16 & 227.18 & 242.41 & 256.46 \\
\hline 213 & 78.10 & 67.14 & 60.73 & 56.18 & 52.65 & 49.76 & 163.63 & 190.35 & 210.45 & 227.49 & 242.74 & 256.81 \\
\hline 214 & 78.53 & 67.51 & 61.06 & 56.49 & 52.94 & 50.04 & 163.50 & 190.20 & 210.29 & 227.32 & 242.55 & 256.61 \\
\hline 215 & 78.78 & 67.72 & 61.25 & 56.67 & 53.11 & 50.20 & 163.74 & 190.48 & 210.60 & 227.65 & 242.91 & 256.99 \\
\hline 216 & 79.02 & 67.93 & 61.44 & 56.84 & 53.27 & 50.35 & 164.01 & 190.79 & 210.94 & 228.02 & 243.31 & 257.40 \\
\hline 217 & 79.31 & 68.18 & 61.67 & 57.05 & 53.46 & 50.53 & 164.17 & 190.97 & 211.14 & 228.24 & 243.54 & 257.65 \\
\hline 218 & 79.71 & 68.52 & 61.98 & 57.34 & 53.73 & 50.79 & 164.09 & 190.88 & 211.04 & 228.13 & 243.42 & 257.53 \\
\hline 219 & 80.05 & 68.81 & 62.24 & 57.58 & 53.96 & 51.00 & 164.15 & 190.96 & 211.12 & 228.22 & 243.52 & 257.63 \\
\hline 220 & 80.24 & 68.97 & 62.39 & 57.71 & 54.09 & 51.12 & 164.52 & 191.38 & 211.59 & 228.72 & 244.06 & 258.20 \\
\hline 221 & 80.64 & 69.32 & 62.70 & 58.00 & 54.36 & 51.38 & 164.44 & 191.29 & 211.49 & 228.62 & 243.94 & 258.08 \\
\hline 222 & 80.88 & 69.53 & 62.89 & 58.17 & 54.52 & 51.53 & 164.69 & 191.58 & 211.81 & 228.97 & 244.32 & 258.47 \\
\hline 223 & 81.22 & 69.82 & 63.15 & 58.42 & 54.75 & 51.75 & 164.73 & 191.63 & 211.87 & 229.03 & 244.38 & 258.54 \\
\hline 224 & 81.57 & 70.12 & 63.42 & 58.67 & 54.98 & 51.97 & 164.77 & 191.67 & 211.91 & 229.08 & 244.43 & 258.59 \\
\hline 225 & 81.85 & 70.36 & 63.64 & 58.87 & 55.18 & 52.15 & 164.93 & 191.86 & 212.12 & 229.30 & 244.68 & 258.85 \\
\hline 226 & 82.12 & 70.59 & 63.85 & 59.07 & 55.36 & 52.33 & 165.12 & 192.08 & 212.37 & 229.57 & 244.95 & 259.15 \\
\hline 227 & 82.43 & 70.86 & 64.09 & 59.29 & 55.57 & 52.52 & 165.23 & 192.21 & 212.51 & 229.72 & 245.11 & 259.32 \\
\hline 228 & 82.74 & 71.13 & 64.33 & 59.51 & 55.77 & 52.72 & 165.33 & 192.33 & 212.64 & 229.86 & 245.27 & 259.48 \\
\hline 229 & 83.05 & 71.39 & 64.57 & 59.74 & 55.98 & 52.92 & 165.44 & 192.46 & 212.78 & 230.01 & 245.43 & 259.65 \\
\hline 230 & 83.30 & 71.61 & 64.77 & 59.92 & 56.15 & 53.08 & 165.66 & 192.71 & 213.06 & 230.32 & 245.76 & 260.00 \\
\hline 231 & 83.70 & 71.96 & 65.08 & 60.21 & 56.42 & 53.33 & 165.58 & 192.62 & 212.96 & 230.21 & 245.64 & 259.87 \\
\hline 232 & 84.06 & 72.26 & 65.36 & 60.46 & 56.66 & 53.56 & 165.60 & 192.64 & 212.98 & 230.23 & 245.67 & 259.90 \\
\hline
\end{tabular}


Table B.2: Simulation Data for Picking Times (High Rate)—continued.

\begin{tabular}{|c|c|c|c|c|c|c|c|c|c|c|c|c|}
\hline \multirow{2}{*}{$\begin{array}{c}\text { Batch } \\
\text { Size }\end{array}$} & \multicolumn{6}{|c|}{ Picking Time (minutes) } & \multicolumn{6}{|c|}{ Picking Rate (orders/hr) } \\
\hline & 20 & 40 & 60 & 80 & 100 & 120 & 20 & 40 & 60 & 80 & 100 & 120 \\
\hline 233 & 84.28 & 72.45 & 65.53 & 60.62 & 56.81 & 53.70 & 165.88 & 192.96 & 213.34 & 230.62 & 246.08 & 260.34 \\
\hline 234 & 84.57 & 72.70 & 65.76 & 60.83 & 57.01 & 53.89 & 166.01 & 193.12 & 213.51 & 230.81 & 246.28 & 260.55 \\
\hline 235 & 84.94 & 73.02 & 66.04 & 61.10 & 57.26 & 54.12 & 166.00 & 193.10 & 213.50 & 230.79 & 246.26 & 260.53 \\
\hline 236 & 85.27 & 73.31 & 66.30 & 61.34 & 57.48 & 54.33 & 166.05 & 193.16 & 213.56 & 230.86 & 246.34 & 260.61 \\
\hline 237 & 85.61 & 73.59 & 66.56 & 61.58 & 57.71 & 54.55 & 166.11 & 193.23 & 213.63 & 230.93 & 246.41 & 260.69 \\
\hline 238 & 85.70 & 73.67 & 66.64 & 61.64 & 57.77 & 54.61 & 166.62 & 193.83 & 214.29 & 231.65 & 247.18 & 261.50 \\
\hline 239 & 86.11 & 74.02 & 66.95 & 61.93 & 58.04 & 54.86 & 166.54 & 193.73 & 214.19 & 231.53 & 247.05 & 261.37 \\
\hline 240 & 86.45 & 74.31 & 67.21 & 62.18 & 58.27 & 55.08 & 166.58 & 193.78 & 214.24 & 231.59 & 247.12 & 261.44 \\
\hline 241 & 86.74 & 74.57 & 67.45 & 62.39 & 58.47 & 55.27 & 166.70 & 193.92 & 214.40 & 231.76 & 247.30 & 261.63 \\
\hline 242 & 87.09 & 74.86 & 67.71 & 62.64 & 58.70 & 55.49 & 166.73 & 193.96 & 214.44 & 231.81 & 247.35 & 261.68 \\
\hline 243 & 87.37 & 75.11 & 67.93 & 62.85 & 58.90 & 55.67 & 166.87 & 194.12 & 214.62 & 232.00 & 247.55 & 261.90 \\
\hline 244 & 87.65 & 75.35 & 68.15 & 63.05 & 59.09 & 55.85 & 167.02 & 194.29 & 214.81 & 232.21 & 247.77 & 262.13 \\
\hline 245 & 87.89 & 75.55 & 68.34 & 63.22 & 59.24 & 56.00 & 167.26 & 194.57 & 215.12 & 232.54 & 248.13 & 262.50 \\
\hline 246 & 88.33 & 75.94 & 68.68 & 63.54 & 59.54 & 56.28 & 167.09 & 194.38 & 214.90 & 232.31 & 247.88 & 262.24 \\
\hline 247 & 88.58 & 76.15 & 68.87 & 63.71 & 59.71 & 56.44 & 167.31 & 194.63 & 215.18 & 232.61 & 248.20 & 262.58 \\
\hline 248 & 88.85 & 76.38 & 69.08 & 63.91 & 59.89 & 56.61 & 167.48 & 194.82 & 215.40 & 232.84 & 248.45 & 262.85 \\
\hline 249 & 89.15 & 76.64 & 69.32 & 64.12 & 60.10 & 56.80 & 167.58 & 194.95 & 215.53 & 232.99 & 248.61 & 263.01 \\
\hline 250 & 89.40 & 76.85 & 69.51 & 64.31 & 60.27 & 56.96 & 167.78 & 195.18 & 215.79 & 233.26 & 248.90 & 263.32 \\
\hline 251 & 89.71 & 77.12 & 69.75 & 64.52 & 60.47 & 57.16 & 167.88 & 195.29 & 215.91 & 233.40 & 249.04 & 263.47 \\
\hline 252 & 90.09 & 77.44 & 70.05 & 64.80 & 60.73 & 57.40 & 167.83 & 195.24 & 215.86 & 233.34 & 248.98 & 263.41 \\
\hline 253 & 90.45 & 77.75 & 70.33 & 65.06 & 60.97 & 57.63 & 167.83 & 195.23 & 215.85 & 233.33 & 248.97 & 263.40 \\
\hline 254 & 90.66 & 77.94 & 70.49 & 65.21 & 61.11 & 57.77 & 168.10 & 195.54 & 216.19 & 233.70 & 249.37 & 263.82 \\
\hline 255 & 91.00 & 78.23 & 70.76 & 65.45 & 61.34 & 57.98 & 168.13 & 195.58 & 216.24 & 233.75 & 249.42 & 263.87 \\
\hline 256 & 91.40 & 78.57 & 71.06 & 65.74 & 61.61 & 58.23 & 168.06 & 195.50 & 216.15 & 233.65 & 249.32 & 263.76 \\
\hline 257 & 91.53 & 78.68 & 71.17 & 65.84 & 61.70 & 58.32 & 168.47 & 195.97 & 216.67 & 234.22 & 249.92 & 264.40 \\
\hline 258 & 91.85 & 78.95 & 71.41 & 66.06 & 61.91 & 58.52 & 168.54 & 196.06 & 216.77 & 234.32 & 250.03 & 264.52 \\
\hline 259 & 92.28 & 79.33 & 71.75 & 66.37 & 62.20 & 58.80 & 168.40 & 195.90 & 216.59 & 234.13 & 249.82 & 264.30 \\
\hline 260 & 92.53 & 79.54 & 71.94 & 66.55 & 62.37 & 58.95 & 168.60 & 196.13 & 216.84 & 234.41 & 250.12 & 264.61 \\
\hline 261 & 92.80 & 79.78 & 72.16 & 66.75 & 62.56 & 59.13 & 168.74 & 196.30 & 217.02 & 234.60 & 250.33 & 264.83 \\
\hline 262 & 93.10 & 80.03 & 72.39 & 66.96 & 62.76 & 59.32 & 168.85 & 196.42 & 217.17 & 234.76 & 250.49 & 265.01 \\
\hline 263 & 93.52 & 80.39 & 72.72 & 67.27 & 63.04 & 59.59 & 168.73 & 196.28 & 217.01 & 234.59 & 250.31 & 264.82 \\
\hline 264 & 93.77 & 80.61 & 72.91 & 67.44 & 63.21 & 59.75 & 168.93 & 196.51 & 217.26 & 234.86 & 250.60 & 265.12 \\
\hline 265 & 94.02 & 80.83 & 73.11 & 67.63 & 63.38 & 59.91 & 169.11 & 196.72 & 217.49 & 235.11 & 250.87 & 265.40 \\
\hline 266 & 94.32 & 81.08 & 73.34 & 67.84 & 63.58 & 60.10 & 169.21 & 196.84 & 217.63 & 235.25 & 251.02 & 265.57 \\
\hline 267 & 94.67 & 81.38 & 73.61 & 68.09 & 63.82 & 60.32 & 169.22 & 196.85 & 217.63 & 235.26 & 251.03 & 265.58 \\
\hline 268 & 95.06 & 81.72 & 73.92 & 68.38 & 64.08 & 60.57 & 169.15 & 196.77 & 217.54 & 235.16 & 250.93 & 265.47 \\
\hline 269 & 95.31 & 81.93 & 74.10 & 68.55 & 64.25 & 60.73 & 169.35 & 197.00 & 217.80 & 235.44 & 251.23 & 265.78 \\
\hline 270 & 95.59 & 82.17 & 74.33 & 68.76 & 64.44 & 60.91 & 169.47 & 197.14 & 217.96 & 235.61 & 251.41 & 265.98 \\
\hline 271 & 95.83 & 82.38 & 74.51 & 68.92 & 64.59 & 61.06 & 169.68 & 197.39 & 218.23 & 235.91 & 251.72 & 266.31 \\
\hline 272 & 96.16 & 82.66 & 74.77 & 69.16 & 64.82 & 61.27 & 169.72 & 197.43 & 218.28 & 235.96 & 251.78 & 266.37 \\
\hline 273 & 96.46 & 82.92 & 75.00 & 69.38 & 65.02 & 61.46 & 169.82 & 197.55 & 218.41 & 236.10 & 251.92 & 266.52 \\
\hline 274 & 96.83 & 83.24 & 75.29 & 69.65 & 65.27 & 61.70 & 169.78 & 197.50 & 218.35 & 236.04 & 251.86 & 266.46 \\
\hline
\end{tabular}


Table B.2: Simulation Data for Picking Times (High Rate)—continued.

\begin{tabular}{|c|c|c|c|c|c|c|c|c|c|c|c|c|}
\hline \multirow{2}{*}{$\begin{array}{c}\text { Batch } \\
\text { Size }\end{array}$} & \multicolumn{6}{|c|}{ Picking Time (minutes) } & \multicolumn{6}{|c|}{ Picking Rate (orders/hr) } \\
\hline & 20 & 40 & 60 & 80 & 100 & 120 & 20 & 40 & 60 & 80 & 100 & 120 \\
\hline 275 & 97.10 & 83.47 & 75.50 & 69.84 & 65.45 & 61.87 & 169.93 & 197.68 & 218.56 & 236.26 & 252.09 & 266.70 \\
\hline 276 & 97.44 & 83.76 & 75.76 & 70.09 & 65.68 & 62.09 & 169.95 & 197.70 & 218.58 & 236.28 & 252.12 & 266.73 \\
\hline 277 & 97.68 & 83.97 & 75.95 & 70.26 & 65.84 & 62.24 & 170.15 & 197.93 & 218.84 & 236.56 & 252.42 & 267.04 \\
\hline 278 & 97.98 & 84.23 & 76.18 & 70.47 & 66.05 & 62.43 & 170.24 & 198.04 & 218.95 & 236.68 & 252.55 & 267.18 \\
\hline 279 & 98.33 & 84.53 & 76.45 & 70.73 & 66.28 & 62.65 & 170.24 & 198.04 & 218.96 & 236.69 & 252.56 & 267.19 \\
\hline 280 & 98.69 & 84.84 & 76.73 & 70.98 & 66.53 & 62.88 & 170.23 & 198.03 & 218.94 & 236.67 & 252.54 & 267.17 \\
\hline 281 & 98.92 & 85.04 & 76.91 & 71.15 & 66.68 & 63.03 & 170.44 & 198.27 & 219.21 & 236.96 & 252.85 & 267.50 \\
\hline 282 & 99.23 & 85.30 & 77.15 & 71.37 & 66.89 & 63.23 & 170.52 & 198.36 & 219.30 & 237.07 & 252.96 & 267.61 \\
\hline 283 & 99.51 & 85.54 & 77.37 & 71.58 & 67.08 & 63.41 & 170.63 & 198.49 & 219.45 & 237.23 & 253.13 & 267.80 \\
\hline 284 & 99.75 & 85.75 & 77.56 & 71.74 & 67.24 & 63.55 & 170.83 & 198.73 & 219.71 & 237.51 & 253.43 & 268.11 \\
\hline 285 & 100.18 & 86.12 & 77.90 & 72.06 & 67.53 & 63.83 & 170.69 & 198.56 & 219.53 & 237.31 & 253.21 & 267.89 \\
\hline 286 & 100.46 & 86.36 & 78.11 & 72.26 & 67.72 & 64.01 & 170.82 & 198.71 & 219.70 & 237.49 & 253.41 & 268.09 \\
\hline 287 & 100.77 & 86.63 & 78.35 & 72.48 & 67.93 & 64.21 & 170.88 & 198.78 & 219.78 & 237.58 & 253.50 & 268.19 \\
\hline 288 & 101.09 & 86.90 & 78.60 & 72.71 & 68.14 & 64.41 & 170.94 & 198.85 & 219.85 & 237.66 & 253.59 & 268.28 \\
\hline 289 & 101.31 & 87.09 & 78.77 & 72.87 & 68.29 & 64.55 & 171.15 & 199.10 & 220.12 & 237.95 & 253.90 & 268.61 \\
\hline 290 & 101.68 & 87.41 & 79.06 & 73.14 & 68.54 & 64.79 & 171.12 & 199.06 & 220.09 & 237.91 & 253.86 & 268.57 \\
\hline 291 & 101.96 & 87.65 & 79.28 & 73.34 & 68.73 & 64.97 & 171.24 & 199.20 & 220.23 & 238.07 & 254.03 & 268.75 \\
\hline 292 & 102.24 & 87.89 & 79.50 & 73.54 & 68.92 & 65.14 & 171.36 & 199.34 & 220.39 & 238.24 & 254.21 & 268.94 \\
\hline 293 & 102.54 & 88.15 & 79.73 & 73.75 & 69.12 & 65.34 & 171.45 & 199.44 & 220.50 & 238.36 & 254.34 & 269.07 \\
\hline 294 & 102.85 & 88.41 & 79.97 & 73.98 & 69.33 & 65.53 & 171.52 & 199.52 & 220.59 & 238.46 & 254.44 & 269.19 \\
\hline 295 & 103.16 & 88.68 & 80.21 & 74.20 & 69.54 & 65.73 & 171.58 & 199.60 & 220.67 & 238.55 & 254.54 & 269.29 \\
\hline 296 & 103.40 & 88.88 & 80.39 & 74.37 & 69.70 & 65.88 & 171.76 & 199.81 & 220.91 & 238.80 & 254.81 & 269.57 \\
\hline 297 & 103.76 & 89.20 & 80.68 & 74.63 & 69.94 & 66.11 & 171.74 & 199.78 & 220.88 & 238.77 & 254.78 & 269.54 \\
\hline 298 & 104.10 & 89.48 & 80.94 & 74.87 & 70.17 & 66.33 & 171.76 & 199.81 & 220.91 & 238.80 & 254.81 & 269.57 \\
\hline 299 & 104.35 & 89.70 & 81.13 & 75.05 & 70.34 & 66.49 & 171.93 & 200.00 & 221.12 & 239.03 & 255.05 & 269.83 \\
\hline 300 & 104.73 & 90.03 & 81.43 & 75.33 & 70.60 & 66.73 & 171.87 & 199.93 & 221.05 & 238.95 & 254.97 & 269.74 \\
\hline 301 & 105.00 & 90.26 & 81.64 & 75.52 & 70.78 & 66.90 & 172.00 & 200.08 & 221.21 & 239.13 & 255.16 & 269.94 \\
\hline 302 & 105.31 & 90.52 & 81.88 & 75.74 & 70.99 & 67.10 & 172.07 & 200.17 & 221.30 & 239.23 & 255.26 & 270.05 \\
\hline 303 & 105.55 & 90.74 & 82.07 & 75.92 & 71.15 & 67.25 & 172.24 & 200.36 & 221.52 & 239.46 & 255.51 & 270.32 \\
\hline 304 & 105.99 & 91.12 & 82.41 & 76.24 & 71.45 & 67.54 & 172.09 & 200.19 & 221.32 & 239.25 & 255.29 & 270.08 \\
\hline 305 & 106.17 & 91.27 & 82.55 & 76.37 & 71.57 & 67.65 & 172.36 & 200.51 & 221.68 & 239.63 & 255.70 & 270.51 \\
\hline 306 & 106.44 & 91.50 & 82.76 & 76.56 & 71.75 & 67.82 & 172.49 & 200.65 & 221.84 & 239.81 & 255.88 & 270.71 \\
\hline 307 & 106.82 & 91.83 & 83.06 & 76.83 & 72.01 & 68.06 & 172.44 & 200.59 & 221.78 & 239.74 & 255.81 & 270.63 \\
\hline 308 & 107.09 & 92.05 & 83.26 & 77.02 & 72.19 & 68.23 & 172.57 & 200.75 & 221.95 & 239.93 & 256.01 & 270.84 \\
\hline 309 & 107.46 & 92.38 & 83.55 & 77.29 & 72.44 & 68.47 & 172.53 & 200.70 & 221.89 & 239.86 & 255.94 & 270.77 \\
\hline 310 & 107.71 & 92.59 & 83.75 & 77.48 & 72.61 & 68.63 & 172.68 & 200.88 & 222.09 & 240.08 & 256.17 & 271.01 \\
\hline 311 & 108.00 & 92.84 & 83.98 & 77.68 & 72.80 & 68.82 & 172.77 & 200.98 & 222.21 & 240.20 & 256.30 & 271.16 \\
\hline 312 & 108.35 & 93.14 & 84.24 & 77.93 & 73.04 & 69.04 & 172.78 & 200.99 & 222.21 & 240.21 & 256.31 & 271.17 \\
\hline 313 & 108.59 & 93.35 & 84.43 & 78.11 & 73.20 & 69.19 & 172.94 & 201.18 & 222.42 & 240.44 & 256.56 & 271.42 \\
\hline 314 & 108.94 & 93.65 & 84.71 & 78.36 & 73.44 & 69.42 & 172.93 & 201.17 & 222.41 & 240.43 & 256.55 & 271.41 \\
\hline 315 & 109.29 & 93.95 & 84.98 & 78.61 & 73.67 & 69.64 & 172.93 & 201.17 & 222.42 & 240.43 & 256.55 & 271.41 \\
\hline 316 & 109.50 & 94.13 & 85.14 & 78.76 & 73.81 & 69.77 & 173.15 & 201.42 & 222.69 & 240.73 & 256.86 & 271.75 \\
\hline
\end{tabular}


Table B.2: Simulation Data for Picking Times (High Rate)—continued.

\begin{tabular}{|c|c|c|c|c|c|c|c|c|c|c|c|c|}
\hline \multirow{2}{*}{$\begin{array}{c}\text { Batch } \\
\text { Size }\end{array}$} & \multicolumn{6}{|c|}{ Picking Time (minutes) } & \multicolumn{6}{|c|}{ Picking Rate (orders/hr) } \\
\hline & 20 & 40 & 60 & 80 & 100 & 120 & 20 & 40 & 60 & 80 & 100 & 120 \\
\hline 317 & 109.82 & 94.40 & 85.39 & 78.99 & 74.03 & 69.97 & 173.19 & 201.47 & 222.75 & 240.79 & 256.93 & 271.82 \\
\hline 318 & 110.14 & 94.68 & 85.64 & 79.22 & 74.24 & 70.18 & 173.24 & 201.52 & 222.80 & 240.85 & 256.99 & 271.88 \\
\hline 319 & 110.40 & 94.91 & 85.84 & 79.41 & 74.42 & 70.34 & 173.37 & 201.67 & 222.97 & 241.03 & 257.19 & 272.09 \\
\hline 320 & 110.76 & 95.22 & 86.12 & 79.67 & 74.66 & 70.57 & 173.34 & 201.65 & 222.94 & 241.00 & 257.15 & 272.05 \\
\hline 321 & 110.99 & 95.41 & 86.30 & 79.84 & 74.82 & 70.72 & 173.52 & 201.86 & 223.17 & 241.25 & 257.42 & 272.34 \\
\hline 322 & 111.40 & 95.76 & 86.62 & 80.13 & 75.09 & 70.98 & 173.43 & 201.74 & 223.05 & 241.11 & 257.28 & 272.18 \\
\hline 323 & 111.63 & 95.96 & 86.80 & 80.29 & 75.25 & 71.13 & 173.61 & 201.96 & 223.28 & 241.37 & 257.55 & 272.47 \\
\hline 324 & 111.91 & 96.20 & 87.01 & 80.49 & 75.44 & 71.30 & 173.71 & 202.08 & 223.42 & 241.51 & 257.70 & 272.64 \\
\hline 325 & 112.28 & 96.52 & 87.30 & 80.76 & 75.69 & 71.54 & 173.67 & 202.02 & 223.36 & 241.45 & 257.63 & 272.56 \\
\hline 326 & 112.56 & 96.76 & 87.52 & 80.96 & 75.88 & 71.72 & 173.77 & 202.15 & 223.49 & 241.59 & 257.79 & 272.73 \\
\hline 327 & 112.84 & 97.00 & 87.73 & 81.16 & 76.06 & 71.90 & 173.88 & 202.27 & 223.63 & 241.74 & 257.95 & 272.89 \\
\hline 328 & 113.11 & 97.23 & 87.95 & 81.36 & 76.25 & 72.07 & 173.99 & 202.40 & 223.77 & 241.90 & 258.11 & 273.07 \\
\hline 329 & 113.47 & 97.54 & 88.23 & 81.62 & 76.49 & 72.30 & 173.96 & 202.37 & 223.74 & 241.86 & 258.07 & 273.03 \\
\hline 330 & 113.83 & 97.85 & 88.51 & 81.88 & 76.73 & 72.53 & 173.94 & 202.34 & 223.71 & 241.83 & 258.04 & 272.99 \\
\hline 331 & 114.11 & 98.09 & 88.72 & 82.08 & 76.92 & 72.71 & 174.04 & 202.46 & 223.84 & 241.97 & 258.19 & 273.15 \\
\hline 332 & 114.42 & 98.36 & 88.96 & 82.30 & 77.13 & 72.90 & 174.10 & 202.53 & 223.92 & 242.05 & 258.28 & 273.24 \\
\hline 333 & 114.69 & 98.59 & 89.18 & 82.49 & 77.31 & 73.08 & 174.21 & 202.65 & 224.05 & 242.20 & 258.43 & 273.41 \\
\hline 334 & 115.07 & 98.92 & 89.47 & 82.76 & 77.56 & 73.32 & 174.16 & 202.60 & 223.99 & 242.13 & 258.36 & 273.34 \\
\hline 335 & 115.22 & 99.04 & 89.58 & 82.87 & 77.67 & 73.41 & 174.45 & 202.94 & 224.37 & 242.54 & 258.80 & 273.79 \\
\hline 336 & 115.58 & 99.36 & 89.87 & 83.14 & 77.91 & 73.65 & 174.42 & 202.90 & 224.33 & 242.50 & 258.75 & 273.74 \\
\hline 337 & 115.86 & 99.60 & 90.08 & 83.33 & 78.10 & 73.82 & 174.52 & 203.02 & 224.46 & 242.64 & 258.90 & 273.90 \\
\hline 338 & 116.18 & 99.87 & 90.33 & 83.57 & 78.32 & 74.03 & 174.55 & 203.06 & 224.50 & 242.68 & 258.95 & 273.95 \\
\hline 339 & 116.49 & 100.14 & 90.57 & 83.79 & 78.52 & 74.22 & 174.61 & 203.12 & 224.57 & 242.76 & 259.03 & 274.04 \\
\hline 340 & 116.83 & 100.43 & 90.84 & 84.03 & 78.75 & 74.44 & 174.61 & 203.13 & 224.58 & 242.76 & 259.04 & 274.05 \\
\hline 341 & 117.11 & 100.67 & 91.06 & 84.23 & 78.94 & 74.62 & 174.71 & 203.23 & 224.70 & 242.89 & 259.18 & 274.19 \\
\hline 342 & 117.37 & 100.89 & 91.26 & 84.42 & 79.12 & 74.78 & 174.84 & 203.38 & 224.86 & 243.07 & 259.37 & 274.40 \\
\hline 343 & 117.67 & 101.16 & 91.49 & 84.64 & 79.32 & 74.98 & 174.89 & 203.45 & 224.93 & 243.15 & 259.45 & 274.48 \\
\hline 344 & 118.01 & 101.45 & 91.76 & 84.88 & 79.55 & 75.19 & 174.90 & 203.46 & 224.94 & 243.16 & 259.46 & 274.49 \\
\hline 345 & 118.27 & 101.67 & 91.96 & 85.07 & 79.72 & 75.36 & 175.03 & 203.60 & 225.10 & 243.34 & 259.65 & 274.69 \\
\hline 346 & 118.65 & 102.00 & 92.26 & 85.34 & 79.98 & 75.60 & 174.96 & 203.53 & 225.03 & 243.25 & 259.56 & 274.60 \\
\hline 347 & 118.98 & 102.28 & 92.51 & 85.58 & 80.21 & 75.81 & 174.98 & 203.55 & 225.05 & 243.27 & 259.58 & 274.62 \\
\hline 348 & 119.26 & 102.52 & 92.73 & 85.78 & 80.39 & 75.99 & 175.08 & 203.66 & 225.17 & 243.41 & 259.72 & 274.77 \\
\hline 349 & 119.48 & 102.71 & 92.90 & 85.94 & 80.54 & 76.13 & 175.26 & 203.88 & 225.41 & 243.67 & 260.00 & 275.07 \\
\hline 350 & 119.77 & 102.96 & 93.13 & 86.15 & 80.74 & 76.31 & 175.33 & 203.96 & 225.50 & 243.76 & 260.10 & 275.18 \\
\hline 351 & 120.14 & 103.28 & 93.42 & 86.42 & 80.99 & 76.55 & 175.29 & 203.91 & 225.44 & 243.70 & 260.04 & 275.11 \\
\hline 352 & 120.40 & 103.50 & 93.62 & 86.60 & 81.16 & 76.72 & 175.41 & 204.06 & 225.60 & 243.88 & 260.22 & 275.30 \\
\hline 353 & 120.71 & 103.77 & 93.86 & 86.82 & 81.37 & 76.91 & 175.46 & 204.11 & 225.67 & 243.94 & 260.30 & 275.38 \\
\hline 354 & 120.97 & 103.99 & 94.06 & 87.01 & 81.54 & 77.08 & 175.58 & 204.25 & 225.82 & 244.11 & 260.48 & 275.57 \\
\hline 355 & 121.31 & 104.28 & 94.32 & 87.26 & 81.77 & 77.30 & 175.58 & 204.25 & 225.82 & 244.11 & 260.47 & 275.56 \\
\hline 356 & 121.60 & 104.53 & 94.55 & 87.47 & 81.97 & 77.48 & 175.66 & 204.34 & 225.91 & 244.21 & 260.58 & 275.68 \\
\hline 357 & 121.97 & 104.85 & 94.83 & 87.73 & 82.22 & 77.71 & 175.62 & 204.30 & 225.87 & 244.16 & 260.53 & 275.63 \\
\hline 358 & 122.13 & 104.99 & 94.96 & 87.84 & 82.33 & 77.82 & 175.88 & 204.60 & 226.20 & 244.52 & 260.92 & 276.03 \\
\hline
\end{tabular}


Table B.2: Simulation Data for Picking Times (High Rate)—continued.

\begin{tabular}{|c|c|c|c|c|c|c|c|c|c|c|c|c|}
\hline \multirow{2}{*}{$\begin{array}{c}\text { Batch } \\
\text { Size }\end{array}$} & \multicolumn{6}{|c|}{ Picking Time (minutes) } & \multicolumn{6}{|c|}{ Picking Rate (orders/hr) } \\
\hline & 20 & 40 & 60 & 80 & 100 & 120 & 20 & 40 & 60 & 80 & 100 & 120 \\
\hline 359 & 122.53 & 105.33 & 95.27 & 88.13 & 82.60 & 78.07 & 175.79 & 204.50 & 226.09 & 244.40 & 260.79 & 275.90 \\
\hline 360 & 122.84 & 105.59 & 95.51 & 8.35 & 82.80 & 78.27 & 175.84 & 204.56 & 226.16 & 244.47 & 260.86 & 275.98 \\
\hline 361 & 123.17 & 105.88 & 95.77 & 88.59 & 83.03 & 78.48 & 175.86 & 204.57 & 226.17 & 244.49 & 260.88 & 276.00 \\
\hline 362 & 123.39 & 106.07 & 95.94 & 88.75 & 83.17 & 78.62 & 176.03 & 204.77 & 226.40 & 244.73 & 261.14 & 276.27 \\
\hline 363 & 123.74 & 106.37 & 96.21 & 89.00 & 83.41 & 78.84 & 176.02 & 204.75 & 226.38 & 244.71 & 261.12 & 276.25 \\
\hline 364 & 124.03 & 106.62 & 96.44 & 89.21 & 83.61 & 79.03 & 176.09 & 204.84 & 226.47 & 244.81 & 261.22 & 276.36 \\
\hline 365 & 124.32 & 106.87 & 96.66 & 89.42 & 83.80 & 79.21 & 176.16 & 204.92 & 226.56 & 244.91 & 261.33 & 276.47 \\
\hline 366 & 124.63 & 107.13 & 96.90 & 89.64 & 84.01 & 79.41 & 176.21 & 204.98 & 226.63 & 244.98 & 261.40 & 276.55 \\
\hline 367 & 124.98 & 107.44 & 97.18 & 89.90 & 84.25 & 79.63 & 176.19 & 204.96 & 226.60 & 244.95 & 261.37 & 276.52 \\
\hline 368 & 125.30 & 107.71 & 97.42 & 90.12 & 84.46 & 79.83 & 176.22 & 205.00 & 226.65 & 245.00 & 261.43 & 276.57 \\
\hline 369 & 125.51 & 107.90 & 97.59 & 90.28 & 84.61 & 79.97 & 176.40 & 205.20 & 226.87 & 245.24 & 261.68 & 276.84 \\
\hline 370 & 125.82 & 108.16 & 97.83 & 90.50 & 84.81 & 80.17 & 176.44 & 205.25 & 226.93 & 245.31 & 261.75 & 276.92 \\
\hline 371 & 126.16 & 108.45 & 98.09 & 90.75 & 85.04 & 80.39 & 176.44 & 205.25 & 226.92 & 245.30 & 261.75 & 276.91 \\
\hline 372 & 126.48 & 108.72 & 98.34 & 90.97 & 85.26 & 80.59 & 176.48 & 205.29 & 226.97 & 245.35 & 261.80 & 276.97 \\
\hline 373 & 126.74 & 108.95 & 98.55 & 91.16 & 85.44 & 80.76 & 176.58 & 205.41 & 227.10 & 245.49 & 261.95 & 277.13 \\
\hline 374 & 127.01 & 109.18 & 98.75 & 91.36 & 85.62 & 80.93 & 176.68 & 205.53 & 227.23 & 245.63 & 262.10 & 277.29 \\
\hline 375 & 127.34 & 109.46 & 99.01 & 91.59 & 85.84 & 81.14 & 176.70 & 205.55 & 227.25 & 245.66 & 262.13 & 277.31 \\
\hline 376 & 127.63 & 109.72 & 99.24 & 91.80 & 86.03 & 81.32 & 176.76 & 205.62 & 227.33 & 245.75 & 262.22 & 277.41 \\
\hline 377 & 127.93 & 109.97 & 99.47 & 92.01 & 86.23 & 81.51 & 176.82 & 205.69 & 227.41 & 245.83 & 262.31 & 277.51 \\
\hline 378 & 128.24 & 110.24 & 99.71 & 92.24 & 86.44 & 81.71 & 176.86 & 205.74 & 227.46 & 245.89 & 262.37 & 277.57 \\
\hline 379 & 128.60 & 110.55 & 99.99 & 92.50 & 86.69 & 81.94 & 176.83 & 205.70 & 227.43 & 245.84 & 262.32 & 277.52 \\
\hline 380 & 128.87 & 110.78 & 100.20 & 92.69 & 86.87 & 82.11 & 176.92 & 205.81 & 227.54 & 245.97 & 262.46 & 277.67 \\
\hline 381 & 129.17 & 111.04 & 100.44 & 92.91 & 87.08 & 82.31 & 176.97 & 205.87 & 227.60 & 246.04 & 262.53 & 277.74 \\
\hline 382 & 129.45 & 111.28 & 100.65 & 93.11 & 87.26 & 82.48 & 177.05 & 205.96 & 227.71 & 246.15 & 262.65 & 277.87 \\
\hline 383 & 129.77 & 111.55 & 100.90 & 93.34 & 87.48 & 82.68 & 177.09 & 206.00 & 227.75 & 246.20 & 262.70 & 277.92 \\
\hline 384 & 130.07 & 111.81 & 101.13 & 93.55 & 87.68 & 82.87 & 177.14 & 206.07 & 227.83 & 246.28 & 262.79 & 278.01 \\
\hline 385 & 130.26 & 111.98 & 101.28 & 93.69 & 87.81 & 83.00 & 177.34 & 206.29 & 228.08 & 246.55 & 263.08 & 278.32 \\
\hline 386 & 130.74 & 112.39 & 101.66 & 94.04 & 88.13 & 83.31 & 177.14 & 206.07 & 227.83 & 246.28 & 262.79 & 278.01 \\
\hline 387 & 130.92 & 112.54 & 101.79 & 94.17 & r & 83.42 & 177.36 & 206.32 & 228.11 & 246.58 & 263.11 & 278.36 \\
\hline 388 & 131.24 & 112.82 & 102.04 & 94.40 & 88.47 & 83.62 & 177.39 & 206.35 & 228.14 & 246.62 & 263.15 & 278.40 \\
\hline 389 & 131.62 & 113.14 & 102.34 & 94.67 & 88.72 & 83.86 & 177.33 & 206.29 & 228.07 & & 263.07 & 278.31 \\
\hline 390 & 131.89 & 113.38 & 102.55 & 94.87 & & & 177.42 & 206.39 & 228.18 & & 263.19 & 278.45 \\
\hline 391 & 132.22 & 113.66 & 102.81 & 95.11 & 89.13 & 84.25 & 177.43 & 206.40 & 228.19 & 246.67 & 263.21 & 278.46 \\
\hline 392 & 132.49 & 113.90 & 103.02 & 95.30 & 89.31 & 84.42 & 177.52 & 206.51 & 228.31 & 246.80 & 263.35 & 278.61 \\
\hline 393 & 132.80 & 114.16 & 103.26 & 95.52 & 89.52 & 84.62 & 177.56 & 206.55 & 228.36 & 246.86 & 263.40 & 278.67 \\
\hline 394 & 133.08 & 114.40 & 103.47 & 95.72 & 89.71 & 84.79 & 177.64 & 206.64 & 228.46 & 246.97 & 263.52 & 278.79 \\
\hline 395 & 133.38 & 114.66 & 103.71 & 95.94 & 89.91 & 84.99 & 177.69 & 206.70 & 228.53 & 247.04 & 263.60 & 278.87 \\
\hline 396 & 133.77 & 114.99 & 104.01 & 96.22 & 90.17 & 85.23 & 177.62 & 206.62 & 228.44 & 246.94 & 263.50 & 278.76 \\
\hline 397 & 133.98 & 115.17 & 104.17 & 96.37 & 90.31 & 85.37 & 177.79 & 206.82 & 228.66 & 247.18 & 263.74 & 279.03 \\
\hline 398 & 134.36 & 115.50 & 104.47 & 96.64 & 90.57 & 85.61 & 177.73 & 206.75 & 228.58 & 247.09 & 263.65 & 278.93 \\
\hline 399 & 134.52 & 115.63 & 104.59 & 96.75 & 90.68 & 85.71 & 177.97 & 207.03 & 228.89 & 247.43 & 264.02 & 279.32 \\
\hline 400 & 134.89 & 115.96 & 104.88 & 97.02 & 90.93 & 85.95 & 177.92 & 206.97 & 228.83 & 247.36 & 263.94 & 279.24 \\
\hline
\end{tabular}


Table B.2: Simulation Data for Picking Times (High Rate)—continued.

\begin{tabular}{|c|c|c|c|c|c|c|c|c|c|c|c|c|}
\hline \multirow{2}{*}{$\begin{array}{c}\text { Batch } \\
\text { Size }\end{array}$} & \multicolumn{6}{|c|}{ Picking Time (minutes) } & \multicolumn{6}{|c|}{ Picking Rate (orders/hr) } \\
\hline & 20 & 40 & 60 & 80 & 100 & 120 & 20 & 40 & 60 & 80 & 100 & 120 \\
\hline 401 & 135.12 & 116.16 & 105.06 & 97.19 & 91.08 & 86.10 & 178.06 & 207.14 & 229.01 & 247.56 & 264.15 & 279.46 \\
\hline 402 & 135.50 & 116.48 & 105.35 & 97.46 & 91.34 & 86.33 & 178.01 & 207.08 & 228.95 & 247.49 & 264.08 & 279.38 \\
\hline 403 & 135.86 & 116.79 & 105.63 & 97.72 & 91.58 & 86.56 & 177.98 & 207.04 & 228.91 & 247.45 & 264.03 & 279.33 \\
\hline 404 & 136.08 & 116.98 & 105.81 & 97.88 & 91.73 & 86.71 & 178.13 & 207.21 & 229.09 & 247.65 & 264.25 & 279.56 \\
\hline 405 & 136.37 & 117.23 & 106.03 & 98.09 & 91.92 & 86.89 & 178.20 & 207.29 & 229.18 & 247.74 & 264.35 & 279.67 \\
\hline 406 & 136.72 & 117.53 & 106.30 & 98.34 & 92.16 & 87.11 & 178.18 & 207.27 & 229.16 & 247.72 & 264.32 & 279.64 \\
\hline 407 & 137.09 & 117.85 & 106.59 & 98.61 & 92.41 & 87.35 & 178.13 & 207.21 & 229.09 & 247.65 & 264.25 & 279.56 \\
\hline 408 & 137.26 & 117.99 & 106.72 & 98.73 & 92.53 & 87.46 & 178.35 & 207.47 & 229.38 & 247.95 & 264.57 & 279.90 \\
\hline 409 & 137.57 & 118.26 & 106.97 & 98.95 & 92.74 & 87.66 & 178.38 & 207.50 & 229.42 & 248.00 & 264.62 & 279.95 \\
\hline 410 & 137.86 & 118.51 & 107.19 & 99.16 & 92.93 & 87.84 & 178.44 & 207.57 & 229.49 & 248.08 & 264.71 & 280.05 \\
\hline 411 & 138.13 & 118.74 & 107.40 & 99.35 & 93.11 & 88.01 & 178.53 & 207.68 & 229.62 & 248.21 & 264.85 & 280.20 \\
\hline 412 & 138.51 & 119.07 & 107.70 & 99.63 & 93.37 & 88.25 & 178.47 & 207.61 & 229.54 & 248.13 & 264.76 & 280.10 \\
\hline 413 & 138.77 & 119.30 & 107.90 & 99.82 & 93.55 & 88.42 & 178.56 & 207.72 & 229.66 & 248.26 & 264.90 & 280.25 \\
\hline 414 & 139.08 & 119.56 & 108.14 & 100.04 & 93.75 & 88.62 & 178.60 & 207.76 & 229.70 & 248.31 & 264.95 & 280.31 \\
\hline 415 & 139.35 & 119.79 & 108.35 & 100.23 & 93.93 & 88.79 & 178.69 & 207.87 & 229.82 & 248.43 & 265.09 & 280.45 \\
\hline 416 & 139.74 & 120.13 & 108.65 & 100.51 & 94.20 & 89.04 & 178.62 & 207.78 & 229.72 & 248.33 & 264.98 & 280.33 \\
\hline 417 & 139.95 & 120.30 & 108.81 & 100.66 & 94.34 & 89.17 & 178.78 & 207.98 & 229.94 & 248.56 & 265.22 & 280.59 \\
\hline 418 & 140.32 & 120.63 & 109.11 & 100.93 & 94.59 & 89.41 & 178.73 & 207.91 & 229.87 & 248.48 & 265.14 & 280.50 \\
\hline 419 & 140.51 & 120.79 & 109.25 & 101.07 & 94.72 & 89.53 & 178.91 & 208.13 & 230.10 & 248.74 & 265.42 & 280.79 \\
\hline 420 & 140.90 & 121.12 & 109.55 & 101.35 & 94.98 & 89.78 & 178.85 & 208.05 & 230.02 & 248.65 & 265.32 & 280.69 \\
\hline 421 & 141.30 & 121.46 & 109.86 & 101.63 & 95.25 & 90.03 & 178.77 & 207.96 & 229.92 & 248.54 & 265.20 & 280.57 \\
\hline 422 & 141.56 & 121.69 & 110.07 & 101.82 & 95.43 & 90.20 & 178.86 & 208.07 & 230.04 & 248.67 & 265.34 & 280.71 \\
\hline 423 & 141.80 & 121.90 & 110.25 & 101.99 & 80.00 & 90.35 & 178.99 & 208.21 & 230.20 & 248.84 & 265.52 & 280.91 \\
\hline 424 & 142.04 & 122.10 & 110.44 & 102.17 & 95.75 & 90.50 & 179.10 & 208.35 & 230.35 & 249.00 & 265.70 & 281.09 \\
\hline 425 & 142.42 & 122.43 & 110.74 & 102.44 & 96.01 & 90.75 & 179.04 & 208.28 & 230.27 & 248.92 & 265.61 & 281.00 \\
\hline 426 & 142.65 & 122.63 & 110.91 & 102.60 & 96.16 & 90.89 & 179.18 & 208.44 & 230.45 & 249.12 & 265.81 & 281.22 \\
\hline 427 & 143.05 & 122.97 & 111.22 & 102.89 & 96.43 & 91.14 & 179.10 & 208.35 & 230.35 & 249.01 & 265.70 & 281.09 \\
\hline 428 & 143.34 & 123.22 & 111.45 & 103.10 & 96.62 & 91.33 & 179.16 & 208.41 & 230.42 & 249.08 & 265.78 & 281.18 \\
\hline 429 & 143.62 & 123.46 & 111.67 & 103.30 & 96.81 & 91.51 & 179.22 & 208.49 & 230.50 & 249.17 & 265.88 & 281.28 \\
\hline 430 & 143.97 & 123.76 & 111.94 & & 97.05 & 91.73 & 179.20 & 208.47 & 230.48 & 249.15 & 265.85 & 281.25 \\
\hline 431 & 144.18 & 123.94 & 112.11 & 103.71 & 97.19 & 91.87 & 179.36 & 208.64 & 230.68 & 249.36 & 266.07 & 281.49 \\
\hline 432 & 144.49 & 124.21 & 112.35 & 103.93 & 97.40 & 92.06 & 179.39 & 208.68 & 230.72 & 249.40 & 266.12 & 281.54 \\
\hline 433 & 144.83 & 124.50 & 112.61 & 104.17 & 97.63 & 92.28 & 179.39 & 208.68 & 230.71 & 249.40 & 266.12 & 281.54 \\
\hline 434 & 145.13 & 124.76 & 112.84 & 104.39 & 97.83 & 92.47 & 179.42 & 208.72 & 230.76 & 249.45 & 266.17 & 281.60 \\
\hline 435 & 145.43 & 125.01 & 113.07 & 104.60 & 98.03 & 92.66 & 179.47 & 208.78 & 230.82 & 249.52 & 266.24 & 281.67 \\
\hline 436 & 145.72 & 125.27 & 113.30 & 104.81 & 98.23 & 92.85 & 179.52 & 208.84 & 230.89 & 249.59 & 266.32 & 281.75 \\
\hline 437 & 145.96 & 125.47 & 113.49 & 104.99 & 98.39 & 93.00 & 179.64 & 208.97 & 231.03 & 249.75 & 266.49 & 281.93 \\
\hline 438 & 146.32 & 125.78 & 113.77 & 105.24 & 98.63 & 93.23 & 179.61 & 208.93 & 231.00 & 249.70 & 266.44 & 281.88 \\
\hline 439 & 146.61 & 126.03 & 113.99 & 105.45 & 98.83 & 93.41 & 179.67 & 209.00 & 231.07 & 249.79 & 266.53 & 281.97 \\
\hline 440 & 146.94 & 126.32 & 114.25 & 105.69 & 99.05 & 93.63 & 179.66 & 209.00 & 231.06 & 249.78 & 266.52 & 281.97 \\
\hline 441 & 147.28 & 126.60 & 114.51 & 105.93 & 99.28 & 93.84 & 179.66 & 209.00 & 231.07 & 249.78 & 266.53 & 281.97 \\
\hline 442 & 147.51 & 126.80 & 114.69 & 106.10 & 99.43 & 93.99 & 179.78 & 209.14 & 231.23 & 249.95 & 266.71 & 282.16 \\
\hline
\end{tabular}


Table B.2: Simulation Data for Picking Times (High Rate)—continued.

\begin{tabular}{|c|c|c|c|c|c|c|c|c|c|c|c|c|}
\hline \multirow{2}{*}{$\begin{array}{c}\text { Batch } \\
\text { Size }\end{array}$} & \multicolumn{6}{|c|}{ Picking Time (minutes) } & \multicolumn{6}{|c|}{ Picking Rate (orders/hr) } \\
\hline & 20 & 40 & 60 & 80 & 100 & 120 & 20 & 40 & 60 & 80 & 100 & 120 \\
\hline 443 & 147.81 & 127.06 & 114.92 & 106.31 & 9.64 & 94.18 & 179.83 & 209.19 & 231.28 & 250.01 & 266.77 & 282.23 \\
\hline 444 & 148.15 & 127.36 & 115.19 & 106.56 & 99.87 & 94.40 & 179.81 & 209.17 & 231.26 & 249.99 & 266.75 & 282.21 \\
\hline 445 & 8.43 & 127.60 & 115.41 & 106.76 & 100.06 & 94.58 & 179.88 & 209.25 & 231.35 & 250.08 & 266.85 & 282.31 \\
\hline 446 & 148.73 & 127.85 & 115.64 & 106.98 & 100.26 & 94.77 & 179.92 & 209.30 & 231.40 & 250.15 & 266.91 & 282.38 \\
\hline 447 & 149.04 & 128.12 & 115.88 & 107.20 & 100.47 & 94.96 & 179.95 & 209.33 & 231.44 & 250.18 & 266.95 & 282.42 \\
\hline 448 & 149.29 & 128.34 & 116.08 & 107.38 & 100.64 & 95.12 & 180.05 & 209.45 & 231.57 & 250.32 & 267.10 & 282.58 \\
\hline 449 & 149.59 & 128.59 & 116.31 & 107.60 & 100.84 & 95.31 & 180.09 & 209.50 & 231.62 & 250.38 & 267.17 & 282.65 \\
\hline 450 & 149.91 & 128.87 & 116.56 & 107.83 & 101.06 & 95.52 & 180.10 & 209.51 & 231.64 & 250.40 & 267.18 & 282.66 \\
\hline 451 & 150.25 & 129.16 & 116.83 & 108.07 & 101.29 & 95.74 & 180.09 & 209.50 & 231.62 & 250.38 & 267.17 & 282.65 \\
\hline 452 & 150.50 & 129.38 & 117.02 & 108.25 & 101.45 & 95.89 & 180.20 & 209.62 & 231.76 & 250.53 & 267.32 & 282.81 \\
\hline 453 & 150.86 & 129.69 & 117.30 & 108.51 & 101.70 & 96.13 & 180.16 & 209.58 & 231.71 & 250.48 & 267.27 & 282.76 \\
\hline 454 & 151.11 & 129.90 & 117.50 & 108.69 & 101.86 & 96.29 & 180.26 & 209.69 & 231.84 & 250.61 & 267.41 & 282.91 \\
\hline 455 & 151.47 & 130.21 & 117.77 & 108.95 & 102.10 & 96.51 & 180.23 & 209.66 & 231.80 & 250.58 & 267.37 & 282.87 \\
\hline 456 & 151.67 & 130.38 & 117.93 & 109.09 & 102.24 & 96.64 & 180.40 & 209.85 & 232.01 & 250.80 & 267.61 & 283.12 \\
\hline 457 & 152.01 & 130.68 & 118.19 & 109.34 & 102.47 & 96.86 & 180.38 & 209.83 & 231.99 & 250.78 & 267.59 & 283.10 \\
\hline 458 & 152.34 & 130.96 & 118.45 & 109.58 & 102.69 & 97.07 & 180.38 & 209.84 & 232.00 & 250.79 & 267.60 & 283.10 \\
\hline 459 & 152.67 & 131.24 & 118.71 & 109.81 & 102.91 & 97.28 & 180.39 & 209.84 & 232.00 & 250.79 & 267.60 & 283.11 \\
\hline 460 & 152.92 & 131.46 & 118.90 & 109.99 & 103.08 & 97.44 & 180.48 & 209.95 & 232.12 & 250.92 & 267.74 & 283.26 \\
\hline 461 & 153.19 & 131.69 & 119.11 & 110.19 & 103.27 & 97.61 & 180.56 & 210.04 & 232.22 & 251.03 & 267.85 & 283.37 \\
\hline 462 & 153.52 & 131.97 & 119.36 & 110.42 & 103.48 & 97.82 & 180.57 & 210.05 & 232.23 & 251.04 & 267.87 & 283.39 \\
\hline 463 & 153.87 & 132.27 & 119.64 & 110.67 & 103.72 & 98.04 & 180.54 & 210.02 & 232.20 & 251.01 & 267.83 & 283.35 \\
\hline 464 & 154.15 & 132.51 & 119.85 & 110.87 & 103.91 & 98.22 & 180.61 & 210.10 & 232.28 & 251.09 & 267.93 & 283.45 \\
\hline 465 & 154.47 & 132.79 & 120.10 & 111.11 & 104.13 & 08 & 180.62 & 210.11 & 232.30 & 251.11 & 267.95 & 283.47 \\
\hline 466 & 154.80 & 133.07 & 120.36 & 111.34 & 104.35 & 98.63 & 180.62 & 210.11 & 232.30 & 251.11 & 267.95 & 283.47 \\
\hline 467 & 155.09 & 133.32 & 120.59 & 111.55 & 104.55 & 98.82 & 180.67 & 210.16 & 232.36 & 251.18 & 268.01 & 283.54 \\
\hline 468 & 155.35 & 133.55 & 120.79 & 111.74 & 104.72 & 98.99 & 180.75 & 210.26 & 232.46 & 251.29 & 268.14 & 283.67 \\
\hline 469 & 155.58 & 133.74 & 120.97 & 111.90 & 104.87 & 99.13 & 180.87 & 210.40 & 232.62 & 251.46 & 268.32 & 283.87 \\
\hline 470 & 155.94 & 134.05 & 121.25 & 112.17 & 105.12 & 99.36 & 180.84 & 210.36 & 232.58 & 251.41 & 268.27 & 283.81 \\
\hline 471 & 156.26 & 134.33 & 121.50 & 112.39 & 105.33 & 99.56 & 180.85 & 210.38 & 232.60 & 251.44 & 268.29 & 283.84 \\
\hline 472 & 156.55 & 134.57 & 121.72 & & & & 180.90 & 210.44 & 232.66 & 251.51 & 268.37 & 283.92 \\
\hline 473 & 156.79 & 134.78 & 121.91 & 112.77 & 105.69 & 99.90 & 181.01 & 210.57 & 232.80 & 251.66 & 268.53 & 284.09 \\
\hline 474 & 157.15 & 135.09 & 122.19 & 113.04 & 105.94 & 100.13 & 180.97 & 210.52 & & & & 284.02 \\
\hline 475 & 157.45 & 135.35 & 122.42 & 113.25 & 106.13 & 100.32 & 181.01 & 210.57 & 232.81 & 251.66 & 268.53 & 284.09 \\
\hline 476 & 157.67 & 135.54 & 122.59 & 113.41 & 106.28 & 100.46 & 181.14 & 210.72 & 232.97 & 251.84 & 268.72 & 284.29 \\
\hline 477 & 158.04 & 135.86 & 122.88 & 113.67 & 106.53 & 100.70 & 181.09 & 210.66 & 232.91 & 251.77 & 268.65 & 284.22 \\
\hline 478 & 158.31 & 136.09 & 123.09 & 113.87 & 106.71 & 100.87 & 181.17 & 210.75 & 233.00 & 251.87 & 268.76 & 284.33 \\
\hline 479 & 158.71 & 136.43 & 123.40 & 114.16 & 106.99 & 101.13 & 181.08 & 210.65 & 232.90 & 251.76 & 268.63 & 284.20 \\
\hline 480 & 158.97 & 136.66 & 123.60 & 114.34 & 107.16 & 101.29 & 181.17 & 210.75 & 233.00 & 251.88 & 268.76 & 284.33 \\
\hline 481 & 159.26 & 136.90 & 123.83 & 114.55 & 107.35 & 101.47 & 181.22 & 210.81 & 233.07 & 251.94 & 268.83 & 284.41 \\
\hline 482 & 159.51 & 137.12 & 124.02 & 114.73 & 107.52 & 101.63 & 181.31 & 210.91 & 233.19 & 252.07 & 268.97 & 284.56 \\
\hline 483 & 159.80 & 137.37 & 124.25 & 114.94 & 107.72 & 101.82 & 181.35 & 210.96 & 233.24 & 252.13 & 269.03 & 284.62 \\
\hline 484 & 160.17 & 137.68 & 124.53 & 115.20 & 107.97 & 102.05 & 181.31 & 210.92 & 233.19 & 252.08 & 268.97 & 284.56 \\
\hline
\end{tabular}


Table B.2: Simulation Data for Picking Times (High Rate)—continued.

\begin{tabular}{|c|c|c|c|c|c|c|c|c|c|c|c|c|}
\hline \multirow{2}{*}{$\begin{array}{c}\text { Batch } \\
\text { Size }\end{array}$} & \multicolumn{6}{|c|}{ Picking Time (minutes) } & \multicolumn{6}{|c|}{ Picking Rate (orders/hr) } \\
\hline & 20 & 40 & 60 & 80 & 100 & 120 & 20 & 40 & 60 & 80 & 100 & 120 \\
\hline 485 & 160.45 & 137.93 & 124.75 & 115.41 & 108.16 & 102.23 & 181.37 & 210.98 & 233.26 & 252.15 & 269.06 & 284.65 \\
\hline 486 & 160.74 & 138.18 & 124.98 & 115.62 & 108.35 & 102.42 & 181.41 & 211.03 & 233.31 & 252.21 & 269.12 & 284.71 \\
\hline 487 & 161.04 & 138.44 & 125.21 & 115.83 & 108.56 & 102.61 & 181.45 & 211.07 & 233.36 & 252.26 & 269.17 & 284.77 \\
\hline 488 & 161.31 & 138.67 & 125.42 & 116.03 & 108.74 & 102.78 & 181.51 & 211.15 & 233.45 & 252.36 & 269.27 & 284.88 \\
\hline 489 & 161.68 & 138.99 & 125.71 & 116.29 & 108.99 & 103.02 & 181.47 & 211.10 & 233.39 & 252.29 & 269.21 & 284.80 \\
\hline 490 & & 139.2 & 125.91 & 116.48 & 109.16 & 103.18 & & 211.20 & 233.50 & 252.41 & 269.33 & 284.94 \\
\hline 491 & 162.24 & 139.47 & 126.15 & 116.70 & 109.36 & 103.37 & 181.58 & 211.23 & 233.54 & 252.45 & 269.38 & 284.98 \\
\hline 492 & 162.57 & 139.76 & 126.41 & 116.94 & 109.59 & 103.59 & 181.58 & 211.23 & 233.53 & 252.45 & 269.37 & 284.98 \\
\hline 493 & 162.81 & 139.96 & 126.59 & 117.11 & 109.75 & 103.74 & 181.68 & 211.35 & 233.67 & 252.59 & 269.52 & 285.14 \\
\hline 494 & 163.16 & 140.26 & 126.86 & 117.35 & 109.98 & 103.96 & 181.67 & 211.33 & 233.64 & 252.57 & 269.50 & 285.11 \\
\hline 495 & 163.46 & 140.52 & 127.10 & 117. & 110.19 & 104.15 & 181. & 211.36 & 233.68 & 252 & 269.54 & 285.16 \\
\hline 496 & 163.73 & 140.75 & 127.31 & 117.77 & 110.37 & 104.33 & & 211.44 & 233.77 & 252.70 & 269.64 & 285.26 \\
\hline 497 & 164.07 & 141.04 & 127.57 & 118.01 & 110.60 & 104.54 & 181.75 & 211.42 & 233.75 & 252.68 & 269.62 & 285.24 \\
\hline 498 & 164.29 & 141.23 & 127.74 & 118.17 & 110.75 & 104.68 & 181.87 & 211.56 & 233.91 & 252.85 & 269.80 & 285.43 \\
\hline 499 & 164.65 & 141.54 & 128.02 & 118.43 & 110.99 & 104.91 & 181.84 & 211.53 & 233.87 & 252.81 & 269.76 & 285.39 \\
\hline 500 & 164.97 & 141.81 & 128.27 & & 111.20 & & 181.8 & 211.55 & 233.89 & 252.83 & 269.78 & 285.41 \\
\hline 501 & 165.32 & 142.12 & 128.54 & 118.91 & 111.44 & 105.34 & 181.83 & 211.52 & 233.85 & 252.79 & 269.74 & 285.37 \\
\hline 502 & 165.52 & 142.29 & 128.70 & 119.05 & 111.57 & 105.46 & 181.97 & 211.69 & 234.04 & 252.99 & 269.95 & 285.60 \\
\hline 503 & & 142.63 & 129.00 & & 111.84 & 105.72 & & 211.60 & 233.95 & 252.89 & 269.85 & 285.48 \\
\hline 504 & 166.19 & 142.86 & 129.22 & & 112.03 & 105.89 & & 211.67 & 234.03 & 252.98 & 269.94 & 285.58 \\
\hline 505 & 166.44 & 143.07 & 129.41 & 119.71 & 112.19 & & 182.05 & 211.78 & 234.14 & 253.10 & 270.07 & 285.72 \\
\hline 506 & 166.80 & 143.39 & 129.69 & & 112.44 & 106.28 & 182.01 & 211.73 & 234.09 & 253.05 & 270.02 & 285.66 \\
\hline 507 & 166.97 & 143.54 & 129.83 & 120.10 & 112.55 & 106.39 & 18210 & 211.93 & 234.31 & 253.29 & 270.27 & 285.93 \\
\hline 508 & 167.33 & 143.85 & 130.11 & 120.36 & 112.80 & 106.62 & 182.15 & 211.89 & 234.27 & 253 & 270.22 & 285.88 \\
\hline 509 & 167.60 & 144.07 & 130.31 & 120.55 & 112.98 & 106.79 & 182.22 & 211.97 & 234.36 & 253.34 & 270.32 & 285.99 \\
\hline 510 & & & & & & & & & & & & 285.94 \\
\hline 511 & 168.25 & 144.64 & 130.82 & 121.02 & 113.42 & 107.21 & 182.23 & 211.98 & 234.37 & 253.35 & 270.33 & 285.99 \\
\hline 512 & 168.52 & 144.86 & & 121.21 & 113.59 & & 18232 & 212.06 & & & 270.44 & 286.11 \\
\hline 513 & 168.83 & 145.14 & 131.27 & 121.44 & 113.81 & 107.58 & 182.31 & 212.08 & 234.47 & 253.46 & 270.45 & 286.12 \\
\hline 514 & 169.22 & 145.47 & 131.57 & 121.71 & 114.07 & 107.82 & 182.25 & 212.01 & 234.40 & 253.38 & 270.37 & 286.03 \\
\hline 515 & 169.44 & 145.66 & 131.75 & 121.88 & & & 1000 & & 234.54 & & 270.53 & 286.21 \\
\hline 516 & 169.77 & 145.94 & 132.00 & 122.11 & 114.44 & 108.17 & 182.36 & 212.14 & 234.54 & 253.54 & 270.53 & 286.21 \\
\hline 517 & 170.02 & 146.15 & 132.19 & 122.29 & 114.61 & & 102.40 & 212.24 & & & 270.67 & 286.35 \\
\hline 518 & 170.34 & 146.43 & 132.44 & & 114.82 & & 182.46 & 212.25 & 234.67 & 253.67 & 270.68 & 286.36 \\
\hline 519 & 170.73 & 146.76 & 132.75 & 122.80 & 115.08 & & 182.40 & 212.18 & & & & 286.26 \\
\hline 520 & & & & & & & & 212.25 & & & 270.67 & 286.36 \\
\hline 521 & 171.26 & 147.22 & 133.16 & 123.19 & 115.45 & 109.12 & 182.53 & 212.33 & 234.75 & 253.76 & 270.77 & 286.46 \\
\hline 522 & 171.46 & 147.39 & 133.32 & 123.33 & 115.58 & 109.25 & 182.67 & 212.49 & 234.93 & 253.96 & 270.98 & 286.69 \\
\hline 523 & 171.85 & 147.73 & 133.62 & 123.61 & 115.84 & 109.50 & 182.60 & 212.41 & 234.84 & 253.86 & 270.88 & 286.58 \\
\hline 524 & 172.14 & 147.97 & 133.84 & 123.81 & 116.03 & 109.68 & 182.65 & 212.47 & 234.91 & 253.93 & 270.95 & 286.65 \\
\hline 525 & 172.43 & 148.23 & 134.07 & 124.03 & 116.24 & 109. & 182.68 & 212.51 & 234.95 & 253.98 & 271.00 & 286.70 \\
\hline 526 & 172.83 & 148.57 & 134.38 & 124.31 & 116.50 & 110.12 & 182.61 & 212.43 & 234.86 & 253.88 & 270.90 & 286.60 \\
\hline
\end{tabular}


Table B.2: Simulation Data for Picking Times (High Rate)—continued.

\begin{tabular}{|c|c|c|c|c|c|c|c|c|c|c|c|c|}
\hline \multirow{2}{*}{$\begin{array}{c}\text { Batch } \\
\text { Size }\end{array}$} & \multicolumn{6}{|c|}{ Picking Time (minutes) } & \multicolumn{6}{|c|}{ Picking Rate (orders/hr) } \\
\hline & 20 & 40 & 60 & 80 & 100 & 120 & 20 & 40 & 60 & 80 & 100 & 120 \\
\hline 527 & 173.02 & 148.73 & 134.52 & 124.45 & 116.63 & 110.24 & 182.76 & 212.60 & 235.05 & 254.09 & 271.12 & 286.83 \\
\hline 528 & 173.48 & 149.13 & 134.88 & 124.78 & 116.94 & 110.53 & 182.62 & 212.44 & 234.87 & 253.89 & 270.91 & 286.61 \\
\hline 529 & 173.69 & 149.31 & 135.05 & 124.93 & 117.08 & 110.67 & 182.74 & 212.58 & 235.03 & 254.07 & 271.10 & 286.80 \\
\hline 530 & 173.99 & 149.57 & 135.28 & 125.15 & 117.28 & 110.86 & 182.77 & 212.61 & 235.06 & 254.10 & 271.14 & 286.85 \\
\hline 531 & 174.22 & 149.76 & 135.46 & 125.31 & 117.44 & 111.01 & 182.88 & 212.74 & 235.20 & 254.25 & 271.29 & 287.01 \\
\hline 532 & 174.57 & 150.07 & 135.73 & 125.56 & 117.68 & 111.23 & 182.85 & 212.70 & 235.16 & 254.21 & 271.25 & 286.97 \\
\hline 533 & 174.86 & 150.31 & 135.96 & 125.77 & 117.87 & 111.41 & 182.89 & 212.75 & 235.22 & 254.27 & 271.32 & 287.04 \\
\hline 534 & 175.14 & 150.56 & 136.18 & 125.97 & 118.06 & 111.59 & 182.94 & 212.81 & 235.28 & 254.34 & 271.39 & 287.11 \\
\hline 535 & 175.48 & 150.85 & 136.44 & 126.22 & 118.29 & 111.81 & 182.93 & 212.80 & 235.27 & 254.32 & 271.37 & 287.09 \\
\hline 536 & 175.80 & 151.12 & 136.69 & 126.45 & 118.50 & 112.01 & 182.94 & 212.81 & 235.28 & 254.33 & 271.38 & 287.11 \\
\hline 537 & 176.07 & 151.36 & 136.90 & 126.65 & 118.69 & 112.19 & 182.99 & 212.87 & 235.35 & 254.41 & 271.46 & 287.19 \\
\hline 538 & 176.45 & 151.68 & 137.19 & 126.91 & 118.94 & 112.43 & 182.94 & 212.82 & 235.29 & 254.35 & 271.39 & 287.12 \\
\hline 539 & 176.64 & 151.85 & 137.34 & 127.05 & 119.07 & 112.55 & 183.08 & 212.98 & 235.47 & 254.54 & 271.60 & 287.34 \\
\hline 540 & 176.92 & 152.09 & 137.56 & 127.25 & 119.26 & 112.73 & 183.14 & 213.04 & 235.53 & 254.61 & 271.68 & 287.42 \\
\hline 541 & 177.32 & 152.43 & 137.87 & 127.54 & 119.53 & 112.98 & 183.06 & 212.95 & 235.44 & 254.51 & 271.57 & 287.30 \\
\hline 542 & 177.62 & 152.69 & 138.10 & 127.76 & 119.73 & 113.17 & 183.09 & 212.98 & 235.47 & 254.55 & 271.61 & 287.35 \\
\hline 543 & 177.88 & 152.91 & 138.31 & 127.94 & 119.91 & 113.34 & 183.16 & 213.06 & 235.56 & 254.64 & 271.71 & 287.46 \\
\hline 544 & 178.26 & 153.24 & 138.60 & 128.22 & 120.16 & 113.58 & 183.11 & 213.00 & 235.50 & 254.57 & 271.64 & 287.38 \\
\hline 545 & 178.40 & 153.36 & 138.71 & 128.32 & 120.25 & 113.67 & 183.30 & 213.23 & 235.75 & 254.84 & 271.92 & 287.68 \\
\hline 546 & 178.75 & 153.66 & 138.98 & 128.57 & 120.49 & 113.89 & 183.27 & 213.20 & 235.71 & 254.80 & 271.88 & 287.64 \\
\hline 547 & 179.07 & 153.94 & 139.24 & 128.80 & 120.71 & 114.10 & 183.28 & 213.20 & 235.72 & 254.81 & 271.89 & 287.64 \\
\hline 548 & 179.34 & 154.17 & 139.44 & 128.99 & 120.89 & 114.27 & 183.34 & 213.28 & 235.80 & 254.89 & 271.98 & 287.74 \\
\hline 549 & 179.66 & 154.45 & 139.69 & & & 114.48 & 18224 & 213.28 & 235.80 & 254.90 & & 287.75 \\
\hline 550 & 180.01 & 154.75 & 139.97 & 129.48 & 121.35 & 114.70 & 183.32 & 213.25 & 235.77 & 254.87 & 271.95 & 287.71 \\
\hline 551 & 180.29 & 154.98 & 140.18 & 129.68 & 121.53 & 114.87 & 183.37 & 213.32 & 235.84 & 254.94 & 272.03 & 287.79 \\
\hline 552 & 180.59 & 155.25 & 140.42 & 129.90 & 121.74 & 115.07 & 183.39 & 213.34 & 235.87 & 254.97 & 272.06 & 287.83 \\
\hline 553 & 180.84 & 155.46 & 140.61 & 130.07 & 121.90 & 115.23 & 183.48 & 213.43 & 235.97 & 255.09 & 272.18 & 287.96 \\
\hline 554 & 181.20 & 155.77 & 140.89 & 130.33 & 122.15 & 115.46 & 100.44 & 213.40 & 235.93 & 255.04 & 272.14 & 287.90 \\
\hline 555 & 181.51 & 156.03 & 141.13 & 130.56 & 122.35 & 115.65 & 183.46 & 213.42 & 235.95 & 255.06 & 272.16 & 287.93 \\
\hline 556 & 181.74 & 156.23 & & & 122.51 & & 183.55 & & 236.07 & & 272.30 & 288.08 \\
\hline 557 & 182.11 & 156.54 & 141.59 & 130.98 & 122.76 & 116.03 & 183.52 & 213.49 & 236.03 & 255.15 & 272.25 & 288.02 \\
\hline 558 & 182.37 & 156.78 & 141.80 & & 122.94 & & 183.58 & 213.55 & 236.10 & & & 288.12 \\
\hline 559 & 182.64 & 157.01 & 142.01 & 131.37 & 123.12 & 116.37 & 183.64 & 213.62 & 236.18 & 255.31 & 272.42 & 288.21 \\
\hline 560 & 183.02 & 157.33 & 142.30 & 131.64 & 123.37 & 116.61 & 183.59 & 213.57 & 236.12 & 255.24 & 272.35 & 288.14 \\
\hline 561 & 183.22 & 157.50 & 142.46 & 131.79 & 123.51 & 116.74 & 183.71 & 213.71 & 236.28 & 255.41 & 272.54 & 288.33 \\
\hline 562 & 183.52 & 157.76 & 142.69 & 132.00 & 123.71 & 116.93 & 183.74 & 213.74 & 236.31 & 255.45 & 272.58 & 288.37 \\
\hline 563 & 183.82 & 158.02 & 142.93 & 132.22 & 123.91 & 117.13 & 183.76 & 213.77 & 236.34 & 255.48 & 272.61 & 288.40 \\
\hline 564 & 184.18 & 158.33 & 143.21 & 132.48 & 124.16 & 117.36 & 183.73 & 213.73 & 236.30 & 255.44 & 272.56 & 288.35 \\
\hline 565 & 184.53 & 158.63 & 143.48 & 132.73 & 124.39 & 117.58 & 183.71 & 213.71 & 236.28 & 255.41 & 272.53 & 288.33 \\
\hline 566 & 184.8 & 158.88 & 143.70 & 132.93 & 124.58 & 117.76 & 183.75 & 213.75 & 236.32 & 255.46 & 272.59 & 288.38 \\
\hline 567 & 185.06 & 159.08 & 143.89 & 133.11 & 124.75 & 117.91 & 183.83 & 213.85 & 236.43 & 255.58 & 272.71 & 288.51 \\
\hline 568 & 185.30 & 159.29 & 144.08 & 133.28 & 124.91 & 118.07 & 183.92 & 213.95 & 236.54 & 255.70 & 272.84 & 288.65 \\
\hline
\end{tabular}


Table B.2: Simulation Data for Picking Times (High Rate)—continued.

\begin{tabular}{|c|c|c|c|c|c|c|c|c|c|c|c|c|}
\hline \multirow{2}{*}{$\begin{array}{c}\text { Batch } \\
\text { Size }\end{array}$} & \multicolumn{6}{|c|}{ Picking Time (minutes) } & \multicolumn{6}{|c|}{ Picking Rate (orders/hr) } \\
\hline & 20 & 40 & 60 & 80 & 100 & 120 & 20 & 40 & 60 & 80 & 100 & 120 \\
\hline 569 & 185.73 & 159.66 & 144.41 & 133.59 & 125.20 & 118.34 & 183.82 & 213.83 & 236.41 & 255.56 & 272.69 & 288.49 \\
\hline 570 & 186.00 & 159.89 & 144.62 & 133.78 & 125.38 & 118.51 & 183.87 & 213.90 & 236.48 & 255.64 & 272.77 & 288.58 \\
\hline 571 & 36.31 & 160.15 & 144.86 & 134.00 & 125.59 & 118.71 & 183.89 & 213.92 & 236.51 & 255.66 & 272.80 & 288.61 \\
\hline 572 & 186.53 & 160.35 & 145.03 & 134.17 & 125.74 & 118.85 & 183.99 & 214.03 & 236.63 & 255.80 & 272.95 & 288.76 \\
\hline 573 & 186.78 & 160.56 & 145.23 & 134.35 & 125.91 & 119.01 & 184.07 & 214.12 & 236.73 & 255.91 & 273.06 & 288.88 \\
\hline 574 & 187.24 & 160.96 & 145.58 & 134.67 & 126.21 & 119.30 & 183.94 & 213.97 & 236.57 & 255.73 & 272.87 & 288.68 \\
\hline 575 & 187.47 & 161.16 & 145.77 & 134.84 & 126.37 & 119.45 & 184.03 & 214.08 & 236.68 & 255.85 & 273.00 & 288.82 \\
\hline 576 & 187.76 & 161.41 & 145.99 & 135.05 & 126.57 & 119.64 & 184.06 & 214.12 & 236.73 & 255.90 & 273.05 & 288.88 \\
\hline 577 & 188.09 & 161.69 & 146.25 & 135.29 & 126.79 & 119.85 & 184.06 & 214.11 & 236.72 & 255.90 & 273.05 & 288.87 \\
\hline 578 & 188.37 & 161.93 & 146.47 & 135.49 & 126.98 & 120.03 & 184.10 & 214.16 & 236.78 & 255.96 & 273.11 & 288.94 \\
\hline 579 & 188.65 & 162.17 & 146.68 & 135.69 & 127.17 & 120.20 & 184.15 & 214.22 & 236.84 & 256.02 & 273.19 & 289.02 \\
\hline 580 & 188.97 & 162.44 & 146.93 & 135.92 & 127.38 & 120.40 & 184.16 & 214.23 & 236.85 & 256.04 & 273.20 & 289.03 \\
\hline 581 & 189.29 & 162.72 & 147.18 & 136.15 & 127.60 & 120.61 & 184.16 & 214.23 & 236.85 & 256.03 & 273.20 & 289.03 \\
\hline 582 & 189.51 & 162.91 & 147.35 & 136.31 & 127.75 & 120.75 & 184.26 & 214.35 & 236.98 & 256.18 & 273.35 & 289.19 \\
\hline 583 & 189.86 & 163.21 & 147.63 & 136.56 & 127.99 & 120.98 & 184.24 & 214.32 & 236.95 & 256.14 & 273.31 & 289.15 \\
\hline 584 & 190.13 & 163.44 & 147.83 & 136.76 & 128.17 & 121.15 & 184.29 & 214.38 & 237.02 & 256.22 & 273.40 & 289.24 \\
\hline 585 & 190.52 & 163.78 & 148.13 & 137.04 & 128.43 & 121.39 & 184.23 & 214.31 & 236.95 & 256.14 & 273.31 & 289.14 \\
\hline 586 & 190.83 & 164.04 & 148.38 & 137.26 & 128.64 & 121.59 & 184.25 & 214.33 & 236.97 & 256.16 & 273.33 & 289.17 \\
\hline 587 & 191.03 & 164.22 & 148.53 & 137.40 & 128.77 & 121.72 & 184.37 & 214.47 & 237.12 & 256.33 & 273.51 & 289.36 \\
\hline 588 & 191.39 & 164.52 & 148.81 & 137.66 & 129.01 & 121.95 & 184.34 & 214.44 & 237.08 & 256.28 & 273.46 & 289.31 \\
\hline 589 & 191.64 & 164.74 & 149.00 & 137.84 & 129.18 & 122.11 & 184.41 & 214.52 & 237.18 & 256.38 & 273.57 & 289.42 \\
\hline 590 & 191.90 & 164.96 & 149.21 & 138.03 & 129.36 & 122.27 & 184.47 & 214.59 & 237.25 & 256.47 & 273.66 & 289.52 \\
\hline 591 & 192.31 & 165.32 & 149.53 & & 129.64 & 122.54 & 184.39 & 214.49 & 237.14 & 256.35 & 273.53 & 289.38 \\
\hline 592 & 192.55 & 165.52 & 149.71 & 138.50 & 129.79 & 122.69 & 184.47 & 214.59 & 237.25 & 256.47 & 273.66 & 289.52 \\
\hline 593 & 192.90 & 165.82 & 149.98 & 138.75 & 130.03 & 122.91 & 184.45 & 214.57 & 237.23 & 256.44 & 273.63 & 289.48 \\
\hline 594 & 193.13 & 166.03 & 150.17 & 138.92 & 130.19 & 123.06 & 184.53 & 214.67 & 237.33 & 256.56 & 273.75 & 289.62 \\
\hline 595 & 193.41 & 166.27 & 150.38 & 139.12 & 130.38 & 123.24 & 184.58 & 214.72 & 237.39 & 256.62 & 273.82 & 289.69 \\
\hline 596 & 193.72 & 166.53 & 150.62 & 139.33 & 130.58 & 123.43 & 184.60 & 214.74 & 237.42 & 256.65 & 273.85 & 289.72 \\
\hline 597 & 194.07 & 166.83 & 150.90 & 139.59 & 130.82 & 123.66 & 184.57 & 214.71 & 237.38 & 256.61 & 273.81 & 289.67 \\
\hline 598 & 194.40 & & & & 131.04 & & 184.57 & 214.70 & 237.37 & 256.60 & & 289.66 \\
\hline 599 & 194.70 & 167.37 & 151.38 & 140.04 & 131.24 & 124.05 & 184.59 & 214.74 & 237.41 & 256.64 & 273.84 & 289.71 \\
\hline 600 & 194.93 & 167.57 & 151.57 & & 131.40 & 124.21 & 184.68 & 214.83 & 237.52 & & & 289.84 \\
\hline 601 & 195.28 & 167.87 & 151.84 & 140.46 & 131.64 & 124.43 & 184.66 & 214.81 & 237.49 & 256.72 & 273.93 & 289.81 \\
\hline 602 & 195.52 & 168.07 & 152.02 & 140.63 & 131.80 & 124.58 & 184.74 & 214.90 & 237.60 & 256.84 & 274.06 & 289.94 \\
\hline 603 & 195.77 & 168.29 & 152.21 & 140.81 & 131.96 & 124.74 & 184.81 & 214.99 & 237.69 & 256.94 & 274.17 & 290.05 \\
\hline 604 & 196.18 & 168.64 & 152.54 & 141.11 & 132.24 & 125.00 & 184.73 & 214.89 & 237.58 & 256.82 & 274.04 & 289.92 \\
\hline 605 & 196.54 & 168.95 & 152.82 & 141.37 & 132.49 & 125.23 & 184.69 & 214.85 & 237.54 & 256.78 & 273.99 & 289.87 \\
\hline 606 & 196.84 & 169.21 & 153.05 & 141.58 & 132.68 & 125.42 & 184.72 & 214.88 & 237.58 & 256.82 & 274.03 & 289.91 \\
\hline 607 & 197.14 & 169.47 & 153.28 & 141.80 & 132.89 & 125.61 & 184.74 & 214.91 & 237.60 & 256.84 & 274.06 & 289.94 \\
\hline 608 & 197.40 & 169.69 & 153.48 & 141.98 & 133.06 & 125.78 & 184.80 & 214.98 & 237.68 & 256.93 & 274.15 & 290.04 \\
\hline 609 & 197.80 & 170.04 & 153.80 & 142.27 & 133.34 & 126.03 & 184.73 & 214.89 & 237.58 & 256.83 & 274.04 & 289.92 \\
\hline 610 & 197.99 & 170.20 & 153.95 & 142.41 & 133.47 & 126.16 & 184.85 & 215.04 & 237.74 & 257.00 & 274.23 & 290.12 \\
\hline
\end{tabular}


Table B.2: Simulation Data for Picking Times (High Rate)—continued.

\begin{tabular}{|c|c|c|c|c|c|c|c|c|c|c|c|c|}
\hline \multirow{2}{*}{$\begin{array}{c}\text { Batch } \\
\text { Size }\end{array}$} & \multicolumn{6}{|c|}{ Picking Time (minutes) } & \multicolumn{6}{|c|}{ Picking Rate (orders/hr) } \\
\hline & 20 & 40 & 60 & 80 & 100 & 120 & 20 & 40 & 60 & 80 & 100 & 120 \\
\hline 611 & 198.28 & 170.45 & 154.17 & 142.62 & 133.66 & 126.34 & 184.89 & 215.08 & 237.79 & 257.05 & 274.28 & 290.17 \\
\hline 612 & 198.64 & 170.75 & 154.45 & 142.87 & 133.90 & 126.56 & 184.86 & 215.04 & 237.75 & 257.01 & 274.24 & 290.13 \\
\hline 613 & 198.90 & 170.98 & 154.65 & 143.06 & 134.07 & 126.73 & 184.92 & 215.11 & 237.83 & 257.09 & 274.33 & 290.22 \\
\hline 614 & 199.30 & 171.32 & 154.96 & 143.35 & 134.34 & 126.99 & 184.85 & 215.03 & 237.74 & 257.00 & 274.22 & 290.11 \\
\hline 615 & 199.46 & 171.47 & 155.09 & 143.47 & 134.46 & 127.09 & 185.00 & 215.20 & 237.93 & 257.20 & 274.44 & 290.34 \\
\hline 616 & 199.75 & 171.71 & 155.31 & 143.68 & 134.65 & 127.27 & 185.03 & 215.24 & 237.97 & 257.25 & 274.49 & 290.40 \\
\hline 617 & 200.06 & 171.98 & 155.55 & 143.90 & 134.86 & 127.47 & 185.04 & 215.26 & 237.99 & 257.26 & 274.51 & 290.42 \\
\hline 618 & 200.45 & 172.32 & 155.86 & 144.18 & 135.12 & 127.72 & 184.98 & 215.19 & 237.91 & 257.18 & 274.42 & 290.32 \\
\hline 619 & 200.72 & 172.54 & 156.06 & 144.37 & 135.30 & 127.89 & 185.04 & 215.25 & 237.98 & 257.26 & 274.50 & 290.41 \\
\hline 620 & 200.98 & 172.77 & 156.27 & 144.56 & 135.48 & 128.06 & 185.09 & 215.31 & 238.05 & 257.33 & 274.58 & 290.49 \\
\hline 621 & 201.31 & 173.05 & 156.52 & 144.79 & 135.70 & 128.27 & 185.09 & 215.31 & 238.05 & 257.33 & 274.58 & 290.49 \\
\hline 622 & 201.64 & 173.34 & 156.78 & 145.04 & 135.92 & 128.48 & 185.08 & 215.30 & 238.04 & 257.32 & 274.56 & 290.47 \\
\hline 623 & 201.81 & 173.48 & 156.91 & 145.16 & 136.04 & 128.59 & 185.22 & 215.47 & 238.22 & 257.51 & 274.78 & 290.70 \\
\hline 624 & 202.26 & 173.87 & 157.27 & 145.48 & 136.34 & 128.88 & 185.11 & 215.33 & 238.07 & 257.35 & 274.60 & 290.51 \\
\hline 625 & 202.43 & 174.01 & 157.39 & 145.60 & 136.45 & 128.98 & 185.25 & 215.50 & 238.26 & 257.56 & 274.82 & 290.74 \\
\hline 626 & 202.82 & 174.35 & 157.70 & 145.88 & 136.72 & 129.23 & 185.19 & 215.43 & 238.18 & 257.47 & 274.72 & 290.64 \\
\hline 627 & 203.10 & 174.60 & 157.92 & 146.09 & 136.91 & 129.41 & 185.23 & 215.47 & 238.22 & 257.52 & 274.78 & 290.70 \\
\hline 628 & 203.35 & 174.81 & 158.11 & 146.27 & 137.08 & 129.57 & 185.29 & 215.55 & 238.31 & 257.61 & 274.88 & 290.81 \\
\hline 629 & 203.70 & 175.11 & 158.38 & 146.52 & 137.31 & 129.79 & 185.27 & 215.52 & 238.28 & 257.58 & 274.85 & 290.77 \\
\hline 630 & 204.04 & 175.40 & 158.64 & 146.76 & 137.54 & 130.01 & 185.26 & 215.51 & 238.27 & 257.57 & 274.83 & 290.76 \\
\hline 631 & 204.19 & 175.53 & 158.76 & 146.87 & 137.64 & 130.10 & 185.42 & 215.70 & 238.47 & 257.79 & 275.07 & 291.01 \\
\hline 632 & 204.57 & 175.86 & 159.06 & 147.14 & 137.90 & 130.34 & 185.37 & 215.63 & 238.40 & 257.71 & 274.99 & 290.92 \\
\hline 633 & 204.97 & 176.20 & 159.37 & 147.43 & 138.16 & 130.60 & 18530 & 215.56 & & 257.62 & & 290.82 \\
\hline 634 & 205.20 & 176.40 & 159.55 & 147.59 & 138.32 & 130.75 & 185.38 & 215.65 & 238.42 & 257.74 & 275.01 & 290.95 \\
\hline 635 & 205.55 & 176.70 & 159.82 & 147.85 & 138.56 & 130.97 & 185.35 & 215.62 & 238.39 & 257.70 & 274.97 & 290.90 \\
\hline 636 & 205.83 & 176.94 & 160.04 & 148.05 & 138.75 & 131.15 & 185.39 & 215.67 & 238.44 & 257.75 & 275.03 & 290.97 \\
\hline 637 & 206.14 & 177.21 & 160.28 & 148.27 & 138.96 & 131.35 & 185.41 & 215.68 & 238.45 & 257.77 & 275.05 & 290.98 \\
\hline 638 & 206.41 & 177.44 & 160.49 & 148.47 & 139.14 & 131.52 & 185.45 & 215.73 & 238.52 & 257.83 & & 291.06 \\
\hline 639 & 206.80 & 177.77 & 160.79 & 148.75 & 139.40 & 131.77 & 185.40 & 215.67 & 238.44 & 257.76 & 275.03 & 290.97 \\
\hline 640 & 206.99 & 177.93 & & & & 131.88 & 185.52 & & & 257.93 & & 291.16 \\
\hline 641 & 207.41 & 178.30 & 161.27 & 149.18 & 139.81 & 132.15 & 185.43 & 215.71 & 238.49 & 257.80 & 275.08 & 291.02 \\
\hline 642 & 207.46 & & & 149.22 & 139.85 & 132.19 & 185.67 & 215.99 & 238.80 & & & 291.40 \\
\hline 643 & 207.91 & 178.73 & 161.66 & 149.55 & 140.15 & 132.48 & 185.56 & 215.86 & 238.65 & 257.98 & 275.27 & 291.22 \\
\hline 644 & 208.13 & 178.92 & 161.83 & 149.70 & 140.30 & 132.62 & 185.65 & 215.96 & 238.77 & 258.11 & 275.41 & 291.37 \\
\hline 645 & 208.54 & 179.27 & 162.15 & 150.00 & 140.57 & 132.87 & 185.58 & 215.88 & 238.67 & 258.00 & 275.30 & 291.25 \\
\hline 646 & 208.77 & 179.47 & 162.33 & 150.16 & 140.73 & 133.02 & 185.66 & 215.97 & 238.78 & 258.12 & 275.42 & 291.38 \\
\hline 647 & 209.03 & 179.69 & 162.52 & 150.35 & 140.90 & 133.19 & 185.72 & 216.04 & 238.86 & 258.20 & 275.51 & 291.47 \\
\hline 648 & 209.43 & 180.03 & 162.84 & 150.64 & 141.17 & 133.44 & 185.65 & 215.96 & 238.77 & 258.11 & 275.41 & 291.37 \\
\hline 649 & 209.66 & 180.23 & 163.01 & 150.80 & 141.33 & 133.59 & 185.73 & 216.06 & 238.88 & 258.22 & 275.53 & 291.50 \\
\hline 650 & 210.07 & 180.59 & 163.34 & 151.10 & 141.61 & 133.85 & 185.65 & 215.96 & 238.77 & 258.11 & 275.41 & 291.37 \\
\hline 651 & 210.16 & 180.67 & 163.41 & 151.17 & 141.67 & 133.91 & 185.85 & 216.20 & 239.03 & 258.39 & 275.71 & 291.69 \\
\hline 652 & 210.60 & 181.04 & 163.75 & 151.48 & 141.97 & 134.19 & 185.75 & 216.08 & 238.90 & 258.25 & 275.56 & 291.53 \\
\hline
\end{tabular}


Table B.2: Simulation Data for Picking Times (High Rate)—continued.

\begin{tabular}{|c|c|c|c|c|c|c|c|c|c|c|c|c|}
\hline \multirow{2}{*}{$\begin{array}{c}\text { Batch } \\
\text { Size }\end{array}$} & \multicolumn{6}{|c|}{ Picking Time (minutes) } & \multicolumn{6}{|c|}{ Picking Rate (orders/hr) } \\
\hline & 20 & 40 & 60 & 80 & 100 & 120 & 20 & 40 & 60 & 80 & 100 & 120 \\
\hline 653 & 210.89 & 181.29 & 163.98 & 151.69 & 142.16 & 134.37 & 185.78 & 216.12 & 238.94 & 258.29 & 275.60 & 291.57 \\
\hline 654 & 211.18 & 181.54 & 164.20 & 151.90 & 142.35 & 134.56 & 185.81 & 216.15 & 238.98 & 258.33 & 275.65 & 291.62 \\
\hline 655 & 211.54 & 181.85 & 164.48 & 152.15 & 142.59 & 134.78 & 185.78 & 216.12 & 238.94 & 258.29 & 275.61 & 291.58 \\
\hline 656 & 211.76 & 182.04 & 164.65 & 152.31 & 142.74 & 134.93 & 185.87 & 216.22 & 239.05 & 258.41 & 275.74 & 291.71 \\
\hline 657 & 212.06 & 182.30 & 164.89 & 152.53 & 142.95 & 135.12 & 185.89 & 216.24 & 239.08 & 258.44 & 275.76 & 291.74 \\
\hline 658 & 212.42 & 182.60 & 165.16 & 152.79 & 143.19 & 135.35 & 185.86 & 216.21 & 239.04 & 258.40 & 275.72 & 291.70 \\
\hline 659 & 212.62 & 182.77 & 165.32 & 152.93 & 143.32 & 135.47 & 185.97 & 216.33 & 239.18 & 258.55 & 275.88 & 291.87 \\
\hline 660 & 212.97 & 183.08 & 166.27 & 153.17 & 143.54 & 135.69 & 185.94 & 216.30 & 238.16 & 258.53 & 275.89 & 291.85 \\
\hline 661 & 213.31 & 183.37 & 165.86 & 153.43 & 143.79 & 135.91 & 185.93 & 216.28 & 239.12 & 258.49 & 275.82 & 291.80 \\
\hline 662 & 213.55 & 183.57 & 166.04 & 153.60 & 143.95 & 136.06 & 186.00 & 216.37 & 239.22 & 258.60 & 275.93 & 291.92 \\
\hline 663 & 213.85 & 183.85 & 166.29 & 153.83 & 144.17 & 136.27 & 186.02 & 216.37 & 239.22 & 258.60 & 275.93 & 291.92 \\
\hline 664 & 214.15 & 184.09 & 166.51 & 154.03 & 144.36 & 136.45 & 186.04 & 216.41 & 239.27 & 258.64 & 275.98 & 291.97 \\
\hline 665 & 214.49 & 184.38 & 166.77 & 154.28 & 144.59 & 136.67 & 186.02 & 216.40 & 239.25 & 258.63 & 275.96 & 291.95 \\
\hline 666 & 214.71 & 184.57 & 166.94 & 154.43 & 144.73 & 136.80 & 186.11 & 216.50 & 239.37 & 258.75 & 276.10 & 292.10 \\
\hline 667 & 215.03 & 184.85 & 167.20 & 154.66 & 144.94 & 137.00 & 186.12 & 216.50 & 239.36 & 258.77 & 276.11 & 292.11 \\
\hline 668 & 215.31 & 185.10 & 167.42 & 154.86 & 145.13 & 137.18 & 186.15 & 216.53 & 239.39 & 258.81 & 276.16 & 292.16 \\
\hline 669 & 215.14 & 185.36 & 167.65 & 155.07 & 145.32 & 137.37 & 186.58 & 216.55 & 239.42 & 258.86 & 276.21 & 292.21 \\
\hline 670 & 215.95 & 185.61 & 167.88 & 155.27 & 145.52 & 137.55 & 186.15 & 216.58 & 239.45 & 258.90 & 276.26 & 292.26 \\
\hline 671 & 216.31 & 185.94 & 168.18 & 155.58 & 145.81 & 137.82 & 186.13 & 216.52 & 239.38 & 258.77 & 276.11 & 292.11 \\
\hline 672 & 216.75 & 186.33 & 168.53 & 155.90 & 146.11 & 138.11 & 186.02 & 216.40 & 239.25 & 258.62 & 275.96 & 291.95 \\
\hline 673 & 216.95 & 186.50 & 168.69 & 156.05 & 146.24 & 138.23 & 186.13 & 216.52 & 239.38 & 258.77 & 276.12 & 292.11 \\
\hline 674 & 217.23 & 186.74 & 168.90 & 156.25 & 146.43 & 138.41 & 186.16 & 216.56 & 239.43 & 258.82 & 276.17 & 292.17 \\
\hline 675 & 217.48 & 186.96 & 169.10 & 156.43 & 146.60 & 138.57 & 186.22 & 216.63 & 239.50 & 258.90 & & 292.26 \\
\hline 676 & 217.79 & 187.22 & 169.34 & 156.65 & 146.81 & 138.77 & 186.23 & 216.64 & 239.52 & 258.92 & 276.27 & 292.28 \\
\hline 677 & 218.01 & 187.41 & 169.51 & 156.81 & 146.96 & 138.91 & 186.32 & 216.74 & 239.63 & 259.04 & 276.40 & 292.42 \\
\hline 678 & 218.43 & 187.77 & 169.83 & 157.11 & 147.24 & 139.18 & 186.24 & 216.65 & 239.53 & 258.93 & 276.28 & 292.29 \\
\hline 679 & 218.68 & 187.99 & 170.03 & 157.29 & 147.41 & 139.34 & 186.30 & 216.71 & 239.60 & 259.00 & 276.37 & 292.38 \\
\hline 680 & 218.92 & 188.19 & 170.22 & 157.46 & 147.57 & 139.49 & 186.37 & 216.80 & 239.70 & 259.11 & 276.48 & 292.50 \\
\hline 681 & 219.31 & 188.52 & 170.52 & 157.74 & 147.83 & 139.73 & 186.32 & 216.74 & 239.62 & 259.03 & 276.40 & 292.41 \\
\hline 682 & 219.58 & & 170.73 & & & & & & & & & 292.47 \\
\hline 683 & 219.91 & 189.04 & 170.99 & 158.18 & 148.24 & 140.12 & 186.35 & 216.78 & 239.67 & 259.08 & 276.45 & 292.46 \\
\hline 684 & 220.19 & & 171.20 & 158.38 & 148.43 & & 186.38 & 216.82 & 239.71 & & & 292.52 \\
\hline 685 & 220.52 & 189.56 & 171.46 & 158.61 & 148.65 & 140.51 & 186.38 & 216.81 & 239.71 & 259.12 & 276.49 & 292.51 \\
\hline 686 & 220.77 & 189.78 & 171.65 & 158.79 & 148.82 & 140.67 & 186.44 & 216.88 & 239.79 & 259.21 & 276.58 & 292.61 \\
\hline 687 & 221.04 & 190.01 & 171.86 & 158.99 & 149.00 & 140.84 & 186.48 & 216.93 & 239.84 & 259.27 & 276.65 & 292.68 \\
\hline 688 & 221.44 & 190.36 & 172.18 & 159.28 & 149.27 & 141.09 & 186.42 & 216.85 & 239.75 & 259.17 & 276.55 & 292.57 \\
\hline 689 & 221.71 & 190.59 & 172.38 & 159.47 & 149.45 & 141.26 & 186.46 & 216.91 & 239.81 & 259.24 & 276.61 & 292.64 \\
\hline 690 & 221.80 & 190.67 & 172.46 & 159.54 & 149.51 & 141.33 & 186.65 & 217.13 & 240.06 & 259.50 & 276.90 & 292.94 \\
\hline 691 & 222.32 & 191.12 & 172.86 & 159.91 & 149.87 & 141.66 & 186.48 & 216.93 & 239.84 & 259.27 & 276.65 & 292.68 \\
\hline 692 & 222.56 & 191.32 & 173.05 & 160.08 & 150.02 & 141.81 & 186.56 & 217.02 & 239.94 & 259.37 & 276.76 & 292.79 \\
\hline 693 & 222.85 & 191.57 & 173.27 & 160.29 & 150.22 & 141.99 & 186.58 & 217.05 & 239.97 & 259.40 & 276.79 & 292.83 \\
\hline 694 & 223.24 & 191.90 & 173.57 & 160.57 & 150.48 & 142.24 & 186.53 & 216.98 & 239.90 & 259.33 & 276.71 & 292.74 \\
\hline
\end{tabular}


Table B.2: Simulation Data for Picking Times (High Rate)—continued.

\begin{tabular}{|c|c|c|c|c|c|c|c|c|c|c|c|c|}
\hline \multirow{2}{*}{$\begin{array}{c}\text { Batch } \\
\text { Size }\end{array}$} & \multicolumn{6}{|c|}{ Picking Time (minutes) } & \multicolumn{6}{|c|}{ Picking Rate (orders/hr) } \\
\hline & 20 & 40 & 60 & 80 & 100 & 120 & 20 & 40 & 60 & 80 & 100 & 120 \\
\hline 695 & 223.43 & 192.07 & 173.73 & 160.71 & 150.61 & 142.37 & 186.63 & 217.11 & 240.03 & 259.47 & 276.87 & 292.91 \\
\hline 696 & 223.74 & 192.34 & 173.97 & 160.93 & 150.82 & 142.56 & 186.64 & 217.12 & 240.05 & 259.49 & 276.88 & 292.93 \\
\hline 697 & 224.12 & 192.66 & 174.26 & 161.20 & 151.08 & 142.80 & 186.60 & 217.07 & 239.99 & 259.42 & 276.81 & 292.85 \\
\hline 698 & 224.37 & 192.88 & 174.46 & 161.39 & 151.25 & 142.96 & 186.65 & 217.13 & 240.06 & 259.50 & 276.90 & 292.94 \\
\hline 699 & 224.66 & 193.13 & 174.68 & 161.59 & 151.44 & 143.15 & 186.68 & 217.16 & 240.09 & 259.54 & 276.94 & 292.99 \\
\hline 700 & 225.01 & 193.43 & 174.95 & 161.85 & 151.68 & 143.37 & 186.66 & 217.13 & 240.06 & 259.50 & 276.90 & 292.95 \\
\hline 701 & 225.33 & 193.70 & 175.20 & 162.07 & 151.89 & 143.57 & 186.66 & 217.14 & 240.07 & 259.52 & 276.91 & 292.96 \\
\hline 702 & 225.63 & 193.96 & 175.44 & 162.29 & 152.10 & 143.77 & 186.67 & 217.15 & 240.09 & 259.53 & 276.93 & 292.97 \\
\hline 703 & 225.96 & 194.25 & 175.69 & 162.53 & 152.32 & 143.98 & 186.67 & 217.15 & 240.08 & 259.52 & 276.92 & 292.97 \\
\hline 704 & 226.28 & 194.52 & 175.94 & 162.75 & 152.53 & 144.18 & 186.67 & 217.15 & 240.09 & 259.53 & 276.93 & 292.97 \\
\hline 705 & 226.44 & 194.66 & 176.06 & 162.87 & 152.64 & 144.28 & 186.80 & 217.31 & 240.25 & 259.71 & 277.12 & 293.18 \\
\hline 706 & 226.83 & 194.99 & 176.37 & 163.15 & 152.90 & 144.53 & 186.75 & 217.24 & 240.18 & 259.63 & 277.04 & 293.09 \\
\hline 707 & 227.14 & 195.26 & 176.61 & 163.38 & 153.11 & 144.73 & 186.76 & 217.25 & 240.19 & 259.65 & 277.05 & 293.11 \\
\hline 708 & 227.50 & 195.57 & 176.89 & 163.63 & 153.35 & 144.95 & 186.73 & 217.22 & 240.15 & 259.60 & 277.01 & 293.06 \\
\hline 709 & 227.73 & 195.76 & 177.07 & 163.80 & 153.51 & 145.10 & 186.80 & 217.30 & 240.25 & 259.71 & 277.12 & 293.17 \\
\hline 710 & 227.98 & 195.98 & 177.26 & 163.98 & 153.68 & 145.26 & 186.86 & 217.37 & 240.32 & 259.79 & 277.20 & 293.26 \\
\hline 711 & 228.15 & 196.13 & 177.40 & 164.10 & 153.80 & 145.37 & 186.98 & 217.51 & 240.48 & 259.96 & 277.38 & 293.45 \\
\hline 712 & 228.65 & 196.56 & 177.78 & 164.46 & 154.13 & 145.69 & 186.83 & 217.34 & 240.29 & 259.75 & 277.17 & 293.23 \\
\hline 713 & 228.98 & 196.84 & 178.04 & 164.70 & 154.35 & 145.90 & 186.83 & 217.34 & 240.29 & 259.75 & 277.16 & 293.22 \\
\hline 714 & 229.29 & 197.11 & 178.28 & 164.92 & 154.56 & 146.10 & 186.84 & 217.34 & 240.29 & 259.76 & 277.17 & 293.23 \\
\hline 715 & 229.49 & 197.28 & 178.43 & 165.06 & 154.70 & 146.22 & 186.94 & 217.46 & 240.43 & 259.90 & 277.32 & 293.39 \\
\hline 716 & 229.75 & 197.50 & 178.63 & 165.25 & 154.87 & 146.39 & 186.99 & 217.52 & 240.49 & 259.97 & 277.40 & 293.47 \\
\hline 717 & 230.17 & 197.86 & 178.96 & 165.55 & 155.15 & 146.66 & 186.91 & 217.43 & 240.39 & & 277.27 & 293.34 \\
\hline 718 & 230.36 & 198.03 & 179.11 & 165.69 & 155.28 & 146.78 & 187.01 & 217.55 & 240.52 & 260.00 & 277.43 & 293.50 \\
\hline 719 & 230.74 & 198.36 & 179.41 & 165.97 & 155.54 & 147.02 & 186.96 & 217.49 & 240.45 & 259.93 & 277.35 & 293.42 \\
\hline 720 & 230.94 & 198.53 & 179.57 & 166.11 & 155.68 & 147.15 & 187.06 & 217.60 & 240.58 & 260.06 & 277.50 & 293.58 \\
\hline 721 & 231.24 & 198.78 & 179.80 & 166.33 & 155.88 & 147.34 & 187.08 & 217.62 & 240.60 & 260.09 & 277.53 & 293.61 \\
\hline 722 & 231.55 & 199.05 & 180.03 & 166.55 & 156.08 & 147.53 & 101.09 & 217.64 & 240.62 & & 277.55 & 293.63 \\
\hline 723 & 231.82 & 199.28 & 180.25 & 166.75 & 156.27 & 147.71 & 187.12 & 217.68 & 240.66 & 260.16 & 277.60 & 293.68 \\
\hline 724 & 232.14 & & & & 156.48 & & 187.13 & & 240.67 & & 277.60 & 293.68 \\
\hline 725 & 232.58 & 199.93 & 180.84 & 167.29 & 156.78 & 148.19 & 187.03 & 217.57 & 240.55 & 260.03 & 277.46 & 293.54 \\
\hline 726 & 232.81 & & & & 156.93 & & 187.11 & 217.66 & 240.64 & & & \\
\hline 727 & 233.20 & 200.47 & 181.32 & 167.73 & 157.20 & 148.59 & 187.05 & 217.59 & 240.57 & 260.05 & 277.49 & 293.57 \\
\hline 728 & 233.36 & 200.60 & 181.44 & 167.85 & 157.30 & 148.69 & 187.18 & 217.74 & 240.74 & 260.23 & 277.68 & 293.77 \\
\hline 729 & 233.76 & 200.94 & 181.75 & 168.13 & 157.57 & 148.94 & 187.12 & 217.67 & 240.66 & 260.15 & 277.59 & 293.67 \\
\hline 730 & 233.96 & 201.12 & 181.91 & 168.28 & 157.71 & 149.07 & 187.21 & 217.78 & 240.78 & 260.28 & 277.72 & 293.82 \\
\hline 731 & 234.28 & 201.39 & 182.16 & 168.51 & 157.92 & 149.27 & 187.22 & 217.78 & 240.78 & 260.28 & 277.73 & 293.82 \\
\hline 732 & 234.60 & 201.67 & 182.41 & 168.74 & 158.14 & 149.48 & 187.21 & 217.78 & 240.77 & 260.28 & 277.72 & 293.82 \\
\hline 733 & 234.83 & 201.87 & 182.58 & 168.90 & 158.29 & 149.62 & 187.29 & 217.87 & 240.87 & 260.38 & 277.84 & 293.94 \\
\hline 734 & 235.15 & 202.14 & 182.84 & 169.14 & 158.51 & 149.83 & 187.28 & 217.86 & 240.87 & 260.38 & 277.83 & 293.93 \\
\hline 735 & 235.41 & 202.36 & 183.04 & 169.32 & 158.69 & 149.99 & 187.34 & 217.92 & 240.94 & 260.45 & 277.91 & 294.01 \\
\hline 736 & 235.81 & 202.71 & 183.35 & 169.61 & 158.96 & 150.25 & 187.27 & 217.84 & 240.85 & 260.36 & 277.81 & 293.91 \\
\hline
\end{tabular}


Table B.2: Simulation Data for Picking Times (High Rate)—continued.

\begin{tabular}{|c|c|c|c|c|c|c|c|c|c|c|c|c|}
\hline \multirow{2}{*}{$\begin{array}{c}\text { Batch } \\
\text { Size }\end{array}$} & \multicolumn{6}{|c|}{ Picking Time (minutes) } & \multicolumn{6}{|c|}{ Picking Rate (orders/hr) } \\
\hline & 20 & 40 & 60 & 80 & 100 & 120 & 20 & 40 & 60 & 80 & 100 & 120 \\
\hline 737 & 236.06 & 202.92 & 183.54 & 169.79 & 159.12 & 150.41 & 187.33 & 217.91 & 240.93 & 260.44 & 277.90 & 294.00 \\
\hline 738 & 236.45 & 203.26 & 183.85 & 170.07 & 159.39 & 150.66 & 187.27 & 217.85 & 240.85 & 260.36 & 277.81 & 293.91 \\
\hline 739 & 236.69 & 203.47 & 184.03 & 170.24 & 159.55 & 150.81 & 187.34 & 217.92 & 240.94 & 260.45 & 277.91 & 294.01 \\
\hline 740 & 236.99 & 203.73 & 184.27 & 170.46 & 159.75 & 151.00 & 187.35 & 217.94 & 240.95 & 260.47 & 277.93 & 294.03 \\
\hline 741 & 237.28 & 203.97 & 184.49 & 170.67 & 159.95 & 151.19 & 187.38 & 217.97 & 240.99 & 260.51 & 277.97 & 294.08 \\
\hline 742 & 237.68 & 204.32 & 184.80 & 170.96 & 160.22 & 151.44 & 187.31 & 217.90 & 240.90 & 260.42 & 277.87 & 293.97 \\
\hline 743 & 237.88 & 204.49 & 184.96 & 171.10 & 160.35 & 151.57 & 187.40 & 218.00 & 241.02 & 260.55 & 278.01 & 294.12 \\
\hline 744 & 238.19 & 204.76 & 185.20 & 171.33 & 160.56 & 151.77 & 187.41 & 218.01 & 241.03 & 260.55 & 278.02 & 294.13 \\
\hline 745 & 238.62 & 205.13 & 185.53 & 171.63 & 160.85 & 152.04 & 187.33 & 217.92 & 240.93 & 260.44 & 277.90 & 294.00 \\
\hline 746 & 238.78 & 205.26 & 185.66 & 171.75 & 160.96 & 152.14 & 187.46 & 218.06 & 241.09 & 260.62 & 278.09 & 294.20 \\
\hline 747 & 239.20 & 205.62 & 185.98 & 172.05 & 161.24 & 152.41 & 187.38 & 217.97 & 240.99 & 260.51 & 277.97 & 294.08 \\
\hline 748 & 239.33 & 205.74 & 186.09 & 172.15 & 161.33 & 152.49 & 187.52 & 218.14 & 241.18 & 260.71 & 278.19 & 294.31 \\
\hline 749 & 239.68 & 206.04 & 186.36 & 172.40 & 161.57 & 152.72 & 187.50 & 218.11 & 241.14 & 260.67 & 278.15 & 294.27 \\
\hline 750 & 240.08 & 206.38 & 186.67 & 172.69 & 161.84 & 152.97 & 187.43 & 218.04 & 241.06 & 260.59 & 278.06 & 294.17 \\
\hline
\end{tabular}

Table B.3: Simulation Data for Picking Times (Low Rate).

\begin{tabular}{|c|c|c|c|c|c|c|c|c|c|c|c|c|}
\hline \multirow{2}{*}{$\begin{array}{c}\text { Batch } \\
\text { Size }\end{array}$} & \multicolumn{6}{|c|}{ Picking Time (minutes) } & \multicolumn{6}{|c|}{ Picking Rate (orders/hr) } \\
\hline & 20 & 40 & 60 & 80 & 100 & 120 & 20 & 40 & 60 & 80 & 100 & 120 \\
\hline 1 & 2.34 & 2.01 & 1.82 & 1.68 & 1.58 & 1.49 & 25.65 & 29.84 & 32.99 & 35.66 & 38.05 & 40.26 \\
\hline 2 & 3.80 & 3.26 & 2.95 & 2.73 & 2.56 & 2.42 & 31.62 & 36.78 & 40.67 & 43.96 & 46.91 & 49.62 \\
\hline 3 & 5.09 & 4.38 & 3.96 & 3.66 & 3.43 & 3.24 & 35.36 & 41.14 & 45.48 & 49.17 & 52.46 & 55.50 \\
\hline 4 & 6.31 & 5.42 & 4.90 & 4.54 & 4.25 & 4.02 & 38.06 & 44.28 & 48.95 & 52.92 & 56.46 & 59.74 \\
\hline 5 & 7.53 & 6.48 & 5.86 & 5.42 & 5.08 & 4.80 & 39.83 & 46.33 & 51.22 & 55.37 & 59.08 & 62.51 \\
\hline 6 & 8.69 & 7.47 & 6.76 & 6.25 & 5.86 & 5.54 & 41.42 & 48.19 & 53.27 & 57.59 & 61.45 & 65.01 \\
\hline 7 & 9.69 & 8.33 & 7.54 & 6.97 & 6.53 & 6.17 & 43.34 & 50.41 & 55.74 & 60.25 & 64.29 & 68.02 \\
\hline 8 & 10.42 & 8.95 & 8.10 & 7.49 & 7.02 & 6.64 & 46.09 & 53.61 & 59.27 & 64.07 & 68.37 & 72.33 \\
\hline 9 & 11.70 & 10.06 & 9.10 & 8.42 & 7.89 & 7.46 & 46.14 & 53.67 & 59.34 & 64.14 & 68.44 & 72.41 \\
\hline 10 & 12.76 & 10.97 & 9.92 & 9.18 & 8.60 & 8.13 & 47.02 & 54.70 & 60.48 & 65.38 & 69.76 & 73.80 \\
\hline 11 & 13.56 & 11.66 & 10.54 & 9.75 & 9.14 & 8.64 & 48.68 & 56.62 & 62.60 & 67.67 & 72.21 & 76.39 \\
\hline 12 & 14.64 & 12.58 & 11.38 & 10.53 & 9.87 & 9.33 & 49.19 & 57.22 & 63.26 & 68.39 & 72.97 & 77.20 \\
\hline 13 & 15.58 & 13.39 & 12.11 & 11.21 & 10.50 & 9.93 & 50.07 & 58.24 & 64.40 & 69.61 & 74.28 & 78.58 \\
\hline 14 & 16.42 & 14.11 & 12.77 & 11.81 & 11.07 & 10.46 & 51.16 & 59.52 & 65.80 & 71.13 & 75.90 & 80.30 \\
\hline 15 & 17.39 & 14.95 & 13.52 & 12.51 & 11.72 & 11.08 & 51.76 & 60.21 & 66.57 & 71.96 & 76.78 & 81.23 \\
\hline 16 & 17.91 & 15.39 & 13.92 & 12.88 & 12.07 & 11.41 & 53.61 & 62.37 & 68.95 & 74.54 & 79.54 & 84.14 \\
\hline 17 & 18.76 & 16.13 & 14.59 & 13.49 & 12.65 & 11.95 & 54.37 & 63.24 & 69.92 & 75.59 & 80.65 & 85.33 \\
\hline 18 & 19.06 & 16.39 & 14.82 & 13.71 & 12.85 & 12.15 & 56.65 & 65.90 & 72.86 & 78.76 & 84.04 & 88.91 \\
\hline 19 & 20.28 & 17.43 & 15.77 & 14.59 & 13.67 & 12.92 & 56.22 & 65.40 & 72.30 & 78.16 & 83.40 & 88.23 \\
\hline 20 & 21.26 & 18.28 & 16.53 & 15.29 & 14.33 & 13.55 & 56.43 & 65.65 & 72.58 & 78.46 & 83.72 & 88.57 \\
\hline 21 & 21.82 & 18.76 & 16.96 & 15.69 & 14.71 & 13.90 & 57.75 & 67.18 & 74.28 & 80.29 & 85.67 & 90.64 \\
\hline 22 & 22.96 & 19.74 & 17.85 & 16.52 & 15.48 & 14.63 & 57.48 & 66.87 & 73.93 & 79.92 & 85.27 & 90.22 \\
\hline
\end{tabular}


Table B.3: Simulation Data for Picking Times (Low Rate) - continued.

\begin{tabular}{|c|c|c|c|c|c|c|c|c|c|c|c|c|}
\hline \multirow{2}{*}{$\begin{array}{c}\text { Batch } \\
\text { Size }\end{array}$} & \multicolumn{6}{|c|}{ Picking Time (minutes) } & \multicolumn{6}{|c|}{ Picking Rate (orders/hr) } \\
\hline & 20 & 40 & 60 & 80 & 100 & 120 & 20 & 40 & 60 & 80 & 100 & 120 \\
\hline 23 & 23.01 & 19.78 & 17.89 & 16.55 & 15.51 & 14.66 & 59.97 & 69.76 & 77.12 & 83.37 & 88.96 & 94.11 \\
\hline 24 & 24.54 & 21.09 & 19.08 & 17.65 & 16.54 & 15.64 & 58.68 & 68.26 & 75.47 & 81.59 & 87.05 & 92.10 \\
\hline 25 & 24.50 & 21.06 & 19.05 & 17.62 & 16.52 & 15.61 & 61.22 & 71.22 & 78.74 & 85.12 & 90.82 & 96.09 \\
\hline 26 & 25.31 & 21.76 & 19.68 & 18.21 & 17.06 & 16.13 & 61.63 & 71.69 & 79.26 & 85.68 & 91.43 & 96.73 \\
\hline 27 & 26.06 & 22.40 & 20.26 & 18.75 & 17.57 & 16.61 & 62.16 & 72.31 & 79.95 & 86.42 & 92.21 & 97.56 \\
\hline 28 & 26.53 & 22.81 & 20.63 & 19.09 & 17.89 & 16.91 & 63.32 & 73.65 & 81.43 & 88.03 & 93.93 & 99.37 \\
\hline 29 & 26.98 & 23.20 & 20.98 & 19.41 & 18.19 & 17.19 & 64.49 & 75.01 & 82.94 & 89.65 & 95.66 & 101.21 \\
\hline 30 & 27.96 & 24.03 & 21.74 & 20.11 & 18.85 & 17.81 & 64.38 & 74.89 & 82.80 & 89.51 & 95.51 & 101.04 \\
\hline 31 & 28.27 & 24.30 & 21.98 & 20.34 & 19.06 & 18.01 & 65.79 & 76.53 & 84.61 & 91.46 & 97.59 & 103.25 \\
\hline 32 & 28.90 & 24.84 & 22.47 & 20.78 & 19.48 & 18.41 & 66.45 & 77.29 & 85.46 & 92.38 & 98.57 & 104.28 \\
\hline 33 & 29.73 & 25.55 & 23.11 & 21.38 & 20.04 & 18.94 & 66.61 & 77.48 & 85.67 & 92.61 & 98.81 & 104.54 \\
\hline 34 & 30.31 & 26.05 & 23.56 & 21.80 & 20.43 & 19.31 & 67.31 & 78.31 & 86.57 & 93.59 & 99.86 & 105.65 \\
\hline 35 & 31.15 & 26.78 & 24.22 & 22.40 & 21.00 & 19.85 & 67.42 & 78.43 & 86.71 & 93.74 & 100.02 & 105.81 \\
\hline 36 & 31.72 & 27.27 & 24.66 & 22.81 & 21.38 & 20.21 & 68.10 & 79.22 & 87.58 & 94.68 & 101.02 & 106.88 \\
\hline 37 & 32.04 & 27.55 & 24.91 & 23.05 & 21.60 & 20.42 & 69.28 & 80.59 & 89.10 & 96.32 & 102.78 & 108.73 \\
\hline 38 & 33.16 & 28.51 & 25.78 & 23.85 & 22.35 & 21.13 & 68.76 & 79.98 & 88.43 & 95.59 & 102.00 & 107.91 \\
\hline 39 & 32.92 & 28.30 & 25.60 & 23.68 & 22.19 & 20.98 & 71.08 & 82.68 & 91.42 & 98.82 & 105.44 & 111.55 \\
\hline 40 & 33.56 & 28.85 & 26.10 & 24.14 & 22.63 & 21.39 & 71.50 & 83.18 & 91.96 & 99.41 & 106.08 & 112.22 \\
\hline 41 & 34.21 & 29.41 & 26.60 & 24.61 & 23.06 & 21.80 & 71.91 & 83.65 & 92.49 & 99.98 & 106.68 & 112.86 \\
\hline 42 & 35.32 & 30.36 & 27.46 & 25.40 & 23.81 & 22.50 & 71.35 & 83.00 & 91.76 & 99.19 & 105.84 & 111.98 \\
\hline 43 & 35.28 & 30.32 & 27.43 & 25.37 & 23.78 & 22.48 & 73.14 & 85.08 & 94.07 & 101.68 & 108.50 & 114.79 \\
\hline 44 & 36.20 & 31.12 & 28.15 & 26.04 & 24.40 & 23.07 & 72.93 & 84.84 & 93.79 & 101.39 & 108.19 & 114.46 \\
\hline 45 & 36.59 & 31.45 & 28.45 & 26.32 & 24.66 & 23.31 & 73.80 & 85.85 & 94.91 & 102.60 & 109.48 & 115.82 \\
\hline 46 & 37.63 & 32.35 & 29.26 & 27.07 & 25.36 & 23.98 & 73.35 & 85.33 & 94.34 & 101.98 & 108.81 & 115.12 \\
\hline 47 & 38.05 & 32.71 & 29.59 & 27.37 & 25.65 & 24.25 & 74.11 & 86.21 & 95.31 & 103.03 & 109.94 & 116.31 \\
\hline 48 & 37.82 & 32.51 & 29.41 & 27.20 & 25.49 & 24.10 & 76.15 & 88.59 & 97.94 & 105.87 & 112.97 & 119.52 \\
\hline 49 & 39.19 & 33.69 & 30.47 & 28.19 & 26.42 & 24.97 & 75.01 & 87.26 & 96.48 & 104.29 & 111.28 & 117.73 \\
\hline 50 & 39.16 & 33.67 & 30.45 & 28.17 & 26.40 & 24.95 & 76.60 & 89.11 & 98.52 & 106.50 & 113.64 & 120.23 \\
\hline 51 & 39.98 & 34.37 & 31.08 & 28.76 & 26.95 & 25.47 & 76.54 & 89.04 & 98.44 & 106.42 & 113.55 & 120.13 \\
\hline 52 & 40.25 & 34.60 & 31.30 & 28.95 & 27.13 & 25.65 & 77.51 & 90.17 & 99.69 & 107.76 & 114.98 & 121.65 \\
\hline 53 & 40.65 & 34.95 & 31.61 & 29.24 & 27.40 & 25.90 & 78.22 & 90.99 & 100.60 & 108.75 & 116.04 & 122.77 \\
\hline 54 & 41.43 & 35.62 & 32.21 & 29.80 & 27.93 & 26.40 & 78.20 & 90.97 & 100.58 & 108.73 & 116.01 & 122.74 \\
\hline 55 & 42.17 & 36.25 & 32.79 & 30.33 & 28.42 & 26.87 & 78.26 & 91.04 & 100.65 & 108.80 & 116.10 & 122.82 \\
\hline 56 & 42.32 & 36.38 & 32.90 & 30.44 & 28.53 & 26.96 & 79.40 & 92.36 & 102.12 & 110.39 & 117.79 & 124.61 \\
\hline 57 & 42.92 & 36.89 & 33.37 & 30.87 & 28.93 & 27.35 & 79.69 & 92.70 & 102.49 & 110.79 & 118.21 & 125.06 \\
\hline 58 & 43.33 & 37.25 & 33.69 & 31.17 & 29.21 & 27.61 & 80.31 & 93.43 & 103.29 & 111.66 & 119.14 & 126.04 \\
\hline 59 & 44.27 & 38.05 & 34.42 & 31.84 & 29.84 & 28.21 & 79.97 & 93.02 & 102.85 & 111.18 & 118.63 & 125.50 \\
\hline 60 & 44.50 & 38.25 & 34.60 & 32.00 & 29.99 & 28.35 & 80.91 & 94.12 & 104.06 & 112.48 & 120.02 & 126.98 \\
\hline 61 & 44.98 & 38.67 & 34.97 & 32.35 & 30.32 & 28.66 & 81.37 & 94.66 & 104.65 & 113.13 & 120.71 & 127.71 \\
\hline 62 & 45.56 & 39.16 & 35.42 & 32.77 & 30.71 & 29.03 & 81.65 & 94.98 & 105.02 & 113.52 & 121.13 & 128.15 \\
\hline 63 & 46.10 & 39.63 & 35.85 & 33.16 & 31.08 & 29.38 & 81.99 & 95.38 & 105.45 & 113.99 & 121.63 & 128.68 \\
\hline 64 & 46.56 & 40.02 & 36.20 & 33.49 & 31.38 & 29.66 & 82.48 & 95.95 & 106.08 & 114.67 & 122.36 & 129.45 \\
\hline
\end{tabular}


Table B.3: Simulation Data for Picking Times (Low Rate) - continued.

\begin{tabular}{|c|c|c|c|c|c|c|c|c|c|c|c|c|}
\hline \multirow{2}{*}{$\begin{array}{c}\text { Batch } \\
\text { Size }\end{array}$} & \multicolumn{6}{|c|}{ Picking Time (minutes) } & \multicolumn{6}{|c|}{ Picking Rate (orders/hr) } \\
\hline & 20 & 40 & 60 & 80 & 100 & 120 & 20 & 40 & 60 & 80 & 100 & 120 \\
\hline 65 & 46.81 & 40.24 & 36.40 & 33.67 & 31.55 & 29.83 & 83.32 & 96.92 & 107.15 & 115.83 & 123.60 & 130.76 \\
\hline 66 & 47.18 & 40.56 & 36.68 & 33.94 & 31.80 & 30.06 & 83.93 & 97.64 & 107.95 & 116.69 & 124.51 & 131.73 \\
\hline 67 & 47.91 & 41.18 & 37.25 & 34.46 & 32.29 & 30.53 & 83.91 & 97.61 & 107.92 & 116.66 & 124.48 & 131.70 \\
\hline 68 & 48.24 & 41.47 & 37.51 & 34.70 & 32.52 & 30.74 & 84.57 & 98.38 & 108.77 & 117.58 & 125.46 & 132.73 \\
\hline 69 & 48.27 & 41.50 & 37.53 & 34.72 & 32.54 & 30.76 & 85.77 & 99.77 & 110.31 & 119.24 & 127.23 & 134.61 \\
\hline 70 & 49.30 & 42.38 & 38.33 & 35.46 & 33.23 & 31.41 & 85.20 & 99.11 & 109.58 & 118.45 & 126.39 & 133.72 \\
\hline 71 & 49.52 & 42.57 & 38.50 & 35.62 & 33.38 & 31.55 & 86.02 & 100.07 & 110.64 & 119.60 & 127.61 & 135.01 \\
\hline 72 & 49.98 & 42.97 & 38.86 & 35.95 & 33.69 & 31.85 & 86.43 & 100.54 & 111.16 & 120.16 & 128.22 & 135.65 \\
\hline 73 & 50.16 & 43.12 & 39.00 & 36.08 & 33.81 & 31.96 & 87.31 & 101.57 & 112.30 & 121.39 & 129.53 & 137.03 \\
\hline 74 & 51.26 & 44.06 & 39.85 & 36.87 & 34.55 & 32.66 & 86.62 & 100.76 & 111.40 & 120.43 & 128.50 & 135.95 \\
\hline 75 & 51.43 & 44.21 & 39.99 & 36.99 & 34.67 & 32.77 & 87.50 & 101.79 & 112.53 & 121.65 & 129.80 & 137.32 \\
\hline 76 & 51.92 & 44.63 & 40.37 & 37.34 & 35.00 & 33.08 & 87.83 & 102.17 & 112.96 & 122.11 & 130.29 & 137.84 \\
\hline 77 & 52.05 & 44.74 & 40.47 & 37.44 & 35.09 & 33.16 & 88.76 & 103.25 & 114.16 & 123.40 & 131.68 & 139.31 \\
\hline 78 & 52.89 & 45.46 & 41.12 & 38.04 & 35.65 & 33.70 & 88.49 & 102.94 & 113.81 & 123.03 & 131.28 & 138.88 \\
\hline 79 & 53.20 & 45.73 & 41.36 & 38.26 & 35.86 & 33.90 & 89.10 & 103.65 & 114.60 & 123.88 & 132.18 & 139.84 \\
\hline 80 & 53.83 & 46.27 & 41.85 & 38.72 & 36.28 & 34.30 & 89.17 & 103.73 & 114.69 & 123.98 & 132.29 & 139.95 \\
\hline 81 & 53.91 & 46.34 & 41.92 & 38.78 & 36.34 & 34.35 & 90.15 & 104.87 & 115.94 & 125.33 & 133.74 & 141.49 \\
\hline 82 & 54.31 & 46.69 & 42.23 & 39.07 & 36.61 & 34.61 & 90.58 & 105.38 & 116.50 & 125.94 & 134.38 & 142.17 \\
\hline 83 & 55.04 & 47.31 & 42.79 & 39.59 & 37.10 & 35.07 & 90.48 & 105.26 & 116.37 & 125.80 & 134.23 & 142.01 \\
\hline 84 & 55.39 & 47.62 & 43.07 & 39.84 & 37.34 & 35.30 & 90.98 & 105.84 & 117.02 & 126.49 & 134.97 & 142.79 \\
\hline 85 & 55.90 & 48.05 & 43.46 & 40.21 & 37.68 & 35.62 & 91.23 & 106.13 & 117.34 & 126.84 & 135.34 & 143.19 \\
\hline 86 & 55.94 & 48.08 & 43.49 & 40.23 & 37.71 & 35.64 & 92.25 & 107.31 & 118.64 & 128.25 & 136.85 & 144.78 \\
\hline 87 & 56.76 & 48.79 & 44.13 & 40.83 & 38.26 & 36.17 & 91.97 & 106.98 & 118.28 & 127.86 & 136.43 & 144.34 \\
\hline 88 & 56.93 & 48.94 & 44.27 & 40.95 & 38.38 & 36.28 & 92.74 & 107.88 & 119.27 & 128.93 & 137.58 & 145.55 \\
\hline 89 & 57.40 & 49.34 & 44.63 & 41.29 & 38.69 & 36.57 & 93.03 & 108.22 & 119.65 & 129.34 & 138.01 & 146.00 \\
\hline 90 & 57.96 & 49.82 & 45.06 & 41.69 & 39.07 & 36.93 & 93.17 & 108.39 & 119.83 & 129.54 & 138.22 & 146.23 \\
\hline 91 & 58.20 & 50.03 & 45.25 & 41.86 & 39.23 & 37.08 & 93.82 & 109.14 & 120.66 & 130.43 & 139.18 & 147.24 \\
\hline 92 & 58.95 & 50.68 & 45.84 & 42.40 & 39.74 & 37.56 & 93.63 & 108.92 & 120.42 & 130.18 & 138.90 & 146.95 \\
\hline 93 & 59.33 & 51.00 & 46.13 & 42.67 & 39.99 & 37.80 & 94.05 & 109.41 & 120.96 & 130.76 & 139.53 & 147.61 \\
\hline 94 & 59.60 & 51.24 & 46.34 & 42.87 & 40.18 & 37.98 & 94.62 & 110.07 & 121.70 & 131.55 & 140.37 & 148.51 \\
\hline 95 & 60.01 & 51.59 & 46.66 & 43.16 & 40.45 & 38.24 & 94.98 & 110.49 & 122.16 & 132.05 & 140.90 & 149.07 \\
\hline 96 & 60.29 & 51.83 & 46.88 & 43.36 & 40.64 & 38.41 & 95.54 & 111.14 & 122.88 & 132.83 & 141.74 & 149.95 \\
\hline 97 & 61.00 & 52.44 & 47.43 & 43.87 & 41.12 & 38.87 & 95.41 & 110.99 & 122.71 & 132.65 & 141.54 & 149.75 \\
\hline 98 & 61.15 & 52.57 & 47.55 & 43.99 & 41.22 & 38.97 & 96.15 & 111.85 & 123.66 & 133.68 & 142.64 & 150.90 \\
\hline 99 & 61.71 & 53.05 & 47.98 & 44.39 & 41.60 & 39.32 & 96.25 & 111.97 & 123.79 & 133.82 & 142.79 & 151.06 \\
\hline 100 & 62.22 & 53.49 & 48.38 & 44.75 & 41.94 & 39.64 & 96.43 & 112.18 & 124.03 & 134.07 & 143.06 & 151.35 \\
\hline 101 & 62.43 & 53.67 & 48.54 & 44.91 & 42.09 & 39.78 & 97.06 & 112.91 & 124.84 & 134.95 & 143.99 & 152.34 \\
\hline 102 & 62.59 & 53.81 & 48.67 & 45.02 & 42.19 & 39.88 & 97.77 & 113.74 & 125.75 & 135.93 & 145.05 & 153.45 \\
\hline 103 & 63.49 & 54.58 & 49.37 & 45.67 & 42.80 & 40.45 & 97.34 & 113.23 & 125.19 & 135.32 & 144.40 & 152.76 \\
\hline 104 & 63.62 & 54.69 & 49.47 & 45.76 & 42.89 & 40.54 & 98.08 & 114.10 & 126.14 & 136.36 & 145.50 & 153.93 \\
\hline 105 & 63.80 & 54.85 & 49.61 & 45.89 & 43.01 & 40.65 & 98.74 & 114.86 & 126.99 & 137.28 & 146.48 & 154.97 \\
\hline 106 & 64.52 & 55.47 & 50.17 & 46.41 & 43.50 & 41.11 & 98.57 & 114.66 & 126.77 & 137.04 & 146.22 & 154.70 \\
\hline
\end{tabular}


Table B.3: Simulation Data for Picking Times (Low Rate) — continued.

\begin{tabular}{|c|c|c|c|c|c|c|c|c|c|c|c|c|}
\hline \multirow{2}{*}{$\begin{array}{c}\text { Batch } \\
\text { Size }\end{array}$} & \multicolumn{6}{|c|}{ Picking Time (minutes) } & \multicolumn{6}{|c|}{ Picking Rate (orders/hr) } \\
\hline & 20 & 40 & 60 & 80 & 100 & 120 & 20 & 40 & 60 & 80 & 100 & 120 \\
\hline 107 & 64.69 & 55.61 & 50.30 & 46.53 & 43.61 & 41.22 & 99.24 & 115.45 & 127.64 & 137.98 & 147.23 & 155.76 \\
\hline 108 & 65.13 & 55.99 & 50.64 & 46.85 & 43.90 & 41.50 & 99.49 & 115.74 & 127.96 & 138.32 & 147.59 & 156.15 \\
\hline 109 & 65.59 & 56.39 & 51.00 & 47.18 & 44.22 & 41.79 & 99.70 & 115.98 & 128.23 & 138.62 & 147.91 & 156.48 \\
\hline 110 & 65.92 & 56.66 & 51.25 & 47.41 & 44.43 & 42.00 & 100.13 & 116.48 & 128.78 & 139.21 & 148.54 & 157.14 \\
\hline 111 & 66.59 & 57.24 & 51.77 & 47.89 & 44.88 & 42.43 & 100.02 & 116.35 & 128.64 & 139.06 & 148.38 & 156.98 \\
\hline 112 & 66.59 & 57.25 & 51.78 & 47.90 & 44.89 & 42.43 & 100.91 & 117.39 & 129.78 & 140.30 & 149.70 & 158.38 \\
\hline 113 & 67.09 & 57.68 & 52.17 & 48.26 & 45.23 & 42.75 & 101.05 & 117.55 & 129.97 & 140.49 & 149.91 & 158.60 \\
\hline 114 & 67.40 & 57.94 & 52.40 & 48.48 & 45.43 & 42.94 & 101.49 & 118.06 & 130.53 & 141.10 & 150.56 & 159.28 \\
\hline 115 & 68.02 & 58.47 & 52.89 & 48.92 & 45.85 & 43.34 & 101.45 & 118.01 & 130.47 & 141.04 & 150.49 & 159.21 \\
\hline 116 & 67.95 & 58.41 & 52.83 & 48.87 & 45.80 & 43.29 & 102.43 & 119.16 & 131.74 & 142.41 & 151.96 & 160.77 \\
\hline 117 & 68.72 & 59.07 & 53.43 & 49.43 & 46.32 & 43.79 & 102.16 & 118.84 & 131.38 & 142.03 & 151.55 & 160.33 \\
\hline 118 & 69.33 & 59.59 & 53.90 & 49.86 & 46.73 & 44.17 & 102.13 & 118.80 & 131.35 & 141.99 & 151.50 & 160.28 \\
\hline 119 & 69.67 & 59.89 & 54.17 & 50.11 & 46.97 & 44.39 & 102.48 & 119.21 & 131.80 & 142.47 & 152.02 & 160.83 \\
\hline 120 & 69.38 & 59.64 & 53.95 & 49.91 & 46.77 & 44.21 & 103.77 & 120.72 & 133.46 & 144.27 & 153.94 & 162.86 \\
\hline 121 & 70.36 & 60.48 & 54.71 & 50.61 & 47.43 & 44.83 & 103.18 & 120.03 & 132.71 & 143.45 & 153.07 & 161.94 \\
\hline 122 & 70.55 & 60.65 & 54.86 & 50.75 & 47.56 & 44.95 & 103.75 & 120.70 & 133.44 & 144.25 & 153.92 & 162.84 \\
\hline 123 & 70.77 & 60.84 & 55.03 & 50.90 & 47.71 & 45.09 & 104.28 & 121.30 & 134.11 & 144.98 & 154.69 & 163.66 \\
\hline 124 & 71.44 & 61.41 & 55.55 & 51.39 & 48.16 & 45.52 & 104.14 & 121.15 & 133.94 & 144.79 & 154.49 & 163.44 \\
\hline 125 & 71.83 & 61.75 & 55.85 & 51.67 & 48.42 & 45.77 & 104.41 & 121.46 & 134.28 & 145.16 & 154.89 & 163.86 \\
\hline 126 & 72.42 & 62.25 & 56.31 & 52.09 & 48.82 & 46.14 & 104.39 & 121.44 & 134.26 & 145.14 & 154.87 & 163.84 \\
\hline 127 & 72.33 & 62.18 & 56.24 & 52.02 & 48.75 & 46.08 & 105.35 & 122.56 & 135.50 & 146.47 & 156.29 & 165.35 \\
\hline 128 & 72.45 & 62.28 & 56.33 & 52.11 & 48.84 & 46.16 & 106.01 & 123.31 & 136.34 & 147.38 & 157.26 & 166.37 \\
\hline 129 & 73.08 & 62.82 & 56.82 & 52.57 & 49.26 & 46.57 & 105.91 & 123.20 & 136.21 & 147.24 & 157.11 & 166.22 \\
\hline 130 & 73.56 & 63.24 & 57.20 & 52.91 & 49.59 & 46.87 & 106.03 & 123.34 & 136.37 & 147.41 & 157.29 & 166.41 \\
\hline 131 & 73.74 & 63.39 & 57.33 & 53.04 & 49.71 & 46.98 & 106.60 & 124.00 & 137.09 & 148.20 & 158.13 & 167.30 \\
\hline 132 & 74.15 & 63.74 & 57.65 & 53.33 & 49.98 & 47.24 & 106.81 & 124.26 & 137.38 & 148.50 & 158.46 & 167.64 \\
\hline 133 & 74.38 & 63.94 & 57.83 & 53.50 & 50.14 & 47.39 & 107.28 & 124.80 & 137.98 & 149.15 & 159.15 & 168.37 \\
\hline 134 & 74.80 & 64.30 & 58.16 & 53.80 & 50.42 & 47.66 & 107.48 & 125.03 & 138.24 & 149.43 & 159.45 & 168.69 \\
\hline 135 & 75.09 & 64.55 & 58.38 & 54.01 & 50.62 & 47.84 & 107.87 & 125.49 & 138.74 & 149.97 & 160.03 & 169.30 \\
\hline 136 & 75.68 & 65.05 & 58.84 & 54.43 & 51.01 & 48.22 & 107.83 & 125.43 & 138.68 & 149.91 & 159.96 & 169.23 \\
\hline 137 & 75.74 & 65.11 & 58.89 & 54.48 & 51.06 & 48.26 & 108.52 & 126.24 & 139.57 & 150.88 & 160.99 & 170.32 \\
\hline 138 & 76.76 & 65.98 & 59.68 & 55.21 & 51.74 & 48.91 & 107.87 & 125.49 & 138.74 & 149.97 & 160.03 & 169.30 \\
\hline 139 & 76.89 & 66.09 & 59.78 & 55.30 & 51.83 & 48.99 & 108.47 & 126.18 & 139.51 & 150.81 & 160.92 & 170.24 \\
\hline 140 & 77.24 & 66.40 & 60.06 & 55.56 & 52.07 & 49.22 & 108.75 & 126.51 & 139.86 & 151.19 & 161.33 & 170.67 \\
\hline 141 & 77.61 & 66.72 & 60.35 & 55.83 & 52.32 & 49.45 & 109.00 & 126.80 & 140.19 & 151.54 & 161.70 & 171.07 \\
\hline 142 & 77.93 & 67.00 & 60.60 & 56.06 & 52.53 & 49.66 & 109.32 & 127.17 & 140.60 & 151.99 & 162.18 & 171.58 \\
\hline 143 & 78.10 & 67.14 & 60.73 & 56.18 & 52.65 & 49.76 & 109.85 & 127.79 & 141.29 & 152.73 & 162.97 & 172.41 \\
\hline 144 & 78.71 & 67.66 & 61.20 & 56.61 & 53.05 & 50.15 & 109.78 & 127.70 & 141.19 & 152.62 & 162.85 & 172.29 \\
\hline 145 & 78.66 & 67.62 & 61.16 & 56.58 & 53.02 & 50.12 & 110.60 & 128.66 & 142.25 & 153.77 & 164.07 & 173.58 \\
\hline 146 & 79.28 & 68.15 & 61.64 & 57.02 & 53.44 & 50.51 & 110.50 & 128.54 & 142.11 & 153.62 & 163.92 & 173.42 \\
\hline 147 & 79.99 & 68.76 & 62.19 & 57.53 & 53.92 & 50.97 & 110.26 & 128.27 & 141.81 & 153.30 & 163.58 & 173.05 \\
\hline 148 & 80.26 & 69.00 & 62.41 & 57.73 & 54.10 & 51.14 & 110.64 & 128.70 & 142.29 & 153.81 & 164.13 & 173.64 \\
\hline
\end{tabular}


Table B.3: Simulation Data for Picking Times (Low Rate) - continued.

\begin{tabular}{|c|c|c|c|c|c|c|c|c|c|c|c|c|}
\hline \multirow{2}{*}{$\begin{array}{c}\text { Batch } \\
\text { Size }\end{array}$} & \multicolumn{6}{|c|}{ Picking Time (minutes) } & \multicolumn{6}{|c|}{ Picking Rate (orders/hr) } \\
\hline & 20 & 40 & 60 & 80 & 100 & 120 & 20 & 40 & 60 & 80 & 100 & 120 \\
\hline 149 & 80.31 & 69.04 & 62.44 & 57.76 & 54.13 & 51.17 & 111.32 & 129.50 & 143.17 & 154.77 & 165.14 & 174.71 \\
\hline 150 & 80.91 & 69.55 & 62.91 & 58.20 & 54.54 & 51.55 & 111.23 & 129.40 & 143.06 & 154.65 & 165.01 & 174.57 \\
\hline 151 & 81.14 & 69.75 & 63.09 & 58.36 & 54.70 & 51.70 & 111.66 & 129.89 & 143.60 & 155.23 & 165.64 & 175.24 \\
\hline 152 & 81.59 & 70.13 & 63.44 & 58.68 & 55.00 & 51.98 & 111.79 & 130.04 & 143.77 & 155.41 & 165.83 & 175.44 \\
\hline 153 & 81.94 & 70.44 & 63.71 & 58.94 & 55.24 & 52.21 & 112.03 & 130.32 & 144.08 & 155.75 & 166.19 & 175.82 \\
\hline 154 & 82.17 & 70.64 & 63.89 & 59.10 & 55.39 & 52.36 & 112.45 & 130.81 & 144.62 & 156.34 & 166.82 & 176.48 \\
\hline 155 & 82.37 & 70.81 & 64.04 & 59.25 & 55.52 & 52.48 & 112.91 & 131.34 & 145.21 & 156.97 & 167.49 & 177.20 \\
\hline 156 & 82.89 & 71.25 & 64.45 & 59.62 & 55.87 & 52.81 & 112.92 & 131.36 & 145.23 & 156.99 & 167.52 & 177.22 \\
\hline 157 & 83.19 & 71.51 & 64.68 & 59.83 & 56.07 & 53.00 & 113.24 & 131.73 & 145.64 & 157.44 & 167.99 & 177.72 \\
\hline 158 & 83.71 & 71.96 & 65.08 & 60.21 & 56.43 & 53.33 & 113.25 & 131.75 & 145.66 & 157.46 & 168.01 & 177.75 \\
\hline 159 & 84.00 & 72.21 & 65.31 & 60.42 & 56.62 & 53.52 & 113.57 & 132.11 & 146.06 & 157.89 & 168.48 & 178.24 \\
\hline 160 & 84.44 & 72.58 & 65.65 & 60.73 & 56.92 & 53.80 & 113.70 & 132.26 & 146.23 & 158.07 & 168.67 & 178.44 \\
\hline 161 & 84.70 & 72.81 & 65.86 & 60.92 & 57.09 & 53.97 & 114.05 & 132.67 & 146.68 & 158.56 & 169.19 & 179.00 \\
\hline 162 & 84.95 & 73.03 & 66.05 & 61.10 & 57.26 & 54.13 & 114.42 & 133.10 & 147.16 & 159.08 & 169.74 & 179.57 \\
\hline 163 & 85.22 & 73.26 & 66.26 & 61.30 & 57.44 & 54.30 & 114.76 & 133.50 & 147.60 & 159.55 & 170.25 & 180.11 \\
\hline 164 & 85.63 & 73.61 & 66.58 & 61.59 & 57.72 & 54.56 & 114.92 & 133.68 & 147.80 & 159.77 & 170.48 & 180.35 \\
\hline 165 & 85.90 & 73.84 & 66.79 & 61.78 & 57.90 & 54.73 & 115.25 & 134.07 & 148.23 & 160.24 & 170.98 & 180.89 \\
\hline 166 & 86.14 & 74.05 & 66.98 & 61.96 & 58.07 & 54.89 & 115.63 & 134.51 & 148.71 & 160.75 & 171.53 & 181.47 \\
\hline 167 & 86.75 & 74.57 & 67.45 & 62.40 & 58.48 & 55.27 & 115.51 & 134.37 & 148.56 & 160.59 & 171.35 & 181.28 \\
\hline 168 & 87.24 & 75.00 & 67.83 & 62.75 & 58.81 & 55.59 & 115.54 & 134.40 & 148.60 & 160.63 & 171.40 & 181.33 \\
\hline 169 & 87.23 & 74.99 & 67.83 & 62.74 & 58.80 & 55.58 & 116.24 & 135.22 & 149.50 & 161.61 & 172.44 & 182.43 \\
\hline 170 & 87.41 & 75.14 & 67.97 & 62.87 & 58.92 & 55.70 & 116.69 & 135.74 & 150.07 & 162.23 & 173.10 & 183.13 \\
\hline 171 & 88.09 & 75.73 & 68.49 & 63.36 & 59.38 & 56.13 & 116.47 & 135.49 & 149.80 & 161.93 & 172.78 & 182.79 \\
\hline 172 & 88.46 & 76.05 & 68.78 & 63.63 & 59.63 & 56.37 & 116.66 & 135.71 & 150.04 & 162.19 & 173.06 & 183.09 \\
\hline 173 & 88.67 & 76.22 & 68.94 & 63.78 & 59.77 & 56.50 & 117.06 & 136.18 & 150.56 & 162.75 & 173.66 & 183.73 \\
\hline 174 & 88.94 & 76.46 & 69.15 & 63.97 & 59.95 & 56.67 & 117.38 & 136.55 & 150.97 & 163.19 & 174.13 & 184.22 \\
\hline 175 & 89.36 & 76.82 & 69.48 & 64.28 & 60.24 & 56.94 & 117.50 & 136.68 & 151.12 & 163.36 & 174.31 & 184.41 \\
\hline 176 & 89.88 & 77.26 & 69.88 & 64.65 & 60.59 & 57.27 & 117.49 & 136.68 & 151.11 & 163.35 & 174.30 & 184.40 \\
\hline 177 & 89.93 & 77.31 & 69.92 & 64.68 & 60.62 & 57.30 & 118.09 & 137.37 & 151.88 & 164.18 & 175.19 & 185.34 \\
\hline 178 & 90.45 & 77.75 & 70.32 & 65.06 & 60.97 & 57.63 & 118.08 & 137.36 & 151.87 & 164.17 & 175.17 & 185.32 \\
\hline 179 & 90.68 & 77.95 & 70.51 & 65.22 & 61.13 & 57.78 & 118.44 & 137.78 & 152.33 & 164.66 & 175.70 & 185.88 \\
\hline 180 & 90.99 & 78.22 & 70.75 & 65.45 & 61.33 & 57.97 & 118.70 & 138.08 & 152.66 & 165.02 & 176.08 & 186.29 \\
\hline 181 & 91.49 & 78.65 & 71.13 & 65.80 & 61.67 & 58.29 & 118.70 & 138.09 & 152.67 & 165.03 & 176.10 & 186.30 \\
\hline 182 & 91.78 & 78.90 & 71.36 & 66.02 & 61.87 & 58.48 & 118.98 & 138.41 & 153.02 & 165.41 & 176.50 & 186.73 \\
\hline 183 & 92.02 & 79.11 & 71.55 & 66.19 & 62.03 & 58.63 & 119.32 & 138.80 & 153.46 & 165.89 & 177.01 & 187.26 \\
\hline 184 & 92.38 & 79.42 & 71.83 & 66.45 & 62.27 & 58.86 & 119.50 & 139.01 & 153.69 & 166.14 & 177.28 & 187.55 \\
\hline 185 & 92.56 & 79.57 & 71.97 & 66.57 & 62.39 & 58.97 & 119.93 & 139.51 & 154.24 & 166.73 & 177.91 & 188.22 \\
\hline 186 & 93.23 & 80.14 & 72.49 & 67.06 & 62.85 & 59.40 & 119.70 & 139.25 & 153.95 & 166.42 & 177.58 & 187.87 \\
\hline 187 & 93.56 & 80.43 & 72.75 & 67.30 & 63.07 & 59.62 & 119.92 & 139.50 & 154.23 & 166.72 & 177.89 & 188.20 \\
\hline 188 & 93.54 & 80.41 & 72.73 & 67.28 & 63.05 & 59.60 & 120.59 & 140.28 & 155.10 & 167.66 & 178.90 & 189.26 \\
\hline 189 & 93.97 & 80.78 & 73.07 & 67.59 & 63.35 & 59.88 & 120.67 & 140.38 & 155.20 & 167.77 & 179.02 & 189.39 \\
\hline 190 & 94.50 & 81.24 & 73.48 & 67.97 & 63.70 & 60.22 & 120.63 & 140.33 & 155.14 & 167.71 & 178.95 & 189.32 \\
\hline
\end{tabular}


Table B.3: Simulation Data for Picking Times (Low Rate) - continued.

\begin{tabular}{|c|c|c|c|c|c|c|c|c|c|c|c|c|}
\hline \multirow{2}{*}{$\begin{array}{c}\text { Batch } \\
\text { Size }\end{array}$} & \multicolumn{6}{|c|}{ Picking Time (minutes) } & \multicolumn{6}{|c|}{ Picking Rate (orders/hr) } \\
\hline & 20 & 40 & 60 & 80 & 100 & 120 & 20 & 40 & 60 & 80 & 100 & 120 \\
\hline 191 & 94.75 & 81.45 & 73.67 & 68.15 & 63.87 & 60.37 & 120.94 & 140.69 & 155.55 & 168.15 & 179.42 & 189.81 \\
\hline 192 & 95.05 & 81.71 & 73.91 & 68.37 & 64.07 & 60.56 & 121.20 & 140.99 & 155.87 & 168.50 & 179.79 & 190.21 \\
\hline 193 & 95.42 & 82.03 & 74.19 & 68.63 & 64.32 & 60.80 & 121.36 & 141.17 & 156.08 & 168.72 & 180.03 & 190.46 \\
\hline 194 & 95.18 & 81.82 & 74.01 & 68.46 & 64.16 & 60.65 & 122.29 & 142.26 & 157.29 & 170.02 & 181.42 & 191.93 \\
\hline 195 & 95.91 & 82.45 & 74.57 & 68.99 & 64.65 & 61.11 & 121.99 & 141.91 & 156.89 & 169.60 & 180.97 & 191.46 \\
\hline 196 & 96.31 & 82.79 & 74.88 & 69.27 & 64.92 & 61.36 & 122.11 & 142.05 & 157.05 & 169.77 & 181.15 & 191.64 \\
\hline 197 & 96.67 & 83.10 & 75.16 & 69.53 & 65.16 & 61.59 & 122.27 & 142.24 & 157.26 & 170.00 & 181.39 & 191.90 \\
\hline 198 & 96.98 & 83.37 & 75.41 & 69.76 & 65.38 & 61.79 & 122.50 & 142.50 & 157.54 & 170.30 & 181.72 & 192.25 \\
\hline 199 & 97.36 & 83.70 & 75.70 & 70.03 & 65.63 & 62.04 & 122.64 & 142.66 & 157.73 & 170.50 & 181.93 & 192.47 \\
\hline 200 & 97.88 & 84.15 & 76.11 & 70.41 & 65.98 & 62.37 & 122.59 & 142.61 & 157.67 & 170.44 & 181.86 & 192.40 \\
\hline 201 & 98.00 & 84.25 & 76.20 & 70.49 & 66.06 & 62.44 & 123.06 & 143.15 & 158.27 & 171.09 & 182.55 & 193.13 \\
\hline 202 & 98.40 & 84.59 & 76.51 & 70.78 & 66.33 & 62.70 & 123.17 & 143.28 & 158.41 & 171.24 & 182.72 & 193.31 \\
\hline 203 & 98.70 & 84.85 & 76.75 & 71.00 & 66.54 & 62.89 & 123.40 & 143.55 & 158.71 & 171.56 & 183.06 & 193.67 \\
\hline 204 & 98.85 & 84.97 & 76.86 & 71.10 & 66.63 & 62.98 & 123.83 & 144.04 & 159.26 & 172.15 & 183.69 & 194.34 \\
\hline 205 & 99.47 & 85.51 & 77.34 & 71.55 & 67.05 & 63.38 & 123.65 & 143.84 & 159.03 & 171.91 & 183.44 & 194.07 \\
\hline 206 & 99.52 & 85.55 & 77.38 & 71.58 & 67.08 & 63.41 & 124.20 & 144.48 & 159.73 & 172.67 & 184.25 & 194.92 \\
\hline 207 & 100.20 & 86.14 & 77.91 & 72.07 & 67.54 & 63.84 & 123.95 & 144.19 & 159.42 & 172.33 & 183.88 & 194.54 \\
\hline 208 & 100.22 & 86.15 & 77.92 & 72.09 & 67.56 & 63.86 & 124.52 & 144.86 & 160.15 & 173.13 & 184.73 & 195.43 \\
\hline 209 & 100.70 & 86.56 & 78.30 & 72.43 & 67.88 & 64.16 & 124.53 & 144.86 & 160.16 & 173.13 & 184.74 & 195.44 \\
\hline 210 & 100.95 & 86.78 & 78.50 & 72.61 & 68.05 & 64.33 & 124.81 & 145.19 & 160.52 & 173.52 & 185.15 & 195.88 \\
\hline 211 & 101.24 & 87.03 & 78.71 & 72.82 & 68.24 & 64.51 & 125.05 & 145.47 & 160.83 & 173.86 & 185.51 & 196.26 \\
\hline 212 & 101.83 & 87.54 & 79.18 & 73.24 & 68.64 & 64.88 & 124.91 & 145.31 & 160.65 & 173.67 & 185.31 & 196.05 \\
\hline 213 & 101.77 & 87.49 & 79.13 & 73.20 & 68.61 & 64.85 & 125.57 & 146.08 & 161.50 & 174.58 & 186.28 & 197.08 \\
\hline 214 & 102.32 & 87.96 & 79.56 & 73.60 & 68.97 & 65.20 & 125.49 & 145.98 & 161.39 & 174.46 & 186.16 & 196.94 \\
\hline 215 & 102.39 & 88.01 & 79.61 & 73.64 & 69.02 & 65.24 & 125.99 & 146.57 & 162.04 & 175.17 & 186.91 & 197.74 \\
\hline 216 & 103.11 & 88.64 & 80.17 & 74.17 & 69.51 & 65.70 & 125.69 & 146.21 & 161.65 & 174.74 & 186.46 & 197.26 \\
\hline 217 & 103.43 & 88.91 & 80.42 & 74.39 & 69.72 & 65.90 & 125.88 & 146.44 & 161.90 & 175.02 & 186.75 & 197.57 \\
\hline 218 & 103.40 & 88.89 & 80.40 & 74.37 & 69.70 & 65.88 & 126.50 & 147.16 & 162.70 & 175.87 & 187.66 & 198.54 \\
\hline 219 & 103.73 & 89.17 & 80.66 & 74.61 & 69.93 & 66.10 & 126.67 & 147.35 & 162.91 & 176.11 & 187.91 & 198.80 \\
\hline 220 & 104.28 & 89.64 & 81.08 & 75.01 & 70.29 & 66.44 & 126.58 & 147.25 & 162.80 & 175.98 & 187.78 & 198.66 \\
\hline 221 & 104.69 & 90.00 & 81.40 & 75.30 & 70.57 & 66.71 & 126.66 & 147.34 & 162.90 & 176.09 & 187.89 & 198.78 \\
\hline 222 & 104.83 & 90.12 & 81.51 & 75.40 & 70.67 & 66.79 & 127.06 & 147.81 & 163.42 & 176.65 & 188.49 & 199.42 \\
\hline 223 & 105.27 & 90.49 & 81.85 & 75.72 & 70.96 & 67.07 & 127.11 & 147.86 & 163.47 & 176.71 & 188.56 & 199.49 \\
\hline 224 & 105.50 & 90.69 & 82.03 & 75.88 & 71.11 & 67.22 & 127.40 & 148.20 & 163.85 & 177.12 & 188.99 & 199.94 \\
\hline 225 & 105.87 & 91.01 & 82.32 & 76.15 & 71.36 & 67.46 & 127.52 & 148.34 & 164.00 & 177.28 & 189.17 & 200.13 \\
\hline 226 & 105.84 & 90.99 & 82.30 & 76.13 & 71.35 & 67.44 & 128.12 & 149.03 & 164.77 & 178.12 & 190.06 & 201.07 \\
\hline 227 & 106.65 & 91.68 & 82.93 & 76.71 & 71.89 & 67.96 & 127.70 & 148.56 & 164.24 & 177.55 & 189.45 & 200.43 \\
\hline 228 & 106.77 & 91.78 & 83.01 & 76.79 & 71.97 & 68.03 & 128.13 & 149.05 & 164.79 & 178.14 & 190.08 & 201.09 \\
\hline 229 & 107.27 & 92.22 & 83.41 & 77.16 & 72.31 & 68.35 & 128.09 & 149.00 & 164.73 & 178.08 & 190.01 & 201.02 \\
\hline 230 & 107.63 & 92.52 & 83.68 & 77.41 & 72.55 & 68.58 & 128.22 & 149.16 & 164.91 & 178.27 & 190.22 & 201.24 \\
\hline 231 & 107.94 & 92.79 & 83.93 & 77.64 & 72.76 & 68.78 & 128.40 & 149.37 & 165.14 & 178.52 & 190.49 & 201.52 \\
\hline 232 & 108.17 & 92.98 & 84.10 & 77.80 & 72.91 & 68.92 & 128.69 & 149.70 & 165.51 & 178.92 & 190.91 & 201.97 \\
\hline
\end{tabular}


Table B.3: Simulation Data for Picking Times (Low Rate) - continued.

\begin{tabular}{|c|c|c|c|c|c|c|c|c|c|c|c|c|}
\hline \multirow{2}{*}{$\begin{array}{c}\text { Batch } \\
\text { Size }\end{array}$} & \multicolumn{6}{|c|}{ Picking Time (minutes) } & \multicolumn{6}{|c|}{ Picking Rate (orders/hr) } \\
\hline & 20 & 40 & 60 & 80 & 100 & 120 & 20 & 40 & 60 & 80 & 100 & 120 \\
\hline 233 & 108.51 & 93.28 & 84.37 & 78.05 & 73.15 & 69.14 & 128.83 & 149.87 & 165.70 & 179.12 & 191.12 & 202.20 \\
\hline 234 & 108.83 & 93.55 & 84.62 & 78.28 & 73.36 & 69.34 & 129.01 & 150.07 & 165.92 & 179.36 & 191.38 & 202.47 \\
\hline 235 & 109.31 & 93.97 & 84.99 & 78.62 & 73.68 & 69.65 & 128.99 & 150.05 & 165.90 & 179.33 & 191.36 & 202.44 \\
\hline 236 & 109.61 & 94.22 & 85.22 & 78.84 & 73.88 & 69.84 & 129.19 & 150.28 & 166.15 & 179.61 & 191.65 & 202.76 \\
\hline 237 & 109.67 & 94.28 & 85.27 & 78.88 & 73.93 & 69.88 & 129.66 & 150.83 & 166.76 & 180.27 & 192.35 & 203.50 \\
\hline 238 & 110.21 & 94.74 & 85.69 & 79.27 & 74.29 & 70.22 & 129.57 & 150.73 & 166.64 & 180.14 & 192.22 & 203.35 \\
\hline 239 & 110.30 & 94.82 & 85.76 & 79.34 & 74.35 & 70.28 & 130.01 & 151.23 & 167.20 & 180.75 & 192.86 & 204.04 \\
\hline 240 & 110.62 & 95.09 & 86.01 & 79.56 & 74.57 & 70.48 & 130.18 & 151.44 & 167.43 & 180.99 & 193.12 & 204.31 \\
\hline 241 & 110.98 & 95.40 & 86.29 & 79.83 & 74.81 & 70.71 & 130.29 & 151.57 & 167.57 & 181.15 & 193.29 & 204.49 \\
\hline 242 & 111.39 & 95.76 & 86.61 & 80.12 & 75.09 & 70.97 & 130.35 & 151.64 & 167.65 & 181.23 & 193.38 & 204.58 \\
\hline 243 & 111.63 & 95.96 & 86.80 & 80.29 & 75.25 & 71.13 & 130.61 & 151.93 & 167.98 & 181.58 & 193.76 & 204.98 \\
\hline 244 & 111.98 & 96.27 & 87.07 & 80.55 & 75.49 & 71.35 & 130.73 & 152.08 & 168.14 & 181.76 & 193.94 & 205.18 \\
\hline 245 & 112.32 & 96.56 & 87.33 & 80.79 & 75.71 & 71.57 & 130.87 & 152.24 & 168.32 & 181.95 & 194.15 & 205.40 \\
\hline 246 & 112.80 & 96.97 & 87.70 & 81.13 & 76.04 & 71.87 & 130.85 & 152.22 & 168.29 & 181.92 & 194.12 & 205.37 \\
\hline 247 & 113.05 & 97.18 & 87.90 & 81.32 & 76.21 & 72.03 & 131.09 & 152.49 & 168.60 & 182.25 & 194.47 & 205.74 \\
\hline 248 & 113.22 & 97.33 & 88.03 & 81.44 & 76.32 & 72.14 & 131.43 & 152.88 & 169.03 & 182.72 & 194.97 & 206.26 \\
\hline 249 & 113.53 & 97.60 & 88.28 & 81.66 & 76.53 & 72.34 & 131.59 & 153.08 & 169.24 & 182.95 & 195.21 & 206.52 \\
\hline 250 & 113.77 & 97.81 & 88.46 & 81.84 & 76.69 & 72.49 & 131.84 & 153.37 & 169.56 & 183.29 & 195.58 & 206.91 \\
\hline 251 & 114.08 & 98.07 & 88.70 & 82.06 & 76.90 & 72.69 & 132.01 & 153.56 & 169.78 & 183.53 & 195.83 & 207.18 \\
\hline 252 & 114.66 & 98.56 & 89.15 & 82.47 & 77.29 & 73.06 & 131.87 & 153.41 & 169.60 & 183.34 & 195.63 & 206.97 \\
\hline 253 & 114.82 & 98.70 & 89.27 & 82.59 & 77.40 & 73.16 & 132.21 & 153.80 & 170.04 & 183.81 & 196.13 & 207.50 \\
\hline 254 & 115.21 & 99.04 & 89.58 & 82.87 & 77.66 & 73.41 & 132.28 & 153.87 & 170.12 & 183.90 & 196.23 & 207.60 \\
\hline 255 & 115.28 & 99.10 & 89.63 & 82.92 & 77.71 & 73.45 & 132.72 & 154.39 & 170.69 & 184.52 & 196.89 & 208.30 \\
\hline 256 & 115.68 & 99.45 & 89.95 & 83.21 & 77.98 & 73.71 & 132.78 & 154.45 & 170.77 & 184.60 & 196.97 & 208.38 \\
\hline 257 & 115.87 & 99.60 & 90.09 & 83.34 & 78.10 & 73.83 & 133.09 & 154.82 & 171.16 & 185.03 & 197.43 & 208.87 \\
\hline 258 & 116.18 & 99.87 & 90.33 & 83.56 & 78.31 & 74.02 & 133.24 & 155.00 & 171.37 & 185.25 & 197.67 & 209.12 \\
\hline 259 & 116.73 & 100.35 & 90.76 & 83.96 & 78.69 & 74.38 & 133.13 & 154.86 & 171.22 & 185.09 & 197.49 & 208.94 \\
\hline 260 & 116.93 & 100.52 & 90.92 & 84.11 & 78.82 & 74.51 & 133.41 & 155.19 & 171.58 & 185.48 & 197.91 & 209.38 \\
\hline 261 & 117.35 & 100.88 & 91.25 & 84.41 & 79.11 & 74.77 & 133.44 & 155.23 & 171.63 & 185.53 & 197.96 & 209.43 \\
\hline 262 & 117.68 & 101.16 & 91.50 & 84.65 & 79.33 & 74.98 & 133.58 & 155.39 & 171.80 & 185.72 & 198.17 & 209.65 \\
\hline 263 & 117.93 & 101.37 & 91.69 & 84.82 & 79.49 & 75.14 & 133.81 & 155.66 & 172.10 & 186.04 & 198.51 & 210.01 \\
\hline 264 & 118.35 & 101.74 & 92.02 & 85.13 & 79.78 & 75.41 & 133.84 & 155.69 & 172.13 & 186.07 & 198.55 & 210.05 \\
\hline 265 & 118.62 & 101.97 & 92.23 & 85.32 & 79.96 & 75.58 & 134.04 & 155.93 & 172.39 & 186.36 & 198.85 & 210.37 \\
\hline 266 & 118.95 & 102.26 & 92.49 & 85.56 & 80.18 & 75.79 & 134.17 & 156.08 & 172.56 & 186.54 & 199.04 & 210.58 \\
\hline 267 & 119.39 & 102.63 & 92.83 & 85.87 & 80.48 & 76.07 & 134.19 & 156.10 & 172.58 & 186.56 & 199.06 & 210.60 \\
\hline 268 & 119.46 & 102.69 & 92.88 & 85.92 & 80.53 & 76.12 & 134.61 & 156.59 & 173.12 & 187.14 & 199.69 & 211.26 \\
\hline 269 & 119.78 & 102.97 & 93.14 & 86.16 & 80.75 & 76.32 & 134.74 & 156.74 & 173.29 & 187.33 & 199.89 & 211.47 \\
\hline 270 & 120.19 & 103.32 & 93.45 & 86.45 & 81.02 & 76.58 & 134.78 & 156.79 & 173.35 & 187.39 & 199.95 & 211.53 \\
\hline 271 & 120.48 & 103.57 & 93.68 & 86.66 & 81.22 & 76.77 & 134.96 & 156.99 & 173.57 & 187.63 & 200.21 & 211.81 \\
\hline 272 & 120.89 & 103.92 & 94.00 & 86.95 & 81.49 & 77.03 & 135.00 & 157.04 & 173.62 & 187.69 & 200.27 & 211.87 \\
\hline 273 & 121.23 & 104.21 & 94.26 & 87.20 & 81.72 & 77.24 & 135.12 & 157.18 & 173.77 & 187.85 & 200.44 & 212.06 \\
\hline 274 & 121.37 & 104.33 & 94.37 & 87.30 & 81.81 & 77.33 & 135.46 & 157.57 & 174.21 & 188.32 & 200.95 & 212.59 \\
\hline
\end{tabular}


Table B.3: Simulation Data for Picking Times (Low Rate) - continued.

\begin{tabular}{|c|c|c|c|c|c|c|c|c|c|c|c|c|}
\hline \multirow{2}{*}{$\begin{array}{c}\text { Batch } \\
\text { Size }\end{array}$} & \multicolumn{6}{|c|}{ Picking Time (minutes) } & \multicolumn{6}{|c|}{ Picking Rate (orders/hr) } \\
\hline & 20 & 40 & 60 & 80 & 100 & 120 & 20 & 40 & 60 & 80 & 100 & 120 \\
\hline 275 & 121.79 & 104.69 & 94.69 & 87.60 & 82.10 & 77.60 & 135.48 & 157.60 & 174.24 & 188.36 & 200.98 & 212.63 \\
\hline 276 & 121.92 & 104.81 & 94.80 & 87.70 & 82.19 & 77.69 & 135.82 & 158.00 & 174.68 & 188.83 & 201.49 & 213.17 \\
\hline 277 & 122.39 & 105.21 & 95.16 & 88.03 & 82.50 & 77.98 & 135.80 & 157.97 & 174.65 & 188.80 & 201.45 & 213.13 \\
\hline 278 & 122.68 & 105.46 & 95.39 & 88.24 & 82.70 & 78.17 & 135.96 & 158.16 & 174.86 & 189.02 & 201.69 & 213.38 \\
\hline 279 & 123.06 & 105.79 & 95.68 & 88.51 & 82.95 & 78.41 & 136.03 & 158.24 & 174.95 & 189.12 & 201.80 & 213.49 \\
\hline 280 & 123.23 & 105.93 & 95.82 & 88.64 & 83.07 & 78.52 & 136.33 & 158.59 & 175.34 & 189.54 & 202.24 & 213.96 \\
\hline 281 & 123.63 & 106.28 & 96.13 & 88.92 & 83.34 & 78.77 & 136.37 & 158.64 & 175.39 & 189.60 & 202.31 & 214.03 \\
\hline 282 & 123.92 & 106.52 & 96.35 & 89.13 & 83.53 & 78.96 & 136.54 & 158.84 & 175.61 & 189.83 & 202.56 & 214.29 \\
\hline 283 & 124.29 & 106.85 & 96.64 & 89.40 & 83.79 & 79.20 & 136.61 & 158.92 & 175.70 & 189.93 & 202.66 & 214.40 \\
\hline 284 & 124.59 & 107.11 & 96.88 & 89.62 & 83.99 & 79.39 & 136.76 & 159.09 & 175.89 & 190.14 & 202.89 & 214.64 \\
\hline 285 & 124.83 & 107.30 & 97.06 & 89.78 & 84.14 & 79.54 & 136.99 & 159.36 & 176.19 & 190.46 & 203.22 & 215.00 \\
\hline 286 & 125.24 & 107.66 & 97.38 & 90.08 & 84.42 & 79.80 & 137.02 & 159.39 & 176.22 & 190.49 & 203.26 & 215.04 \\
\hline 287 & 125.43 & 107.82 & 97.53 & 90.22 & 84.55 & 79.92 & 137.29 & 159.70 & 176.57 & 190.87 & 203.66 & 215.46 \\
\hline 288 & 125.83 & 108.17 & 97.84 & 90.51 & 84.82 & 80.18 & 137.33 & 159.75 & 176.62 & 190.92 & 203.72 & 215.52 \\
\hline 289 & 126.15 & 108.44 & 98.08 & 90.74 & 85.03 & 80.38 & 137.46 & 159.90 & 176.79 & 191.11 & 203.92 & 215.73 \\
\hline 290 & 126.51 & 108.75 & 98.37 & 91.00 & 85.28 & 80.61 & 137.54 & 159.99 & 176.89 & 191.21 & 204.03 & 215.86 \\
\hline 291 & 126.77 & 108.97 & 98.57 & 91.18 & 85.45 & 80.77 & 137.73 & 160.22 & 177.14 & 191.49 & 204.32 & 216.16 \\
\hline 292 & 127.01 & 109.18 & 98.75 & 91.36 & 85.62 & 80.93 & 137.94 & 160.46 & 177.41 & 191.78 & 204.63 & 216.49 \\
\hline 293 & 127.27 & 109.40 & 98.95 & 91.54 & 85.79 & 81.09 & 138.13 & 160.69 & 177.66 & 192.05 & 204.92 & 216.79 \\
\hline 294 & 127.50 & 109.60 & 99.13 & 91.71 & 85.94 & 81.24 & 138.36 & 160.95 & 177.94 & 192.36 & 205.25 & 217.14 \\
\hline 295 & 128.03 & 110.06 & 99.55 & 92.09 & 86.31 & 81.58 & 138.24 & 160.82 & 177.80 & 192.20 & 205.08 & 216.97 \\
\hline 296 & 128.41 & 110.38 & 99.84 & 92.36 & 86.56 & 81.82 & 138.31 & 160.89 & 177.88 & 192.29 & 205.18 & 217.07 \\
\hline 297 & 128.36 & 110.34 & 99.80 & 92.33 & 86.53 & 81.79 & 138.83 & 161.50 & 178.55 & 193.01 & 205.95 & 217.88 \\
\hline 298 & 128.88 & 110.79 & 100.21 & 92.70 & 86.88 & 82.12 & 138.73 & 161.38 & 178.43 & 192.88 & 205.81 & 217.73 \\
\hline 299 & 129.37 & 111.21 & 100.59 & 93.05 & 87.21 & 82.43 & 138.67 & 161.32 & 178.35 & 192.80 & 205.72 & 217.64 \\
\hline 300 & 129.62 & 111.43 & 100.78 & 93.23 & 87.37 & 82.59 & 138.87 & 161.54 & 178.60 & 193.07 & 206.01 & 217.95 \\
\hline 301 & 129.82 & 111.59 & 100.94 & 93.37 & 87.51 & 82.71 & 139.12 & 161.84 & 178.93 & 193.42 & 206.38 & 218.34 \\
\hline 302 & 130.15 & 111.89 & 101.20 & 93.62 & 87.74 & 82.93 & 139.22 & 161.95 & 179.05 & 193.56 & 206.53 & 218.50 \\
\hline 303 & 130.48 & 112.16 & 101.45 & 93.85 & 87.95 & 83.14 & 139.33 & 162.08 & 179.20 & 193.71 & 206.70 & 218.67 \\
\hline 304 & 130.78 & 112.42 & 101.68 & 94.07 & 88.16 & 83.33 & 139.47 & 162.25 & 179.38 & 193.91 & 206.91 & 218.90 \\
\hline 305 & 130.89 & 112.52 & 101.77 & 94.15 & 88.23 & 83.40 & 139.81 & 162.64 & 179.81 & 194.37 & 207.40 & 219.42 \\
\hline 306 & 131.46 & 113.01 & 102.21 & 94.56 & 88.62 & 83.76 & 139.66 & 162.47 & 179.62 & 194.17 & 207.19 & 219.19 \\
\hline 307 & 131.64 & 113.16 & 102.36 & 94.69 & 88.74 & 83.88 & 139.93 & 162.77 & 179.96 & 194.54 & 207.58 & 219.61 \\
\hline 308 & 132.14 & 113.59 & 102.74 & 95.04 & 89.07 & 84.19 & 139.86 & 162.69 & 179.87 & 194.44 & 207.47 & 219.50 \\
\hline 309 & 132.24 & 113.68 & 102.82 & 95.12 & 89.14 & 84.26 & 140.20 & 163.09 & 180.32 & 194.92 & 207.99 & 220.04 \\
\hline 310 & 132.70 & 114.08 & 103.18 & 95.45 & 89.45 & 84.55 & 140.16 & 163.05 & 180.27 & 194.87 & 207.93 & 219.98 \\
\hline 311 & 132.85 & 114.20 & 103.29 & 95.55 & 89.55 & 84.65 & 140.46 & 163.40 & 180.65 & 195.28 & 208.37 & 220.45 \\
\hline 312 & 133.23 & 114.53 & 103.59 & 95.83 & 89.81 & 84.89 & 140.51 & 163.46 & 180.72 & 195.35 & 208.45 & 220.53 \\
\hline 313 & 133.35 & 114.63 & 103.68 & 95.92 & 89.89 & 84.97 & 140.83 & 163.83 & 181.13 & 195.80 & 208.92 & 221.03 \\
\hline 314 & 133.78 & 115.00 & 104.02 & 96.23 & 90.18 & 85.24 & 140.83 & 163.82 & 181.12 & 195.79 & 208.91 & 221.02 \\
\hline 315 & 134.29 & 115.44 & 104.42 & 96.59 & 90.53 & 85.57 & 140.74 & 163.71 & 181.00 & 195.66 & 208.78 & 220.88 \\
\hline 316 & 134.40 & 115.54 & 104.50 & 96.67 & 90.60 & 85.64 & 141.07 & 164.11 & 181.43 & 196.13 & 209.28 & 221.40 \\
\hline
\end{tabular}


Table B.3: Simulation Data for Picking Times (Low Rate) - continued.

\begin{tabular}{|c|c|c|c|c|c|c|c|c|c|c|c|c|}
\hline \multirow{2}{*}{$\begin{array}{c}\text { Batch } \\
\text { Size }\end{array}$} & \multicolumn{6}{|c|}{ Picking Time (minutes) } & \multicolumn{6}{|c|}{ Picking Rate (orders/hr) } \\
\hline & 20 & 40 & 60 & 80 & 100 & 120 & 20 & 40 & 60 & 80 & 100 & 120 \\
\hline 317 & 134.82 & 115.89 & 104.82 & 96.97 & 90.88 & 85.90 & 141.08 & 164.12 & 181.45 & 196.14 & 209.29 & 221.42 \\
\hline 318 & 135.24 & 116.26 & 105.15 & 97.28 & 91.17 & 86.17 & 141.08 & 164.12 & 181.45 & 196.14 & 209.29 & 221.42 \\
\hline 319 & 135.44 & 116.43 & 105.31 & 97.42 & 91.30 & 86.30 & 141.31 & 164.39 & 181.75 & 196.47 & 209.64 & 221.78 \\
\hline 320 & 135.79 & 116.73 & 105.58 & 97.67 & 91.53 & 86.52 & 141.40 & 164.48 & 181.85 & 196.58 & 209.76 & 221.91 \\
\hline 321 & 136.08 & 116.98 & 105.80 & 97.88 & 91.73 & 86.70 & 141.54 & 164.65 & 182.04 & 196.78 & 209.97 & 222.14 \\
\hline 322 & 136.25 & 117.12 & 105.94 & 98.00 & 91.84 & 86.81 & 141.80 & 164.95 & 182.37 & 197.14 & 210.36 & 222.55 \\
\hline 323 & 136.58 & 117.41 & 106.19 & 98.24 & 92.06 & 87.02 & 141.90 & 165.07 & 182.50 & 197.28 & 210.50 & 222.70 \\
\hline 324 & 136.99 & 117.76 & 106.51 & 98.53 & 92.34 & 87.29 & 141.91 & 165.08 & 182.51 & 197.29 & 210.52 & 222.72 \\
\hline 325 & 137.49 & 118.19 & 106.90 & 98.89 & 92.68 & 87.60 & 141.83 & 164.99 & 182.41 & 197.19 & 210.41 & 222.60 \\
\hline 326 & 137.62 & 118.30 & 107.01 & 98.99 & 92.77 & 87.69 & 142.13 & 165.34 & 182.80 & 197.60 & 210.85 & 223.06 \\
\hline 327 & 137.84 & 118.49 & 107.17 & 99.14 & 92.92 & 87.83 & 142.34 & 165.58 & 183.07 & 197.89 & 211.16 & 223.40 \\
\hline 328 & 138.30 & 118.88 & 107.53 & 99.47 & 93.22 & 88.12 & 142.30 & 165.54 & 183.02 & 197.84 & 211.10 & 223.34 \\
\hline 329 & 138.28 & 118.87 & 107.52 & 99.46 & 93.22 & 88.11 & 142.75 & 166.06 & 183.59 & 198.46 & 211.77 & 224.04 \\
\hline 330 & 138.95 & 119.45 & 108.04 & 99.94 & 93.67 & 88.53 & 142.50 & 165.76 & 183.27 & 198.11 & 211.39 & 223.64 \\
\hline 331 & 139.10 & 119.58 & 108.15 & 100.05 & 93.77 & 88.63 & 142.78 & 166.09 & 183.63 & 198.50 & 211.80 & 224.08 \\
\hline 332 & 139.39 & 119.82 & 108.38 & 100.26 & 93.96 & 88.81 & 142.91 & 166.25 & 183.80 & 198.69 & 212.01 & 224.29 \\
\hline 333 & 139.92 & 120.28 & 108.79 & 100.64 & 94.32 & 89.15 & 142.80 & 166.12 & 183.66 & 198.53 & 211.84 & 224.12 \\
\hline 334 & 139.49 & 119.91 & 108.46 & 100.33 & 94.03 & 88.88 & 143.67 & 167.13 & 184.77 & 199.74 & 213.13 & 225.48 \\
\hline 335 & 139.83 & 120.20 & 108.72 & 100.58 & 94.26 & 89.10 & 143.74 & 167.21 & 184.87 & 199.85 & 213.24 & 225.60 \\
\hline 336 & 140.12 & 120.46 & 108.95 & 100.79 & 94.46 & 89.28 & 143.87 & 167.36 & 185.04 & 200.02 & 213.43 & 225.80 \\
\hline 337 & 140.49 & 120.77 & 109.24 & 101.05 & 94.71 & 89.52 & 143.92 & 167.42 & 185.10 & 200.09 & 213.50 & 225.88 \\
\hline 338 & 140.74 & 120.99 & 109.43 & 101.23 & 94.87 & 89.68 & 144.09 & 167.62 & 185.32 & 200.33 & 213.76 & 226.15 \\
\hline 339 & 141.20 & 121.38 & 109.79 & 101.56 & 95.18 & 89.97 & 144.05 & 167.57 & 185.26 & 200.27 & 213.69 & 226.07 \\
\hline 340 & 141.50 & 121.64 & 110.02 & 101.78 & 95.39 & 90.16 & 144.17 & 167.70 & 185.41 & 200.43 & 213.87 & 226.26 \\
\hline 341 & 141.59 & 121.71 & 110.09 & 101.84 & 95.44 & 90.22 & 144.50 & 168.10 & 185.85 & 200.90 & 214.37 & 226.79 \\
\hline 342 & 142.15 & 122.19 & 110.52 & 102.24 & 95.82 & 90.57 & 144.36 & 167.93 & 185.66 & & 214.15 & 226.56 \\
\hline 343 & 142.29 & 122.32 & 110.63 & 102.35 & 95.92 & 90.66 & 144.63 & 168.25 & 186.02 & 201.08 & 214.56 & 227.00 \\
\hline 344 & 142.46 & 122.46 & 110.77 & 102.47 & ר & 0077 & 144.88 & 168.54 & 186.34 & 201.43 & 214.93 & 227.38 \\
\hline 345 & 143.02 & 122.95 & 111.21 & 102.87 & 96.41 & 91.13 & 144.73 & 168.36 & 186.14 & 201.22 & 214.71 & 227.15 \\
\hline 346 & 143.30 & 123.18 & 111.42 & 103.07 & 96.59 & 91.30 & 144.87 & 168.53 & 186.33 & 201.42 & 214.92 & 227.37 \\
\hline 347 & 143.48 & 123.34 & 111.56 & 103.20 & 96.72 & 91.42 & 145.11 & 168.80 & 186.63 & 201.74 & 215.26 & 227.74 \\
\hline 348 & 143.91 & 123.71 & 111.89 & 103.51 & 97.01 & 91.69 & 145.09 & 168.78 & 186.61 & 201.72 & 215.24 & 227.71 \\
\hline 349 & 144.22 & 123.98 & 112.13 & 103.73 & 97.22 & 91.89 & 145.20 & 168.90 & 186.74 & 201.86 & 215.40 & 227.88 \\
\hline 350 & 144.35 & 124.09 & 112.24 & 103.83 & 97.30 & 91.98 & 145.48 & 169.23 & 187.10 & 202.26 & 215.82 & 228.32 \\
\hline 351 & 144.76 & 124.44 & 112.55 & 104.12 & 97.58 & 92.23 & 145.48 & 169.24 & 187.11 & 202.27 & 215.82 & 228.33 \\
\hline 352 & 145.10 & 124.73 & 112.82 & 104.37 & 97.81 & 92.45 & 145.55 & 169.32 & 187.20 & 202.36 & 215.93 & 228.44 \\
\hline 353 & 145.38 & 124.97 & 113.04 & 104.57 & 98.00 & 92.63 & 145.69 & 169.48 & 187.37 & 202.55 & 216.13 & 228.65 \\
\hline 354 & 145.61 & 125.17 & 113.22 & 104.74 & 98.16 & 92.78 & 145.87 & 169.68 & 187.60 & 202.80 & 216.39 & 228.93 \\
\hline 355 & 146.09 & 125.59 & 113.59 & 105.08 & 98.48 & 93.09 & 145.80 & 169.60 & 187.51 & 202.70 & 216.29 & 228.82 \\
\hline 356 & 146.28 & 125.75 & 113.74 & 105.21 & 98.60 & 93.20 & 146.02 & 169.87 & 187.80 & 203.01 & 216.62 & 229.18 \\
\hline 357 & 146.54 & 125.97 & 113.94 & 105.40 & 98.78 & 93.37 & 146.17 & 170.04 & 188.00 & 203.22 & 216.85 & 229.41 \\
\hline 358 & 146.88 & 126.27 & 114.21 & 105.65 & 99.01 & 93.59 & 146.24 & 170.12 & 188.08 & 203.31 & 216.94 & 229.51 \\
\hline
\end{tabular}


Table B.3: Simulation Data for Picking Times (Low Rate) — continued.

\begin{tabular}{|c|c|c|c|c|c|c|c|c|c|c|c|c|}
\hline \multirow{2}{*}{$\begin{array}{c}\text { Batch } \\
\text { Size }\end{array}$} & \multicolumn{6}{|c|}{ Picking Time (minutes) } & \multicolumn{6}{|c|}{ Picking Rate (orders/hr) } \\
\hline & 20 & 40 & 60 & 80 & 100 & 120 & 20 & 40 & 60 & 80 & 100 & 120 \\
\hline 359 & 147.30 & 126.63 & 114.53 & 105.95 & 9.30 & 93.86 & 146.23 & 170.10 & 188.07 & 203.30 & 216.93 & 229.50 \\
\hline 360 & 147.57 & 126.86 & 114.74 & 106.14 & 99.47 & 94.03 & 146.37 & 170.27 & 188.25 & 203.50 & 217.14 & 229.72 \\
\hline 361 & 17.73 & 127.00 & 114.87 & 106.26 & 99.58 & 94.13 & 146.62 & 170.56 & 188.57 & 203.84 & 217.50 & 230.11 \\
\hline 362 & 148.06 & 127.28 & 115.12 & 106.50 & 99.81 & 94.34 & 146.69 & 170.65 & 188.67 & 203.95 & 217.62 & 230.23 \\
\hline 363 & 148.39 & 127.56 & 115.38 & 106.73 & 100.03 & 94.55 & 146.78 & 170.74 & 188.77 & 204.06 & 217.74 & 230.36 \\
\hline 364 & 148.68 & 127.81 & 115.61 & 106.94 & 100.23 & 94.74 & 146.89 & 170.87 & 188.92 & 204.22 & 217.91 & 230.54 \\
\hline 365 & 148.90 & 128.00 & 115.78 & 107.10 & 100.37 & 94.88 & 147.08 & 171.09 & 189.16 & 204.48 & 218.19 & 230.83 \\
\hline 366 & 149.34 & 128.38 & 116.12 & 107.42 & 100.67 & 95.16 & 147.05 & 171.06 & 189.12 & 204.44 & 218.14 & 230.78 \\
\hline 367 & 149.69 & 128.68 & 116.39 & 107.67 & 100.90 & 95.38 & 147.11 & 171.13 & 189.20 & 204.52 & 218.23 & 230.88 \\
\hline 368 & 149.95 & 128.90 & 116.59 & 107.86 & 101.08 & 95.54 & 147.25 & 171.29 & 189.38 & 204.72 & 218.44 & 231.10 \\
\hline 369 & 150.19 & 129.11 & 116.78 & 108.03 & 101.24 & 95.70 & 147.41 & 171.48 & 189.59 & 204.95 & 218.69 & 231.36 \\
\hline 370 & 150.59 & 129.46 & 117.09 & 108.32 & 101.51 & 95.95 & 147.42 & 171.49 & 189.60 & 204.95 & 218.69 & 231.36 \\
\hline 371 & 150.72 & 129.56 & 117.19 & 108.41 & 101.60 & 96.03 & 147.69 & 171.81 & 189.95 & 205.34 & 219.10 & 231.80 \\
\hline 372 & 151.13 & 129.91 & 117.51 & 108.70 & 101.87 & 96.29 & 147.69 & 171.81 & 189.95 & 205.33 & 219.10 & 231.79 \\
\hline 373 & 151.51 & 130.24 & 117.80 & 108.97 & 102.13 & 96.53 & 147.72 & 171.84 & 189.98 & 205.37 & 219.14 & 231.83 \\
\hline 374 & 151.85 & 130.53 & 118.07 & 109.22 & 102.36 & 96.75 & 147.78 & 171.91 & 190.06 & 205.46 & 219.23 & 231.93 \\
\hline 375 & 152.03 & 130.69 & 118.21 & 109.35 & 102.48 & 96.87 & 148.00 & 172.16 & 190.35 & 205.76 & 219.55 & 232.28 \\
\hline 376 & 152.43 & 131.03 & 118.52 & 109.64 & 102.75 & 97.12 & 148.01 & 172.17 & 190.35 & 205.77 & 219.56 & 232.29 \\
\hline 377 & 152.63 & 131.20 & 118.67 & 109.78 & 102.88 & 97.25 & 148.21 & 172.41 & 190.61 & 206.05 & 219.86 & 232.60 \\
\hline 378 & 152.85 & 131.39 & 118.84 & 109.94 & 103.03 & 97.39 & 148.38 & 172.61 & 190.84 & 206.30 & 220.12 & 232.88 \\
\hline 379 & 153.31 & 131.80 & 119.21 & 110.28 & 103.35 & 97.69 & 148.32 & 172.54 & 190.76 & 206.21 & 220.03 & 232.78 \\
\hline 380 & 153.49 & 131.95 & 119.34 & 110.40 & 103.47 & 97.80 & 148.54 & 172.80 & 191.04 & 206.52 & 220.36 & 233.13 \\
\hline 381 & 153.81 & 132.22 & 119.59 & 110.63 & 103.68 & 0800 & 148.63 & 172.89 & 191.15 & 206.63 & 220.49 & 233.26 \\
\hline 382 & 154.20 & 132.56 & 119.89 & 110.91 & 103.94 & 98.25 & 148.64 & 172.91 & 191.17 & 206.65 & 220.50 & 233.28 \\
\hline 383 & 154.55 & 132.85 & 120.16 & 111.16 & 104.18 & 98.47 & 148.69 & 172.97 & 191.24 & 206.73 & 220.59 & 233.37 \\
\hline 384 & 154.83 & 133.09 & 120.38 & 111.36 & 104.37 & 98.65 & 148.81 & 173.11 & 191.39 & 206.89 & 220.76 & 233.55 \\
\hline 385 & 155.08 & 133.31 & 120.58 & 111.54 & 104.53 & 98.81 & 148.96 & 173.28 & 191.58 & 207.10 & 220.98 & 233.78 \\
\hline 386 & 155.30 & 133.50 & 120.75 & 111.70 & 104.69 & 98.95 & 149.13 & 173.48 & 191.80 & 207.33 & 221.23 & 234.05 \\
\hline 387 & 155.72 & 133.86 & 121.08 & 112.01 & 104.97 & 99.22 & 149.11 & 173.46 & 191.78 & 207.31 & 221.21 & 234.03 \\
\hline 388 & 155.99 & 134.10 & 121.29 & & & 99.39 & & 173.61 & 191.94 & & 221.39 & 234.22 \\
\hline 389 & 156.37 & 134.42 & 121.58 & 112.47 & 105.41 & 99.63 & 149.26 & 173.64 & 191.97 & 207.52 & 221.43 & 234.26 \\
\hline 390 & 156.67 & 134.68 & 121.81 & 112.69 & 105.61 & 99.82 & 149.36 & 173.75 & 192.10 & 207.66 & 221.58 & 234.42 \\
\hline 391 & 156.95 & 134.92 & 122.04 & 112.89 & 105.80 & 100.01 & 149.47 & 173.88 & 192.24 & 207.81 & 221.74 & 234.59 \\
\hline 392 & 157.28 & 135.20 & 122.29 & 113.12 & 106.02 & 100.21 & 149.55 & 173.96 & 192.33 & 207.91 & 221.85 & 234.70 \\
\hline 393 & 157.57 & 135.46 & 122.52 & 113.34 & 106.22 & 100.40 & 149.64 & 174.08 & 192.46 & 208.05 & 221.99 & 234.86 \\
\hline 394 & 157.89 & 135.73 & 122.76 & 113.56 & 106.43 & 100.60 & 149.73 & 174.17 & 192.57 & 208.16 & 222.12 & 234.99 \\
\hline 395 & 158.01 & 135.83 & 122.86 & 113.65 & 106.51 & 100.68 & 149.99 & 174.48 & 192.91 & 208.53 & 222.51 & 235.41 \\
\hline 396 & 158.46 & 136.22 & 123.21 & 113.98 & 106.82 & 100.96 & 149.94 & 174.43 & 192.85 & 208.47 & 222.44 & 235.33 \\
\hline 397 & 158.80 & 136.51 & 123.48 & 114.22 & 107.05 & 101.19 & 150.00 & 174.49 & 192.91 & 208.54 & 222.52 & 235.41 \\
\hline 398 & 158.99 & 136.68 & 123.62 & 114.36 & 107.18 & 101.31 & 150.20 & 174.72 & 193.17 & 208.81 & 222.81 & 235.72 \\
\hline 399 & 159.42 & 137.05 & 123.96 & 114.67 & 107.47 & 101.58 & 150.17 & 174.69 & 193.13 & 208.78 & 222.77 & 235.68 \\
\hline 400 & 159.62 & 137.21 & 124.11 & 114.81 & 107.60 & 101.70 & 150.36 & 174.91 & 193.38 & 209.04 & 223.06 & 235.98 \\
\hline
\end{tabular}


Table B.3: Simulation Data for Picking Times (Low Rate) - continued.

\begin{tabular}{|c|c|c|c|c|c|c|c|c|c|c|c|c|}
\hline \multirow{2}{*}{$\begin{array}{c}\text { Batch } \\
\text { Size }\end{array}$} & \multicolumn{6}{|c|}{ Picking Time (minutes) } & \multicolumn{6}{|c|}{ Picking Rate (orders/hr) } \\
\hline & 20 & 40 & 60 & 80 & 100 & 120 & 20 & 40 & 60 & 80 & 100 & 120 \\
\hline 401 & 159.97 & 137.52 & 124.38 & 115.06 & 107.83 & 101.93 & 150.40 & 174.96 & 193.44 & 209.10 & 223.12 & 236.05 \\
\hline 402 & 160.18 & 137.70 & 124.54 & 115.21 & 107.97 & 102.06 & 150.58 & 175.17 & 193.67 & 209.35 & 223.39 & 236.33 \\
\hline 403 & 160.63 & 138.08 & 124.89 & 115.53 & 108.28 & 102.35 & 150.54 & 175.12 & 193.61 & 209.29 & 223.32 & 236.26 \\
\hline 404 & 160.83 & 138.25 & 125.05 & 115.68 & 108.41 & 102.47 & 150.72 & 175.33 & 193.84 & 209.54 & 223.59 & 236.55 \\
\hline 405 & 161.13 & 138.51 & 125.28 & 115.89 & 108.61 & 102.66 & 150.81 & 175.44 & 193.96 & 209.67 & 223.73 & 236.69 \\
\hline 406 & 161.50 & 138.83 & 125.57 & 116.17 & 108.87 & 102.90 & 150.83 & 175.46 & 193.99 & 209.70 & 223.76 & 236.72 \\
\hline 407 & 161.71 & 139.01 & 125.73 & 116.31 & 109.01 & 103.04 & 151.01 & 175.67 & 194.22 & 209.95 & 224.02 & 237.01 \\
\hline 408 & 162.08 & 139.33 & 126.02 & 116.58 & 109.26 & 103.27 & 151.03 & 175.70 & 194.25 & 209.98 & 224.06 & 237.04 \\
\hline 409 & 162.38 & 139.59 & 126.25 & 116.80 & 109.46 & 103.46 & 151.13 & 175.80 & 194.37 & 210.11 & 224.20 & 237.19 \\
\hline 410 & 162.69 & 139.85 & 126.50 & 117.02 & 109.67 & 103.66 & 151.21 & 175.90 & 194.47 & 210.22 & 224.31 & 237.31 \\
\hline 411 & 163.14 & 140.24 & 126.84 & 117.34 & 109.97 & 103.94 & 151.16 & 175.84 & 194.41 & 210.16 & 224.25 & 237.24 \\
\hline 412 & 163.31 & 140.39 & 126.98 & 117.47 & 110.09 & 104.06 & 151.37 & 176.08 & 194.68 & 210.44 & 224.55 & 237.56 \\
\hline 413 & 163.56 & 140.60 & 127.17 & 117.65 & 110.26 & 104.22 & 151.50 & 176.24 & 194.85 & 210.63 & 224.75 & 237.77 \\
\hline 414 & 163.89 & 140.89 & 127.43 & 117.88 & 110.48 & 104.43 & 151.56 & 176.31 & 194.93 & 210.72 & 224.84 & 237.87 \\
\hline 415 & 164.29 & 141.23 & 127.74 & 118.17 & 110.74 & 104.68 & 151.56 & 176.31 & 194.93 & 210.72 & 224.84 & 237.87 \\
\hline 416 & 164.53 & 141.44 & 127.93 & 118.35 & 110.91 & 104.84 & 151.70 & 176.47 & 195.11 & 210.91 & 225.05 & 238.09 \\
\hline 417 & 164.72 & 141.60 & 128.08 & 118.48 & 111.04 & 104.96 & 151.89 & 176.69 & 195.35 & 211.17 & 225.33 & 238.39 \\
\hline 418 & 165.19 & 142.00 & 128.44 & 118.81 & 111.35 & 105.25 & 151.83 & 176.62 & 195.27 & 211.09 & 225.24 & 238.29 \\
\hline 419 & 165.44 & 142.22 & 128.64 & 119.00 & 111.52 & 105.41 & 151.96 & 176.77 & 195.43 & 211.26 & 225.42 & 238.49 \\
\hline 420 & 165.87 & 142.58 & 128.97 & 119.30 & 111.81 & 105.68 & 151.93 & 176.74 & 195.40 & 211.23 & 225.39 & 238.45 \\
\hline 421 & 166.06 & 142.75 & 129.11 & 119.44 & 111.94 & 105.81 & 152.12 & 176.95 & 195.64 & 211.49 & 225.66 & 238.74 \\
\hline 422 & 166.20 & 142.87 & 129.23 & 119.54 & 112.03 & 105.90 & 152.35 & 177.22 & 195.94 & 211.81 & 226.00 & 239.10 \\
\hline 423 & 166.64 & 143.25 & 129.57 & 119.86 & & 106.18 & 152.31 & & 195.88 & & & 239.04 \\
\hline 424 & 166.97 & 143.54 & 129.83 & 120.10 & 112.56 & 106.39 & 152.36 & 177.24 & 195.95 & 211.82 & 226.02 & 239.12 \\
\hline 425 & 167.29 & 143.81 & 130.07 & 120.33 & 112.77 & 106.59 & 152.43 & 177.32 & 196.04 & 211.92 & 226.13 & 239.23 \\
\hline 426 & 167.58 & 144.06 & 130.30 & 120.54 & 112.97 & 106.78 & 152.52 & 177.42 & 196.16 & 212.05 & 226.26 & 239.37 \\
\hline 427 & 167.88 & 144.32 & 130.54 & 120.76 & 113.17 & 106.97 & 152.61 & 177.52 & 196.27 & 212.16 & 226.39 & 239.50 \\
\hline 428 & 168.16 & 144.56 & 130.75 & 120.95 & 113.35 & 107.15 & 152.71 & 177.65 & 196.41 & 212.31 & 226.55 & 239.67 \\
\hline 429 & 168.36 & 144.73 & 130.90 & 121.10 & 113.49 & 107.27 & 152.89 & 177.85 & 196.63 & 212.56 & 226.81 & 239.95 \\
\hline 430 & 168.72 & 145.04 & & & & & 152.92 & 177.89 & 196.67 & 212.60 & & 240.00 \\
\hline 431 & 168.98 & 145.26 & 131.39 & 121.54 & 113.91 & 107.67 & 153.04 & 178.02 & 196.82 & 212.76 & 227.03 & 240.18 \\
\hline 432 & 169.40 & 145.62 & & 121.85 & 114.19 & 107.94 & 153.01 & 177.99 & 196.79 & & & 240.14 \\
\hline 433 & 169.64 & 145.83 & 131.90 & 122.02 & 114.35 & 108.09 & 153.15 & 178.15 & 196.97 & 212.92 & 227.19 & 240.36 \\
\hline 434 & 170.01 & 146.15 & 132.19 & 122.28 & 114.60 & 108.32 & 153.17 & 178.18 & 196.99 & 212.95 & 227.22 & 240.39 \\
\hline 435 & 170.25 & 146.36 & 132.38 & 122.46 & 114.77 & 108.48 & 153.30 & 178.33 & 197.16 & 213.13 & 227.42 & 240.60 \\
\hline 436 & 170.53 & 146.60 & 132.60 & 122.66 & 114.95 & 108.66 & 153.40 & 178.45 & 197.29 & 213.27 & 227.57 & 240.75 \\
\hline 437 & 170.90 & 146.91 & 132.88 & 122.92 & 115.20 & 108.89 & 153.43 & 178.48 & 197.33 & 213.31 & 227.61 & 240.79 \\
\hline 438 & 171.15 & 147.13 & 133.07 & 123.10 & 115.37 & 109.05 & 153.55 & 178.62 & 197.49 & 213.48 & 227.79 & 240.99 \\
\hline 439 & 171.48 & 147.41 & 133.33 & 123.34 & 115.59 & 109.26 & 153.61 & 178.69 & 197.55 & 213.56 & 227.87 & 241.07 \\
\hline 440 & 171.84 & 147.72 & 133.61 & 123.60 & 115.84 & 109.49 & 153.63 & 178.72 & 197.59 & 213.59 & 227.91 & 241.11 \\
\hline 441 & 172.02 & 147.88 & 133.75 & 123.73 & 115.96 & 109.61 & 153.82 & 178.93 & 197.83 & 213.85 & 228.18 & 241.40 \\
\hline 442 & 172.39 & 148.20 & 134.04 & 124.00 & 116.21 & 109.84 & 153.83 & 178.95 & 197.85 & 213.87 & 228.21 & 241.43 \\
\hline
\end{tabular}


Table B.3: Simulation Data for Picking Times (Low Rate) - continued.

\begin{tabular}{|c|c|c|c|c|c|c|c|c|c|c|c|c|}
\hline \multirow{2}{*}{$\begin{array}{c}\text { Batch } \\
\text { Size }\end{array}$} & \multicolumn{6}{|c|}{ Picking Time (minutes) } & \multicolumn{6}{|c|}{ Picking Rate (orders/hr) } \\
\hline & 20 & 40 & 60 & 80 & 100 & 120 & 20 & 40 & 60 & 80 & 100 & 120 \\
\hline 443 & 172.73 & 148.49 & 134.31 & 124.24 & 116.44 & 110.06 & 153.88 & 179.00 & 197.91 & 213.93 & 228.28 & 241.50 \\
\hline 444 & 172.94 & 148.66 & 134.46 & 124.39 & 116.58 & 110.19 & & 179.20 & 198.12 & 214.17 & 228.52 & 241.76 \\
\hline 445 & 173.22 & 148.90 & 134.68 & 124.59 & 116.76 & 110.37 & 154.14 & 179.31 & 198.24 & 214.30 & 228.67 & 241.92 \\
\hline 446 & 17 & 149. & 134. & 124. & 117 & & 154 & 179.35 & 198. & 14.35 & 228.72 & 41.97 \\
\hline 447 & 173.90 & 149.49 & 135.21 & 125.08 & 117.22 & 110.80 & 154.23 & 179.41 & 198.36 & 214.42 & 228.80 & 242.06 \\
\hline 448 & 174.16 & 149.72 & 135.42 & 125.27 & 117.40 & 110.97 & 154.34 & 179.54 & 198.50 & 214.58 & 228.96 & 242.23 \\
\hline 449 & 174.46 & 149.97 & 135.65 & 125.48 & 117.60 & 111.16 & 154.42 & 179.63 & 198.60 & 214.69 & 229.08 & 242.35 \\
\hline 450 & 174.74 & 150.21 & 135.87 & 125.69 & 117.79 & 111.34 & 154.51 & 179.74 & 198.72 & 214.82 & 229.22 & 242.50 \\
\hline 451 & 175.08 & 150.51 & 136. & 125.93 & 118. & 111.56 & 154. & 179.79 & 198.77 & 214.87 & 229 . & 242.56 \\
\hline 452 & 175.29 & 150.69 & 136.29 & 126.08 & 118.16 & 111.69 & 154.72 & 179.98 & 198.98 & 215.10 & 229.52 & 242.82 \\
\hline 453 & 175.58 & 150.93 & 136.52 & 126.29 & 118.36 & 111.87 & 154.80 & 180.08 & 199.09 & 215.22 & 229.65 & 242.95 \\
\hline 454 & 175.97 & 151.27 & 136.82 & 126.57 & 118.62 & 112.12 & 154.80 & 180.07 & 199.09 & 215.21 & 229.64 & 242.95 \\
\hline 455 & 176.25 & 151.51 & 137.04 & 126.77 & 118.81 & 112.30 & 154.89 & 180.18 & 199.21 & 215.34 & 229.78 & 243.09 \\
\hline 456 & 176.59 & 151.80 & 137.30 & 127.02 & 119.0 & 112.52 & 154. & 180.23 & 199.27 & 215.40 & 229.84 & 243.16 \\
\hline 457 & 176.95 & 152.11 & 137.58 & 127.27 & 119.28 & 112.75 & 154.96 & & 199.30 & 215.44 & & 243.20 \\
\hline 458 & 177.35 & 152.46 & 137.89 & 127.56 & 119.55 & 113.00 & 154.95 & 180.25 & 199.28 & 215.42 & 229.86 & 243.18 \\
\hline 459 & 177.57 & 152.65 & 138.07 & 127.72 & 119.70 & 113.14 & 155.09 & 180.42 & 199.47 & 215.62 & 230.08 & 243.41 \\
\hline 460 & 177.86 & 152.89 & 138.29 & 127.93 & 119.89 & 113.32 & 155.18 & 180.52 & 199.58 & 215.75 & 230.21 & 243.55 \\
\hline 461 & 178.09 & 153.09 & 138.47 & 128.09 & 120.05 & 113.47 & 155.32 & 180.68 & 199.76 & 215.94 & 230.41 & 243.76 \\
\hline 462 & 178.45 & 153.40 & 138.75 & 128.36 & & 113.70 & 155.34 & 180.70 & 199.78 & 215.96 & 230.44 & 243.79 \\
\hline 463 & 178.68 & 153.60 & 138.93 & 128.52 & 120.45 & 113.85 & 155.47 & 180.86 & 199.96 & 216.15 & 230.64 & 244.00 \\
\hline 464 & 179.08 & 153.94 & 139.24 & 128.81 & 120.71 & 114.10 & 155.46 & 180.85 & 199.94 & 216.14 & 230.63 & 243.99 \\
\hline 465 & 179.37 & 154.19 & 139.46 & 129.01 & 120.91 & 114.29 & 155.55 & 180.95 & 200.05 & 216.26 & 230.75 & 244.12 \\
\hline 466 & 179.55 & 154.35 & 139.61 & 129.15 & 121.03 & 114.41 & 155.72 & 181.15 & & 216.50 & & 244.39 \\
\hline 467 & 179.91 & 154.65 & 139.88 & 129.40 & 121.27 & 114.63 & 155.75 & 181.18 & 200.31 & 216.53 & 231.05 & 244.44 \\
\hline 468 & 180.24 & 154.94 & 140.14 & 129.64 & 121.50 & 114.84 & 155.79 & 181.23 & 200.37 & 216.59 & 231.11 & 244.51 \\
\hline 469 & 180.62 & 155.27 & 140.44 & 129.91 & 121.75 & 115.08 & 155.80 & 181.24 & 200.38 & 216.61 & 231.13 & 244.52 \\
\hline 470 & 180.81 & 155.43 & 140.58 & 130.05 & 121.88 & 115.21 & 155.97 & 181.43 & 200.59 & 216.84 & 231.37 & 244.78 \\
\hline 471 & & 155.69 & 140.82 & & 122.08 & & & & & & & \\
\hline 472 & 181.45 & 155.98 & 141.08 & 130.51 & 122.31 & 115.61 & 156.08 & 181.56 & 200.73 & 216.99 & 231.54 & 244.95 \\
\hline 473 & & & & & & & & & & & & \\
\hline 474 & & & & & & & & & & & & \\
\hline 475 & 182.33 & 156.74 & 141.77 & 131.14 & 122.90 & 116.17 & 156.31 & 181.83 & 201.04 & 217.32 & 231.89 & 245.32 \\
\hline 476 & 182.77 & 157.11 & 142.11 & 131.46 & 123.20 & & 156.27 & 181.78 & 200.98 & 217.25 & 231.82 & 245.25 \\
\hline 477 & 183.01 & 157.32 & 142.30 & 131.63 & 123.36 & 116.61 & 156.39 & 181.92 & 201.13 & 217.42 & 232.00 & 245.44 \\
\hline 478 & 183.20 & 157.48 & 142.44 & 131.77 & 123.49 & 116.73 & 156.55 & 182.12 & 201.35 & 217.66 & 232.25 & 245.70 \\
\hline 479 & 183.58 & 157.81 & 142.74 & 132.04 & 123.7 & 116.97 & 156.55 & 182.12 & 201.35 & 217.65 & 232.25 & 245.70 \\
\hline 480 & 183.81 & 158.01 & 142.91 & 132.21 & 123.90 & 117.11 & 156.69 & 182.27 & 201.52 & 217.84 & 232.44 & 245.91 \\
\hline 481 & 184.15 & 158.30 & 143.18 & 132.46 & 124.1 & 117.34 & 156.72 & 182.31 & 201.56 & 217.89 & 232.49 & 245.96 \\
\hline 482 & 184.47 & 158.57 & 143.43 & 132.68 & 124.35 & 117.54 & 156.78 & 182.38 & 201.63 & 217.96 & 232.58 & 246.05 \\
\hline 483 & 184.79 & 158.85 & 143.68 & 132.92 & 124.57 & 117.74 & 156.83 & 182.43 & 201.70 & 218.03 & 232.65 & 246.13 \\
\hline 484 & 185.03 & 159.06 & 143.87 & 133.09 & 124.73 & 117.90 & 156.94 & 182.57 & 201.85 & 218.20 & 232.82 & 246.32 \\
\hline
\end{tabular}


Table B.3: Simulation Data for Picking Times (Low Rate) - continued.

\begin{tabular}{|c|c|c|c|c|c|c|c|c|c|c|c|c|}
\hline \multirow{2}{*}{$\begin{array}{c}\text { Batch } \\
\text { Size }\end{array}$} & \multicolumn{6}{|c|}{ Picking Time (minutes) } & \multicolumn{6}{|c|}{ Picking Rate (orders/hr) } \\
\hline & 20 & 40 & 60 & 80 & 100 & 120 & 20 & 40 & 60 & 80 & 100 & 120 \\
\hline 485 & 185.41 & 159.38 & 144.16 & 133.36 & 124.98 & 118.13 & 156.95 & 182.58 & 201.86 & 218.21 & 232.84 & 246.33 \\
\hline 486 & 185.69 & 159.63 & 144.38 & 133.57 & 125.17 & 118.32 & 157.03 & 182.67 & 201.96 & 218.32 & 232.96 & 246.45 \\
\hline 487 & 35.95 & 159.85 & 144.58 & 133.75 & 125.34 & 118.48 & 157.14 & 182.80 & 202.10 & 218.47 & 233.12 & 246.63 \\
\hline 488 & 186.21 & 160.08 & 144.79 & 133.94 & 125.52 & 118.65 & 157.24 & 182.91 & 202.23 & 218.61 & 233.26 & 246.78 \\
\hline 489 & 186.58 & 160.39 & 145.07 & 134.20 & 125.77 & 118.88 & 157.25 & 182.93 & 202.24 & 218.62 & 233.28 & 246.79 \\
\hline 490 & 186.75 & 160.54 & 145.20 & 134.32 & 125.88 & 118.99 & 157.43 & 183.14 & 202.48 & 218.87 & 233.55 & 247.08 \\
\hline 491 & 187.08 & 160.82 & 145.46 & 134.56 & 126.11 & 119.20 & 157.48 & 183.19 & 202.53 & 218.94 & 233.61 & 247.15 \\
\hline 492 & 187.48 & 161.17 & 145.77 & 134.85 & 126.38 & 119.46 & 157.45 & 183.16 & 202.50 & 218.91 & 233.58 & 247.11 \\
\hline 493 & 187.81 & 161.45 & 146.03 & 135.09 & 126.60 & 119.67 & 157.50 & 183.22 & 202.56 & 218.97 & 233.65 & 247.19 \\
\hline 494 & 188.12 & 161.72 & 146.27 & 135.31 & 126.81 & 119.86 & 157.56 & 183.29 & 202.64 & 219.05 & 233.74 & 247.28 \\
\hline 495 & 188.31 & 161.88 & 146.42 & 135.45 & 126.94 & 119.99 & 157.72 & 183.47 & 202.84 & 219.27 & 233.97 & 247.53 \\
\hline 496 & 188.66 & 162.18 & 146.69 & 135.70 & 127.17 & 120.21 & 157.74 & 183.50 & 202.88 & 219.31 & 234.01 & 247.57 \\
\hline 497 & 188.98 & 162.45 & 146.94 & 135.93 & 127.39 & 120.41 & 157.80 & 183.56 & 202.95 & 219.38 & 234.09 & 247.65 \\
\hline 498 & 189.23 & 162.67 & 147.14 & 136.11 & 127.56 & 120.57 & 157.90 & 183.68 & 203.08 & 219.53 & 234.24 & 247.81 \\
\hline 499 & 189.62 & 163.01 & 147.44 & 136.39 & 127.82 & 120.82 & 157.89 & 183.67 & 203.07 & 219.51 & 234.23 & 247.80 \\
\hline 500 & 189.85 & 163.20 & 147.61 & 136.55 & 127.97 & 120.97 & 158.02 & 183.82 & 203.23 & 219.70 & 234.42 & 248.01 \\
\hline 501 & 190.11 & 163.43 & 147.82 & 136.74 & 128.15 & 121.13 & 158.12 & 183.94 & 203.36 & 219.83 & 234.57 & 248.16 \\
\hline 502 & 190.46 & 163.73 & 148.09 & 137.00 & 128.39 & 121.36 & 158.14 & 183.96 & 203.39 & 219.86 & 234.60 & 248.19 \\
\hline 503 & 190.75 & 163.98 & 148.32 & 137.20 & 128.58 & 121.54 & 158.22 & 184.05 & 203.49 & 219.97 & 234.71 & 248.31 \\
\hline 504 & 191.09 & 164.27 & 148.58 & 137.45 & 128.81 & 121.76 & 158.25 & 184.09 & 203.53 & 220.02 & 234.76 & 248.37 \\
\hline 505 & 191.37 & 164.51 & 148.80 & 137.65 & 129.00 & 121.93 & 158.33 & 184.18 & 203.63 & 220.13 & 234.88 & 248.49 \\
\hline 506 & 191.59 & 164.70 & 148.97 & 137.80 & 129.15 & 122.07 & 158.46 & 184.34 & 203.81 & 220.31 & 235.08 & 248.70 \\
\hline 507 & 191.96 & 165.02 & 149.26 & 138.07 & 129.40 & 122.31 & 158.47 & 184.35 & 203.81 & 220.32 & 235.09 & \\
\hline 508 & 192.29 & 165.30 & 149.51 & 138.31 & 129.62 & 122.52 & 158.51 & 184.39 & 203.86 & 220.37 & 235.15 & 248.77 \\
\hline 509 & 192.51 & 165.49 & 149.68 & 138.46 & 129.77 & 122.66 & 158.64 & 184.55 & 204.04 & 220.56 & 235.35 & 248.98 \\
\hline 510 & 192.86 & 165.79 & 149.96 & 138.72 & 130.01 & 122.89 & 158.66 & 184.57 & 204.06 & 220.59 & 235.37 & 249.01 \\
\hline 511 & 193.20 & 166.09 & 150.22 & 138.97 & 130.24 & 123.10 & 158.69 & 184.60 & 204.10 & 220.63 & 235.42 & 249.06 \\
\hline 512 & 193.58 & 166.41 & 150.51 & 139.24 & 130.49 & 123.34 & 158.70 & 184.61 & 204.10 & 220.63 & 235.42 & 249.07 \\
\hline 513 & 193.80 & 166.60 & 150.69 & 139.40 & 130.64 & 123.48 & 158.82 & 184.75 & 204.26 & 220.81 & 235.61 & 249.26 \\
\hline 514 & 194.06 & & 150.89 & & 130.82 & & & 184.86 & 204.39 & & & 249.41 \\
\hline 515 & 194.32 & 167.04 & 151.09 & 139.77 & 130.99 & 123.81 & 159.02 & 184.98 & 204.52 & 221.08 & 235.90 & 249.57 \\
\hline 516 & 194.66 & 167.34 & 151.36 & & 131.22 & 124.03 & 159.05 & 185.01 & 204.55 & & & 249.61 \\
\hline 517 & 194.99 & 167.62 & 151.61 & 140.25 & 131.44 & 124.24 & 159.08 & 185.06 & 204.60 & 221.17 & 236.00 & 249.67 \\
\hline 518 & 195.24 & 167.84 & 151.81 & 140.43 & 131.61 & 124.40 & 159.19 & 185.18 & 204.73 & 221.31 & 236.15 & 249.83 \\
\hline 519 & 195.58 & 168.13 & 152.07 & 140.68 & 131.84 & 124.62 & 159.22 & 185.21 & 204.77 & 221.36 & 236.19 & 249.88 \\
\hline 520 & 195.90 & 168.40 & 152.31 & 140.90 & 132.05 & 124.82 & 159.27 & 185.27 & 204.84 & 221.43 & 236.27 & 249.96 \\
\hline 521 & 196.15 & 168.62 & 152.51 & 141.08 & 132.22 & 124.98 & 159.37 & 185.39 & 204.97 & 221.57 & 236.42 & 250.12 \\
\hline 522 & 196.42 & 168.85 & 152.73 & 141.28 & 132.41 & 125.16 & 159.45 & 185.49 & 205.07 & 221.68 & 236.54 & 250.25 \\
\hline 523 & 196.77 & 169.15 & 153.00 & 141.54 & 132.64 & 125.38 & 159.47 & 185.51 & 205.10 & 221.71 & 236.57 & 250.28 \\
\hline 524 & 197.04 & 169.39 & 153.21 & 141.73 & 132.82 & 125.55 & 159.56 & 185.61 & 205.21 & 221.83 & 236.70 & 250.42 \\
\hline 525 & 197.34 & 169.64 & 153.44 & 141.94 & 133.03 & 125.74 & 159.62 & 185.68 & 205.29 & 221.92 & 236.80 & 250.52 \\
\hline 526 & 197.70 & 169.95 & 153.72 & 142.20 & 133.27 & 125.97 & 159.64 & 185.70 & 205.31 & 221.94 & 236.82 & 250.54 \\
\hline
\end{tabular}


Table B.3: Simulation Data for Picking Times (Low Rate) - continued.

\begin{tabular}{|c|c|c|c|c|c|c|c|c|c|c|c|c|}
\hline \multirow{2}{*}{$\begin{array}{c}\text { Batch } \\
\text { Size }\end{array}$} & \multicolumn{6}{|c|}{ Picking Time (minutes) } & \multicolumn{6}{|c|}{ Picking Rate (orders/hr) } \\
\hline & 20 & 40 & 60 & 80 & 100 & 120 & 20 & 40 & 60 & 80 & 100 & 120 \\
\hline 527 & 198.03 & 170.23 & 153.97 & 142.43 & 133.49 & 126.18 & 159.68 & 185.75 & 205.36 & 222.00 & 236.88 & 250.60 \\
\hline 528 & 198.29 & 170.46 & 154.18 & 142.63 & 133.67 & 126.34 & 159.77 & 185.85 & 205.48 & 222.12 & 237.01 & 250.74 \\
\hline 529 & 198.55 & 170.68 & 154.38 & 142.81 & 133.84 & 126.51 & 159.86 & 185.96 & 205.60 & 222.25 & 237.15 & 250.89 \\
\hline 530 & 198.99 & 171.06 & 154.72 & 143.13 & 134.13 & 126.79 & 159.81 & 185.90 & 205.54 & 222.18 & 237.08 & 250.81 \\
\hline 531 & 199.22 & 171.26 & 154.90 & 143.30 & 134.29 & 126.94 & 159.92 & 186.03 & 205.68 & 222.34 & 237.24 & 250.99 \\
\hline 532 & 199.46 & 171.47 & 155.09 & 143.47 & 134.46 & 127.09 & 160.03 & 186.16 & 205.82 & 222.49 & 237.40 & 251.16 \\
\hline 533 & 199.85 & 171.80 & 155.39 & 143.75 & 134.72 & 127.34 & 160.02 & 186.15 & 205.80 & 222.47 & 237.39 & 251.14 \\
\hline 534 & 200.03 & 171.96 & 155.53 & 143.88 & 134.84 & 127.46 & 160.17 & 186.33 & 206.00 & 222.69 & 237.61 & 251.38 \\
\hline 535 & 200.47 & 172.34 & 155.88 & 144.20 & 135.14 & 127.74 & 160.12 & 186.26 & 205.93 & 222.61 & 237.54 & 251.30 \\
\hline 536 & 200.75 & 172.57 & 156.09 & 144.39 & 135.32 & 127.91 & 160.20 & 186.36 & 206.04 & 222.73 & 237.66 & 251.43 \\
\hline 537 & 201.03 & 172.81 & 156.31 & 144.60 & 135.51 & 128.09 & 160.27 & 186.44 & 206.13 & 222.83 & 237.76 & 251.54 \\
\hline 538 & 201.37 & 173.11 & 156.57 & 144.84 & 135.74 & 128.31 & 160.30 & 186.47 & 206.17 & 222.86 & 237.80 & 251.58 \\
\hline 539 & 201.49 & 173.21 & 156.66 & 144.93 & 135.82 & 128.38 & 160.51 & 186.71 & 206.43 & 223.15 & 238.11 & 251.90 \\
\hline 540 & 201.96 & 173.61 & 157.03 & 145.26 & 136.14 & 128.68 & 160.43 & 186.63 & 206.33 & 223.05 & 238.00 & 251.79 \\
\hline 541 & 202.18 & 173.80 & 157.20 & 145.42 & 136.29 & 128.82 & 160.55 & 186.77 & 206.49 & 223.21 & 238.18 & 251.98 \\
\hline 542 & 202.50 & 174.08 & 157.45 & 145.66 & 136.51 & 129.03 & 160.59 & 186.81 & 206.54 & 223.27 & 238.23 & 252.04 \\
\hline 543 & 202.85 & 174.38 & 157.72 & 145.90 & 136.74 & 129.25 & 160.61 & 186.84 & 206.57 & 223.30 & 238.26 & 252.07 \\
\hline 544 & 203.14 & 174.62 & 157.95 & 146.11 & 136.93 & 129.43 & 160.68 & 186.92 & 206.65 & 223.39 & 238.37 & 252.18 \\
\hline 545 & 203.43 & 174.87 & 158.17 & 146.32 & 137.13 & 129.62 & 160.75 & 186.99 & 206.74 & 223.48 & 238.46 & 252.28 \\
\hline 546 & 203.65 & 175.07 & 158.34 & 146.48 & 137.28 & 129.76 & 160.86 & 187.13 & 206.89 & 223.65 & 238.64 & 252.47 \\
\hline 547 & 204.16 & 175.50 & 158.74 & 146.84 & 137.62 & 130.08 & 160.76 & 187.01 & 206.76 & 223.50 & 238.48 & 252.30 \\
\hline 548 & 204.20 & 175.54 & 158.77 & 146.88 & 137.65 & 130.11 & 161.02 & 187.31 & 207.09 & 223.86 & 238.87 & 252.71 \\
\hline 549 & 204.58 & 175.87 & 159.07 & & & 130.36 & 161.01 & 187.30 & 207.08 & 223.85 & & 252.69 \\
\hline 550 & 204.91 & 176.14 & 159.32 & 147.38 & 138.12 & 130.56 & 161.05 & 187.35 & 207.13 & 223.91 & 238.91 & 252.76 \\
\hline 551 & 205.20 & 176.39 & 159.55 & 147.59 & 138.32 & 130.74 & 161.11 & 187.42 & 207.21 & 223.99 & 239.01 & 252.86 \\
\hline 552 & 205.49 & 176.65 & 159.78 & 147.81 & 138.52 & 130.93 & 161.17 & 187.49 & 207.29 & 224.08 & 239.10 & 252.95 \\
\hline 553 & 205.76 & 176.88 & 159.99 & 148.00 & 138.70 & 131.11 & 161.25 & 187.58 & 207.39 & 224.19 & 239.22 & 253.08 \\
\hline 554 & 206.14 & 177.20 & 160.28 & 148.27 & 138.95 & 131.34 & 161.25 & 187.58 & 207.39 & 224.19 & 239.22 & 253.08 \\
\hline 555 & 206.44 & 177.46 & 160.51 & 148.49 & 139.16 & 131.54 & 161.31 & 187.65 & 207.46 & 224.26 & 239.30 & 253.16 \\
\hline 556 & 206.79 & 177.76 & 160.78 & & & & 161.33 & 187.67 & 207.48 & 224.29 & & 253.19 \\
\hline 557 & 207.08 & 178.02 & 161.01 & 148.95 & 139.59 & 131.95 & 161.38 & 187.73 & 207.56 & 224.37 & 239.41 & 253.28 \\
\hline 558 & 207.34 & 178.24 & & & 139.76 & & 161.48 & 187.84 & 207.68 & 224.50 & & 253.43 \\
\hline 559 & 207.70 & 178.55 & 161.50 & 149.40 & 140.01 & 132.34 & 161.48 & 187.85 & 207.68 & 224.50 & 239.55 & 253.43 \\
\hline 560 & 207.98 & 178.79 & 161.71 & 149.60 & 140.20 & 132.52 & 161.55 & 187.93 & 207.78 & 224.61 & 239.66 & 253.55 \\
\hline 561 & 208.22 & 178.99 & 161.90 & 149.77 & 140.36 & 132.67 & 161.66 & 188.05 & 207.91 & 224.75 & 239.81 & 253.71 \\
\hline 562 & 208.51 & 179.25 & 162.12 & 149.98 & 140.56 & 132.86 & 161.72 & 188.12 & 207.99 & 224.83 & 239.90 & 253.81 \\
\hline 563 & 208.77 & 179.46 & 162.32 & 150.16 & 140.73 & 133.02 & 161.81 & 188.23 & 208.11 & 224.96 & 240.04 & 253.95 \\
\hline 564 & 209.16 & 179.80 & 162.63 & 150.45 & 140.99 & 133.27 & 161.79 & 188.20 & 208.08 & 224.93 & 240.01 & 253.92 \\
\hline 565 & 209.37 & 179.98 & 162.79 & 150.59 & 141.13 & 133.40 & 161.92 & 188.36 & 208.25 & 225.11 & 240.20 & 254.12 \\
\hline 566 & 209.65 & 180.22 & 163.01 & 150.80 & 141.32 & 133.58 & 161.98 & 188.43 & 208.33 & 225.20 & 240.30 & 254.22 \\
\hline 567 & 210.05 & 180.57 & 163.32 & 151.09 & 141.59 & 133.84 & 161.96 & 188.41 & 208.30 & 225.17 & 240.27 & 254.19 \\
\hline 568 & 210.30 & 180.78 & 163.51 & 151.26 & 141.76 & 133.99 & 162.06 & 188.52 & 208.42 & 225.31 & 240.41 & 254.34 \\
\hline
\end{tabular}


Table B.3: Simulation Data for Picking Times (Low Rate) - continued.

\begin{tabular}{|c|c|c|c|c|c|c|c|c|c|c|c|c|}
\hline \multirow{2}{*}{$\begin{array}{c}\text { Batch } \\
\text { Size }\end{array}$} & \multicolumn{6}{|c|}{ Picking Time (minutes) } & \multicolumn{6}{|c|}{ Picking Rate (orders/hr) } \\
\hline & 20 & 40 & 60 & 80 & 100 & 120 & 20 & 40 & 60 & 80 & 100 & 120 \\
\hline 569 & 210.68 & 181.11 & 163.81 & 151.54 & 142.02 & 134.24 & 162.05 & 188.51 & 208.41 & 225.29 & 240.39 & 254.32 \\
\hline 570 & 211.05 & 181.43 & 164.10 & 151.81 & 142.27 & 134.48 & 162.04 & 188.50 & 208.41 & 225.29 & 240.39 & 254.32 \\
\hline 571 & 211.24 & 181.59 & 164.25 & 151.94 & 142.40 & 134.60 & 162.18 & 188.67 & 208.59 & 225.48 & 240.60 & 254.54 \\
\hline 572 & 211.52 & 181.83 & 164.46 & 152.14 & 142.58 & 134.77 & 162.25 & 188.75 & 208.68 & 225.58 & 240.70 & 254.65 \\
\hline 573 & 211.83 & 182.09 & 164.70 & 152.36 & 142.79 & 134.97 & 162.30 & 188.80 & 208.74 & 225.65 & 240.77 & 254.72 \\
\hline 574 & 212.15 & 182.37 & 164.95 & 152.59 & 143.01 & 135.17 & 162.34 & 188.85 & 208.79 & 225.70 & 240.83 & 254.78 \\
\hline 575 & 212.45 & 182.63 & 165.19 & 152.81 & 143.21 & 135.37 & 162.39 & 188.91 & 208.86 & 225.77 & 240.91 & 254.87 \\
\hline 576 & 212.74 & 182.88 & 165.41 & 153.02 & 143.40 & 135.55 & 162.45 & 188.98 & 208.94 & 225.86 & 241.00 & 254.96 \\
\hline 577 & 213.15 & 183.23 & 165.73 & 153.31 & 143.68 & 135.81 & 162.42 & 188.94 & 208.89 & 225.81 & 240.95 & 254.91 \\
\hline 578 & 213.38 & 183.43 & 165.91 & 153.48 & 143.83 & 135.96 & 162.53 & 189.07 & 209.03 & 225.96 & 241.11 & 255.08 \\
\hline 579 & 213.56 & 183.58 & 166.05 & 153.61 & 143.96 & 136.07 & 162.67 & 189.24 & 209.22 & 226.16 & 241.32 & 255.31 \\
\hline 580 & 213.89 & 183.87 & 166.31 & 153.85 & 144.18 & 136.29 & 162.70 & 189.26 & 209.25 & 226.20 & 241.36 & 255.34 \\
\hline 581 & 214.18 & 184.12 & 166.53 & 154.05 & 144.38 & 136.47 & 162.76 & 189.34 & 209.33 & 226.29 & 241.45 & 255.44 \\
\hline 582 & 214.59 & 184.47 & 166.85 & 154.35 & 144.65 & 136.73 & 162.73 & 189.30 & 209.29 & 226.24 & 241.41 & 255.40 \\
\hline 583 & 214.86 & 184.70 & 167.06 & 154.54 & 144.84 & 136.90 & 162.80 & 189.39 & 209.38 & 226.34 & 241.52 & 255.51 \\
\hline 584 & 215.18 & 184.98 & 167.31 & 154.77 & 145.05 & 137.11 & 162.84 & 189.43 & 209.43 & 226.40 & 241.57 & 255.57 \\
\hline 585 & 215.51 & 185.26 & 167.57 & 155.01 & 145.27 & 137.32 & 162.87 & 189.46 & 209.47 & 226.43 & 241.61 & 255.61 \\
\hline 586 & 215.82 & 185.53 & 167.81 & 155.23 & 145.48 & 137.51 & 162.91 & 189.52 & 209.53 & 226.50 & 241.68 & 255.68 \\
\hline 587 & 216.09 & 185.76 & 168.02 & 155.43 & 145.67 & 137.69 & 162.99 & 189.60 & 209.62 & 226.60 & 241.79 & 255.80 \\
\hline 588 & 216.49 & 186.10 & 168.33 & 155.72 & 145.93 & 137.94 & 162.96 & 189.57 & 209.59 & 226.57 & 241.75 & 255.76 \\
\hline 589 & 216.60 & 186.19 & 168.41 & 155.79 & 146.00 & 138.01 & 163.16 & 189.80 & 209.85 & 226.84 & 242.05 & 256.07 \\
\hline 590 & 216.94 & 186.49 & 168.68 & 156.04 & 146.24 & 138.23 & 163.18 & 189.82 & 209.86 & 226.86 & 242.07 & 256.09 \\
\hline 591 & 217.26 & 186.76 & 168.92 & 156.27 & 146.45 & 138.43 & 163.22 & 189.87 & 209.92 & 226.92 & 242.13 & 256.16 \\
\hline 592 & 217.50 & 186.97 & 169.11 & 156.44 & 146.61 & 138.58 & 163.31 & 189.98 & 210.04 & 227.05 & 242.27 & 256.31 \\
\hline 593 & 217.87 & 187.29 & 169.40 & 156.71 & 146.87 & 138.82 & 163.31 & 189.97 & 210.03 & 227.04 & 242.26 & 256.30 \\
\hline 594 & 218.13 & 187.51 & 169.60 & 156.89 & 147.04 & 138.98 & 163.39 & 190.07 & 210.14 & 227.16 & 242.39 & 256.43 \\
\hline 595 & 218.45 & 187.79 & 169.85 & 157.13 & 147.26 & 139.19 & 163.42 & 190.11 & 210.18 & 227.20 & 242.43 & 256.48 \\
\hline 596 & 218.81 & 188.10 & 170.13 & 157.39 & 147.50 & 139.42 & 163.43 & 190.11 & 210.19 & 227.21 & 242.44 & 256.49 \\
\hline 597 & 219.08 & 188.33 & 170.34 & 157.58 & 147.68 & 139.59 & 163.50 & 190.20 & 210.29 & 227.32 & 242.56 & 256.61 \\
\hline 598 & 219.37 & & 170.56 & & & & & & 210.36 & 227.40 & & 256.70 \\
\hline 599 & 219.66 & 188.83 & 170.79 & 158.00 & 148.07 & 139.96 & 163.62 & 190.33 & 210.43 & 227.47 & 242.72 & 256.79 \\
\hline 600 & 219.98 & & 171.04 & 158.23 & 148.29 & & 163.65 & 190.37 & 210.47 & 227.52 & & 256.84 \\
\hline 601 & 220.27 & 189.36 & 171.27 & 158.44 & 148.48 & 140.35 & 163.71 & 190.44 & 210.54 & 227.60 & 242.85 & 256.93 \\
\hline 602 & 220.55 & 189.59 & 171.48 & 158.63 & 148.67 & 140.53 & 163.77 & 190.52 & 210.63 & 227.69 & 242.96 & 257.03 \\
\hline 603 & 220.88 & 189.87 & 171.74 & 158.87 & 148.89 & 140.74 & 163.80 & 190.55 & 210.67 & 227.73 & 243.00 & 257.08 \\
\hline 604 & 221.09 & 190.06 & 171.90 & 159.02 & 149.03 & 140.87 & 163.92 & 190.68 & 210.82 & 227.89 & 243.17 & 257.26 \\
\hline 605 & 221.37 & 190.30 & 172.13 & 159.23 & 149.23 & 141.05 & 163.98 & 190.75 & 210.89 & 227.97 & 243.26 & 257.35 \\
\hline 606 & 221.72 & 190.60 & 172.39 & 159.48 & 149.46 & 141.27 & 163.99 & 190.77 & 210.91 & 227.99 & 243.28 & 257.37 \\
\hline 607 & 222.05 & 190.88 & 172.65 & 159.72 & 149.68 & 141.48 & 164.02 & 190.80 & 210.94 & 228.03 & 243.31 & 257.41 \\
\hline 608 & 222.47 & 191.25 & 172.98 & 160.02 & 149.97 & 141.75 & 163.97 & 190.75 & 210.89 & 227.97 & 243.25 & 257.35 \\
\hline 609 & 222.74 & 191.47 & 173.19 & 160.21 & 150.15 & 141.92 & 164.05 & 190.84 & 210.99 & 228.08 & 243.36 & 257.47 \\
\hline 610 & 222.93 & 191.64 & 173.34 & 160.35 & 150.28 & 142.05 & 164.17 & 190.98 & 211.15 & 228.25 & 243.55 & 257.66 \\
\hline
\end{tabular}


Table B.3: Simulation Data for Picking Times (Low Rate) - continued.

\begin{tabular}{|c|c|c|c|c|c|c|c|c|c|c|c|c|}
\hline \multirow{2}{*}{$\begin{array}{c}\text { Batch } \\
\text { Size }\end{array}$} & \multicolumn{6}{|c|}{ Picking Time (minutes) } & \multicolumn{6}{|c|}{ Picking Rate (orders/hr) } \\
\hline & 20 & 40 & 60 & 80 & 100 & 120 & 20 & 40 & 60 & 80 & 100 & 120 \\
\hline 611 & 223.25 & 191.92 & 173.59 & 160.58 & 150.49 & 142.25 & 164.21 & 191.02 & 211.19 & 228.30 & 243.60 & 257.72 \\
\hline 612 & 223.48 & 192.11 & 173.76 & 160.75 & 150.65 & 142.40 & 164.31 & 191.14 & 211.32 & 228.44 & 243.75 & 257.87 \\
\hline 613 & 223.81 & 192.40 & 174.02 & 160.98 & 150.87 & 142.60 & 164.34 & 191.17 & 211.36 & 228.47 & 243.79 & 257.92 \\
\hline 614 & 224.17 & 192.71 & 174.30 & 161.24 & 151.11 & 142.83 & 164.34 & 191.17 & 211.36 & 228.48 & 243.79 & 257.92 \\
\hline 615 & 224.52 & 193.01 & 174.57 & 161.49 & 151.35 & 143.06 & 164.35 & 191.19 & 211.38 & 228.49 & 243.81 & 257.94 \\
\hline 616 & 224.81 & 193.25 & 174.79 & 161.70 & 151.54 & 143.24 & 164.41 & 191.25 & 211.45 & 228.57 & 243.90 & 258.03 \\
\hline 617 & 225.16 & 193.56 & 175.07 & 161.95 & 151.78 & 143.46 & 164.42 & 191.26 & 211.46 & 228.59 & 243.91 & 258.04 \\
\hline 618 & 225.43 & 193.79 & 175.28 & 162.15 & 151. & 143.64 & 164.49 & 191.34 & 211.55 & 228.68 & 244.01 & 258.15 \\
\hline 619 & 225.71 & 194.03 & 175.50 & 162.35 & 152.15 & 143.82 & 164.54 & 191.41 & 211.62 & 228.76 & 244.10 & 258.24 \\
\hline 620 & 226.00 & 194.28 & 175.72 & 162.55 & 152.34 & 144.00 & 164.60 & 191.48 & 211.70 & 228.85 & 244.19 & 258.34 \\
\hline 621 & 226.18 & 194.43 & 175.86 & 162.69 & 152.47 & 144.12 & 164.74 & 191.63 & 211.87 & 229.03 & 244.38 & 258.54 \\
\hline 622 & 226.48 & 194.69 & 176.09 & 162.90 & 152.67 & 144.30 & 164.78 & 191.69 & 211.93 & 229.10 & 244.46 & 258.62 \\
\hline 623 & 226.85 & 195.01 & 176.38 & 163.17 & 152.92 & 144.54 & 164.78 & 191.68 & 211.92 & 229.09 & 244.44 & 258.61 \\
\hline 624 & 227.11 & 195.23 & 176.58 & 163.35 & 153.09 & 144.71 & 164.86 & 191.77 & 212.03 & 229.20 & 244.56 & 258.73 \\
\hline 625 & 227.55 & 195.61 & 176.92 & 163.67 & 153.39 & 144.98 & 164.80 & 191.71 & 211.96 & 229.12 & 244.48 & 258.65 \\
\hline 626 & 227.79 & 195.81 & 177.11 & 163.84 & 153.55 & 145.14 & 164.89 & 191.81 & 212.07 & 229.25 & 244.61 & 258.79 \\
\hline 627 & 228.02 & 196.01 & 177.29 & 164.01 & 153.70 & 145.28 & 164.99 & 191.93 & 212.20 & 229.38 & 244.76 & 258.94 \\
\hline 628 & 228.34 & 196.29 & 177.54 & 164.24 & 153.92 & 145.49 & 165.02 & 191.96 & 212.23 & 229.42 & 244.80 & 258.99 \\
\hline 629 & 228.64 & 196.54 & 177.77 & 164.45 & 154.12 & 145.68 & 165.07 & 192.02 & 212.30 & 229.49 & 244.87 & 259.06 \\
\hline 630 & 229.04 & 196.89 & 178.09 & 164.74 & 154.39 & 145.94 & 165.04 & 191.98 & 212.26 & 229.45 & 244.83 & 259.02 \\
\hline 631 & 229.29 & 197.11 & 178.28 & 164.93 & 154.56 & 146.10 & 165.12 & 192.08 & 212.36 & 229.56 & 244.95 & 259.14 \\
\hline 632 & 229.53 & 197.31 & 178.47 & 165.10 & 154.72 & 146.25 & 165.21 & 192.18 & 212.48 & 229.69 & 245.08 & 259.28 \\
\hline 633 & 229.94 & 197.67 & 178.79 & 165.39 & 155.00 & 146.51 & 165.17 & 192.14 & 212.43 & & & 259.23 \\
\hline 634 & 230.35 & 198.02 & 179.11 & 165.69 & 155.28 & 146.77 & 165.14 & 192.10 & 212.39 & 229.59 & 244.98 & 259.17 \\
\hline 635 & 230.49 & 198.14 & 179.21 & 165.79 & 155.37 & 146.86 & 165.30 & 192.29 & 212.60 & 229.82 & 245.22 & 259.43 \\
\hline 636 & 230.72 & 198.33 & 179.39 & 165.95 & 155.52 & 147.00 & 165.40 & 192.41 & 212.72 & 229.95 & 245.37 & 259.58 \\
\hline 637 & 231.00 & 198.58 & 179.61 & 166.15 & 155.72 & 147.19 & 165.45 & 192.47 & 212.79 & 230.03 & 245.45 & 259.67 \\
\hline 638 & 231.43 & 198.95 & 179.95 & 166.46 & 156.01 & 147.46 & 165.40 & 192.41 & 212.73 & 229.96 & 245.37 & 259.59 \\
\hline 639 & 231.57 & 199.07 & 180.05 & 166.56 & 156.10 & 147.55 & 165.56 & 192.60 & 212.94 & 230.18 & 245.61 & 259.84 \\
\hline 640 & 231.95 & 199.39 & & & 156.35 & & & 192.59 & & & & 259.83 \\
\hline 641 & 232.24 & 199.64 & 180.57 & 167.05 & 156.55 & 147.98 & 165.60 & 192.64 & 212.99 & 230.24 & 245.67 & 259.91 \\
\hline 642 & 232.60 & 199.95 & & & 156.79 & & 165.61 & 192.65 & 212.99 & & & 259.91 \\
\hline 643 & 232.87 & 200.19 & 181.07 & 167.50 & 156.98 & 148.38 & 165.67 & 192.72 & 213.07 & 230.33 & 245.77 & 260.01 \\
\hline 644 & 233.13 & 200.40 & 181.26 & 167.68 & 157.15 & 148.54 & 165.75 & 192.81 & 213.17 & 230.44 & 245.88 & 260.13 \\
\hline 645 & 233.45 & 200.69 & 181.52 & 167.92 & 157.37 & 148.75 & 165.77 & 192.84 & 213.20 & 230.47 & 245.92 & 260.17 \\
\hline 646 & 233.83 & 201.01 & 181.81 & 168.19 & 157.62 & 148.99 & 165.76 & 192.83 & 213.19 & 230.46 & 245.91 & 260.16 \\
\hline 647 & 234.00 & 201.15 & 181.94 & 168.31 & 157.74 & 149.10 & 165.90 & 192.99 & 213.37 & 230.65 & 246.11 & 260.37 \\
\hline 648 & 234.37 & 201.47 & 182.23 & 168.58 & 157.99 & 149.33 & 165.89 & 192.98 & 213.36 & 230.64 & 246.10 & 260.36 \\
\hline 649 & 234.65 & 201.71 & 182.45 & 168.78 & 158.17 & 149.51 & 165.95 & 193.05 & 213.43 & 230.72 & 246.19 & 260.45 \\
\hline 650 & 235.08 & 202.08 & 182.78 & 169.08 & 158.46 & 149.78 & 165.90 & 192.99 & 213.37 & 230.65 & 246.12 & 260.38 \\
\hline 651 & 235.21 & 202.20 & 182.88 & 169.18 & 158.55 & 149.87 & 166.06 & 193.18 & 213.58 & 230.88 & 246.35 & 260.63 \\
\hline 652 & 235.52 & 202.47 & 183.13 & 169.41 & 158.76 & 150.07 & 166.10 & 193.22 & 213.62 & 230.92 & 246.40 & 260.68 \\
\hline
\end{tabular}


Table B.3: Simulation Data for Picking Times (Low Rate) - continued.

\begin{tabular}{|c|c|c|c|c|c|c|c|c|c|c|c|c|}
\hline \multirow{2}{*}{$\begin{array}{c}\text { Batch } \\
\text { Size }\end{array}$} & \multicolumn{6}{|c|}{ Picking Time (minutes) } & \multicolumn{6}{|c|}{ Picking Rate (orders/hr) } \\
\hline & 20 & 40 & 60 & 80 & 100 & 120 & 20 & 40 & 60 & 80 & 100 & 120 \\
\hline 653 & 235.85 & 202.74 & 183.38 & 169.64 & 158.98 & 150.27 & 166.12 & 193.25 & 213.66 & 230.96 & 246.44 & 260.72 \\
\hline 654 & 236.11 & 202.97 & 183.58 & 169.83 & 159.16 & 150.44 & 166.20 & 193.33 & 213.75 & 231.06 & 246.55 & 260.84 \\
\hline 655 & 236.47 & 203.28 & 183.86 & 170.09 & 159.40 & 150.67 & 166.20 & 193.33 & 213.75 & 231.06 & 246.55 & 260.83 \\
\hline 656 & 236.75 & 203.52 & 184.08 & 170.29 & 159.59 & 150.85 & 166.25 & 193.40 & 213.82 & 231.14 & 246.63 & 260.92 \\
\hline 657 & 237.09 & 203.81 & 184.35 & 170.53 & 159.82 & 151.07 & 166.27 & 193.41 & 213.84 & 231.16 & 246.65 & 260.94 \\
\hline 658 & 237.36 & 204.04 & 184.55 & 170.73 & 160.00 & 151.24 & 166.33 & 193.49 & 213.92 & 231.25 & 246.75 & 261.05 \\
\hline 659 & 237.54 & 204.20 & 184.70 & 170.86 & 160.12 & 151.35 & 166.46 & 193.63 & 214.08 & 231.42 & 246.93 & 261.24 \\
\hline 660 & 237.99 & 204.58 & 185.04 & 171.18 & 160.42 & 151.64 & 166.40 & 193.56 & 214.01 & 231.34 & 246.85 & 261.15 \\
\hline 661 & 238.24 & 204.80 & 185.24 & 171.36 & 160.60 & 151.80 & 166.47 & 193.65 & 214.10 & 231.44 & 246.95 & 261.26 \\
\hline 662 & 238.63 & 205.14 & 185.54 & 171.64 & 160.86 & 152.05 & 166.45 & 193.63 & 214.07 & 231.41 & 246.92 & 261.23 \\
\hline 663 & 238.85 & 205.32 & 185.71 & 171.80 & 161.01 & 152.19 & 166.55 & 193.74 & 214.20 & 231.55 & 247.07 & 261.39 \\
\hline 664 & 239.23 & 205.65 & 186.01 & 172.07 & 161.26 & 152.43 & 166.53 & 193.73 & 214.18 & 231.53 & 247.05 & 261.37 \\
\hline 665 & 239.53 & 205.91 & 186.24 & 172.29 & 161.46 & 152.62 & 166.58 & 193.78 & 214.24 & 231.59 & 247.12 & 261.44 \\
\hline 666 & 239.78 & 206.12 & 186.44 & 172.47 & 161.63 & 152.78 & 166.65 & 193.87 & 214.34 & 231.70 & 247.23 & 261.55 \\
\hline 667 & 239.97 & 206.29 & 186.58 & 172.61 & 161.76 & 152.90 & 166.77 & 194.00 & 214.49 & 231.86 & 247.40 & 261.74 \\
\hline 668 & 240.35 & 206.62 & 186.88 & 172.88 & 162.02 & 153.15 & 166.75 & 193.98 & 214.47 & 231.84 & 247.38 & 261.71 \\
\hline 669 & 240.66 & 206.88 & 187.12 & 173.10 & 162.22 & 153.34 & 166.79 & 194.03 & 214.52 & 231.89 & 247.44 & 261.77 \\
\hline 670 & 240.88 & 207.07 & 187.29 & 173.26 & 162.37 & 153.48 & 166.89 & 194.14 & 214.64 & 232.02 & 247.58 & 261.92 \\
\hline 671 & 241.33 & 207.46 & 187.64 & 173.59 & 162.68 & 153.77 & 166.82 & 194.06 & 214.55 & 231.93 & 247.48 & 261.82 \\
\hline 672 & 241.64 & 207.72 & 187.88 & 173.80 & 162.89 & 153.96 & 166.86 & 194.11 & 214.60 & 231.98 & 247.54 & 261.88 \\
\hline 673 & 241.91 & 207.95 & 188.09 & 174.00 & 163.07 & 154.13 & 166.92 & 194.18 & 214.69 & 232.07 & 247.63 & 261.98 \\
\hline 674 & 242.17 & 208.18 & 188.30 & 174.19 & 163.25 & 154.31 & 166.99 & 194.25 & 214.77 & 232.16 & 247.72 & 262.08 \\
\hline 675 & 242.53 & 208.49 & 188.58 & 174.45 & 163.49 & 154.53 & 166.99 & 194.25 & 214.77 & 232.16 & 247.72 & 262.08 \\
\hline 676 & 242.76 & 208.68 & 188.75 & 174.61 & 163.64 & 154.68 & 167.08 & 194.36 & 214.89 & 232.29 & 247.86 & 262.22 \\
\hline 677 & 242.99 & 208.89 & 188.94 & 174.78 & 163.80 & 154.83 & 167.16 & 194.46 & 214.99 & 232.41 & 247.99 & 262.36 \\
\hline 678 & 243.42 & 209.25 & 189.26 & 175.08 & 164.08 & 155.10 & 167.12 & 194.41 & 214.94 & 232.35 & 247.92 & 262.29 \\
\hline 679 & 243.63 & 209.43 & 189.43 & 175.24 & 164.23 & 155.23 & 167.22 & 194.53 & 215.07 & 232.49 & 248.07 & 262.45 \\
\hline 680 & 243.97 & 209.72 & 189.69 & 175.48 & 164.46 & 155.45 & 167.24 & 194.54 & 215.08 & 232.51 & & 262.47 \\
\hline 681 & 244.25 & 209.97 & 189.91 & 175.68 & 164.65 & 155.63 & 167.29 & 194.60 & 215.15 & 232.58 & 248.17 & 262.55 \\
\hline 682 & 244.76 & & & 176.05 & & & 167.18 & 194.48 & 215.02 & 232.43 & & 262.38 \\
\hline 683 & 244.80 & 210.44 & 190.34 & 176.08 & 165.02 & 155.98 & 167.40 & 194.73 & 215.30 & 232.73 & 248.33 & 262.72 \\
\hline 684 & 245.19 & 210.77 & & 176.36 & 165.28 & & 167.38 & 194.71 & 215.27 & & & 262.70 \\
\hline 685 & 245.49 & 211.03 & 190.87 & 176.57 & 165.48 & 156.42 & 167.42 & 194.76 & 215.32 & 232.76 & 248.37 & 262.76 \\
\hline 686 & 245.83 & 211.32 & 191.14 & 176.82 & 165.71 & 156.63 & 167.44 & 194.77 & 215.34 & 232.78 & 248.39 & 262.78 \\
\hline 687 & 246.10 & 211.56 & 191.35 & 177.01 & 165.89 & 156.81 & 167.49 & 194.84 & 215.42 & 232.87 & 248.47 & 262.87 \\
\hline 688 & 246.39 & 211.80 & 191.57 & 177.22 & 166.09 & 156.99 & 167.54 & 194.90 & 215.48 & 232.93 & 248.54 & 262.95 \\
\hline 689 & 246.56 & 211.95 & 191.71 & 177.35 & 166.21 & 157.10 & 167.67 & 195.04 & 215.64 & 233.10 & 248.73 & 263.14 \\
\hline 690 & 247.03 & 212.36 & 192.08 & 177.68 & 166.52 & 157.40 & 167.59 & 194.95 & 215.54 & 233.00 & 248.62 & 263.02 \\
\hline 691 & 247.31 & 212.60 & 192.29 & 177.88 & 166.71 & 157.58 & 167.64 & 195.02 & 215.61 & 233.07 & 248.70 & 263.11 \\
\hline 692 & 247.57 & 212.82 & 192.49 & 178.07 & 166.88 & 157.74 & 167.71 & 195.10 & 215.70 & 233.17 & 248.80 & 263.22 \\
\hline 693 & 247.88 & 213.09 & 192.74 & 178.30 & 167.09 & 157.94 & 167.74 & 195.13 & 215.74 & 233.21 & 248.84 & 263.26 \\
\hline 694 & 248.01 & 213.20 & 192.84 & 178.39 & 167.18 & 158.03 & 167.90 & 195.31 & 215.93 & 233.42 & 249.07 & 263.50 \\
\hline
\end{tabular}


Table B.3: Simulation Data for Picking Times (Low Rate) - continued.

\begin{tabular}{|c|c|c|c|c|c|c|c|c|c|c|c|c|}
\hline \multirow{2}{*}{$\begin{array}{c}\text { Batch } \\
\text { Size }\end{array}$} & \multicolumn{6}{|c|}{ Picking Time (minutes) } & \multicolumn{6}{|c|}{ Picking Rate (orders/hr) } \\
\hline & 20 & 40 & 60 & 80 & 100 & 120 & 20 & 40 & 60 & 80 & 100 & 120 \\
\hline 695 & 248.47 & 213.59 & 193.19 & 178.72 & 167.49 & 158.32 & 167.83 & 195.23 & 215.85 & 233.33 & 248.97 & 263.40 \\
\hline 696 & 248.86 & 213.93 & 193.50 & 179.00 & 167.76 & 158.57 & 167.80 & 195.20 & 215.82 & 233.29 & 248.93 & 263.36 \\
\hline 697 & 249.02 & 214.06 & 193.62 & 179.11 & 167.86 & 158.67 & 167.94 & 195.36 & 215.99 & 233.48 & 249.14 & 263.57 \\
\hline 698 & 249.40 & 214.39 & 193.92 & 179.39 & 168.12 & 158.91 & 167.92 & 195.34 & 215.97 & 233.46 & 249.11 & 263.55 \\
\hline 699 & 249.70 & 214.66 & 194.15 & 179.61 & 168.32 & 159.10 & 167.96 & 195.38 & 216.02 & 233.51 & 249.16 & 263.60 \\
\hline 700 & 250.97 & 215.74 & 195.14 & 180.52 & 169.17 & 159.91 & 167.35 & 194.68 & 215.23 & 232.67 & 248.26 & 262.65 \\
\hline 701 & 251.26 & 215.99 & 195.36 & 180.72 & 169.37 & 160.09 & 167.40 & 194.73 & 215.29 & 232.73 & 248.33 & 262.72 \\
\hline 702 & 251.42 & 216.13 & 195.48 & 180.84 & 169.48 & 160.20 & 167.53 & 194.88 & 215.46 & 232.92 & 248.53 & 262.93 \\
\hline 703 & 251.70 & 216.37 & 195.71 & 181.04 & 169.67 & 160.38 & 167.58 & 194.94 & 215.53 & 232.98 & 248.60 & 263.01 \\
\hline 704 & 252.07 & 216.68 & 195.99 & 181.30 & 169.91 & 160.61 & 167.58 & 194.94 & 215.52 & 232.98 & 248.60 & 263.00 \\
\hline 705 & 252.47 & 217.03 & 196.30 & 181.59 & 170.18 & 160.86 & 167.55 & 194.90 & 215.49 & 232.94 & 248.55 & 262.96 \\
\hline 706 & 252.80 & 217.32 & 196.56 & 181.83 & 170.41 & 161.08 & 167.56 & 194.92 & 215.51 & 232.96 & 248.58 & 262.98 \\
\hline 707 & 252.93 & 217.43 & 196.66 & 181.93 & 170.50 & 161.16 & 167.71 & 195.10 & 215.70 & 233.17 & 248.80 & 263.21 \\
\hline 708 & 253.25 & 217.70 & 196.91 & 182.15 & 170.71 & 161.36 & 167.74 & 195.13 & 215.74 & 233.21 & 248.84 & 263.26 \\
\hline 709 & 253.62 & 218.02 & 197.20 & 182.42 & 170.96 & 161.60 & 167.73 & 195.12 & 215.72 & 233.19 & 248.83 & 263.24 \\
\hline 710 & 253.84 & 218.21 & 197.37 & 182.58 & 171.11 & 161.74 & 167.82 & 195.22 & 215.84 & 233.32 & 248.96 & 263.39 \\
\hline 711 & 254.24 & 218.55 & 197.68 & 182.87 & 171.38 & 161.99 & 167.80 & 195.19 & 215.81 & 233.28 & 248.92 & 263.35 \\
\hline 712 & 254.41 & 218.70 & 197.82 & 182.99 & 171.50 & 162.11 & 167.91 & 195.33 & 215.96 & 233.45 & 249.10 & 263.53 \\
\hline 713 & 254.78 & 219.02 & 198.10 & 183.25 & 171.74 & 162.34 & 167.91 & 195.33 & 215.95 & 233.45 & 249.09 & 263.53 \\
\hline 714 & 254.96 & 219.17 & 198.24 & 183.38 & 171.86 & 162.45 & 168.03 & 195.46 & 216.11 & 233.61 & 249.27 & 263.71 \\
\hline 715 & 255.41 & 219.56 & 198.59 & 183.71 & 172.17 & 162.74 & 167.96 & 195.39 & 216.02 & 233.52 & 249.17 & 263.61 \\
\hline 716 & 255.65 & 219.77 & 198.78 & 183.88 & 172.33 & 162.89 & 168.04 & 195.48 & 216.12 & 233.63 & 249.29 & 263.73 \\
\hline 717 & 256.03 & 220.10 & 199.07 & & 172.59 & 163.14 & 168.03 & 195.46 & & & & 263.71 \\
\hline 718 & 256.27 & 220.30 & 199.26 & 184.33 & 172.75 & 163.29 & 168.10 & 195.55 & 216.20 & 233.71 & 249.38 & 263.83 \\
\hline 719 & 256.62 & 220.60 & 199.53 & 184.58 & 172.99 & 163.51 & 168.11 & 195.56 & 216.21 & 233.72 & 249.39 & 263.84 \\
\hline 720 & 256.83 & 220.78 & 199.69 & 184.73 & 173.13 & 163.64 & 168.20 & 195.67 & 216.33 & 233.85 & 249.53 & 263.99 \\
\hline 721 & 257.19 & 221.09 & 199.97 & 184.99 & 173.37 & 163.87 & 168.20 & 195.67 & 216.33 & 233.85 & 249.53 & 263.99 \\
\hline 722 & 257.45 & 221.31 & 200.17 & 185.18 & 173.54 & 164.04 & 168.27 & 195.74 & 216.41 & & 249.62 & 264.09 \\
\hline 723 & 257.79 & 221.61 & 200.44 & 185.42 & 173.77 & 164.26 & 168.28 & 195.75 & 216.42 & 233.95 & 249.63 & 264.10 \\
\hline 724 & 258.07 & 221.85 & 200.66 & & 173.97 & & & & 216.48 & & & 264.17 \\
\hline 725 & 258.36 & 222.10 & 200.88 & 185.83 & 174.16 & 164.62 & 168.37 & 195.86 & 216.54 & 234.08 & 249.77 & 264.25 \\
\hline 726 & 258.70 & 222.39 & & & 174.39 & & 168.38 & 195.87 & 216.56 & & & 264.26 \\
\hline 727 & 258.98 & 222.63 & 201.37 & 186.28 & 174.58 & 165.01 & 168.43 & 195.93 & 216.62 & 234.17 & 249.86 & 264.34 \\
\hline 728 & 259.38 & 222.97 & 201.68 & 186.57 & 174.85 & 165.27 & 168.40 & 195.90 & 216.58 & 234.12 & 249.82 & 264.29 \\
\hline 729 & 259.60 & 223.16 & 201.85 & 186.73 & 174.99 & 165.41 & 168.49 & 196.00 & 216.70 & 234.25 & 249.95 & 264.43 \\
\hline 730 & 259.84 & 223.37 & 202.03 & 186.89 & 175.15 & 165.56 & 168.57 & 196.09 & 216.80 & 234.36 & 250.07 & 264.56 \\
\hline 731 & 260.18 & 223.66 & 202.30 & 187.14 & 175.39 & 165.78 & 168.57 & 196.10 & 216.81 & 234.36 & 250.08 & 264.57 \\
\hline 732 & 260.45 & 223.89 & 202.51 & 187.34 & 175.57 & 165.95 & 168.63 & 196.16 & 216.88 & 234.44 & 250.16 & 264.66 \\
\hline 733 & 260.71 & 224.12 & 202.71 & 187.52 & 175.74 & 166.12 & 168.69 & 196.24 & 216.96 & 234.53 & 250.25 & 264.76 \\
\hline 734 & 261.02 & 224.39 & 202.95 & 187.75 & 175.95 & 166.32 & 168.72 & 196.27 & 216.99 & 234.57 & 250.29 & 264.80 \\
\hline 735 & 261.48 & 224.78 & 203.31 & 188.07 & 176.26 & 166.61 & 168.66 & 196.19 & 216.91 & 234.48 & 250.20 & 264.70 \\
\hline 736 & 261.75 & 225.01 & 203.52 & 188.27 & 176.44 & 166.78 & 168.71 & 196.26 & 216.99 & 234.56 & 250.28 & 264.79 \\
\hline
\end{tabular}


Table B.3: Simulation Data for Picking Times (Low Rate) — continued.

\begin{tabular}{|c|c|c|c|c|c|c|c|c|c|c|c|c|}
\hline \multirow{2}{*}{$\begin{array}{c}\text { Batch } \\
\text { Size }\end{array}$} & \multicolumn{6}{|c|}{ Picking Time (minutes) } & \multicolumn{6}{|c|}{ Picking Rate (orders/hr) } \\
\hline & 20 & 40 & 60 & 80 & 100 & 120 & 20 & 40 & 60 & 80 & 100 & 120 \\
\hline 737 & 261.92 & 225.15 & 203.65 & 188.39 & 176.55 & 166.88 & 168.83 & 196.40 & 217.14 & 234.73 & 250.46 & 264.97 \\
\hline 738 & 261.31 & 224.63 & 203.18 & 187.96 & 176.15 & 166.50 & 169.45 & 197.12 & 217.94 & 235.59 & 251.38 & 265.95 \\
\hline 739 & 261.68 & 224.95 & 203.46 & 188.22 & 176.39 & 166.73 & 169.45 & 197.11 & 217.93 & 235.58 & 251.37 & 265.93 \\
\hline 740 & 261.90 & 225.14 & 203.64 & 188.38 & 176.55 & 166.88 & 169.53 & 197.21 & 218.03 & 235.69 & 251.49 & 266.07 \\
\hline 741 & 262.29 & 225.47 & 203.94 & 188.66 & 176.81 & 167.12 & 169.51 & 197.18 & 218.01 & 235.66 & 251.46 & 266.03 \\
\hline 742 & 262.52 & 225.67 & 204.12 & 188.82 & 176.96 & 167.27 & 169.59 & 197.28 & 218.11 & 235.77 & 251.58 & 266.16 \\
\hline 743 & 262.84 & 225.94 & 204.36 & 189.05 & 177.18 & 167.47 & 169.61 & 197.30 & 218.14 & 235.81 & 251.61 & 266.19 \\
\hline 744 & 263.16 & 226.22 & 204.61 & 189.28 & 177.39 & 167.67 & 169.63 & 197.33 & 218.17 & 235.84 & 251.65 & 266.23 \\
\hline 745 & 263.52 & 226.54 & 204.90 & 189.55 & 177.64 & 167.91 & 169.62 & 197.32 & 218.16 & 235.83 & 251.63 & 266.22 \\
\hline 746 & 263.91 & 226.87 & 205.20 & 189.82 & 177.90 & 168.15 & 169.60 & 197.30 & 218.13 & 235.80 & 251.60 & 266.18 \\
\hline 747 & 264.01 & 226.95 & 205.27 & 189.89 & 177.96 & 168.22 & 169.77 & 197.49 & 218.34 & 236.03 & 251.85 & 266.44 \\
\hline 748 & 264.41 & 227.29 & 205.58 & 190.18 & 178.23 & 168.47 & 169.74 & 197.45 & 218.30 & 235.99 & 251.80 & 266.39 \\
\hline 749 & 264.67 & 227.52 & 205.79 & 190.37 & 178.41 & 168.64 & 169.79 & 197.52 & 218.38 & 236.06 & 251.89 & 266.48 \\
\hline 750 & 264.96 & 227.77 & 206.01 & 190.58 & 178.60 & 168.82 & 169.84 & 197.57 & 218.43 & 236.13 & 251.95 & 266.55 \\
\hline
\end{tabular}

Table B.4: Manual Packing Times and Rates.

\begin{tabular}{|c|c|c|c|c|c|c|}
\hline \multirow[t]{2}{*}{$\begin{array}{c}\text { Batch } \\
\text { Size }\end{array}$} & \multicolumn{3}{|c|}{$\begin{array}{l}\text { Packing Times } \\
\quad \text { (minutes) }\end{array}$} & \multicolumn{3}{|c|}{$\begin{array}{c}\text { Packing Rates } \\
\text { (orders/hr) }\end{array}$} \\
\hline & Low & Med. & High & Low & Med. & High \\
\hline 1 & 1.31 & 0.91 & 0.56 & 38.85 & 56.95 & 78.79 \\
\hline 2 & 2.61 & 1.83 & 1.12 & 38.08 & 52.67 & 71.86 \\
\hline 3 & 4.37 & 2.98 & 1.70 & 36.36 & 50.65 & 67.14 \\
\hline 4 & 5.82 & 3.98 & 2.27 & 35.74 & 48.35 & 63.76 \\
\hline 5 & 7.66 & 5.18 & 2.86 & 34.47 & 46.65 & 61.98 \\
\hline 6 & 9.19 & 6.21 & 3.43 & 33.51 & 46.46 & 59.63 \\
\hline 7 & 11.08 & 7.44 & 4.04 & 34.18 & 45.59 & 57.64 \\
\hline 8 & 12.66 & 8.50 & 4.62 & 34.01 & 44.73 & 56.64 \\
\hline 9 & 14.61 & 9.75 & 5.25 & 32.46 & 42.73 & 55.37 \\
\hline 10 & 16.23 & 10.84 & 5.83 & 32.74 & 42.14 & 54.55 \\
\hline 11 & 18.21 & 12.11 & 6.48 & 32.03 & 42.18 & 53.30 \\
\hline 12 & 19.86 & 13.21 & 7.07 & 31.18 & 41.49 & 52.34 \\
\hline 13 & 21.88 & 14.49 & 7.73 & 31.73 & 41.33 & 51.71 \\
\hline 14 & 23.56 & 15.61 & 8.32 & 31.08 & 40.80 & 50.73 \\
\hline 15 & 25.60 & 16.91 & 9.00 & 30.08 & 39.84 & 49.57 \\
\hline 16 & 27.31 & 18.04 & 9.60 & 30.52 & 39.10 & 49.43 \\
\hline 17 & 29.37 & 19.35 & 10.30 & 29.86 & 39.36 & 48.32 \\
\hline 18 & 31.10 & 20.49 & 10.91 & 29.24 & 38.87 & 47.52 \\
\hline 19 & 33.19 & 21.82 & 11.62 & 30.45 & 38.72 & 47.61 \\
\hline 20 & 34.94 & 22.97 & 12.23 & 29.66 & 37.96 & 46.71 \\
\hline 21 & 37.05 & 24.30 & 12.97 & 29.87 & 37.22 & 46.62 \\
\hline
\end{tabular}


Table B.4: Manual Packing Times and Rates - continued.

\begin{tabular}{|c|c|c|c|c|c|c|}
\hline \multirow[t]{2}{*}{$\begin{array}{l}\text { Batch } \\
\text { Size }\end{array}$} & \multicolumn{3}{|c|}{$\begin{array}{l}\text { Packing Times } \\
\text { (minutes) }\end{array}$} & \multicolumn{3}{|c|}{$\begin{array}{c}\text { Packing Rates } \\
\text { (orders/hr) }\end{array}$} \\
\hline & Low & Med. & High & Low & Med. & High \\
\hline 22 & 38.81 & 25.46 & 13.58 & 28.98 & 37.28 & 45.94 \\
\hline 23 & 40.94 & 26.81 & 14.33 & 29.23 & 36.55 & 45.35 \\
\hline 24 & 42.72 & 27.97 & 14.96 & 28.32 & 36.65 & 44.76 \\
\hline 25 & 44.87 & 29.33 & 15.73 & 28.46 & 36.81 & 44.51 \\
\hline 26 & 46.66 & 30.50 & 16.35 & 28.99 & 36.44 & 44.32 \\
\hline 27 & 48.83 & 31.86 & 17.14 & 27.85 & 36.04 & 43.99 \\
\hline 28 & 50.64 & 33.04 & 17.78 & 28.64 & 35.24 & 43.54 \\
\hline 29 & 52.82 & 34.41 & 18.58 & 28.02 & 35.48 & 42.70 \\
\hline 30 & 54.64 & 35.60 & 19.22 & 27.59 & 34.78 & 42.49 \\
\hline 31 & 56.83 & 36.98 & 20.05 & 27.75 & 35.05 & 42.46 \\
\hline 32 & 58.66 & 38.17 & 20.69 & 28.03 & 35.03 & 42.24 \\
\hline 33 & 60.87 & 39.55 & 21.54 & 27.10 & 34.69 & 41.73 \\
\hline 34 & 62.72 & 40.75 & 22.19 & 27.47 & 34.14 & 41.19 \\
\hline 35 & 64.94 & 42.14 & 23.05 & 26.75 & 34.20 & 41.06 \\
\hline 36 & 66.79 & 43.35 & 23.71 & 27.00 & 33.80 & 41.11 \\
\hline 37 & 69.02 & 44.75 & 24.59 & 26.57 & 33.46 & 40.60 \\
\hline 38 & 70.89 & 45.95 & 25.25 & 26.50 & 33.31 & 40.42 \\
\hline 39 & 73.13 & 47.36 & 26.15 & 26.99 & 33.37 & 40.00 \\
\hline 40 & 75.01 & 48.57 & 26.82 & 26.94 & 33.14 & 39.84 \\
\hline 41 & 77.26 & 49.98 & 27.74 & 26.66 & 33.13 & 39.67 \\
\hline 42 & 79.15 & 51.20 & 28.42 & 25.87 & 32.81 & 39.49 \\
\hline 43 & 81.42 & 52.61 & 29.35 & 26.59 & 32.87 & 39.03 \\
\hline 44 & 83.31 & 53.84 & 30.03 & 26.20 & 32.79 & 38.96 \\
\hline 45 & 85.58 & 55.25 & 30.99 & 26.46 & 32.44 & 38.47 \\
\hline 46 & 87.49 & 56.48 & 31.68 & 25.98 & 32.02 & 38.61 \\
\hline 47 & 89.77 & 57.91 & 32.65 & 25.90 & 31.95 & 38.05 \\
\hline 48 & 91.68 & 59.14 & 33.35 & 25.46 & 32.30 & 38.26 \\
\hline 49 & 93.98 & 60.57 & 34.34 & 25.84 & 31.56 & 37.98 \\
\hline 50 & 95.90 & 61.80 & 35.04 & 25.53 & 31.84 & 37.79 \\
\hline 51 & 98.20 & 63.23 & 36.05 & 26.07 & 31.84 & 37.67 \\
\hline 52 & 100.13 & 64.47 & 36.76 & 25.78 & 31.38 & 37.42 \\
\hline 53 & 102.44 & 65.91 & 37.79 & 24.89 & 31.45 & 36.98 \\
\hline 54 & 104.37 & 67.15 & 38.50 & 25.63 & 31.10 & 36.97 \\
\hline 55 & 106.69 & 68.59 & 39.55 & 25.59 & 30.88 & 36.72 \\
\hline 56 & 108.63 & 69.84 & 40.26 & 25.47 & 31.24 & 36.44 \\
\hline 57 & 110.96 & 71.29 & 41.33 & 25.52 & 30.88 & 36.67 \\
\hline 58 & 112.91 & 72.54 & 42.06 & 25.01 & 30.88 & 36.29 \\
\hline 59 & 115.25 & 73.99 & 43.14 & 24.88 & 30.65 & 36.14 \\
\hline 60 & 117.20 & 75.24 & 43.87 & 25.09 & 30.25 & 35.98 \\
\hline 61 & 119.55 & 76.69 & 44.97 & 25.21 & 30.68 & 35.65 \\
\hline 62 & 121.51 & 77.95 & 45.71 & 24.67 & 30.04 & 35.87 \\
\hline
\end{tabular}


Table B.4: Manual Packing Times and Rates - continued.

\begin{tabular}{|c|c|c|c|c|c|c|}
\hline \multirow[t]{2}{*}{$\begin{array}{l}\text { Batch } \\
\text { Size }\end{array}$} & \multicolumn{3}{|c|}{$\begin{array}{l}\text { Packing Times } \\
\text { (minutes) }\end{array}$} & \multicolumn{3}{|c|}{$\begin{array}{c}\text { Packing Rates } \\
\text { (orders/hr) }\end{array}$} \\
\hline & Low & Med. & High & Low & Med. & High \\
\hline 63 & 123.86 & 79.40 & 46.83 & 24.93 & 29.99 & 35.49 \\
\hline 64 & 125.83 & 80.67 & 47.57 & 24.65 & 30.23 & 35.56 \\
\hline 65 & 128.19 & 82.12 & 48.71 & 24.77 & 30.14 & 35.26 \\
\hline 66 & 130.16 & 83.39 & 49.46 & 24.85 & 30.13 & 35.13 \\
\hline 67 & 132.53 & 84.85 & 50.61 & 24.01 & 29.98 & 35.11 \\
\hline 68 & 134.50 & 86.12 & 51.37 & 24.09 & 29.64 & 34.98 \\
\hline 69 & 136.88 & 87.58 & 52.54 & 24.17 & 29.65 & 34.76 \\
\hline 70 & 138.86 & 88.85 & 53.30 & 24.54 & 29.61 & 34.62 \\
\hline 71 & 141.24 & 90.32 & 54.49 & 24.26 & 29.19 & 34.53 \\
\hline 72 & 143.23 & 91.60 & 55.25 & 23.86 & 29.51 & 34.43 \\
\hline 73 & 145.62 & 93.07 & 56.46 & 24.17 & 29.24 & 34.17 \\
\hline 74 & 147.62 & 94.34 & 57.23 & 24.01 & 29.03 & 34.21 \\
\hline 75 & 150.01 & 95.82 & 58.45 & 23.44 & 29.19 & 33.88 \\
\hline 76 & 152.01 & 97.10 & 59.23 & 23.94 & 29.19 & 33.84 \\
\hline 77 & 154.41 & 98.58 & 60.47 & 23.27 & 28.74 & 33.57 \\
\hline 78 & 156.42 & 99.86 & 61.25 & 23.52 & 29.00 & 33.56 \\
\hline 79 & 158.82 & 101.34 & 62.50 & 23.39 & 28.91 & 33.60 \\
\hline 80 & 160.83 & 102.62 & 63.29 & 24.00 & 28.43 & 33.50 \\
\hline 81 & 163.24 & 104.11 & 64.56 & 23.11 & 28.37 & 33.27 \\
\hline 82 & 165.26 & 105.39 & 65.35 & 23.81 & 28.70 & 33.24 \\
\hline 83 & 167.68 & 106.88 & 66.63 & 23.33 & 28.26 & 32.91 \\
\hline 84 & 169.70 & 108.17 & 67.43 & 23.70 & 28.19 & 33.00 \\
\hline 85 & 172.12 & 109.66 & 68.73 & 22.90 & 28.20 & 33.01 \\
\hline 86 & 174.15 & 110.95 & 69.53 & 23.57 & 28.38 & 32.76 \\
\hline 87 & 176.58 & 112.44 & 70.84 & 22.84 & 27.84 & 32.75 \\
\hline 88 & 178.61 & 113.73 & 71.65 & 23.47 & 28.10 & 32.41 \\
\hline 89 & 181.04 & 115.23 & 72.97 & 23.39 & 27.74 & 32.53 \\
\hline 90 & 183.08 & 116.52 & 73.79 & 22.78 & 27.98 & 32.46 \\
\hline 91 & 185.52 & 118.02 & 75.12 & 23.14 & 27.81 & 32.18 \\
\hline 92 & 187.55 & 119.32 & 75.95 & 22.84 & 27.77 & 32.03 \\
\hline 93 & 190.00 & 120.82 & 77.29 & 23.03 & 27.41 & 32.00 \\
\hline 94 & 192.04 & 122.12 & 78.12 & 22.60 & 27.44 & 31.84 \\
\hline 95 & 194.49 & 123.62 & 79.47 & 22.55 & 27.33 & 31.99 \\
\hline 96 & 196.54 & 124.92 & 80.30 & 22.67 & 27.20 & 31.66 \\
\hline 97 & 199.00 & 126.43 & 81.66 & 22.79 & 27.36 & 31.75 \\
\hline 98 & 201.05 & 127.73 & 82.51 & 22.93 & 27.48 & 31.58 \\
\hline 99 & 203.51 & 129.24 & 83.88 & 22.98 & 27.44 & 31.47 \\
\hline 100 & 205.56 & 130.54 & 84.72 & 22.44 & 27.06 & 31.38 \\
\hline 101 & 208.03 & 132.06 & 86.10 & 22.27 & 26.93 & 31.38 \\
\hline 102 & 210.09 & 133.36 & 86.95 & 22.83 & 26.95 & 31.23 \\
\hline 103 & 212.56 & 134.88 & 88.34 & 22.86 & 27.06 & 31.37 \\
\hline
\end{tabular}


Table B.4: Manual Packing Times and Rates - continued.

\begin{tabular}{c|ccc|ccc}
\hline \multirow{2}{*}{$\begin{array}{c}\text { Batch } \\
\text { Size }\end{array}$} & \multicolumn{3}{|c|}{ Packing Times } & \multicolumn{3}{c}{ Packing Rates } \\
\cline { 2 - 7 } (minutes) & Low & Med. & High & Low & Med. & High \\
\hline 104 & 214.62 & 136.18 & 89.19 & 22.47 & 26.90 & 31.13 \\
105 & 217.10 & 137.70 & 90.59 & 22.23 & 27.00 & 31.22 \\
106 & 219.17 & 139.01 & 91.45 & 22.20 & 26.88 & 30.97 \\
107 & 221.65 & 140.53 & 92.85 & 21.93 & 26.83 & 30.79 \\
108 & 223.72 & 141.84 & 93.71 & 22.06 & 26.70 & 30.72 \\
109 & 226.20 & 143.36 & 95.11 & 21.97 & 26.83 & 30.82 \\
110 & 228.28 & 144.68 & 95.99 & 22.07 & 26.69 & 30.82 \\
111 & 230.76 & 146.20 & 97.39 & 21.87 & 26.37 & 30.68 \\
112 & 232.84 & 147.52 & 98.27 & 22.00 & 26.51 & 30.60 \\
113 & 235.34 & 149.04 & 99.68 & 21.94 & 26.37 & 30.49 \\
114 & 237.42 & 150.36 & 100.56 & 22.26 & 26.22 & 30.29 \\
115 & 239.92 & 151.88 & 101.97 & 22.32 & 26.09 & 30.36 \\
116 & 242.00 & 153.20 & 102.86 & 21.92 & 26.42 & 30.10 \\
117 & 244.50 & 154.73 & 104.27 & 22.05 & 26.29 & 30.10 \\
118 & 246.59 & 156.06 & 105.16 & 21.68 & 26.25 & 30.22 \\
119 & 249.10 & 157.59 & 106.57 & 21.80 & 26.26 & 30.08 \\
120 & 251.19 & 158.91 & 107.46 & 21.96 & 26.12 & 29.87 \\
121 & 253.70 & 160.44 & 108.87 & 21.41 & 26.21 & 29.87 \\
122 & 255.80 & 161.77 & 109.77 & 21.51 & 25.87 & 29.72 \\
123 & 258.31 & 163.30 & 111.18 & 21.57 & 25.84 & 29.68 \\
124 & 260.41 & 164.63 & 112.08 & 21.62 & 25.73 & 29.80 \\
125 & 262.93 & 166.16 & 113.49 & 21.54 & 25.60 & 29.48 \\
126 & 265.04 & 167.49 & 114.39 & 21.45 & 25.87 & 29.65 \\
127 & 267.56 & 169.03 & 115.79 & 21.34 & 25.77 & 29.35 \\
128 & 269.66 & 170.36 & 116.70 & 21.81 & 25.47 & 29.38 \\
129 & 272.19 & 171.90 & 118.10 & 21.52 & 25.40 & 29.36 \\
130 & 274.30 & 173.23 & 119.01 & 21.58 & 25.76 & 29.20 \\
131 & 276.83 & 174.78 & 120.40 & 21.20 & 25.36 & 29.12 \\
\hline & & & & & & \\
\hline & & & & & \\
$110 y y y y$ &
\end{tabular}

Table B.5: Simulation Data for Automated Packing with Overlapping Waves.

\begin{tabular}{r|ccc|ccc}
\hline \multirow{2}{*}{$\begin{array}{c}\text { Batch } \\
\text { Size }\end{array}$} & \multicolumn{3}{|c|}{$\begin{array}{c}\text { Packing Times } \\
\text { (minutes) }\end{array}$} & \multicolumn{3}{c}{$\begin{array}{c}\text { Packing Rates } \\
\text { (orders/hr) }\end{array}$} \\
\cline { 2 - 7 } & Low & Med. & High & Low & Med. & High \\
\hline 1 & 1.26 & 0.85 & 0.50 & 47.6929 & 70.2520 & 118.89 \\
2 & 2.52 & 1.71 & 1.01 & 47.6928 & 70.1936 & 118.77 \\
3 & 3.77 & 2.57 & 1.52 & 47.6928 & 70.1594 & 118.70 \\
4 & 5.03 & 3.42 & 2.02 & 47.6927 & 70.1352 & 118.65 \\
5 & 6.29 & 4.28 & 2.53 & 47.6926 & 70.1165 & 118.61 \\
6 & 7.55 & 5.14 & 3.04 & 47.6925 & 70.1011 & 118.58 \\
\hline
\end{tabular}


Table B.5: Simulation Data for Automated Packing with Overlapping Waves - continued.

\begin{tabular}{|c|c|c|c|c|c|c|}
\hline \multirow[t]{2}{*}{$\begin{array}{l}\text { Batch } \\
\text { Size }\end{array}$} & \multicolumn{3}{|c|}{$\begin{array}{l}\text { Packing Times } \\
\text { (minutes) }\end{array}$} & \multicolumn{3}{|c|}{$\begin{array}{c}\text { Packing Rates } \\
\text { (orders/hr) }\end{array}$} \\
\hline & Low & Med. & High & Low & Med. & High \\
\hline 7 & 8.81 & 5.99 & 3.54 & 47.6924 & 70.0881 & 118.56 \\
\hline 8 & 10.06 & 6.85 & 4.05 & 47.6924 & 70.0769 & 118.53 \\
\hline 9 & 11.32 & 7.71 & 4.56 & 47.6923 & 70.0670 & 118.51 \\
\hline 10 & 12.58 & 8.56 & 5.06 & 47.6922 & 70.0582 & 118.50 \\
\hline 11 & 13.84 & 9.42 & 5.57 & 47.6921 & 70.0501 & 118.48 \\
\hline 12 & 15.10 & 10.28 & 6.08 & 47.6920 & 70.0428 & 118.47 \\
\hline 13 & 16.35 & 11.14 & 6.58 & 47.6920 & 70.0361 & 118.45 \\
\hline 14 & 17.61 & 11.99 & 7.09 & 47.6919 & 70.0299 & 118.44 \\
\hline 15 & 18.87 & 12.85 & 7.60 & 47.6918 & 70.0241 & 118.43 \\
\hline 16 & 20.13 & 13.71 & 8.11 & 47.6917 & 70.0187 & 118.42 \\
\hline 17 & 21.39 & 14.57 & 8.61 & 47.6916 & 70.0136 & 118.41 \\
\hline 18 & 22.65 & 15.43 & 9.12 & 47.6916 & 70.0088 & 118.40 \\
\hline 19 & 23.90 & 16.28 & 9.63 & 47.6915 & 70.0042 & 118.39 \\
\hline 20 & 25.16 & 17.14 & 10.14 & 47.6914 & 69.9999 & 118.38 \\
\hline 21 & 26.42 & 18.00 & 10.64 & 47.6913 & 69.9958 & 118.37 \\
\hline 22 & 27.68 & 18.86 & 11.15 & 47.6912 & 69.9919 & 118.36 \\
\hline 23 & 28.94 & 19.72 & 11.66 & 47.6912 & 69.9882 & 118.35 \\
\hline 24 & 30.19 & 20.58 & 12.17 & 47.6911 & 69.9846 & 118.35 \\
\hline 25 & 31.45 & 21.43 & 12.68 & 47.6910 & 69.9812 & 118.34 \\
\hline 26 & 32.71 & 22.29 & 13.18 & 47.6909 & 69.9779 & 118.33 \\
\hline 27 & 33.97 & 23.15 & 13.69 & 47.6908 & 69.9747 & 118.33 \\
\hline 28 & 35.23 & 24.01 & 14.20 & 47.6908 & 69.9716 & 118.32 \\
\hline 29 & 36.49 & 24.87 & 14.71 & 47.6907 & 69.9687 & 118.31 \\
\hline 30 & 37.74 & 25.73 & 15.21 & 47.6906 & 69.9659 & 118.31 \\
\hline 31 & 39.00 & 26.59 & 15.72 & 47.6905 & 69.9631 & 118.30 \\
\hline 32 & 40.26 & 27.44 & 16.23 & 47.6904 & 69.9604 & 118.30 \\
\hline 33 & 41.52 & 28.30 & 16.74 & 47.6904 & 69.9579 & 118.29 \\
\hline 34 & 42.78 & 29.16 & 17.25 & 47.6903 & 69.9553 & 118.29 \\
\hline 35 & 44.03 & 30.02 & 17.75 & 47.6902 & 69.9529 & 118.28 \\
\hline 36 & 45.29 & 30.88 & 18.26 & 47.6901 & 69.9505 & 118.28 \\
\hline 37 & 46.55 & 31.74 & 18.77 & 47.6900 & 69.9482 & 118.27 \\
\hline 38 & 47.81 & 32.60 & 19.28 & 47.6900 & 69.9460 & 118.27 \\
\hline 39 & 49.07 & 33.46 & 19.79 & 47.6899 & 69.9438 & 118.26 \\
\hline 40 & 50.33 & 34.31 & 20.29 & 47.6898 & 69.9417 & 118.26 \\
\hline 41 & 51.58 & 35.17 & 20.80 & 47.6897 & 69.9396 & 118.25 \\
\hline 42 & 52.84 & 36.03 & 21.31 & 47.6896 & 69.9376 & 118.25 \\
\hline 43 & 54.10 & 36.89 & 21.82 & 47.6896 & 69.9356 & 118.25 \\
\hline 44 & 55.36 & 37.75 & 22.33 & 47.6895 & 69.9337 & 118.24 \\
\hline 45 & 56.62 & 38.61 & 22.84 & 47.6894 & 69.9318 & 118.24 \\
\hline 46 & 57.87 & 39.47 & 23.34 & 47.6893 & 69.9300 & 118.24 \\
\hline
\end{tabular}


Table B.5: Simulation Data for Automated Packing with Overlapping Waves - continued.

\begin{tabular}{|c|c|c|c|c|c|c|}
\hline \multirow[t]{2}{*}{$\begin{array}{l}\text { Batch } \\
\text { Size }\end{array}$} & \multicolumn{3}{|c|}{$\begin{array}{l}\text { Packing Times } \\
\text { (minutes) }\end{array}$} & \multicolumn{3}{|c|}{$\begin{array}{c}\text { Packing Rates } \\
\text { (orders/hr) }\end{array}$} \\
\hline & Low & Med. & High & Low & Med. & High \\
\hline 47 & 59.13 & 40.33 & 23.85 & 47.6892 & 69.9282 & 118.23 \\
\hline 48 & 60.39 & 41.19 & 24.36 & 47.6892 & 69.9264 & 118.23 \\
\hline 49 & 61.65 & 42.05 & 24.87 & 47.6891 & 69.9247 & 118.22 \\
\hline 50 & 62.91 & 42.90 & 25.38 & 47.6890 & 69.9230 & 118.22 \\
\hline 51 & 64.17 & 43.76 & 25.88 & 47.6889 & 69.9213 & 118.22 \\
\hline 52 & 65.42 & 44.62 & 26.39 & 47.6888 & 69.9197 & 118.21 \\
\hline 53 & 66.68 & 45.48 & 26.90 & 47.6888 & 69.9181 & 118.21 \\
\hline 54 & 67.94 & 46.34 & 27.41 & 47.6887 & 69.9165 & 118.21 \\
\hline 55 & 69.20 & 47.20 & 27.92 & 47.6886 & 69.9150 & 118.20 \\
\hline 56 & 70.46 & 48.06 & 28.43 & 47.6885 & 69.9135 & 118.20 \\
\hline 57 & 71.72 & 48.92 & 28.93 & 47.6884 & 69.9120 & 118.20 \\
\hline 58 & 72.97 & 49.78 & 29.44 & 47.6884 & 69.9105 & 118.20 \\
\hline 59 & 74.23 & 50.64 & 29.95 & 47.6883 & 69.9091 & 118.19 \\
\hline 60 & 75.49 & 51.50 & 30.46 & 47.6882 & 69.9077 & 118.19 \\
\hline 61 & 76.75 & 52.36 & 30.97 & 47.6881 & 69.9063 & 118.19 \\
\hline 62 & 78.01 & 53.22 & 31.48 & 47.6880 & 69.9049 & 118.18 \\
\hline 63 & 79.27 & 54.07 & 31.98 & 47.6880 & 69.9036 & 118.18 \\
\hline 64 & 80.52 & 54.93 & 32.49 & 47.6879 & 69.9023 & 118.18 \\
\hline 65 & 81.78 & 55.79 & 33.00 & 47.6878 & 69.9010 & 118.18 \\
\hline 66 & 83.04 & 56.65 & 33.51 & 47.6877 & 69.8997 & 118.17 \\
\hline 67 & 84.30 & 57.51 & 34.02 & 47.6876 & 69.8984 & 118.17 \\
\hline 68 & 85.56 & 58.37 & 34.53 & 47.6876 & 69.8972 & 118.17 \\
\hline 69 & 86.82 & 59.23 & 35.04 & 47.6875 & 69.8960 & 118.17 \\
\hline 70 & 88.07 & 60.09 & 35.54 & 47.6874 & 69.8948 & 118.16 \\
\hline 71 & 89.33 & 60.95 & 36.05 & 47.6873 & 69.8936 & 118.16 \\
\hline 72 & 90.59 & 61.81 & 36.56 & 47.6872 & 69.8924 & 118.16 \\
\hline 73 & 91.85 & 62.67 & 37.07 & 47.6872 & 69.8912 & 118.16 \\
\hline 74 & 93.11 & 63.53 & 37.58 & 47.6871 & 69.8901 & 118.15 \\
\hline 75 & 94.37 & 64.39 & 38.09 & 47.6870 & 69.8890 & 118.15 \\
\hline 76 & 95.62 & 65.25 & 38.60 & 47.6869 & 69.8879 & 118.15 \\
\hline 77 & 96.88 & 66.11 & 39.10 & 47.6868 & 69.8868 & 118.15 \\
\hline 78 & 98.14 & 66.97 & 39.61 & 47.6868 & 69.8857 & 118.15 \\
\hline 79 & 99.40 & 67.83 & 40.12 & 47.6867 & 69.8846 & 118.14 \\
\hline 80 & 100.66 & 68.69 & 40.63 & 47.6866 & 69.8836 & 118.14 \\
\hline 81 & 101.92 & 69.55 & 41.14 & 47.6865 & 69.8825 & 118.14 \\
\hline 82 & 103.17 & 70.40 & 41.65 & 47.6864 & 69.8815 & 118.14 \\
\hline 83 & 104.43 & 71.26 & 42.16 & 47.6864 & 69.8805 & 118.13 \\
\hline 84 & 105.69 & 72.12 & 42.66 & 47.6863 & 69.8795 & 118.13 \\
\hline 85 & 106.95 & 72.98 & 43.17 & 47.6862 & 69.8785 & 118.13 \\
\hline 86 & 108.21 & 73.84 & 43.68 & 47.6861 & 69.8775 & 118.13 \\
\hline
\end{tabular}


Table B.5: Simulation Data for Automated Packing with Overlapping Waves - continued.

\begin{tabular}{|c|c|c|c|c|c|c|}
\hline \multirow[t]{2}{*}{$\begin{array}{c}\text { Batch } \\
\text { Size }\end{array}$} & \multicolumn{3}{|c|}{$\begin{array}{l}\text { Packing Times } \\
\quad \text { (minutes) }\end{array}$} & \multicolumn{3}{|c|}{$\begin{array}{c}\text { Packing Rates } \\
\text { (orders/hr) }\end{array}$} \\
\hline & Low & Med. & High & Low & Med. & High \\
\hline 87 & 109.47 & 74.70 & 44.19 & 47.6860 & 69.8765 & 118.13 \\
\hline 88 & 110.72 & 75.56 & 44.70 & 47.6860 & 69.8756 & 118.12 \\
\hline 89 & 111.98 & 76.42 & 45.21 & 47.6859 & 69.8746 & 118.12 \\
\hline 90 & 113.24 & 77.28 & 45.72 & 47.6858 & 69.8737 & 118.12 \\
\hline 91 & 114.50 & 78.14 & 46.22 & 47.6857 & 69.8728 & 118.12 \\
\hline 92 & 115.76 & 79.00 & 46.73 & 47.6856 & 69.8718 & 118.12 \\
\hline 93 & 117.02 & 79.86 & 47.24 & 47.6856 & 69.8709 & 118.11 \\
\hline 94 & 118.27 & 80.72 & 47.75 & 47.6855 & 69.8700 & 118.11 \\
\hline 95 & 119.53 & 81.58 & 48.26 & 47.6854 & 69.8691 & 118.11 \\
\hline 96 & 120.79 & 82.44 & 48.77 & 47.6853 & 69.8683 & 118.11 \\
\hline 97 & 122.05 & 83.30 & 49.28 & 47.6852 & 69.8674 & 118.11 \\
\hline 98 & 123.31 & 84.16 & 49.79 & 47.6852 & 69.8665 & 118.11 \\
\hline 99 & 124.57 & 85.02 & 50.29 & 47.6851 & 69.8657 & 118.10 \\
\hline 100 & 125.83 & 85.88 & 50.80 & 47.6850 & 69.8648 & 118.10 \\
\hline 101 & 127.08 & 86.74 & 51.31 & 47.6849 & 69.8640 & 118.10 \\
\hline 102 & 128.34 & 87.60 & 51.82 & 47.6848 & 69.8632 & 118.10 \\
\hline 103 & 129.60 & 88.46 & 52.33 & 47.6848 & 69.8624 & 118.10 \\
\hline 104 & 130.86 & 89.32 & 52.84 & 47.6847 & 69.8616 & 118.10 \\
\hline 105 & 132.12 & 90.18 & 53.35 & 47.6846 & 69.8608 & 118.09 \\
\hline 106 & 133.38 & 91.04 & 53.86 & 47.6845 & 69.8600 & 118.09 \\
\hline 107 & 134.64 & 91.90 & 54.36 & 47.6844 & 69.8592 & 118.09 \\
\hline 108 & 135.89 & 92.76 & 54.87 & 47.6844 & 69.8584 & 118.09 \\
\hline 109 & 137.15 & 93.62 & 55.38 & 47.6843 & 69.8576 & 118.09 \\
\hline 110 & 138.41 & 94.48 & 55.89 & 47.6842 & 69.8569 & 118.09 \\
\hline 111 & 139.67 & 95.34 & 56.40 & 47.6841 & 69.8561 & 118.08 \\
\hline 112 & 140.93 & 96.20 & 56.91 & 47.6840 & 69.8553 & 118.08 \\
\hline 113 & 142.19 & 97.06 & 57.42 & 47.6840 & 69.8546 & 118.08 \\
\hline 114 & 143.44 & 97.92 & 57.93 & 47.6839 & 69.8539 & 118.08 \\
\hline 115 & 144.70 & 98.78 & 58.44 & 47.6838 & 69.8531 & 118.08 \\
\hline 116 & 145.96 & 99.64 & 58.94 & 47.6837 & 69.8524 & 118.08 \\
\hline 117 & 147.22 & 100.50 & 59.45 & 47.6836 & 69.8517 & 118.08 \\
\hline 118 & 148.48 & 101.36 & 59.96 & 47.6836 & 69.8510 & 118.07 \\
\hline 119 & 149.74 & 102.22 & 60.47 & 47.6835 & 69.8503 & 118.07 \\
\hline 120 & 151.00 & 103.08 & 60.98 & 47.6834 & 69.8496 & 118.07 \\
\hline 121 & 152.25 & 103.94 & 61.49 & 47.6833 & 69.8489 & 118.07 \\
\hline 122 & 153.51 & 104.80 & 62.00 & 47.6832 & 69.8482 & 118.07 \\
\hline 123 & 154.77 & 105.66 & 62.51 & 47.6832 & 69.8475 & 118.07 \\
\hline 124 & 156.03 & 106.52 & 63.02 & 47.6831 & 69.8468 & 118.07 \\
\hline 125 & 157.29 & 107.38 & 63.52 & 47.6830 & 69.8461 & 118.06 \\
\hline 126 & 158.55 & 108.24 & 64.03 & 47.6829 & 69.8455 & 118.06 \\
\hline
\end{tabular}


Table B.5: Simulation Data for Automated Packing with Overlapping Waves - continued.

\begin{tabular}{|c|c|c|c|c|c|c|}
\hline \multirow[t]{2}{*}{$\begin{array}{l}\text { Batch } \\
\text { Size }\end{array}$} & \multicolumn{3}{|c|}{$\begin{array}{l}\text { Packing Times } \\
\quad \text { (minutes) }\end{array}$} & \multicolumn{3}{|c|}{$\begin{array}{c}\text { Packing Rates } \\
\text { (orders/hr) }\end{array}$} \\
\hline & Low & Med. & High & Low & Med. & High \\
\hline 127 & 159.81 & 109.10 & 64.54 & 47.6828 & 69.8448 & 118.06 \\
\hline 128 & 161.06 & 109.96 & 65.05 & 47.6828 & 69.8442 & 118.06 \\
\hline 129 & 162.32 & 110.82 & 65.56 & 47.6827 & 69.8435 & 118.06 \\
\hline 130 & 163.58 & 111.68 & 66.07 & 47.6826 & 69.8429 & 118.06 \\
\hline 131 & 164.84 & 112.54 & 66.58 & 47.6825 & 69.8422 & 118.06 \\
\hline 132 & 166.10 & 113.40 & 67.09 & 47.6824 & 69.8416 & 118.06 \\
\hline 133 & 167.36 & 114.26 & 67.60 & 47.6824 & 69.8409 & 118.05 \\
\hline 134 & 168.62 & 115.12 & 68.11 & 47.6823 & 69.8403 & 118.05 \\
\hline 135 & 169.87 & 115.98 & 68.61 & 47.6822 & 69.8397 & 118.05 \\
\hline 136 & 171.13 & 116.84 & 69.12 & 47.6821 & 69.8391 & 118.05 \\
\hline 137 & 172.39 & 117.70 & 69.63 & 47.6820 & 69.8385 & 118.05 \\
\hline 138 & 173.65 & 118.56 & 70.14 & 47.6820 & 69.8378 & 118.05 \\
\hline 139 & 174.91 & 119.42 & 70.65 & 47.6819 & 69.8372 & 118.05 \\
\hline 140 & 176.17 & 120.28 & 71.16 & 47.6818 & 69.8366 & 118.04 \\
\hline 141 & 177.43 & 121.14 & 71.67 & 47.6817 & 69.8360 & 118.04 \\
\hline 142 & 178.69 & 122.00 & 72.18 & 47.6816 & 69.8355 & 118.04 \\
\hline 143 & 179.94 & 122.86 & 72.69 & 47.6816 & 69.8349 & 118.04 \\
\hline 144 & 181.20 & 123.72 & 73.20 & 47.6815 & 69.8343 & 118.04 \\
\hline 145 & 182.46 & 124.58 & 73.70 & 47.6814 & 69.8337 & 118.04 \\
\hline 146 & 183.72 & 125.44 & 74.21 & 47.6813 & 69.8331 & 118.04 \\
\hline 147 & 184.98 & 126.30 & 74.72 & 47.6812 & 69.8326 & 118.04 \\
\hline 148 & 186.24 & 127.16 & 75.23 & 47.6812 & 69.8320 & 118.04 \\
\hline 149 & 187.50 & 128.02 & 75.74 & 47.6811 & 69.8314 & 118.03 \\
\hline 150 & 188.75 & 128.88 & 76.25 & 47.6810 & 69.8309 & 118.03 \\
\hline 151 & 190.01 & 129.74 & 76.76 & 47.6809 & 69.8303 & 118.03 \\
\hline 152 & 191.27 & 130.60 & 77.27 & 47.6808 & 69.8297 & 118.03 \\
\hline 153 & 192.53 & 131.46 & 77.78 & 47.6808 & 69.8292 & 118.03 \\
\hline 154 & 193.79 & 132.32 & 78.29 & 47.6807 & 69.8287 & 118.03 \\
\hline 155 & 195.05 & 133.18 & 78.80 & 47.6806 & 69.8281 & 118.03 \\
\hline 156 & 196.31 & 134.04 & 79.30 & 47.6805 & 69.8276 & 118.03 \\
\hline 157 & 197.57 & 134.90 & 79.81 & 47.6804 & 69.8270 & 118.03 \\
\hline 158 & 198.82 & 135.77 & 80.32 & 47.6804 & 69.8265 & 118.02 \\
\hline 159 & 200.08 & 136.63 & 80.83 & 47.6803 & 69.8260 & 118.02 \\
\hline 160 & 201.34 & 137.49 & 81.34 & 47.6802 & 69.8255 & 118.02 \\
\hline 161 & 202.60 & 138.35 & 81.85 & 47.6801 & 69.8249 & 118.02 \\
\hline 162 & 203.86 & 139.21 & 82.36 & 47.6800 & 69.8244 & 118.02 \\
\hline 163 & 205.12 & 140.07 & 82.87 & 47.6800 & 69.8239 & 118.02 \\
\hline 164 & 206.38 & 140.93 & 83.38 & 47.6799 & 69.8234 & 118.02 \\
\hline 165 & 207.64 & 141.79 & 83.89 & 47.6798 & 69.8229 & 118.02 \\
\hline 166 & 208.89 & 142.65 & 84.40 & 47.6797 & 69.8224 & 118.02 \\
\hline
\end{tabular}


Table B.5: Simulation Data for Automated Packing with Overlapping Waves - continued.

\begin{tabular}{|c|c|c|c|c|c|c|}
\hline \multirow[t]{2}{*}{$\begin{array}{l}\text { Batch } \\
\text { Size }\end{array}$} & \multicolumn{3}{|c|}{$\begin{array}{l}\text { Packing Times } \\
\quad \text { (minutes) }\end{array}$} & \multicolumn{3}{|c|}{$\begin{array}{c}\text { Packing Rates } \\
\text { (orders/hr) }\end{array}$} \\
\hline & Low & Med. & High & Low & Med. & High \\
\hline 167 & 210.15 & 143.51 & 84.90 & 47.6796 & 69.8219 & 118.01 \\
\hline 168 & 211.41 & 144.37 & 85.41 & 47.6796 & 69.8214 & 118.01 \\
\hline 169 & 212.67 & 145.23 & 85.92 & 47.6795 & 69.8209 & 118.01 \\
\hline 170 & 213.93 & 146.09 & 86.43 & 47.6794 & 69.8204 & 118.01 \\
\hline 171 & 215.19 & 146.95 & 86.94 & 47.6793 & 69.8199 & 118.01 \\
\hline 172 & 216.45 & 147.81 & 87.45 & 47.6792 & 69.8194 & 118.01 \\
\hline 173 & 217.71 & 148.67 & 87.96 & 47.6792 & 69.8189 & 118.01 \\
\hline 174 & 218.96 & 149.53 & 88.47 & 47.6791 & 69.8184 & 118.01 \\
\hline 175 & 220.22 & 150.39 & 88.98 & 47.6790 & 69.8179 & 118.01 \\
\hline 176 & 221.48 & 151.25 & 89.49 & 47.6789 & 69.8175 & 118.01 \\
\hline 177 & 222.74 & 152.11 & 90.00 & 47.6788 & 69.8170 & 118.00 \\
\hline 178 & 224.00 & 152.97 & 90.51 & 47.6788 & 69.8165 & 118.00 \\
\hline 179 & 225.26 & 153.83 & 91.01 & 47.6787 & 69.8160 & 118.00 \\
\hline 180 & 226.52 & 154.69 & 91.52 & 47.6786 & 69.8156 & 118.00 \\
\hline 181 & 227.78 & 155.55 & 92.03 & 47.6785 & 69.8151 & 118.00 \\
\hline 182 & 229.03 & 156.41 & 92.54 & 47.6784 & 69.8147 & 118.00 \\
\hline 183 & 230.29 & 157.27 & 93.05 & 47.6784 & 69.8142 & 118.00 \\
\hline 184 & 231.55 & 158.14 & 93.56 & 47.6783 & 69.8137 & 118.00 \\
\hline 185 & 232.81 & 159.00 & 94.07 & 47.6782 & 69.8133 & 118.00 \\
\hline 186 & 234.07 & 159.86 & 94.58 & 47.6781 & 69.8128 & 118.00 \\
\hline 187 & 235.33 & 160.72 & 95.09 & 47.6780 & 69.8124 & 118.00 \\
\hline 188 & 236.59 & 161.58 & 95.60 & 47.6780 & 69.8119 & 117.99 \\
\hline 189 & 237.85 & 162.44 & 96.11 & 47.6779 & 69.8115 & 117.99 \\
\hline 190 & 239.10 & 163.30 & 96.62 & 47.6778 & 69.8111 & 117.99 \\
\hline 191 & 240.36 & 164.16 & 97.13 & 47.6777 & 69.8106 & 117.99 \\
\hline 192 & 241.62 & 165.02 & 97.63 & 47.6776 & 69.8102 & 117.99 \\
\hline 193 & 242.88 & 165.88 & 98.14 & 47.6776 & 69.8097 & 117.99 \\
\hline 194 & 244.14 & 166.74 & 98.65 & 47.6775 & 69.8093 & 117.99 \\
\hline 195 & 245.40 & 167.60 & 99.16 & 47.6774 & 69.8089 & 117.99 \\
\hline 196 & 246.66 & 168.46 & 99.67 & 47.6773 & 69.8084 & 117.99 \\
\hline 197 & 247.92 & 169.32 & 100.18 & 47.6772 & 69.8080 & 117.99 \\
\hline 198 & 249.18 & 170.18 & 100.69 & 47.6772 & 69.8076 & 117.99 \\
\hline 199 & 250.43 & 171.04 & 101.20 & 47.6771 & 69.8072 & 117.98 \\
\hline 200 & 251.69 & 171.90 & 101.71 & 47.6770 & 69.8068 & 117.98 \\
\hline 201 & 252.95 & 172.76 & 102.22 & 47.6769 & 69.8063 & 117.98 \\
\hline 202 & 254.21 & 173.62 & 102.73 & 47.6768 & 69.8059 & 117.98 \\
\hline 203 & 255.47 & 174.48 & 103.24 & 47.6768 & 69.8055 & 117.98 \\
\hline 204 & 256.73 & 175.35 & 103.75 & 47.6767 & 69.8051 & 117.98 \\
\hline 205 & 257.99 & 176.21 & 104.26 & 47.6766 & 69.8047 & 117.98 \\
\hline 206 & 259.25 & 177.07 & 104.76 & 47.6765 & 69.8043 & 117.98 \\
\hline
\end{tabular}


Table B.5: Simulation Data for Automated Packing with Overlapping Waves - continued.

\begin{tabular}{|c|c|c|c|c|c|c|}
\hline \multirow[t]{2}{*}{$\begin{array}{c}\text { Batch } \\
\text { Size }\end{array}$} & \multicolumn{3}{|c|}{$\begin{array}{l}\text { Packing Times } \\
\text { (minutes) }\end{array}$} & \multicolumn{3}{|c|}{$\begin{array}{c}\text { Packing Rates } \\
\text { (orders/hr) }\end{array}$} \\
\hline & Low & Med. & High & Low & Med. & High \\
\hline 207 & 260.51 & 177.93 & 105.27 & 47.6764 & 69.8039 & 117.98 \\
\hline 208 & 261.76 & 178.79 & 105.78 & 47.6764 & 69.8035 & 117.98 \\
\hline 209 & 263.02 & 179.65 & 106.29 & 47.6763 & 69.8031 & 117.98 \\
\hline 210 & 264.28 & 180.51 & 106.80 & 47.6762 & 69.8027 & 117.98 \\
\hline 211 & 265.54 & 181.37 & 107.31 & 47.6761 & 69.8023 & 117.97 \\
\hline 212 & 266.80 & 182.23 & 107.82 & 47.6760 & 69.8019 & 117.97 \\
\hline 213 & 268.06 & 183.09 & 108.33 & 47.6760 & 69.8015 & 117.97 \\
\hline 214 & 269.32 & 183.95 & 108.84 & 47.6759 & 69.8011 & 117.97 \\
\hline 215 & 270.58 & 184.81 & 109.35 & 47.6758 & 69.8007 & 117.97 \\
\hline 216 & 271.84 & 185.67 & 109.86 & 47.6757 & 69.8003 & 117.97 \\
\hline 217 & 273.10 & 186.53 & 110.37 & 47.6756 & 69.7999 & 117.97 \\
\hline 218 & 274.35 & 187.39 & 110.88 & 47.6756 & 69.7995 & 117.97 \\
\hline 219 & 275.61 & 188.25 & 111.39 & 47.6755 & 69.7992 & 117.97 \\
\hline 220 & 276.87 & 189.12 & 111.90 & 47.6754 & 69.7988 & 117.97 \\
\hline 221 & 278.13 & 189.98 & 112.40 & 47.6753 & 69.7984 & 117.97 \\
\hline 222 & 279.39 & 190.84 & 112.91 & 47.6752 & 69.7980 & 117.97 \\
\hline 223 & 280.65 & 191.70 & 113.42 & 47.6752 & 69.7976 & 117.97 \\
\hline 224 & 281.91 & 192.56 & 113.93 & 47.6751 & 69.7973 & 117.96 \\
\hline 225 & 283.17 & 193.42 & 114.44 & 47.6750 & 69.7969 & 117.96 \\
\hline 226 & 284.43 & 194.28 & 114.95 & 47.6749 & 69.7965 & 117.96 \\
\hline 227 & 285.69 & 195.14 & 115.46 & 47.6748 & 69.7961 & 117.96 \\
\hline 228 & 286.94 & 196.00 & 115.97 & 47.6748 & 69.7958 & 117.96 \\
\hline 229 & 288.20 & 196.86 & 116.48 & 47.6747 & 69.7954 & 117.96 \\
\hline 230 & 289.46 & 197.72 & 116.99 & 47.6746 & 69.7950 & 117.96 \\
\hline 231 & 290.72 & 198.58 & 117.50 & 47.6745 & 69.7947 & 117.96 \\
\hline 232 & 291.98 & 199.44 & 118.01 & 47.6744 & 69.7943 & 117.96 \\
\hline 233 & 293.24 & 200.30 & 118.52 & 47.6744 & 69.7940 & 117.96 \\
\hline 234 & 294.50 & 201.16 & 119.03 & 47.6743 & 69.7936 & 117.96 \\
\hline 235 & 295.76 & 202.03 & 119.54 & 47.6742 & 69.7932 & 117.96 \\
\hline 236 & 297.02 & 202.89 & 120.05 & 47.6741 & 69.7929 & 117.96 \\
\hline 237 & 298.28 & 203.75 & 120.55 & 47.6740 & 69.7925 & 117.95 \\
\hline 238 & 299.53 & 204.61 & 121.06 & 47.6740 & 69.7922 & 117.95 \\
\hline 239 & 300.79 & 205.47 & 121.57 & 47.6739 & 69.7918 & 117.95 \\
\hline 240 & 302.05 & 206.33 & 122.08 & 47.6738 & 69.7915 & 117.95 \\
\hline
\end{tabular}


Table B.6: Simulation Data for Automated Packing with Non-Overlapping Waves.

\begin{tabular}{|c|c|c|c|c|c|c|}
\hline \multirow[t]{2}{*}{$\begin{array}{c}\text { Batch } \\
\text { Size }\end{array}$} & \multicolumn{3}{|c|}{$\begin{array}{l}\text { Packing Times } \\
\quad \text { (minutes) }\end{array}$} & \multicolumn{3}{|c|}{$\begin{array}{c}\text { Packing Rates } \\
\text { (orders/hr) }\end{array}$} \\
\hline & Low & Med. & High & Low & Med. & High \\
\hline 1 & 1.31 & 0.91 & 0.56 & 45.92 & 65.68 & 106.88 \\
\hline 2 & 2.80 & 1.93 & 1.13 & 42.88 & 62.24 & 106.37 \\
\hline 3 & 4.37 & 2.98 & 1.70 & 41.20 & 60.31 & 105.86 \\
\hline 4 & 5.99 & 4.07 & 2.28 & 40.05 & 58.98 & 105.35 \\
\hline 5 & 7.66 & 5.18 & 2.86 & 39.18 & 57.96 & 104.85 \\
\hline 6 & 9.36 & 6.30 & 3.45 & 38.48 & 57.15 & 104.35 \\
\hline 7 & 11.08 & 7.44 & 4.04 & 37.90 & 56.47 & 103.85 \\
\hline 8 & 12.83 & 8.59 & 4.64 & 37.40 & 55.88 & 103.35 \\
\hline 9 & 14.61 & 9.75 & 5.25 & 36.97 & 55.37 & 102.86 \\
\hline 10 & 16.40 & 10.92 & 5.86 & 36.59 & 54.92 & 102.37 \\
\hline 11 & 18.21 & 12.11 & 6.48 & 36.25 & 54.52 & 101.88 \\
\hline 12 & 20.03 & 13.30 & 7.10 & 35.94 & 54.15 & 101.40 \\
\hline 13 & 21.88 & 14.49 & 7.73 & 35.66 & 53.81 & 100.92 \\
\hline 14 & 23.73 & 15.70 & 8.36 & 35.40 & 53.51 & 100.44 \\
\hline 15 & 25.60 & 16.91 & 9.00 & 35.16 & 53.22 & 99.97 \\
\hline 16 & 27.48 & 18.13 & 9.65 & 34.93 & 52.95 & 99.50 \\
\hline 17 & 29.37 & 19.35 & 10.30 & 34.73 & 52.70 & 99.03 \\
\hline 18 & 31.28 & 20.58 & 10.96 & 34.53 & 52.47 & 98.56 \\
\hline 19 & 33.19 & 21.82 & 11.62 & 34.35 & 52.25 & 98.10 \\
\hline 20 & 35.11 & 23.06 & 12.29 & 34.17 & 52.04 & 97.64 \\
\hline 21 & 37.05 & 24.30 & 12.97 & 34.01 & 51.85 & 97.18 \\
\hline 22 & 38.99 & 25.55 & 13.65 & 33.85 & 51.66 & 96.73 \\
\hline 23 & 40.94 & 26.81 & 14.33 & 33.71 & 51.48 & 96.28 \\
\hline 24 & 42.90 & 28.06 & 15.03 & 33.56 & 51.31 & 95.83 \\
\hline 25 & 44.87 & 29.33 & 15.73 & 33.43 & 51.15 & 95.39 \\
\hline 26 & 46.85 & 30.59 & 16.43 & 33.30 & 50.99 & 94.95 \\
\hline 27 & 48.83 & 31.86 & 17.14 & 33.18 & 50.84 & 94.51 \\
\hline 28 & 50.82 & 33.14 & 17.86 & 33.06 & 50.70 & 94.07 \\
\hline 29 & 52.82 & 34.41 & 18.58 & 32.94 & 50.56 & 93.64 \\
\hline 30 & 54.82 & 35.69 & 19.31 & 32.83 & 50.43 & 93.21 \\
\hline 31 & 56.83 & 36.98 & 20.05 & 32.73 & 50.30 & 92.78 \\
\hline 32 & 58.85 & 38.26 & 20.79 & 32.63 & 50.18 & 92.36 \\
\hline 33 & 60.87 & 39.55 & 21.54 & 32.53 & 50.06 & 91.94 \\
\hline 34 & 62.90 & 40.85 & 22.29 & 32.43 & 49.94 & 91.52 \\
\hline 35 & 64.94 & 42.14 & 23.05 & 32.34 & 49.83 & 91.11 \\
\hline 36 & 66.98 & 43.44 & 23.82 & 32.25 & 49.72 & 90.70 \\
\hline 37 & 69.02 & 44.75 & 24.59 & 32.16 & 49.61 & 90.29 \\
\hline 38 & 71.08 & 46.05 & 25.37 & 32.08 & 49.51 & 89.88 \\
\hline 39 & 73.13 & 47.36 & 26.15 & 32.00 & 49.41 & 89.48 \\
\hline
\end{tabular}


Table B.6: Simulation Data for Automated Packing with Non-Overlapping

Waves - continued.

\begin{tabular}{|c|c|c|c|c|c|c|}
\hline \multirow[t]{2}{*}{$\begin{array}{c}\text { Batch } \\
\text { Size }\end{array}$} & \multicolumn{3}{|c|}{$\begin{array}{l}\text { Packing Times } \\
\text { (minutes) }\end{array}$} & \multicolumn{3}{|c|}{$\begin{array}{c}\text { Packing Rates } \\
\text { (orders/hr) }\end{array}$} \\
\hline & Low & Med. & High & Low & Med. & High \\
\hline 40 & 75.20 & 48.67 & 26.94 & 31.92 & 49.31 & 89.08 \\
\hline 41 & 77.26 & 49.98 & 27.74 & 31.84 & 49.22 & 88.68 \\
\hline 42 & 79.34 & 51.29 & 28.54 & 31.76 & 49.13 & 88.29 \\
\hline 43 & 81.42 & 52.61 & 29.35 & 31.69 & 49.04 & 87.90 \\
\hline 44 & 83.50 & 53.93 & 30.17 & 31.62 & 48.95 & 87.51 \\
\hline 45 & 85.58 & 55.25 & 30.99 & 31.55 & 48.87 & 87.13 \\
\hline 46 & 87.68 & 56.58 & 31.82 & 31.48 & 48.78 & 86.75 \\
\hline 47 & 89.77 & 57.91 & 32.65 & 31.41 & 48.70 & 86.37 \\
\hline 48 & 91.87 & 59.23 & 33.49 & 31.35 & 48.62 & 85.99 \\
\hline 49 & 93.98 & 60.57 & 34.34 & 31.28 & 48.54 & 85.62 \\
\hline 50 & 96.09 & 61.90 & 35.19 & 31.22 & 48.47 & 85.25 \\
\hline 51 & 98.20 & 63.23 & 36.05 & 31.16 & 48.39 & 84.88 \\
\hline 52 & 100.32 & 64.57 & 36.91 & 31.10 & 48.32 & 84.52 \\
\hline 53 & 102.44 & 65.91 & 37.79 & 31.04 & 48.25 & 84.16 \\
\hline 54 & 104.56 & 67.25 & 38.66 & 30.99 & 48.18 & 83.80 \\
\hline 55 & 106.69 & 68.59 & 39.55 & 30.93 & 48.11 & 83.45 \\
\hline 56 & 108.83 & 69.94 & 40.44 & 30.87 & 48.04 & 83.10 \\
\hline 57 & 110.96 & 71.29 & 41.33 & 30.82 & 47.98 & 82.75 \\
\hline 58 & 113.10 & 72.63 & 42.23 & 30.77 & 47.91 & 82.40 \\
\hline 59 & 115.25 & 73.99 & 43.14 & 30.72 & 47.85 & 82.06 \\
\hline 60 & 117.40 & 75.34 & 44.05 & 30.67 & 47.78 & 81.72 \\
\hline 61 & 119.55 & 76.69 & 44.97 & 30.62 & 47.72 & 81.38 \\
\hline 62 & 121.70 & 78.05 & 45.90 & 30.57 & 47.66 & 81.05 \\
\hline 63 & 123.86 & 79.40 & 46.83 & 30.52 & 47.60 & 80.72 \\
\hline 64 & 126.02 & 80.76 & 47.77 & 30.47 & 47.55 & 80.39 \\
\hline 65 & 128.19 & 82.12 & 48.71 & 30.42 & 47.49 & 80.07 \\
\hline 66 & 130.36 & 83.49 & 49.66 & 30.38 & 47.43 & 79.75 \\
\hline 67 & 132.53 & 84.85 & 50.61 & 30.33 & 47.38 & 79.43 \\
\hline 68 & 134.70 & 86.22 & 51.57 & 30.29 & 47.32 & 79.11 \\
\hline 69 & 136.88 & 87.58 & 52.54 & 30.25 & 47.27 & 78.80 \\
\hline 70 & 139.06 & 88.95 & 53.51 & 30.20 & 47.22 & 78.49 \\
\hline 71 & 141.24 & 90.32 & 54.49 & 30.16 & 47.16 & 78.18 \\
\hline 72 & 143.43 & 91.69 & 55.47 & 30.12 & 47.11 & 77.88 \\
\hline 73 & 145.62 & 93.07 & 56.46 & 30.08 & 47.06 & 77.58 \\
\hline 74 & 147.81 & 94.44 & 57.45 & 30.04 & 47.01 & 77.28 \\
\hline 75 & 150.01 & 95.82 & 58.45 & 30.00 & 46.96 & 76.99 \\
\hline 76 & 152.21 & 97.20 & 59.46 & 29.96 & 46.92 & 76.70 \\
\hline 77 & 154.41 & 98.58 & 60.47 & 29.92 & 46.87 & 76.41 \\
\hline 78 & 156.61 & 99.96 & 61.48 & 29.88 & 46.82 & 76.12 \\
\hline 79 & 158.82 & 101.34 & 62.50 & 29.84 & 46.77 & 75.84 \\
\hline
\end{tabular}


Table B.6: Simulation Data for Automated Packing with Non-Overlapping

Waves - continued.

\begin{tabular}{|c|c|c|c|c|c|c|}
\hline \multirow[t]{2}{*}{$\begin{array}{c}\text { Batch } \\
\text { Size }\end{array}$} & \multicolumn{3}{|c|}{$\begin{array}{l}\text { Packing Times } \\
\quad \text { (minutes) }\end{array}$} & \multicolumn{3}{|c|}{$\begin{array}{c}\text { Packing Rates } \\
\text { (orders/hr) }\end{array}$} \\
\hline & Low & Med. & High & Low & Med. & High \\
\hline 80 & 161.03 & 102.72 & 63.53 & 29.81 & 46.73 & 75.56 \\
\hline 81 & 163.24 & 104.11 & 64.56 & 29.77 & 46.68 & 75.28 \\
\hline 82 & 165.46 & 105.49 & 65.59 & 29.74 & 46.64 & 75.01 \\
\hline 83 & 167.68 & 106.88 & 66.63 & 29.70 & 46.60 & 74.74 \\
\hline 84 & 169.90 & 108.27 & 67.68 & 29.66 & 46.55 & 74.47 \\
\hline 85 & 172.12 & 109.66 & 68.73 & 29.63 & 46.51 & 74.21 \\
\hline 86 & 174.35 & 111.05 & 69.78 & 29.60 & 46.47 & 73.95 \\
\hline 87 & 176.58 & 112.44 & 70.84 & 29.56 & 46.43 & 73.69 \\
\hline 88 & 178.81 & 113.83 & 71.90 & 29.53 & 46.38 & 73.43 \\
\hline 89 & 181.04 & 115.23 & 72.97 & 29.50 & 46.34 & 73.18 \\
\hline 90 & 183.28 & 116.62 & 74.04 & 29.46 & 46.30 & 72.93 \\
\hline 91 & 185.52 & 118.02 & 75.12 & 29.43 & 46.26 & 72.68 \\
\hline 92 & 187.76 & 119.42 & 76.20 & 29.40 & 46.22 & 72.44 \\
\hline 93 & 190.00 & 120.82 & 77.29 & 29.37 & 46.19 & 72.20 \\
\hline 94 & 192.25 & 122.22 & 78.37 & 29.34 & 46.15 & 71.96 \\
\hline 95 & 194.49 & 123.62 & 79.47 & 29.31 & 46.11 & 71.73 \\
\hline 96 & 196.74 & 125.02 & 80.56 & 29.28 & 46.07 & 71.50 \\
\hline 97 & 199.00 & 126.43 & 81.66 & 29.25 & 46.03 & 71.27 \\
\hline 98 & 201.25 & 127.83 & 82.77 & 29.22 & 46.00 & 71.04 \\
\hline 99 & 203.51 & 129.24 & 83.88 & 29.19 & 45.96 & 70.82 \\
\hline 100 & 205.77 & 130.65 & 84.99 & 29.16 & 45.93 & 70.60 \\
\hline 101 & 208.03 & 132.06 & 86.10 & 29.13 & 45.89 & 70.38 \\
\hline 102 & 210.29 & 133.46 & 87.22 & 29.10 & 45.85 & 70.17 \\
\hline 103 & 212.56 & 134.88 & 88.34 & 29.07 & 45.82 & 69.96 \\
\hline 104 & 214.83 & 136.29 & 89.46 & 29.05 & 45.79 & 69.75 \\
\hline 105 & 217.10 & 137.70 & 90.59 & 29.02 & 45.75 & 69.55 \\
\hline 106 & 219.37 & 139.11 & 91.71 & 28.99 & 45.72 & 69.35 \\
\hline 107 & 221.65 & 140.53 & 92.85 & 28.97 & 45.68 & 69.15 \\
\hline 108 & 223.92 & 141.94 & 93.98 & 28.94 & 45.65 & 68.95 \\
\hline 109 & 226.20 & 143.36 & 95.11 & 28.91 & 45.62 & 68.76 \\
\hline 110 & 228.48 & 144.78 & 96.25 & 28.89 & 45.59 & 68.57 \\
\hline 111 & 230.76 & 146.20 & 97.39 & 28.86 & 45.55 & 68.38 \\
\hline 112 & 233.05 & 147.62 & 98.53 & 28.84 & 45.52 & 68.20 \\
\hline 113 & 235.34 & 149.04 & 99.68 & 28.81 & 45.49 & 68.02 \\
\hline 114 & 237.63 & 150.46 & 100.82 & 28.78 & 45.46 & 67.84 \\
\hline 115 & 239.92 & 151.88 & 101.97 & 28.76 & 45.43 & 67.67 \\
\hline 116 & 242.21 & 153.31 & 103.12 & 28.74 & 45.40 & 67.50 \\
\hline 117 & 244.50 & 154.73 & 104.27 & 28.71 & 45.37 & 67.33 \\
\hline 118 & 246.80 & 156.16 & 105.42 & 28.69 & 45.34 & 67.16 \\
\hline 119 & 249.10 & 157.59 & 106.57 & 28.66 & 45.31 & 67.00 \\
\hline
\end{tabular}


Table B.6: Simulation Data for Automated Packing with Non-Overlapping

Waves - continued.

\begin{tabular}{|c|c|c|c|c|c|c|}
\hline \multirow[t]{2}{*}{$\begin{array}{c}\text { Batch } \\
\text { Size }\end{array}$} & \multicolumn{3}{|c|}{$\begin{array}{l}\text { Packing Times } \\
\quad \text { (minutes) }\end{array}$} & \multicolumn{3}{|c|}{$\begin{array}{c}\text { Packing Rates } \\
\text { (orders/hr) }\end{array}$} \\
\hline & Low & Med. & High & Low & Med. & High \\
\hline 120 & 251.40 & 159.01 & 107.72 & 28.64 & 45.28 & 66.84 \\
\hline 121 & 253.70 & 160.44 & 108.87 & 28.62 & 45.25 & 66.68 \\
\hline 122 & 256.01 & 161.87 & 110.03 & 28.59 & 45.22 & 66.53 \\
\hline 123 & 258.31 & 163.30 & 111.18 & 28.57 & 45.19 & 66.38 \\
\hline 124 & 260.62 & 164.73 & 112.33 & 28.55 & 45.16 & 66.23 \\
\hline 125 & 262.93 & 166.16 & 113.49 & 28.52 & 45.14 & 66.09 \\
\hline 126 & 265.24 & 167.60 & 114.64 & 28.50 & 45.11 & 65.95 \\
\hline 127 & 267.56 & 169.03 & 115.79 & 28.48 & 45.08 & 65.81 \\
\hline 128 & 269.87 & 170.47 & 116.94 & 28.46 & 45.05 & 65.67 \\
\hline 129 & 272.19 & 171.90 & 118.10 & 28.44 & 45.03 & 65.54 \\
\hline 130 & 274.51 & 173.34 & 119.25 & 28.41 & 45.00 & 65.41 \\
\hline 131 & 276.83 & 174.78 & 120.40 & 28.39 & 44.97 & 65.28 \\
\hline 132 & 279.15 & 176.21 & 121.55 & 28.37 & 44.95 & 65.16 \\
\hline 133 & 281.48 & 177.65 & 122.69 & 28.35 & 44.92 & 65.04 \\
\hline 134 & 283.80 & 179.09 & 123.84 & 28.33 & 44.89 & 64.92 \\
\hline 135 & 286.13 & 180.53 & 124.99 & 28.31 & 44.87 & 64.81 \\
\hline 136 & 288.46 & 181.98 & 126.13 & 28.29 & 44.84 & 64.70 \\
\hline 137 & 290.79 & 183.42 & 127.27 & 28.27 & 44.82 & 64.59 \\
\hline 138 & 293.12 & 184.86 & 128.41 & 28.25 & 44.79 & 64.48 \\
\hline 139 & 295.46 & 186.31 & 129.54 & 28.23 & 44.77 & 64.38 \\
\hline 140 & 297.79 & 187.75 & 130.68 & 28.21 & 44.74 & 64.28 \\
\hline 141 & 300.13 & 189.20 & 131.81 & 28.19 & 44.72 & 64.18 \\
\hline 142 & 302.47 & 190.64 & 132.94 & 28.17 & 44.69 & 64.09 \\
\hline 143 & 304.81 & 192.09 & 134.06 & 28.15 & 44.67 & 64.00 \\
\hline 144 & 307.15 & 193.54 & 135.19 & 28.13 & 44.64 & 63.91 \\
\hline 145 & 309.50 & 194.99 & 136.30 & 28.11 & 44.62 & 63.83 \\
\hline 146 & 311.84 & 196.44 & 137.42 & 28.09 & 44.59 & 63.75 \\
\hline 147 & 314.19 & 197.89 & 138.53 & 28.07 & 44.57 & 63.67 \\
\hline 148 & 316.54 & 199.34 & 139.64 & 28.05 & 44.55 & 63.59 \\
\hline 149 & 318.89 & 200.79 & 140.74 & 28.03 & 44.52 & 63.52 \\
\hline 150 & 321.24 & 202.24 & 141.84 & 28.02 & 44.50 & 63.45 \\
\hline
\end{tabular}


Table B.7: Manual Picking and Packing Rate Combinations.

\begin{tabular}{|c|c|c|c|c|c|c|c|c|c|c|}
\hline Wave & $\overline{\mathrm{H}}$ & $\overline{\mathrm{H}}$ & $\overline{\mathrm{H}}$ & $\bar{L}$ & $\bar{L}$ & $\overline{\mathrm{H}}$ & $\bar{L}$ & $\bar{L}$ & $\bar{M}$ & $\mathrm{M}$ \\
\hline Length & Picking & Packing & Picking & Packing & Picking & Packing & Picking & Packing & Picking & Packing \\
\hline 20 & 63.33 & 63.76 & 63.33 & 35.74 & 31.62 & 71.86 & 31.62 & 38.08 & 42.51 & 50.65 \\
\hline 20 & 68.69 & 61.98 & 68.69 & 34.47 & 35.36 & 67.14 & 35.36 & 36.36 & 48.20 & 48.35 \\
\hline 20 & 70.45 & 59.63 & 70.45 & 33.51 & 38.06 & 63.76 & 35.36 & 36.36 & 49.80 & 46.65 \\
\hline 20 & 77.82 & 55.37 & 77.82 & 32.46 & 39.83 & 61.98 & 39.83 & 34.47 & 51.34 & 46.46 \\
\hline 20 & 90.32 & 49.57 & 90.32 & 30.08 & 43.34 & 57.64 & 43.34 & 34.18 & 59.60 & 42.14 \\
\hline 20 & 113.12 & 40.42 & 113.12 & 26.50 & 56.22 & 47.61 & 56.22 & 30.45 & 78.21 & 36.04 \\
\hline 40 & 97.65 & 53.30 & 97.65 & 32.03 & 48.19 & 59.63 & 48.19 & 33.51 & 66.85 & 44.73 \\
\hline 40 & 103.95 & 50.73 & 103.95 & 31.08 & 50.41 & 57.64 & 50.41 & 34.18 & 66.51 & 42.73 \\
\hline 40 & 111.04 & 47.61 & 111.04 & 30.45 & 53.67 & 55.37 & 53.67 & 32.46 & 70.98 & 41.49 \\
\hline 40 & 121.89 & 43.99 & 121.89 & 27.85 & 58.24 & 51.71 & 58.24 & 31.73 & 80.87 & 38.87 \\
\hline 40 & 138.54 & 38.61 & 138.54 & 25.98 & 68.26 & 44.76 & 68.26 & 28.32 & 95.43 & 35.03 \\
\hline 40 & 169.05 & 30.49 & 169.05 & 21.94 & 95.95 & 35.56 & 95.95 & 24.65 & 128.47 & 28.38 \\
\hline 60 & 126.16 & 46.62 & 126.16 & 29.87 & 62.60 & 53.30 & 62.60 & 32.03 & 86.07 & 39.84 \\
\hline 60 & 134.76 & 43.99 & 134.76 & 27.85 & 64.40 & 51.71 & 64.40 & 31.73 & 89.40 & 38.87 \\
\hline 60 & 144.40 & 41.11 & 144.40 & 27.00 & 72.30 & 47.61 & 72.30 & 30.45 & 98.35 & 36.81 \\
\hline 60 & 158.14 & 36.98 & 158.14 & 24.89 & 79.95 & 43.99 & 79.95 & 27.85 & 110.54 & 33.46 \\
\hline 60 & 178.15 & 32.53 & 178.15 & 23.39 & 96.48 & 37.98 & 96.48 & 25.84 & 130.75 & 30.13 \\
\hline 60 & 209.81 & 25.61 & 209.81 & 19.68 & 139.86 & 28.80 & 139.86 & 21.00 & 173.58 & 23.70 \\
\hline 80 & 155.33 & 41.06 & 155.33 & 26.75 & 75.59 & 48.32 & 75.59 & 29.86 & 105.19 & 36.65 \\
\hline 80 & 164.69 & 38.47 & 164.69 & 26.46 & 79.92 & 45.94 & 79.92 & 28.98 & 112.47 & 34.78 \\
\hline 80 & 177.15 & 36.14 & 177.15 & 24.88 & 89.51 & 42.49 & 89.51 & 27.59 & 122.62 & 33.13 \\
\hline 80 & 190.21 & 33.01 & 190.21 & 22.90 & 101.98 & 38.61 & 101.98 & 25.98 & 138.31 & 30.04 \\
\hline 80 & 212.09 & 28.49 & 212.09 & 21.13 & 126.84 & 33.01 & 126.84 & 22.90 & 164.20 & 26.69 \\
\hline 80 & 241.11 & 22.61 & 241.11 & 17.44 & 181.23 & 24.55 & 181.23 & 18.75 & 211.35 & 20.97 \\
\hline 100 & 181.07 & 37.67 & 181.07 & 26.07 & 91.43 & 44.32 & 91.43 & 28.99 & 125.26 & 34.20 \\
\hline 100 & 191.01 & 35.56 & 191.01 & 24.65 & 98.81 & 41.73 & 98.81 & 27.10 & 134.80 & 32.44 \\
\hline 100 & 202.96 & 33.01 & 202.96 & 22.90 & 108.81 & 38.61 & 108.81 & 25.98 & 147.58 & 30.04 \\
\hline 100 & 219.12 & 29.72 & 219.12 & 21.51 & 127.61 & 34.53 & 127.61 & 24.26 & 166.91 & 27.41 \\
\hline 100 & 239.88 & 25.98 & 239.88 & 19.34 & 158.46 & 29.14 & 158.46 & 21.24 & 196.83 & 24.23 \\
\hline 100 & 266.85 & 20.47 & 266.85 & 16.35 & 217.74 & 21.73 & 217.74 & 17.33 & 242.19 & 19.08 \\
\hline 120 & 205.54 & 34.76 & 205.54 & 24.17 & 106.88 & 41.11 & 106.88 & 27.00 & 147.74 & 31.84 \\
\hline 120 & 216.00 & 32.75 & 216.00 & 22.84 & 116.31 & 38.05 & 116.31 & 25.90 & 157.20 & 29.99 \\
\hline 120 & 229.64 & 30.36 & 229.64 & 22.32 & 131.73 & 35.13 & 131.73 & 24.85 & 173.43 & 27.84 \\
\hline 120 & 245.08 & 27.57 & 245.08 & 20.25 & 152.76 & 31.37 & 152.76 & 22.86 & 195.03 & 25.76 \\
\hline 120 & 265.57 & 23.98 & 265.57 & 18.29 & 189.32 & 26.39 & 189.32 & 20.05 & 118.96 & 22.45 \\
\hline 120 & 288.94 & 18.96 & 288.94 & 15.49 & 247.57 & 19.81 & 247.57 & 15.89 & 268.23 & 17.63 \\
\hline
\end{tabular}

Table B.8: Automated Picking and Packing Rate Combinations.

\begin{tabular}{|c|c|c|c|c|c|c|c|}
\hline & WL & \multicolumn{2}{|c|}{ Picking } & \multicolumn{2}{|c|}{ Packing (Overlap) } & \multicolumn{2}{|c|}{$\overline{\text { Packing (No Overlap) }}$} \\
\hline & $(\min )$ & Rate & Batch Size & Rate & Batch Size & Rate & Batch Size \\
\hline \multirow{6}{*}{$\mathrm{HH}$} & 20 & 113.45 & 37 & 118.26 & 39 & 93.21 & 30 \\
\hline & 40 & 169.48 & 112 & 118.15 & 78 & 83.45 & 55 \\
\hline & 60 & 209.78 & 209 & 118.07 & 118 & 76.70 & 76 \\
\hline & 80 & 241.25 & 321 & 118.03 & 157 & 71.73 & 95 \\
\hline & 100 & 266.75 & 444 & 117.99 & 196 & 68.02 & 113 \\
\hline & 120 & 288.87 & 577 & 117.96 & 235 & 65.41 & 130 \\
\hline \multirow{6}{*}{ HL } & 20 & 113.45 & 37 & 47.709 & 15 & 35.94 & 12 \\
\hline & 40 & 169.48 & 92 & 47.704 & 31 & 33.85 & 22 \\
\hline & 60 & 209.78 & 153 & 47.699 & 47 & 32.63 & 32 \\
\hline & 80 & 241.25 & 218 & 47.694 & 63 & 31.76 & 42 \\
\hline & 100 & 266.75 & 284 & 47.689 & 79 & 31.16 & 51 \\
\hline & 120 & 288.87 & 350 & 47.685 & 95 & 30.62 & 61 \\
\hline \multirow{6}{*}{$\mathrm{LH}$} & 20 & 56.65 & 18 & 118.26 & 39 & 93.21 & 30 \\
\hline & 40 & 95.38 & 63 & 118.15 & 78 & 83.45 & 55 \\
\hline & 60 & 139.51 & 139 & 118.07 & 118 & 76.70 & 76 \\
\hline & 80 & 181.15 & 241 & 118.03 & 157 & 71.73 & 95 \\
\hline & 100 & 217.62 & 362 & 117.99 & 196 & 68.02 & 113 \\
\hline & 120 & 247.53 & 495 & 117.96 & 235 & 65.41 & 130 \\
\hline \multirow{6}{*}{ LL } & 20 & 56.65 & 18 & 47.692 & 15 & 35.94 & 12 \\
\hline & 40 & 95.38 & 63 & 47.691 & 31 & 33.85 & 22 \\
\hline & 60 & 139.51 & 139 & 47.689 & 47 & 32.63 & 32 \\
\hline & 80 & 181.15 & 241 & 47.688 & 63 & 31.76 & 42 \\
\hline & 100 & 217.62 & 362 & 47.687 & 79 & 31.16 & 51 \\
\hline & 120 & 247.53 & 495 & 47.685 & 95 & 30.62 & 61 \\
\hline \multirow{6}{*}{ MM } & 20 & 78.23 & 26 & 69.99 & 23 & 52.70 & 17 \\
\hline & 40 & 127.67 & 85 & 69.93 & 46 & 50.06 & 33 \\
\hline & 60 & 173.16 & 173 & 69.90 & 69 & 48.62 & 48 \\
\hline & 80 & 211.19 & 281 & 69.87 & 93 & 47.60 & 63 \\
\hline & 100 & 242.16 & 403 & 69.85 & 116 & 46.82 & 78 \\
\hline & 120 & 268.06 & 536 & 69.84 & 139 & 46.22 & 92 \\
\hline
\end{tabular}




\section{Appendix C}

\section{Deterministic Spreadsheet Model}

\section{Results}

The deterministic spreadsheet model calculates the total annual system cost for a set of given parameters for six wave lengths ranging from 20 minutes to 2 hours. Within each wave length, a range of picking and packing rate combinations are calculated. The lowest annual cost is determined and the system with this cost is recommended. Table C.1 presents the spreadsheet analyses for an automated system at a demand level of 1100 orders per hour. The first six rows represent the system with overlap, and the bottom six rows present the data for a system with no wave overlap. Table C.2 presents the spreadsheet analyses for a manual system at this demand level. In this analyses, an average of 3 items per order was used. Refer to Section 4.1 for all notation and units labeling each column.

The spreadsheets presented in this appendix are from the initial model discussed in Chapter 4. The picking and packing rate combinations are estimated from data gathered from several distribution centers. Appendix F presents the same model with combinations obtained from simulation data and are used in the throughput simulations. The italicized total annual costs represent the best annual solution within the wave length for the manual system, and the best solution for the overlap or no wave overlap system for the automated system. The boldface total annual cost represents the best overall solution. 
Table C.1: Automated System Deterministic Model Calculations (Demand $=1100$ orders/hr, Sorter Cost $=\$ 250,000)$.

\begin{tabular}{ccccccccccccccccccc}
\hline$W$ & $p(n)$ & $q(m)$ & $t$ & $r_{p}$ & $r_{q}$ & $N_{q}$ & $r_{a}$ & $r_{t}$ & $r_{s}$ & $T_{\ell}^{a}$ & $r_{q} c_{q}$ & $r_{s} c_{t}$ & $C_{t}^{s}$ & $C_{t}^{s} * A$ \\
\hline 20 & 75 & 75 & 20 & 15 & 15 & 25 & 15 & 3 & 1 & 990,000 & 150,000 & 50,000 & 450,000 & 150,480 & $1,140,480$ \\
40 & 90 & 75 & 20 & 13 & 15 & 25 & 30 & 3 & 1 & 930,000 & 300,000 & 50,000 & 600,000 & 200,640 & $1,130,640$ \\
60 & 105 & 75 & 20 & 11 & 15 & 25 & 44 & 3 & 1 & 870,000 & 440,000 & 50,000 & 740,000 & 247,456 & $1,117,456$ \\
80 & 120 & 75 & 20 & 10 & 15 & 25 & 59 & 3 & 1 & 840,000 & 590,000 & 50,000 & 890,000 & 297,616 & $1,137,616$ & 1600 \\
100 & 135 & 75 & 20 & 9 & 15 & 25 & 74 & 3 & 1 & 810,000 & 740,000 & 50,000 & $1,040,000$ & 347,776 & $1,157,776$ & 1,000 \\
120 & 150 & 75 & 20 & 8 & 15 & 25 & 88 & 3 & 1 & 780,000 & 880,000 & 50,000 & $1,180,000$ & 394,592 & $1,174,592$ \\
\hline 20 & 75 & 75 & 20 & 15 & 15 & 25 & 15 & 3 & 1 & 990,000 & 150,000 & 50,000 & 450,000 & 150,480 & $\mathbf{1 , 1 4 0 , 4 8 0}$ \\
40 & 90 & 73 & 20 & 13 & 16 & 25 & 30 & 3 & 1 & 960,000 & 300,000 & 50,000 & 600,000 & 200,640 & $1,160,640$ \\
60 & 105 & 71 & 20 & 11 & 16 & 25 & 44 & 3 & 1 & 900,000 & 440,000 & 50,000 & 740,000 & 247,456 & $1,147,456$ \\
80 & 120 & 69 & 20 & 10 & 16 & 25 & 59 & 3 & 1 & 870,000 & 590,000 & 50,000 & 890,000 & 297,616 & $1,167,616$ \\
100 & 135 & 67 & 20 & 9 & 17 & 25 & 74 & 3 & 1 & 870,000 & 740,000 & 50,000 & $1,040,000$ & 347,776 & $1,217,776$ \\
120 & 150 & 65 & 20 & 8 & 17 & 25 & 88 & 3 & 1 & 840,000 & 880,000 & 50,000 & $1,180,000$ & 394,592 & $1,234,592$ \\
\hline
\end{tabular}

Table C.2: Manual System Deterministic Model Calculations.

\begin{tabular}{|c|c|c|c|c|c|c|c|c|c|c|c|c|}
\hline $\bar{D}$ & $\bar{W}$ & $\overline{N_{O}}$ & $\overline{p(n)}$ & $q(m)$ & $r_{p}$ & $r_{q}$ & $\overline{N_{q}}$ & $n_{q}$ & $r_{a}$ & $r_{a} c_{q}$ & $T_{\ell}^{m}$ & $T^{m}$ \\
\hline 1100 & 20 & 3 & 50 & 45.1 & 22 & 25 & 25 & 15 & 25 & 5,852 & $1,410,000$ & $1,415,852$ \\
\hline 1100 & 20 & 3 & 70 & 39.9 & 16 & 28 & 25 & 15 & 28 & 6,554 & $1,320,000$ & $1,326,554$ \\
\hline 1100 & 20 & 3 & 90 & 35.5 & 13 & 31 & 25 & 15 & 31 & 7,256 & $1,320,000$ & $1,327,256$ \\
\hline 1100 & 20 & 3 & 110 & 31.5 & 10 & 35 & 25 & 15 & 35 & 8,193 & $1,350,000$ & $1,358,193$ \\
\hline 1100 & 20 & 3 & 130 & 28.0 & 9 & 40 & 25 & 15 & 40 & 9,363 & $1,470,000$ & $1,479,363$ \\
\hline 1100 & 20 & 3 & 150 & 25.0 & 8 & 44 & 25 & 15 & 44 & 10,300 & $1,560,000$ & $1,570,300$ \\
\hline 1100 & 40 & 3 & 60 & 45.0 & 19 & 25 & 25 & 30 & 30 & 7,022 & $1,320,000$ & $1,327,022$ \\
\hline 1100 & 40 & 3 & 80 & 39.9 & 14 & 28 & 25 & 30 & 30 & 7,022 & $1,260,000$ & 1,267,022 \\
\hline 1100 & 40 & 3 & 100 & 35.5 & 11 & 31 & 25 & 30 & 31 & 7,256 & $1,260,000$ & $1,267,256$ \\
\hline 1100 & 40 & 3 & 120 & 31.5 & 10 & 35 & 25 & 30 & 35 & 8,193 & $1,350,000$ & $1,358,193$ \\
\hline 1100 & 40 & 3 & 140 & 28.0 & 8 & 40 & 25 & 30 & 40 & 9,363 & $1,440,000$ & $1,449,363$ \\
\hline 1100 & 40 & 3 & 160 & 25.0 & 7 & 44 & 25 & 30 & 44 & 10,300 & $1,530,000$ & $1,540,300$ \\
\hline 1100 & 60 & 3 & 70 & 45.0 & 16 & 25 & 25 & 44 & 44 & 10,300 & $1,230,000$ & $1,240,300$ \\
\hline 1100 & 60 & 3 & 90 & 39.9 & 13 & 28 & 25 & 44 & 44 & 10,300 & $1,230,000$ & $1,240,300$ \\
\hline 1100 & 60 & 3 & 110 & 35.5 & 10 & 31 & 25 & 44 & 44 & 10,300 & $1,230,000$ & $1,240,300$ \\
\hline 1100 & 60 & 3 & 130 & 31.5 & 9 & 35 & 25 & 44 & 44 & 10,300 & $1,320,000$ & $1,330,300$ \\
\hline 1100 & 60 & 3 & 150 & 28.0 & 8 & 40 & 25 & 44 & 44 & 10,300 & $1,440,000$ & $1,450,300$ \\
\hline 1100 & 60 & 3 & 170 & 25.0 & 7 & 44 & 25 & 44 & 44 & 10,300 & $1,530,000$ & $1,540,300$ \\
\hline 1100 & 80 & 3 & 80 & 45.0 & 14 & 25 & 25 & 59 & 59 & 13,811 & $1,170,000$ & $1,183,811$ \\
\hline 1100 & 80 & 3 & 100 & 39.9 & 11 & 28 & 25 & 59 & 59 & 13,811 & $1,170,000$ & $1,183,811$ \\
\hline 1100 & 80 & 3 & 120 & 35.5 & 10 & 31 & 25 & 59 & 59 & 13,811 & $1,230,000$ & $1,243,811$ \\
\hline 1100 & 80 & 3 & 140 & 31.5 & 8 & 35 & 25 & 59 & 59 & 13,811 & $1,290,000$ & $1,303,811$ \\
\hline 1100 & 80 & 3 & 160 & 28.0 & 7 & 40 & 25 & 59 & 59 & 13,811 & $1,410,000$ & $1,423,811$ \\
\hline 1100 & 80 & 3 & 180 & 25.0 & 7 & 44 & 25 & 59 & 59 & 13,811 & $1,530,000$ & $1,543,811$ \\
\hline 1100 & 100 & 3 & 90 & 45.0 & 13 & 25 & 25 & 74 & 74 & 17,322 & $1,140,000$ & $1,157,322$ \\
\hline 1100 & 100 & 3 & 110 & 39.9 & 10 & 28 & 25 & 74 & 74 & 17,322 & $1,140,000$ & 1,157,322 \\
\hline 1100 & 100 & 3 & 130 & 35.5 & 9 & 31 & 25 & 74 & 74 & 17,322 & $1,200,000$ & $1,217,322$ \\
\hline 1100 & 100 & 3 & 150 & 31.5 & 8 & 35 & 25 & 74 & 74 & 17,322 & $1,290,000$ & $1,307,322$ \\
\hline 1100 & 100 & 3 & 170 & 28.0 & 7 & 40 & 25 & 74 & 74 & 17,322 & $1,410,000$ & $1,427,322$ \\
\hline 1100 & 100 & 3 & 190 & 25.0 & 6 & 44 & 25 & 74 & 74 & 17,322 & $1,500,000$ & $1,517,322$ \\
\hline 1100 & 120 & 3 & 100 & 45.0 & 11 & 25 & 25 & 88 & 88 & 20,599 & $1,080,000$ & $\mathbf{1 , 1 0 0 , 5 9 9}$ \\
\hline 1100 & 120 & 3 & 120 & 39.9 & 10 & 28 & 25 & 88 & 88 & 20,599 & $1,140,000$ & $1,160,599$ \\
\hline 1100 & 120 & 3 & 140 & 35.5 & 8 & 31 & 25 & 88 & 88 & 20,599 & $1,170,000$ & $1,190,599$ \\
\hline 1100 & 120 & 3 & 160 & 31.5 & 7 & 35 & 25 & 88 & 88 & 20,599 & $1,260,000$ & $1,280,599$ \\
\hline 1100 & 120 & 3 & 180 & 28.0 & 7 & 40 & 25 & 88 & 88 & 20,599 & $1,410,000$ & $1,430,599$ \\
\hline 1100 & 120 & 3 & 200 & 25.0 & 6 & 44 & 25 & 88 & 88 & 20,599 & $1,500,000$ & $1,520,599$ \\
\hline
\end{tabular}




\section{Appendix D}

\section{Sensitivity Analysis Data and Results}

Sensitivity analysis results are presented in this appendix. For each demand level (550, 1100, 2200 , and 3300 orders per hour), we present the resulting best manual and automated solution when other parameters are varied, as well as the corresponding wave length (WL) and difference between the two systems, manual (M) and automated (A). A value in parenthesis represents a negative number. In that case, the manual system is less expensive than the automated system, and thus, the difference is negative. 
Table D.1: Sensitivity Analysis for Demand $=550$ orders $/$ hour.

\begin{tabular}{|c|c|c|c|c|c|c|c|c|}
\hline $\begin{array}{c}\text { Demand } \\
\text { Level } \\
\text { (orders/hr) }\end{array}$ & $\begin{array}{l}\text { Labor } \\
\text { Cost } \\
(\$)\end{array}$ & $\begin{array}{c}\text { PS Cost } \\
\text { (Auto) } \\
(\$)\end{array}$ & $\begin{array}{c}\text { Induction } \\
\text { Rate } \\
\text { (items/min) }\end{array}$ & $\begin{array}{c}\text { Manual } \\
\text { Best } \\
(\$)\end{array}$ & $\begin{array}{c}\text { WL } \\
\text { Manual } \\
\text { (min) }\end{array}$ & $\begin{array}{c}\text { Automated } \\
\text { Best } \\
(\$)\end{array}$ & $\begin{array}{l}\text { WL } \\
\text { Auto } \\
\text { (min) }\end{array}$ & $\begin{array}{c}\text { Difference } \\
(\mathrm{M}-\mathrm{A}) \\
(\$)\end{array}$ \\
\hline 250000 & 18000 & 2,500 & 20 & 350,661 & 100 & 389,104 & 120 & $(38,443)$ \\
\hline 250000 & 18000 & 2,500 & 60 & 350,661 & 100 & 371,104 & 120 & $(20,443)$ \\
\hline 250000 & 18000 & 10,000 & 20 & 350,661 & 100 & 451,072 & 20 & $(100,411)$ \\
\hline 250000 & 18000 & 10,000 & 60 & 350,661 & 100 & 433,072 & 20 & $(82,411)$ \\
\hline 250000 & 18000 & 20,000 & 20 & 350,661 & 100 & 477,824 & 20 & $(127,163)$ \\
\hline 250000 & 18000 & 20,000 & 60 & 350,661 & 100 & 459,824 & 20 & $(109,163)$ \\
\hline 250000 & 30000 & 2,500 & 20 & 578,661 & 100 & 557,104 & 120 & 21,557 \\
\hline 250000 & 30000 & 2,500 & 60 & 578,661 & 100 & 527,104 & 120 & 51,557 \\
\hline 250000 & 30000 & 10,000 & 20 & 578,661 & 100 & 650,640 & 80 & $(71,979)$ \\
\hline 250000 & 30000 & 10,000 & 60 & 578,661 & 100 & 620,640 & 80 & $(41,979)$ \\
\hline 250000 & 30000 & 20,000 & 20 & 578,661 & 100 & 693,824 & 20 & $(115,163)$ \\
\hline 250000 & 30000 & 20,000 & 60 & 578,661 & 100 & 663,824 & 20 & $(85,163)$ \\
\hline 500000 & 18000 & 2,500 & 20 & 350,661 & 100 & 472,704 & 120 & $(122,043)$ \\
\hline 500000 & 18000 & 2,500 & 60 & 350,661 & 100 & 454,704 & 120 & $(104,043)$ \\
\hline 500000 & 18000 & 10,000 & 20 & 350,661 & 100 & 534,672 & 20 & $(184,011)$ \\
\hline 500000 & 18000 & 10,000 & 60 & 350,661 & 100 & 516,672 & 20 & $(166,011)$ \\
\hline 500000 & 18000 & 20,000 & 20 & 350,661 & 100 & 561,424 & 20 & $(210,763)$ \\
\hline 500000 & 18000 & 20,000 & 60 & 350,661 & 100 & 543,424 & 20 & $(192,763)$ \\
\hline 500000 & 30000 & 2,500 & 20 & 578,661 & 100 & 640,704 & 120 & $(62,043)$ \\
\hline 500000 & 30000 & 2,500 & 60 & 578,661 & 100 & 610,704 & 120 & $(32,043)$ \\
\hline 500000 & 30000 & 10,000 & 20 & 578,661 & 100 & 734,240 & 80 & $(155,579)$ \\
\hline 500000 & 30000 & 10,000 & 60 & 578,661 & 100 & 704,240 & 80 & $(125,579)$ \\
\hline 500000 & 30000 & 20,000 & 20 & 578,661 & 100 & 777,424 & 20 & $(198,763)$ \\
\hline 500000 & 30000 & 20,000 & 60 & 578,661 & 100 & 747,424 & 20 & $(168,763)$ \\
\hline 1000000 & 18000 & 2,500 & 20 & 350,661 & 100 & 639,904 & 120 & $(289,243)$ \\
\hline 1000000 & 18000 & 2,500 & 60 & 350,661 & 100 & 621,904 & 120 & $(271,243)$ \\
\hline 1000000 & 18000 & 10,000 & 20 & 350,661 & 100 & 701,872 & 20 & $(351,211)$ \\
\hline 1000000 & 18000 & 10,000 & 60 & 350,661 & 100 & 683,872 & 20 & $(333,211)$ \\
\hline 1000000 & 18000 & 20,000 & 20 & 350,661 & 100 & 728,624 & 20 & $(377,963)$ \\
\hline 1000000 & 18000 & 20,000 & 60 & 350,661 & 100 & 710,624 & 20 & $(359,963)$ \\
\hline 1000000 & 30000 & 2,500 & 20 & 578,661 & 100 & 807,904 & 120 & $(229,243)$ \\
\hline 1000000 & 30000 & 2,500 & 60 & 578,661 & 100 & 777,904 & 120 & $(199,243)$ \\
\hline 1000000 & 30000 & 10,000 & 20 & 578,661 & 100 & 901,440 & 60 & $(322,779)$ \\
\hline 1000000 & 30000 & 10,000 & 60 & 578,661 & 100 & 871,440 & 60 & $(292,779)$ \\
\hline 1000000 & 30000 & 20,000 & 20 & 578,661 & 100 & 994,624 & 20 & $(415,963)$ \\
\hline 1000000 & 30000 & 20,000 & 60 & 578,661 & 100 & 914,624 & 20 & $(335,963)$ \\
\hline
\end{tabular}


Table D.2: Sensitivity Analysis for Demand $=1100$ orders/hour.

\begin{tabular}{|c|c|c|c|c|c|c|c|c|}
\hline $\begin{array}{c}\text { Demand } \\
\text { Level } \\
\text { (orders/hr) }\end{array}$ & $\begin{array}{l}\text { Labor } \\
\text { Cost } \\
(\$)\end{array}$ & $\begin{array}{c}\text { PS Cost } \\
\text { (Auto) } \\
\text { (\$) }\end{array}$ & $\begin{array}{c}\text { Induction } \\
\text { Rate } \\
\text { (items } / \mathrm{min} \text { ) }\end{array}$ & $\begin{array}{c}\text { Manual } \\
\text { Best } \\
(\$)\end{array}$ & $\begin{array}{c}\text { WL } \\
\text { Manual } \\
\text { (min) }\end{array}$ & $\begin{array}{c}\text { Automated } \\
\text { Best } \\
(\$)\end{array}$ & $\begin{array}{l}\text { WL } \\
\text { Auto } \\
\text { (min) }\end{array}$ & $\begin{array}{c}\text { Difference } \\
(\mathrm{M}-\mathrm{A}) \\
(\$)\end{array}$ \\
\hline 250000 & 18000 & 2,500 & 20 & 668,599 & 120 & 641,888 & 120 & 26,711 \\
\hline 250000 & 18000 & 2,500 & 60 & 668,599 & 120 & 605,888 & 120 & 62,711 \\
\hline 250000 & 18000 & 10,000 & 20 & 668,599 & 120 & 744,480 & 20 & $(75,881)$ \\
\hline 250000 & 18000 & 10,000 & 60 & 668,599 & 120 & 708,480 & 20 & $(39,881)$ \\
\hline 250000 & 18000 & 20,000 & 20 & 668,599 & 120 & 794,640 & 20 & $(126,041)$ \\
\hline 250000 & 18000 & 20,000 & 60 & 668,599 & 120 & 758,640 & 20 & $(90,041)$ \\
\hline 250000 & 30000 & 2,500 & 20 & $1,100,599$ & 120 & 953,888 & 120 & 146,711 \\
\hline 250000 & 30000 & 2,500 & 60 & $1,100,599$ & 120 & 893,888 & 120 & 206,711 \\
\hline 250000 & 30000 & 10,000 & 20 & $1,100,599$ & 120 & $1,117,456$ & 60 & $(16,857)$ \\
\hline 250000 & 30000 & 10,000 & 60 & $1,100,599$ & 120 & $1,057,456$ & 60 & 43,143 \\
\hline 250000 & 30000 & 20,000 & 20 & $1,100,599$ & 120 & $1,190,640$ & 20 & $(90,041)$ \\
\hline 250000 & 30000 & 20,000 & 60 & $1,100,599$ & 120 & $1,130,640$ & 20 & $(30,041)$ \\
\hline 500000 & 18000 & 2,500 & 20 & 668,599 & 120 & 725,488 & 120 & $(56,889)$ \\
\hline 500000 & 18000 & 2,500 & 60 & 668,599 & 120 & 689,488 & 120 & $(20,889)$ \\
\hline 500000 & 18000 & 10,000 & 20 & 668,599 & 120 & 828,080 & 20 & $(159,481)$ \\
\hline 500000 & 18000 & 10,000 & 60 & 668,599 & 120 & 792,080 & 20 & $(123,481)$ \\
\hline 500000 & 18000 & 20,000 & 20 & 668,599 & 120 & 878,240 & 20 & $(209,641)$ \\
\hline 500000 & 18000 & 20,000 & 60 & 668,599 & 120 & 842,240 & 20 & $(173,641)$ \\
\hline 500000 & 30000 & 2,500 & 20 & $1,100,599$ & 120 & $1,037,488$ & 120 & 63,111 \\
\hline 500000 & 30000 & 2,500 & 60 & $1,100,599$ & 120 & 977,488 & 120 & 123,111 \\
\hline 500000 & 30000 & 10,000 & 20 & $1,100,599$ & 120 & $1,201,056$ & 60 & $(100,457)$ \\
\hline 500000 & 30000 & 10,000 & 60 & $1,100,599$ & 120 & $1,141,056$ & 60 & $(40,457)$ \\
\hline 500000 & 30000 & 20,000 & 20 & $1,100,599$ & 120 & $1,274,240$ & 20 & $(173,641)$ \\
\hline 500000 & 30000 & 20,000 & 60 & $1,100,599$ & 120 & $1,214,240$ & 20 & $(113,641)$ \\
\hline 1000000 & 18000 & 2,500 & 20 & 668,599 & 120 & 892,668 & 120 & $(224,069)$ \\
\hline 1000000 & 18000 & 2,500 & 60 & 668,599 & 120 & 856,688 & 120 & $(188,089)$ \\
\hline 1000000 & 18000 & 10,000 & 20 & 668,599 & 120 & 995,280 & 20 & $(326,681)$ \\
\hline 1000000 & 18000 & 10,000 & 60 & 668,599 & 120 & 959,280 & 20 & $(290,681)$ \\
\hline 1000000 & 18000 & 20,000 & 20 & 668,599 & 120 & $1,045,440$ & 20 & $(376,841)$ \\
\hline 1000000 & 18000 & 20,000 & 60 & 668,599 & 120 & $1,009,440$ & 20 & $(340,841)$ \\
\hline 1000000 & 30000 & 2,500 & 20 & $1,100,599$ & 120 & $1,204,668$ & 120 & $(104,069)$ \\
\hline 1000000 & 30000 & 2,500 & 60 & $1,100,599$ & 120 & $1,144,688$ & 120 & $(44,089)$ \\
\hline 1000000 & 30000 & 10,000 & 20 & $1,100,599$ & 120 & $1,368,256$ & 60 & $(267,657)$ \\
\hline 1000000 & 30000 & 10,000 & 60 & $1,100,599$ & 120 & $1,308,256$ & 60 & $(207,657)$ \\
\hline 1000000 & 30000 & 20,000 & 20 & $1,100,599$ & 120 & $1,441,440$ & 20 & $(340,841)$ \\
\hline 1000000 & 30000 & 20,000 & 60 & $1,100,599$ & 120 & $1,381,440$ & 20 & $(280,841)$ \\
\hline
\end{tabular}


Table D.3: Sensitivity Analysis for Demand = 2200 orders/hour.

\begin{tabular}{|c|c|c|c|c|c|c|c|c|}
\hline $\begin{array}{l}\text { Demand } \\
\text { Level } \\
\text { (orders/hr) }\end{array}$ & $\begin{array}{l}\text { Labor } \\
\text { Cost } \\
(\$)\end{array}$ & $\begin{array}{l}\text { PS Cost } \\
\text { (Auto) } \\
\text { (\$) }\end{array}$ & $\begin{array}{c}\text { Induction } \\
\text { Rate } \\
\text { (items } / \mathrm{min} \text { ) }\end{array}$ & $\begin{array}{l}\text { Manual } \\
\text { Best } \\
(\$)\end{array}$ & $\begin{array}{c}\text { WL } \\
\text { Manual } \\
\text { (min) }\end{array}$ & $\begin{array}{c}\text { Automated } \\
\text { Best } \\
(\$)\end{array}$ & $\begin{array}{l}\text { WL } \\
\text { Auto } \\
\text { (min) }\end{array}$ & $\begin{array}{c}\text { Difference } \\
(\mathrm{M}-\mathrm{A}) \\
(\$)\end{array}$ \\
\hline 250000 & 18000 & 2,500 & 20 & $1,319,198$ & 120 & $1,254,176$ & 120 & 65,022 \\
\hline 250000 & 18000 & 2,500 & 60 & $1,319,198$ & 120 & $1,146,176$ & 120 & 173,022 \\
\hline 250000 & 18000 & 10,000 & 20 & $1,319,198$ & 120 & $1,477,360$ & 20 & $(158,162)$ \\
\hline 250000 & 18000 & 10,000 & 60 & $1,319,198$ & 120 & $1,369,360$ & 20 & $(50,162)$ \\
\hline 250000 & 18000 & 20,000 & 20 & $1,319,198$ & 120 & $1,577,680$ & 20 & $(258,482)$ \\
\hline 250000 & 18000 & 20,000 & 60 & $1,319,198$ & 120 & $1,469,680$ & 20 & $(150,482)$ \\
\hline 250000 & 30000 & 2,500 & 20 & $2,171,198$ & 120 & $1,914,176$ & 120 & 257,022 \\
\hline 250000 & 30000 & 2,500 & 60 & $2,171,198$ & 120 & $1,734,176$ & 120 & 437,022 \\
\hline 250000 & 30000 & 10,000 & 20 & $2,171,198$ & 120 & $2,241,312$ & 60 & $(70,114)$ \\
\hline 250000 & 30000 & 10,000 & 60 & $2,171,198$ & 120 & $2,061,312$ & 60 & 109,886 \\
\hline 250000 & 30000 & 20,000 & 20 & $2,171,198$ & 120 & $2,417,680$ & 20 & $(246,482)$ \\
\hline 250000 & 30000 & 20,000 & 60 & $2,171,198$ & 120 & $2,237,680$ & 20 & $(66,482)$ \\
\hline 500000 & 18000 & 2,500 & 20 & $1,319,198$ & 120 & $1,337,776$ & 120 & $(18,578)$ \\
\hline 500000 & 18000 & 2,500 & 60 & $1,319,198$ & 120 & $1,229,776$ & 120 & 89,422 \\
\hline 500000 & 18000 & 10,000 & 20 & $1,319,198$ & 120 & $1,560,960$ & 20 & $(241,762)$ \\
\hline 500000 & 18000 & 10,000 & 60 & $1,319,198$ & 120 & $1,452,960$ & 20 & $(133,762)$ \\
\hline 500000 & 18000 & 20,000 & 20 & $1,319,198$ & 120 & $1,661,280$ & 20 & $(342,082)$ \\
\hline 500000 & 18000 & 20,000 & 60 & $1,319,198$ & 120 & $1,553,280$ & 20 & $(234,082)$ \\
\hline 500000 & 30000 & 2,500 & 20 & $2,171,198$ & 120 & $1,997,776$ & 120 & 173,422 \\
\hline 500000 & 30000 & 2,500 & 60 & $2,171,198$ & 120 & $1,817,776$ & 120 & 353,422 \\
\hline 500000 & 30000 & 10,000 & 20 & $2,171,198$ & 120 & $2,324,912$ & 60 & $(153,714)$ \\
\hline 500000 & 30000 & 10,000 & 60 & $2,171,198$ & 120 & $2,144,912$ & 60 & 26,286 \\
\hline 500000 & 30000 & 20,000 & 20 & $2,171,198$ & 120 & $2,501,280$ & 20 & $(330,082)$ \\
\hline 500000 & 30000 & 20,000 & 60 & $2,171,198$ & 120 & $2,321,280$ & 20 & $(150,082)$ \\
\hline 1000000 & 18000 & 2,500 & 20 & $1,319,198$ & 120 & $1,504,976$ & 120 & $(185,778)$ \\
\hline 1000000 & 18000 & 2,500 & 60 & $1,319,198$ & 120 & $1,396,976$ & 20 & $(77,778)$ \\
\hline 1000000 & 18000 & 10,000 & 20 & $1,319,198$ & 120 & $1,728,160$ & 20 & $(408,962)$ \\
\hline 1000000 & 18000 & 10,000 & 60 & $1,319,198$ & 120 & $1,620,160$ & 20 & $(300,962)$ \\
\hline 1000000 & 18000 & 20,000 & 20 & $1,319,198$ & 120 & $1,828,480$ & 20 & $(509,282)$ \\
\hline 1000000 & 18000 & 20,000 & 60 & $1,319,198$ & 120 & $1,720,480$ & 20 & $(401,282)$ \\
\hline 1000000 & 30000 & 2,500 & 20 & $2,171,198$ & 120 & $2,164,976$ & 120 & 6,222 \\
\hline 1000000 & 30000 & 2,500 & 60 & $2,171,198$ & 120 & $1,984,976$ & 120 & 186,222 \\
\hline 1000000 & 30000 & 10,000 & 20 & $2,171,198$ & 120 & $2,492,112$ & 60 & $(320,914)$ \\
\hline 1000000 & 30000 & 10,000 & 60 & $2,171,198$ & 120 & $2,312,112$ & 60 & $(140,914)$ \\
\hline 1000000 & 30000 & 20,000 & 20 & $2,171,198$ & 120 & $2,668,480$ & 20 & $(497,282)$ \\
\hline 1000000 & 30000 & 20,000 & 60 & $2,171,198$ & 120 & $2,488,480$ & 20 & $(317,282)$ \\
\hline
\end{tabular}


Table D.4: Sensitivity Analysis for Demand = 3300 orders/hour.

\begin{tabular}{|c|c|c|c|c|c|c|c|c|}
\hline $\begin{array}{c}\text { Demand } \\
\text { Level } \\
\text { (orders/hr) }\end{array}$ & $\begin{array}{l}\text { Labor } \\
\text { Cost } \\
(\$)\end{array}$ & $\begin{array}{c}\text { PS Cost } \\
\text { (Auto) } \\
\text { (\$) }\end{array}$ & $\begin{array}{c}\text { Induction } \\
\text { Rate } \\
\text { (items } / \mathrm{min} \text { ) }\end{array}$ & $\begin{array}{c}\text { Manual } \\
\text { Best } \\
(\$)\end{array}$ & $\begin{array}{c}\text { WL } \\
\text { Manual } \\
\text { (min) }\end{array}$ & $\begin{array}{c}\text { Automated } \\
\text { Best } \\
(\$)\end{array}$ & $\begin{array}{l}\text { WL } \\
\text { Auto } \\
\text { (min) }\end{array}$ & $\begin{array}{c}\text { Difference } \\
(\mathrm{M}-\mathrm{A}) \\
(\$)\end{array}$ \\
\hline 250000 & 18000 & 2,500 & 20 & $1,987,797$ & 120 & $2,025,904$ & 120 & $(38,107)$ \\
\hline 250000 & 18000 & 2,500 & 60 & $1,987,797$ & 120 & $1,755,904$ & 120 & 231,893 \\
\hline 250000 & 18000 & 10,000 & 20 & $1,987,797$ & 120 & $2,348,336$ & 20 & $(360,539)$ \\
\hline 250000 & 18000 & 10,000 & 60 & $1,987,797$ & 120 & $2,078,336$ & 20 & $(90,539)$ \\
\hline 250000 & 18000 & 20,000 & 20 & $1,987,797$ & 120 & $2,495,472$ & 20 & $(507,675)$ \\
\hline 250000 & 18000 & 20,000 & 60 & $1,987,797$ & 120 & $2,225,472$ & 20 & $(237,675)$ \\
\hline 250000 & 30000 & 2,500 & 20 & $3,271,797$ & 120 & $3,117,904$ & 120 & 153,893 \\
\hline 250000 & 30000 & 2,500 & 60 & $3,271,797$ & 120 & $2,667,904$ & 120 & 603,893 \\
\hline 250000 & 30000 & 10,000 & 20 & $3,271,797$ & 120 & $3,638,608$ & 60 & $(366,811)$ \\
\hline 250000 & 30000 & 10,000 & 60 & $3,271,797$ & 120 & $3,188,608$ & 60 & 83,189 \\
\hline 250000 & 30000 & 20,000 & 20 & $3,271,797$ & 120 & $3,851,472$ & 20 & $(579,675)$ \\
\hline 250000 & 30000 & 20,000 & 60 & $3,271,797$ & 120 & $3,401,472$ & 20 & $(129,675)$ \\
\hline 500000 & 18000 & 2,500 & 20 & $1,987,797$ & 120 & $2,109,504$ & 120 & $(121,707)$ \\
\hline 500000 & 18000 & 2,500 & 60 & $1,987,797$ & 120 & $1,839,504$ & 120 & 148,293 \\
\hline 500000 & 18000 & 10,000 & 20 & $1,987,797$ & 120 & $2,431,936$ & 20 & $(444,139)$ \\
\hline 500000 & 18000 & 10,000 & 60 & $1,987,797$ & 120 & $2,309,072$ & 20 & $(321,275)$ \\
\hline 500000 & 18000 & 20,000 & 20 & $1,987,797$ & 120 & $2,579,072$ & 20 & $(591,275)$ \\
\hline 500000 & 18000 & 20,000 & 60 & $1,987,797$ & 120 & $2,309,072$ & 20 & $(321,275)$ \\
\hline 500000 & 30000 & 2,500 & 20 & $3,271,797$ & 120 & $3,201,504$ & 120 & 70,293 \\
\hline 500000 & 30000 & 2,500 & 60 & $3,271,797$ & 120 & $2,751,504$ & 120 & 520,293 \\
\hline 500000 & 30000 & 10,000 & 20 & $3,271,797$ & 120 & $3,722,208$ & 60 & $(450,411)$ \\
\hline 500000 & 30000 & 10,000 & 60 & $3,271,797$ & 120 & $3,272,208$ & 60 & (411) \\
\hline 500000 & 30000 & 20,000 & 20 & $3,271,797$ & 120 & $3,935,072$ & 20 & $(663,275)$ \\
\hline 500000 & 30000 & 20,000 & 60 & $3,271,797$ & 120 & $3,485,072$ & 20 & $(213,275)$ \\
\hline 1000000 & 18000 & 2,500 & 20 & $1,987,797$ & 120 & $2,276,704$ & 120 & $(288,907)$ \\
\hline 1000000 & 18000 & 2,500 & 60 & $1,987,797$ & 120 & $2,006,704$ & 120 & $(18,907)$ \\
\hline 1000000 & 18000 & 10,000 & 20 & $1,987,797$ & 120 & $2,599,136$ & 20 & $(611,339)$ \\
\hline 1000000 & 18000 & 10,000 & 60 & $1,987,797$ & 120 & $2,329,136$ & 20 & $(341,339)$ \\
\hline 1000000 & 18000 & 20,000 & 20 & $1,987,797$ & 120 & $2,746,272$ & 20 & $(758,475)$ \\
\hline 1000000 & 18000 & 20,000 & 60 & $1,987,797$ & 120 & $2,476,272$ & 20 & $(488,475)$ \\
\hline 1000000 & 30000 & 2,500 & 20 & $3,271,797$ & 120 & $3,368,704$ & 120 & $(96,907)$ \\
\hline 1000000 & 30000 & 2,500 & 60 & $3,271,797$ & 120 & $2,918,704$ & 120 & 353,093 \\
\hline 1000000 & 30000 & 10,000 & 20 & $3,271,797$ & 120 & $3,889,408$ & 60 & $(617,611)$ \\
\hline 1000000 & 30000 & 10,000 & 60 & $3,271,797$ & 120 & $3,439,408$ & 60 & $(167,611)$ \\
\hline 1000000 & 30000 & 20,000 & 20 & $3,271,797$ & 120 & $4,102,272$ & 20 & $(830,475)$ \\
\hline 1000000 & 30000 & 20,000 & 60 & $3,271,797$ & 120 & $3,652,272$ & 20 & $(380,475)$ \\
\hline
\end{tabular}




\section{Appendix E}

\section{Proof of Result 1.}

Result 1 The expression, (5.9), yields the optimal value for $p_{1}^{*}$ when $p_{1}$ and $p_{2}$ are constrained to be greater than zero.

\section{Proof:}

Taking the derivative of (5.6) and setting it equal to zero yields a quadratic equation in terms of $p_{1}$. The two roots are expressed as:

$$
p_{1}=\frac{-2 b m \pm \sqrt{4 b^{2} m^{2}-4 b^{2} m(m+1)}}{2 m(m+1)} .
$$

We now refer to the first root as the positive root, and the second root as the negative root. Since $p_{1}$ must be positive, we know that:

$$
\frac{-2 b m \pm \sqrt{4 b^{2} m^{2}-4 b^{2} m(m+1)}}{2 m(m+1)}>0 .
$$

Also, since $p_{2}=b+m p_{1}>0$, we know that:

$$
\frac{-2 b m \pm \sqrt{4 b^{2} m^{2}-4 b^{2} m(m+1)}}{2 m(m+1)}<\frac{-b}{m}
$$


Consider the inequality given by (E.2). Simplification leads to the following expression:

$$
\begin{aligned}
& \frac{-2 b m \pm \sqrt{-4 b^{2} m}}{2 m(m+1)}>0 \\
& \frac{-b m \pm b \sqrt{-m}}{m(m+1)}>0 .
\end{aligned}
$$

The correct root is dependent on the sign of the denominator, $m(m+1)$.

Case 1: $-1<m<0$. This results in $m(m+1)<0$. In order to satisfy (E.4), we need to determine the root that results in a negative numerator as well.

$$
\begin{gathered}
-b m \pm b \sqrt{-m}<0 \\
\pm b \sqrt{-m}<b m \\
\pm \sqrt{-m}<m .
\end{gathered}
$$

Since $-1<m<0,-\sqrt{-m}<m$. Therefore, the second root is feasible for $p_{1}$ and the first root is not. Using the second root, we now show that $p_{2}>0$ by substituting (E.4) into $p_{2}=b+m p_{1}$ :

$$
b+m\left(\frac{-b m-b \sqrt{-m}}{m(m+1)}\right)>0 .
$$

This reduces to the following:

$$
\frac{b m+b-b m-b \sqrt{-m}}{m+1}>0
$$

The simplified inequality is

$$
\frac{b-b \sqrt{-m}}{m+1}>0
$$

Since $-1<m<0$, we know that $m+1>0$, and therefore $b-b \sqrt{-m}$ must be positive in order to satisfy the inequality. Since $b>0, b>b \sqrt{-m}$, therefore the numerator is also positive, ensuring the second root is valid for $p_{2}$ as well.

Case 2: $m=-1$. This results in a denominator of 0 . Therefore, we must prove Result 1 
using the original equation for $p_{1}$ by substituting $m=-1$ into (5.6):

$$
\begin{gathered}
z^{\prime}=\frac{-D}{p_{1}^{2}}+\frac{-D m}{\left(m p_{1}+b\right)^{2}}=0 ; \\
\frac{-D m}{\left(m p_{1}+b\right)^{2}}=\frac{D}{p_{1}^{2}} .
\end{gathered}
$$

Substituting $m=-1$ results in the following:

$$
\frac{D}{\left(-p_{1}+b\right)^{2}}=\frac{D}{p_{1}^{2}}
$$

which is solved by:

$$
p_{1}=\frac{b}{2}
$$

This equation for $p_{1}$ is only valid for $m=-1$. Note that $p_{2}=-\frac{b}{2}+b=\frac{b}{2}$, which is obviously greater than zero.

Case 3: $m<-1$. In this case, $m(m+1)>0$. Therefore, $-b m \pm b \sqrt{-m}$ must be greater than zero in order to ensure $p_{1}>0$. The correct root is determined by the following:

$$
\begin{aligned}
& \pm b \sqrt{-m}>b m \\
& \pm \sqrt{-m}>m
\end{aligned}
$$

Since $m<-1$, both roots lead to the required result. Therefore, we must determine which root results in a positive value for $p_{2}$, where $p_{2}=b+m p_{1}$ :

$$
\begin{gathered}
m\left(\frac{-2 b m \pm \sqrt{-4 b^{2} m}}{2 m(m+1)}\right)+b>0 \\
\frac{-b m \pm b \sqrt{-m}}{m+1}+\frac{b m+b}{m+1}>0 .
\end{gathered}
$$

This reduces to the following:

$$
\frac{b \pm b \sqrt{-m}}{m+1}>0
$$

Since $m<-1, m+1<0$, and therefore $b \pm b \sqrt{-m}$ must be negative. Since $b>0$, 
and $b \sqrt{-m}>0$, the following inequality must hold:

$$
\pm b \sqrt{-m}<-b
$$

This inequality can only be satisfied by the second root for $p_{1}$.

From Cases 1 and 3, we see that the second root for $p_{1}$ is the only valid root. Thus, $p_{1}^{*}$ is equal to the second root when $m \neq 1$. Case 2 completes the proof by showing $p_{1}^{*}=\frac{b}{2}$ when $m=-1$. 


\section{Appendix F}

\section{Throughput Simulation Results}

In this appendix we present the results from several throughput simulation models, as well as the spreadsheet models containing data for the efficiency curves developed using the simulation and time study data. The spreadsheet models represent different levels and combinations of picking and packing rates. Again, in Tables F.1 through F.10, the italicized total annual cost represents the best cost in the wavelength (or overlap/no overlap automated system) and the boldface total annual cost is the best overall cost in the manual or automated system. Fifteen scenarios were developed to test the system design recommended by the prescriptive model in order to see if the desired throughput could be met. Each level of picking and packing rate combinations were tested, as shown in the spreadsheet model calculations presented in Tables F.1 through F.10. Again, for the automated system results, the top six rows represent a system with wave overlap, and the bottom six rows represent a system with no wave overlap.

The models presented are for various levels of demand and sorter costs, as described in the table caption. Each of the fifteen scenarios run have a different set of input parameters, therefore we did not present every combination of this data. The main purpose of this presentation is to show the picking and packing rates from the efficiency curves developed from the data in Appendix B that are used in the model. Resulting picking, packing, and induction times were recorded for 100 replications. Picking and packing data are provided in 
Tables F.11 through F.14. In order to see the effects of rounding the batch size, additional simulations were run. The results from these simulation runs are presented in Tables F.15 through F.18. Induction data are recorded in Tables F.19 through F.24. 
Table F.1: Automated System Prescriptive Model Calculations (High Picking and High Packing Levels, Demand $=550$ orders $/$ hr, Sorter Cost $=\$ 250,000$ ).

\begin{tabular}{cccccccccccccccc}
\hline$W$ & $p(n)$ & $q(m)$ & $t$ & $r_{p}$ & $r_{q}$ & $N_{q}$ & $r_{a}$ & $r_{t}$ & $r_{s}$ & $T_{\ell}^{a}$ & \multicolumn{1}{c}{$r_{q} c_{q}$} & $r_{s} c_{t}$ & $C_{t}^{s}$ & $C_{t}^{s} * A$ \\
\hline 20 & 113.45 & 118.26 & 120 & 5 & 5 & 25 & 8 & 1 & 1 & 330,000 & 20,000 & 50,000 & 320,000 & 84,415 & 414,415 \\
40 & 169.48 & 118.15 & 120 & 4 & 5 & 25 & 15 & 1 & 1 & 300,000 & 37,500 & 50,000 & 337,500 & 89,032 & 389,032 \\
60 & 209.78 & 118.07 & 120 & 3 & 5 & 25 & 22 & 1 & 1 & 270,000 & 55,000 & 50,000 & 355,000 & 93,648 & 363,648 \\
80 & 241.25 & 118.03 & 120 & 3 & 5 & 25 & 30 & 1 & 1 & 270,000 & 75,000 & 50,000 & 375,000 & 98,924 & 368,924 \\
100 & 266.75 & 117.99 & 120 & 3 & 5 & 25 & 37 & 1 & 1 & 270,000 & 92,500 & 50,000 & 392,500 & 103,541 & 373,541 \\
120 & 288.87 & 117.96 & 120 & 2 & 5 & 25 & 44 & 1 & 1 & 240,000 & 110,000 & 50,000 & 410,000 & 108,157 & $\mathbf{3 4 8 , 1 5 7}$ \\
\hline 20 & 113.45 & 93.21 & 120 & 5 & 6 & 25 & 8 & 1 & 1 & 360,000 & 20,000 & 50,000 & 320,000 & 84,415 & 444,415 \\
40 & 169.48 & 83.45 & 120 & 4 & 7 & 25 & 15 & 1 & 1 & 360,000 & 37,500 & 50,000 & 337,500 & 89,032 & 449,032 \\
60 & 209.78 & 76.70 & 120 & 3 & 8 & 25 & 22 & 1 & 1 & 360,000 & 55,000 & 50,000 & 355,000 & 93,648 & 453,648 \\
80 & 241.25 & 71.73 & 120 & 3 & 8 & 25 & 30 & 1 & 1 & 360,000 & 75,000 & 50,000 & 375,000 & 98,924 & 458,924 \\
100 & 266.75 & 68.02 & 120 & 3 & 9 & 25 & 37 & 1 & 1 & 390,000 & 92,500 & 50,000 & 392,500 & 103,541 & 493,541 \\
120 & 288.87 & 65.41 & 120 & 2 & 9 & 25 & 44 & 1 & 1 & 360,000 & 110,000 & 50,000 & 410,000 & 108,157 & 468,157 \\
\hline
\end{tabular}

Table F.2: Manual System Prescriptive Model Calculations (High Picking and High Packing Levels).

\begin{tabular}{|c|c|c|c|c|c|c|c|c|c|c|c|c|}
\hline$D$ & $W$ & $N_{o}$ & $p(n)$ & $q(m)$ & $r_{p}$ & $r_{q}$ & $\overline{N_{q}}$ & $n_{q}$ & $r_{a}$ & $r_{a} c_{q}$ & $T_{\ell}^{m}$ & $T^{m}$ \\
\hline 550 & 20 & 3 & 61.65 & 67.14 & 9 & 9 & 25 & 8 & 9 & 2,107 & $\frac{\ell}{540,000}$ & 542,107 \\
\hline 550 & 20 & 3 & 63.33 & 63.76 & 9 & 9 & 25 & 8 & 9 & 2,107 & 540,000 & 542,107 \\
\hline 550 & 20 & 3 & 68.69 & 61.98 & 9 & 9 & 25 & 8 & 9 & 2,107 & 540,000 & 542,107 \\
\hline 550 & 20 & 3 & 76.25 & 56.64 & 8 & 10 & 25 & 8 & 10 & 2,341 & 540,000 & 542,341 \\
\hline 550 & 20 & 3 & 89.36 & 50.73 & 7 & 11 & 25 & 8 & 11 & 2,575 & 540,000 & 542,575 \\
\hline 550 & 20 & 3 & 113.45 & 40.60 & 5 & 14 & 25 & 8 & 14 & 3,277 & 570,000 & 573,277 \\
\hline 550 & 40 & 3 & 94.54 & 54.55 & 6 & 11 & 25 & 15 & 15 & 3,511 & 510,000 & 513,511 \\
\hline 550 & 40 & 3 & 100.63 & 51.71 & 6 & 11 & 25 & 15 & 15 & 3,511 & 510,000 & 513,511 \\
\hline 550 & 40 & 3 & 109.09 & 47.52 & 6 & 12 & 25 & 15 & 15 & 3,511 & 540,000 & 543,511 \\
\hline 550 & 40 & 3 & 120.70 & 44.32 & 5 & 13 & 25 & 15 & 15 & 3,511 & 540,000 & 543,511 \\
\hline 550 & 40 & 3 & 137.80 & 38.47 & 4 & 15 & 25 & 15 & 15 & 3,511 & 570,000 & 573,511 \\
\hline 550 & 40 & 3 & 169.48 & 30.60 & 4 & 18 & 25 & 15 & 18 & 4,213 & 660,000 & 664,213 \\
\hline 550 & 60 & 3 & 123.39 & 46.71 & 5 & 12 & 25 & 22 & 22 & 5,150 & 510,000 & 515,150 \\
\hline 550 & 60 & 3 & 133.45 & 44.32 & 5 & 13 & 25 & 22 & 22 & 5,150 & 540,000 & 545,150 \\
\hline 550 & 60 & 3 & 143.69 & 41.06 & 4 & 14 & 25 & 22 & 22 & 5,150 & 540,000 & 545,150 \\
\hline 550 & 60 & 3 & 158.04 & 37.42 & 4 & 15 & 25 & 22 & 22 & 5,150 & 570,000 & 575,150 \\
\hline 550 & 60 & 3 & 177.71 & 32.41 & 4 & 17 & 25 & 22 & 22 & 5,150 & 630,000 & 635,150 \\
\hline 550 & 60 & 3 & 209.78 & 25.55 & 3 & 22 & 25 & 22 & 22 & 5,150 & 750,000 & 755,150 \\
\hline 550 & 80 & 3 & 154.61 & 41.19 & 4 & 14 & 25 & 30 & 30 & 7,022 & 540,000 & 547,022 \\
\hline 550 & 80 & 3 & 165.34 & 38.96 & 4 & 15 & 25 & 30 & 30 & 7,022 & 570,000 & 577,022 \\
\hline 550 & 80 & 3 & 175.99 & 36.29 & 4 & 16 & 25 & 30 & 30 & 7,022 & 600,000 & 607,022 \\
\hline 550 & 80 & 3 & 190.16 & 33.00 & 3 & 17 & 25 & 30 & 30 & 7,022 & 600,000 & 607,022 \\
\hline 550 & 80 & 3 & 212.05 & 28.60 & 3 & 20 & 25 & 30 & 30 & 7,022 & 690,000 & 697,022 \\
\hline 550 & 80 & 3 & 241.25 & 22.62 & 3 & 25 & 25 & 30 & 30 & 7,022 & 840,000 & 847,022 \\
\hline 550 & 100 & 3 & 180.80 & 37.79 & 4 & 15 & 25 & 37 & 37 & 8,661 & 570,000 & 578,661 \\
\hline 550 & 100 & 3 & 190.18 & 35.49 & 3 & 16 & 25 & 37 & 37 & 8,661 & 570,000 & 578,661 \\
\hline 550 & 100 & 3 & 202.90 & 33.00 & 3 & 17 & 25 & 37 & 37 & 8,661 & 600,000 & 608,661 \\
\hline 550 & 100 & 3 & 218.98 & 29.87 & 3 & 19 & 25 & 37 & 37 & 8,661 & 660,000 & 668,661 \\
\hline 550 & 100 & 3 & 240.07 & 25.91 & 3 & 22 & 25 & 37 & 37 & 8,661 & 750,000 & 758,661 \\
\hline 550 & 100 & 3 & 266.75 & 20.56 & 3 & 27 & 25 & 37 & 37 & 8,661 & 900,000 & 908,661 \\
\hline 550 & 120 & 3 & 204.89 & 34.98 & 3 & 16 & 25 & 44 & 44 & $\overline{10,300}$ & 570,000 & 580,300 \\
\hline 550 & 120 & 3 & 215.91 & 32.76 & 3 & 17 & 25 & 44 & 44 & 10,300 & 600,000 & 610,300 \\
\hline 550 & 120 & 3 & 228.78 & 30.29 & 3 & 19 & 25 & 44 & 44 & 10,300 & 660,000 & 670,300 \\
\hline 550 & 120 & 3 & 245.16 & 27.53 & 3 & 20 & 25 & 44 & 44 & 10,300 & 690,000 & 700,300 \\
\hline 550 & 120 & 3 & 265.40 & 23.88 & 3 & 24 & 25 & 44 & 44 & 10,300 & 810,000 & 820,300 \\
\hline 550 & 120 & 3 & 288.87 & 18.91 & 2 & 30 & 25 & 44 & 44 & 10,300 & 960,000 & 970,300 \\
\hline
\end{tabular}


Table F.3: Automated System Prescriptive Model Calculations (High Picking and Low Packing Levels, Demand $=550$ orders $/$ hr, Sorter Cost $=\$ 1,000,000)$.

\begin{tabular}{cccccccccccccccc}
\hline$W$ & $p(n)$ & $q(m)$ & $t$ & $r_{p}$ & $r_{q}$ & $N_{q}$ & $r_{a}$ & $r_{t}$ & $r_{s}$ & $T_{\ell}^{a}$ & \multicolumn{1}{c}{$r_{q} c_{q}$} & \multicolumn{1}{c}{$r_{s} c_{t}$} & $C_{t}^{s}$ & $C_{t}^{s} * A$ \\
\hline 20 & 113.45 & 118.26 & 120 & 5 & 5 & 25 & 8 & 1 & 1 & 330,000 & 20,000 & 50,000 & 320,000 & 84,415 & 414,415 \\
40 & 169.48 & 118.15 & 120 & 4 & 5 & 25 & 15 & 1 & 1 & 300,000 & 37,500 & 50,000 & 337,500 & 89,032 & 389,032 \\
60 & 209.78 & 118.07 & 120 & 3 & 5 & 25 & 22 & 1 & 1 & 270,000 & 55,000 & 50,000 & 355,000 & 93,648 & 363,648 \\
80 & 241.25 & 118.03 & 120 & 3 & 5 & 25 & 30 & 1 & 1 & 270,000 & 75,000 & 50,000 & 375,000 & 98,924 & 368,924 \\
100 & 266.75 & 117.99 & 120 & 3 & 5 & 25 & 37 & 1 & 1 & 270,000 & 92,500 & 50,000 & 392,500 & 103,541 & 373,541 \\
120 & 288.87 & 117.96 & 120 & 2 & 5 & 25 & 44 & 1 & 1 & 240,000 & 110,000 & 50,000 & 410,000 & 108,157 & $\mathbf{3 4 8 , 1 5 7}$ \\
\hline 20 & 113.45 & 93.21 & 120 & 5 & 6 & 25 & 8 & 1 & 1 & 360,000 & 20,000 & 50,000 & 320,000 & 84,415 & 444,415 \\
40 & 169.48 & 83.45 & 120 & 4 & 7 & 25 & 15 & 1 & 1 & 360,000 & 37,500 & 50,000 & 337,500 & 89,032 & 449,032 \\
60 & 209.78 & 76.70 & 120 & 3 & 8 & 25 & 22 & 1 & 1 & 360,000 & 55,000 & 50,000 & 355,000 & 93,648 & 453,648 \\
80 & 241.25 & 71.73 & 120 & 3 & 8 & 25 & 30 & 1 & 1 & 360,000 & 75,000 & 50,000 & 375,000 & 98,924 & 458,924 \\
100 & 266.75 & 68.02 & 120 & 3 & 9 & 25 & 37 & 1 & 1 & 390,000 & 92,500 & 50,000 & 392,500 & 103,541 & 493,541 \\
120 & 288.87 & 65.41 & 120 & 2 & 9 & 25 & 44 & 1 & 1 & 360,000 & 110,000 & 50,000 & 410,000 & 108,157 & 468,157 \\
\hline
\end{tabular}

Table F.4: Manual System Prescriptive Model Calculations (High Picking and Low Packing Levels).

\begin{tabular}{|c|c|c|c|c|c|c|c|c|c|c|c|c|}
\hline$D$ & $W$ & $\overline{N_{o}}$ & $p(n)$ & $q(m)$ & $r_{p}$ & $r_{q}$ & $N_{q}$ & $n_{q}$ & $r_{a}$ & $r_{a} c_{q}$ & $T_{\ell}^{m}$ & $T^{m}$ \\
\hline 1100 & 20 & 3 & 61.65 & 36.36 & 18 & 31 & 25 & 15 & 31 & 5,724 & $1,470,000$ & $1,475,724$ \\
\hline 1100 & 20 & 3 & 63.33 & 35.74 & 18 & 31 & 25 & 15 & 31 & 5,724 & $1,470,000$ & $1,475,724$ \\
\hline 1100 & 20 & 3 & 68.69 & 34.47 & 17 & 32 & 25 & 15 & 32 & 5,909 & $1,470,000$ & $1,475,909$ \\
\hline 1100 & 20 & 3 & 76.25 & 34.01 & 15 & 33 & 25 & 15 & 33 & 6,094 & $1,440,000$ & $1,446,094$ \\
\hline 1100 & 20 & 3 & 89.36 & 31.08 & 13 & 36 & 25 & 15 & 36 & 6,648 & $1,470,000$ & $1,476,648$ \\
\hline 1100 & 20 & 3 & 113.45 & 26.57 & 10 & 42 & 25 & 15 & 42 & 7,756 & $1,560,000$ & $1,567,756$ \\
\hline 1100 & 40 & 3 & 94.54 & 32.74 & 12 & 34 & 25 & 30 & 34 & 6,278 & $1,380,000$ & $1,386,278$ \\
\hline 1100 & 40 & 3 & 100.63 & 31.73 & 11 & 35 & 25 & 30 & 35 & 6,463 & $1,380,000$ & $1,386,463$ \\
\hline 1100 & 40 & 3 & 109.09 & 29.24 & 11 & 38 & 25 & 30 & 38 & 7,017 & $1,470,000$ & $1,477,017$ \\
\hline 1100 & 40 & 3 & 120.70 & 28.99 & 10 & 38 & 25 & 30 & 38 & 7,017 & $1,440,000$ & $1,447,017$ \\
\hline 1100 & 40 & 3 & 137.80 & 26.46 & 8 & 42 & 25 & 30 & 42 & 7,756 & $1,500,000$ & $1,507,756$ \\
\hline 1100 & 40 & 3 & 169.48 & 22.00 & 7 & 50 & 25 & 30 & 50 & 9,233 & $1,710,000$ & $1,719,233$ \\
\hline 1100 & 60 & 3 & 123.39 & 29.66 & 9 & 38 & 25 & 44 & 44 & 8,125 & $1,410,000$ & $1,418,125$ \\
\hline 1100 & 60 & 3 & 133.45 & 28.99 & 9 & 38 & 25 & 44 & 44 & 8,125 & $1,410,000$ & $1,418,125$ \\
\hline 1100 & 60 & 3 & 143.69 & 26.75 & 8 & 42 & 25 & 44 & 44 & 8,125 & $1,500,000$ & $1,508,125$ \\
\hline 1100 & 60 & 3 & 158.04 & 25.78 & 7 & 43 & 25 & 44 & 44 & 8,125 & $1,500,000$ & $1,508,125$ \\
\hline 1100 & 60 & 3 & 177.71 & 23.47 & 7 & 47 & 25 & 44 & 47 & 8,679 & $1,620,000$ & $1,628,679$ \\
\hline 1100 & 60 & 3 & 209.78 & 19.40 & 6 & 57 & 25 & 44 & 57 & 10,526 & $1,890,000$ & $1,900,526$ \\
\hline 1100 & 80 & 3 & 154.61 & 27.47 & 8 & 41 & 25 & $\overline{59}$ & 59 & 10,895 & $1,470,000$ & $1,480,895$ \\
\hline 1100 & 80 & 3 & 165.34 & 26.20 & 7 & 42 & 25 & 59 & 59 & 10,895 & $1,470,000$ & $1,480,895$ \\
\hline 1100 & 80 & 3 & 175.99 & 25.01 & 7 & 44 & 25 & 59 & 59 & 10,895 & $1,530,000$ & $1,540,895$ \\
\hline 1100 & 80 & 3 & 190.16 & 23.70 & 6 & 47 & 25 & 59 & 59 & 10,895 & $1,590,000$ & $1,600,895$ \\
\hline 1100 & 80 & 3 & 212.05 & 20.98 & 6 & 53 & 25 & 59 & 59 & 10,895 & $1,770,000$ & $1,780,895$ \\
\hline 1100 & 80 & 3 & 241.25 & 17.86 & 5 & 62 & 25 & 59 & 62 & 11,449 & $2,010,000$ & $2,021,449$ \\
\hline 1100 & 100 & 3 & 180.80 & 25.53 & 7 & 44 & 25 & 74 & 74 & 13,665 & $1,530,000$ & $1,543,665$ \\
\hline 1100 & 100 & 3 & 190.18 & 24.93 & 6 & 45 & 25 & 74 & 74 & 13,665 & $1,530,000$ & $1,543,665$ \\
\hline 1100 & 100 & 3 & 202.90 & 23.70 & 6 & 47 & 25 & 74 & 74 & 13,665 & $1,590,000$ & $1,603,665$ \\
\hline 1100 & 100 & 3 & 218.98 & 21.41 & 6 & 52 & 25 & 74 & 74 & 13,665 & $1,740,000$ & $1,753,665$ \\
\hline 1100 & 100 & 3 & 240.07 & 19.59 & 5 & 57 & 25 & 74 & 74 & 13,665 & $1,860,000$ & $1,873,665$ \\
\hline 1100 & 100 & 3 & 266.75 & 16.19 & 5 & 68 & 25 & 74 & 74 & 13,665 & $2,190,000$ & $2,203,665$ \\
\hline 1100 & 120 & 3 & 204.89 & 24.09 & 6 & 46 & 25 & 88 & 88 & 16,250 & $1,560,000$ & $1,576,250$ \\
\hline 1100 & 120 & 3 & 215.91 & 23.57 & 6 & 47 & 25 & 88 & 88 & 16,250 & $1,590,000$ & $1,606,250$ \\
\hline 1100 & 120 & 3 & 228.78 & 22.26 & 5 & 50 & 25 & 88 & 88 & 16,250 & $1,650,000$ & $1,666,250$ \\
\hline 1100 & 120 & 3 & 245.16 & 20.42 & 5 & 54 & 25 & 88 & 88 & 16,250 & $1,770,000$ & $1,786,250$ \\
\hline 1100 & 120 & 3 & 265.40 & 18.58 & 5 & 60 & 25 & 88 & 88 & 16,250 & $1,950,000$ & $1,966,250$ \\
\hline 1100 & 120 & 3 & 288.87 & 15.52 & 4 & 71 & 25 & 88 & 88 & 16,250 & $2,250,000$ & $2,266,250$ \\
\hline
\end{tabular}


Table F.5: Automated System Prescriptive Model Calculations (Medium Picking and Medium Packing Levels, Demand $=2200$ orders $/$ hr, Sorter Cost $=\$ 250,000$ ).

\begin{tabular}{|c|c|c|c|c|c|c|c|c|c|c|c|c|c|c|c|}
\hline$W$ & $p(n)$ & $q(m)$ & $t$ & $r_{p}$ & $r_{q}$ & $N_{q}$ & $r_{a}$ & $r_{t}$ & $r_{s}$ & $T_{\ell}^{a}$ & $r_{q} c_{q}$ & $r_{s} c_{t}$ & $C_{t}^{S}$ & $C_{t}^{S} * A$ & $T^{a}$ \\
\hline 20 & 78.23 & 69.99 & 20 & 29 & 32 & 25 & 30 & 8 & 4 & $2,070,000$ & 600,000 & 200,000 & $1,050,000$ & 351,099 & $2,421,099$ \\
\hline 40 & 127.67 & 69.93 & 20 & 18 & 32 & 25 & 59 & 8 & 4 & $1,740,000$ & $1,180,000$ & 200,000 & $1,630,000$ & 545,039 & $2,285,039$ \\
\hline 60 & 173.16 & 69.90 & 20 & 13 & 32 & 25 & 88 & 8 & 4 & $1,590,000$ & $1,760,000$ & 200,000 & $2,210,000$ & 738,979 & $2,328,979$ \\
\hline 80 & 211.19 & 69.87 & 20 & 11 & 32 & 25 & 118 & 8 & 4 & $1,530,000$ & $2,360,000$ & 200,000 & $2,810,000$ & 939,607 & $2,469,607$ \\
\hline 100 & 242.16 & 69.85 & 20 & 10 & 32 & 25 & 147 & 8 & 4 & $1,500,000$ & $2,940,000$ & 200,000 & $3,390,000$ & $1,133,547$ & $2,633,547$ \\
\hline 120 & 268.06 & 69.84 & 20 & 9 & 32 & 25 & 176 & 8 & 4 & $1,470,000$ & $3,520,000$ & 200,000 & $3,970,000$ & $1,327,487$ & $2,797,487$ \\
\hline 20 & 78.23 & 52.70 & 20 & 29 & 42 & 25 & 30 & 8 & 4 & $2,370,000$ & 600,000 & 200,000 & $1,050,000$ & 351,099 & $2,721,099$ \\
\hline 40 & 127.67 & 50.06 & 20 & 18 & 44 & 25 & 59 & 8 & 4 & $2,100,000$ & $1,180,000$ & 200,000 & $1,630,000$ & 545,039 & $2,645,039$ \\
\hline 60 & 173.16 & 48.62 & 20 & 13 & 46 & 25 & 88 & 8 & 4 & $2,010,000$ & $1,760,000$ & 200,000 & $2,210,000$ & 738,979 & $2,748,979$ \\
\hline 80 & 211.19 & 47.60 & 20 & 11 & 47 & 25 & 118 & 8 & 4 & $1,980,000$ & $2,360,000$ & 200,000 & $2,810,000$ & 939,607 & $2,919,607$ \\
\hline 120 & 268.06 & 46.22 & 20 & 9 & 48 & 25 & 176 & 8 & 4 & $1,950,000$ & $3,520,000$ & 200,000 & $3,970,000$ & $1,327,487$ & $3,277,487$ \\
\hline
\end{tabular}

Table F.6: Manual System Prescriptive Model Calculations (Medium Picking and Medium Packing Levels).

\begin{tabular}{|c|c|c|c|c|c|c|c|c|c|c|c|c|}
\hline$D$ & $W$ & $N_{o}$ & $p(n)$ & $q(m)$ & $r_{p}$ & $r_{q}$ & $N_{q}$ & $n_{q}$ & $r_{a}$ & $r_{a} c_{q}$ & $T_{\ell}^{m}$ & $T^{m}$ \\
\hline 2200 & 20 & 3 & 40.47 & 52.67 & 55 & 42 & $\frac{4}{25}$ & 30 & 42 & 9,831 & $2,910,000$ & $2,919,831$ \\
\hline 2200 & 20 & 3 & 40.47 & 52.67 & 55 & 42 & 25 & 30 & 42 & 9,831 & $2,910,000$ & $2,919,831$ \\
\hline 2200 & 20 & 3 & 48.20 & 48.35 & 46 & 46 & 25 & 30 & 46 & 10,768 & $2,760,000$ & $2,770,768$ \\
\hline 2200 & 20 & 3 & 49.80 & 46.65 & 45 & 48 & 25 & 30 & 48 & 11,236 & $2,790,000$ & $2,801,236$ \\
\hline 2200 & 20 & 3 & 57.17 & 42.73 & 39 & 52 & 25 & 30 & 52 & 12,172 & $2,730,000$ & $2,742,172$ \\
\hline 2200 & 20 & 3 & 78.23 & 36.44 & 29 & 61 & 25 & 30 & 61 & 14,279 & $2,700,000$ & $2,714,279$ \\
\hline 2200 & 40 & 3 & 64.88 & 45.59 & 34 & 49 & 25 & 59 & 59 & 13,811 & $2,490,000$ & $2,503,811$ \\
\hline 2200 & 40 & 3 & 66.85 & 44.73 & 33 & 50 & 25 & 59 & 59 & 13,811 & $2,490,000$ & $2,503,811$ \\
\hline 2200 & 40 & 3 & 71.02 & 42.18 & 31 & 53 & 25 & 59 & 59 & 13,811 & $2,520,000$ & $2,533,811$ \\
\hline 2200 & 40 & 3 & 79.88 & 39.36 & 28 & 56 & 25 & 59 & 59 & 811 & $2,520,000$ & $2,533,811$ \\
\hline 2200 & 40 & 3 & 94.55 & 35.05 & 24 & 63 & 25 & 59 & 63 & 14,747 & $2,610,000$ & $2,624,747$ \\
\hline 2200 & 40 & 3 & 127.67 & 28.20 & 18 & 79 & 25 & 59 & 79 & 18,492 & $2,910,000$ & $2,928,492$ \\
\hline 2200 & 60 & 3 & 84.49 & 40.80 & 27 & 54 & 25 & 88 & 88 & 20,599 & $2,430,000$ & $2,450,599$ \\
\hline 2200 & 60 & 3 & 88.32 & 39.36 & 25 & 56 & 25 & 88 & 88 & 20,599 & $2,430,000$ & $2,450,599$ \\
\hline 2200 & 60 & 3 & 97.31 & 36.65 & 23 & 61 & 25 & 88 & 88 & 20,599 & $2,520,000$ & $2,450,599$ \\
\hline 2200 & 60 & 3 & 110.22 & 33.80 & 20 & 66 & 25 & 88 & 88 & 20,599 & $2,580,000$ & $2,600,599$ \\
\hline 2200 & 60 & 3 & 130.87 & 30.14 & 17 & 73 & 25 & 88 & 88 & 20,599 & $2,700,000$ & $2,720,599$ \\
\hline 2200 & 60 & 3 & 173.16 & 23.75 & 13 & 93 & 25 & 88 & 93 & 21,769 & $3,180,000$ & $3,201,769$ \\
\hline 2200 & 80 & 3 & 104.37 & 36.55 & 2 & 61 & 25 & 118 & 118 & 27,621 & $2,490,000$ & $2,517,621$ \\
\hline 2200 & 80 & 3 & 111.28 & 35.48 & 20 & 63 & 25 & 118 & 118 & 27,621 & $2,490,000$ & $2,517,621$ \\
\hline 2200 & 80 & 3 & 121.78 & 33.14 & 19 & 67 & 25 & 118 & 118 & 27,621 & $2,580,000$ & $2,607,621$ \\
\hline 2200 & 80 & 3 & 138.64 & 30.68 & 16 & 72 & 25 & 118 & 118 & 27,621 & $2,640,000$ & $2,667,621$ \\
\hline 2200 & 80 & 3 & 164.50 & 26.83 & 14 & 82 & 25 & 118 & 118 & 27,621 & $2,880,000$ & $2,907,621$ \\
\hline 2200 & 80 & 3 & 211.19 & 21.14 & 11 & 105 & 25 & 118 & 118 & 27,621 & $3,480,000$ & $3,507,621$ \\
\hline 2200 & 100 & 3 & 123.77 & 34.14 & 18 & 65 & 25 & 147 & 147 & 34,410 & $2,490,000$ & $2,524,410$ \\
\hline 2200 & 100 & 3 & 135.46 & 32.79 & 17 & 68 & 25 & 147 & 147 & 34,410 & $2,550,000$ & $2,584,410$ \\
\hline 2200 & 100 & 3 & 147.93 & 30.68 & 15 & 72 & 25 & 147 & 147 & 34,410 & $2,610,000$ & $2,644,410$ \\
\hline 2200 & 100 & 3 & 166.56 & 27.77 & 14 & 80 & 25 & 147 & 147 & 34,410 & $2,820,000$ & $2,854,410$ \\
\hline 2200 & 100 & 3 & 196.52 & 24.06 & 12 & 92 & 25 & 147 & 147 & 34,410 & $3,120,000$ & $3,154,410$ \\
\hline 2200 & 100 & 3 & 242.16 & 19.06 & 10 & 116 & 25 & 147 & 147 & 34, & $3,780,000$ & $3,814,410$ \\
\hline 2200 & 120 & 3 & 147.80 & 31.56 & 15 & 70 & 25 & 176 & 176 & 41,198 & $2,550,000$ & $2,591,198$ \\
\hline 2200 & 120 & 3 & 156.13 & 30.04 & 15 & 74 & 25 & 176 & 176 & 41,198 & $2,670,000$ & $2,711,198$ \\
\hline 2200 & 120 & 3 & 173.32 & 28.38 & 13 & 78 & 25 & 176 & 176 & & $2,730,000$ & $2,771,198$ \\
\hline 2200 & 120 & 3 & 194.01 & 25.40 & 12 & 87 & 25 & 176 & 176 & & $2,970,000$ & $3,011,198$ \\
\hline 2200 & 120 & 3 & 225.00 & 22.36 & 10 & 99 & 25 & 176 & 176 & 41,198 & $3,270,000$ & $3,311,198$ \\
\hline 2200 & 120 & 3 & 268.06 & 17.69 & 9 & 125 & 25 & 176 & 176 & 41,198 & $4,020,000$ & $4,061,198$ \\
\hline
\end{tabular}


Table F.7: Automated System Prescriptive Model Calculations (Low Picking and High Packing Levels, Demand $=3300$ orders $/ \mathrm{hr}$, Sorter Cost $=\$ 250,000$ ).

\begin{tabular}{ccccccccccrrrrrr}
\hline$W$ & $p(n)$ & $q(m)$ & $t$ & $r_{p}$ & $r_{q}$ & $N_{q}$ & $r_{a}$ & $r_{t}$ & $r_{s}$ & $T_{\ell}^{a}$ & \multicolumn{1}{c}{$r_{q} c_{q}$} & $r_{s} c_{t}$ \\
\hline 20 & 56.65 & 118.26 & 60 & 59 & 28 & 25 & 44 & 4 & 1 & $2,730,000$ & 440,000 & 50,000 & 740,000 & $C_{t}^{S} * A$ \\
40 & 95.38 & 118.15 & 60 & 35 & 28 & 25 & 88 & 4 & 1 & $2,010,000$ & 880,000 & 50,000 & $1,180,000$ & 281,457 & $2,906,507$ \\
60 & 139.51 & 118.07 & 60 & 24 & 28 & 25 & 132 & 4 & 1 & $1,680,000$ & $1,320,000$ & 50,000 & $1,620,000$ & 386,407 & $2,291,457$ \\
80 & 181.15 & 118.03 & 60 & 19 & 28 & 25 & 176 & 4 & 1 & $1,530,000$ & $1,760,000$ & 50,000 & $2,060,000$ & 491,357 & $\mathbf{2 , 0 2 1 , 3 5 7}$ \\
100 & 217.62 & 117.99 & 60 & 16 & 28 & 25 & 220 & 4 & 1 & $1,440,000$ & $2,200,000$ & 50,000 & $2,500,000$ & 596,307 & $2,036,307$ \\
120 & 247.53 & 117.96 & 60 & 14 & 28 & 25 & 264 & 4 & 1 & $1,380,000$ & $2,640,000$ & 50,000 & $2,940,000$ & 701,257 & $2,081,257$ \\
\hline 20 & 56.65 & 93.21 & 60 & 59 & 36 & 25 & 44 & 4 & 1 & $2,970,000$ & 440,000 & 50,000 & 740,000 & 176,507 & $3,146,507$ \\
40 & 95.38 & 83.45 & 60 & 35 & 40 & 25 & 88 & 4 & 1 & $2,370,000$ & 880,000 & 50,000 & $1,180,000$ & 281,457 & $2,651,457$ \\
60 & 139.51 & 76.70 & 60 & 24 & 44 & 25 & 132 & 4 & 1 & $2,160,000$ & $1,320,000$ & 50,000 & $1,620,000$ & 386,407 & $2,546,407$ \\
80 & 181.15 & 71.73 & 60 & 19 & 47 & 25 & 176 & 4 & 1 & $2,100,000$ & $1,760,000$ & 50,000 & $2,060,000$ & 491,357 & $2,591,357$ \\
100 & 217.62 & 68.02 & 60 & 16 & 49 & 25 & 220 & 4 & 1 & $2,070,000$ & $2,200,000$ & 50,000 & $2,500,000$ & 596,307 & $2,666,307$ \\
120 & 247.53 & 65.41 & 60 & 14 & 51 & 25 & 264 & 4 & 1 & $2,070,000$ & $2,640,000$ & 50,000 & $2,940,000$ & 701,257 & $2,771,257$ \\
\hline
\end{tabular}

Table F.8: Manual System Prescriptive Model Calculations (Low Picking and High Packing Levels).

\begin{tabular}{|c|c|c|c|c|c|c|c|c|c|c|c|c|}
\hline$D$ & $W$ & $N_{o}$ & $p(n)$ & $q(m)$ & $r_{p}$ & $r_{q}$ & $N_{q}$ & $n_{q}$ & $r_{a}$ & $r_{a} c_{q}$ & $T_{\ell}^{m}$ & $T^{m}$ \\
\hline 3300 & 20 & 3 & 25.65 & 78.79 & 129 & $\frac{1}{42}$ & 25 & 44 & 44 & 7,346 & $5,130,000$ & $5,137,346$ \\
\hline 3300 & 20 & 3 & 31.62 & 71.86 & 105 & 46 & 25 & 44 & 46 & 7,680 & $4,530,000$ & $4,537,680$ \\
\hline 3300 & 20 & 3 & 31.62 & 71.86 & 105 & 46 & 25 & 44 & 46 & 7,680 & $4,530,000$ & $4,537,680$ \\
\hline 3300 & 20 & 3 & 38.06 & 63.76 & 87 & 52 & 25 & 44 & 52 & 8,681 & $4,170,000$ & $4,178,681$ \\
\hline 3300 & 20 & 3 & 41.42 & 59.63 & 80 & 56 & 25 & 44 & 56 & 9,349 & $4,080,000$ & $4,089,349$ \\
\hline 3300 & 20 & 3 & 56.65 & 47.52 & 59 & 70 & 25 & 44 & 70 & 11,687 & $3,870,000$ & $3,881,687$ \\
\hline 3300 & 40 & 3 & 46.33 & 61.98 & 72 & 54 & 25 & 88 & 88 & 14,692 & $3,780,000$ & $3,794,692$ \\
\hline 3300 & 40 & 3 & 48.19 & 59.63 & 69 & 56 & 25 & 88 & 88 & 14,692 & $3,750,000$ & $3,764,692$ \\
\hline 3300 & 40 & 3 & 53.61 & 56.64 & 62 & 59 & 25 & 88 & 88 & 14,692 & $3,630,000$ & $3,644,692$ \\
\hline 3300 & 40 & 3 & 57.22 & 52.34 & 58 & 64 & 25 & 88 & 88 & 14,692 & $3,660,000$ & $3,674,692$ \\
\hline 3300 & 40 & 3 & 69.76 & 45.35 & 48 & 73 & 25 & 88 & 88 & 14,692 & $3,630,000$ & $3,644,692$ \\
\hline 3300 & 40 & 3 & 95.38 & 35.49 & 35 & 93 & 25 & 88 & 93 & 15,526 & $3,840,000$ & $3,855,526$ \\
\hline 3300 & 60 & 3 & 60.48 & 54.55 & 55 & 61 & 25 & 132 & 132 & 22,037 & $3,480,000$ & $3,502,037$ \\
\hline 3300 & 60 & 3 & 63.26 & 52.34 & 53 & 64 & 25 & 132 & 132 & 22,037 & $3,510,000$ & $3,532,037$ \\
\hline 3300 & 60 & 3 & 72.86 & 47.52 & 46 & 70 & 25 & 132 & 132 & 22,037 & $3,480,000$ & $3,502,037$ \\
\hline 3300 & 60 & 3 & 79.26 & 44.32 & 42 & 75 & 25 & 132 & 132 & 22,037 & $3,510,000$ & $3,532,037$ \\
\hline 3300 & 60 & 3 & 97.94 & 38.26 & 34 & 87 & 25 & 132 & 132 & 22,037 & $3,630,000$ & $3,652,037$ \\
\hline 3300 & 60 & 3 & 139.51 & 28.67 & 24 & 116 & 25 & 132 & 132 & 22,037 & $4,200,000$ & $4,222,037$ \\
\hline 3300 & 80 & 3 & 74.54 & 49.43 & 45 & 67 & 25 & 176 & 176 & 29,383 & $3,360,000$ & $3,389,383$ \\
\hline 3300 & 80 & 3 & 80.29 & 46.62 & 42 & 71 & 25 & 176 & 176 & 29,383 & $3,390,000$ & $3,419,383$ \\
\hline 3300 & 80 & 3 & 89.65 & 42.70 & 37 & 78 & 25 & 176 & 176 & 29,383 & $3,450,000$ & $3,479,383$ \\
\hline 3300 & 80 & 3 & 102.60 & 38.47 & 33 & 86 & 25 & 176 & 176 & 29,383 & $3,570,000$ & $3,599,383$ \\
\hline 3300 & 80 & 3 & 126.49 & 33.00 & 27 & 101 & 25 & 176 & 176 & 29,383 & $3,840,000$ & $3,869,383$ \\
\hline 3300 & 80 & 3 & 181.15 & 24.51 & 19 & 135 & 25 & 176 & 176 & 29,383 & $4,620,000$ & $4,649,383$ \\
\hline 3300 & 100 & 3 & 90.82 & 44.51 & 37 & 75 & 25 & 220 & 220 & 36,729 & $3,360,000$ & $3,396,729$ \\
\hline 3300 & 100 & 3 & 98.57 & 42.24 & 34 & 79 & 25 & 220 & 220 & 36,729 & $3,390,000$ & $3,426,729$ \\
\hline 3300 & 100 & 3 & 109.48 & 38.47 & 31 & 86 & 25 & 220 & 220 & 36,729 & $3,510,000$ & $3,546,729$ \\
\hline 3300 & 100 & 3 & 126.39 & 34.62 & 27 & 96 & 25 & 220 & 220 & 36,729 & $3,690,000$ & $3,726,729$ \\
\hline 3300 & 100 & 3 & 158.13 & 29.12 & 21 & 114 & 25 & 220 & 220 & 36,729 & $4,050,000$ & $4,086,729$ \\
\hline 3300 & 100 & 3 & 217.62 & 21.88 & 16 & 151 & 25 & 220 & 220 & 36,729 & $5,010,000$ & $5,046,729$ \\
\hline 3300 & 120 & 3 & 105.81 & 41.06 & 32 & 81 & 25 & 264 & 264 & 44,075 & $3,390,000$ & $3,434,075$ \\
\hline 3300 & 120 & 3 & 115.12 & 38.61 & 29 & 86 & 25 & 264 & 264 & 44,075 & $3,450,000$ & $3,494,075$ \\
\hline 3300 & 120 & 3 & 130.76 & 35.26 & 26 & 94 & 25 & 264 & 264 & 44,075 & $3,600,000$ & $3,644,075$ \\
\hline 3300 & 120 & 3 & 153.45 & 31.23 & 22 & 106 & 25 & 264 & 264 & 44,075 & $3,840,000$ & $3,884,075$ \\
\hline 3300 & 120 & 3 & 189.39 & 26.32 & 18 & 126 & 25 & 264 & 264 & 44,075 & $4,320,000$ & $4,364,075$ \\
\hline 3300 & 120 & 3 & 247.53 & 19.92 & 14 & 166 & 25 & 264 & 264 & 44,075 & $5,400,000$ & $5,444,075$ \\
\hline
\end{tabular}


Table F.9: Automated System Prescriptive Model Calculations (Low Picking and Low Packing Levels, Demand $=2200$ orders $/$ hr, Sorter Cost $=\$ 500,000$ ).

\begin{tabular}{|c|c|c|c|c|c|c|c|c|c|c|c|c|c|c|c|}
\hline$W$ & $p(n)$ & $q(m)$ & $t$ & $r_{p}$ & $r_{q}$ & $N_{q}$ & $r_{a}$ & $r_{t}$ & $r_{s}$ & $T_{\ell}^{a}$ & $r_{q} c_{q}$ & $r_{s} c_{t}$ & $C_{t}^{s}$ & $C_{t}^{S} * A$ & $T^{a}$ \\
\hline 20 & 56.65 & $\overline{47.71}$ & 7200 & 39 & 47 & 25 & 30 & 2 & 2 & $2,640,000$ & 75,000 & 100,000 & 675,000 & 178,063 & $2,818,063$ \\
\hline 40 & 95.38 & 47.70 & 7200 & 24 & 47 & 25 & 59 & 2 & 2 & $2,190,000$ & 147,500 & 100,000 & 747,500 & 197,189 & $2,387,189$ \\
\hline 60 & 139.51 & 47.70 & 7200 & 16 & 47 & 25 & 88 & 2 & 2 & $1,950,000$ & 220,000 & 100,000 & 820,000 & 216,314 & $2,166,314$ \\
\hline 80 & 181.15 & 47.69 & 7200 & 13 & 47 & 25 & 118 & 2 & 2 & $1,860,000$ & 295,000 & 100,000 & 895,000 & 236,099 & $2,096,099$ \\
\hline 100 & 217.62 & 47.69 & 7200 & 11 & 47 & 25 & 147 & 2 & 2 & $1,800,000$ & 367,500 & 100,000 & 967,500 & 255,224 & $2,055,224$ \\
\hline 120 & 247.53 & 47.68 & 7200 & 9 & 47 & 25 & 176 & 2 & 2 & $1,740,000$ & 440,000 & 100,000 & $1,040,000$ & 274,349 & $2,014,349$ \\
\hline 20 & 56.65 & 35.94 & 7200 & 39 & 62 & 25 & 30 & 2 & 2 & $3,090,000$ & 75,000 & 100,000 & 675,000 & 178,063 & $3,268,063$ \\
\hline 40 & 95.38 & 33.85 & 7200 & 24 & 65 & 25 & 59 & 2 & 2 & $2,730,000$ & 147,500 & 100,000 & 747,500 & 197,189 & $2,927,189$ \\
\hline 60 & 139.51 & 32.63 & 7200 & 16 & 68 & 25 & 88 & 2 & 2 & $2,580,000$ & 220,000 & 100,000 & 820,000 & 216,314 & $2,796,314$ \\
\hline 80 & 181.15 & 31.76 & 7200 & 13 & 70 & 25 & 118 & 2 & 2 & $2,550,000$ & 295,000 & 100,000 & 895,000 & 236,099 & $2,786,099$ \\
\hline 100 & 217.62 & 31.16 & 7200 & 11 & 71 & 25 & 147 & 2 & 2 & $2,520,000$ & 367,500 & 100,000 & 967,500 & 255,224 & $2,775,224$ \\
\hline 120 & 247.53 & 30.62 & 7200 & 9 & 72 & 25 & 176 & 2 & 2 & $2,490,000$ & 440,000 & 100,000 & $1,040,000$ & 274,349 & $2,764,349$ \\
\hline
\end{tabular}

Table F.10: Manual System Prescriptive Model Calculations (Low Picking and Low Packing Levels).

\begin{tabular}{|c|c|c|c|c|c|c|c|c|c|c|c|c|}
\hline$D$ & $\bar{W}$ & $N_{o}$ & $p(n)$ & $q(m)$ & $r_{p}$ & $r_{q}$ & $N_{q}$ & $n_{q}$ & $r_{a}$ & $r_{a} c_{q}$ & $T_{\ell}^{m}$ & $T^{m}$ \\
\hline 2200 & 20 & 3 & 25.65 & 38.85 & 86 & 57 & 25 & 30 & 57 & 10,526 & $4,290,000$ & $4,300,526$ \\
\hline 2200 & 20 & 3 & 31.62 & 38.08 & 70 & 58 & 25 & 30 & 58 & 10,710 & $3,840,000$ & $3,850,710$ \\
\hline 2200 & 20 & 3 & 31.62 & 38.08 & 70 & 58 & 25 & 30 & 58 & 10,710 & $3,840,000$ & $3,850,710$ \\
\hline 2200 & 20 & 3 & 38.06 & 35.74 & 58 & 62 & 25 & 30 & 62 & 11,449 & $3,600,000$ & $3,611,449$ \\
\hline 2200 & 20 & 3 & 41.42 & 33.51 & 54 & 66 & 25 & 30 & 66 & 12,188 & $3,600,000$ & $3,612,188$ \\
\hline 2200 & 20 & 3 & 56.65 & 29.24 & 39 & 76 & 25 & 30 & 76 & 14,034 & $3,450,000$ & $3,464,034$ \\
\hline 2200 & 40 & 3 & 46.33 & 34.47 & 48 & 64 & 25 & 59 & 64 & 11,818 & $3,360,000$ & $3,371,818$ \\
\hline 2200 & 40 & 3 & 48.19 & 33.51 & 46 & 66 & 25 & 59 & 66 & 12,188 & $3,360,000$ & $3,372,188$ \\
\hline 2200 & 40 & 3 & 53.61 & 34.01 & 42 & 65 & 25 & 59 & 65 & 12,003 & $3,210,000$ & 3,222,003 \\
\hline 2200 & 40 & 3 & 57.22 & 31.18 & 39 & 71 & 25 & 59 & 71 & 13,111 & $3,300,000$ & $3,313,111$ \\
\hline 2200 & 40 & 3 & 69.76 & 29.23 & 32 & 76 & 25 & 59 & 76 & 14,034 & $3,240,000$ & $3,254,034$ \\
\hline 2200 & 40 & 3 & 95.38 & 24.93 & 24 & 89 & 25 & 59 & 89 & 16,435 & $3,390,000$ & $3,406,435$ \\
\hline 2200 & 60 & 3 & 60.48 & 32.74 & 37 & 68 & 25 & 88 & 88 & 16,250 & $3,150,000$ & $3,166,250$ \\
\hline 2200 & 60 & 3 & 63.26 & 31.18 & 35 & 71 & 25 & 88 & 88 & 16,250 & $3,180,000$ & $3,196,250$ \\
\hline 2200 & 60 & 3 & 72.86 & 29.24 & 31 & 76 & 25 & 88 & 88 & 16,250 & $3,210,000$ & $3,226,250$ \\
\hline 2200 & 60 & 3 & 79.26 & 28.99 & 28 & 76 & 25 & 88 & 88 & 16,250 & $3,120,000$ & $3,136,250$ \\
\hline 2200 & 60 & 3 & 97.94 & 25.46 & 23 & 87 & 25 & 88 & 88 & 16,250 & $3,300,000$ & $3,316,250$ \\
\hline 2200 & 60 & 3 & 139.51 & 21.49 & 16 & 103 & 25 & 88 & 103 & 19,020 & $3,570,000$ & $3,589,020$ \\
\hline 2200 & 80 & 3 & 74.54 & 30.52 & 30 & 73 & 25 & 118 & 118 & 21,790 & $3,090,000$ & $3,111,790$ \\
\hline 2200 & 80 & 3 & 80.29 & 29.87 & 28 & 74 & 25 & 118 & 118 & 21,790 & $3,060,000$ & $3,081,790$ \\
\hline 2200 & 80 & 3 & 89.65 & 28.02 & 25 & 79 & 25 & 118 & 118 & 21,790 & $3,120,000$ & $3,141,790$ \\
\hline 2200 & 80 & 3 & 102.60 & 26.46 & 22 & 84 & 25 & 118 & 118 & 21,790 & $3,180,000$ & $3,201,790$ \\
\hline 2200 & 80 & 3 & 126.49 & 23.70 & 18 & 93 & 25 & 118 & 118 & 21,790 & $3,330,000$ & $3,351,790$ \\
\hline 2200 & 80 & 3 & 181.15 & 18.87 & 13 & 117 & 25 & 118 & 118 & 21,790 & $3,900,000$ & $3,921,790$ \\
\hline 2200 & 100 & 3 & 90.82 & 28.46 & 25 & 78 & 25 & 147 & 147 & 27,145 & $3,090,000$ & $3,117,145$ \\
\hline 2200 & 100 & 3 & 98.57 & 28.03 & 23 & 79 & 25 & 147 & 147 & 27,145 & $3,060,000$ & $3,087,145$ \\
\hline 2200 & 100 & 3 & 109.48 & 26.46 & 21 & 84 & 25 & 147 & 147 & 27,145 & $3,150,000$ & $3,177,145$ \\
\hline 2200 & 100 & 3 & 126.39 & 24.54 & 18 & 90 & 25 & 147 & 147 & 27,145 & $3,240,000$ & $3,267,145$ \\
\hline 2200 & 100 & 3 & 158.13 & 21.20 & 14 & 104 & 25 & 147 & 147 & 27,145 & $3,540,000$ & $3,567,145$ \\
\hline 2200 & 100 & 3 & 217.62 & 17.03 & 11 & 130 & 25 & 147 & 147 & 27,145 & $4,230,000$ & $4,257,145$ \\
\hline 2200 & 120 & 3 & 105.81 & 26.75 & 21 & 83 & 25 & 176 & 176 & 32,500 & $3,120,000$ & $3,152,500$ \\
\hline 2200 & 120 & 3 & 115.12 & 25.98 & 20 & 85 & 25 & 176 & 176 & 32,500 & $3,150,000$ & $3,182,500$ \\
\hline 2200 & 120 & 3 & 130.76 & 24.77 & 17 & 89 & 25 & 176 & 176 & 32,500 & $3,180,000$ & $3,212,500$ \\
\hline 2200 & 120 & 3 & 153.45 & 22.83 & 15 & 97 & 25 & 176 & 176 & 32,500 & $3,360,000$ & $3,392,500$ \\
\hline 2200 & 120 & 3 & 189.39 & 19.98 & 12 & 111 & 25 & 176 & 176 & 32,500 & $3,690,000$ & $3,722,500$ \\
\hline 2200 & 120 & 3 & 247.53 & 16.11 & 9 & 137 & 25 & 176 & 176 & 32,500 & $4,380,000$ & $4,412,500$ \\
\hline
\end{tabular}


Table F.11: Throughput Simulation Picking Times (Overlapping Waves).

\begin{tabular}{|c|c|c|c|c|c|c|c|c|c|c|c|c|c|c|}
\hline 1 & 2 & 3 & 4 & 5 & 6 & 7 & 8 & 9 & 10 & 11 & 12 & 13 & 14 & 15 \\
\hline 114.29 & 39.54 & 59.08 & 39.36 & 64.70 & 73.74 & 108.52 & 93.83 & 59.87 & 57.92 & 114.25 & 77.08 & 54.16 & 90.83 & 57.61 \\
\hline 113.35 & 36.37 & 58.44 & 40.54 & 68.72 & 73.82 & 109.19 & 79.24 & 64.50 & 59.20 & 114.66 & 68.32 & 53.15 & 83.16 & 59.69 \\
\hline 114.06 & 37.63 & 59.74 & 40.79 & 55.93 & 73.89 & 109.81 & 78.90 & 57.14 & 58.46 & 114.99 & 52.87 & 54.16 & 76.42 & 64.33 \\
\hline 113.49 & 40.23 & 57.02 & 39.68 & 64.91 & 73.98 & 109.93 & 76.14 & 59.18 & 59.78 & 114.36 & 61.12 & 53.70 & 89.07 & 64.38 \\
\hline 113.46 & 39.56 & 59.18 & 39.64 & 60.27 & 74.15 & 109.40 & 76.70 & 63.03 & 60.38 & 114.25 & 54.59 & 53.42 & 91.25 & 55.69 \\
\hline 114.28 & 38.70 & 57.44 & 39.70 & 61.90 & 73.50 & 109.26 & 99.66 & 66.39 & 58.63 & 114.97 & 64.09 & 53.93 & 76.83 & 67.14 \\
\hline 113.65 & 36.37 & 59.25 & 40.63 & 70.53 & 74.44 & 109.38 & 73.79 & 61.38 & 59.71 & 115.01 & 64.54 & 54.35 & 67.53 & 67.65 \\
\hline 113.45 & 34.40 & 58.55 & 39.43 & 66.45 & 74.48 & 109.37 & 83.33 & 54.01 & 60.95 & 114.28 & 64.79 & 53.42 & 97.33 & 55.20 \\
\hline 114.20 & 38.19 & 57.04 & 40.04 & 62.74 & 74.40 & 109.66 & 85.51 & 60.81 & 57.92 & 114.89 & 59.90 & 53.89 & 84.97 & 65.73 \\
\hline 113.83 & 38.41 & 59.43 & 39.62 & 67.69 & 73.92 & 109.88 & 74.18 & 57.29 & 60.42 & 114.68 & 73.61 & 54.14 & 83.01 & 71.94 \\
\hline 113.46 & 38.30 & 58.88 & 39.65 & 71.87 & 74.16 & 109.71 & 99.69 & 59.00 & 57.86 & 114.35 & 70.86 & 53.93 & 77.85 & 59.42 \\
\hline 114.09 & 42.83 & 57.84 & 40.16 & 66.65 & 74.30 & 109.24 & 88.65 & 66.57 & 59.11 & 114.50 & 65.71 & 54.08 & 88.91 & 66.80 \\
\hline 114.13 & 42.81 & 57.13 & 38.75 & 61.99 & 73.18 & 109.82 & 72.69 & 64.94 & 58.70 & 114.23 & 58.64 & 54.20 & 95.84 & 80.73 \\
\hline 113.65 & 43.55 & 57.64 & 40.25 & 67.22 & 74.30 & 109.70 & 65.04 & 63.10 & 58.18 & 114.46 & 54.39 & 53.80 & 76.13 & 54.35 \\
\hline 113.76 & 39.43 & 58.86 & 39.62 & 63.95 & 74.05 & 109.80 & 80.26 & 68.71 & 59.75 & 114.94 & 60.67 & 54.17 & 73.65 & 50.23 \\
\hline 113.92 & 36.29 & 58.85 & 40.42 & 61.98 & 74.05 & 109.43 & 80.80 & 52.25 & 59.25 & 114.34 & 55.54 & 54.27 & 79.03 & 63.31 \\
\hline 113.45 & 41.00 & 58.29 & 39.42 & 57.57 & 73.60 & 109.58 & 82.87 & 57.06 & 58.39 & 114.40 & 51.77 & 53.61 & 85.42 & 65.87 \\
\hline 114.06 & 40.89 & 57.81 & 40.27 & 56.78 & 73.92 & 109.26 & 79.08 & 56.05 & 59.26 & 115.07 & 72.36 & 53.09 & 79.15 & 81.02 \\
\hline 113.91 & 40.34 & 58.44 & 39.47 & 57.82 & 74.23 & 109.10 & 68.00 & 58.43 & 59.16 & 115.13 & 69.88 & 53.81 & 76.06 & 61.96 \\
\hline 113.59 & 37.56 & 57.99 & 40.57 & 69.04 & 74.18 & 109.20 & 94.02 & 57.84 & 60.30 & 114.85 & 57.31 & 54.20 & 88.29 & 57.54 \\
\hline 113.96 & 37.59 & 57.50 & 39.88 & 51.55 & 73.75 & 109.29 & 86.24 & 54.48 & 58.02 & 114.95 & 66.10 & 53.56 & 70.72 & 57.37 \\
\hline 113.73 & 36.35 & 56.80 & 41.01 & 63.03 & 73.77 & 109.72 & 84.10 & 58.56 & 58.52 & 114.62 & 71.37 & 53.34 & 70.57 & 53.38 \\
\hline 114.24 & 38.19 & 56.52 & 40.81 & 70.13 & 73.76 & 109.50 & 83.94 & 61.02 & 60.85 & 114.87 & 53.13 & 52.82 & 63.38 & 58.26 \\
\hline 114.21 & 39.48 & 58.62 & 39.43 & 57.55 & 74.51 & 109.18 & 77.59 & 65.88 & 58.48 & 114.94 & 69.07 & 54.02 & 74.47 & 57.53 \\
\hline 114.16 & 37.47 & 58.35 & 38.64 & 54.05 & 73.99 & 108.81 & 89.71 & 62.04 & 60.96 & 114.81 & 65.84 & 54.00 & 93.69 & 49.17 \\
\hline 114.06 & 41.51 & 58.23 & 39.72 & 64.62 & 74.23 & 108.96 & 80.10 & 59.41 & 58.62 & 114.41 & 66.61 & 54.32 & 86.10 & 57.48 \\
\hline 113.44 & 42.84 & 58.50 & 39.27 & 53.46 & 73.96 & 109.71 & 75.35 & 55.29 & 60.80 & 115.04 & 63.03 & 53.43 & 85.12 & 70.52 \\
\hline 114.19 & 37.05 & 59.51 & 40.38 & 57.95 & 73.83 & 109.20 & 76.17 & 59.76 & 57.46 & 114.91 & 75.50 & 53.57 & 85.45 & 58.35 \\
\hline 114.40 & 39.02 & 59.55 & 39.02 & 65.59 & 74.11 & 109.96 & 87.94 & 63.36 & 60.31 & 114.17 & 74.98 & 53.71 & 88.82 & 59.90 \\
\hline 113.66 & 40.27 & 57.67 & 39.64 & 64.31 & 74.41 & 109.46 & 81.34 & 60.53 & 58.60 & 115.18 & 60.89 & 53.87 & 81.47 & 55.28 \\
\hline 114.24 & 39.59 & 56.32 & 39.83 & 66.89 & 73.56 & 109.65 & 70.23 & 63.52 & 56.77 & 114.85 & 65.00 & 53.56 & 75.41 & 61.39 \\
\hline 114.00 & 41.96 & 56.99 & 39.59 & 69.54 & 73.88 & 108.90 & 85.07 & 58.43 & 59.19 & 114.98 & 69.78 & 53.44 & 83.15 & 72.09 \\
\hline 114.16 & 39.63 & 59.37 & 39.19 & 67.14 & 73.99 & 109.46 & 72.59 & 57.20 & 57.83 & 114.50 & 63.11 & 53.12 & 68.92 & 57.36 \\
\hline 114.10 & 39.75 & 57.54 & 38.83 & 71.51 & 73.93 & 108.47 & 65.24 & 61.11 & 58.71 & 114.59 & 61.07 & 53.89 & 78.43 & 55.09 \\
\hline 114.23 & 39.48 & 57.81 & 39.91 & 79.04 & 74.32 & 109.09 & 89.73 & 61.86 & 59.31 & 114.14 & 69.19 & 53.69 & 85.61 & 62.58 \\
\hline 114.37 & 36.97 & 58.14 & 38.36 & 71.69 & 74.22 & 109.80 & 77.17 & 61.36 & 60.33 & 114.87 & 68.83 & 53.72 & 88.57 & 54.90 \\
\hline 114.13 & 42.07 & 58.77 & 39.09 & 60.61 & 74.33 & 109.30 & 88.22 & 63.70 & 57.47 & 114.32 & 55.89 & 54.39 & 88.97 & 68.16 \\
\hline 113.60 & 36.25 & 58.63 & 39.26 & 72.69 & 73.98 & 109.70 & 74.38 & 60.56 & 59.64 & 114.31 & 65.60 & 53.67 & 72.74 & 77.17 \\
\hline 114.05 & 41.61 & 57.50 & 40.36 & 59.21 & 74.23 & 109.44 & 72.14 & 58.47 & 59.86 & 114.78 & 52.97 & 52.73 & 73.61 & 53.92 \\
\hline 114.01 & 41.38 & 58.99 & 39.88 & 63.03 & 73.67 & 109.58 & 79.77 & 62.80 & 59.89 & 114.67 & 63.40 & 53.50 & 91.96 & 46.09 \\
\hline 113.56 & 42.27 & 57.47 & 38.92 & 72.72 & 73.99 & 109.61 & 68.30 & 59.05 & 59.92 & 114.55 & 57.55 & 53.79 & 83.90 & 52.98 \\
\hline 113.99 & 37.61 & 59.19 & 39.17 & 54.02 & 73.75 & 109.93 & 81.72 & 59.93 & 57.97 & 114.60 & 65.15 & 54.07 & 79.41 & 56.19 \\
\hline 114.25 & 39.56 & 58.44 & 39.20 & 67.36 & 74.35 & 109.83 & 85.08 & 55.29 & 58.10 & 114.56 & 59.15 & 53.15 & 88.55 & 66.71 \\
\hline 114.26 & 40.95 & 58.26 & 39.23 & 53.16 & 74.17 & 110.00 & 74.90 & 62.89 & 58.12 & 114.71 & 61.90 & 53.73 & 89.25 & 68.67 \\
\hline 113.43 & 37.45 & 58.77 & 39.44 & 65.42 & 73.82 & 108.75 & 101.28 & 60.98 & 60.37 & 114.40 & 58.29 & 54.03 & 76.07 & 66.78 \\
\hline 113.77 & 40.82 & 58.32 & 39.68 & 57.84 & 74.51 & 109.62 & 63.88 & 62.84 & 57.54 & 114.26 & 64.46 & 53.50 & 79.48 & 65.22 \\
\hline 113.87 & 41.49 & 58.20 & 40.95 & 60.57 & 73.55 & 109.39 & 88.04 & 57.37 & 59.03 & 114.61 & 60.16 & 53.49 & 91.26 & 72.87 \\
\hline 113.86 & 38.91 & 58.09 & 39.69 & 61.13 & 74.15 & 109.07 & 83.88 & 61.25 & 58.73 & 114.59 & 66.54 & 53.73 & 91.23 & 58.92 \\
\hline 113.68 & 40.11 & 58.30 & 39.47 & 62.84 & 74.52 & 109.84 & 80.97 & 64.31 & 58.76 & 114.38 & 57.67 & 53.81 & 76.49 & 69.55 \\
\hline 114.19 & 36.47 & 58.63 & 39.88 & 61.21 & 73.60 & 109.21 & 75.24 & 55.41 & 57.57 & 114.93 & 54.66 & 53.82 & 79.61 & 60.80 \\
\hline 113.73 & 38.87 & 58.73 & 39.16 & 74.28 & 74.06 & 109.27 & 77.50 & 63.10 & 59.91 & 114.95 & 68.04 & 53.71 & 67.88 & 71.20 \\
\hline 113.62 & 37.06 & 58.09 & 39.49 & 60.65 & 73.96 & 109.27 & 75.41 & 65.33 & 60.47 & 114.34 & 52.70 & 53.86 & 80.94 & 62.56 \\
\hline 114.28 & 41.55 & 59.20 & 39.94 & 53.75 & 74.04 & 109.28 & 89.63 & 62.62 & 57.37 & 114.36 & 69.79 & 54.06 & 68.16 & 60.27 \\
\hline 113.60 & 38.73 & 57.76 & 40.12 & 58.48 & 74.00 & 109.16 & 83.43 & 59.68 & 59.89 & 115.07 & 67.89 & 54.11 & 84.20 & 63.71 \\
\hline 114.17 & 40.31 & 58.21 & 40.16 & 55.37 & 74.25 & 110.01 & 83.17 & 63.19 & 56.29 & 114.29 & 66.99 & 53.48 & 71.50 & 69.02 \\
\hline 114.10 & 38.77 & 57.94 & 38.82 & 60.10 & 73.84 & 108.72 & 62.04 & 65.98 & 58.51 & 114.78 & 67.88 & 53.83 & 86.54 & 62.37 \\
\hline 113.54 & 41.00 & 58.88 & 39.90 & 51.85 & 73.82 & 109.86 & 86.11 & 57.43 & 61.61 & 114.48 & 55.34 & 53.61 & 82.11 & 67.62 \\
\hline 113.88 & 38.28 & 58.73 & 39.07 & 65.70 & 73.45 & 109.38 & 78.80 & 62.53 & 57.58 & 114.48 & 59.25 & 54.09 & 89.11 & 58.83 \\
\hline
\end{tabular}


Table F.11: Throughput Simulation Picking Times (Overlapping Waves) — continued.

\begin{tabular}{|c|c|c|c|c|c|c|c|c|c|c|c|c|c|c|}
\hline 1 & 2 & 3 & 4 & 5 & 6 & 7 & 8 & 9 & 10 & 11 & 12 & 13 & 14 & 15 \\
\hline 113.41 & 40.67 & 59.41 & 39.77 & 67.15 & 74.20 & 109.36 & 70.28 & 61.33 & 60.89 & 114.66 & 69.70 & 53.92 & 78.31 & 68.85 \\
\hline 114.11 & 41.58 & 59.38 & 39.95 & 61.28 & 74.11 & 109.60 & 80.52 & 60.93 & 61.01 & 114.77 & 69.47 & 54.56 & 73.64 & 64.22 \\
\hline 114.23 & 38.96 & 58.67 & 40.39 & 58.87 & 73.95 & 109.13 & 69.64 & 63.72 & 57.80 & 115.18 & 61.73 & 53.60 & 85.27 & 66.71 \\
\hline 113.92 & 40.79 & 58.55 & 40.29 & 68.26 & 74.35 & 109.25 & 93.35 & 60.46 & 61.57 & 114.65 & 58.05 & 53.64 & 89.05 & 67.96 \\
\hline 113.89 & 40.17 & 57.55 & 40.32 & 52.18 & 73.92 & 109.56 & 89.98 & 66.13 & 61.35 & 114.80 & 60.34 & 53.59 & 90.99 & 61.08 \\
\hline 113.63 & 39.55 & 58.96 & 40.12 & 73.67 & 74.26 & 108.69 & 95.55 & 62.94 & 59.37 & 114.90 & 64.62 & 54.48 & 71.46 & 61.15 \\
\hline 113.63 & 42.14 & 57.74 & 40.07 & 66.05 & 74.53 & 109.00 & 92.58 & 65.16 & 58.48 & 114.84 & 59.69 & 54.35 & 86.61 & 59.82 \\
\hline 114.25 & 36.89 & 58.53 & 40.11 & 57.93 & 73.91 & 109.67 & 89.87 & 59.30 & 57.89 & 114.94 & 71.43 & 53.23 & 88.54 & 62.83 \\
\hline 113.61 & 37.39 & 58.18 & 39.47 & 62.32 & 73.98 & 108.99 & 79.53 & 62.27 & 60.83 & 114.72 & 67.23 & 53.73 & 85.81 & 48.56 \\
\hline 114.20 & 38.29 & 58.24 & 39.48 & 54.07 & 73.57 & 109.81 & 82.68 & 64.15 & 57.95 & 115.07 & 58.36 & 53.95 & 72.09 & 47.90 \\
\hline 113.50 & 38.88 & 58.16 & 39.80 & 66.99 & 73.37 & 109.81 & 70.51 & 54.00 & 59.65 & 114.84 & 64.76 & 53.88 & 88.14 & 66.00 \\
\hline 113.93 & 39.59 & 59.54 & 39.33 & 59.79 & 73.96 & 109.31 & 80.16 & 57.43 & 57.99 & 114.89 & 50.43 & 54.29 & 79.23 & 65.50 \\
\hline 113.72 & 40.96 & 58.97 & 40.50 & 68.89 & 74.21 & 109.46 & 100.49 & 56.69 & 61.63 & 114.32 & 57.43 & 54.05 & 73.63 & 78.57 \\
\hline 113.37 & 41.53 & 56.82 & 39.91 & 70.77 & 73.52 & 109.66 & 93.84 & 58.89 & 59.24 & 114.28 & 52.61 & 53.73 & 88.23 & 69.45 \\
\hline 114.25 & 37.04 & 59.18 & 39.12 & 63.26 & 73.85 & 109.40 & 84.53 & 59.52 & 60.52 & 115.07 & 55.74 & 54.36 & 73.93 & 57.74 \\
\hline 114.39 & 36.76 & 57.08 & 40.11 & 63.87 & 73.73 & 108.70 & 87.93 & 61.60 & 58.48 & 114.60 & 65.56 & 52.80 & 88.34 & 59.62 \\
\hline 114.36 & 37.73 & 57.67 & 39.28 & 65.80 & 73.84 & 109.73 & 81.12 & 64.78 & 59.01 & 114.40 & 64.09 & 54.24 & 78.86 & 68.35 \\
\hline 113.45 & 40.93 & 58.74 & 40.48 & 72.79 & 73.63 & 109.80 & 79.62 & 62.15 & 61.56 & 114.67 & 66.74 & 54.07 & 78.75 & 54.25 \\
\hline 113.71 & 41.40 & 58.25 & 39.74 & 73.08 & 73.51 & 109.69 & 85.92 & 51.73 & 59.69 & 114.72 & 61.61 & 53.71 & 83.41 & 67.03 \\
\hline 113.46 & 40.76 & 57.69 & 39.92 & 68.97 & 73.93 & 109.77 & 90.09 & 61.09 & 59.16 & 114.97 & 65.69 & 53.69 & 91.25 & 59.19 \\
\hline 114.13 & 40.98 & 58.23 & 39.41 & 57.60 & 73.97 & 109.02 & 81.11 & 60.70 & 59.63 & 114.44 & 60.94 & 53.94 & 89.01 & 67.57 \\
\hline 113.42 & 38.96 & 58.97 & 38.89 & 63.26 & 74.10 & 109.62 & 79.08 & 56.91 & 59.87 & 114.25 & 55.36 & 53.48 & 75.67 & 64.31 \\
\hline 113.68 & 36.92 & 59.09 & 39.32 & 66.49 & 73.57 & 109.86 & 75.79 & 63.12 & 60.98 & 115.20 & 69.85 & 53.79 & 77.02 & 59.41 \\
\hline 113.59 & 42.09 & 60.28 & 39.66 & 66.24 & 74.50 & 109.94 & 96.81 & 55.19 & 62.03 & 114.80 & 64.89 & 53.62 & 81.22 & 62.03 \\
\hline 113.47 & 39.39 & 57.18 & 39.73 & 58.95 & 73.80 & 109.70 & 86.41 & 62.36 & 59.90 & 114.67 & 69.10 & 54.48 & 84.47 & 55.42 \\
\hline 113.70 & 35.22 & 58.74 & 40.32 & 65.82 & 74.28 & 109.32 & 68.75 & 61.53 & 59.64 & 114.44 & 70.35 & 53.60 & 75.22 & 74.60 \\
\hline 113.47 & 39.59 & 57.79 & 40.92 & 49.82 & 73.54 & 109.10 & 87.47 & 61.28 & 58.41 & 114.51 & 67.78 & 53.18 & 72.49 & 65.26 \\
\hline 114.12 & 42.16 & 59.51 & 39.53 & 66.93 & 74.17 & 109.37 & 63.92 & 62.39 & 58.71 & 115.02 & 71.18 & 54.02 & 92.71 & 73.98 \\
\hline 114.29 & 35.66 & 58.54 & 40.34 & 59.51 & 74.47 & 108.92 & 86.22 & 53.20 & 60.31 & 115.17 & 51.72 & 54.36 & 85.41 & 62.36 \\
\hline 113.47 & 35.49 & 57.22 & 40.52 & 67.84 & 73.81 & 109.84 & 82.81 & 58.82 & 60.23 & 115.06 & 59.12 & 54.06 & 81.88 & 74.73 \\
\hline 113.53 & 42.94 & 58.13 & 40.33 & 66.65 & 74.29 & 108.79 & 82.22 & 65.78 & 57.39 & 114.29 & 71.86 & 53.96 & 82.63 & 57.62 \\
\hline 113.32 & 38.24 & 57.47 & 39.63 & 50.36 & 73.72 & 108.84 & 81.28 & 57.38 & 59.14 & 114.78 & 64.04 & 53.55 & 68.87 & 53.28 \\
\hline 113.92 & 36.94 & 58.23 & 40.65 & 64.48 & 74.16 & 108.97 & 68.05 & 59.57 & 57.95 & 115.11 & 62.61 & 54.13 & 73.31 & 59.27 \\
\hline 113.97 & 40.27 & 58.25 & 40.30 & 58.96 & 73.64 & 109.13 & 79.09 & 60.14 & 61.44 & 114.82 & 64.21 & 54.12 & 76.35 & 62.99 \\
\hline 114.13 & 33.65 & 58.63 & 40.08 & 73.97 & 74.14 & 109.80 & 93.01 & 58.21 & 59.68 & 114.41 & 64.02 & 53.36 & 81.56 & 71.07 \\
\hline 114.22 & 38.18 & 59.27 & 40.03 & 62.66 & 73.93 & 108.95 & 80.64 & 59.59 & 59.84 & 114.25 & 71.43 & 53.32 & 96.95 & 65.58 \\
\hline 113.89 & 40.23 & 58.15 & 38.93 & 60.92 & 74.13 & 109.34 & 70.15 & 59.38 & 60.23 & 114.17 & 72.93 & 54.40 & 71.38 & 57.74 \\
\hline 113.89 & 40.17 & 58.83 & 39.71 & 70.84 & 74.27 & 109.79 & 71.33 & 56.92 & 58.15 & 114.98 & 63.62 & 53.80 & 75.93 & 65.13 \\
\hline 113.68 & 40.21 & 58.29 & 40.26 & 53.32 & 73.78 & 109.55 & 78.16 & 57.86 & 59.84 & 114.96 & 75.50 & 54.00 & 74.54 & 66.70 \\
\hline 114.04 & 42.64 & 58.92 & 40.63 & 64.46 & 73.91 & 109.87 & 67.34 & 61.13 & 60.28 & 115.00 & 50.52 & 53.79 & 71.40 & 58.07 \\
\hline 113.61 & 37.49 & 57.59 & 39.64 & 45.56 & 73.65 & 109.95 & 69.11 & 61.95 & 56.73 & 115.18 & 58.82 & 54.37 & 88.80 & 59.27 \\
\hline 113.76 & 41.61 & 58.69 & 40.56 & 55.56 & 73.75 & 109.51 & 72.02 & 59.13 & 58.74 & 114.83 & 68.37 & 54.06 & 70.26 & 60.62 \\
\hline
\end{tabular}

Table F.12: Throughput Simulation Packing Times (Overlapping Waves).

\begin{tabular}{rrrrrrrrrrrrrrrr}
\hline 1 & 2 & 3 & 4 & 5 & 6 & 7 & 8 & 9 & 10 & 11 & 12 & 13 & 14 & 15 \\
\hline 118.15 & 42.79 & 59.81 & 38.82 & Man. & 78.60 & 115.55 & Man. & Man. & 57.29 & 112.76 & Man. & 56.12 & Man. & Man. \\
118.10 & 39.83 & 60.44 & 38.69 & Man. & 79.14 & 114.83 & Man. & Man. & 58.25 & 110.78 & Man. & 56.92 & Man. & Man. \\
116.88 & 40.45 & 60.27 & 39.41 & Man. & 77.76 & 115.08 & Man. & Man. & 57.80 & 110.78 & Man. & 56.17 & Man. & Man. \\
119.03 & 40.87 & 60.00 & 38.97 & Man. & 76.25 & 116.41 & Man. & Man. & 58.15 & 112.03 & Man. & 55.57 & Man. & Man. \\
117.76 & 39.86 & 59.97 & 39.87 & Man. & 78.85 & 115.69 & Man. & Man. & 57.75 & 111.40 & Man. & 56.73 & Man. & Man. \\
118.81 & 40.04 & 59.81 & 38.97 & Man. & 76.57 & 117.73 & Man. & Man. & 58.09 & 112.23 & Man. & 56.03 & Man. & Man. \\
117.62 & 41.47 & 60.14 & 38.53 & Man. & 77.18 & 117.39 & Man. & Man. & 58.24 & 110.43 & Man. & 55.92 & Man. & Man. \\
116.49 & 39.31 & 60.50 & 39.34 & Man. & 80.28 & 116.28 & Man. & Man. & 56.78 & 111.82 & Man. & 56.26 & Man. & Man. \\
117.27 & 39.94 & 60.85 & 39.28 & Man. & 79.58 & 117.56 & Man. & Man. & 57.07 & 111.09 & Man. & 56.08 & Man. & Man. \\
117.96 & 39.94 & 59.71 & 39.73 & Man. & 78.23 & 115.96 & Man. & Man. & 58.85 & 111.93 & Man. & 56.34 & Man. & Man. \\
117.32 & 39.87 & 59.93 & 39.12 & Man. & 79.24 & 117.03 & Man. & Man. & 57.77 & 112.22 & Man. & 56.53 & Man. & Man. \\
119.11 & 40.73 & 59.76 & 39.50 & Man. & 77.42 & 114.79 & Man. & Man. & 57.85 & 112.86 & Man. & 57.56 & Man. & Man. \\
\hline
\end{tabular}


Table F.12: Throughput Simulation Packing Times (Overlapping Waves) - continued.

\begin{tabular}{|c|c|c|c|c|c|c|c|c|c|c|c|c|c|c|}
\hline 1 & 2 & 3 & 4 & 5 & 6 & 7 & 8 & 9 & 10 & 11 & 12 & 13 & 14 & 15 \\
\hline 117.82 & 39.71 & 60.25 & 39.55 & Man. & 78.17 & 117.48 & Man. & Man. & 58.52 & 112.54 & Man. & 55.46 & Man. & Man. \\
\hline 117.38 & 40.65 & 59.89 & 39.42 & Man. & 75.74 & 115.47 & Man. & Man. & 59.08 & 112.48 & Man. & 55.08 & Man. & Man. \\
\hline 118.57 & 40.79 & 60.51 & 39.78 & Man. & 78.56 & 115.76 & Man. & Man. & 59.35 & 113.94 & Man. & 56.62 & Man. & Man. \\
\hline 117.51 & 39.43 & 59.85 & 39.45 & Man. & 78.62 & 115.76 & Man. & Man. & 59.48 & 112.33 & Man. & 56.39 & Man. & Man. \\
\hline 117.91 & 40.54 & 60.15 & 38.78 & Man. & 78.86 & 114.35 & Man. & Man. & 58.41 & 112.07 & Man. & 55.67 & Man. & Man. \\
\hline 118.20 & 40.53 & 60.38 & 39.05 & Man. & 79.14 & 117.16 & Man. & Man. & 57.47 & 112.15 & Man. & 55.50 & Man. & Man. \\
\hline 117.49 & 39.34 & 60.11 & 39.66 & Man. & 77.91 & 115.72 & Man. & Man. & 59.64 & 111.80 & Man. & 56.68 & Man. & Man. \\
\hline 116.33 & 40.01 & 60.15 & 39.13 & Man. & 78.20 & 116.12 & Man. & Man. & 57.56 & 110.96 & Man. & 55.54 & Man. & Man. \\
\hline 116.91 & 41.05 & 59.95 & 39.36 & Man. & 77.10 & 112.64 & Man. & Man. & 56.26 & 110.32 & Man. & 55.13 & Man. & Man. \\
\hline 118.48 & 39.30 & 60.43 & 39.48 & Man. & 77.90 & 115.10 & Man. & Man. & 55.67 & 112.85 & Man. & 56.12 & Man. & Man. \\
\hline 115.90 & 40.66 & 60.03 & 39.38 & Man. & 77.17 & 117.78 & Man. & Man. & 57.32 & 112.66 & Man. & 56.42 & Man. & Man. \\
\hline 117.55 & 39.42 & 59.69 & 38.99 & Man. & 77.60 & 115.46 & Man. & Man. & 56.77 & 112.84 & Man. & 56.69 & Man. & Man. \\
\hline 116.51 & 40.66 & 59.91 & 39.73 & Man. & 78.73 & 116.16 & Man. & Man. & 58.29 & 113.15 & Man. & 55.00 & Man. & Man. \\
\hline 118.88 & 40.04 & 60.14 & 39.10 & Man. & 76.41 & 118.01 & Man. & Man. & 57.04 & 110.89 & Man. & 56.08 & Man. & Man. \\
\hline 118.98 & 40.47 & 60.40 & 38.50 & Man. & 79.22 & 115.21 & Man. & Man. & 58.82 & 112.09 & Man. & 55.65 & Man. & Man. \\
\hline 117.36 & 40.52 & 60.07 & 39.69 & Man. & 76.52 & 118.89 & Man. & Man. & 56.99 & 112.27 & Man. & 55.97 & Man. & Man. \\
\hline 117.13 & 39.65 & 60.14 & 39.49 & Man. & 78.59 & 113.11 & Man. & Man. & 57.32 & 111.56 & Man. & 55.64 & Man. & Man. \\
\hline 116.86 & 39.78 & 59.86 & 39.09 & Man. & 77.21 & 119.92 & Man. & Man. & 57.76 & 111.04 & Man. & 55.93 & Man. & Man. \\
\hline 117.17 & 39.14 & 60.32 & 39.43 & Man. & 78.24 & 113.07 & Man. & Man. & 59.37 & 112.07 & Man. & 55.87 & Man. & Man. \\
\hline 118.03 & 39.15 & 59.93 & 39.28 & Man. & 78.35 & 114.63 & Man. & Man. & 57.12 & 111.20 & Man. & 55.23 & Man. & Man. \\
\hline 116.36 & 39.75 & 60.05 & 38.61 & Man. & 77.40 & 113.82 & Man. & Man. & 57.54 & 110.19 & Man. & 55.75 & Man. & Man. \\
\hline 116.92 & 41.06 & 60.38 & 39.42 & Man. & 78.70 & 118.19 & Man. & Man. & 56.80 & 111.42 & Man. & 56.63 & Man. & Man. \\
\hline 117.76 & 41.76 & 60.18 & 38.54 & Man. & 78.31 & 115.37 & Man. & Man. & 57.92 & 110.37 & Man. & 55.44 & Man. & Man. \\
\hline 116.55 & 41.19 & 60.25 & 39.23 & Man. & 78.54 & 115.85 & Man. & Man. & 59.23 & 112.05 & Man. & 55.52 & Man. & Man. \\
\hline 115.90 & 40.18 & 60.31 & 38.98 & Man. & 81.15 & 115.82 & Man. & Man. & 58.61 & 111.85 & Man. & 56.15 & Man. & Man. \\
\hline 118.31 & 40.00 & 60.04 & 39.73 & Man. & 76.65 & 113.68 & Man. & Man. & 57.78 & 111.75 & Man. & 56.05 & Man. & Man. \\
\hline 116.96 & 40.69 & 59.98 & 39.39 & Man. & 78.43 & 114.44 & Man. & Man. & 59.55 & 113.51 & Man. & 56.15 & Man. & Man. \\
\hline 117.83 & 41.14 & 60.16 & 38.39 & Man. & 80.36 & 116.21 & Man. & Man. & 57.61 & 111.12 & Man. & 55.91 & Man. & Man. \\
\hline 115.34 & 39.70 & 59.97 & 39.50 & Man. & 79.77 & 116.06 & Man. & Man. & 57.20 & 111.20 & Man. & 56.05 & Man. & Man. \\
\hline 118.04 & 39.84 & 60.14 & 38.98 & Man. & 81.14 & 115.22 & Man. & Man. & 59.20 & 109.50 & Man. & 56.91 & Man. & Man. \\
\hline 117.25 & 40.12 & 59.99 & 38.43 & Man. & 79.91 & 114.62 & Man. & Man. & 58.08 & 112.92 & Man. & 56.97 & Man. & Man. \\
\hline 117.68 & 39.14 & 59.97 & 39.26 & Man. & 77.96 & 116.77 & Man. & Man. & 59.18 & 112.51 & Man. & 56.41 & Man. & Man. \\
\hline 116.10 & 40.22 & 59.71 & 39.07 & Man. & 76.71 & 112.96 & Man. & Man. & 60.88 & 110.67 & Man. & 56.92 & Man. & Man. \\
\hline 116.76 & 40.54 & 60.13 & 39.20 & Man. & 76.35 & 115.34 & Man. & Man. & 57.54 & 112.31 & Man. & 56.33 & Man. & Man. \\
\hline 115.38 & 41.67 & 60.26 & 38.69 & Man. & 79.63 & 116.12 & Man. & Man. & 59.69 & 112.07 & Man. & 56.04 & Man. & Man. \\
\hline 117.81 & 38.57 & 60.15 & 39.09 & Man. & 77.89 & 115.02 & Man. & Man. & 56.79 & 111.02 & Man. & 56.70 & Man. & Man. \\
\hline 116.67 & 39.79 & 59.87 & 39.92 & Man. & 79.38 & 115.20 & Man. & Man. & 56.38 & 111.78 & Man. & 55.65 & Man. & Man. \\
\hline 116.68 & 38.79 & 60.41 & 39.35 & Man. & 78.70 & 115.70 & Man. & Man. & 58.74 & 113.12 & Man. & 56.88 & Man. & Man. \\
\hline 116.28 & 39.37 & 60.19 & 39.35 & Man. & 77.19 & 116.69 & Man. & Man. & 58.37 & 112.29 & Man. & 54.83 & Man. & Man. \\
\hline 116.69 & 40.61 & 59.61 & 39.30 & Man. & 80.43 & 117.23 & Man. & Man. & 59.38 & 111.55 & Man. & 56.17 & Man. & Man. \\
\hline 117.58 & 40.23 & 60.25 & 39.62 & Man. & 78.00 & 116.21 & Man. & Man. & 57.20 & 113.04 & Man. & 55.54 & Man. & Man. \\
\hline 117.23 & 41.63 & 60.26 & 39.38 & Man. & 78.46 & 115.34 & Man. & Man. & 59.08 & 111.22 & Man. & 56.05 & Man. & Man. \\
\hline 117.20 & 39.84 & 59.53 & 38.88 & Man. & 79.41 & 115.53 & Man. & Man. & 56.72 & 112.06 & Man. & 55.66 & Man. & Man. \\
\hline 116.55 & 39.29 & 60.22 & 38.27 & Man. & 77.34 & 115.54 & Man. & Man. & 57.55 & 111.77 & Man. & 56.74 & Man. & Man. \\
\hline 117.37 & 41.18 & 59.73 & 40.13 & Man. & 79.27 & 113.96 & Man. & Man. & 58.17 & 111.27 & Man. & 57.51 & Man. & Man. \\
\hline 117.53 & 39.47 & 60.28 & 39.30 & Man. & 77.09 & 113.58 & Man. & Man. & 57.87 & 111.78 & Man. & 56.58 & Man. & Man. \\
\hline 118.60 & 39.74 & 59.87 & 38.43 & Man. & 78.20 & 115.38 & Man. & Man. & 58.17 & 112.45 & Man. & 56.61 & Man. & Man. \\
\hline 117.79 & 40.03 & 60.13 & 40.03 & Man. & 79.43 & 117.79 & Man. & Man. & 58.93 & 112.88 & Man. & 55.99 & Man. & Man. \\
\hline 116.51 & 40.12 & 60.07 & 39.03 & Man. & 77.94 & 113.99 & Man. & Man. & 57.77 & 112.69 & Man. & 55.64 & Man. & Man. \\
\hline 118.97 & 40.67 & 60.10 & 39.47 & Man. & 79.58 & 119.32 & Man. & Man. & 57.59 & 111.66 & Man. & 56.47 & Man. & Man. \\
\hline 116.59 & 39.39 & 60.32 & 39.04 & Man. & 77.84 & 115.51 & Man. & Man. & 57.55 & 111.58 & Man. & 55.84 & Man. & Man. \\
\hline 116.28 & 40.27 & 59.88 & 39.26 & Man. & 78.60 & 118.14 & Man. & Man. & 58.02 & 111.76 & Man. & 57.10 & Man. & Man. \\
\hline 117.48 & 41.48 & 60.20 & 37.97 & Man. & 78.31 & 116.66 & Man. & Man. & 58.47 & 111.05 & Man. & 56.09 & Man. & Man. \\
\hline 118.45 & 39.60 & 60.14 & 38.76 & Man. & 77.10 & 115.91 & Man. & Man. & 58.36 & 112.58 & Man. & 54.96 & Man. & Man. \\
\hline 116.60 & 39.84 & 59.99 & 39.48 & Man. & 76.64 & 115.29 & Man. & Man. & 59.53 & 111.48 & Man. & 56.07 & Man. & Man. \\
\hline 117.59 & 39.96 & 60.14 & 38.89 & Man. & 78.13 & 115.84 & Man. & Man. & 56.82 & 111.88 & Man. & 54.88 & Man. & Man. \\
\hline 118.88 & 39.68 & 60.60 & 39.24 & Man. & 77.50 & 114.21 & Man. & Man. & 58.80 & 112.11 & Man. & 54.88 & Man. & Man. \\
\hline 118.52 & 39.71 & 60.26 & 39.07 & Man. & 79.06 & 117.40 & Man. & Man. & 57.28 & 111.94 & Man. & 56.03 & Man. & Man. \\
\hline 118.00 & 41.40 & 60.49 & 38.91 & Man. & 78.41 & 115.62 & Man. & Man. & 57.96 & 112.16 & Man. & 56.21 & Man. & Man. \\
\hline
\end{tabular}


Table F.12: Throughput Simulation Packing Times (Overlapping Waves) — continued.

\begin{tabular}{|c|c|c|c|c|c|c|c|c|c|c|c|c|c|c|}
\hline 1 & 2 & 3 & 4 & 5 & 6 & 7 & 8 & 9 & 10 & 11 & 12 & 13 & 14 & 15 \\
\hline 117.18 & 39.67 & 59.76 & 39.87 & Man. & 76.45 & 115.87 & Man. & Man. & 58.92 & 112.44 & Man. & 55.55 & Man. & Man. \\
\hline 119.08 & 40.18 & 59.78 & 39.00 & Man. & 78.15 & 116.00 & Man. & Man. & 57.41 & 111.39 & Man. & 56.34 & Man. & Man. \\
\hline 114.97 & 41.06 & 60.01 & 39.37 & Man. & 77.01 & 118.04 & Man. & Man. & 58.18 & 112.34 & Man. & 54.76 & Man. & Man. \\
\hline 118.66 & 41.22 & 60.05 & 38.78 & Man. & 77.35 & 117.07 & Man. & Man. & 58.06 & 112.04 & Man. & 55.29 & Man. & Man. \\
\hline 116.82 & 40.14 & 59.88 & 39.65 & Man. & 79.74 & 118.26 & Man. & Man. & 57.35 & 112.30 & Man. & 57.18 & Man. & Man. \\
\hline 116.03 & 39.59 & 60.45 & 38.51 & Man. & 77.56 & 117.04 & Man. & Man. & 57.91 & 110.85 & Man. & 56.63 & Man. & Man. \\
\hline 119.51 & 39.94 & 60.13 & 39.04 & Man. & 73.43 & 115.41 & Man. & Man. & 57.71 & 111.24 & Man. & 54.05 & Man. & Man. \\
\hline 116.95 & 40.35 & 60.10 & 39.11 & Man. & 78.35 & 114.31 & Man. & Man. & 56.98 & 110.86 & Man. & 55.21 & Man. & Man. \\
\hline 117.92 & 39.80 & 60.04 & 39.08 & Man. & 78.92 & 115.16 & Man. & Man. & 57.45 & 111.36 & Man. & 57.28 & Man. & Man. \\
\hline 117.70 & 40.69 & 60.15 & 39.26 & Man. & 75.54 & 115.30 & Man. & Man. & 58.19 & 113.41 & Man. & 56.43 & Man. & Man. \\
\hline 115.88 & 41.37 & 60.29 & 38.93 & Man. & 78.44 & 115.45 & Man. & Man. & 55.85 & 112.49 & Man. & 55.91 & Man. & Man. \\
\hline 116.45 & 40.81 & 59.82 & 38.67 & Man. & 76.58 & 117.70 & Man. & Man. & 57.79 & 110.28 & Man. & 56.40 & Man. & Man. \\
\hline 117.07 & 41.43 & 60.20 & 39.20 & Man. & 75.96 & 114.21 & Man. & Man. & 58.47 & 112.62 & Man. & 54.96 & Man. & Man. \\
\hline 117.40 & 40.48 & 60.18 & 38.59 & Man. & 75.82 & 115.65 & Man. & Man. & 56.49 & 109.85 & Man. & 56.59 & Man. & Man. \\
\hline 116.53 & 39.72 & 59.88 & 39.82 & Man. & 78.26 & 118.64 & Man. & Man. & 59.22 & 109.93 & Man. & 56.10 & Man. & Man. \\
\hline 116.90 & 42.15 & 60.05 & 39.28 & Man. & 78.55 & 113.73 & Man. & Man. & 57.75 & 113.01 & Man. & 54.83 & Man. & Man. \\
\hline 116.39 & 41.12 & 59.84 & 39.49 & Man. & 78.18 & 115.59 & Man. & Man. & 56.66 & 111.55 & Man. & 55.67 & Man. & Man. \\
\hline 118.80 & 39.30 & 59.93 & 39.29 & Man. & 77.70 & 117.75 & Man. & Man. & 56.98 & 111.93 & Man. & 56.71 & Man. & Man. \\
\hline 118.09 & 40.23 & 59.94 & 38.49 & Man. & 78.24 & 116.95 & Man. & Man. & 56.85 & 110.96 & Man. & 55.86 & Man. & Man. \\
\hline 118.25 & 39.98 & 60.04 & 39.69 & Man. & 78.95 & 115.19 & Man. & Man. & 59.07 & 112.98 & Man. & 56.57 & Man. & Man. \\
\hline 117.92 & 40.39 & 60.12 & 40.30 & Man. & 77.78 & 115.89 & Man. & Man. & 55.16 & 111.45 & Man. & 57.16 & Man. & Man. \\
\hline 119.00 & 39.35 & 60.09 & 39.30 & Man. & 78.16 & 117.45 & Man. & Man. & 57.58 & 110.92 & Man. & 55.10 & Man. & Man. \\
\hline 116.71 & 40.58 & 60.24 & 38.82 & Man. & 80.53 & 115.59 & Man. & Man. & 59.26 & 111.79 & Man. & 56.13 & Man. & Man. \\
\hline 119.00 & 40.58 & 59.51 & 39.27 & Man. & 76.44 & 115.99 & Man. & Man. & 59.91 & 112.89 & Man. & 55.33 & Man. & Man. \\
\hline 116.80 & 41.74 & 60.01 & 39.28 & Man. & 76.89 & 116.16 & Man. & Man. & 58.81 & 112.55 & Man. & 56.21 & Man. & Man. \\
\hline 116.31 & 41.25 & 59.96 & 39.09 & Man. & 78.75 & 115.66 & Man. & Man. & 58.27 & 112.43 & Man. & 56.07 & Man. & Man. \\
\hline 118.98 & 39.98 & 60.16 & 39.76 & Man. & 75.86 & 114.43 & Man. & Man. & 56.47 & 111.52 & Man. & 57.32 & Man. & Man. \\
\hline 118.12 & 39.46 & 59.86 & 38.78 & Man. & 78.27 & 115.88 & Man. & Man. & 58.90 & 110.19 & Man. & 56.71 & Man. & Man. \\
\hline 117.41 & 40.21 & 60.33 & 39.55 & Man. & 76.38 & 114.78 & Man. & Man. & 57.32 & 111.94 & Man. & 56.09 & Man. & Man. \\
\hline
\end{tabular}

Table F.13: Throughput Simulation Picking Times (Non-Overlapping Waves).

\begin{tabular}{|c|c|c|c|c|c|c|c|c|c|c|c|c|c|c|}
\hline 1 & 2 & 3 & 4 & 5 & 6 & 7 & 8 & 9 & 10 & 11 & 12 & 13 & 14 & 15 \\
\hline 39.34 & 113.02 & 71.11 & 19.83 & 80.21 & 37.44 & 108.52 & 55.84 & 63.54 & 102.35 & 18.31 & 61.78 & 21.43 & 72.38 & 61.15 \\
\hline 39.63 & 95.28 & 51.41 & 19.74 & 67.08 & 37.90 & 109.19 & 89.71 & 59.08 & 96.07 & 19.44 & 57.51 & 19.22 & 83.52 & 56.97 \\
\hline 40.20 & 104.01 & 60.27 & 20.20 & 66.89 & 37.42 & 109.81 & 95.75 & 58.78 & 107.54 & 18.81 & 58.28 & 19.63 & 70.57 & 47.31 \\
\hline 39.77 & 91.59 & 68.30 & 21.13 & 60.18 & 36.64 & 109.93 & 64.71 & 61.20 & 89.49 & 18.88 & 63.84 & 20.74 & 83.09 & 63.14 \\
\hline 39.23 & 111.03 & 55.54 & 20.20 & 80.23 & 37.36 & 109.40 & 70.34 & 56.84 & 88.65 & 19.50 & 60.22 & 19.55 & 83.35 & 60.97 \\
\hline 39.32 & 101.56 & 55.07 & 20.42 & 47.12 & 38.40 & 109.26 & 90.52 & 56.18 & 110.97 & 18.99 & 54.76 & 19.28 & 93.99 & 63.75 \\
\hline 39.62 & 107.43 & 72.15 & 19.13 & 66.96 & 37.50 & 109.38 & 64.94 & 60.01 & 107.58 & 19.53 & 48.06 & 19.03 & 74.19 & 61.75 \\
\hline 39.09 & 114.16 & 63.14 & 20.21 & 61.52 & 37.93 & 109.37 & 80.34 & 58.41 & 115.81 & 19.18 & 59.34 & 19.85 & 83.73 & 62.46 \\
\hline 39.49 & 107.19 & 65.11 & 20.49 & 70.45 & 37.94 & 109.66 & 69.42 & 68.98 & 102.05 & 19.85 & 52.74 & 18.94 & 81.11 & 52.56 \\
\hline 39.88 & 101.69 & 59.47 & 18.44 & 62.02 & 37.27 & 109.88 & 78.29 & 59.50 & 116.42 & 20.80 & 67.05 & 19.15 & 70.00 & 65.71 \\
\hline 40.10 & 106.53 & 65.74 & 20.24 & 69.06 & 38.35 & 109.71 & 60.86 & 59.64 & 106.54 & 18.83 & 56.67 & 20.05 & 81.19 & 59.15 \\
\hline 39.57 & 92.47 & 49.88 & 19.03 & 62.20 & 37.33 & 109.24 & 84.38 & 65.67 & 92.97 & 20.33 & 56.26 & 19.17 & 77.57 & 76.41 \\
\hline 39.76 & 93.79 & 62.78 & 20.14 & 67.41 & 37.26 & 109.82 & 76.21 & 60.06 & 103.48 & 17.29 & 68.56 & 20.05 & 63.50 & 60.10 \\
\hline 39.44 & 92.20 & 59.94 & 20.73 & 58.54 & 37.74 & 109.70 & 82.51 & 60.12 & 108.52 & 20.39 & 70.81 & 19.49 & 74.67 & 54.95 \\
\hline 40.44 & 90.29 & 64.48 & 19.46 & 63.28 & 37.36 & 109.80 & 76.32 & 56.33 & 99.08 & 18.75 & 65.96 & 18.42 & 70.47 & 58.57 \\
\hline 39.44 & 109.66 & 63.75 & 20.22 & 65.07 & 38.19 & 109.43 & 90.55 & 52.12 & 97.66 & 20.34 & 62.65 & 21.00 & 88.02 & 56.29 \\
\hline 39.85 & 98.53 & 67.40 & 19.72 & 52.72 & 37.09 & 109.58 & 82.55 & 60.93 & 103.95 & 18.76 & 63.85 & 19.75 & 86.41 & 51.19 \\
\hline 39.07 & 109.52 & 66.86 & 20.02 & 71.24 & 36.93 & 109.26 & 79.77 & 59.03 & 110.90 & 19.45 & 70.37 & 19.92 & 80.75 & 68.41 \\
\hline 40.27 & 91.53 & 75.81 & 18.24 & 59.62 & 37.67 & 109.10 & 80.42 & 62.43 & 103.16 & 20.75 & 79.60 & 18.52 & 88.37 & 51.85 \\
\hline 39.38 & 113.73 & 59.75 & 19.10 & 74.72 & 37.81 & 109.20 & 70.48 & 60.10 & 87.06 & 19.05 & 64.32 & 21.10 & 87.12 & 67.67 \\
\hline 38.48 & 95.81 & 67.14 & 18.56 & 61.14 & 38.56 & 109.29 & 83.54 & 59.82 & 109.76 & 19.69 & 61.31 & 18.93 & 63.06 & 65.64 \\
\hline 39.39 & 100.39 & 57.10 & 21.26 & 49.17 & 39.18 & 109.72 & 79.50 & 58.51 & 107.74 & 19.41 & 64.18 & 19.06 & 87.75 & 48.26 \\
\hline 40.44 & 99.45 & 70.93 & 20.22 & 63.93 & 36.29 & 109.50 & 66.41 & 59.75 & 90.48 & 20.78 & 61.02 & 21.04 & 80.88 & 64.23 \\
\hline 39.21 & 95.30 & 66.63 & 19.93 & 58.85 & 38.16 & 109.18 & 92.74 & 58.29 & 104.22 & 18.01 & 67.98 & 20.45 & 77.77 & 70.59 \\
\hline 40.34 & 94.16 & 67.83 & 19.62 & 68.82 & 38.15 & 108.81 & 54.63 & 52.88 & 99.21 & 19.46 & 60.16 & 19.51 & 74.36 & 51.51 \\
\hline
\end{tabular}


Table F.13: Throughput Simulation Picking Times (Non-Overlapping Waves) — continued.

\begin{tabular}{|c|c|c|c|c|c|c|c|c|c|c|c|c|c|c|}
\hline 1 & 2 & 3 & 4 & 5 & 6 & 7 & 8 & 9 & 10 & 11 & 12 & 13 & 14 & 15 \\
\hline 39.80 & 93.33 & 70.49 & 20.19 & 58.51 & 37.39 & 108.96 & 90.46 & 60.25 & 101.23 & 19.82 & 70.59 & 18.80 & 80.67 & 58.18 \\
\hline 39.89 & 104.93 & 58.51 & 20.38 & 62.69 & 37.00 & 109.71 & 82.78 & 61.77 & 101.17 & 18.59 & 59.33 & 18.05 & 68.11 & 55.74 \\
\hline 39.08 & 121.92 & 57.63 & 19.36 & 76.93 & 37.88 & 109.20 & 86.46 & 60.46 & 104.90 & 21.88 & 61.02 & 19.76 & 79.88 & 55.76 \\
\hline 39.48 & 94.15 & 59.16 & 18.30 & 59.35 & 37.57 & 109.96 & 75.30 & 63.14 & 97.53 & 18.92 & 59.49 & 19.99 & 86.00 & 69.14 \\
\hline 40.07 & 98.92 & 69.41 & 20.82 & 59.95 & 37.46 & 109.46 & 82.37 & 60.22 & 104.74 & 19.85 & 61.64 & 19.65 & 79.22 & 62.33 \\
\hline 38.73 & 99.74 & 65.92 & 20.87 & 47.39 & 38.16 & 109.65 & 83.56 & 55.94 & 100.40 & 19.34 & 65.13 & 19.50 & 76.22 & 58.36 \\
\hline 40.55 & 97.55 & 66.85 & 19.61 & 58.50 & 37.45 & 108.90 & 86.33 & 60.09 & 98.43 & 19.71 & 62.81 & 20.15 & 81.94 & 51.56 \\
\hline 39.44 & 100.01 & 70.91 & 20.42 & 70.69 & 38.66 & 109.46 & 78.08 & 58.64 & 96.18 & 22.20 & 66.88 & 19.48 & 90.92 & 54.88 \\
\hline 39.91 & 98.21 & 58.35 & 21.19 & 68.48 & 37.55 & 108.47 & 80.42 & 59.59 & 89.16 & 20.34 & 56.21 & 19.00 & 86.25 & 62.95 \\
\hline 39.84 & 96.45 & 71.17 & 21.10 & 73.19 & 38.51 & 109.09 & 63.94 & 55.33 & 102.02 & 18.77 & 55.85 & 21.22 & 74.39 & 65.78 \\
\hline 39.21 & 107.65 & 73.10 & 20.16 & 70.25 & 39.24 & 109.80 & 78.69 & 57.09 & 104.42 & 18.40 & 66.54 & 19.79 & 72.37 & 59.49 \\
\hline 40.24 & 101.89 & 62.47 & 19.21 & 52.88 & 37.73 & 109.30 & 80.74 & 50.41 & 82.16 & 20.33 & 55.79 & 20.04 & 85.86 & 64.86 \\
\hline 40.66 & 110.27 & 54.54 & 18.01 & 60.70 & 37.51 & 109.70 & 75.94 & 58.65 & 97.77 & 18.98 & 67.75 & 19.60 & 77.14 & 59.71 \\
\hline 39.54 & 93.34 & 62.28 & 20.80 & 63.21 & 36.35 & 109.44 & 73.76 & 55.91 & 113.69 & 20.04 & 72.14 & 20.69 & 83.66 & 63.45 \\
\hline 39.28 & 90.62 & 66.65 & 20.17 & 63.73 & 37.63 & 109.58 & 83.56 & 57.21 & 110.25 & 19.49 & 56.45 & 19.27 & 73.09 & 58.74 \\
\hline 39.52 & 88.68 & 60.40 & 19.95 & 62.35 & 38.07 & 109.61 & 88.99 & 60.55 & 99.14 & 18.64 & 61.21 & 19.17 & 86.49 & 56.62 \\
\hline 39.19 & 112.75 & 57.18 & 20.36 & 66.52 & 38.34 & 109.93 & 78.14 & 63.01 & 89.18 & 20.14 & 61.42 & 22.04 & 76.15 & 73.30 \\
\hline 40.14 & 104.66 & 65.88 & 19.67 & 64.66 & 37.85 & 109.83 & 84.94 & 57.97 & 103.35 & 20.59 & 51.59 & 21.02 & 82.52 & 76.46 \\
\hline 39.89 & 107.50 & 56.97 & 20.33 & 60.45 & 36.43 & 110.00 & 80.83 & 63.57 & 111.38 & 18.82 & 66.11 & 18.24 & 82.25 & 58.83 \\
\hline 40.16 & 103.42 & 76.44 & 20.41 & 64.80 & 36.81 & 108.75 & 90.39 & 59.84 & 103.86 & 18.68 & 55.65 & 18.34 & 94.39 & 74.49 \\
\hline 39.75 & 98.74 & 69.37 & 19.46 & 65.11 & 37.53 & 109.62 & 87.20 & 64.19 & 100.85 & 19.30 & 55.38 & 19.20 & 69.71 & 56.83 \\
\hline 38.46 & 91.82 & 49.37 & 20.84 & 56.06 & 37.33 & 109.39 & 71.15 & 56.39 & 97.57 & 19.98 & 56.38 & 21.08 & 83.90 & 59.95 \\
\hline 39.97 & 116.95 & 63.89 & 19.99 & 73.89 & 37.73 & 109.07 & 82.75 & 61.14 & 109.08 & 19.24 & 63.85 & 18.21 & 78.72 & 65.77 \\
\hline 39.89 & 107.53 & 58.50 & 21.08 & 66.92 & 36.78 & 109.84 & 68.00 & 70.55 & 110.33 & 18.23 & 70.48 & 19.45 & 74.99 & 65.93 \\
\hline 38.74 & 104.89 & 58.83 & 20.66 & 66.56 & 38.38 & 109.21 & 83.46 & 62.92 & 62.26 & 20.15 & 56.12 & 18.96 & 79.14 & 57.14 \\
\hline 39.99 & 98.58 & 60.52 & 19.70 & 58.62 & 37.74 & 109.27 & 75.75 & 63.53 & 104.82 & 20.57 & 52.21 & 20.73 & 82.27 & 53.74 \\
\hline 40.10 & 98.43 & 67.48 & 18.50 & 54.15 & 37.71 & 109.27 & 73.78 & 54.23 & 90.11 & 19.54 & 59.40 & 18.47 & 91.26 & 51.07 \\
\hline 39.77 & 107.84 & 57.48 & 19.10 & 57.25 & 37.30 & 109.28 & 72.41 & 52.34 & 89.71 & 18.51 & 60.12 & 20.06 & 75.32 & 61.89 \\
\hline 39.71 & 113.54 & 52.20 & 19.17 & 53.01 & 36.61 & 109.16 & 72.92 & 59.46 & 117.01 & 19.90 & 67.17 & 19.55 & 66.98 & 64.82 \\
\hline 39.50 & 111.00 & 62.12 & 19.33 & 54.77 & 37.51 & 110.01 & 79.76 & 58.10 & 100.66 & 20.14 & 74.85 & 19.91 & 73.19 & 48.33 \\
\hline 38.45 & 100.00 & 71.00 & 19.62 & 56.98 & 37.06 & 108.72 & 74.15 & 60.70 & 97.24 & 19.30 & 60.97 & 19.91 & 83.36 & 67.20 \\
\hline 39.55 & 103.95 & 71.31 & 21.40 & 68.12 & 37.40 & 109.86 & 85.85 & 54.77 & 101.98 & 20.54 & 60.26 & 19.34 & 79.47 & 63.75 \\
\hline 39.80 & 103.18 & 62.04 & 20.65 & 72.04 & 38.31 & 109.38 & 75.64 & 50.51 & 96.81 & 18.38 & 58.59 & 18.90 & 94.30 & 57.92 \\
\hline 39.75 & 114.34 & 67.57 & 20.51 & 67.36 & 37.53 & 109.36 & 86.20 & 56.01 & 107.11 & 19.37 & 78.15 & 18.05 & 88.02 & 73.91 \\
\hline 39.88 & 104.66 & 60.75 & 19.88 & 61.76 & 38.47 & 109.60 & 79.77 & 54.75 & 113.57 & 19.79 & 58.15 & 19.25 & 86.29 & 64.82 \\
\hline 39.58 & 119.82 & 56.72 & 19.92 & 60.42 & 37.52 & 109.13 & 90.50 & 61.17 & 98.55 & 18.90 & 67.89 & 19.42 & 82.77 & 63.16 \\
\hline 38.98 & 108.77 & 62.89 & 19.35 & 54.42 & 38.06 & 109.25 & 89.02 & 61.32 & 91.79 & 19.12 & 66.40 & 19.20 & 76.36 & 66.01 \\
\hline 39.41 & 101.42 & 63.71 & 21.10 & 63.95 & 37.57 & 109.56 & 81.72 & 52.15 & 109.91 & 20.18 & 73.92 & 18.64 & 62.49 & 55.84 \\
\hline 39.70 & 110.93 & 60.65 & 19.36 & 67.88 & 37.27 & 108.69 & 82.20 & 63.57 & 104.66 & 19.89 & 58.14 & 18.89 & 75.77 & 69.05 \\
\hline 39.81 & 117.11 & 71.77 & 20.78 & 65.74 & 37.97 & 109.00 & 59.10 & 61.90 & 97.66 & 18.97 & 50.86 & 19.43 & 93.57 & 67.63 \\
\hline 39.84 & 100.85 & 58.13 & 19.95 & 56.24 & 38.64 & 109.67 & 85.85 & 63.12 & 111.12 & 18.54 & 73.49 & 20.04 & 86.16 & 64.41 \\
\hline 40.08 & 106.83 & 65.60 & 18.52 & 62.92 & 37.45 & 108.99 & 89.64 & 52.75 & 98.39 & 18.50 & 59.75 & 20.35 & 69.83 & 75.74 \\
\hline 40.22 & 104.54 & 59.99 & 20.21 & 70.07 & 37.33 & 109.81 & 81.65 & 55.31 & 93.91 & 19.97 & 52.78 & 19.05 & 93.06 & 73.47 \\
\hline 39.55 & 80.27 & 58.51 & 17.95 & 76.77 & 38.25 & 109.81 & 83.26 & 55.82 & 97.06 & 19.53 & 61.43 & 19.82 & 91.81 & 64.85 \\
\hline 40.13 & 95.35 & 61.09 & 19.29 & 65.56 & 37.14 & 109.31 & 88.80 & 63.69 & 114.24 & 18.07 & 69.81 & 19.74 & 90.52 & 60.46 \\
\hline 39.56 & 110.79 & 58.76 & 19.90 & 54.36 & 38.42 & 109.46 & 73.08 & 55.30 & 97.32 & 19.81 & 66.78 & 19.82 & 80.50 & 71.89 \\
\hline 39.25 & 95.16 & 62.46 & 20.29 & 56.42 & 37.41 & 109.66 & 92.58 & 56.17 & 106.88 & 19.82 & 66.11 & 21.01 & 73.83 & 66.19 \\
\hline 39.73 & 88.63 & 56.60 & 16.82 & 56.85 & 37.91 & 109.40 & 67.76 & 58.76 & 99.83 & 18.95 & 76.69 & 18.50 & 71.91 & 84.98 \\
\hline 39.91 & 95.39 & 71.70 & 19.53 & 64.17 & 38.33 & 108.70 & 80.17 & 56.42 & 118.85 & 18.49 & 56.67 & 19.30 & 81.70 & 57.70 \\
\hline 39.94 & 90.20 & 59.42 & 19.97 & 59.00 & 37.17 & 109.73 & 79.99 & 59.80 & 107.99 & 20.33 & 55.53 & 19.70 & 66.10 & 60.51 \\
\hline 39.93 & 113.92 & 65.83 & 20.21 & 52.47 & 38.64 & 109.80 & 70.99 & 64.26 & 107.89 & 20.75 & 60.22 & 19.86 & 77.28 & 69.13 \\
\hline 39.16 & 100.65 & 72.14 & 20.25 & 54.82 & 37.06 & 109.69 & 96.46 & 59.61 & 107.59 & 19.46 & 69.97 & 18.77 & 82.98 & 52.34 \\
\hline 39.73 & 111.88 & 60.94 & 20.99 & 72.34 & 36.97 & 109.77 & 86.95 & 66.81 & 117.03 & 19.83 & 59.67 & 20.32 & 90.17 & 75.64 \\
\hline 39.76 & 99.78 & 59.53 & 18.73 & 55.60 & 37.84 & 109.02 & 85.60 & 66.19 & 106.33 & 18.85 & 56.02 & 19.33 & 77.26 & 54.34 \\
\hline 40.73 & 115.97 & 61.37 & 20.68 & 78.38 & 38.47 & 109.62 & 63.29 & 58.67 & 107.28 & 20.25 & 63.24 & 19.44 & 79.46 & 67.81 \\
\hline 39.65 & 100.46 & 61.44 & 20.01 & 67.12 & 38.58 & 109.86 & 82.56 & 55.21 & 91.76 & 19.43 & 64.53 & 20.10 & 88.39 & 69.56 \\
\hline 39.21 & 110.45 & 64.19 & 20.05 & 71.47 & 38.33 & 109.94 & 83.28 & 56.07 & 117.60 & 19.75 & 58.93 & 20.11 & 73.44 & 54.68 \\
\hline 39.49 & 94.94 & 63.23 & 20.13 & 72.02 & 38.25 & 109.70 & 90.16 & 64.27 & 100.25 & 19.51 & 65.09 & 17.88 & 85.73 & 61.41 \\
\hline 40.24 & 91.73 & 59.21 & 21.00 & 61.07 & 37.13 & 109.32 & 69.27 & 59.33 & 110.14 & 19.74 & 48.44 & 19.06 & 85.94 & 63.84 \\
\hline
\end{tabular}


Table F.13: Throughput Simulation Picking Times (Non-Overlapping Waves) — continued.

\begin{tabular}{rrrrrrrrrrrrrrrrr}
\hline 1 & 2 & 3 & 4 & 5 & 6 & 7 & 8 & 9 & 10 & 11 & 12 & 13 \\
\hline 39.48 & 107.90 & 62.36 & 19.67 & 66.58 & 36.85 & 109.10 & 79.73 & 60.36 & 116.70 & 19.10 & 64.54 & 19.54 & 81.17 & 58.56 \\
39.18 & 92.98 & 63.84 & 19.32 & 58.16 & 38.34 & 109.37 & 81.25 & 62.45 & 113.14 & 19.65 & 58.98 & 19.44 & 84.95 & 70.24 \\
38.94 & 113.77 & 60.86 & 19.43 & 67.60 & 37.74 & 108.92 & 69.15 & 59.17 & 111.62 & 19.25 & 57.42 & 19.73 & 77.64 & 65.95 \\
39.49 & 113.45 & 68.12 & 20.15 & 55.22 & 37.06 & 109.84 & 76.25 & 59.31 & 97.79 & 19.20 & 57.25 & 17.93 & 78.26 & 61.67 \\
38.97 & 112.72 & 61.32 & 20.49 & 60.74 & 38.05 & 108.79 & 65.69 & 55.67 & 109.56 & 20.18 & 56.96 & 19.55 & 87.36 & 66.96 \\
40.17 & 75.10 & 65.03 & 19.31 & 58.54 & 38.81 & 108.84 & 83.07 & 69.47 & 101.63 & 19.27 & 61.11 & 18.61 & 71.51 & 56.18 \\
40.18 & 95.55 & 74.32 & 21.58 & 62.73 & 38.49 & 108.97 & 85.83 & 61.86 & 113.38 & 20.07 & 55.28 & 20.19 & 88.25 & 62.12 \\
39.83 & 110.84 & 66.40 & 21.65 & 71.78 & 36.96 & 109.13 & 77.57 & 60.46 & 95.13 & 20.05 & 65.87 & 19.62 & 85.27 & 54.72 \\
40.30 & 107.30 & 67.36 & 18.91 & 62.03 & 38.43 & 109.80 & 82.91 & 61.93 & 105.08 & 18.01 & 60.63 & 19.44 & 87.95 & 61.54 \\
40.81 & 105.05 & 71.47 & 20.68 & 66.85 & 37.66 & 108.95 & 88.13 & 59.85 & 102.07 & 18.94 & 67.03 & 19.18 & 70.56 & 59.93 \\
39.17 & 101.43 & 64.23 & 18.14 & 66.08 & 37.86 & 109.34 & 93.89 & 57.10 & 116.21 & 19.71 & 72.15 & 19.21 & 70.36 & 50.47 \\
39.56 & 104.61 & 69.48 & 19.66 & 64.61 & 37.80 & 109.79 & 90.10 & 58.59 & 95.51 & 17.88 & 69.24 & 19.45 & 82.15 & 60.39 \\
39.79 & 105.98 & 60.32 & 19.06 & 64.38 & 38.11 & 109.55 & 73.97 & 67.14 & 113.58 & 21.46 & 60.12 & 19.37 & 86.06 & 58.32 \\
39.38 & 107.93 & 66.40 & 20.22 & 66.34 & 37.45 & 109.87 & 70.14 & 56.09 & 120.64 & 20.74 & 73.45 & 20.45 & 85.72 & 68.19 \\
38.64 & 108.00 & 52.75 & 19.63 & 62.25 & 36.55 & 109.95 & 70.96 & 58.27 & 110.89 & 21.52 & 67.47 & 20.23 & 79.85 & 50.23 \\
39.86 & 94.75 & 62.66 & 19.13 & 56.52 & 38.13 & 109.51 & 82.62 & 57.20 & 116.06 & 19.89 & 62.10 & 20.02 & 83.31 & 62.77 \\
\hline
\end{tabular}

Table F.14: Throughput Simulation Packing Times (Non-Overlapping Waves).

\begin{tabular}{|c|c|c|c|c|c|c|c|c|c|c|c|c|c|c|}
\hline 1 & 2 & 3 & 4 & 5 & 6 & 7 & 8 & 9 & 10 & 11 & 12 & 13 & 14 & 15 \\
\hline 41.00 & Man. & Man. & 20.33 & Man. & 40.32 & 123.47 & Man. & Man. & Man. & 20.99 & Man. & 22.56 & Man. & Man. \\
\hline 43.70 & Man. & Man. & 20.72 & Man. & 41.77 & 120.77 & Man. & Man. & Man. & 20.34 & Man. & 19.66 & Man. & Man. \\
\hline 38.10 & Man. & Man. & 19.83 & Man. & 40.43 & 120.98 & Man. & Man. & Man. & 19.92 & Man. & 20.94 & Man. & Man. \\
\hline 41.84 & Man. & Man. & 18.80 & Man. & 35.46 & 123.36 & Man. & Man. & Man. & 21.98 & Man. & 18.76 & Man. & Man. \\
\hline 40.62 & Man. & Man. & 20.67 & Man. & 38.17 & 120.54 & Man. & Man. & Man. & 20.55 & Man. & 21.58 & Man. & Man. \\
\hline 42.21 & Man. & Man. & 20.56 & Man. & 42.83 & 120.58 & Man. & Man. & Man. & 23.32 & Man. & 19.51 & Man. & Man. \\
\hline 42.28 & Man. & Man. & 20.46 & Man. & 42.57 & 118.72 & Man. & Man. & Man. & 20.17 & Man. & 20.19 & Man. & Man. \\
\hline 40.49 & Man. & Man. & 20.35 & Man. & 42.64 & 116.33 & Man. & Man. & Man. & 20.54 & Man. & 19.94 & Man. & Man. \\
\hline 41.27 & Man. & Man. & 22.04 & Man. & 42.09 & 118.99 & Man. & Man. & Man. & 20.51 & Man. & 20.05 & Man. & Man. \\
\hline 41.29 & Man. & Man. & 20.15 & Man. & 41.35 & 120.56 & Man. & Man. & Man. & 19.79 & Man. & 19.74 & Man. & Man. \\
\hline 43.14 & Man. & Man. & 20.12 & Man. & 40.83 & 122.03 & Man. & Man. & Man. & 20.89 & Man. & 20.75 & Man. & Man. \\
\hline 41.41 & Man. & Man. & 20.70 & Man. & 38.62 & 121.12 & Man. & Man. & Man. & 23.46 & Man. & 20.45 & Man. & Man. \\
\hline 39.42 & Man. & Man. & 19.61 & Man. & 41.87 & 123.93 & Man. & Man. & Man. & 18.80 & Man. & 20.97 & Man. & Man. \\
\hline 40.07 & Man. & Man. & 20.03 & Man. & 40.76 & 123.15 & Man. & Man. & Man. & 20.88 & Man. & 21.12 & Man. & Man. \\
\hline 39.89 & Man. & Man. & 20.04 & Man. & 43.09 & 123.16 & Man. & Man. & Man. & 20.02 & Man. & 21.44 & Man. & Man. \\
\hline 41.41 & Man. & Man. & 21.05 & Man. & 40.95 & 123.84 & Man. & Man. & Man. & 21.14 & Man. & 20.08 & Man. & Man. \\
\hline 39.21 & Man. & Man. & 20.29 & Man. & 41.71 & 120.82 & Man. & Man. & Man. & 20.36 & Man. & 21.09 & Man. & Man. \\
\hline 38.11 & Man. & Man. & 21.17 & Man. & 39.28 & 122.55 & Man. & Man. & Man. & 20.27 & Man. & 21.06 & Man. & Man. \\
\hline 39.43 & Man. & Man. & 21.42 & Man. & 40.99 & 119.65 & Man. & Man. & Man. & 20.43 & Man. & 21.03 & Man. & Man. \\
\hline 41.34 & Man. & Man. & 21.30 & Man. & 41.47 & 122.84 & Man. & Man. & Man. & 20.92 & Man. & 20.57 & Man. & Man. \\
\hline 38.46 & Man. & Man. & 21.21 & Man. & 42.20 & 123.47 & Man. & Man. & Man. & 19.84 & Man. & 20.53 & Man. & Man. \\
\hline 41.03 & Man. & Man. & 20.25 & Man. & 41.14 & 120.02 & Man. & Man. & Man. & 21.76 & Man. & 21.88 & Man. & Man. \\
\hline 39.86 & Man. & Man. & 21.46 & Man. & 43.03 & 120.72 & Man. & Man. & Man. & 21.22 & Man. & 21.96 & Man. & Man. \\
\hline 39.98 & Man. & Man. & 20.57 & Man. & 39.81 & 124.96 & Man. & Man. & Man. & 20.04 & Man. & 19.98 & Man. & Man. \\
\hline 41.72 & Man. & Man. & 20.38 & Man. & 39.75 & 125.49 & Man. & Man. & Man. & 20.43 & Man. & 18.89 & Man. & Man. \\
\hline 40.33 & Man. & Man. & 20.55 & Man. & 42.00 & 121.62 & Man. & Man. & Man. & 20.44 & Man. & 20.43 & Man. & Man. \\
\hline 41.48 & Man. & Man. & 20.47 & Man. & 41.36 & 122.75 & Man. & Man. & Man. & 19.85 & Man. & 22.66 & Man. & Man. \\
\hline 40.45 & Man. & Man. & 20.30 & Man. & 45.16 & 120.97 & Man. & Man. & Man. & 20.80 & Man. & 20.22 & Man. & Man. \\
\hline 41.07 & Man. & Man. & 20.54 & Man. & 41.64 & 119.64 & Man. & Man. & Man. & 19.68 & Man. & 20.54 & Man. & Man. \\
\hline 40.88 & Man. & Man. & 21.70 & Man. & 42.90 & 119.16 & Man. & Man. & Man. & 20.87 & Man. & 19.33 & Man. & Man. \\
\hline 41.50 & Man. & Man. & 20.50 & Man. & 38.41 & 122.17 & Man. & Man. & Man. & 21.70 & Man. & 21.07 & Man. & Man. \\
\hline 40.58 & Man. & Man. & 19.05 & Man. & 39.35 & 115.89 & Man. & Man. & Man. & 20.86 & Man. & 22.81 & Man. & Man. \\
\hline 40.79 & Man. & Man. & 21.17 & Man. & 40.94 & 119.26 & Man. & Man. & Man. & 19.98 & Man. & 21.95 & Man. & Man. \\
\hline 41.22 & Man. & Man. & 20.56 & Man. & 38.84 & 122.00 & Man. & Man. & Man. & 20.39 & Man. & 18.03 & Man. & Man. \\
\hline 41.73 & Man. & Man. & 20.39 & Man. & 39.03 & 117.46 & Man. & Man. & Man. & 21.11 & Man. & 21.23 & Man. & Man. \\
\hline 40.55 & Man. & Man. & 19.74 & Man. & 44.10 & 116.52 & Man. & Man. & Man. & 22.11 & Man. & 21.09 & Man. & Man. \\
\hline 40.01 & Man. & Man. & 21.40 & Man. & 40.18 & 119.41 & Man. & Man. & Man. & 20.58 & Man. & 21.10 & Man. & Man. \\
\hline 40.29 & Man. & Man. & 21.65 & Man. & 41.33 & 121.80 & Man. & Man. & Man. & 20.65 & Man. & 20.25 & Man. & Man. \\
\hline
\end{tabular}


Table F.14: Throughput Simulation Packing Times (Non-Overlapping Waves) - continued.

\begin{tabular}{|c|c|c|c|c|c|c|c|c|c|c|c|c|c|c|}
\hline 1 & 2 & 3 & 4 & 5 & 6 & 7 & 8 & 9 & 10 & 11 & 12 & 13 & 14 & 15 \\
\hline 39.97 & Man. & Man. & 20.45 & Man. & 37.77 & 120.52 & Man. & Man. & Man. & 19.23 & Man. & 21.50 & Man. & Man. \\
\hline 41.11 & Man. & Man. & 20.79 & Man. & 41.18 & 115.38 & Man. & Man. & Man. & 22.11 & Man. & 20.71 & Man. & Man. \\
\hline 39.02 & Man. & Man. & 21.14 & Man. & 39.61 & 121.80 & Man. & Man. & Man. & 21.51 & Man. & 21.16 & Man. & Man. \\
\hline 40.70 & Man. & Man. & 21.45 & Man. & 41.25 & 121.54 & Man. & Man. & Man. & 20.15 & Man. & 20.87 & Man. & Man. \\
\hline 41.04 & Man. & Man. & 20.69 & Man. & 43.56 & 120.58 & Man. & Man. & Man. & 20.21 & Man. & 19.70 & Man. & Man. \\
\hline 39.84 & Man. & Man. & 20.26 & Man. & 42.23 & 122.07 & Man. & Man. & Man. & 20.90 & Man. & 19.81 & Man. & Man. \\
\hline 40.72 & Man. & Man. & 20.91 & Man. & 40.73 & 126.17 & Man. & Man. & Man. & 19.86 & Man. & 18.31 & Man. & Man. \\
\hline 41.91 & Man. & Man. & 21.14 & Man. & 39.84 & 118.50 & Man. & Man. & Man. & 18.83 & Man. & 19.89 & Man. & Man. \\
\hline 40.75 & Man. & Man. & 20.03 & Man. & 43.28 & 123.20 & Man. & Man. & Man. & 20.52 & Man. & 22.30 & Man. & Man. \\
\hline 41.95 & Man. & Man. & 18.54 & Man. & 40.86 & 120.49 & Man. & Man. & Man. & 21.81 & Man. & 20.95 & Man. & Man. \\
\hline 40.03 & Man. & Man. & 20.58 & Man. & 43.15 & 109.78 & Man. & Man. & Man. & 20.54 & Man. & 20.84 & Man. & Man. \\
\hline 40.54 & Man. & Man. & 20.81 & Man. & 43.63 & 118.76 & Man. & Man. & Man. & 22.30 & Man. & 19.26 & Man. & Man. \\
\hline 41.05 & Man. & Man. & 21.25 & Man. & 36.86 & 117.60 & Man. & Man. & Man. & 21.23 & Man. & 20.67 & Man. & Man. \\
\hline 41.41 & Man. & Man. & 20.20 & Man. & 39.46 & 119.29 & Man. & Man. & Man. & 19.21 & Man. & 21.80 & Man. & Man. \\
\hline 41.01 & Man. & Man. & 20.46 & Man. & 40.41 & 121.07 & Man. & Man. & Man. & 21.60 & Man. & 20.60 & Man. & Man. \\
\hline 41.04 & Man. & Man. & 20.76 & Man. & 39.93 & 120.74 & Man. & Man. & Man. & 21.15 & Man. & 22.00 & Man. & Man. \\
\hline 42.60 & Man. & Man. & 20.53 & Man. & 42.00 & 121.12 & Man. & Man. & Man. & 20.71 & Man. & 21.08 & Man. & Man. \\
\hline 41.36 & Man. & Man. & 21.03 & Man. & 40.52 & 120.94 & Man. & Man. & Man. & 19.70 & Man. & 20.09 & Man. & Man. \\
\hline 40.06 & Man. & Man. & 20.65 & Man. & 40.33 & 115.56 & Man. & Man. & Man. & 22.85 & Man. & 20.86 & Man. & Man. \\
\hline 39.95 & Man. & Man. & 21.75 & Man. & 43.84 & 113.04 & Man. & Man. & Man. & 22.36 & Man. & 20.68 & Man. & Man. \\
\hline 40.35 & Man. & Man. & 20.83 & Man. & 41.01 & 121.21 & Man. & Man. & Man. & 22.27 & Man. & 21.62 & Man. & Man. \\
\hline 42.05 & Man. & Man. & 20.52 & Man. & 41.19 & 122.82 & Man. & Man. & Man. & 20.66 & Man. & 21.64 & Man. & Man. \\
\hline 40.93 & Man. & Man. & 21.42 & Man. & 39.39 & 119.56 & Man. & Man. & Man. & 22.12 & Man. & 20.82 & Man. & Man. \\
\hline 40.65 & Man. & Man. & 20.28 & Man. & 41.66 & 122.82 & Man. & Man. & Man. & 21.49 & Man. & 21.52 & Man. & Man. \\
\hline 41.40 & Man. & Man. & 20.54 & Man. & 42.50 & 116.32 & Man. & Man. & Man. & 20.81 & Man. & 20.64 & Man. & Man. \\
\hline 42.41 & Man. & Man. & 20.31 & Man. & 36.87 & 121.10 & Man. & Man. & Man. & 21.31 & Man. & 21.45 & Man. & Man. \\
\hline 41.57 & Man. & Man. & 21.89 & Man. & 42.09 & 123.55 & Man. & Man. & Man. & 23.39 & Man. & 20.24 & Man. & Man. \\
\hline 41.12 & Man. & Man. & 20.25 & Man. & 41.03 & 120.90 & Man. & Man. & Man. & 19.67 & Man. & 20.71 & Man. & Man. \\
\hline 40.40 & Man. & Man. & 20.09 & Man. & 41.72 & 116.29 & Man. & Man. & Man. & 21.20 & Man. & 18.72 & Man. & Man. \\
\hline 42.04 & Man. & Man. & 20.82 & Man. & 39.44 & 121.16 & Man. & Man. & Man. & 21.74 & Man. & 21.28 & Man. & Man. \\
\hline 40.08 & Man. & Man. & 21.48 & Man. & 40.90 & 123.12 & Man. & Man. & Man. & 23.38 & Man. & 22.33 & Man. & Man. \\
\hline 42.13 & Man. & Man. & 20.60 & Man. & 39.08 & 118.86 & Man. & Man. & Man. & 19.82 & Man. & 21.08 & Man. & Man. \\
\hline 40.95 & Man. & Man. & 20.76 & Man. & 38.78 & 120.40 & Man. & Man. & Man. & 19.98 & Man. & 21.97 & Man. & Man. \\
\hline 41.08 & Man. & Man. & 21.10 & Man. & 40.86 & 120.99 & Man. & Man. & Man. & 21.26 & Man. & 20.32 & Man. & Man. \\
\hline 40.66 & Man. & Man. & 20.75 & Man. & 38.77 & 120.82 & Man. & Man. & Man. & 20.83 & Man. & 20.14 & Man. & Man. \\
\hline 40.33 & Man. & Man. & 20.83 & Man. & 41.28 & 122.99 & Man. & Man. & Man. & 19.40 & Man. & 20.13 & Man. & Man. \\
\hline 40.33 & Man. & Man. & 20.77 & Man. & 42.33 & 121.72 & Man. & Man. & Man. & 19.49 & Man. & 19.97 & Man. & Man. \\
\hline 41.36 & Man. & Man. & 20.18 & Man. & 44.45 & 120.48 & Man. & Man. & Man. & 20.99 & Man. & 20.16 & Man. & Man. \\
\hline 40.39 & Man. & Man. & 21.10 & Man. & 40.80 & 120.51 & Man. & Man. & Man. & 21.18 & Man. & 21.05 & Man. & Man. \\
\hline 42.11 & Man. & Man. & 20.81 & Man. & 40.27 & 120.11 & Man. & Man. & Man. & 19.45 & Man. & 20.69 & Man. & Man. \\
\hline 40.03 & Man. & Man. & 21.74 & Man. & 39.86 & 120.41 & Man. & Man. & Man. & 22.30 & Man. & 21.15 & Man. & Man. \\
\hline 41.41 & Man. & Man. & 21.52 & Man. & 40.30 & 119.69 & Man. & Man. & Man. & 20.24 & Man. & 21.29 & Man. & Man. \\
\hline 40.72 & Man. & Man. & 21.41 & Man. & 38.88 & 119.27 & Man. & Man. & Man. & 20.74 & Man. & 19.85 & Man. & Man. \\
\hline 40.80 & Man. & Man. & 20.79 & Man. & 39.93 & 122.58 & Man. & Man. & Man. & 22.51 & Man. & 20.56 & Man. & Man. \\
\hline 41.61 & Man. & Man. & 20.90 & Man. & 39.36 & 120.23 & Man. & Man. & Man. & 20.38 & Man. & 20.75 & Man. & Man. \\
\hline 40.11 & Man. & Man. & 21.59 & Man. & 40.88 & 120.86 & Man. & Man. & Man. & 22.19 & Man. & 20.95 & Man. & Man. \\
\hline 41.07 & Man. & Man. & 19.08 & Man. & 37.53 & 119.17 & Man. & Man. & Man. & 20.75 & Man. & 21.56 & Man. & Man. \\
\hline 40.48 & Man. & Man. & 20.20 & Man. & 41.46 & 118.50 & Man. & Man. & Man. & 20.48 & Man. & 19.32 & Man. & Man. \\
\hline 40.30 & Man. & Man. & 20.00 & Man. & 39.55 & 117.98 & Man. & Man. & Man. & 20.18 & Man. & 22.25 & Man. & Man. \\
\hline 40.67 & Man. & Man. & 20.03 & Man. & 40.28 & 120.06 & Man. & Man. & Man. & 20.30 & Man. & 21.84 & Man. & Man. \\
\hline 41.09 & Man. & Man. & 20.40 & Man. & 44.87 & 120.37 & Man. & Man. & Man. & 20.46 & Man. & 21.89 & Man. & Man. \\
\hline 41.70 & Man. & Man. & 20.47 & Man. & 36.66 & 121.17 & Man. & Man. & Man. & 19.19 & Man. & 20.17 & Man. & Man. \\
\hline 41.33 & Man. & Man. & 20.38 & Man. & 44.42 & 120.38 & Man. & Man. & Man. & 22.01 & Man. & 20.23 & Man. & Man. \\
\hline 41.73 & Man. & Man. & 21.13 & Man. & 40.13 & 120.22 & Man. & Man. & Man. & 21.81 & Man. & 21.99 & Man. & Man. \\
\hline 40.37 & Man. & Man. & 20.84 & Man. & 40.49 & 121.01 & Man. & Man. & Man. & 21.43 & Man. & 21.02 & Man. & Man. \\
\hline 40.42 & Man. & Man. & 20.02 & Man. & 39.29 & 121.07 & Man. & Man. & Man. & 20.18 & Man. & 20.43 & Man. & Man. \\
\hline 40.52 & Man. & Man. & 19.76 & Man. & 41.24 & 118.56 & Man. & Man. & Man. & 21.15 & Man. & 19.57 & Man. & Man. \\
\hline 40.01 & Man. & Man. & 20.36 & Man. & 40.63 & 127.49 & Man. & Man. & Man. & 20.05 & Man. & 22.42 & Man. & Man. \\
\hline 42.95 & Man. & Man. & 21.10 & Man. & 42.81 & 121.22 & Man. & Man. & Man. & 20.54 & Man. & 20.93 & Man. & Man. \\
\hline
\end{tabular}


Table F.14: Throughput Simulation Packing Times (Non-Overlapping Waves) - continued.

\begin{tabular}{rrrrrrrrrrrrrrrrr}
\hline 1 & 2 & 3 & 4 & 5 & 6 & 7 & 8 & 9 & 10 & 11 & 12 & 13 & 14 & 15 \\
\hline 41.66 & Man. & Man. & 20.81 & Man. & 41.80 & 125.30 & Man. & Man. & Man. & 20.27 & Man. & 21.65 & Man. & Man. \\
40.18 & Man. & Man. & 22.48 & Man. & 41.21 & 124.01 & Man. & Man. & Man. & 21.20 & Man. & 20.04 & Man. & Man. \\
41.87 & Man. & Man. & 20.39 & Man. & 41.00 & 119.76 & Man. & Man. & Man. & 21.56 & Man. & 20.80 & Man. & Man. \\
\hline
\end{tabular}

Table F.15: Throughput Simulation Scaled Picking Times (Overlapping Waves).

\begin{tabular}{|c|c|c|c|c|c|c|c|c|c|c|c|c|c|c|}
\hline 1 & 2 & 3 & 4 & 5 & 6 & 7 & 8 & 9 & 10 & 11 & 12 & 13 & 14 & 15 \\
\hline 114.17 & 39.23 & 58.97 & 39.05 & 61.62 & 73.57 & 108.52 & 89.94 & 58.08 & 57.71 & 114.25 & 67.29 & 53.96 & 86.50 & 50.30 \\
\hline 113.23 & 36.08 & 58.33 & 40.22 & 65.45 & 73.65 & 109.19 & 75.96 & 62.57 & 58.98 & 114.66 & 59.65 & 52.96 & 79.20 & 52.11 \\
\hline 113.95 & 37.34 & 59.62 & 40.46 & 53.27 & 73.73 & 109.81 & 75.63 & 55.43 & 58.24 & 114.99 & 46.16 & 53.96 & 72.78 & 56.16 \\
\hline 113.38 & 39.91 & 56.91 & 39.36 & 61.82 & 73.81 & 109.93 & 72.99 & 57.41 & 59.57 & 114.36 & 53.36 & 53.51 & 84.83 & 56.20 \\
\hline 113.35 & 39.25 & 59.06 & 39.32 & 57.40 & 73.98 & 109.40 & 73.52 & 61.14 & 60.16 & 114.25 & 47.66 & 53.23 & 86.90 & 48.62 \\
\hline 114.16 & 38.39 & 57.33 & 39.39 & 58.95 & 73.34 & 109.26 & 95.54 & 64.40 & 58.42 & 114.97 & 55.95 & 53.73 & 73.17 & 58.62 \\
\hline 113.54 & 36.08 & 59.14 & 40.31 & 67.17 & 74.27 & 109.38 & 70.74 & 59.54 & 59.49 & 115.01 & 56.35 & 54.15 & 64.31 & 59.06 \\
\hline 113.33 & 34.12 & 58.43 & 39.12 & 63.28 & 74.31 & 109.37 & 79.88 & 52.39 & 60.73 & 114.28 & 56.56 & 53.22 & 92.69 & 48.19 \\
\hline 114.08 & 37.88 & 56.93 & 39.72 & 59.75 & 74.23 & 109.66 & 81.97 & 58.99 & 57.71 & 114.89 & 52.29 & 53.69 & 80.93 & 57.38 \\
\hline 113.72 & 38.10 & 59.31 & 39.30 & 64.47 & 73.75 & 109.88 & 71.11 & 55.57 & 60.20 & 114.68 & 64.26 & 53.94 & 79.06 & 62.80 \\
\hline 113.34 & 38.00 & 58.76 & 39.34 & 68.45 & 73.99 & 109.71 & 95.57 & 57.23 & 57.65 & 114.35 & 61.86 & 53.74 & 74.14 & 51.88 \\
\hline 113.98 & 42.49 & 57.73 & 39.85 & 63.48 & 74.14 & 109.24 & 84.99 & 64.57 & 58.90 & 114.50 & 57.37 & 53.88 & 84.67 & 58.32 \\
\hline 114.01 & 42.47 & 57.01 & 38.45 & 59.03 & 73.02 & 109.82 & 69.69 & 63.00 & 58.49 & 114.23 & 51.19 & 54.01 & 91.28 & 70.48 \\
\hline 113.54 & 43.21 & 57.52 & 39.93 & 64.02 & 74.13 & 109.70 & 62.35 & 61.20 & 57.97 & 114.46 & 47.48 & 53.61 & 72.51 & 47.45 \\
\hline 113.65 & 39.12 & 58.74 & 39.31 & 60.90 & 73.89 & 109.80 & 76.94 & 66.65 & 59.53 & 114.94 & 52.96 & 53.97 & 70.14 & 43.85 \\
\hline 113.81 & 36.01 & 58.74 & 40.10 & 59.03 & 73.88 & 109.43 & 77.46 & 50.69 & 59.04 & 114.34 & 48.49 & 54.07 & 75.26 & 55.27 \\
\hline 113.34 & 40.68 & 58.17 & 39.11 & 54.83 & 73.43 & 109.58 & 79.44 & 55.35 & 58.17 & 114.40 & 45.20 & 53.42 & 81.35 & 57.51 \\
\hline 113.95 & 40.57 & 57.69 & 39.95 & 54.07 & 73.75 & 109.26 & 75.81 & 54.37 & 59.05 & 115.07 & 63.17 & 52.90 & 75.38 & 70.74 \\
\hline 113.80 & 40.02 & 58.32 & 39.16 & 55.06 & 74.06 & 109.10 & 65.18 & 56.68 & 58.94 & 115.13 & 61.01 & 53.61 & 72.44 & 54.10 \\
\hline 113.48 & 37.26 & 57.87 & 40.25 & 65.76 & 74.01 & 109.20 & 90.13 & 56.10 & 60.09 & 114.85 & 50.04 & 54.00 & 84.08 & 50.23 \\
\hline 113.85 & 37.29 & 57.39 & 39.56 & 49.09 & 73.58 & 109.29 & 82.67 & 52.85 & 57.81 & 114.95 & 57.71 & 53.36 & 67.36 & 50.09 \\
\hline 113.62 & 36.06 & 56.69 & 40.69 & 60.03 & 73.60 & 109.72 & 80.62 & 56.80 & 58.30 & 114.62 & 62.31 & 53.15 & 67.21 & 46.60 \\
\hline 114.12 & 37.89 & 56.40 & 40.48 & 66.79 & 73.59 & 109.50 & 80.46 & 59.19 & 60.63 & 114.87 & 46.38 & 52.63 & 60.36 & 50.86 \\
\hline 114.10 & 39.17 & 58.50 & 39.12 & 54.81 & 74.34 & 109.18 & 74.37 & 63.90 & 58.26 & 114.94 & 60.30 & 53.82 & 70.93 & 50.23 \\
\hline 114.04 & 37.17 & 58.23 & 38.34 & 51.48 & 73.83 & 108.81 & 86.00 & 60.18 & 60.74 & 114.81 & 57.48 & 53.81 & 89.23 & 42.93 \\
\hline 113.94 & 41.18 & 58.11 & 39.41 & 61.54 & 74.06 & 108.96 & 76.79 & 57.63 & 58.41 & 114.41 & 58.15 & 54.12 & 82.00 & 50.18 \\
\hline 113.33 & 42.50 & 58.38 & 38.96 & 50.91 & 73.79 & 109.71 & 72.23 & 53.63 & 60.58 & 115.04 & 55.03 & 53.24 & 81.06 & 61.57 \\
\hline 114.07 & 36.76 & 59.40 & 40.06 & 55.19 & 73.66 & 109.20 & 73.02 & 57.97 & 57.25 & 114.91 & 65.92 & 53.37 & 81.38 & 50.94 \\
\hline 114.28 & 38.71 & 59.43 & 38.71 & 62.47 & 73.94 & 109.96 & 84.30 & 61.46 & 60.09 & 114.17 & 65.46 & 53.52 & 84.60 & 52.29 \\
\hline 113.55 & 39.95 & 57.55 & 39.33 & 61.25 & 74.24 & 109.46 & 77.98 & 58.72 & 58.39 & 115.18 & 53.16 & 53.68 & 77.59 & 48.26 \\
\hline 114.12 & 39.28 & 56.20 & 39.52 & 63.70 & 73.40 & 109.65 & 67.32 & 61.61 & 56.56 & 114.85 & 56.75 & 53.36 & 71.82 & 53.59 \\
\hline 113.89 & 41.63 & 56.87 & 39.28 & 66.23 & 73.71 & 108.90 & 81.55 & 56.68 & 58.97 & 114.98 & 60.92 & 53.25 & 79.20 & 62.94 \\
\hline 114.05 & 39.32 & 59.25 & 38.88 & 63.94 & 73.82 & 109.46 & 69.58 & 55.49 & 57.62 & 114.50 & 55.10 & 52.93 & 65.64 & 50.08 \\
\hline 113.99 & 39.43 & 57.43 & 38.52 & 68.11 & 73.76 & 108.47 & 62.54 & 59.28 & 58.50 & 114.59 & 53.32 & 53.70 & 74.69 & 48.10 \\
\hline 114.12 & 39.16 & 57.70 & 39.59 & 75.27 & 74.16 & 109.09 & 86.01 & 60.00 & 59.10 & 114.14 & 60.40 & 53.49 & 81.53 & 54.63 \\
\hline 114.26 & 36.67 & 58.02 & 38.05 & 68.28 & 74.05 & 109.80 & 73.97 & 59.52 & 60.11 & 114.87 & 60.09 & 53.52 & 84.35 & 47.93 \\
\hline 114.02 & 41.74 & 58.66 & 38.78 & 57.72 & 74.16 & 109.30 & 84.57 & 61.79 & 57.27 & 114.32 & 48.79 & 54.19 & 84.73 & 59.50 \\
\hline 113.48 & 35.97 & 58.52 & 38.95 & 69.23 & 73.81 & 109.70 & 71.30 & 58.74 & 59.42 & 114.31 & 57.27 & 53.48 & 69.28 & 67.37 \\
\hline 113.94 & 41.28 & 57.38 & 40.04 & 56.39 & 74.06 & 109.44 & 69.16 & 56.72 & 59.64 & 114.78 & 46.24 & 52.54 & 70.11 & 47.08 \\
\hline 113.89 & 41.05 & 58.87 & 39.56 & 60.03 & 73.50 & 109.58 & 76.47 & 60.91 & 59.67 & 114.67 & 55.35 & 53.31 & 87.58 & 40.24 \\
\hline 113.45 & 41.93 & 57.36 & 38.61 & 69.26 & 73.82 & 109.61 & 65.47 & 57.28 & 59.70 & 114.55 & 50.24 & 53.59 & 79.91 & 46.25 \\
\hline 113.88 & 37.31 & 59.07 & 38.86 & 51.44 & 73.58 & 109.93 & 78.33 & 58.13 & 57.76 & 114.60 & 56.88 & 53.87 & 75.63 & 49.05 \\
\hline 114.14 & 39.24 & 58.33 & 38.89 & 64.15 & 74.19 & 109.83 & 81.55 & 53.63 & 57.89 & 114.56 & 51.64 & 52.96 & 84.34 & 58.24 \\
\hline 114.14 & 40.63 & 58.15 & 38.92 & 50.63 & 74.00 & 110.00 & 71.80 & 61.01 & 57.91 & 114.71 & 54.04 & 53.53 & 85.00 & 59.95 \\
\hline 113.32 & 37.16 & 58.65 & 39.13 & 62.30 & 73.65 & 108.75 & 97.09 & 59.15 & 60.15 & 114.40 & 50.89 & 53.84 & 72.45 & 58.30 \\
\hline 113.65 & 40.50 & 58.20 & 39.36 & 55.08 & 74.34 & 109.62 & 61.24 & 60.96 & 57.33 & 114.26 & 56.27 & 53.30 & 75.69 & 56.94 \\
\hline 113.76 & 41.16 & 58.08 & 40.62 & 57.69 & 73.38 & 109.39 & 84.40 & 55.65 & 58.82 & 114.61 & 52.52 & 53.29 & 86.91 & 63.62 \\
\hline 113.75 & 38.60 & 57.98 & 39.38 & 58.22 & 73.98 & 109.07 & 80.40 & 59.41 & 58.51 & 114.59 & 58.09 & 53.54 & 86.88 & 51.44 \\
\hline 113.57 & 39.79 & 58.18 & 39.15 & 59.85 & 74.35 & 109.84 & 77.62 & 62.38 & 58.54 & 114.38 & 50.35 & 53.62 & 72.85 & 60.72 \\
\hline 114.08 & 36.18 & 58.52 & 39.57 & 58.29 & 73.43 & 109.21 & 72.13 & 53.75 & 57.36 & 114.93 & 47.72 & 53.63 & 75.82 & 53.08 \\
\hline 113.62 & 38.56 & 58.61 & 38.84 & 70.74 & 73.89 & 109.27 & 74.29 & 61.21 & 59.69 & 114.95 & 59.40 & 53.51 & 64.65 & 62.16 \\
\hline
\end{tabular}


Table F.15: Throughput Simulation Scaled Picking Times (Overlapping Waves) - contin-

ued.

\begin{tabular}{|c|c|c|c|c|c|c|c|c|c|c|c|c|c|c|}
\hline 1 & 2 & 3 & 4 & 5 & 6 & 7 & 8 & 9 & 10 & 11 & 12 & 13 & 14 & 15 \\
\hline 113.50 & 36.76 & 57.97 & 39.18 & 57.76 & 73.79 & 109.27 & 72.29 & 63.38 & 60.25 & 114.34 & 46.01 & 53.66 & 77.08 & 54.61 \\
\hline 114.17 & 41.22 & 59.09 & 39.63 & 51.19 & 73.87 & 109.28 & 85.92 & 60.75 & 57.17 & 114.36 & 60.92 & 53.86 & 64.91 & 52.62 \\
\hline 113.49 & 38.42 & 57.65 & 39.80 & 55.70 & 73.83 & 109.16 & 79.98 & 57.89 & 59.67 & 115.07 & 59.27 & 53.92 & 80.19 & 55.62 \\
\hline 114.06 & 39.99 & 58.10 & 39.84 & 52.73 & 74.08 & 110.01 & 79.73 & 61.30 & 56.08 & 114.29 & 58.48 & 53.29 & 68.10 & 60.26 \\
\hline 113.98 & 38.46 & 57.82 & 38.51 & 57.24 & 73.67 & 108.72 & 59.47 & 64.01 & 58.29 & 114.78 & 59.26 & 53.63 & 82.42 & 54.45 \\
\hline 113.42 & 40.67 & 58.76 & 39.58 & 49.38 & 73.65 & 109.86 & 82.55 & 55.71 & 61.39 & 114.48 & 48.31 & 53.41 & 78.20 & 59.03 \\
\hline 113.77 & 37.97 & 58.62 & 38.76 & 62.58 & 73.28 & 109.38 & 75.53 & 60.65 & 57.37 & 114.48 & 51.73 & 53.89 & 84.87 & 51.36 \\
\hline 113.30 & 40.35 & 59.29 & 39.46 & 63.95 & 74.04 & 109.36 & 67.37 & 59.49 & 60.67 & 114.66 & 60.85 & 53.72 & 74.58 & 60.11 \\
\hline 114.00 & 41.25 & 59.26 & 39.63 & 58.36 & 73.94 & 109.60 & 77.18 & 59.10 & 60.79 & 114.77 & 60.65 & 54.36 & 70.13 & 56.07 \\
\hline 114.12 & 38.65 & 58.55 & 40.07 & 56.06 & 73.78 & 109.13 & 66.76 & 61.81 & 57.59 & 115.18 & 53.89 & 53.40 & 81.21 & 58.24 \\
\hline 113.81 & 40.47 & 58.43 & 39.97 & 65.01 & 74.18 & 109.25 & 89.48 & 58.65 & 61.35 & 114.65 & 50.68 & 53.45 & 84.81 & 59.33 \\
\hline 113.78 & 39.85 & 57.44 & 40.00 & 49.70 & 73.75 & 109.56 & 86.26 & 64.15 & 61.12 & 114.80 & 52.68 & 53.39 & 86.66 & 53.33 \\
\hline 113.51 & 39.24 & 58.84 & 39.81 & 70.16 & 74.09 & 108.69 & 91.60 & 61.05 & 59.15 & 114.90 & 56.42 & 54.28 & 68.06 & 53.38 \\
\hline 113.51 & 41.81 & 57.62 & 39.76 & 62.91 & 74.36 & 109.00 & 88.75 & 63.21 & 58.27 & 114.84 & 52.11 & 54.15 & 82.48 & 52.22 \\
\hline 114.13 & 36.59 & 58.41 & 39.79 & 55.17 & 73.75 & 109.67 & 86.15 & 57.53 & 57.68 & 114.94 & 62.36 & 53.03 & 84.33 & 54.85 \\
\hline 113.49 & 37.10 & 58.06 & 39.16 & 59.35 & 73.81 & 108.99 & 76.24 & 60.41 & 60.61 & 114.72 & 58.69 & 53.53 & 81.72 & 42.40 \\
\hline 114.09 & 37.99 & 58.13 & 39.16 & 51.49 & 73.40 & 109.81 & 79.25 & 62.22 & 57.74 & 115.07 & 50.95 & 53.75 & 68.65 & 41.82 \\
\hline 113.39 & 38.57 & 58.04 & 39.49 & 63.80 & 73.20 & 109.81 & 67.59 & 52.38 & 59.43 & 114.84 & 56.54 & 53.69 & 83.95 & 57.62 \\
\hline 113.82 & 39.27 & 59.42 & 39.02 & 56.95 & 73.79 & 109.31 & 76.85 & 55.71 & 57.78 & 114.89 & 44.03 & 54.09 & 75.46 & 57.19 \\
\hline 113.61 & 40.63 & 58.85 & 40.18 & 65.61 & 74.05 & 109.46 & 96.33 & 54.99 & 61.41 & 114.32 & 50.14 & 53.86 & 70.12 & 68.59 \\
\hline 113.26 & 41.20 & 56.71 & 39.60 & 67.40 & 73.35 & 109.66 & 89.96 & 57.12 & 59.03 & 114.28 & 45.93 & 53.53 & 84.03 & 60.63 \\
\hline 114.13 & 36.75 & 59.06 & 38.81 & 60.25 & 73.69 & 109.40 & 81.04 & 57.73 & 60.30 & 115.07 & 48.66 & 54.16 & 70.41 & 50.41 \\
\hline 114.28 & 36.47 & 56.97 & 39.79 & 60.83 & 73.57 & 108.70 & 84.29 & 59.75 & 58.26 & 114.60 & 57.23 & 52.61 & 84.13 & 52.05 \\
\hline 114.25 & 37.43 & 57.56 & 38.96 & 62.66 & 73.67 & 109.73 & 77.76 & 62.83 & 58.80 & 114.40 & 55.95 & 54.04 & 75.11 & 59.67 \\
\hline 113.34 & 40.60 & 58.62 & 40.16 & 69.32 & 73.46 & 109.80 & 76.32 & 60.29 & 61.33 & 114.67 & 58.27 & 53.88 & 75.00 & 47.36 \\
\hline 113.59 & 41.07 & 58.13 & 39.43 & 69.60 & 73.34 & 109.69 & 82.36 & 50.18 & 59.47 & 114.72 & 53.78 & 53.52 & 79.44 & 58.52 \\
\hline 113.34 & 40.43 & 57.58 & 39.60 & 65.69 & 73.76 & 109.77 & 86.36 & 59.25 & 58.95 & 114.97 & 57.35 & 53.50 & 86.91 & 51.67 \\
\hline 114.02 & 40.66 & 58.12 & 39.09 & 54.86 & 73.80 & 109.02 & 77.76 & 58.88 & 59.42 & 114.44 & 53.21 & 53.75 & 84.77 & 58.99 \\
\hline 113.30 & 38.65 & 58.85 & 38.58 & 60.25 & 73.93 & 109.62 & 75.81 & 55.20 & 59.65 & 114.25 & 48.33 & 53.29 & 72.07 & 56.14 \\
\hline 113.56 & 36.63 & 58.97 & 39.00 & 63.32 & 73.40 & 109.86 & 72.65 & 61.23 & 60.76 & 115.20 & 60.98 & 53.60 & 73.35 & 51.87 \\
\hline 113.47 & 41.76 & 60.16 & 39.34 & 63.08 & 74.33 & 109.94 & 92.80 & 53.53 & 61.81 & 114.80 & 56.65 & 53.43 & 77.35 & 54.15 \\
\hline 113.36 & 39.08 & 57.07 & 39.41 & 56.14 & 73.63 & 109.70 & 82.84 & 60.49 & 59.68 & 114.67 & 60.33 & 54.29 & 80.44 & 48.39 \\
\hline 113.58 & 34.94 & 58.62 & 40.00 & 62.68 & 74.11 & 109.32 & 65.90 & 59.69 & 59.42 & 114.44 & 61.42 & 53.40 & 71.64 & 65.13 \\
\hline 113.36 & 39.27 & 57.68 & 40.59 & 47.45 & 73.37 & 109.10 & 83.85 & 59.44 & 58.20 & 114.51 & 59.17 & 52.99 & 69.04 & 56.97 \\
\hline 114.00 & 41.82 & 59.39 & 39.22 & 63.74 & 74.00 & 109.37 & 61.27 & 60.52 & 58.50 & 115.02 & 62.14 & 53.82 & 88.29 & 64.59 \\
\hline 114.17 & 35.38 & 58.42 & 40.02 & 56.68 & 74.30 & 108.92 & 82.65 & 51.60 & 60.09 & 115.17 & 45.15 & 54.17 & 81.34 & 54.44 \\
\hline 113.36 & 35.20 & 57.11 & 40.19 & 64.61 & 73.64 & 109.84 & 79.39 & 57.06 & 60.01 & 115.06 & 51.61 & 53.86 & 77.98 & 65.24 \\
\hline 113.42 & 42.60 & 58.01 & 40.01 & 63.47 & 74.12 & 108.79 & 78.82 & 63.81 & 57.19 & 114.29 & 62.74 & 53.76 & 78.70 & 50.31 \\
\hline 113.21 & 37.94 & 57.35 & 39.31 & 47.96 & 73.56 & 108.84 & 77.92 & 55.66 & 58.93 & 114.78 & 55.91 & 53.36 & 65.59 & 46.51 \\
\hline 113.81 & 36.65 & 58.12 & 40.33 & 61.41 & 73.99 & 108.97 & 65.23 & 57.78 & 57.74 & 115.11 & 54.66 & 53.93 & 69.82 & 51.74 \\
\hline 113.86 & 39.95 & 58.13 & 39.98 & 56.15 & 73.47 & 109.13 & 75.82 & 58.34 & 61.22 & 114.82 & 56.06 & 53.92 & 72.71 & 55.00 \\
\hline 114.02 & 33.38 & 58.51 & 39.77 & 70.45 & 73.97 & 109.80 & 89.16 & 56.46 & 59.46 & 114.41 & 55.89 & 53.17 & 77.67 & 62.05 \\
\hline 114.11 & 37.88 & 59.15 & 39.71 & 59.68 & 73.76 & 108.95 & 77.30 & 57.80 & 59.62 & 114.25 & 62.36 & 53.13 & 92.33 & 57.25 \\
\hline 113.78 & 39.91 & 58.03 & 38.63 & 58.02 & 73.97 & 109.34 & 67.25 & 57.60 & 60.01 & 114.17 & 63.67 & 54.20 & 67.98 & 50.41 \\
\hline 113.77 & 39.85 & 58.71 & 39.40 & 67.47 & 74.10 & 109.79 & 68.38 & 55.21 & 57.94 & 114.98 & 55.54 & 53.60 & 72.32 & 56.86 \\
\hline 113.57 & 39.89 & 58.18 & 39.94 & 50.78 & 73.62 & 109.55 & 74.93 & 56.12 & 59.62 & 114.96 & 65.92 & 53.80 & 70.99 & 58.23 \\
\hline 113.92 & 42.30 & 58.81 & 40.31 & 61.39 & 73.75 & 109.87 & 64.55 & 59.30 & 60.06 & 115.00 & 44.11 & 53.60 & 68.00 & 50.70 \\
\hline 113.49 & 37.19 & 57.48 & 39.33 & 43.39 & 73.48 & 109.95 & 66.25 & 60.09 & 56.53 & 115.18 & 51.35 & 54.18 & 84.57 & 51.75 \\
\hline 113.64 & 41.28 & 58.58 & 40.24 & 52.92 & 73.58 & 109.51 & 69.04 & 57.36 & 58.52 & 114.83 & 59.68 & 53.87 & 66.92 & 52.92 \\
\hline
\end{tabular}

Table F.16: Throughput Simulation Scaled Packing Times (Overlapping Waves).

\begin{tabular}{rrrrrrrrrrrrrrrrrr}
\hline 1 & 2 & 3 & 4 & 5 & 6 & 7 & 8 & 9 & 10 & 11 & 12 & 13 \\
\hline 118.03 & 42.451 & 59.613 & 38.783 & Man. & 77.469 & 115.13 & Man. & Man. & 57.078 & 112.76 & Man. & 56.119 & Man. & Man. \\
117.98 & 39.511 & 60.235 & 38.652 & Man. & 78.009 & 114.42 & Man. & Man. & 58.04 & 110.78 & Man. & 56.919 & Man. & Man. \\
116.76 & 40.129 & 60.065 & 39.374 & Man. & 76.648 & 114.66 & Man. & Man. & 57.592 & 110.78 & Man. & 56.165 & Man. & Man. \\
118.91 & 40.55 & 59.8 & 38.927 & Man. & 75.157 & 115.99 & Man. & Man. & 57.941 & 112.03 & Man. & 55.575 & Man. & Man. \\
\hline
\end{tabular}


Table F.16: Throughput Simulation Scaled Packing Times (Overlapping Waves) - contin-

ued.

\begin{tabular}{|c|c|c|c|c|c|c|c|c|c|c|c|c|c|c|}
\hline 1 & 2 & 3 & 4 & 5 & 6 & 7 & 8 & 9 & 10 & 11 & 12 & 13 & 14 & 15 \\
\hline 117.64 & 39.544 & 59.769 & 39.828 & Man. & 77.719 & 115.27 & Man. & Man. & 57.537 & 111.4 & Man. & 56.733 & Man. & Man. \\
\hline 118.69 & 39.723 & 59.613 & 38.931 & Man. & 75.47 & 117.3 & Man. & Man. & 57.882 & 112.23 & Man. & 56.03 & Man. & Man. \\
\hline 117.5 & 41.141 & 59.943 & 38.49 & Man. & 76.077 & 116.97 & Man. & Man. & 58.025 & 110.43 & Man. & 55.916 & Man. & Man. \\
\hline 116.37 & 38.997 & 60.299 & 39.298 & Man. & 79.126 & 115.85 & Man. & Man. & 56.575 & 111.82 & Man. & 56.26 & Man. & Man. \\
\hline 117.15 & 39.619 & 60.649 & 39.246 & Man. & 78.441 & 117.13 & Man. & Man. & 56.863 & 111.09 & Man. & 56.084 & Man. & Man. \\
\hline 117.84 & 39.624 & 59.515 & 39.695 & Man. & 77.109 & 115.54 & Man. & Man. & 58.638 & 111.93 & Man. & 56.336 & Man. & Man. \\
\hline 117.2 & 39.55 & 59.73 & 39.081 & Man. & 78.101 & 116.6 & Man. & Man. & 57.559 & 112.22 & Man. & 56.528 & Man. & Man. \\
\hline 118.99 & 40.403 & 59.563 & 39.461 & Man. & 76.313 & 114.37 & Man. & Man. & 57.638 & 112.86 & Man. & 57.559 & Man. & Man. \\
\hline 117.7 & 39.391 & 60.051 & 39.514 & Man. & 77.047 & 117.05 & Man. & Man. & 58.305 & 112.54 & Man. & 55.465 & Man. & Man. \\
\hline 117.26 & 40.323 & 59.69 & 39.376 & Man. & 74.658 & 115.05 & Man. & Man. & 58.87 & 112.48 & Man. & 55.083 & Man. & Man. \\
\hline 118.45 & 40.464 & 60.308 & 39.738 & Man. & 77.435 & 115.34 & Man. & Man. & 59.134 & 113.94 & Man. & 56.624 & Man. & Man. \\
\hline 117.39 & 39.116 & 59.655 & 39.413 & Man. & 77.494 & 115.34 & Man. & Man. & 59.266 & 112.33 & Man. & 56.386 & Man. & Man. \\
\hline 117.79 & 40.217 & 59.955 & 38.743 & Man. & 77.726 & 113.94 & Man. & Man. & 58.195 & 112.07 & Man. & 55.668 & Man. & Man. \\
\hline 118.08 & 40.211 & 60.18 & 39.009 & Man. & 78.001 & 116.73 & Man. & Man. & 57.263 & 112.15 & Man. & 55.497 & Man. & Man. \\
\hline 117.37 & 39.028 & 59.907 & 39.616 & Man. & 76.794 & 115.3 & Man. & Man. & 59.421 & 111.8 & Man. & 56.676 & Man. & Man. \\
\hline 116.21 & 39.694 & 59.955 & 39.095 & Man. & 77.076 & 115.7 & Man. & Man. & 57.355 & 110.96 & Man. & 55.539 & Man. & Man. \\
\hline 116.8 & 40.723 & 59.749 & 39.322 & Man. & 75.991 & 112.23 & Man. & Man. & 56.051 & 110.32 & Man. & 55.126 & Man. & Man. \\
\hline 118.36 & 38.991 & 60.226 & 39.441 & Man. & 76.781 & 114.69 & Man. & Man. & 55.467 & 112.85 & Man. & 56.118 & Man. & Man. \\
\hline 115.78 & 40.339 & 59.832 & 39.344 & Man. & 76.06 & 117.35 & Man. & Man. & 57.115 & 112.66 & Man. & 56.424 & Man. & Man. \\
\hline 117.43 & 39.107 & 59.496 & 38.955 & Man. & 76.491 & 115.04 & Man. & Man. & 56.565 & 112.84 & Man. & 56.695 & Man. & Man. \\
\hline 116.39 & 40.333 & 59.707 & 39.693 & Man. & 77.606 & 115.74 & Man. & Man. & 58.077 & 113.15 & Man. & 55.004 & Man. & Man. \\
\hline 118.76 & 39.724 & 59.939 & 39.062 & Man. & 75.312 & 117.58 & Man. & Man. & 56.834 & 110.89 & Man. & 56.077 & Man. & Man. \\
\hline 118.86 & 40.15 & 60.195 & 38.465 & Man. & 78.085 & 114.79 & Man. & Man. & 58.611 & 112.09 & Man. & 55.645 & Man. & Man. \\
\hline 117.24 & 40.197 & 59.869 & 39.646 & Man. & 75.425 & 118.46 & Man. & Man. & 56.785 & 112.27 & Man. & 55.971 & Man. & Man. \\
\hline 117.02 & 39.336 & 59.942 & 39.451 & Man. & 77.463 & 112.7 & Man. & Man. & 57.116 & 111.56 & Man. & 55.643 & Man. & Man. \\
\hline 116.74 & 39.464 & 59.659 & 39.055 & Man. & 76.105 & 119.49 & Man. & Man. & 57.553 & 111.04 & Man. & 55.929 & Man. & Man. \\
\hline 117.05 & 38.832 & 60.115 & 39.392 & Man. & 77.12 & 112.66 & Man. & Man. & 59.151 & 112.07 & Man. & 55.868 & Man. & Man. \\
\hline 117.91 & 38.838 & 59.731 & 39.244 & Man. & 77.225 & 114.22 & Man. & Man. & 56.915 & 111.2 & Man. & 55.227 & Man. & Man. \\
\hline 116.24 & 39.439 & 59.85 & 38.567 & Man. & 76.289 & 113.41 & Man. & Man. & 57.331 & 110.19 & Man. & 55.751 & Man. & Man. \\
\hline 116.8 & 40.736 & 60.174 & 39.384 & Man. & 77.573 & 117.76 & Man. & Man. & 56.597 & 111.42 & Man. & 56.626 & Man. & Man. \\
\hline 117.64 & 41.431 & 59.977 & 38.506 & Man. & 77.187 & 114.95 & Man. & Man. & 57.714 & 110.37 & Man. & 55.439 & Man. & Man. \\
\hline 116.43 & 40.867 & 60.049 & 39.188 & Man. & 77.414 & 115.43 & Man. & Man. & 59.019 & 112.05 & Man. & 55.52 & Man. & Man. \\
\hline 115.78 & 39.863 & 60.11 & 38.938 & Man. & 79.986 & 115.4 & Man. & Man. & 58.393 & 111.85 & Man. & 56.146 & Man. & Man. \\
\hline 118.19 & 39.681 & 59.841 & 39.693 & Man. & 75.552 & 113.26 & Man. & Man. & 57.574 & 111.75 & Man. & 56.055 & Man. & Man. \\
\hline 116.84 & 40.367 & 59.786 & 39.351 & Man. & 77.302 & 114.03 & Man. & Man. & 59.337 & 113.51 & Man. & 56.145 & Man. & Man. \\
\hline 117.71 & 40.814 & 59.961 & 38.349 & Man. & 79.205 & 115.79 & Man. & Man. & 57.403 & 111.12 & Man. & 55.909 & Man. & Man. \\
\hline 115.23 & 39.39 & 59.772 & 39.461 & Man. & 78.625 & 115.64 & Man. & Man. & 56.99 & 111.2 & Man. & 56.046 & Man. & Man. \\
\hline 117.92 & 39.526 & 59.937 & 38.942 & Man. & 79.978 & 114.8 & Man. & Man. & 58.986 & 109.5 & Man. & 56.912 & Man. & Man. \\
\hline 117.13 & 39.806 & 59.786 & 38.39 & Man. & 78.76 & 114.2 & Man. & Man. & 57.871 & 112.92 & Man. & 56.975 & Man. & Man. \\
\hline 117.56 & 38.829 & 59.772 & 39.223 & Man. & 76.842 & 116.35 & Man. & Man. & 58.965 & 112.51 & Man. & 56.41 & Man. & Man. \\
\hline 115.98 & 39.896 & 59.513 & 39.035 & Man. & 75.608 & 112.56 & Man. & Man. & 60.662 & 110.67 & Man. & 56.915 & Man. & Man. \\
\hline 116.64 & 40.216 & 59.932 & 39.157 & Man. & 75.251 & 114.92 & Man. & Man. & 57.327 & 112.31 & Man. & 56.333 & Man. & Man. \\
\hline 115.26 & 41.335 & 60.058 & 38.648 & Man. & 78.489 & 115.7 & Man. & Man. & 59.477 & 112.07 & Man. & 56.038 & Man. & Man. \\
\hline 117.69 & 38.268 & 59.954 & 39.051 & Man. & 76.774 & 114.6 & Man. & Man. & 56.582 & 111.02 & Man. & 56.699 & Man. & Man. \\
\hline 116.55 & 39.47 & 59.67 & 39.877 & Man. & 78.245 & 114.79 & Man. & Man. & 56.176 & 111.78 & Man. & 55.653 & Man. & Man. \\
\hline 116.57 & 38.484 & 60.207 & 39.313 & Man. & 77.575 & 115.28 & Man. & Man. & 58.525 & 113.12 & Man. & 56.876 & Man. & Man. \\
\hline 116.16 & 39.062 & 59.992 & 39.313 & Man. & 76.088 & 116.27 & Man. & Man. & 58.159 & 112.29 & Man. & 54.833 & Man. & Man. \\
\hline 116.58 & 40.292 & 59.414 & 39.26 & Man. & 79.281 & 116.8 & Man. & Man. & 59.167 & 111.55 & Man. & 56.17 & Man. & Man. \\
\hline 117.46 & 39.914 & 60.053 & 39.583 & Man. & 76.884 & 115.79 & Man. & Man. & 56.998 & 113.04 & Man. & 55.536 & Man. & Man. \\
\hline 117.12 & 41.301 & 60.06 & 39.344 & Man. & 77.337 & 114.92 & Man. & Man. & 58.862 & 111.22 & Man. & 56.046 & Man. & Man. \\
\hline 117.09 & 39.524 & 59.328 & 38.841 & Man. & 78.272 & 115.11 & Man. & Man. & 56.513 & 112.06 & Man. & 55.665 & Man. & Man. \\
\hline 116.44 & 38.974 & 60.019 & 38.23 & Man. & 76.231 & 115.12 & Man. & Man. & 57.341 & 111.77 & Man. & 56.738 & Man. & Man. \\
\hline 117.26 & 40.854 & 59.53 & 40.091 & Man. & 78.131 & 113.55 & Man. & Man. & 57.957 & 111.27 & Man. & 57.512 & Man. & Man. \\
\hline 117.42 & 39.158 & 60.08 & 39.263 & Man. & 75.984 & 113.17 & Man. & Man. & 57.658 & 111.78 & Man. & 56.58 & Man. & Man. \\
\hline 118.48 & 39.421 & 59.674 & 38.394 & Man. & 77.076 & 114.97 & Man. & Man. & 57.957 & 112.45 & Man. & 56.611 & Man. & Man. \\
\hline 117.67 & 39.714 & 59.931 & 39.986 & Man. & 78.296 & 117.37 & Man. & Man. & 58.717 & 112.88 & Man. & 55.992 & Man. & Man. \\
\hline 116.39 & 39.803 & 59.871 & 38.99 & Man. & 76.826 & 113.57 & Man. & Man. & 57.562 & 112.69 & Man. & 55.643 & Man. & Man. \\
\hline 118.85 & 40.349 & 59.903 & 39.428 & Man. & 78.436 & 118.89 & Man. & Man. & 57.383 & 111.66 & Man. & 56.471 & Man. & Man. \\
\hline
\end{tabular}


Table F.16: Throughput Simulation Scaled Packing Times (Overlapping Waves) - contin-

ued.

\begin{tabular}{|c|c|c|c|c|c|c|c|c|c|c|c|c|c|c|}
\hline 1 & 2 & 3 & 4 & 5 & 6 & 7 & 8 & 9 & 10 & 11 & 12 & 13 & 14 & 15 \\
\hline 116.47 & 39.077 & 60.119 & 39 & Man. & 76.729 & 115.09 & Man. & Man. & 57.337 & 111.58 & Man. & 55.838 & Man. & Man. \\
\hline 116.17 & 39.951 & 59.682 & 39.225 & Man. & 77.473 & 117.71 & Man. & Man. & 57.808 & 111.76 & Man. & 57.103 & Man. & Man. \\
\hline 117.36 & 41.152 & 60.001 & 37.934 & Man. & 77.191 & 116.24 & Man. & Man. & 58.258 & 111.05 & Man. & 56.093 & Man. & Man. \\
\hline 118.33 & 39.288 & 59.936 & 38.717 & Man. & 75.993 & 115.49 & Man. & Man. & 58.148 & 112.58 & Man. & 54.955 & Man. & Man. \\
\hline 116.48 & 39.522 & 59.789 & 39.443 & Man. & 75.545 & 114.87 & Man. & Man. & 59.312 & 111.48 & Man. & 56.07 & Man. & Man. \\
\hline 117.47 & 39.646 & 59.941 & 38.847 & Man. & 77.007 & 115.42 & Man. & Man. & 56.619 & 111.88 & Man. & 54.875 & Man. & Man. \\
\hline 118.76 & 39.361 & 60.396 & 39.198 & Man. & 76.386 & 113.79 & Man. & Man. & 58.59 & 112.11 & Man. & 54.876 & Man. & Man. \\
\hline 118.4 & 39.39 & 60.06 & 39.032 & Man. & 77.925 & 116.98 & Man. & Man. & 57.07 & 111.94 & Man. & 56.034 & Man. & Man. \\
\hline 117.89 & 41.069 & 60.293 & 38.875 & Man. & 77.283 & 115.2 & Man. & Man. & 57.749 & 112.16 & Man. & 56.206 & Man. & Man. \\
\hline 117.07 & 39.36 & 59.561 & 39.826 & Man. & 75.353 & 115.45 & Man. & Man. & 58.707 & 112.44 & Man. & 55.553 & Man. & Man. \\
\hline 118.96 & 39.863 & 59.577 & 38.956 & Man. & 77.034 & 115.58 & Man. & Man. & 57.201 & 111.39 & Man. & 56.343 & Man. & Man. \\
\hline 114.86 & 40.733 & 59.813 & 39.335 & Man. & 75.902 & 117.61 & Man. & Man. & 57.965 & 112.34 & Man. & 54.757 & Man. & Man. \\
\hline 118.54 & 40.89 & 59.855 & 38.746 & Man. & 76.239 & 116.65 & Man. & Man. & 57.846 & 112.04 & Man. & 55.286 & Man. & Man. \\
\hline 116.7 & 39.817 & 59.683 & 39.612 & Man. & 78.601 & 117.83 & Man. & Man. & 57.147 & 112.3 & Man. & 57.177 & Man. & Man. \\
\hline 115.91 & 39.279 & 60.246 & 38.47 & Man. & 76.446 & 116.62 & Man. & Man. & 57.705 & 110.85 & Man. & 56.626 & Man. & Man. \\
\hline 119.39 & 39.619 & 59.931 & 39 & Man. & 72.379 & 114.99 & Man. & Man. & 57.5 & 111.24 & Man. & 54.054 & Man. & Man. \\
\hline 116.83 & 40.031 & 59.897 & 39.075 & Man. & 77.223 & 113.89 & Man. & Man. & 56.776 & 110.86 & Man. & 55.209 & Man. & Man. \\
\hline 117.8 & 39.488 & 59.837 & 39.037 & Man. & 77.788 & 114.74 & Man. & Man. & 57.243 & 111.36 & Man. & 57.282 & Man. & Man. \\
\hline 117.58 & 40.363 & 59.955 & 39.219 & Man. & 74.456 & 114.88 & Man. & Man. & 57.98 & 113.41 & Man. & 56.433 & Man. & Man. \\
\hline 115.76 & 41.044 & 60.086 & 38.893 & Man. & 77.311 & 115.03 & Man. & Man. & 55.644 & 112.49 & Man. & 55.91 & Man. & Man. \\
\hline 116.33 & 40.49 & 59.622 & 38.631 & Man. & 75.484 & 117.27 & Man. & Man. & 57.581 & 110.28 & Man. & 56.401 & Man. & Man. \\
\hline 116.96 & 41.103 & 60.001 & 39.163 & Man. & 74.873 & 113.79 & Man. & Man. & 58.259 & 112.62 & Man. & 54.962 & Man. & Man. \\
\hline 117.28 & 40.16 & 59.981 & 38.552 & Man. & 74.732 & 115.24 & Man. & Man. & 56.29 & 109.85 & Man. & 56.586 & Man. & Man. \\
\hline 116.42 & 39.408 & 59.686 & 39.777 & Man. & 77.143 & 118.21 & Man. & Man. & 59.003 & 109.93 & Man. & 56.098 & Man. & Man. \\
\hline 116.78 & 41.812 & 59.849 & 39.244 & Man. & 77.426 & 113.32 & Man. & Man. & 57.545 & 113.01 & Man. & 54.828 & Man. & Man. \\
\hline 116.27 & 40.795 & 59.642 & 39.452 & Man. & 77.055 & 115.17 & Man. & Man. & 56.453 & 111.55 & Man. & 55.665 & Man. & Man. \\
\hline 118.68 & 38.992 & 59.731 & 39.255 & Man. & 76.59 & 117.32 & Man. & Man. & 56.772 & 111.93 & Man. & 56.715 & Man. & Man. \\
\hline 117.97 & 39.912 & 59.744 & 38.449 & Man. & 77.115 & 116.53 & Man. & Man. & 56.642 & 110.96 & Man. & 55.859 & Man. & Man. \\
\hline 118.14 & 39.659 & 59.844 & 39.646 & Man. & 77.82 & 114.77 & Man. & Man. & 58.852 & 112.98 & Man. & 56.57 & Man. & Man. \\
\hline 117.8 & 40.071 & 59.924 & 40.256 & Man. & 76.662 & 115.47 & Man. & Man. & 54.96 & 111.45 & Man. & 57.156 & Man. & Man. \\
\hline 118.89 & 39.039 & 59.889 & 39.259 & Man. & 77.036 & 117.02 & Man. & Man. & 57.369 & 110.92 & Man. & 55.101 & Man. & Man. \\
\hline 116.59 & 40.262 & 60.042 & 38.783 & Man. & 79.377 & 115.17 & Man. & Man. & 59.049 & 111.79 & Man. & 56.13 & Man. & Man. \\
\hline 118.88 & 40.262 & 59.313 & 39.232 & Man. & 75.344 & 115.57 & Man. & Man. & 59.689 & 112.89 & Man. & 55.332 & Man. & Man. \\
\hline 116.68 & 41.411 & 59.814 & 39.238 & Man. & 75.784 & 115.74 & Man. & Man. & 58.602 & 112.55 & Man. & 56.214 & Man. & Man. \\
\hline 116.19 & 40.923 & 59.758 & 39.053 & Man. & 77.623 & 115.24 & Man. & Man. & 58.06 & 112.43 & Man. & 56.067 & Man. & Man. \\
\hline 118.86 & 39.663 & 59.955 & 39.723 & Man. & 74.77 & 114.02 & Man. & Man. & 56.267 & 111.52 & Man. & 57.323 & Man. & Man. \\
\hline 118 & 39.147 & 59.663 & 38.741 & Man. & 77.152 & 115.46 & Man. & Man. & 58.685 & 110.19 & Man. & 56.714 & Man. & Man. \\
\hline 117.3 & 39.893 & 60.129 & 39.508 & Man. & 75.282 & 114.36 & Man. & Man. & 57.115 & 111.94 & Man. & 56.092 & Man. & Man. \\
\hline
\end{tabular}

Table F.17: Throughput Simulation Scaled Picking Times (Non-Overlapping Waves).

\begin{tabular}{|c|c|c|c|c|c|c|c|c|c|c|c|c|c|c|}
\hline 1 & 2 & 3 & 4 & 5 & 6 & 7 & 8 & 9 & 10 & 11 & 12 & 13 & 14 & 15 \\
\hline 39.02 & 109.771 & 67.72 & 19.33 & 76.39 & 37.35 & 108.52 & 53.53 & 61.64 & 92.524 & 18.15 & 53.93 & 21.23 & 68.94 & 53.39 \\
\hline 39.32 & 92.5401 & 48.96 & 19.24 & 63.89 & 37.82 & 109.19 & 86 & 57.31 & 86.85 & 19.27 & 50.21 & 19.05 & 79.55 & 49.74 \\
\hline 39.88 & 101.02 & 57.4 & 19.69 & 63.7 & 37.33 & 109.81 & 91.78 & 57.01 & 97.219 & 18.64 & 50.88 & 19.45 & 67.21 & 41.31 \\
\hline 39.46 & 88.9527 & 65.04 & 20.59 & 57.32 & 36.55 & 109.93 & 62.03 & 59.37 & 80.903 & 18.71 & 55.73 & 20.56 & 79.13 & 55.12 \\
\hline 38.92 & 107.838 & 52.9 & 19.69 & 76.41 & 37.28 & 109.4 & 67.43 & 55.14 & 80.136 & 19.33 & 52.57 & 19.37 & 79.38 & 53.23 \\
\hline 39.01 & 98.6416 & 52.44 & 19.9 & 44.88 & 38.31 & 109.26 & 86.77 & 54.5 & 100.32 & 18.82 & 47.81 & 19.11 & 89.52 & 55.66 \\
\hline 39.31 & 104.341 & 68.71 & 18.65 & 63.77 & 37.42 & 109.38 & 62.25 & 58.21 & 97.256 & 19.36 & 41.96 & 18.86 & 70.66 & 53.91 \\
\hline 38.78 & 110.881 & 60.14 & 19.7 & 58.59 & 37.85 & 109.37 & 77.02 & 56.66 & 104.69 & 19.01 & 51.81 & 19.67 & 79.75 & 54.53 \\
\hline 39.18 & 104.107 & 62.01 & 19.97 & 67.1 & 37.85 & 109.66 & 66.54 & 66.91 & 92.252 & 19.67 & 46.04 & 18.77 & 77.25 & 45.88 \\
\hline 39.56 & 98.7624 & 56.64 & 17.97 & 59.07 & 37.19 & 109.88 & 75.05 & 57.71 & 105.24 & 20.61 & 58.53 & 18.98 & 66.66 & 57.37 \\
\hline 39.78 & 103.471 & 62.61 & 19.73 & 65.77 & 38.26 & 109.71 & 58.34 & 57.86 & 96.317 & 18.66 & 49.48 & 19.87 & 77.33 & 51.64 \\
\hline 39.26 & 89.8118 & 47.51 & 18.54 & 59.23 & 37.24 & 109.24 & 80.89 & 63.7 & 84.047 & 20.15 & 49.12 & 19 & 73.88 & 66.7 \\
\hline 39.44 & 91.0965 & 59.79 & 19.63 & 64.2 & 37.17 & 109.82 & 73.05 & 58.26 & 93.544 & 17.13 & 59.86 & 19.87 & 60.48 & 52.47 \\
\hline 39.13 & 89.5472 & 57.09 & 20.2 & 55.75 & 37.66 & 109.7 & 79.1 & 58.32 & 98.105 & 20.21 & 61.82 & 19.32 & 71.12 & 47.98 \\
\hline 40.12 & 87.6955 & 61.41 & 18.97 & 60.27 & 37.27 & 109.8 & 73.17 & 54.64 & 89.573 & 18.58 & 57.59 & 18.25 & 67.11 & 51.14 \\
\hline
\end{tabular}


Table F.17: Throughput Simulation Scaled Picking Times (Non-Overlapping Waves) continued.

\begin{tabular}{|c|c|c|c|c|c|c|c|c|c|c|c|c|c|c|}
\hline 1 & 2 & 3 & 4 & 5 & 6 & 7 & 8 & 9 & 10 & 11 & 12 & 13 & 14 & 15 \\
\hline 39.13 & 106.503 & 60.71 & 19.7 & 61.97 & 38.1 & 109.43 & 86.8 & 50.55 & 88.282 & 20.16 & 54.69 & 20.81 & 83.83 & 49.14 \\
\hline 39.54 & 95.6937 & 64.19 & 19.22 & 50.21 & 37 & 109.58 & 79.13 & 59.11 & 93.976 & 18.59 & 55.74 & 19.58 & 82.3 & 44.69 \\
\hline 38.76 & 106.371 & 63.67 & 19.51 & 67.85 & 36.85 & 109.26 & 76.47 & 57.26 & 100.25 & 19.28 & 61.44 & 19.74 & 76.91 & 59.72 \\
\hline 39.95 & 88.8949 & 72.2 & 17.77 & 56.78 & 37.58 & 109.1 & 77.09 & 60.56 & 93.26 & 20.56 & 69.49 & 18.36 & 84.16 & 45.26 \\
\hline 39.07 & 110.456 & 56.9 & 18.61 & 71.16 & 37.73 & 109.2 & 67.57 & 58.29 & 78.703 & 18.88 & 56.15 & 20.91 & 82.97 & 59.08 \\
\hline 38.17 & 93.0562 & 63.95 & 18.09 & 58.23 & 38.47 & 109.29 & 80.08 & 58.03 & 99.222 & 19.52 & 53.53 & 18.76 & 60.06 & 57.3 \\
\hline 39.07 & 97.5033 & 54.38 & 20.72 & 46.83 & 39.09 & 109.72 & 76.21 & 56.76 & 97.398 & 19.24 & 56.03 & 18.89 & 83.57 & 42.13 \\
\hline 40.12 & 96.589 & 67.56 & 19.71 & 60.89 & 36.2 & 109.5 & 63.66 & 57.96 & 81.798 & 20.59 & 53.27 & 20.85 & 77.03 & 56.08 \\
\hline 38.9 & 92.5648 & 63.45 & 19.42 & 56.05 & 38.07 & 109.18 & 88.9 & 56.54 & 94.22 & 17.85 & 59.35 & 20.27 & 74.07 & 61.62 \\
\hline 40.02 & 91.4534 & 64.6 & 19.12 & 65.55 & 38.07 & 108.81 & 52.37 & 51.3 & 89.685 & 19.28 & 52.52 & 19.34 & 70.82 & 44.97 \\
\hline 39.49 & 90.6439 & 67.14 & 19.68 & 55.73 & 37.31 & 108.96 & 86.71 & 58.44 & 91.511 & 19.64 & 61.63 & 18.64 & 76.83 & 50.79 \\
\hline 39.58 & 101.915 & 55.73 & 19.86 & 59.71 & 36.91 & 109.71 & 79.36 & 59.92 & 91.459 & 18.42 & 51.8 & 17.89 & 64.86 & 48.66 \\
\hline 38.77 & 118.413 & 54.89 & 18.87 & 73.26 & 37.8 & 109.2 & 82.88 & 58.65 & 94.83 & 21.69 & 53.27 & 19.58 & 76.07 & 48.68 \\
\hline 39.17 & 91.4432 & 56.34 & 17.83 & 56.53 & 37.49 & 109.96 & 72.18 & 61.25 & 88.169 & 18.75 & 51.93 & 19.81 & 81.9 & 60.36 \\
\hline 39.75 & 96.0741 & 66.11 & 20.29 & 57.09 & 37.37 & 109.46 & 78.96 & 58.41 & 94.689 & 19.67 & 53.81 & 19.47 & 75.45 & 54.42 \\
\hline 38.42 & 96.8714 & 62.78 & 20.34 & 45.13 & 38.07 & 109.65 & 80.1 & 54.26 & 90.763 & 19.17 & 56.86 & 19.33 & 72.59 & 50.95 \\
\hline 40.23 & 94.741 & 63.67 & 19.11 & 55.71 & 37.37 & 108.9 & 82.76 & 58.28 & 88.985 & 19.53 & 54.83 & 19.97 & 78.03 & 45.02 \\
\hline 39.13 & 97.1351 & 67.53 & 19.9 & 67.32 & 38.58 & 109.46 & 74.85 & 56.88 & 86.948 & 22 & 58.39 & 19.3 & 86.59 & 47.91 \\
\hline 39.6 & 95.3865 & 55.58 & 20.65 & 65.22 & 37.47 & 108.47 & 77.09 & 57.8 & 80.599 & 20.16 & 49.07 & 18.83 & 82.15 & 54.95 \\
\hline 39.52 & 93.6732 & 67.78 & 20.56 & 69.71 & 38.42 & 109.09 & 61.3 & 53.67 & 92.227 & 18.6 & 48.76 & 21.03 & 70.85 & 57.42 \\
\hline 38.9 & 104.559 & 69.62 & 19.65 & 66.91 & 39.15 & 109.8 & 75.43 & 55.38 & 94.397 & 18.23 & 58.09 & 19.61 & 68.92 & 51.94 \\
\hline 39.92 & 98.9651 & 59.49 & 18.73 & 50.36 & 37.64 & 109.3 & 77.4 & 48.9 & 74.272 & 20.15 & 48.71 & 19.86 & 81.77 & 56.62 \\
\hline 40.33 & 107.104 & 51.94 & 17.55 & 57.81 & 37.42 & 109.7 & 72.79 & 56.89 & 88.383 & 18.81 & 59.14 & 19.42 & 73.46 & 52.13 \\
\hline 39.23 & 90.6587 & 59.32 & 20.28 & 60.2 & 36.27 & 109.44 & 70.71 & 54.23 & 102.78 & 19.86 & 62.98 & 20.5 & 79.67 & 55.39 \\
\hline 38.97 & 88.0106 & 63.48 & 19.66 & 60.7 & 37.54 & 109.58 & 80.1 & 55.49 & 99.664 & 19.32 & 49.29 & 19.1 & 69.61 & 51.28 \\
\hline 39.2 & 86.128 & 57.52 & 19.45 & 59.38 & 37.99 & 109.61 & 85.3 & 58.73 & 89.627 & 18.47 & 53.44 & 19 & 82.37 & 49.43 \\
\hline 38.88 & 109.513 & 54.46 & 19.84 & 63.35 & 38.26 & 109.93 & 74.9 & 61.12 & 80.62 & 19.96 & 53.62 & 21.85 & 72.52 & 63.99 \\
\hline 39.82 & 101.652 & 62.74 & 19.17 & 61.59 & 37.77 & 109.83 & 81.42 & 56.23 & 93.433 & 20.4 & 45.04 & 20.83 & 78.59 & 66.75 \\
\hline 39.57 & 104.407 & 54.26 & 19.82 & 57.57 & 36.35 & 110 & 77.48 & 61.66 & 100.69 & 18.65 & 57.71 & 18.07 & 78.33 & 51.36 \\
\hline 39.84 & 100.445 & 72.8 & 19.89 & 61.71 & 36.72 & 108.75 & 86.64 & 58.04 & 93.893 & 18.52 & 48.58 & 18.17 & 89.89 & 65.03 \\
\hline 39.44 & 95.8998 & 66.07 & 18.97 & 62.01 & 37.44 & 109.62 & 83.59 & 62.27 & 91.165 & 19.13 & 48.35 & 19.02 & 66.39 & 49.62 \\
\hline 38.15 & 89.1839 & 47.02 & 20.31 & 53.39 & 37.24 & 109.39 & 68.2 & 54.7 & 88.202 & 19.8 & 49.22 & 20.89 & 79.9 & 52.34 \\
\hline 39.66 & 113.584 & 60.85 & 19.48 & 70.37 & 37.64 & 109.07 & 79.33 & 59.31 & 98.614 & 19.07 & 55.74 & 18.05 & 74.97 & 57.42 \\
\hline 39.57 & 104.443 & 55.72 & 20.54 & 63.73 & 36.7 & 109.84 & 65.18 & 68.44 & 99.74 & 18.06 & 61.53 & 19.28 & 71.42 & 57.56 \\
\hline 38.44 & 101.87 & 56.03 & 20.13 & 63.39 & 38.29 & 109.21 & 80 & 61.03 & 56.285 & 19.96 & 48.99 & 18.78 & 75.37 & 49.88 \\
\hline 39.67 & 95.7448 & 57.63 & 19.2 & 55.83 & 37.65 & 109.27 & 72.62 & 61.62 & 94.761 & 20.38 & 45.58 & 20.54 & 78.36 & 46.92 \\
\hline 39.79 & 95.6011 & 64.26 & 18.04 & 51.57 & 37.62 & 109.27 & 70.72 & 52.61 & 81.464 & 19.36 & 51.86 & 18.31 & 86.92 & 44.59 \\
\hline 39.46 & 104.739 & 54.74 & 18.62 & 54.53 & 37.22 & 109.28 & 69.42 & 50.77 & 81.099 & 18.34 & 52.49 & 19.88 & 71.73 & 54.03 \\
\hline 39.4 & 110.277 & 49.72 & 18.68 & 50.48 & 36.53 & 109.16 & 69.9 & 57.68 & 105.78 & 19.72 & 58.64 & 19.37 & 63.79 & 56.59 \\
\hline 39.19 & 107.811 & 59.16 & 18.84 & 52.17 & 37.42 & 110.01 & 76.46 & 56.35 & 91.001 & 19.96 & 65.34 & 19.73 & 69.7 & 42.2 \\
\hline 38.15 & 97.1264 & 67.62 & 19.13 & 54.27 & 36.98 & 108.72 & 71.09 & 58.88 & 87.91 & 19.13 & 53.22 & 19.73 & 79.39 & 58.67 \\
\hline 39.24 & 100.966 & 67.91 & 20.86 & 64.87 & 37.32 & 109.86 & 82.29 & 53.13 & 92.189 & 20.35 & 52.61 & 19.16 & 75.68 & 55.65 \\
\hline 39.48 & 100.215 & 59.09 & 20.13 & 68.61 & 38.23 & 109.38 & 72.51 & 49 & 87.516 & 18.21 & 51.15 & 18.73 & 89.81 & 50.56 \\
\hline 39.43 & 111.05 & 64.35 & 19.99 & 64.15 & 37.44 & 109.36 & 82.64 & 54.33 & 96.826 & 19.19 & 68.23 & 17.89 & 83.83 & 64.52 \\
\hline 39.56 & 101.655 & 57.85 & 19.38 & 58.82 & 38.38 & 109.6 & 76.47 & 53.11 & 102.67 & 19.61 & 50.77 & 19.08 & 82.18 & 56.59 \\
\hline 39.26 & 116.38 & 54.02 & 19.42 & 57.54 & 37.43 & 109.13 & 86.76 & 59.33 & 89.09 & 18.73 & 59.27 & 19.24 & 78.83 & 55.14 \\
\hline 38.68 & 105.645 & 59.89 & 18.86 & 51.83 & 37.98 & 109.25 & 85.33 & 59.48 & 82.983 & 18.95 & 57.97 & 19.03 & 72.72 & 57.63 \\
\hline 39.09 & 98.5079 & 60.68 & 20.57 & 60.91 & 37.48 & 109.56 & 78.34 & 50.59 & 99.362 & 20 & 64.54 & 18.48 & 59.51 & 48.75 \\
\hline 39.39 & 107.741 & 57.76 & 18.87 & 64.64 & 37.19 & 108.69 & 78.79 & 61.66 & 94.612 & 19.71 & 50.76 & 18.72 & 72.16 & 60.28 \\
\hline 39.49 & 113.743 & 68.35 & 20.25 & 62.61 & 37.89 & 109 & 56.65 & 60.05 & 88.287 & 18.8 & 44.4 & 19.26 & 89.12 & 59.04 \\
\hline 39.52 & 97.9526 & 55.37 & 19.45 & 53.57 & 38.55 & 109.67 & 82.29 & 61.23 & 100.45 & 18.37 & 64.16 & 19.86 & 82.05 & 56.23 \\
\hline 39.77 & 103.76 & 62.48 & 18.05 & 59.92 & 37.36 & 108.99 & 85.93 & 51.17 & 88.947 & 18.33 & 52.17 & 20.17 & 66.51 & 66.13 \\
\hline 39.9 & 101.537 & 57.13 & 19.7 & 66.74 & 37.25 & 109.81 & 78.27 & 53.65 & 84.899 & 19.79 & 46.08 & 18.88 & 88.62 & 64.14 \\
\hline 39.24 & 77.9577 & 55.73 & 17.49 & 73.11 & 38.16 & 109.81 & 79.82 & 54.14 & 87.741 & 19.35 & 53.63 & 19.64 & 87.44 & 56.61 \\
\hline 39.81 & 92.6132 & 58.18 & 18.8 & 62.43 & 37.05 & 109.31 & 85.12 & 61.78 & 103.27 & 17.91 & 60.94 & 19.56 & 86.21 & 52.78 \\
\hline 39.24 & 107.607 & 55.96 & 19.39 & 51.77 & 38.33 & 109.46 & 70.06 & 53.64 & 87.98 & 19.63 & 58.3 & 19.65 & 76.67 & 62.76 \\
\hline 38.94 & 92.422 & 59.48 & 19.78 & 53.73 & 37.32 & 109.66 & 88.75 & 54.48 & 96.618 & 19.64 & 57.71 & 20.82 & 70.31 & 57.79 \\
\hline 39.41 & 86.0805 & 53.91 & 16.39 & 54.15 & 37.82 & 109.4 & 64.96 & 57 & 90.244 & 18.78 & 66.95 & 18.34 & 68.49 & 74.19 \\
\hline
\end{tabular}


Table F.17: Throughput Simulation Scaled Picking Times (Non-Overlapping Waves) continued.

\begin{tabular}{|c|c|c|c|c|c|c|c|c|c|c|c|c|c|c|}
\hline 1 & 2 & 3 & 4 & 5 & 6 & 7 & 8 & 9 & 10 & 11 & 12 & 13 & 14 & 15 \\
\hline 39.59 & 92.6521 & 68.28 & 19.04 & 61.11 & 38.24 & 108.7 & 76.85 & 54.73 & 107.44 & 18.32 & 49.47 & 19.12 & 77.81 & 50.37 \\
\hline 39.62 & 87.6035 & 56.59 & 19.47 & 56.19 & 37.08 & 109.73 & 76.68 & 58 & 97.62 & 20.14 & 48.48 & 19.52 & 62.95 & 52.82 \\
\hline 39.61 & 110.647 & 62.69 & 19.7 & 49.97 & 38.55 & 109.8 & 68.05 & 62.34 & 97.538 & 20.56 & 52.57 & 19.68 & 73.6 & 60.35 \\
\hline 38.85 & 97.7612 & 68.7 & 19.74 & 52.21 & 36.97 & 109.69 & 92.47 & 57.82 & 97.265 & 19.28 & 61.08 & 18.6 & 79.03 & 45.69 \\
\hline 39.41 & 108.66 & 58.03 & 20.46 & 68.89 & 36.89 & 109.77 & 83.35 & 64.8 & 105.8 & 19.65 & 52.09 & 20.14 & 85.87 & 66.03 \\
\hline 39.45 & 96.91 & 56.7 & 18.25 & 52.95 & 37.76 & 109.02 & 82.05 & 64.2 & 96.127 & 18.68 & 48.91 & 19.16 & 73.58 & 47.44 \\
\hline 40.41 & 112.633 & 58.45 & 20.15 & 74.65 & 38.39 & 109.62 & 60.67 & 56.91 & 96.982 & 20.07 & 55.21 & 19.27 & 75.67 & 59.2 \\
\hline 39.34 & 97.5731 & 58.52 & 19.51 & 63.93 & 38.49 & 109.86 & 79.14 & 53.55 & 82.948 & 19.25 & 56.33 & 19.92 & 84.18 & 60.73 \\
\hline 38.9 & 107.277 & 61.13 & 19.54 & 68.07 & 38.24 & 109.94 & 79.83 & 54.39 & 106.32 & 19.57 & 51.45 & 19.93 & 69.94 & 47.73 \\
\hline 39.18 & 92.2154 & 60.22 & 19.62 & 68.59 & 38.16 & 109.7 & 86.43 & 62.34 & 90.627 & 19.34 & 56.82 & 17.72 & 81.65 & 53.62 \\
\hline 39.92 & 89.0886 & 56.39 & 20.47 & 58.16 & 37.04 & 109.32 & 66.41 & 57.55 & 99.566 & 19.56 & 42.29 & 18.89 & 81.84 & 55.73 \\
\hline 39.17 & 104.795 & 59.39 & 19.17 & 63.41 & 36.77 & 109.1 & 76.43 & 58.55 & 105.5 & 18.93 & 56.35 & 19.36 & 77.3 & 51.13 \\
\hline 38.87 & 90.3038 & 60.8 & 18.83 & 55.39 & 38.25 & 109.37 & 77.89 & 60.58 & 102.28 & 19.47 & 51.49 & 19.27 & 80.9 & 61.32 \\
\hline 38.63 & 110.501 & 57.96 & 18.94 & 64.38 & 37.66 & 108.92 & 66.28 & 57.4 & 100.9 & 19.07 & 50.13 & 19.55 & 73.94 & 57.57 \\
\hline 39.18 & 110.185 & 64.88 & 19.64 & 52.59 & 36.97 & 109.84 & 73.1 & 57.53 & 88.4 & 19.02 & 49.98 & 17.77 & 74.53 & 53.83 \\
\hline 38.66 & 109.475 & 58.4 & 19.97 & 57.85 & 37.96 & 108.79 & 62.97 & 54 & 99.044 & 20 & 49.72 & 19.38 & 83.2 & 58.46 \\
\hline 39.86 & 72.9368 & 61.93 & 18.82 & 55.75 & 38.72 & 108.84 & 79.64 & 67.38 & 91.876 & 19.1 & 53.35 & 18.44 & 68.1 & 49.04 \\
\hline 39.86 & 92.799 & 70.78 & 21.04 & 59.74 & 38.4 & 108.97 & 82.27 & 60 & 102.49 & 19.89 & 48.26 & 20.01 & 84.05 & 54.24 \\
\hline 39.51 & 107.656 & 63.24 & 21.1 & 68.36 & 36.87 & 109.13 & 74.36 & 58.64 & 85.997 & 19.87 & 57.5 & 19.44 & 81.21 & 47.77 \\
\hline 39.98 & 104.212 & 64.15 & 18.43 & 59.08 & 38.35 & 109.8 & 79.48 & 60.07 & 94.99 & 17.85 & 52.93 & 19.27 & 83.76 & 53.73 \\
\hline 40.49 & 102.03 & 68.07 & 20.16 & 63.66 & 37.57 & 108.95 & 84.48 & 58.05 & 92.276 & 18.77 & 58.52 & 19.01 & 67.2 & 52.32 \\
\hline 38.86 & 98.5128 & 61.17 & 17.68 & 62.93 & 37.77 & 109.34 & 90 & 55.39 & 105.06 & 19.53 & 62.99 & 19.04 & 67.01 & 44.06 \\
\hline 39.24 & 101.603 & 66.17 & 19.16 & 61.53 & 37.71 & 109.79 & 86.37 & 56.83 & 86.341 & 17.71 & 60.44 & 19.28 & 78.23 & 52.72 \\
\hline 39.47 & 102.93 & 57.45 & 18.57 & 61.31 & 38.03 & 109.55 & 70.9 & 65.13 & 102.68 & 21.27 & 52.49 & 19.2 & 81.96 & 50.92 \\
\hline 39.06 & 104.83 & 63.24 & 19.71 & 63.18 & 37.37 & 109.87 & 67.23 & 54.41 & 109.06 & 20.55 & 64.12 & 20.27 & 81.64 & 59.53 \\
\hline 38.34 & 104.896 & 50.24 & 19.13 & 59.29 & 36.47 & 109.95 & 68.02 & 56.52 & 100.24 & 21.32 & 58.9 & 20.05 & 76.04 & 43.85 \\
\hline 39.54 & 92.0295 & 59.68 & 18.64 & 53.83 & 38.04 & 109.51 & 79.2 & 55.48 & 104.92 & 19.71 & 54.22 & 19.84 & 79.34 & 54.8 \\
\hline
\end{tabular}

Table F.18: Throughput Simulation Scaled Packing Times (Non-Overlapping Waves).

\begin{tabular}{|c|c|c|c|c|c|c|c|c|c|c|c|c|c|c|}
\hline 1 & 2 & 3 & 4 & 5 & 6 & 7 & 8 & 9 & 10 & 11 & 12 & 13 & 14 & 15 \\
\hline 38.605 & Man. & Man. & 19.874 & Man. & 38.954 & 121.7 & Man. & Man. & Man. & 20.686 & Man. & 22.233 & Man. & Man. \\
\hline 41.149 & Man. & Man. & 20.253 & Man. & 40.357 & 119.04 & Man. & Man. & Man. & 20.052 & Man. & 19.378 & Man. & Man. \\
\hline 35.874 & Man. & Man. & 19.385 & Man. & 39.065 & 119.24 & Man. & Man. & Man. & 19.639 & Man. & 20.638 & Man. & Man. \\
\hline 39.397 & Man. & Man. & 18.382 & Man. & 34.26 & 121.6 & Man. & Man. & Man. & 21.668 & Man. & 18.491 & Man. & Man. \\
\hline 38.253 & Man. & Man. & 20.21 & Man. & 36.877 & 118.82 & Man. & Man. & Man. & 20.258 & Man. & 21.274 & Man. & Man. \\
\hline 39.744 & Man. & Man. & 20.096 & Man. & 41.377 & 118.85 & Man. & Man. & Man. & 22.984 & Man. & 19.227 & Man. & Man. \\
\hline 39.814 & Man. & Man. & 20.004 & Man. & 41.131 & 117.02 & Man. & Man. & Man. & 19.879 & Man. & 19.901 & Man. & Man. \\
\hline 38.13 & Man. & Man. & 19.893 & Man. & 41.198 & 114.66 & Man. & Man. & Man. & 20.247 & Man. & 19.65 & Man. & Man. \\
\hline 38.859 & Man. & Man. & 21.543 & Man. & 40.669 & 117.28 & Man. & Man. & Man. & 20.218 & Man. & 19.764 & Man. & Man. \\
\hline 38.88 & Man. & Man. & 19.702 & Man. & 39.953 & 118.83 & Man. & Man. & Man. & 19.51 & Man. & 19.46 & Man. & Man. \\
\hline 40.623 & Man. & Man. & 19.667 & Man. & 39.448 & 120.28 & Man. & Man. & Man. & 20.592 & Man. & 20.448 & Man. & Man. \\
\hline 38.988 & Man. & Man. & 20.231 & Man. & 37.312 & 119.38 & Man. & Man. & Man. & 23.124 & Man. & 20.152 & Man. & Man. \\
\hline 37.115 & Man. & Man. & 19.172 & Man. & 40.457 & 122.16 & Man. & Man. & Man. & 18.533 & Man. & 20.669 & Man. & Man. \\
\hline 37.732 & Man. & Man. & 19.579 & Man. & 39.386 & 121.38 & Man. & Man. & Man. & 20.577 & Man. & 20.814 & Man. & Man. \\
\hline 37.563 & Man. & Man. & 19.585 & Man. & 41.635 & 121.39 & Man. & Man. & Man. & 19.736 & Man. & 21.135 & Man. & Man. \\
\hline 38.989 & Man. & Man. & 20.581 & Man. & 39.566 & 122.07 & Man. & Man. & Man. & 20.836 & Man. & 19.796 & Man. & Man. \\
\hline 36.919 & Man. & Man. & 19.835 & Man. & 40.299 & 119.09 & Man. & Man. & Man. & 20.063 & Man. & 20.785 & Man. & Man. \\
\hline 35.886 & Man. & Man. & 20.692 & Man. & 37.955 & 120.79 & Man. & Man. & Man. & 19.975 & Man. & 20.755 & Man. & Man. \\
\hline 37.132 & Man. & Man. & 20.938 & Man. & 39.602 & 117.94 & Man. & Man. & Man. & 20.14 & Man. & 20.733 & Man. & Man. \\
\hline 38.924 & Man. & Man. & 20.82 & Man. & 40.067 & 121.08 & Man. & Man. & Man. & 20.623 & Man. & 20.279 & Man. & Man. \\
\hline 36.214 & Man. & Man. & 20.729 & Man. & 40.777 & 121.7 & Man. & Man. & Man. & 19.554 & Man. & 20.238 & Man. & Man. \\
\hline 38.637 & Man. & Man. & 19.79 & Man. & 39.744 & 118.3 & Man. & Man. & Man. & 21.444 & Man. & 21.563 & Man. & Man. \\
\hline 37.534 & Man. & Man. & 20.974 & Man. & 41.573 & 118.99 & Man. & Man. & Man. & 20.915 & Man. & 21.65 & Man. & Man. \\
\hline 37.649 & Man. & Man. & 20.104 & Man. & 38.467 & 123.17 & Man. & Man. & Man. & 19.749 & Man. & 19.69 & Man. & Man. \\
\hline 39.281 & Man. & Man. & 19.921 & Man. & 38.405 & 123.69 & Man. & Man. & Man. & 20.138 & Man. & 18.616 & Man. & Man. \\
\hline 37.977 & Man. & Man. & 20.09 & Man. & 40.581 & 119.87 & Man. & Man. & Man. & 20.145 & Man. & 20.134 & Man. & Man. \\
\hline
\end{tabular}


Table F.18: Throughput Simulation Scaled Packing Times (Non-Overlapping Waves) continued.

\begin{tabular}{|c|c|c|c|c|c|c|c|c|c|c|c|c|c|c|}
\hline 1 & 2 & 3 & 4 & 5 & 6 & 7 & 8 & 9 & 10 & 11 & 12 & 13 & 14 & 15 \\
\hline 39.063 & Man. & Man. & 20.01 & Man. & 39.966 & 120.99 & Man. & Man. & Man. & 19.566 & Man. & 22.338 & Man. & Man \\
\hline 38.092 & Man. & Man. & 19.846 & Man. & 43.633 & 119.23 & Man. & Man. & Man. & 20.503 & Man. & 19.93 & Man. & Man \\
\hline 38.669 & Man. & Man. & 20.077 & Man. & 40.232 & 117.93 & Man. & Man. & Man. & 19.4 & Man. & 20.242 & Man. & Man \\
\hline 38.491 & Man. & Man. & 21.213 & Man. & 41.448 & 117.45 & Man. & Man. & Man. & 20.576 & Man. & 19.052 & Man. & Man \\
\hline 39.081 & Man. & Man. & 20.037 & Man. & 37.114 & 120.42 & Man. & Man. & Man. & 21.388 & Man. & 20.765 & Man. & Man \\
\hline 38.212 & Man. & Man. & 18.622 & Man. & 38.019 & 114.23 & Man. & Man. & Man. & 20.557 & Man. & 22.482 & Man. & Man \\
\hline 38.405 & Man. & Man. & 20.694 & Man. & 39.555 & 117.55 & Man. & Man. & Man. & 19.695 & Man. & 21.633 & Man. & Man \\
\hline 38.814 & Man. & Man. & 20.097 & Man. & 37.527 & 120.25 & Man. & Man. & Man. & 20.101 & Man. & 17.775 & Man. & Man \\
\hline 39.295 & Man. & Man. & 19.93 & Man. & 37.713 & 115.77 & Man. & Man. & Man. & 20.807 & Man. & 20.921 & Man. & Man \\
\hline 38.181 & Man. & Man. & 19.298 & Man. & 42.605 & 114.85 & Man. & Man. & Man. & 21.791 & Man. & 20.786 & Man. & Man \\
\hline 37.673 & Man. & Man. & 20.923 & Man. & 38.822 & 117.7 & Man. & Man. & Man. & 20.288 & Man. & 20.802 & Man. & Man \\
\hline 37.935 & Man. & Man. & 21.16 & Man. & 39.93 & 120.05 & Man. & Man. & Man. & 20.349 & Man. & 19.957 & Man. & Man \\
\hline 37.64 & Man. & Man. & 19.989 & Man. & 36.488 & 118.79 & Man. & Man. & Man. & 18.951 & Man. & 21.196 & Man. & Man \\
\hline 38.706 & Man. & Man. & 20.324 & Man. & 39.784 & 113.73 & Man. & Man. & Man. & 21.792 & Man. & 20.408 & Man. & Man \\
\hline 36.74 & Man. & Man. & 20.669 & Man. & 38.268 & 120.05 & Man. & Man. & Man. & 21.206 & Man. & 20.857 & Man. & Man \\
\hline 38.321 & Man. & Man. & 20.97 & Man. & 39.859 & 119.8 & Man. & Man. & Man. & 19.862 & Man. & 20.569 & Man. & Man \\
\hline 38.64 & Man. & Man. & 20.22 & Man. & 42.091 & 118.86 & Man. & Man. & Man. & 19.919 & Man. & 19.417 & Man. & Man \\
\hline 37.516 & Man. & Man. & 19.805 & Man. & 40.797 & 120.32 & Man. & Man. & Man. & 20.598 & Man. & 19.53 & Man. & Man \\
\hline 38.346 & Man. & Man. & 20.441 & Man. & 39.354 & 124.36 & Man. & Man. & Man. & 19.576 & Man. & 18.051 & Man. & Man \\
\hline 39.459 & Man. & Man. & 20.661 & Man. & 38.489 & 116.8 & Man. & Man. & Man. & 18.555 & Man. & 19.607 & Man. & Man \\
\hline 38.374 & Man. & Man. & 19.582 & Man. & 41.816 & 121.44 & Man. & Man. & Man. & 20.223 & Man. & 21.979 & Man. & Man \\
\hline 39.496 & Man. & Man. & 18.122 & Man. & 39.479 & 118.76 & Man. & Man. & Man. & 21.499 & Man. & 20.649 & Man. & Man \\
\hline 37.696 & Man. & Man. & 20.117 & Man. & 41.686 & 108.21 & Man. & Man. & Man. & 20.246 & Man. & 20.544 & Man. & Man \\
\hline 38.175 & Man. & Man. & 20.341 & Man. & 42.154 & 117.06 & Man. & Man. & Man. & 21.982 & Man. & 18.985 & Man. & Man \\
\hline 38.651 & Man. & Man. & 20.775 & Man. & 35.613 & 115.92 & Man. & Man. & Man. & 20.923 & Man. & 20.377 & Man. & Man \\
\hline 38.991 & Man. & Man. & 19.744 & Man. & 38.128 & 117.58 & Man. & Man. & Man. & 18.931 & Man. & 21.483 & Man. & Man \\
\hline 38.614 & Man. & Man. & 19.995 & Man. & 39.041 & 119.33 & Man. & Man. & Man. & 21.291 & Man. & 20.305 & Man. & Man \\
\hline 38.64 & Man. & Man. & 20.292 & Man. & 38.58 & 119.01 & Man. & Man. & Man. & 20.846 & Man. & 21.681 & Man. & Man \\
\hline 40.114 & Man. & Man. & 20.068 & Man. & 40.584 & 119.39 & Man. & Man. & Man. & 20.417 & Man. & 20.775 & Man. & Man \\
\hline 38.948 & Man. & Man. & 20.561 & Man. & 39.15 & 119.21 & Man. & Man. & Man. & 19.416 & Man. & 19.8 & Man. & Man \\
\hline 37.72 & Man. & Man. & 20.188 & Man. & 38.969 & 113.91 & Man. & Man. & Man. & 22.525 & Man. & 20.561 & Man. & Man \\
\hline 37.615 & Man. & Man. & 21.263 & Man. & 42.359 & 111.42 & Man. & Man. & Man. & 22.044 & Man. & 20.385 & Man. & Man \\
\hline 37.994 & Man. & Man. & 20.36 & Man. & 39.626 & 119.47 & Man. & Man. & Man. & 21.95 & Man. & 21.307 & Man. & Man \\
\hline 39.594 & Man. & Man. & 20.057 & Man. & 39.799 & 121.06 & Man. & Man. & Man. & 20.365 & Man. & 21.326 & Man. & Man \\
\hline 38.54 & Man. & Man. & 20.941 & Man. & 38.061 & 117.84 & Man. & Man. & Man. & 21.804 & Man. & 20.519 & Man. & Man \\
\hline 38.272 & Man. & Man. & 19.827 & Man. & 40.252 & 121.06 & Man. & Man. & Man. & 21.18 & Man. & 21.212 & Man. & Man \\
\hline 38.987 & Man. & Man. & 20.075 & Man. & 41.06 & 114.65 & Man. & Man. & Man. & 20.514 & Man. & 20.342 & Man. & Man. \\
\hline 39.937 & Man. & Man. & 19.85 & Man. & 35.625 & 119.37 & Man. & Man. & Man. & 21.007 & Man. & 21.146 & Man. & Man \\
\hline 39.144 & Man. & Man. & 21.394 & Man. & 40.663 & 121.78 & Man. & Man. & Man. & 23.054 & Man. & 19.95 & Man. & Man. \\
\hline 38.724 & Man. & Man. & 19.799 & Man. & 39.641 & 119.17 & Man. & Man. & Man. & 19.387 & Man. & 20.415 & Man. & Man \\
\hline 38.04 & Man. & Man. & 19.641 & Man. & 40.313 & 114.63 & Man. & Man. & Man. & 20.9 & Man. & 18.452 & Man. & Man. \\
\hline 39.581 & Man. & Man. & 20.357 & Man. & 38.106 & 119.42 & Man. & Man. & Man. & 21.432 & Man. & 20.976 & Man. & Man \\
\hline 37.741 & Man. & Man. & 20.997 & Man. & 39.52 & 121.35 & Man. & Man. & Man. & 23.047 & Man. & 22.014 & Man. & Man. \\
\hline 39.666 & Man. & Man. & 20.136 & Man. & 37.763 & 117.16 & Man. & Man. & Man. & 19.534 & Man. & 20.777 & Man. & Man \\
\hline 38.561 & Man. & Man. & 20.296 & Man. & 37.466 & 118.67 & Man. & Man. & Man. & 19.69 & Man. & 21.651 & Man. & Man. \\
\hline 38.685 & Man. & Man. & 20.63 & Man. & 39.479 & 119.25 & Man. & Man. & Man. & 20.96 & Man. & 20.031 & Man. & Man \\
\hline 38.289 & Man. & Man. & 20.286 & Man. & 37.458 & 119.09 & Man. & Man. & Man. & 20.535 & Man. & 19.852 & Man. & Man \\
\hline 37.973 & Man. & Man. & 20.359 & Man. & 39.885 & 121.23 & Man. & Man. & Man. & 19.119 & Man. & 19.843 & Man. & Man \\
\hline 37.971 & Man. & Man. & 20.299 & Man. & 40.903 & 119.97 & Man. & Man. & Man. & 19.212 & Man. & 19.684 & Man. & Man \\
\hline 38.947 & Man. & Man. & 19.722 & Man. & 42.951 & 118.75 & Man. & Man. & Man. & 20.688 & Man. & 19.87 & Man. & Man. \\
\hline 38.033 & Man. & Man. & 20.63 & Man. & 39.422 & 118.78 & Man. & Man. & Man. & 20.881 & Man. & 20.747 & Man. & Man \\
\hline 39.649 & Man. & Man. & 20.338 & Man. & 38.912 & 118.39 & Man. & Man. & Man. & 19.168 & Man. & 20.39 & Man. & Man \\
\hline 37.694 & Man. & Man. & 21.251 & Man. & 38.51 & 118.68 & Man. & Man. & Man. & 21.977 & Man. & 20.847 & Man. & Man \\
\hline 38.99 & Man. & Man. & 21.034 & Man. & 38.932 & 117.97 & Man. & Man. & Man. & 19.949 & Man. & 20.981 & Man. & Man \\
\hline 38.339 & Man. & Man. & 20.933 & Man. & 37.566 & 117.56 & Man. & Man. & Man. & 20.442 & Man. & 19.564 & Man. & Man \\
\hline 38.417 & Man. & Man. & 20.321 & Man. & 38.582 & 120.83 & Man. & Man. & Man. & 22.19 & Man. & 20.264 & Man. & Man \\
\hline 39.182 & Man. & Man. & 20.433 & Man. & 38.028 & 118.51 & Man. & Man. & Man. & 20.091 & Man. & 20.452 & Man. & Man \\
\hline 37.769 & Man. & Man. & 21.104 & Man. & 39.498 & 119.13 & Man. & Man. & Man. & 21.87 & Man. & 20.652 & Man. & Man. \\
\hline
\end{tabular}


Table F.18: Throughput Simulation Scaled Packing Times (Non-Overlapping Waves) continued.

\begin{tabular}{|c|c|c|c|c|c|c|c|c|c|c|c|c|c|c|}
\hline 1 & 2 & 3 & 4 & 5 & 6 & 7 & 8 & 9 & 10 & 11 & 12 & 13 & 14 & 15 \\
\hline 38.674 & Man. & Man. & 18.652 & Man. & 36.263 & 117.46 & Man. & Man. & Man. & 20.457 & Man. & 21.253 & Man. & Man. \\
\hline 38.12 & Man. & Man. & 19.746 & Man. & 40.061 & 116.8 & Man. & Man. & Man. & 20.19 & Man. & 19.038 & Man. & Man \\
\hline 37.947 & Man. & Man. & 19.554 & Man. & 38.214 & 116.29 & Man. & Man. & Man. & 19.894 & Man. & 21.926 & Man. & Man \\
\hline 38.297 & Man. & Man. & 19.582 & Man. & 38.916 & 118.34 & Man. & Man. & Man. & 20.009 & Man. & 21.523 & Man. & Man \\
\hline 38.688 & Man. & Man. & 19.94 & Man. & 43.348 & 118.64 & Man. & Man. & Man. & 20.164 & Man. & 21.573 & Man. & Man \\
\hline 39.265 & Man. & Man. & 20.008 & Man. & 35.422 & 119.44 & Man. & Man. & Man. & 18.918 & Man. & 19.877 & Man. & Man \\
\hline 38.921 & Man. & Man. & 19.92 & Man. & 42.914 & 118.65 & Man. & Man. & Man. & 21.697 & Man. & 19.94 & Man. & Man \\
\hline 39.298 & Man. & Man. & 20.658 & Man. & 38.769 & 118.5 & Man. & Man. & Man. & 21.498 & Man. & 21.676 & Man. & Man \\
\hline 38.015 & Man. & Man. & 20.371 & Man. & 39.121 & 119.28 & Man. & Man. & Man. & 21.124 & Man. & 20.721 & Man. & Man \\
\hline 38.061 & Man. & Man. & 19.566 & Man. & 37.96 & 119.34 & Man. & Man. & Man. & 19.889 & Man. & 20.136 & Man. & Man \\
\hline 38.152 & Man. & Man. & 19.312 & Man. & 39.843 & 116.86 & Man. & Man. & Man. & 20.843 & Man. & 19.293 & Man. & Man \\
\hline 37.671 & Man. & Man. & 19.903 & Man. & 39.256 & 125.66 & Man. & Man. & Man. & 19.765 & Man. & 22.097 & Man. & Man \\
\hline 40.441 & Man. & Man. & 20.625 & Man. & 41.361 & 119.48 & Man. & Man. & Man. & 20.245 & Man. & 20.634 & Man. & Man \\
\hline 39.232 & Man. & Man. & 20.343 & Man. & 40.39 & 123.51 & Man. & Man. & Man. & 19.977 & Man. & 21.338 & Man. & Man \\
\hline 37.836 & Man. & Man. & 21.97 & Man. & 39.815 & 122.23 & Man. & Man. & Man. & 20.892 & Man. & 19.757 & Man. & Man \\
\hline 39.423 & Man. & Man. & 19.933 & Man. & 39.611 & 118.04 & Man. & Man. & Man. & 21.254 & Man. & 20.504 & Man. & Man \\
\hline
\end{tabular}

Table F.19: Throughput Simulation Induction Times (Overlapping Waves - c.v. $=0.2$ ).

\begin{tabular}{|c|c|c|c|c|c|c|c|c|c|c|c|c|c|c|}
\hline 1 & 2 & 3 & 4 & 5 & 6 & 7 & 8 & 9 & 10 & 11 & 12 & 13 & 14 & 15 \\
\hline 90.00 & 30.01 & 60.00 & 36.46 & Man. & 37.76 & 66.00 & Man. & Man. & 55.26 & 28.35 & Man. & 27.20 & Man. & Man. \\
\hline 90.01 & 30.00 & 60.00 & 36.44 & Man. & 37.68 & 66.00 & Man. & Man. & 55.85 & 28.33 & Man. & 27.58 & Man. & Man. \\
\hline 90.00 & 30.00 & 60.00 & 36.40 & Man. & 37.60 & 66.00 & Man. & Man. & 55.82 & 28.29 & Man. & 27.57 & Man. & Man. \\
\hline 90.00 & 30.01 & 60.00 & 36.56 & Man. & 37.50 & 66.00 & Man. & Man. & 55.88 & 28.17 & Man. & 27.40 & Man. & Man. \\
\hline 90.01 & 30.00 & 59.99 & 36.47 & Man. & 37.87 & 66.00 & Man. & Man. & 55.79 & 28.41 & Man. & 27.48 & Man. & Man. \\
\hline 90.00 & 30.00 & 59.99 & 36.41 & Man. & 37.77 & 66.00 & Man. & Man. & 55.43 & 28.43 & Man. & 27.42 & Man. & Man. \\
\hline 90.01 & 30.01 & 59.99 & 36.36 & Man. & 37.56 & 66.00 & Man. & Man. & 55.78 & 28.25 & Man. & 27.27 & Man. & Man. \\
\hline 90.01 & 30.01 & 60.00 & 36.33 & Man. & 37.81 & 66.00 & Man. & Man. & 55.73 & 28.30 & Man. & 27.25 & Man. & Man. \\
\hline 90.01 & 30.00 & 60.00 & 36.46 & Man. & 37.65 & 66.00 & Man. & Man. & 55.57 & 28.34 & Man. & 27.39 & Man. & Man. \\
\hline 90.01 & 30.00 & 59.99 & 36.52 & Man. & 37.61 & 66.00 & Man. & Man. & 55.26 & 28.24 & Man. & 27.33 & Man. & Man. \\
\hline 90.01 & 30.00 & 59.99 & 36.46 & Man. & 37.70 & 66.00 & Man. & Man. & 55.66 & 28.34 & Man. & 27.45 & Man. & Man. \\
\hline 90.00 & 30.01 & 59.99 & 36.49 & Man. & 37.59 & 66.00 & Man. & Man. & 55.63 & 28.24 & Man. & 27.41 & Man. & Man. \\
\hline 90.00 & 30.01 & 59.99 & 36.38 & Man. & 37.86 & 66.00 & Man. & Man. & 55.62 & 28.46 & Man. & 27.65 & Man. & Man. \\
\hline 90.00 & 30.00 & 59.99 & 36.46 & Man. & 37.74 & 66.00 & Man. & Man. & 55.82 & 28.25 & Man. & 27.77 & Man. & Man. \\
\hline 90.01 & 30.01 & 60.00 & 36.45 & Man. & 37.51 & 66.00 & Man. & Man. & 55.57 & 28.13 & Man. & 27.53 & Man. & Man. \\
\hline 90.01 & 30.01 & 59.99 & 36.50 & Man. & 37.67 & 66.00 & Man. & Man. & 55.54 & 28.30 & Man. & 27.50 & Man. & Man. \\
\hline 90.00 & 30.01 & 59.99 & 36.48 & Man. & 37.67 & 66.00 & Man. & Man. & 55.71 & 28.34 & Man. & 27.64 & Man. & Man. \\
\hline 90.01 & 30.00 & 59.99 & 36.59 & Man. & 37.52 & 66.00 & Man. & Man. & 55.70 & 28.09 & Man. & 27.57 & Man. & Man. \\
\hline 90.00 & 30.00 & 59.99 & 36.34 & Man. & 37.53 & 66.00 & Man. & Man. & 55.85 & 28.17 & Man. & 27.43 & Man. & Man. \\
\hline 90.00 & 30.00 & 59.99 & 36.41 & Man. & 37.77 & 66.00 & Man. & Man. & 54.88 & 28.35 & Man. & 27.54 & Man. & Man. \\
\hline 90.01 & 30.01 & 59.99 & 36.48 & Man. & 37.64 & 66.00 & Man. & Man. & 55.83 & 28.16 & Man. & 27.49 & Man. & Man. \\
\hline 90.00 & 30.01 & 60.00 & 36.44 & Man. & 37.68 & 66.00 & Man. & Man. & 55.35 & 28.24 & Man. & 27.51 & Man. & Man. \\
\hline 90.01 & 30.00 & 59.99 & 36.46 & Man. & 37.50 & 66.00 & Man. & Man. & 55.89 & 28.05 & Man. & 27.50 & Man. & Man. \\
\hline 90.01 & 30.01 & 59.99 & 36.43 & Man. & 37.68 & 66.00 & Man. & Man. & 55.49 & 28.34 & Man. & 27.40 & Man. & Man. \\
\hline 90.00 & 30.00 & 59.99 & 36.53 & Man. & 37.39 & 66.00 & Man. & Man. & 55.52 & 28.07 & Man. & 27.67 & Man. & Man. \\
\hline 90.00 & 30.01 & 60.00 & 36.30 & Man. & 37.65 & 66.00 & Man. & Man. & 55.41 & 28.24 & Man. & 27.25 & Man. & Man. \\
\hline 90.00 & 30.00 & 59.99 & 36.59 & Man. & 37.88 & 66.00 & Man. & Man. & 55.22 & 28.29 & Man. & 27.47 & Man. & Man. \\
\hline 90.00 & 30.00 & 60.00 & 36.35 & Man. & 37.79 & 66.00 & Man. & Man. & 55.90 & 28.41 & Man. & 27.45 & Man. & Man. \\
\hline 90.01 & 30.01 & 60.00 & 36.44 & Man. & 37.86 & 66.00 & Man. & Man. & 55.62 & 28.38 & Man. & 27.31 & Man. & Man. \\
\hline 90.01 & 30.01 & 60.00 & 36.48 & Man. & 37.63 & 66.00 & Man. & Man. & 55.60 & 28.25 & Man. & 27.45 & Man. & Man. \\
\hline 90.00 & 30.00 & 60.00 & 36.46 & Man. & 37.65 & 66.00 & Man. & Man. & 56.05 & 28.19 & Man. & 27.55 & Man. & Man. \\
\hline 90.00 & 30.00 & 60.00 & 36.47 & Man. & 37.63 & 66.00 & Man. & Man. & 55.44 & 28.29 & Man. & 27.47 & Man. & Man. \\
\hline 90.00 & 30.00 & 59.99 & 36.38 & Man. & 37.49 & 66.00 & Man. & Man. & 54.89 & 28.19 & Man. & 27.53 & Man. & Man. \\
\hline 90.01 & 30.01 & 60.00 & 36.42 & Man. & 37.59 & 66.00 & Man. & Man. & 55.47 & 28.18 & Man. & 27.57 & Man. & Man. \\
\hline 90.01 & 30.00 & 59.99 & 36.42 & Man. & 37.38 & 66.00 & Man. & Man. & 55.03 & 28.01 & Man. & 27.63 & Man. & Man. \\
\hline 90.01 & 30.00 & 60.01 & 36.47 & Man. & 37.81 & 66.00 & Man. & Man. & 55.46 & 28.39 & Man. & 27.49 & Man. & Man. \\
\hline 90.00 & 30.00 & 59.99 & 36.40 & Man. & 37.53 & 66.00 & Man. & Man. & 55.59 & 28.07 & Man. & 27.57 & Man. & Man. \\
\hline
\end{tabular}


Table F.19: Throughput Simulation Induction Times (Overlapping Waves - c.v. $=0.2)-$ continued.

\begin{tabular}{|c|c|c|c|c|c|c|c|c|c|c|c|c|c|c|}
\hline 1 & 2 & 3 & 4 & 5 & 6 & 7 & 8 & 9 & 10 & 11 & 12 & 13 & 14 & 15 \\
\hline 90.01 & 30.00 & 60.00 & 36.35 & Man. & 37.78 & 66.00 & Man. & Man. & 55.57 & 28.28 & Man. & 27.37 & Man. & Man. \\
\hline 90.00 & 30.01 & 59.99 & 36.44 & Man. & 37.41 & 66.00 & Man. & Man. & 54.97 & 28.09 & Man. & 27.61 & Man. & Man. \\
\hline 90.00 & 30.00 & 59.99 & 36.47 & Man. & 37.71 & 66.00 & Man. & Man. & 55.86 & 28.26 & Man. & 27.47 & Man. & Man. \\
\hline 90.00 & 30.01 & 60.00 & 36.51 & Man. & 37.81 & 66.00 & Man. & Man. & 54.95 & 28.45 & Man. & 27.45 & Man. & Man. \\
\hline 90.00 & 30.00 & 59.99 & 36.53 & Man. & 37.62 & 66.00 & Man. & Man. & 55.23 & 28.28 & Man. & 27.62 & Man. & Man. \\
\hline 90.00 & 30.00 & 60.00 & 36.43 & Man. & 37.57 & 66.00 & Man. & Man. & 55.76 & 28.09 & Man. & 27.45 & Man. & Man. \\
\hline 90.00 & 30.00 & 59.99 & 36.36 & Man. & 37.86 & 66.00 & Man. & Man. & 55.55 & 28.44 & Man. & 27.29 & Man. & Man. \\
\hline 90.01 & 30.00 & 59.99 & 36.51 & Man. & 37.60 & 66.00 & Man. & Man. & 55.77 & 28.14 & Man. & 27.47 & Man. & Man. \\
\hline 90.00 & 30.00 & 59.99 & 36.39 & Man. & 37.80 & 66.00 & Man. & Man. & 55.68 & 28.22 & Man. & 27.29 & Man. & Man. \\
\hline 90.00 & 30.00 & 60.00 & 36.41 & Man. & 37.66 & 66.00 & Man. & Man. & 55.41 & 28.14 & Man. & 27.38 & Man. & Man. \\
\hline 90.01 & 30.00 & 60.00 & 36.32 & Man. & 37.62 & 66.00 & Man. & Man. & 55.67 & 28.22 & Man. & 27.75 & Man. & Man \\
\hline 90.01 & 30.00 & 60.00 & 36.36 & Man. & 37.55 & 66.00 & Man. & Man. & 55.94 & 28.20 & Man. & 27.19 & Man. & Man. \\
\hline 90.00 & 30.01 & 60.00 & 36.43 & Man. & 37.62 & 66.00 & Man. & Man. & 55.42 & 28.26 & Man. & 27.51 & Man. & Man \\
\hline 90.00 & 30.00 & 59.99 & 36.41 & Man. & 37.65 & 66.00 & Man. & Man. & 55.85 & 28.19 & Man. & 27.34 & Man. & Man. \\
\hline 90.00 & 30.01 & 59.99 & 36.42 & Man. & 37.73 & 66.00 & Man. & Man. & 55.70 & 28.27 & Man. & 27.28 & Man. & Man. \\
\hline 90.00 & 30.00 & 59.99 & 36.42 & Man. & 37.57 & 66.00 & Man. & Man. & 55.55 & 28.12 & Man. & 27.59 & Man. & Man. \\
\hline 90.00 & 30.01 & 59.99 & 36.49 & Man. & 37.60 & 66.00 & Man. & Man. & 56.23 & 28.29 & Man. & 27.51 & Man. & Man. \\
\hline 90.00 & 30.00 & 60.00 & 36.47 & Man. & 37.51 & 66.00 & Man. & Man. & 55.54 & 28.24 & Man. & 27.43 & Man. & Man. \\
\hline 90.01 & 30.00 & 59.99 & 36.42 & Man. & 37.80 & 66.00 & Man. & Man. & 56.10 & 28.28 & Man. & 27.67 & Man. & Man \\
\hline 90.01 & 30.01 & 60.01 & 36.54 & Man. & 37.75 & 66.00 & Man. & Man. & 55.74 & 28.41 & Man. & 27.71 & Man. & Man \\
\hline 90.00 & 30.00 & 59.99 & 36.35 & Man. & 37.74 & 66.00 & Man. & Man. & 55.79 & 28.38 & Man. & 27.59 & Man. & Man. \\
\hline 90.01 & 30.00 & 60.00 & 36.44 & Man. & 37.62 & 66.00 & Man. & Man. & 55.62 & 28.20 & Man. & 27.41 & Man. & Man \\
\hline 90.00 & 30.00 & 60.00 & 36.40 & Man. & 37.89 & 66.00 & Man. & Man. & 56.17 & 28.44 & Man. & 27.33 & Man. & Man. \\
\hline 90.01 & 30.01 & 60.01 & 36.57 & Man. & 37.82 & 66.00 & Man. & Man. & 55.76 & 28.39 & Man. & 27.44 & Man. & Man \\
\hline 90.01 & 30.01 & 60.00 & 36.54 & Man. & 37.35 & 66.00 & Man. & Man. & 55.86 & 28.20 & Man. & 27.54 & Man. & Man. \\
\hline 90.00 & 30.01 & 59.99 & 36.52 & Man. & 37.40 & 66.00 & Man. & Man. & 55.95 & 28.12 & Man. & 27.59 & Man. & Man \\
\hline 90.01 & 30.01 & 60.00 & 36.42 & Man. & 37.83 & 66.00 & Man. & Man. & 55.88 & 28.31 & Man. & 27.54 & Man. & Man. \\
\hline 90.01 & 30.00 & 60.00 & 36.37 & Man. & 37.45 & 66.00 & Man. & Man. & 55.62 & 28.21 & Man. & 27.42 & Man. & Man. \\
\hline 90.01 & 30.01 & 60.00 & 36.46 & Man. & 37.64 & 66.00 & Man. & Man. & 55.80 & 28.26 & Man. & 27.50 & Man. & Man. \\
\hline 90.00 & 30.00 & 59.99 & 36.43 & Man. & 37.55 & 66.00 & Man. & Man. & 55.85 & 28.18 & Man. & 27.51 & Man. & Man. \\
\hline 90.00 & 30.01 & 59.99 & 36.44 & Man. & 37.61 & 66.00 & Man. & Man. & 55.77 & 28.25 & Man. & 27.26 & Man. & Man. \\
\hline 90.01 & 30.01 & 60.00 & 36.39 & Man. & 37.45 & 66.00 & Man. & Man. & 55.63 & 28.06 & Man. & 27.55 & Man. & Man. \\
\hline 90.01 & 30.01 & 59.99 & 36.36 & Man. & 37.91 & 66.00 & Man. & Man. & 55.71 & 28.36 & Man. & 27.53 & Man. & Man. \\
\hline 90.00 & 30.01 & 60.00 & 36.38 & Man. & 37.48 & 66.00 & Man. & Man. & 54.99 & 28.02 & Man. & 27.49 & Man. & Man \\
\hline 90.00 & 30.01 & 60.00 & 36.34 & Man. & 37.59 & 66.00 & Man. & Man. & 55.80 & 28.08 & Man. & 27.73 & Man. & Man \\
\hline 90.00 & 30.00 & 59.99 & 36.37 & Man. & 37.57 & 66.00 & Man. & Man. & 55.56 & 28.23 & Man. & 27.29 & Man. & Man. \\
\hline 90.00 & 30.00 & 60.01 & 36.48 & Man. & 37.56 & 66.00 & Man. & Man. & 55.70 & 28.11 & Man. & 27.53 & Man. & Man. \\
\hline 90.01 & 30.01 & 60.00 & 36.44 & Man. & 37.69 & 66.00 & Man. & Man. & 55.87 & 28.28 & Man. & 27.48 & Man. & Man \\
\hline 90.00 & 30.01 & 59.99 & 36.39 & Man. & 37.86 & 66.00 & Man. & Man. & 55.75 & 28.41 & Man. & 27.43 & Man. & Man \\
\hline 90.00 & 30.00 & 60.00 & 36.45 & Man. & 37.83 & 66.00 & Man. & Man. & 55.83 & 28.54 & Man. & 27.77 & Man. & Man \\
\hline 90.01 & 30.00 & 59.99 & 36.48 & Man. & 37.72 & 66.00 & Man. & Man. & 55.86 & 28.31 & Man. & 27.23 & Man. & Man. \\
\hline 90.00 & 30.01 & 59.99 & 36.45 & Man. & 37.44 & 66.00 & Man. & Man. & 55.65 & 28.06 & Man. & 27.19 & Man. & Man \\
\hline 90.00 & 30.00 & 60.00 & 36.50 & Man. & 37.62 & 66.00 & Man. & Man. & 55.67 & 28.20 & Man. & 27.57 & Man. & Man. \\
\hline 90.00 & 30.01 & 59.99 & 36.51 & Man. & 37.69 & 66.00 & Man. & Man. & 55.59 & 28.34 & Man. & 27.66 & Man. & Man \\
\hline 90.00 & 30.01 & 60.00 & 36.48 & Man. & 37.81 & 66.00 & Man. & Man. & 55.82 & 28.36 & Man. & 27.35 & Man. & Man \\
\hline 90.00 & 30.01 & 60.00 & 36.39 & Man. & 37.72 & 66.00 & Man. & Man. & 56.18 & 28.30 & Man. & 27.40 & Man. & Man \\
\hline 90.01 & 30.01 & 60.00 & 36.44 & Man. & 37.65 & 66.00 & Man. & Man. & 55.69 & 28.29 & Man. & 27.58 & Man. & Man \\
\hline 90.00 & 30.01 & 59.99 & 36.37 & Man. & 37.94 & 66.00 & Man. & Man. & 55.66 & 28.43 & Man. & 27.37 & Man. & Man \\
\hline 90.01 & 30.00 & 60.00 & 36.55 & Man. & 37.60 & 66.00 & Man. & Man. & 55.89 & 28.24 & Man. & 27.29 & Man. & Man. \\
\hline 90.00 & 30.00 & 59.99 & 36.43 & Man. & 37.67 & 66.00 & Man. & Man. & 55.59 & 28.17 & Man. & 27.48 & Man. & Man. \\
\hline 90.00 & 30.00 & 59.99 & 36.47 & Man. & 37.53 & 66.00 & Man. & Man. & 55.76 & 28.16 & Man. & 27.53 & Man. & Man \\
\hline 90.00 & 30.00 & 59.99 & 36.36 & Man. & 37.74 & 66.00 & Man. & Man. & 55.62 & 28.30 & Man. & 27.86 & Man. & Man \\
\hline 90.00 & 30.00 & 60.00 & 36.45 & Man. & 37.62 & 66.00 & Man. & Man. & 55.91 & 28.23 & Man. & 27.53 & Man. & Man \\
\hline 90.01 & 30.00 & 60.00 & 36.39 & Man. & 37.59 & 66.00 & Man. & Man. & 55.50 & 28.07 & Man. & 27.49 & Man. & Man. \\
\hline 90.00 & 30.01 & 60.00 & 36.43 & Man. & 37.52 & 66.00 & Man. & Man. & 55.63 & 28.23 & Man. & 27.37 & Man. & Man. \\
\hline 90.00 & 30.01 & 60.00 & 36.36 & Man. & 37.51 & 66.00 & Man. & Man. & 55.62 & 28.12 & Man. & 27.63 & Man. & Man. \\
\hline 90.00 & 30.00 & 59.99 & 36.41 & Man. & 37.58 & 66.00 & Man. & Man. & 55.94 & 28.08 & Man. & 27.36 & Man. & Man. \\
\hline 90.00 & 30.01 & 59.99 & 36.35 & Man. & 37.67 & 66.00 & Man. & Man. & 55.68 & 28.25 & Man. & 27.68 & Man. & Man. \\
\hline
\end{tabular}


Table F.19: Throughput Simulation Induction Times (Overlapping Waves - c.v. $=0.2)-$

continued.

\begin{tabular}{rrrrrrrrrrrrrrrrrr}
\hline 1 & 2 & 3 & 4 & 5 & 6 & 7 & 8 & 9 & 10 & 11 & 12 & 13 \\
\hline 90.00 & 30.00 & 59.99 & 36.39 & Man. & 37.61 & 66.00 & Man. & Man. & 55.69 & 28.28 & Man. & 27.48 & Man. & Man. \\
90.00 & 30.01 & 60.00 & 36.35 & Man. & 37.58 & 66.00 & Man. & Man. & 55.95 & 28.07 & Man. & 27.59 & Man. & Man. \\
90.00 & 30.00 & 60.00 & 36.46 & Man. & 37.57 & 66.00 & Man. & Man. & 55.59 & 28.20 & Man. & 27.27 & Man. & Man. \\
90.00 & 30.00 & 60.00 & 36.24 & Man. & 37.86 & 66.00 & Man. & Man. & 55.74 & 28.44 & Man. & 27.71 & Man. & Man. \\
90.01 & 30.00 & 59.99 & 36.42 & Man. & 37.61 & 66.00 & Man. & Man. & 55.86 & 28.32 & Man. & 27.59 & Man. & Man. \\
\hline
\end{tabular}

Table F.20: Throughput Simulation Induction Times (Non-Overlapping Waves - c.v. = $0.2)$.

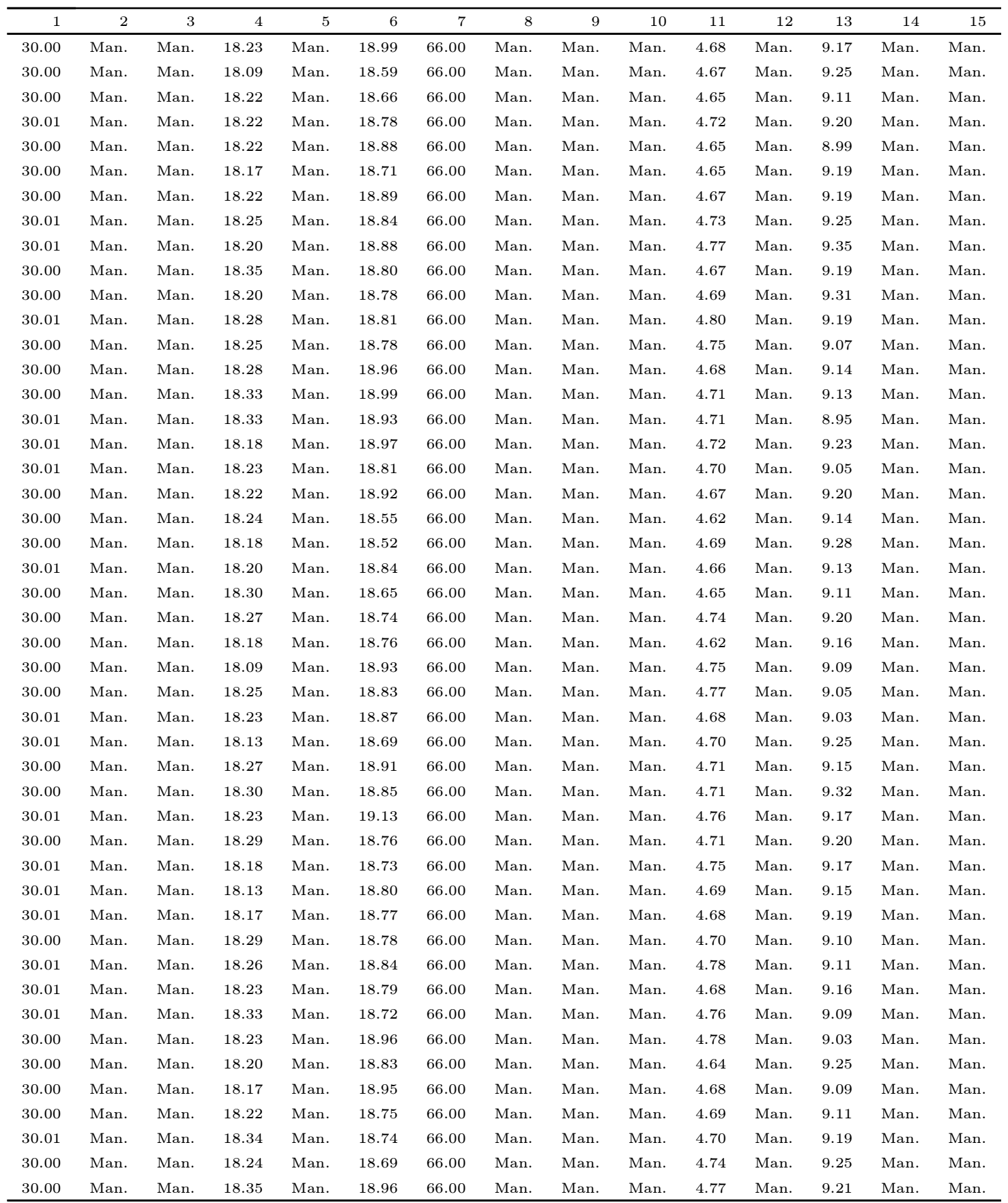


Table F.20: Throughput Simulation Induction Times (Non-Overlapping Waves - c.v. $=$ $0.2)$ - continued.

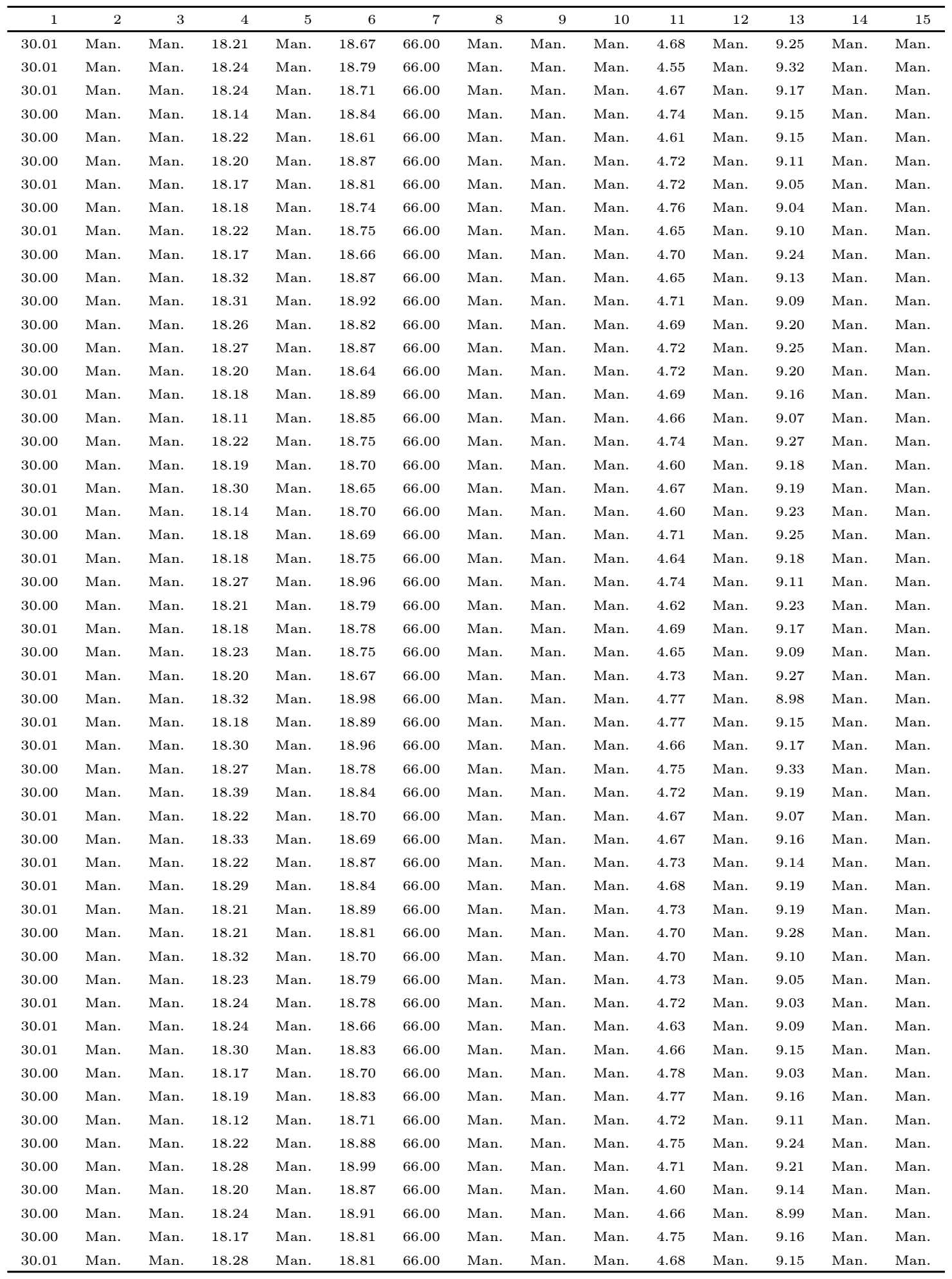


Table F.21: Throughput Simulation Induction Times (Overlapping Waves - c.v. =0.4).

\begin{tabular}{|c|c|c|c|c|c|c|c|c|c|c|c|c|c|c|}
\hline 1 & 2 & 3 & 4 & 5 & 6 & 7 & 8 & 9 & 10 & 11 & 12 & 13 & 14 & 15 \\
\hline 90.00 & 30.00 & 60.00 & 37.45 & Man. & 35.42 & 66.00 & Man. & Man. & 56.09 & 26.55 & Man. & 27.15 & Man. & Man. \\
\hline 90.01 & 30.00 & 60.00 & 37.45 & Man. & 35.18 & 66.00 & Man. & Man. & 55.75 & 26.38 & Man. & 27.45 & Man. & Man. \\
\hline 90.00 & 30.00 & 60.00 & 37.25 & Man. & 35.53 & 66.00 & Man. & Man. & 55.66 & 26.77 & Man. & 27.52 & Man. & Man. \\
\hline 90.00 & 30.00 & 60.00 & 37.23 & Man. & 35.21 & 66.00 & Man. & Man. & 56.35 & 26.42 & Man. & 27.67 & Man. & Man. \\
\hline 90.00 & 30.00 & 60.00 & 37.32 & Man. & 35.17 & 66.00 & Man. & Man. & 56.15 & 26.41 & Man. & 27.37 & Man. & Man. \\
\hline 90.00 & 30.00 & 59.99 & 37.23 & Man. & 35.19 & 66.00 & Man. & Man. & 55.62 & 26.31 & Man. & 27.55 & Man. & Man. \\
\hline 90.00 & 30.00 & 60.00 & 37.40 & Man. & 35.18 & 66.00 & Man. & Man. & 55.94 & 26.45 & Man. & 27.25 & Man. & Man. \\
\hline 90.01 & 30.01 & 60.00 & 37.28 & Man. & 35.35 & 66.00 & Man. & Man. & 55.01 & 26.43 & Man. & 27.10 & Man. & Man. \\
\hline 90.00 & 30.00 & 59.99 & 37.32 & Man. & 35.38 & 66.00 & Man. & Man. & 55.04 & 26.60 & Man. & 27.29 & Man. & Man. \\
\hline 90.00 & 30.01 & 60.00 & 37.60 & Man. & 35.38 & 66.00 & Man. & Man. & 54.94 & 26.51 & Man. & 27.10 & Man. & Man. \\
\hline 90.00 & 30.00 & 59.99 & 37.70 & Man. & 35.50 & 66.00 & Man. & Man. & 56.35 & 26.63 & Man. & 27.51 & Man. & Man. \\
\hline 90.00 & 30.01 & 60.00 & 37.38 & Man. & 35.33 & 66.00 & Man. & Man. & 54.79 & 26.50 & Man. & 27.43 & Man. & Man \\
\hline 90.00 & 30.00 & 59.99 & 37.24 & Man. & 34.98 & 66.00 & Man. & Man. & 55.68 & 26.38 & Man. & 27.84 & Man. & Man \\
\hline 90.00 & 30.00 & 59.99 & 37.27 & Man. & 35.14 & 66.00 & Man. & Man. & 56.03 & 26.42 & Man. & 27.96 & Man. & Man \\
\hline 90.01 & 30.01 & 60.00 & 37.25 & Man. & 35.25 & 66.00 & Man. & Man. & 56.04 & 26.43 & Man. & 27.61 & Man. & Man \\
\hline 90.00 & 30.00 & 59.99 & 37.37 & Man. & 35.42 & 66.00 & Man. & Man. & 56.06 & 26.54 & Man. & 27.35 & Man. & Man \\
\hline 90.00 & 30.01 & 60.00 & 37.17 & Man. & 35.45 & 66.00 & Man. & Man. & 54.67 & 26.52 & Man. & 27.55 & Man. & Man. \\
\hline 90.01 & 30.00 & 60.00 & 37.70 & Man. & 35.34 & 66.00 & Man. & Man. & 55.54 & 26.45 & Man. & 27.70 & Man. & Man \\
\hline 90.00 & 30.00 & 60.01 & 37.52 & Man. & 35.23 & 66.00 & Man. & Man. & 56.08 & 26.46 & Man. & 27.51 & Man. & Man. \\
\hline 90.00 & 30.00 & 60.00 & 37.56 & Man. & 35.58 & 66.00 & Man. & Man. & 55.93 & 26.60 & Man. & 27.58 & Man. & Man \\
\hline 90.00 & 30.00 & 59.99 & 37.21 & Man. & 35.27 & 66.00 & Man. & Man. & 55.45 & 26.47 & Man. & 27.42 & Man. & Man \\
\hline 90.00 & 30.01 & 60.00 & 37.54 & Man. & 35.46 & 66.00 & Man. & Man. & 55.49 & 26.61 & Man. & 27.39 & Man. & Man. \\
\hline 90.00 & 30.00 & 59.99 & 37.18 & Man. & 35.20 & 66.00 & Man. & Man. & 54.80 & 26.43 & Man. & 27.47 & Man. & Man. \\
\hline 90.01 & 30.01 & 59.99 & 37.68 & Man. & 35.31 & 66.00 & Man. & Man. & 56.15 & 26.52 & Man. & 27.35 & Man. & Man \\
\hline 90.00 & 30.01 & 59.99 & 37.38 & Man. & 35.19 & 66.00 & Man. & Man. & 55.81 & 26.45 & Man. & 27.61 & Man. & Man \\
\hline 90.00 & 30.01 & 60.00 & 37.61 & Man. & 35.33 & 66.00 & Man. & Man. & 56.30 & 26.55 & Man. & 27.20 & Man. & Man \\
\hline 90.00 & 30.00 & 59.99 & 37.42 & Man. & 35.36 & 66.00 & Man. & Man. & 55.70 & 26.48 & Man. & 27.22 & Man. & Man \\
\hline 90.00 & 30.00 & 59.99 & 37.53 & Man. & 35.37 & 66.00 & Man. & Man. & 55.10 & 26.48 & Man. & 27.64 & Man. & Man. \\
\hline 90.00 & 30.01 & 60.00 & 37.43 & Man. & 35.39 & 66.00 & Man. & Man. & 56.19 & 26.47 & Man. & 27.16 & Man. & Man \\
\hline 90.00 & 30.01 & 60.01 & 37.44 & Man. & 35.54 & 66.00 & Man. & Man. & 55.58 & 26.66 & Man. & 27.66 & Man. & Man. \\
\hline 90.00 & 30.00 & 60.00 & 37.54 & Man. & 35.46 & 66.00 & Man. & Man. & 55.58 & 26.67 & Man. & 27.69 & Man. & Man \\
\hline 90.00 & 30.00 & 60.01 & 37.29 & Man. & 35.11 & 66.00 & Man. & Man. & 55.90 & 26.34 & Man. & 27.29 & Man. & Man. \\
\hline 90.00 & 30.00 & 59.99 & 37.48 & Man. & 34.91 & 66.00 & Man. & Man. & 55.26 & 26.11 & Man. & 27.37 & Man. & Man \\
\hline 90.01 & 30.00 & 60.01 & 37.34 & Man. & 35.48 & 66.00 & Man. & Man. & 55.93 & 26.59 & Man. & 27.59 & Man. & Man \\
\hline 90.00 & 30.00 & 60.00 & 37.83 & Man. & 35.26 & 66.00 & Man. & Man. & 55.81 & 26.29 & Man. & 27.63 & Man. & Man \\
\hline 90.01 & 30.00 & 60.00 & 37.36 & Man. & 35.54 & 66.00 & Man. & Man. & 56.29 & 26.61 & Man. & 27.27 & Man. & Man. \\
\hline 90.00 & 30.00 & 59.99 & 37.50 & Man. & 35.11 & 66.00 & Man. & Man. & 54.94 & 26.33 & Man. & 27.57 & Man. & Man. \\
\hline 90.01 & 30.00 & 60.00 & 37.30 & Man. & 35.48 & 66.00 & Man. & Man. & 56.33 & 26.53 & Man. & 27.27 & Man. & Man. \\
\hline 90.00 & 30.00 & 59.99 & 37.37 & Man. & 35.32 & 66.00 & Man. & Man. & 55.88 & 26.41 & Man. & 27.68 & Man. & Man \\
\hline 90.00 & 30.00 & 60.00 & 37.22 & Man. & 35.29 & 66.00 & Man. & Man. & 55.76 & 26.42 & Man. & 27.55 & Man. & Man \\
\hline 90.00 & 30.01 & 60.00 & 37.27 & Man. & 35.63 & 66.00 & Man. & Man. & 55.89 & 26.67 & Man. & 27.43 & Man. & Man. \\
\hline 90.00 & 30.00 & 59.99 & 37.23 & Man. & 35.21 & 66.00 & Man. & Man. & 55.86 & 26.38 & Man. & 27.64 & Man. & Man \\
\hline 90.00 & 30.00 & 60.01 & 37.25 & Man. & 35.31 & 66.00 & Man. & Man. & 56.25 & 26.62 & Man. & 27.38 & Man. & Man \\
\hline 90.00 & 30.00 & 59.99 & 37.27 & Man. & 35.52 & 66.00 & Man. & Man. & 54.83 & 26.62 & Man. & 27.01 & Man. & Man. \\
\hline 90.01 & 30.00 & 59.99 & 37.24 & Man. & 35.28 & 66.00 & Man. & Man. & 55.87 & 26.61 & Man. & 27.57 & Man. & Man. \\
\hline 90.00 & 30.00 & 59.99 & 37.27 & Man. & 35.77 & 66.00 & Man. & Man. & 55.44 & 26.71 & Man. & 27.21 & Man. & Man. \\
\hline 90.00 & 30.00 & 60.00 & 37.30 & Man. & 35.45 & 66.00 & Man. & Man. & 56.17 & 26.57 & Man. & 27.17 & Man. & Man. \\
\hline 90.00 & 30.00 & 59.99 & 37.31 & Man. & 35.41 & 66.00 & Man. & Man. & 55.58 & 26.58 & Man. & 27.77 & Man. & Man. \\
\hline 90.00 & 30.00 & 59.99 & 37.66 & Man. & 35.29 & 66.00 & Man. & Man. & 55.35 & 26.51 & Man. & 27.04 & Man. & Man. \\
\hline 90.00 & 30.01 & 60.00 & 37.39 & Man. & 35.47 & 66.00 & Man. & Man. & 55.96 & 26.52 & Man. & 27.47 & Man. & Man. \\
\hline 90.00 & 30.00 & 60.00 & 37.72 & Man. & 35.45 & 66.00 & Man. & Man. & 56.07 & 26.57 & Man. & 27.29 & Man. & Man \\
\hline 90.00 & 30.00 & 59.99 & 37.37 & Man. & 35.45 & 66.00 & Man. & Man. & 55.54 & 26.62 & Man. & 27.15 & Man. & Man \\
\hline 90.00 & 30.00 & 60.01 & 37.34 & Man. & 35.24 & 66.00 & Man. & Man. & 55.63 & 26.44 & Man. & 27.83 & Man. & Man \\
\hline 90.00 & 30.01 & 59.99 & 37.29 & Man. & 35.32 & 66.00 & Man. & Man. & 56.14 & 26.49 & Man. & 27.59 & Man. & Man \\
\hline 90.00 & 30.00 & 60.00 & 37.42 & Man. & 35.42 & 66.00 & Man. & Man. & 55.54 & 26.54 & Man. & 27.36 & Man. & Man \\
\hline 90.00 & 30.00 & 60.00 & 37.42 & Man. & 35.44 & 66.00 & Man. & Man. & 55.36 & 26.62 & Man. & 27.65 & Man. & Man. \\
\hline 90.01 & 30.00 & 60.01 & 37.31 & Man. & 35.40 & 66.00 & Man. & Man. & 55.73 & 26.57 & Man. & 27.91 & Man. & Man \\
\hline 90.00 & 30.00 & 59.99 & 37.59 & Man. & 35.55 & 66.00 & Man. & Man. & 56.42 & 26.64 & Man. & 27.61 & Man. & Man. \\
\hline
\end{tabular}


Table F.21: Throughput Simulation Induction Times (Overlapping Waves - c.v. $=0.4$ ) continued.

\begin{tabular}{|c|c|c|c|c|c|c|c|c|c|c|c|c|c|c|}
\hline 1 & 2 & 3 & 4 & 5 & 6 & 7 & 8 & 9 & 10 & 11 & 12 & 13 & 14 & 15 \\
\hline 90.01 & 30.00 & 60.00 & 37.53 & Man. & 35.08 & 66.00 & Man. & Man. & 55.20 & 26.28 & Man. & 27.45 & Man. & Man. \\
\hline 90.00 & 30.00 & 59.99 & 37.22 & Man. & 35.31 & 66.00 & Man. & Man. & 55.65 & 26.66 & Man. & 27.11 & Man. & Man. \\
\hline 90.01 & 30.00 & 60.01 & 37.32 & Man. & 35.34 & 66.00 & Man. & Man. & 55.42 & 26.42 & Man. & 27.21 & Man. & Man. \\
\hline 90.00 & 30.01 & 60.01 & 37.19 & Man. & 35.22 & 66.00 & Man. & Man. & 56.35 & 26.44 & Man. & 27.58 & Man. & Man. \\
\hline 90.00 & 30.01 & 60.00 & 37.17 & Man. & 35.36 & 66.00 & Man. & Man. & 55.95 & 26.52 & Man. & 27.77 & Man. & Man. \\
\hline 90.01 & 30.01 & 60.00 & 37.48 & Man. & 35.24 & 66.00 & Man. & Man. & 55.48 & 26.32 & Man. & 27.80 & Man. & Man. \\
\hline 90.01 & 30.00 & 60.00 & 37.53 & Man. & 35.37 & 66.00 & Man. & Man. & 55.89 & 26.50 & Man. & 27.25 & Man. & Man \\
\hline 90.01 & 30.00 & 60.00 & 37.34 & Man. & 35.31 & 66.00 & Man. & Man. & 55.70 & 26.41 & Man. & 27.42 & Man. & Man. \\
\hline 90.00 & 30.00 & 60.01 & 37.19 & Man. & 35.27 & 66.00 & Man. & Man. & 55.87 & 26.35 & Man. & 27.47 & Man. & Man. \\
\hline 90.01 & 30.01 & 59.99 & 37.35 & Man. & 35.29 & 66.00 & Man. & Man. & 55.50 & 26.62 & Man. & 27.13 & Man. & Man. \\
\hline 90.01 & 30.00 & 60.00 & 37.26 & Man. & 35.19 & 66.00 & Man. & Man. & 54.98 & 26.34 & Man. & 27.59 & Man. & Man. \\
\hline 90.00 & 30.00 & 59.99 & 37.28 & Man. & 35.59 & 66.00 & Man. & Man. & 55.77 & 26.75 & Man. & 27.49 & Man. & Man. \\
\hline 90.00 & 30.00 & 60.00 & 37.69 & Man. & 35.00 & 66.00 & Man. & Man. & 55.97 & 26.34 & Man. & 27.59 & Man. & Man. \\
\hline 90.00 & 30.01 & 60.00 & 37.25 & Man. & 35.23 & 66.00 & Man. & Man. & 55.89 & 26.40 & Man. & 27.77 & Man. & Man. \\
\hline 90.00 & 30.00 & 60.00 & 37.28 & Man. & 35.36 & 66.00 & Man. & Man. & 55.24 & 26.54 & Man. & 27.11 & Man. & Man. \\
\hline 90.00 & 30.01 & 60.00 & 37.53 & Man. & 34.96 & 66.00 & Man. & Man. & 55.91 & 26.19 & Man. & 27.74 & Man. & Man. \\
\hline 90.00 & 30.01 & 60.01 & 37.18 & Man. & 35.53 & 66.00 & Man. & Man. & 55.08 & 26.62 & Man. & 27.43 & Man. & Man. \\
\hline 90.00 & 30.01 & 59.99 & 37.17 & Man. & 35.74 & 66.00 & Man. & Man. & 56.01 & 26.66 & Man. & 27.44 & Man. & Man \\
\hline 90.00 & 30.00 & 60.01 & 37.54 & Man. & 35.65 & 66.00 & Man. & Man. & 56.07 & 26.65 & Man. & 27.85 & Man. & Man. \\
\hline 90.01 & 30.01 & 60.00 & 37.64 & Man. & 35.33 & 66.00 & Man. & Man. & 55.85 & 26.43 & Man. & 27.21 & Man. & Man. \\
\hline 90.00 & 30.01 & 60.00 & 37.55 & Man. & 35.07 & 66.00 & Man. & Man. & 55.18 & 26.25 & Man. & 27.18 & Man. & Man. \\
\hline 90.00 & 30.01 & 59.99 & 37.79 & Man. & 35.44 & 66.00 & Man. & Man. & 55.77 & 26.47 & Man. & 27.46 & Man. & Man. \\
\hline 90.00 & 30.01 & 60.00 & 37.30 & Man. & 35.32 & 66.00 & Man. & Man. & 55.98 & 26.39 & Man. & 27.89 & Man. & Man. \\
\hline 90.00 & 30.01 & 60.01 & 37.67 & Man. & 35.55 & 66.00 & Man. & Man. & 55.50 & 26.61 & Man. & 27.31 & Man. & Man. \\
\hline 90.00 & 30.01 & 60.01 & 37.07 & Man. & 35.32 & 66.00 & Man. & Man. & 55.23 & 26.56 & Man. & 27.39 & Man. & Man. \\
\hline 90.01 & 30.01 & 59.99 & 37.65 & Man. & 35.34 & 66.00 & Man. & Man. & 55.63 & 26.56 & Man. & 27.79 & Man. & Man. \\
\hline 90.00 & 30.01 & 60.00 & 37.15 & Man. & 35.39 & 66.00 & Man. & Man. & 55.74 & 26.47 & Man. & 27.48 & Man. & Man. \\
\hline 90.00 & 30.00 & 59.99 & 37.33 & Man. & 35.19 & 66.00 & Man. & Man. & 55.50 & 26.37 & Man. & 27.31 & Man. & Man. \\
\hline 90.00 & 30.00 & 60.00 & 37.22 & Man. & 35.54 & 66.00 & Man. & Man. & 56.01 & 26.63 & Man. & 27.71 & Man. & Man. \\
\hline 90.00 & 30.00 & 59.99 & 37.28 & Man. & 35.22 & 66.00 & Man. & Man. & 55.73 & 26.43 & Man. & 27.36 & Man. & Man. \\
\hline 90.01 & 30.00 & 59.99 & 37.28 & Man. & 35.55 & 66.00 & Man. & Man. & 56.04 & 26.57 & Man. & 27.80 & Man. & Man. \\
\hline 90.00 & 30.00 & 59.99 & 37.44 & Man. & 35.21 & 66.00 & Man. & Man. & 56.03 & 26.40 & Man. & 27.49 & Man. & Man. \\
\hline 90.01 & 30.00 & 60.00 & 37.31 & Man. & 35.24 & 66.00 & Man. & Man. & 55.34 & 26.39 & Man. & 27.35 & Man. & Man. \\
\hline 90.00 & 30.01 & 60.01 & 37.23 & Man. & 35.43 & 66.00 & Man. & Man. & 55.04 & 26.42 & Man. & 27.18 & Man. & Man \\
\hline 90.00 & 30.01 & 60.00 & 37.51 & Man. & 35.43 & 66.00 & Man. & Man. & 55.82 & 26.59 & Man. & 27.73 & Man. & Man \\
\hline 90.00 & 30.00 & 60.00 & 37.23 & Man. & 35.44 & 66.00 & Man. & Man. & 55.12 & 26.57 & Man. & 27.29 & Man. & Man \\
\hline 90.00 & 30.00 & 59.99 & 37.24 & Man. & 35.40 & 66.00 & Man. & Man. & 56.02 & 26.68 & Man. & 27.79 & Man. & Man \\
\hline 90.00 & 30.00 & 59.99 & 37.24 & Man. & 35.42 & 66.00 & Man. & Man. & 54.98 & 26.60 & Man. & 27.17 & Man. & Man \\
\hline 90.00 & 30.01 & 60.00 & 37.22 & Man. & 35.26 & 66.00 & Man. & Man. & 55.76 & 26.45 & Man. & 27.81 & Man. & Man \\
\hline 90.00 & 30.00 & 59.99 & 37.30 & Man. & 35.27 & 66.00 & Man. & Man. & 55.96 & 26.45 & Man. & 26.89 & Man. & Man. \\
\hline 90.00 & 30.00 & 60.00 & 37.36 & Man. & 35.32 & 66.00 & Man. & Man. & 56.24 & 26.43 & Man. & 27.95 & Man. & Man. \\
\hline 90.01 & 30.01 & 60.01 & 37.30 & Man. & 35.41 & 66.00 & Man. & Man. & 55.66 & 26.54 & Man. & 27.67 & Man. & Man. \\
\hline
\end{tabular}

Table F.22: Throughput Simulation Induction Times (Non-Overlapping Waves - c.v. $=$ $0.4)$.

\begin{tabular}{rllrlrrrrrrrrrrrrr}
\hline 1 & 2 & 3 & 4 & 5 & 6 & 7 & 8 & 9 & 10 & 11 & 12 & 13 & 14 \\
\hline 30.00 & Man. & Man. & 18.74 & Man. & 17.69 & 66.00 & Man. & Man. & Man. & 4.41 & Man. & 9.15 & Man. & Man. \\
30.00 & Man. & Man. & 18.68 & Man. & 17.64 & 66.00 & Man. & Man. & Man. & 4.32 & Man. & 9.21 & Man. & Man. \\
30.01 & Man. & Man. & 18.59 & Man. & 17.75 & 66.00 & Man. & Man. & Man. & 4.51 & Man. & 9.01 & Man. & Man. \\
30.01 & Man. & Man. & 18.72 & Man. & 17.51 & 66.00 & Man. & Man. & Man. & 4.46 & Man. & 9.09 & Man. & Man. \\
30.00 & Man. & Man. & 18.91 & Man. & 17.50 & 66.00 & Man. & Man. & Man. & 4.40 & Man. & 8.91 & Man. & Man. \\
30.00 & Man. & Man. & 18.61 & Man. & 17.51 & 66.00 & Man. & Man. & Man. & 4.40 & Man. & 9.19 & Man. & Man. \\
30.00 & Man. & Man. & 18.78 & Man. & 17.65 & 66.00 & Man. & Man. & Man. & 4.41 & Man. & 9.25 & Man. & Man. \\
30.01 & Man. & Man. & 18.67 & Man. & 17.69 & 66.00 & Man. & Man. & Man. & 4.39 & Man. & 9.39 & Man. & Man. \\
30.00 & Man. & Man. & 18.71 & Man. & 17.57 & 66.00 & Man. & Man. & Man. & 4.45 & Man. & 9.55 & Man. & Man. \\
30.00 & Man. & Man. & 18.75 & Man. & 17.75 & 66.00 & Man. & Man. & Man. & 4.41 & Man. & 9.23 & Man. & Man. \\
\hline
\end{tabular}


Table F.22: Throughput Simulation Induction Times (Non-Overlapping Waves - c.v. $=$ 0.4) - continued.

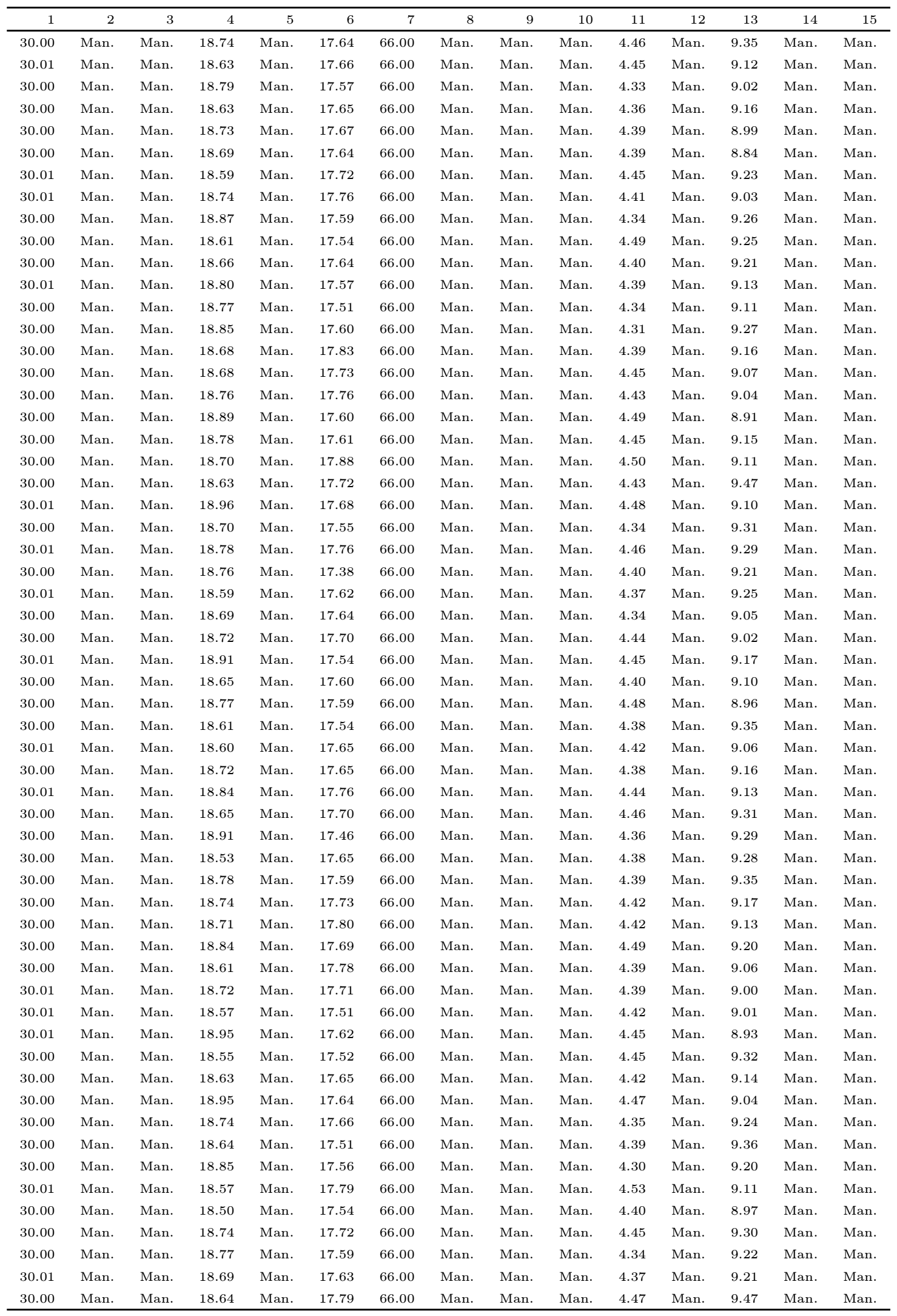


Table F.22: Throughput Simulation Induction Times (Non-Overlapping Waves - c.v. = $0.4)$ - continued.

\begin{tabular}{|c|c|c|c|c|c|c|c|c|c|c|c|c|c|c|}
\hline 1 & 2 & 3 & 4 & 5 & 6 & 7 & 8 & 9 & 10 & 11 & 12 & 13 & 14 & 15 \\
\hline 30.00 & Man. & Man. & 18.58 & Man. & 17.77 & 66.00 & Man. & Man. & Man. & 4.54 & Man. & 9.41 & Man. & Man. \\
\hline 30.00 & Man. & Man. & 18.58 & Man. & 17.74 & 66.00 & Man. & Man. & Man. & 4.43 & Man. & 9.24 & Man. & Man. \\
\hline 30.00 & Man. & Man. & 18.73 & Man. & 17.66 & 66.00 & Man. & Man. & Man. & 4.38 & Man. & 9.12 & Man. & Man. \\
\hline 30.00 & Man. & Man. & 18.78 & Man. & 17.54 & 66.00 & Man. & Man. & Man. & 4.48 & Man. & 9.23 & Man. & Man. \\
\hline 30.00 & Man. & Man. & 18.58 & Man. & 17.57 & 66.00 & Man. & Man. & Man. & 4.38 & Man. & 9.13 & Man. & Man. \\
\hline 30.00 & Man. & Man. & 18.75 & Man. & 17.75 & 66.00 & Man. & Man. & Man. & 4.52 & Man. & 9.13 & Man. & Man. \\
\hline 30.01 & Man. & Man. & 18.76 & Man. & 17.59 & 66.00 & Man. & Man. & Man. & 4.42 & Man. & 9.31 & Man. & Man. \\
\hline 30.00 & Man. & Man. & 18.65 & Man. & 17.51 & 66.00 & Man. & Man. & Man. & 4.38 & Man. & 8.85 & Man. & Man. \\
\hline 30.01 & Man. & Man. & 18.61 & Man. & 17.54 & 66.00 & Man. & Man. & Man. & 4.38 & Man. & 9.08 & Man. & Man. \\
\hline 30.01 & Man. & Man. & 18.70 & Man. & 17.66 & 66.00 & Man. & Man. & Man. & 4.43 & Man. & 9.15 & Man. & Man. \\
\hline 30.00 & Man. & Man. & 18.61 & Man. & 17.56 & 66.00 & Man. & Man. & Man. & 4.38 & Man. & 9.31 & Man. & Man. \\
\hline 30.00 & Man. & Man. & 18.83 & Man. & 17.62 & 66.00 & Man. & Man. & Man. & 4.28 & Man. & 9.35 & Man. & Man. \\
\hline 30.01 & Man. & Man. & 18.75 & Man. & 17.71 & 66.00 & Man. & Man. & Man. & 4.41 & Man. & 9.03 & Man. & Man. \\
\hline 30.00 & Man. & Man. & 18.68 & Man. & 17.74 & 66.00 & Man. & Man. & Man. & 4.43 & Man. & 9.29 & Man. & Man. \\
\hline 30.00 & Man. & Man. & 18.78 & Man. & 17.61 & 66.00 & Man. & Man. & Man. & 4.50 & Man. & 9.22 & Man. & Man. \\
\hline 30.00 & Man. & Man. & 18.63 & Man. & 17.89 & 66.00 & Man. & Man. & Man. & 4.45 & Man. & 9.24 & Man. & Man. \\
\hline 30.01 & Man. & Man. & 18.53 & Man. & 17.64 & 66.00 & Man. & Man. & Man. & 4.45 & Man. & 9.25 & Man. & Man. \\
\hline 30.00 & Man. & Man. & 18.54 & Man. & 17.72 & 66.00 & Man. & Man. & Man. & 4.37 & Man. & 9.33 & Man. & Man. \\
\hline 30.00 & Man. & Man. & 18.68 & Man. & 17.95 & 66.00 & Man. & Man. & Man. & 4.45 & Man. & 9.07 & Man. & Man. \\
\hline 30.00 & Man. & Man. & 18.67 & Man. & 17.63 & 66.00 & Man. & Man. & Man. & 4.37 & Man. & 8.86 & Man. & Man. \\
\hline 30.01 & Man. & Man. & 18.58 & Man. & 17.55 & 66.00 & Man. & Man. & Man. & 4.31 & Man. & 8.99 & Man. & Man. \\
\hline 30.00 & Man. & Man. & 18.56 & Man. & 17.68 & 66.00 & Man. & Man. & Man. & 4.39 & Man. & 9.05 & Man. & Man. \\
\hline 30.01 & Man. & Man. & 18.61 & Man. & 17.78 & 66.00 & Man. & Man. & Man. & 4.37 & Man. & 9.13 & Man. & Man. \\
\hline 30.00 & Man. & Man. & 18.59 & Man. & 17.52 & 66.00 & Man. & Man. & Man. & 4.39 & Man. & 9.03 & Man. & Man. \\
\hline 30.00 & Man. & Man. & 18.71 & Man. & 17.42 & 66.00 & Man. & Man. & Man. & 4.37 & Man. & 9.26 & Man. & Man. \\
\hline 30.00 & Man. & Man. & 18.67 & Man. & 17.66 & 66.00 & Man. & Man. & Man. & 4.39 & Man. & 9.12 & Man. & Man. \\
\hline 30.00 & Man. & Man. & 18.91 & Man. & 17.66 & 66.00 & Man. & Man. & Man. & 4.36 & Man. & 9.21 & Man. & Man. \\
\hline 30.00 & Man. & Man. & 18.68 & Man. & 17.65 & 66.00 & Man. & Man. & Man. & 4.36 & Man. & 9.17 & Man. & Man. \\
\hline 30.01 & Man. & Man. & 18.69 & Man. & 17.74 & 66.00 & Man. & Man. & Man. & 4.36 & Man. & 9.14 & Man. & Man. \\
\hline 30.00 & Man. & Man. & 18.61 & Man. & 17.61 & 66.00 & Man. & Man. & Man. & 4.44 & Man. & 8.91 & Man. & Man. \\
\hline 30.00 & Man. & Man. & 18.70 & Man. & 17.58 & 66.00 & Man. & Man. & Man. & 4.44 & Man. & 9.09 & Man. & Man. \\
\hline 30.01 & Man. & Man. & 18.63 & Man. & 17.50 & 66.00 & Man. & Man. & Man. & 4.43 & Man. & 9.15 & Man. & Man. \\
\hline
\end{tabular}


Table F.23: Number of Items Inducted (Overlapping Waves).

\begin{tabular}{r|rrrr|rrrr}
\hline Trial & \multicolumn{4}{|c|}{ c.v $=0.2$} & \multicolumn{4}{c}{ c.v =0.4 } \\
\cline { 2 - 8 } No. & \multicolumn{1}{|c|}{ Ind. 1 } & Ind. 2 & Ind. 3 & Ind. 4 & Ind. 1 & Ind. 2 & Ind. 3 & Ind. 4 \\
\hline 1 & 10524.0 & 7476.0 & & & 11210.1 & 6789.9 & & \\
2 & 3509.3 & 2490.7 & & & 3737.4 & 2262.6 & & \\
3 & 7007.4 & 1992.7 & & & 6462.0 & 2538.0 & & \\
4 & 2178.1 & 2028.3 & 1793.6 & & 2238.1 & 2046.3 & 1715.6 & \\
5 & Man. & Man. & Man. & Man. & Man. & Man. & Man. & Man. \\
6 & 4400.0 & & & & 4400.0 & & & \\
7 & 6600.0 & & & & 6600.0 & & & \\
8 & Man. & Man. & Man. & Man. & Man. & Man. & Man. & Man. \\
9 & Man. & Man. & Man. & Man. & Man. & Man. & Man. & Man. \\
10 & 1111.2 & 1100.9 & 1087.9 & & 1111.8 & 1100.7 & 1087.5 & \\
11 & 3300.0 & & & & 3300.0 & & & \\
12 & Man. & Man. & Man. & Man. & Man. & Man. & Man. & Man. \\
13 & 1650.0 & & & & 1650.0 & & & \\
14 & Man. & Man. & Man. & Man. & Man. & Man. & Man. & Man. \\
15 & Man. & Man. & Man. & Man. & Man. & Man. & Man. & Man. \\
\hline
\end{tabular}

Table F.24: Number of Items Inducted (Non-overlapping Waves).

\begin{tabular}{r|rrrr|rrrr}
\hline Trial & \multicolumn{4}{|c|}{ c.v $=0.2$} & \multicolumn{4}{c}{ c.v = 0.4 } \\
\cline { 2 - 8 } & Ind. 1 & Ind. 2 & Ind. 3 & Ind. 4 & Ind. 1 & Ind. 2 & Ind. 3 & Ind. 4 \\
\hline 1 & 3509.0 & 2491.1 & & & 3740.4 & 2259.6 & & \\
2 & Man. & Man. & Man. & Man. & Man. & Man. & Man. & Man. \\
3 & Man. & Man. & Man. & Man. & Man. & Man. & Man. & Man. \\
4 & 1089.5 & 1013.5 & 897.0 & & 1119.2 & 1023.2 & 857.6 & \\
5 & Man. & Man. & Man. & Man. & Man. & Man. & Man. & Man. \\
6 & 2200.0 & & & & 2200.0 & & & \\
7 & 6600.0 & & & & 6600.0 & & & \\
8 & Man. & Man. & Man. & Man. & Man. & Man. & Man. & Man. \\
9 & Man. & Man. & Man. & Man. & Man. & Man. & Man. & Man. \\
10 & Man. & Man. & Man. & Man. & Man. & Man. & Man. & Man. \\
11 & 550.0 & & & & 550.0 & & & \\
12 & Man. & Man. & Man. & Man. & Man. & Man. & Man. & Man. \\
13 & 550.0 & & & & 550.0 & & & \\
14 & Man. & Man. & Man. & Man. & Man. & Man. & Man. & Man. \\
15 & Man. & Man. & Man. & Man. & Man. & Man. & Man. & Man. \\
\hline
\end{tabular}




\section{Appendix G}

\section{Adjusted Induction Model}

In order to calculate the number of inductors needed to meet demand in a system, the effective induction rate for each inductor must be used. The effective induction rate for each inductor decreases as the number of inductors increases. That is, the first inductor can reach its maximum induction rate, while the second, third, and fourth inductors successively will be blocked. Tables G.1 through G.3 present the effective rates of the inductors in a system for three different conveyors speeds (100, 150, and 200 trays per minute) and induction rates ranging from 10 to 120 trays per minute. The left column defines the number of induction stations in the system, and the next column is the nominal induction rate. The first set of data is the effective rates of each inductor (1-4). The second set of data is the percentage of the actual rate that the inductor can achieve.

The number of inductors is now calculated by first determining the number of items that

must be inducted per minute in order to meet demand, $\frac{D * N_{o}}{60}$. Using the appropriate table based on the conveyor speed, the number of inductors is determined by first selecting the number of stations and then the nominal inductor rate. The number of stations required is determined by the following equation (as presented in Chapter 4).

$$
r_{s}= \begin{cases}1 & \text { if } x \leq s \\ \lceil x /(2 s-x)\rceil & \text { if } x>s\end{cases}
$$


Dividing the required throughput per minute needed to meet demand by $r_{s}$ yields the total effective induction rate needed at each station. Finally, the effective rates for one inductor, two inductors, etc., are added until the required demand per minute is met. This model assumes that only four inductors can be placed at one station, and a sorter can have no more than four induction stations.

In order to illustrate, consider a system with a demand level of 3300 orders per hour, a sorter speed of 150 trays per minute, and an induction rate of 60 trays per minute. Spreadsheet calculations based on the total throughput required per minute and (G.1) result in the requirement of two induction stations. Looking at Table G.2 (sorter speed of 150 trays per minute), we first find data corresponding to two induction stations. Then, we calculate the required number of items per minute that must be inducted at each station in order to meet demand, $\frac{1}{2}(3300 * 3 / 60)$, or 82.5 items per minute at each station. Using the data for two induction stations, we look at the effective rate of the first inductor with an actual rate of 60 items per minute. The rate for the first inductor is 40 items per minute. Since this is less than 82.5 , we must add another inductor. With two inductors, the total effective rate is 74.5 items per minute at each station. Three inductors are therefore required to meet demand, at a total effective induction rate of 100 items per minute at each station. With six inductors total (three at each station), this results in 200 items per minute total, which exceeds the demand requirements of 165 items per minute.

The following examples were developed in order to test the adjusted induction model with more than one induction station. Each of the examples tested in the throughput simulations required only one induction station. The examples presented in this appendix provide us with confidence that this model accurately predicts the number of inductors and induction stations necessary to meet demand. Table G.4 provides the details of the example experiments. Tables G.5 and G.6 present the results for each example. Finally, Table G.7 provides the time to induct the assigned items for each replication. 
Table G.1: Effective Induction Rates for Conveyor Speed of 100 Trays per Minute.

\begin{tabular}{|c|c|c|c|c|c|c|c|c|c|}
\hline & \multirow{2}{*}{$\begin{array}{c}\text { Nominal } \\
\text { Induction Rate } \\
\text { (tpm) }\end{array}$} & \multicolumn{4}{|c|}{$\begin{array}{l}\text { Effective Induction Rate } \\
\text { (tpm) }\end{array}$} & \multicolumn{4}{|c|}{$\begin{array}{c}\% \text { of Nominal Induction Rate } \\
(\%)\end{array}$} \\
\hline & & 1 & 2 & 3 & 4 & 1 & 2 & 3 & 4 \\
\hline \multirow{12}{*}{1 Station } & 10 & 10.00 & 9.90 & 9.80 & 9.60 & 100.00 & 99.00 & 98.00 & 96.00 \\
\hline & 20 & 20.00 & 19.20 & 18.00 & 16.40 & 100.00 & 96.00 & 90.00 & 82.00 \\
\hline & 30 & 30.00 & 27.50 & 23.60 & 18.70 & 100.00 & 91.67 & 78.67 & 62.33 \\
\hline & 40 & 40.00 & 34.50 & 25.50 & 0.00 & 100.00 & 86.25 & 63.75 & 0.00 \\
\hline & 50 & 50.00 & 40.00 & 10.00 & 0.00 & 100.00 & 80.00 & 20.00 & 0.00 \\
\hline & 60 & 60.00 & 40.00 & 0.00 & 0.00 & 100.00 & 66.67 & 0.00 & 0.00 \\
\hline & 70 & 70.00 & 30.00 & 0.00 & 0.00 & 100.00 & 42.86 & 0.00 & 0.00 \\
\hline & 80 & 80.00 & 20.00 & 0.00 & 0.00 & 100.00 & 25.00 & 0.00 & 0.00 \\
\hline & 90 & 90.00 & 10.00 & 0.00 & 0.00 & 100.00 & 11.11 & 0.00 & 0.00 \\
\hline & 100 & 100.00 & 0.00 & 0.00 & 0.00 & 100.00 & 0.00 & 0.00 & 0.00 \\
\hline & 110 & 100.00 & 0.00 & 0.00 & 0.00 & 90.91 & 0.00 & 0.00 & 0.00 \\
\hline & 120 & 100.00 & 0.00 & 0.00 & 0.00 & 83.33 & 0.00 & 0.00 & 0.00 \\
\hline \multirow{12}{*}{2 Stations } & 10 & 9.30 & 9.20 & 9.10 & 9.00 & 93.00 & 92.00 & 91.00 & 90.00 \\
\hline & 20 & 16.40 & 15.70 & 14.80 & 13.40 & 82.00 & 78.50 & 74.00 & 67.00 \\
\hline & 30 & 20.00 & 18.40 & 15.70 & 12.50 & 66.67 & 61.33 & 52.33 & 41.67 \\
\hline & 40 & 26.70 & 23.00 & 17.00 & 0.00 & 66.75 & 57.50 & 42.50 & 0.00 \\
\hline & 50 & 33.30 & 26.70 & 6.70 & 0.00 & 66.60 & 53.40 & 13.40 & 0.00 \\
\hline & 60 & 40.00 & 26.70 & 0.00 & 0.00 & 66.67 & 44.50 & 0.00 & 0.00 \\
\hline & 70 & 46.70 & 20.00 & 0.00 & 0.00 & 66.71 & 28.57 & 0.00 & 0.00 \\
\hline & 80 & 53.30 & 13.30 & 0.00 & 0.00 & 66.63 & 16.63 & 0.00 & 0.00 \\
\hline & 90 & 60.00 & 6.70 & 0.00 & 0.00 & 66.67 & 7.44 & 0.00 & 0.00 \\
\hline & 100 & 66.70 & 0.00 & 0.00 & 0.00 & 66.70 & 0.00 & 0.00 & 0.00 \\
\hline & 110 & 66.70 & 0.00 & 0.00 & 0.00 & 60.64 & 0.00 & 0.00 & 0.00 \\
\hline & 120 & 66.70 & 0.00 & 0.00 & 0.00 & 55.58 & 0.00 & 0.00 & 0.00 \\
\hline \multirow{12}{*}{3 Stations } & 10 & 8.50 & 8.40 & 8.30 & 8.20 & 85.00 & 84.00 & 83.00 & 82.00 \\
\hline & 20 & 13.10 & 12.60 & 11.80 & 10.80 & 65.50 & 63.00 & 59.00 & 54.00 \\
\hline & 30 & 15.00 & 13.80 & 11.80 & 9.40 & 50.00 & 46.00 & 39.33 & 31.33 \\
\hline & 40 & 20.00 & 17.20 & 12.80 & 0.00 & 50.00 & 43.00 & 32.00 & 0.00 \\
\hline & 50 & 25.00 & 20.00 & 5.00 & 0.00 & 50.00 & 40.00 & 10.00 & 0.00 \\
\hline & 60 & 30.00 & 20.00 & 0.00 & 0.00 & 50.00 & 33.33 & 0.00 & 0.00 \\
\hline & 70 & 35.00 & 15.00 & 0.00 & 0.00 & 50.00 & 21.43 & 0.00 & 0.00 \\
\hline & 80 & 40.00 & 10.00 & 0.00 & 0.00 & 50.00 & 12.50 & 0.00 & 0.00 \\
\hline & 90 & 45.00 & 5.00 & 0.00 & 0.00 & 50.00 & 5.56 & 0.00 & 0.00 \\
\hline & 100 & 50.00 & 0.00 & 0.00 & 0.00 & 50.00 & 0.00 & 0.00 & 0.00 \\
\hline & 110 & 50.00 & 0.00 & 0.00 & 0.00 & 45.45 & 0.00 & 0.00 & 0.00 \\
\hline & 120 & 50.00 & 0.00 & 0.00 & 0.00 & 41.67 & 0.00 & 0.00 & 0.00 \\
\hline \multirow{12}{*}{4 Stations } & 10 & 7.70 & 7.60 & 7.50 & 7.40 & 77.00 & 76.00 & 75.00 & $\overline{74.00}$ \\
\hline & 20 & 10.80 & 10.40 & 9.70 & 8.90 & 54.00 & 52.00 & 48.50 & 44.50 \\
\hline & 30 & 12.00 & 11.00 & 9.40 & 7.50 & 40.00 & 36.67 & 31.33 & 25.00 \\
\hline & 40 & 16.00 & 13.80 & 10.20 & 0.00 & 40.00 & 34.50 & 25.50 & 0.00 \\
\hline & 50 & 20.00 & 16.00 & 4.00 & 0.00 & 40.00 & 32.00 & 8.00 & 0.00 \\
\hline & 60 & 24.00 & 16.00 & 0.00 & 0.00 & 40.00 & 26.67 & 0.00 & 0.00 \\
\hline & 70 & 28.00 & 12.00 & 0.00 & 0.00 & 40.00 & 17.14 & 0.00 & 0.00 \\
\hline & 80 & 32.00 & 8.00 & 0.00 & 0.00 & 40.00 & 10.00 & 0.00 & 0.00 \\
\hline & 90 & 36.00 & 4.00 & 0.00 & 0.00 & 40.00 & 4.44 & 0.00 & 0.00 \\
\hline & 100 & 40.00 & 0.00 & 0.00 & 0.00 & 40.00 & 0.00 & 0.00 & 0.00 \\
\hline & 110 & 40.00 & 0.00 & 0.00 & 0.00 & 36.36 & 0.00 & 0.00 & 0.00 \\
\hline & 120 & 40.00 & 0.00 & 0.00 & 0.00 & 33.33 & 0.00 & 0.00 & 0.00 \\
\hline
\end{tabular}


Table G.2: Effective Induction Rates for Conveyor Speed of 150 Trays per Minute.

\begin{tabular}{|c|c|c|c|c|c|c|c|c|c|}
\hline & \multirow{2}{*}{$\begin{array}{c}\text { Nominal } \\
\text { Induction Rate } \\
(\mathrm{tpm})\end{array}$} & \multicolumn{4}{|c|}{$\begin{array}{l}\text { Effective Induction Rate } \\
(\text { tpm) }\end{array}$} & \multicolumn{4}{|c|}{$\begin{array}{c}\% \text { of Nominal Induction Rate } \\
(\%)\end{array}$} \\
\hline & & 1 & 2 & 3 & 4 & 1 & 2 & 3 & 4 \\
\hline \multirow{12}{*}{1 Station } & 10 & 10.00 & 9.96 & 9.90 & 9.84 & 100.00 & 99.56 & 99.01 & 98.38 \\
\hline & 20 & 20.00 & 19.70 & 19.10 & 18.50 & 100.00 & 98.50 & 95.50 & 92.50 \\
\hline & 30 & 30.00 & 28.85 & 27.05 & 24.64 & 100.00 & 96.15 & 90.15 & 82.13 \\
\hline & 40 & 40.00 & 37.34 & 33.10 & 27.64 & 100.00 & 93.36 & 82.75 & 69.09 \\
\hline & 50 & 50.00 & 45.00 & 37.18 & 17.82 & 100.00 & 90.00 & 74.36 & 35.64 \\
\hline & 60 & 60.00 & 51.70 & 38.30 & 0.00 & 100.00 & 86.17 & 63.83 & 0.00 \\
\hline & 70 & 70.00 & 57.48 & 22.52 & 0.00 & 100.00 & 82.12 & 32.17 & 0.00 \\
\hline & 80 & 80.00 & 62.28 & 7.72 & 0.00 & 100.00 & 77.85 & 9.65 & 0.00 \\
\hline & 90 & 90.00 & 60.00 & 0.00 & 0.00 & 100.00 & 66.67 & 0.00 & 0.00 \\
\hline & 100 & 100.00 & 50.00 & 0.00 & 0.00 & 100.00 & 50.00 & 0.00 & 0.00 \\
\hline & 110 & 110.00 & 40.00 & 0.00 & 0.00 & 100.00 & 36.36 & 0.00 & 0.00 \\
\hline & 120 & 120.00 & 30.00 & 0.00 & 0.00 & 100.00 & 25.00 & 0.00 & 0.00 \\
\hline \multirow{12}{*}{2 Stations } & 10 & 9.67 & 9.63 & 9.58 & 9.52 & 96.72 & 96.30 & 95.76 & 95.16 \\
\hline & 20 & 17.90 & 17.60 & 17.10 & 16.50 & 89.50 & 88.00 & 85.50 & 82.50 \\
\hline & 30 & 24.55 & 23.60 & 22.13 & 20.16 & 81.82 & 78.68 & 73.77 & 67.20 \\
\hline & 40 & 28.97 & 27.05 & 23.97 & 20.01 & 72.42 & 67.61 & 59.93 & 50.04 \\
\hline & 50 & 33.33 & 30.00 & 24.79 & 11.88 & 66.67 & 60.00 & 49.57 & 23.76 \\
\hline & 60 & 40.00 & 34.50 & 25.50 & 0.00 & 66.67 & 57.50 & 42.50 & 0.00 \\
\hline & 70 & 46.67 & 38.32 & 15.01 & 0.00 & 66.67 & 54.74 & 21.45 & 0.00 \\
\hline & 80 & 53.33 & 41.52 & 5.14 & 0.00 & 66.67 & 51.90 & 6.43 & 0.00 \\
\hline & 90 & 60.00 & 40.00 & 0.00 & 0.00 & 66.67 & 44.44 & 0.00 & 0.00 \\
\hline & 100 & 66.67 & 33.33 & 0.00 & 0.00 & 66.67 & 33.33 & 0.00 & 0.00 \\
\hline & 110 & 73.33 & 26.67 & 0.00 & 0.00 & 66.67 & 24.24 & 0.00 & 0.00 \\
\hline & 120 & 80.00 & 20.00 & 0.00 & 0.00 & 66.67 & 16.67 & 0.00 & 0.00 \\
\hline \multirow{12}{*}{3 Stations } & 10 & 9.25 & 9.21 & 9.16 & 9.10 & 92.54 & 92.13 & 91.61 & 91.04 \\
\hline & 20 & 15.50 & 15.30 & 14.90 & 14.40 & 77.50 & 76.50 & 74.50 & 72.00 \\
\hline & 30 & 19.64 & 18.89 & 17.71 & 16.13 & 65.48 & 62.96 & 59.03 & 53.78 \\
\hline & 40 & 21.73 & 20.28 & 17.98 & 15.01 & 54.32 & 50.71 & 44.95 & 37.53 \\
\hline & 50 & 25.00 & 22.50 & 18.59 & 8.91 & 50.00 & 45.00 & 37.18 & 17.82 \\
\hline & 60 & 30.00 & 25.90 & 19.10 & 0.00 & 50.00 & 43.17 & 31.83 & 0.00 \\
\hline & 70 & 35.00 & 28.74 & 11.26 & 0.00 & 50.00 & 41.06 & 16.08 & 0.00 \\
\hline & 80 & 40.00 & 31.14 & 3.86 & 0.00 & 50.00 & 38.93 & 4.82 & 0.00 \\
\hline & 90 & 45.00 & 30.00 & 0.00 & 0.00 & 50.00 & 33.33 & 0.00 & 0.00 \\
\hline & 100 & 50.00 & 25.00 & 0.00 & 0.00 & 50.00 & 25.00 & 0.00 & 0.00 \\
\hline & 110 & 55.00 & 20.00 & 0.00 & 0.00 & 50.00 & 18.18 & 0.00 & 0.00 \\
\hline & 120 & 60.00 & 15.00 & 0.00 & 0.00 & 50.00 & 12.50 & 0.00 & 0.00 \\
\hline \multirow{12}{*}{4 Stations } & 10 & 8.80 & 8.76 & 8.71 & 8.66 & 88.02 & $\overline{87.63}$ & 87.15 & 86.60 \\
\hline & 20 & 13.50 & 13.30 & 12.90 & 12.50 & 67.50 & 66.50 & 64.50 & 62.50 \\
\hline & 30 & 16.16 & 15.54 & 14.57 & 13.28 & 53.88 & 51.81 & 48.58 & 44.25 \\
\hline & 40 & 17.38 & 16.23 & 14.38 & 12.01 & 43.45 & 40.57 & 35.96 & 30.02 \\
\hline & 50 & 20.00 & 18.00 & 14.87 & 7.13 & 40.00 & 36.00 & 29.74 & 14.26 \\
\hline & 60 & 24.00 & 20.70 & 15.30 & 0.00 & 40.00 & 34.50 & 25.50 & 0.00 \\
\hline & 70 & 28.00 & 22.99 & 9.01 & 0.00 & 40.00 & 32.85 & 12.87 & 0.00 \\
\hline & 80 & 32.00 & 24.91 & 3.09 & 0.00 & 40.00 & 31.14 & 3.86 & 0.00 \\
\hline & 90 & 36.00 & 24.00 & 0.00 & 0.00 & 40.00 & 26.67 & 0.00 & 0.00 \\
\hline & 100 & 40.00 & 20.00 & 0.00 & 0.00 & 40.00 & 20.00 & 0.00 & 0.00 \\
\hline & 110 & 44.00 & 16.00 & 0.00 & 0.00 & 40.00 & 14.55 & 0.00 & 0.00 \\
\hline & 120 & 48.00 & 12.00 & 0.00 & 0.00 & 40.00 & 10.00 & 0.00 & 0.00 \\
\hline
\end{tabular}


Table G.3: Effective Induction Rates for Conveyor Speed of 200 Trays per Minute.

\begin{tabular}{|c|c|c|c|c|c|c|c|c|c|}
\hline & \multirow{2}{*}{$\begin{array}{c}\text { Nominal } \\
\text { Induction Rate } \\
(\mathrm{tpm})\end{array}$} & \multicolumn{4}{|c|}{$\begin{array}{l}\text { Effective Induction Rate } \\
(\text { tpm) }\end{array}$} & \multicolumn{4}{|c|}{$\begin{array}{c}\% \text { of Nominal Induction Rate } \\
(\%)\end{array}$} \\
\hline & & 1 & 2 & 3 & 4 & 1 & 2 & 3 & 4 \\
\hline \multirow{12}{*}{1 Station } & 10 & 10.00 & 9.98 & 9.95 & 9.91 & 100.00 & 99.75 & 99.45 & 99.13 \\
\hline & 20 & 20.00 & 19.80 & 19.50 & 19.20 & 100.00 & 99.00 & 97.50 & 96.00 \\
\hline & 30 & 30.00 & 29.34 & 28.36 & 27.09 & 100.00 & 97.80 & 94.54 & 90.31 \\
\hline & 40 & 40.00 & 38.46 & 36.06 & 32.85 & 100.00 & 96.15 & 90.15 & 82.13 \\
\hline & 50 & 50.00 & 47.06 & 42.37 & 36.22 & 100.00 & 94.12 & 84.73 & 72.44 \\
\hline & 60 & 60.00 & 55.00 & 47.20 & 37.50 & 100.00 & 91.67 & 78.67 & 62.50 \\
\hline & 70 & 70.00 & 62.36 & 50.54 & 17.09 & 100.00 & 89.09 & 72.21 & 24.42 \\
\hline & 80 & 80.00 & 68.97 & 51.03 & 0.00 & 100.00 & 86.21 & 63.79 & 0.00 \\
\hline & 90 & 90.00 & 74.84 & 35.16 & 0.00 & 100.00 & 83.16 & 39.06 & 0.00 \\
\hline & 100 & 100.00 & 80.00 & 20.00 & 0.00 & 100.00 & 80.00 & 20.00 & 0.00 \\
\hline & 110 & 110.00 & 84.45 & 5.55 & 0.00 & 100.00 & 76.78 & 5.04 & 0.00 \\
\hline & 120 & 120.00 & 80.00 & 0.00 & 0.00 & 100.00 & 66.67 & 0.00 & 0.00 \\
\hline \multirow{12}{*}{2 Stations } & 10 & 9.81 & 9.78 & 9.76 & 9.72 & 98.09 & 97.85 & 97.56 & $\overline{97.24}$ \\
\hline & 20 & 18.70 & 18.50 & 18.20 & 17.90 & 93.50 & 92.50 & 91.00 & 89.50 \\
\hline & 30 & 26.22 & 25.65 & 24.79 & 23.68 & 87.41 & 85.49 & 82.64 & 78.94 \\
\hline & 40 & 32.73 & 31.47 & 29.51 & 26.88 & 81.82 & 78.68 & 73.77 & 67.20 \\
\hline & 50 & 37.96 & 35.72 & 32.16 & 27.49 & 75.91 & 71.45 & 64.32 & 54.99 \\
\hline & 60 & 40.10 & 36.80 & 31.50 & 25.00 & 66.83 & 61.33 & 52.50 & 41.67 \\
\hline & 70 & 46.67 & 41.57 & 33.70 & 11.40 & 66.67 & 59.39 & 48.14 & 16.28 \\
\hline & 80 & 53.33 & 45.98 & 34.02 & 0.00 & 66.67 & 57.47 & 42.53 & 0.00 \\
\hline & 90 & 60.00 & 49.90 & 23.44 & 0.00 & 66.67 & 55.44 & 26.04 & 0.00 \\
\hline & 100 & 66.67 & 53.33 & 13.33 & 0.00 & 66.67 & 53.33 & 13.33 & 0.00 \\
\hline & 110 & 73.33 & 56.30 & 3.70 & 0.00 & 66.67 & 51.18 & 3.36 & 0.00 \\
\hline & 120 & 80.00 & 53.30 & 0.00 & 0.00 & 66.67 & 44.42 & 0.00 & 0.00 \\
\hline \multirow{12}{*}{3 Stations } & 10 & 9.57 & 9.54 & 9.52 & 9.48 & 95.68 & $\overline{95.44}$ & 95.15 & 94.85 \\
\hline & 20 & 17.00 & 16.90 & 16.60 & 16.30 & 85.00 & 84.50 & 83.00 & 81.50 \\
\hline & 30 & 22.25 & 21.76 & 21.04 & 20.09 & 74.16 & 72.53 & 70.12 & 66.98 \\
\hline & 40 & 26.19 & 25.19 & 23.61 & 21.51 & 65.48 & 62.96 & 59.03 & 53.78 \\
\hline & 50 & 28.47 & 26.79 & 24.12 & 20.62 & 56.93 & 53.58 & 48.24 & 41.24 \\
\hline & 60 & 30.00 & 27.60 & 23.60 & 18.80 & 50.00 & 46.00 & 39.33 & 31.33 \\
\hline & 70 & 35.00 & 31.18 & 25.27 & 8.55 & 50.00 & 44.54 & 36.10 & 12.21 \\
\hline & 80 & 40.00 & 34.48 & 25.52 & 0.00 & 50.00 & 43.10 & 31.90 & 0.00 \\
\hline & 90 & 45.00 & 37.42 & 17.58 & 0.00 & 50.00 & 41.58 & 19.53 & 0.00 \\
\hline & 100 & 50.00 & 40.00 & 10.00 & 0.00 & 50.00 & 40.00 & 10.00 & 0.00 \\
\hline & 110 & 55.00 & 42.23 & 2.77 & 0.00 & 50.00 & 38.39 & 2.52 & 0.00 \\
\hline & 120 & 60.00 & 40.00 & 0.00 & 0.00 & 50.00 & 33.33 & 0.00 & 0.00 \\
\hline \multirow{12}{*}{4 Stations } & 10 & 9.30 & 9.28 & 9.25 & 9.22 & 93.02 & 92.79 & 92.51 & 92.21 \\
\hline & 20 & 15.40 & 15.30 & 15.10 & 14.80 & 77.00 & 76.50 & 75.50 & 74.00 \\
\hline & 30 & 19.02 & 18.60 & 17.98 & 17.17 & 63.39 & 61.99 & 59.93 & 57.25 \\
\hline & 40 & 21.55 & 20.72 & 19.43 & 17.70 & 53.88 & 51.81 & 48.58 & 44.25 \\
\hline & 50 & 22.77 & 21.43 & 19.30 & 16.50 & 45.55 & 42.87 & 38.59 & 32.99 \\
\hline & 60 & 24.00 & 22.10 & 18.90 & 15.00 & 40.00 & 36.83 & 31.50 & 25.00 \\
\hline & 70 & 28.00 & 24.94 & 20.22 & 6.84 & 40.00 & 35.63 & 28.88 & 9.77 \\
\hline & 80 & 32.00 & 27.59 & 20.41 & 0.00 & 40.00 & 34.48 & 25.52 & 0.00 \\
\hline & 90 & 36.00 & 29.94 & 14.06 & 0.00 & 40.00 & 33.26 & 15.62 & 0.00 \\
\hline & 100 & 40.00 & 32.00 & 8.00 & 0.00 & 40.00 & 32.00 & 8.00 & 0.00 \\
\hline & 110 & 44.00 & 33.78 & 2.22 & 0.00 & 40.00 & 30.71 & 2.02 & 0.00 \\
\hline & 120 & 48.00 & 32.00 & 0.00 & 0.00 & 40.00 & 26.67 & 0.00 & 0.00 \\
\hline
\end{tabular}


Table G.4: Input Data for Example Experiments.

\begin{tabular}{cccccccc}
\hline $\begin{array}{c}\text { Trial } \\
\text { No. }\end{array}$ & $\begin{array}{c}\text { Demand } \\
\text { (orders/hr) }\end{array}$ & $\begin{array}{c}\text { Wave Length } \\
\text { (min) }\end{array}$ & $\begin{array}{c}\text { Reqd Throughput } \\
\text { (items/wave) }\end{array}$ & $\begin{array}{c}\text { Sorter Speed } \\
\text { (trays/min) }\end{array}$ & $\begin{array}{c}\text { Induction Rate } \\
\text { (items/min) }\end{array}$ & $\begin{array}{c}\text { Required } \\
\text { Inductors }\end{array}$ & $\begin{array}{c}\text { Required } \\
\text { Stations }\end{array}$ \\
\hline Example 1 & 1500 & 40 & 3000 & 100 & 20 & 6 & 2 \\
Example 2 & 3000 & 40 & 6000 & 100 & 20 & 16 & 4 \\
Example 3 & 2200 & 40 & 4400 & 100 & 20 & 8 & 2 \\
\hline
\end{tabular}

Table G.5: Induction Results from Example Experiments.

\begin{tabular}{r|rrr|rrr}
\hline & \multicolumn{3}{|c|}{ c.v. $=0.2$} & \multicolumn{3}{c}{ c.v. $=0.4$} \\
\cline { 2 - 7 } & Example 1 & Example 2 & Example 3 & Example 1 & Example 2 & Example 3 \\
\hline Inductor Utilization & 0.7142 & 0.9416 & 0.8877 & 0.7245 & 0.9448 & 0.9005 \\
Maximum Time (min) & 28.86 & 38.13 & 35.87 & 29.48 & 38.26 & 36.51 \\
Minimum Time (min) & 28.27 & 37.22 & 35.12 & 28.67 & 37.14 & 35.64 \\
\hline
\end{tabular}

Table G.6: Inductor Throughput for Example Experiments.

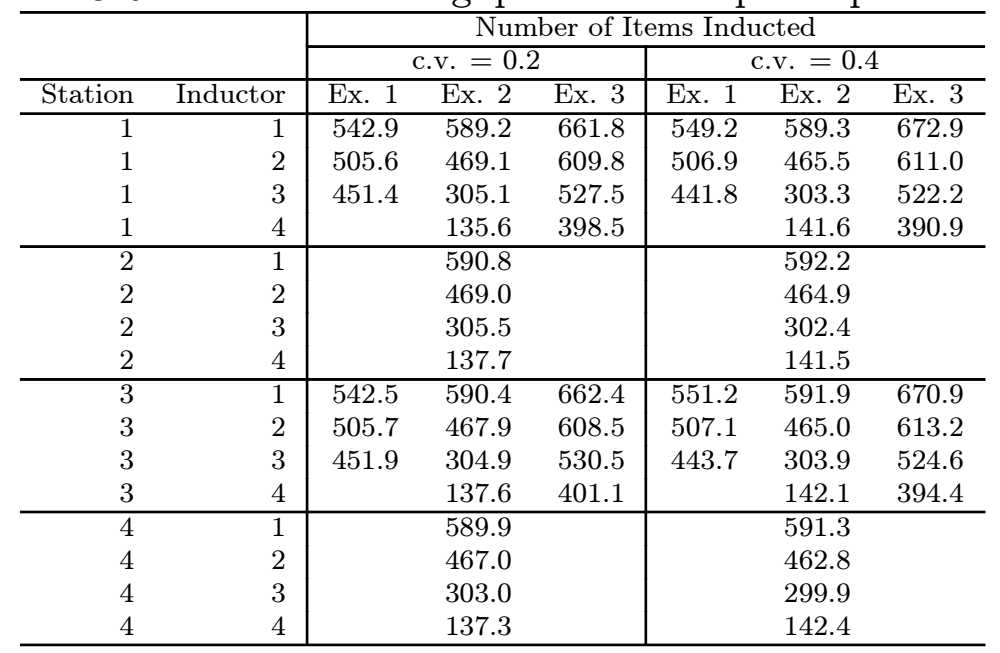


Table G.7: Induction Times for Example Experiments.

\begin{tabular}{|c|c|c|c|c|c|}
\hline \multicolumn{3}{|c|}{ c.v. $=0.2$} & \multicolumn{3}{|c|}{ c.v. $=0.4$} \\
\hline Example 1 & Example 2 & Example 3 & Example 1 & Example 2 & Example 3 \\
\hline 28.38 & 37.41 & 35.41 & 28.91 & 37.63 & 36.25 \\
\hline 28.66 & 37.80 & 35.37 & 29.12 & 37.88 & 35.81 \\
\hline 28.74 & 37.82 & 35.51 & 28.99 & 37.77 & 36.17 \\
\hline 28.46 & 37.44 & 35.28 & 28.80 & 37.14 & 35.93 \\
\hline 28.82 & 37.94 & 35.46 & 29.21 & 38.02 & 36.09 \\
\hline 28.48 & 37.95 & 35.48 & 28.76 & 38.26 & 35.89 \\
\hline 28.43 & 37.71 & 35.68 & 28.82 & 37.72 & 36.09 \\
\hline 28.36 & 37.47 & 35.41 & 28.82 & 37.78 & 36.11 \\
\hline 28.62 & 38.04 & 35.84 & 29.01 & 38.13 & 36.13 \\
\hline 28.85 & 37.56 & 35.30 & 29.10 & 37.84 & 36.00 \\
\hline 28.57 & 38.02 & 35.47 & 28.89 & 38.17 & 36.07 \\
\hline 28.61 & 37.70 & 35.43 & 28.83 & 37.78 & 35.90 \\
\hline 28.68 & 37.74 & 35.46 & 28.81 & 38.03 & 35.96 \\
\hline 28.50 & 37.89 & 35.87 & 29.21 & 37.94 & 36.19 \\
\hline 28.54 & 37.63 & 35.58 & 28.95 & 37.59 & 36.04 \\
\hline 28.46 & 38.04 & 35.48 & 28.84 & 37.72 & 36.11 \\
\hline 28.67 & 37.79 & 35.53 & 28.89 & 37.91 & 36.16 \\
\hline 28.61 & 37.72 & 35.56 & 28.92 & 37.62 & 36.14 \\
\hline 28.54 & 37.74 & 35.34 & 29.16 & 37.53 & 35.89 \\
\hline 28.71 & 37.40 & 35.54 & 28.89 & 37.52 & 35.73 \\
\hline 28.46 & 37.72 & 35.38 & 28.94 & 37.78 & 36.11 \\
\hline 28.60 & 37.29 & 35.34 & 29.10 & 37.70 & 36.04 \\
\hline 28.69 & 37.47 & 35.53 & 28.75 & 37.78 & 36.19 \\
\hline 28.76 & 37.69 & 35.51 & 29.07 & 37.62 & 36.03 \\
\hline 28.41 & 37.53 & 35.59 & 28.91 & 37.61 & 36.11 \\
\hline 28.42 & 37.71 & 35.37 & 28.94 & 37.80 & 36.29 \\
\hline 28.56 & 37.70 & 35.69 & 29.25 & 37.81 & 36.25 \\
\hline 28.86 & 37.54 & 35.59 & 29.12 & 37.40 & 36.21 \\
\hline 28.58 & 37.67 & 35.42 & 28.83 & 37.84 & 36.14 \\
\hline 28.49 & 37.98 & 35.61 & 28.99 & 37.98 & 35.76 \\
\hline 28.54 & 37.60 & 35.48 & 29.14 & 37.70 & 35.93 \\
\hline 28.63 & 37.87 & 35.43 & 28.92 & 38.02 & 35.91 \\
\hline 28.48 & 37.45 & 35.37 & 29.25 & 37.80 & 36.22 \\
\hline 28.50 & 37.62 & 35.64 & 28.98 & 37.91 & 36.24 \\
\hline 28.53 & 37.61 & 35.64 & 29.06 & 37.58 & 35.86 \\
\hline 28.52 & 37.93 & 35.56 & 29.00 & 37.84 & 36.09 \\
\hline 28.57 & 37.36 & 35.50 & 28.82 & 37.71 & 35.90 \\
\hline 28.61 & 37.54 & 35.56 & 28.87 & 37.62 & 36.10 \\
\hline 28.42 & 38.08 & 35.53 & 28.74 & 38.14 & 35.93 \\
\hline 28.54 & 37.45 & 35.63 & 28.83 & 37.55 & 36.07 \\
\hline 28.69 & 37.94 & 35.36 & 28.82 & 37.94 & 35.82 \\
\hline 28.46 & 37.90 & 35.43 & 28.71 & 38.13 & 36.00 \\
\hline 28.63 & 38.13 & 35.73 & 28.88 & 38.15 & 35.97 \\
\hline 28.58 & 37.59 & 35.64 & 28.95 & 37.77 & 35.89 \\
\hline 28.48 & 37.83 & 35.12 & 29.21 & 37.76 & 36.01 \\
\hline 28.60 & 37.55 & 35.61 & 28.92 & 37.67 & 36.17 \\
\hline 28.44 & 37.73 & 35.47 & 29.25 & 37.89 & 36.02 \\
\hline 28.56 & 37.57 & 35.56 & 28.85 & 37.74 & 35.93 \\
\hline 28.64 & 37.72 & 35.81 & 29.00 & 37.61 & 35.99 \\
\hline 28.79 & 37.40 & 35.36 & 28.99 & 37.86 & 35.86 \\
\hline 28.65 & 37.62 & 35.43 & 29.15 & 37.60 & 35.64 \\
\hline 28.45 & 37.40 & 35.46 & 29.18 & 37.66 & 35.90 \\
\hline 28.38 & 37.53 & 35.84 & 28.87 & 37.60 & 36.39 \\
\hline 28.41 & 37.70 & 35.54 & 29.02 & 37.96 & 35.74 \\
\hline 28.58 & 37.40 & 35.76 & 28.76 & 37.64 & 36.51 \\
\hline 28.60 & 37.26 & 35.63 & 29.11 & 37.71 & 36.08 \\
\hline 28.48 & 37.97 & 35.39 & 29.06 & 38.01 & 36.16 \\
\hline
\end{tabular}


Table G.7: Induction Times for Example Experiments — continued.

\begin{tabular}{|c|c|c|c|c|c|}
\hline \multicolumn{3}{|c|}{ c.v. $=0.2$} & \multicolumn{3}{|c|}{ c.v. $=0.4$} \\
\hline Example 1 & Example 2 & Example 3 & Example 1 & Example 2 & Example 3 \\
\hline 28.73 & 37.60 & 35.32 & 29.16 & 37.80 & 35.81 \\
\hline 28.50 & 37.89 & 35.61 & 28.82 & 37.91 & 36.20 \\
\hline 28.60 & 37.56 & 35.34 & 28.84 & 37.85 & 35.89 \\
\hline 28.48 & 37.96 & 35.87 & 29.20 & 37.75 & 36.07 \\
\hline 28.61 & 37.48 & 35.72 & 29.14 & 37.82 & 36.43 \\
\hline 28.33 & 37.64 & 35.49 & 28.70 & 37.66 & 35.89 \\
\hline 28.52 & 37.87 & 35.58 & 28.72 & 38.12 & 36.29 \\
\hline 28.40 & 37.75 & 35.48 & 28.99 & 37.91 & 36.12 \\
\hline 28.55 & 37.42 & 35.68 & 29.02 & 37.67 & 36.20 \\
\hline 28.51 & 37.65 & 35.54 & 29.22 & 37.51 & 36.08 \\
\hline 28.74 & 37.49 & 35.67 & 29.06 & 37.86 & 36.22 \\
\hline 28.60 & 37.86 & 35.65 & 28.82 & 37.89 & 36.25 \\
\hline 28.70 & 37.44 & 35.39 & 29.20 & 37.87 & 35.75 \\
\hline 28.47 & 37.45 & 35.55 & 29.04 & 37.57 & 35.86 \\
\hline 28.61 & 37.37 & 35.54 & 28.96 & 37.69 & 35.91 \\
\hline 28.59 & 37.61 & 35.25 & 28.80 & 37.83 & 35.94 \\
\hline 28.51 & 37.42 & 35.49 & 28.83 & 37.88 & 35.94 \\
\hline 28.57 & 37.68 & 35.39 & 29.48 & 37.95 & 35.96 \\
\hline 28.71 & 37.84 & 35.48 & 29.03 & 37.77 & 36.03 \\
\hline 28.48 & 37.59 & 35.38 & 29.04 & 37.92 & 35.78 \\
\hline 28.65 & 37.73 & 35.57 & 29.06 & 38.05 & 36.11 \\
\hline 28.65 & 37.50 & 35.44 & 28.97 & 37.46 & 36.11 \\
\hline 28.84 & 37.91 & 35.72 & 29.25 & 37.95 & 36.01 \\
\hline 28.43 & 37.46 & 35.68 & 28.85 & 37.84 & 36.14 \\
\hline 28.45 & 37.47 & 35.44 & 29.15 & 37.66 & 35.97 \\
\hline 28.81 & 37.86 & 35.38 & 29.18 & 38.17 & 36.09 \\
\hline 28.60 & 37.22 & 35.25 & 28.95 & 37.29 & 35.92 \\
\hline 28.51 & 37.51 & 35.23 & 28.98 & 37.70 & 35.94 \\
\hline 28.60 & 37.79 & 35.78 & 29.07 & 37.81 & 36.14 \\
\hline 28.60 & 37.91 & 35.62 & 29.19 & 37.92 & 36.21 \\
\hline 28.56 & 37.69 & 35.27 & 28.85 & 37.85 & 36.14 \\
\hline 28.51 & 37.50 & 35.51 & 28.89 & 37.69 & 35.84 \\
\hline 28.77 & 37.77 & 35.41 & 29.10 & 38.17 & 35.98 \\
\hline 28.70 & 37.47 & 35.54 & 28.96 & 37.40 & 36.03 \\
\hline 28.69 & 37.50 & 35.48 & 28.85 & 37.82 & 35.80 \\
\hline 28.50 & 37.95 & 35.31 & 28.67 & 37.71 & 35.99 \\
\hline 28.43 & 37.58 & 35.59 & 29.26 & 37.48 & 35.85 \\
\hline 28.53 & 37.41 & 35.27 & 28.98 & 37.81 & 35.90 \\
\hline 28.27 & 37.79 & 35.49 & 29.11 & 37.87 & 35.80 \\
\hline 28.58 & 37.57 & 35.78 & 28.79 & 37.95 & 35.91 \\
\hline 28.57 & 37.60 & 35.33 & 28.73 & 37.45 & 35.79 \\
\hline 28.49 & 37.80 & 35.67 & 29.04 & 37.82 & 35.78 \\
\hline 28.40 & 38.03 & 35.26 & 28.81 & 38.04 & 35.89 \\
\hline 28.57 & 37.66 & 35.51 & 28.98 & 37.79 & 36.02 \\
\hline
\end{tabular}




\section{Vita}

Mardi Russell was born and raised in Decatur, Alabama. She is the daughter of Kay and Fuller Russell. In May of 1999, she graduated Summa Cum Laude with a Bachelor of Science in Industrial Engineering from Mississippi State University. She began graduate school at Virginia Tech in the Fall of 1999 in the Industrial and Systems Engineering Department with an emphasis in Operations Research. While at Virginia Tech, she received a Material Handling Education Foundation scholarship from FKI Logistex, and won second place in the 2000 team Material Handling Design Competition sponsored by the College-Industry Council on Material Handling Education. She also served as treasurer of the Virginia Tech

Student Chapter of INFORMS. She received a Master's degree from the Virginia Tech Grado Department of Industrial and Systems Engineering in May, 2001. 